Pathologe 2014 · [Suppl 1] · 35:6-164

DOI 10.1007/s00292-014-1944-9

(c) Springer-Verlag Berlin Heidelberg 2014

\title{
98. Jahrestagung der Deutschen Gesellschaft für Pathologie e.V.
}

\author{
Berlin, 12.-15. Juni 2014
}

\section{Molecular pathology and its \\ diagnostic use in bone tumors}

\author{
SA-015 \\ Molecular pathology and its diagnostic use in bone tumors \\ J. Bovée $e^{*}$ \\ LUMC, Pathology, Leiden, Netherlands
}

\begin{abstract}
Most pathologists consider bone tumors difficult diagnostically as they are rare, have overlapping morphology, and need radiological correlation. Moreover, the usefulness of immunohistochemistry is limited, emphasizing the need for better molecular tools to assist in the diagnosis. With the advance of next generation sequencing, also for bone tumours more and more has become known on their molecular background. Three groups of bone tumors are recognized including tumors with specific translocations combined with a relatively simple karyotype involving chromosomal translocations (Ewing sarcoma, aneurysmal bone cyst), tumors with specific gene mutations or amplifications (chondrosarcoma, fibrous dysplasia, chordoma) and sarcomas with genetic instability and as a consequence complex karyotypes (osteosarcoma). Next generation sequencing is expected to reveal more novel alterations in the rare sarcoma subtypes of which the molecular background is so far unknown. Specific genetic aberrations in bone tumors can be applied in molecular diagnostics.
\end{abstract}

\section{The new WHO classification of lung tumors}

\section{SO-056 \\ The 2015 WHO classification of lung tumors}

\section{W.D. Travis*}

Memorial Sloan-Kettering Cancer Center, Department of Cancer Biology \& Genetics, New York, United States

Largely driven by the molecular revolution and therapeutic breakthroughs, the pathologic diagnosis of lung cancer has been transformed over the past decade. The revision of the 2004 WHO Classification of Lung Tumours is being prepared and should be published in 2015. In 2013, the WHO commissioned a panel of editors and an international multidisciplinary panel of authors to develop this classification. Over the past year a series of meetings by an international panel of multidisciplinary experts has been convened by the IASLC and the WHO to develop this classification. The many advances in the clinical, radiological, histologic and genetic aspects of lung cancer over the past decade were reviewed and provide the basis for this revision.
The new classification is summarized in $\bullet$ Tab. 1. A major change is the introduction of immunohistochemistry and genetic testing (EGFR mutation and ALK rearrangement) for many tumors to guide clinicians making personalized therapeutic decisions. These changes impact not only the evaluation of tumors obtained by non-resection specimens in patients with advanced lung cancer, but also tumors obtained by resection specimens. For adenocarcinoma, the IASLC/ATS/ERS lung adenocarcinoma classification including the approach to classification in non-resection specimens was adopted. Unlike previous WHO classifications, the order of the tumors starts with the most common tumor (i.e. invasive adenocarcinoma) and ends with the preinvasive lesion (i.e, atypical adenomatous hyperplasia). For squamous cell carcinoma, the former "Clear cell, Small cell and Papillary" subtypes were dropped and replaced by the keratinizing and nonkeratinizing subtypes with preservation of the basaloid subtype. Neuroendocrine tumors are now put into one group. Large cell carcinoma subtypes from the $2004 \mathrm{WHO}$ classification are discontinued and now this category only includes undifferentiated tumors. The former large cell carcinomas that are pneumocyte marker or mucin positive are moved to adenocarcinoma and the squamous marker positive cases are reclassified as nonkeratinizing squamous cell carcinoma. These changes are supported by a combination of clinical-pathologic studies as well as multiple genetic data including recent comprehensive analyses of lung cancer genome alterations. The landscape of lung cancer pathology and classification has been dramatically altered over the past decade and is now driven by the principle of personalized medicine and genetics.

\section{Omnis cellula e cellula revisited}

\section{FR-K059}

Omnis cellula e cellula revisited: cell biology as the foundation of pathology

\section{N. Wright*}

Centre for Tumour Biology, Barts Cancer Institute, Barts and the London School of Medicine and Dentistry, Queen Mary, University of London, London, United Kingdom

I shall argue strongly that cell biology, in its many disciplines, underpins the foundation of our understanding of the mechanisms of disease-the holy grail of pathology. Our increasing knowledge of the human genome will not be enough to attain this goal without parallel developments in our comprehension of the results, at the cellular level, of these genetic changes. In the end, it is cell biology and cell biologists who will deliver this mission and pathologists who will have to interpret it for the future of our subject. Pari passu, I shall attempt to re-assess the views of Virchow and Remak, together with those of François-Vincent 


\begin{tabular}{|c|c|}
\hline & WHO classification \\
\hline $1-1$ & Classification approach to non-resection specimens \\
\hline $1-2$ & Adenocarcinoma \\
\hline $1-2 \mathrm{~A}$ & Invasive adenocarcinoma \\
\hline $1-2 B$ & Variants of invasive adenocarcinoma \\
\hline $1-2 \mathrm{C}$ & Minimally invasive adenocarcinoma \\
\hline $1-2 \mathrm{D}$ & Preinvasive lesions \\
\hline $1-2 D-i$ & Atypical adenomatous hyperplasia \\
\hline 1-2D-ii & Adenocarcinoma in situ \\
\hline $1-3$ & Squamous cell carcinoma \\
\hline $1-3 \mathrm{~A}$ & Keratinizing and nonkeratinizing squamous cell carcinoma \\
\hline $1-3 B$ & Basaloid carcinoma \\
\hline $1-3 C$ & Preinvasive lesion: Squamous carcinoma in situ \\
\hline $1-4$ & Neuroendocrine Tumours \\
\hline $1-4 \mathrm{~A}$ & Small cell carcinoma \\
\hline $1-4 \mathrm{~B}$ & Large cell neuroendocrine carcinoma \\
\hline $1-4 C$ & Carcinoid tumors \\
\hline $1-4 \mathrm{D}$ & $\begin{array}{l}\text { Preinvasive lesion: Diffuse idiopathic pulmonary neuro- } \\
\text { endocrine cell hyperplasia }\end{array}$ \\
\hline $1-5$ & Large cell carcinoma \\
\hline $1-6$ & Adenosquamous carcinoma \\
\hline $1-7$ & Sarcomatoid carcinoma \\
\hline $1-7 \mathrm{~A}$ & Pleomorphic, spindle cell and giant cell carcinoma \\
\hline $1-7 \mathrm{~B}$ & Carcinosarcoma \\
\hline $1-7 C$ & Pulmonary blastoma \\
\hline $1-8$ & Other carcinomas \\
\hline $1-8 \mathrm{~A}$ & Lymphoepithelioma-like carcinoma \\
\hline $1-8 \mathrm{~B}$ & NUT-carcinoma \\
\hline $1-9$ & Salivary gland tumours \\
\hline $1-9 \mathrm{~A}$ & Mucoepidermoid carcinoma \\
\hline $1-9 B$ & Adenoid cystic carcinoma \\
\hline $1-9 \mathrm{C}$ & Epithelial-myoepithelial carcinoma \\
\hline $1-9 D$ & Pleomorphic adenoma \\
\hline
\end{tabular}

Raspail in this context, who were in reality the first proponents of this fundamental truth.

\section{Japanese-German Symposium on Main Topics I}

\section{FR-K051}

\section{Inflammatory liver tumorigenesis}

\section{P. Schirmacher* \\ Institute of Pathology, University Hospital Heidelberg, Heidelberg, Germany}

Chronic inflammation is a well recognized cause of tumorigenesis and documented for many organ systems. Despite its well documented relevance the mechanisms of inflammatory carcinogenesis are less well defined, are overlapping, and can be largely attributed to three different types of damage.

- Specific oncogenic mechanisms attributable to the etiological agent (infectious, toxic)

- Oncogenic effects exerted by host molecules released or induced due to the inflammatory process, e.g. inflammatory cytokines (e.g. TNF, IL-6)
- Non-specific protumorigenic effects related to continuous cell death and regeneration induced by the necro-inflammatory process Liver is the paradigm of inflammation induced carcinogenesis, with well over $80 \%$ of its carcinomas related to a defined chronic inflammatory process and all three above mentioned mechanisms are active. The target cells of inflammation associated tumorigenesis are liver epithelial cells, i.e. hepatocytes and cholangiocytes, and the respective tumors reflect the affected cell population. Diseases affecting liver parenchymal cells (e.g. chronic hepatitis B/C, alcoholic/non-alcoholic steatohepatitis) predispose to HCC, while chronic inflammation of the biliary tract (e.g. chronic fluke infestation, PSC) has been linked to development of cholangiocarcinoma. Molecular analyses have shown on one hand the specific contribution of the respective inflammatory process and corresponding/complementary host molecular changes, demonstrating that the inflammatory process leaves its specific imprint.

Carcinogenic potential of chronic liver inflammation allows for the definition of (high) risk groups for cancer development, surveillance strategies and secondary preventive measures. There is increasing evidence that sufficient treatment of the underlying chronic viral infection even at the cirrhotic stage or in established HCC improves course of the disease. Long standing chronic inflammation preceding carcinoma development implies the existence of a gradual tumorigenic process including premalignant lesions that have been defined for hepatocytes (Dysplastic Nodules/Foci) and biliary epithelium (BilIN, IPN-B).

Future research concentrates on the definition and relevance of inflammation-induced molecular mechanisms, their diagnosis and specific modes of interaction and resulting preventive and therapeutic strategies.

\section{FR-K052}

\section{Epstein-Barr virus and gastric cancer}

\section{Fukayama* \\ The University of Tokyo, Tokyo, Japan}

Epstein-Barr virus (EBV)-associated gastric cancer (GC), comprising $10 \%$ of total GC, consists of monoclonal growth of EBV-infected epithelial cells. The molecular pathway of cancer development is also characteristic to EBV-associated GC, such as epigenetic and microRNA abnormalities.

Promoter methylation was analyzed using Illumina's Infinium bead array, demonstrating that gastric cancers were classified into three subgroups: the low- and high-methylation epigenotypes in EBV-negative cases, and the markedly higher methylation epigenotype which was completely matched to EBV-associated GC. EBV-methylation epigenotypes were characterized by the simultaneous methylation of three groups of genes, such as EBV-markers, High-markers, and Commonmarkers. When $\mathrm{MKN} 7$, a GC cell line of low methylation epigenotype, was infected with EBV, the infection induced extensive DNA methylation in both High- and EBV-markers. Furthermore, time-course analysis demonstrated that viral DNA-methylation and its completion preceded those of host DNA. CpG methylation may be a part of the cellular defense mechanism against DNA-virus, and it is overdriven to involve the host genome in EBV-associated GC.

We next focused on CpG-island methylation of PTEN promoter and subsequent loss of expression. Both of methylation and expression loss were reproduced in EBV-infected $\mathrm{MKN} 1$ and $\mathrm{MKN}$ 7. EBV-latent protein, $\mathrm{LMP}_{2} \mathrm{~A}$, induced phosphorylation of $\mathrm{STAT}_{3}$ and thereby upregulated DNA methyltransferase 1 (DNMT1), which resulted in promoter methylation of PTEN. LMP2A-induced PSTAT3 appeared to be independent of gpizo/IL6 pathway.

MicroRNA (miRNA) abnormalities are also involved in EBV-associated GC and its tumor microenvironment. We evaluated the expression of epithelial-to-mesenchymal transition (EMT)-related microRNAs, miR-20oa and miR-20ob. Expression of MiR-20o family was decreased in EBV-associated GC. Downregulation of miR-20o family was found 
in GC cell lines infected with recombinant EBV, which was accompanied by the loss of cell adhesion, reduction of E-cadherin expression, and upregulation of ZEB1 and ZEB2. E-cadherin expression was partially restored by transfection of EBV-infected cells with miR-20o family precursors.

Two abnormalities, epigenetics and microRNA, are based on the strategy of EBV for its survival, and the study of them will clarify the defense mechanisms of stomach cells.

\section{Japanese-German Symposium on Main Topics II}

\section{FR-K053 \\ Activation of Akt-mTOR pathway and its therapeutic implication in spindle cell soft tissue sarcomas \\ Y. Oda* \\ Department of Anatomic Pathology, Kyushu University, Fukuoka, Japan}

Background. The systemic chemotherapy and radiotherapy are effective for round cell sarcomas such as rhabdomyosarcoma and Ewing sarcoma. However, no effective systemic therapy is currently available for unresectable or metastatic spindle cell soft tissue sarcomas. The Akt/ mTOR pathway activation causes protein synthesis, which induces cellular proliferation, survival, motility, invasion, and differentiation and can ultimately lead to tumor initiation and progression. Some preclinical studies have lent credence to targeting this pathway in certain kinds of soft tissue sarcomas.

Materials and methods. The phosphorylation statuses of Akt-mTOR and its related pathway proteins were assessed by immunohistochemistry and western blotting in primary spindle cell sarcomas including 129 leiomyosarcomas (LMSs), 91 malignant peripheral nerve sheath tumors (MPNSTs), 103 synovial sarcomas (SSs), 66 solitary fibrous tumors (SFTs), 68 myxofibrosarcomas (MFSs) and 81 dermatofibrosarcoma protuberans [DFSPs; 52 DFSP, 25 DFSP with fibrosarcoma (FS), 4 superficial FS with COL1A1-PDGFB fusion gene]. The obtained data were compared with clinicopathological and histopathological findings.

Results. The Akt/mTOR pathway was activated in most cases of LMS, MPNST and SS. Activation of this pathway was associated with adverse prognosis or aggressive pathological findings in LMS, MPNST, SS and MFS. No mutations of $\mathrm{PIK}_{3} \mathrm{CA}$ or $\mathrm{AKT}_{1}$ were detected in LMS, SS and MFS. In MPNST its activated status showed no difference between NF1-related and sporadic tumors. mTOR inhibition by Everolimus showed antitumor activity on four MPNST cell lines in vitro. In SFT this pathway was activated in approximately $50 \%$ of the cases and was associated with phosphorylated PDGFR-beta and IFG-1R-beta, which were phosphorylated at different rates. In MFS theMAPK pathway as an alternative activator of the Akt/mTOR pathway. In DFSP Akt and mTOR were more frequently activated in FS component, compared with ordinary DFSP component. There was significant correlation between PFGFR-beta and p-Akt in ordinary DFSP component, however, no such relationship was observed in FS component.

Conclusion and discussion. The Akt/mTOR pathway was activated and was associated with worse clinical behavior and pathologic findings in the examined spindle cell soft tissue sarcomas. In these sarcomas this pathway may be involved in the tumorigenesis and may have potential as a therapeutic target.

\section{Japanese-German Symposium on Main Topics III}

\section{FR-K054}

Stem cells in chronic gastritis and gastric cancer

\section{Röcken*}

Institut für Pathologie, Universitätsklinikum Schleswig-Holstein, ChristianAlbrechts-Universität, Kiel, Germany

Gastric cancer is usually diagnosed in an advanced age with a dismal prognosis. Treatment options are limited in locally advanced and metastatic disease, and a significant number of tumours recur despite initial therapeutic response. A putative explanation of an ineffective therapy is the presence of cancer stem cells (CSC). The CSC hypothesis postulates that a tumour is a conglomerate of heterogeneous cell populations. Only a subpopulation of this conglomerate maintains the capability of colony formation, and hence recurrence and metastatic spread. CSCs are more resistant to chemotherapy, leading to tumour recurrence, progression and ultimately patient death. Chronic gastritis, e.g. due to infection with Helicobacter pylori, leads to metaplasia, which is associated with cancer and is considered a permanent alteration of the progenitor and stem cell pool of the gastric mucosa. Thus, stem cells are already involved in cancer development. While the CSC-model is increasingly accepted, identification and confirmation of so called stem cell markers and stem cell niches in native human tissue is difficult and largely missing. Recently, the leucine-rich, G-protein coupled receptor and Wnt target gene LGR5 was identified as a novel stem cell marker of the small intestine, colon as well as in the stomach. In addition, $\mathrm{ADAM}_{17}, \mathrm{CD}_{44}$, Musashi-1 and Troy were reported as putative markers of SC or CSC of the stomach. Their functional role in non-neoplastic gastric mucosa and gastric cancer is slowly unravelled and evidence is increasing that the stomach may harbor two distinct stem cell pools.

\section{Neue Entwicklungen bei den Weichgewebstumoren}

\section{FR-K060}

The new WHO classification and further developments in soft tissue tumor pathology

\section{Petersen*}

Institute of Pathology, Jena University Hospital, Jena, Germany

The new World Health Organization (WHO) classification presents a comprehensive description of soft tissue tumors which was published in book format at the beginning of 2013. Changes have been made relating to the allocation of known entities, e.g. undifferentiated sarcomas were included into a new group and are not longer assigned to the so-called fibrohistiocytic tumors. In addition, "new" subgroups were incorporated such as nerve sheath tumors and gastrointestinal stroma tumors which were previously included in the tumor classification of other organ systems. This development is important from the practical point of view as the most relevant soft tissue tumors are now represented and can be found in a single book. There is a rapid increase in the knowledge of the genetics and cell biology of soft tissue tumors and important new findings have been published after completion of the classification. Particularly relevant is the fact that many low grade sarcoma entities can meanwhile be defined by single driver mutations, one recent example is the identification of the recurrent NAB2-STAT6 gene fusion in solitary fibrous tumors. Similarly important is the characterization of the role of specific tumor suppressor genes and their signalling pathways in tumorigenesis, e.g. pRB in adipocytic differentiation and lineage commitement, p16 in peripheral nerve sheath tumor progression, $\mathrm{p} 53$ in chromosomal instable high grade sarcomas or the 
SWI/SNF nucleosome complex in "epigenetically instable" sarcoma. The detection of frequent mutations in the promoter of the hTERT gene in malignant melanoma has generated new insight into the mechanisms of cancer immortality. Generally, the phenotypic and genetic diversity of soft tissue tumors constitute a paradigm for the understanding of cancer biology. Likewise, the combination of a sophisticated morphological analysis with a targeted molecular analysis may set the example for future tumor classification in the era of personalized cancer therapy.

\section{FR-K061}

\section{Value of core needle biopsy in the preoperative diagnosis of soft} tissue sarcomas: possibilities and limitations

\section{A. Agaimy*}

Institute of Pathology, Friedrich-Alexander-University, Erlangen-Nuremberg, Germany

Superficial and deep-seated soft tissue tumors vary greatly in their histogenetic derivation/line of differentiation, biological potentials and hence their treatment modalities. Precise early histological diagnosis and appropriate treatment of soft tissue sarcomas are essential to improve patient's outcome, as radical surgery with wide clear resection margins (Ro resection) is a key prognostic factor for patient survival. Since histopathological verification of diagnosis is essential for optimal treatment planning, preoperative core needle biopsy (PCNB) has emerged as a widely used alternative to the old practice of open surgical biopsy and can be performed by blind or image-guided techniques. While blind PCNB is safe, rapid and cost-effective for peripherally located soft tissue masses, image-guided techniques are more expensive but offer a real-time imaging of the tumor and adjacent structures and they are suitable for deep-seated masses because of the great hazard of injury to adjacent vital structures. There is an increasing evidence that PCNB as a diagnostic modality is superior, quick and save for definitive diagnosis and allow for planning best treatment strategies (primary surgery versus preoperative radiochemotherapy), particularly with regard to the possibility of preoperative therapy for downstaging larger and/or initially inoperable tumors. Tissue material gained by PCNB is generally sufficient for a quick diagnosis and grading of sarcomas. For $\mathrm{PCNB}$ to fulfil these expectations and to be of real diagnostic impact for patients and to minimize waiting time prior to initiation of definitive oncologic-surgical treatment, a well-thought of diagnostic approach incorporating clinicopathological, imaging, immunohistochemical and molecular data/tools and based on sufficient experience and familiarity with the ever increasing entities and subentities of soft tissue neoplasms is mandatory. Recent data illustrate that in the experienced hand and in the context of sufficient clinical and imaging data, preoperative diagnoses on $\mathrm{CNB}$ and final diagnoses on resection specimens are highly concordant. Discordant diagnoses are uncommon when biopsy interpretation is done by pathologists with sufficient experience in soft tissue pathology. However, in a few cases, tumor grading on CNB might be challenged by a great intratumoral heterogeneity leading to discordant results (low versus high-grade) that might have resulted in changing in treatment strategies.

\section{Hier steht eine Anzeige.}

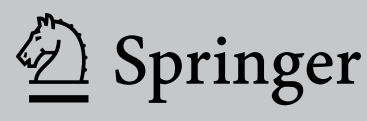




\section{FR-K062}

\section{Tyrosine kinases in soft tissue and bone tumors}

\section{T. Knöse $\left.\right|^{* 1,2}$}

'Institute of Pathology of the University of Munich, Munich, Germany, ${ }^{2}$ Institute of Pathology, Munich, Germany

Aims. Tyrosine kinases are promising targets for individual patients' therapy and new drugs are in phase 2 and phase 3 clinical trials. However the expression in different tumors is not evaluated before therapy as predictive biomarker and only limited studies investigated the expression on protein level. In this study we analyzed a well characterized cohort of soft tissue and bone tumors for different kinases and correlate the results with clinicopathological parameters including survival. Methods and results. 170 soft tissue tumors and bone tumors of the Ludwig-Maximilians-University (LMU) were revisited and catagorized in the actual WHO classification system. High expression of VEGFR1 was detected immunohistochemically in $57 \%$, VEGFR2 (KDR) in $35 \%$, $\mathrm{VEGFR}_{3}$ in $57 \%$, PDGFRA in $62 \%$ and PDGFRB in $59 \%$. High expression of VEGFR1-3 was significantly correlated with higher grading (G2 vs $\mathrm{G}_{3}, \mathrm{p}=0.020, \mathrm{p}=0.004, \mathrm{p}=0.021$, respectively) and high VEGFR2 was significantly correlated with shorter patients survival $(\mathrm{p}<0.001)$. The tumor collective comprises undifferentiated pleomorphic sarcomas $(n=52)$, leiomyosarcomas $(n=30)$, synovial sarcomas $(n=16)$, liposarcomas $(n=21)$, angiosarcomas $(n=25)$ and other soft tissue sarcomas $(\mathrm{n}=26)$.

Conclusions. Tyrosine kinases are differentially expressed in soft tissue and bone sarcomas, and protein expression of tyrosine kinases should be evaluated before targeted therapy as a predictive marker. High expression of VEGFR1-3 is significantly correlated with higher grading and VEGFR2 (KDR) is correlated significantly with shorter patients' survival and might be a prognostic marker.

\section{Neue Entwicklungen bei den Knochentumoren}

\section{SA-016}

\section{Current surgical treatment options in bone tumors}

\section{Andreou*}

Department of General Orthopedics and Tumororthopedics, Muenster University Hospital, Muenster, Germany

Primary bone neoplasms can be classified into benign, locally aggressive/rarely metastasizing and malignant tumors. Patients with benign tumors usually undergo surgical treatment in case of local symptoms, mainly consisting in pain or functional deficits due to compression of important anatomical structures such as nerves or vessels. Locally aggressive/rarely metastasizing tumors exhibit an infiltrative growth pattern, so that surgical treatment is necessary to prevent further destruction of bone leading to local instability. Finally, the surgical treatment of malignant tumors is - with few exceptions - considered to be a prerequisite for long-term survival, either alone or in combination with systemic chemotherapy.

Whereas the main objective of the surgeon in the treatment of benign tumors is the relief of local symptoms with a minimum amount of damage to healthy tissue, and minimizing the risk of local recurrence while ensuring bone stability in locally aggressive/rarely metastasizing tumors, the primary goal in the operative treatment of bone sarcomas is the resection of the tumor with clear surgical margins, followed by the reconstruction of a functional limb. This review examines the current developments in the surgical treatment of primary bone neoplasms, in regard to both the management of the tumors and novel reconstructive options.
SA-017

New developments in the molecular characterization of osteogenic tumors

\section{Baumhoer*}

Institute of Pathology, University Clinic Basel, Basel, Switzerland

Osteogenic tumors comprise a heterogeneous group of benign and malignant lesions of bone. Whereas in benign tumors molecular data is still scarce, osteosarcoma and its distinct subtypes have increasingly been analyzed on a genetic level. Mdm2 amplification for example is a typical feature of low-grade osteosarcoma and can help in the differential diagnosis from benign mimickers, including myositis ossificans. High-grade osteosarcomas on the other hand show complex and diverse structural and numerical chromosomal alterations that at least in a subgroup of tumors seem to be caused by chromothripsis. Nonetheless, there are also recurrent aberrations including amplification of MYC or mutations of $\mathrm{RB}_{1}$ and $\mathrm{TP}_{53}$. New insights into the mechanisms of tumor suppressor gene inactivation as well as microRNA cluster activation in osteosarcoma will be presented and discussed in the context of tumor development and progression.

\section{SA-018}

\section{New aspects in chordoma}

\section{S. Scheil-Bertram}

Institute of Pathology and Cytology, Wiesbaden, Germany,

Introduction. Chordoma is a rare, low-malignant bone tumor. This unique bone tumor has both epithelial and mesenchymal characteristics. Chordomas arise along the spine with hot spots at the upper (skull base 20-30\%) and lower (sacro-coccygeal 50-60\%) end, and are therefore thought to originate from remnants of the notochord. To date, no targeted therapeutic strategies have been established for chordomas. However, a phase II study showed a modest antitumor activity of lapatinib in chordoma. Chordoma characteristically occurs in adolescence and is rarely found in children. Conventional and molecular cytogenetic analyses revealed chromosomal gains of $7 q$ and losses of $1 p$ and $3 p$ to be the most prominent alterations in chordoma. Loss of heterozygosity ( $\mathrm{LOH})$ and genome-wide linkage studies have already been successfully used to define candidate regions for chordoma development on 1p36.13 and 7933. Some studies focused on gene expression analysis in chordoma. Brachyury $(\mathrm{T})$ was one of these candidate genes, which was knocked down in the chordoma cell line $\mathrm{U}-\mathrm{CH}$.

Aims. The molecular basis of chordoma is still poorly understood, particularly with respect to differentially expressed genes involved in the primary origin of chordoma.

Methods. A study based on transcriptional expression profile in chordoma using a high-density oligonucleotide array was performed.

Results. The mRNA level of 65 genes differ significantly ( $p<0.001 ; \geq 6$ fold change). Genes with increased expression in chordoma compared to control as well as chondrosarcoma were most frequently located on chromosomes 2 (11\%), 5 (8\%), 1 and 7 (each 6\%), whereas interphase cytogenetics of 33 chordomas demonstrated gains of chromosomal material most prevalent on $7 \mathrm{q}(42 \%), 12 \mathrm{q}(21 \%), 17 \mathrm{q}(21 \%), 20 \mathrm{q}(27 \%)$, and $22 \mathrm{q}(21 \%)$. The microarray data were confirmed for selected genes by real-time reverse transcription polymerase chain reaction analysis. As in other studies, the expression of brachyury was confirmed.

Conclusions. The expression of new potential candidates for chordoma tumorigenesis, such as CD24, ECRG4, RARRES2, IGFBP2, RAP1, $\mathrm{HAI}_{2}, \mathrm{RAB}_{3} 8$, osteopontin, GalNAc-T3, VAMP8, and others could be identified. Thus, the role of this set of interesting candidate genes in chordoma needs further studies. 


\section{SA-K019}

Pathological and clinical aspects of giant cell tumour of bone including differential diagnostic problems

\section{Szendröi*1, Z. Sápi², I. Antal'}

'Department of Orthopaedics, Semmelweis University, Budapest, Hungary, ${ }^{2}$ Institute of Pathology and Expert Cancer Research, Semmelweis University, Budapest, Hungary

Purpose. Overview about the pathological appearance and clinical aspects of the giant cell tumour of bone (GCT) based on the literary data and on the 303 own cases out of the 6503 bone tumours.

Introduction. The giant cell tumour of the bone is a locally aggressive bone tumour of intermediary character which have a benign course in $90 \%$ of the cases but $4-5 \%$ show malignancy from its onset or undergo malignant transformation through their recurrences and $1-4 \%$ give pulmonary metastases.

Pathology. GCT is consisted of three different cell types: osteoclast-like giant cells, macrophage-like osteoclast precursors and spindel-shaped mesenchymal stromal cells which osteoblast-precursor character. The former cell-lines are reactive and non-neoplastic, the latter cell line is genetically unstable and responsible for the tumorous behaviour of the GCT.

Imaging. GCT appears as pure lytic lesion in the epi-metaphyseal part of the tubular bones. There is no periosteal reaction, the bone is blown up in its active stage but can invade the surrounding tissue in the late aggressive stage. CT is useful for the assessment of the cortical involvement, MRI reveal usually low to intermediate signal intensity on $\mathrm{T} 1$ weighted images and high heterogeneous (necrosis!) signal intensity on T2-weighted images.

Differential diagnostics. Other pure lytic tumours in this location i.g. chondroblastoma, aneurysmal bone cyst, etc. and lytic bone metastases should also be excluded. Histologically all tumours and tumourlikeconditions which contains abundant number of osteoclast-like giant cell may have similar appearance.

Prognostic factors. The histological picture, age, sex, surgical stage are not real prognostic factors of the GCT. Factors which may influence the rate of local recurrences: massive soft tissue component, location of GCT in the distal part of the radius, type of surgery (resection versus curettage), use of adjuvants (phenol, bone cement, cryosurgery), number of mitotic figures, frequency of chromosome abnormalities, number of recurrences in the patient's history.

Conclusion. We should try to keep the joint as long as possible for two reasons: sacrificing the joint means inferior quality of life for the long run and at least $98 \%$ of the cases can be cured by conservative surgical procedures with additional adjuvant therapy. New targeted therapies as bisposphonates and denosumab may further improve the treatment results in GCT.

\section{Nichtonkologische orthopädische Pathologie}

\section{SA-020}

\section{Adverse reactions to wear from total joint arthroplasty}

\author{
C. Lohmann ${ }^{* 1}$, H. Meyer', G. Singh', G. Buchhorn², G. Pflüger ${ }^{3}$ \\ 'Department of Orthopaedics, Magdeburg, Germany, ${ }^{2}$ Department of \\ Orthopaedics, Göttingen, Germany, ${ }^{3}$ EKH Vienna, Vienna, Austria
}

Various wear products from the articulating surfaces or the trunnions of total joint arthroplasties (THA) may induce a periprosthetic tissue response that leads to aseptic loosening. Polyethylene wear particles are stored on macrophages, activate osteoclasts and lead to osteolysis. The era of Metal-on-Metal (MoM) total hip arthroplasty (THA) has left the orthopaedic community with valuable insights and lessons on periprosthetic tissue reactions to metallic debris. Various terms have been used to describe the tissue reactions. Sometimes the nomenclature can be confusing. We present a review of the concepts introduced by Willert and Semlitsch in 1977, along with further developments made in the understanding of periprosthetic tissue reactions to metallic debris. We propose that periprosthetic tissue reactions be thought of as (i) gross (metallosis, necrosis, cyst formation and pseudotumour), (ii) histological (macrophage-dominated, lymphocyte-dominated or mixed) and (iii) molecular (expression of inflammatory mediators and cytokines such as Il- 6 and TNF-alpha. Taper corrosion and modularity are discussed, along with future research directions to elucidate the antigenpresenting pathways and material-specific biomarkers which may allow early detection and intervention in a patient with adverse periprosthetic tissue reactions to wear debris.

\section{SA-K024}

\section{Revised histopathological consensus classification of joint implant-} related pathology

\section{Krenn*1, L. Morawietz ${ }^{2}$}

'Pathologie, Trier, Germany, ${ }^{2}$ Institute of Pathology Potsdam, Potsdam, Germany

The extended classification of joint implant related pathology is an applicatory histopathologic classification based on defined morphological criteria covering the complete spectrum of pathohistologic changes in periprosthetic tissues. To be included here is the established consensus classification of the periprosthetic membrane, with which aseptic and septic prosthesis loosening can be broken down into four histological types (type I to type IV). Further essential pathologies include: endoprosthetic-associated arthrofibrosis (EaaF), adverse reactions (particle-induced immunological and inflammatory reactions and toxic mechanisms) as well as the spectrum of bone tissue pathologies: osteomyelitis, inflammatory particle induced bone alterations and metabolic disorders. These characteristic tissue alterations and their relationships are summarized in a chart as the extended classification. Since particle heterogeneity in periprosthetic tissue is high and particle identification is a necessary part of diagnosis the histopathological particle algorithm is proposed which enables the identification of different types of particles based on 1) Conventional transmitted light microscopy with a guide to sizing, shaping and coloring 2) Polarization optical criteria and 3) enzyme-histochemical properties (oil red staining and Prussian blue reaction). These qualities and the relative importance for particle identification and its differential diagnosis to non prosthesis-materials are summarized in the so called histopathological particle algorithm. A "particle score" is recommended evaluating 1) the predominant prosthesis-material particle type in respect to micro-, and macroparticles, 2) the existence of non prosthesis-material particles and 3) the quantification of particle induced necrosis and inflammatory reactions. This classification, which is based on low cost standard tissue processing and on defined criteria, allows diagnosing implant associated pathologies and particle identification on a histopathologic level giving a stable and reproducible diagnostic tool for pathologist. Since this classification is suitable as a basis for standardized histopathological diagnostics it may also serve as a data standard for joint arthroplasty registers.

\section{SA-K025}

\section{Molecular diagnosis of infections in orthopedic pathology}

\section{J. Kriegsmann*}

MVZ für Histologie, Zytologie und molekulare Diagnostik Trier, Trier, Germany

Diagnosis of infections in orhopedic pathology includes granulomatous and non granulomatous inflammation of the joints, especially synovial membrane as well as infections of the periprosthetic membrane. Fast 
and precise diagnosis of infections is mandatory to prevent functional loss or the requirement for replacing the prosthesis. Methods of germ detection in microbiology are based on bacterial culture. Detection of low-grade infections in periprosthetic membrane requires culture for at least 2 weeks, often germ detection by bacterial culture is frustrane. Histology is mainly based on the amount of neutophils in periprosthetic tissue but do not give any information about the strain of the germ. Gram stain may be used to discriminate infections by gram-positive or gram-negative bacteria. Various molecular methods were developed to detect infectious agents as bacteria, fungi or viruses. Counting neutrophils may be time consuming and is not precise. Neutrophils contain granules with numerous enzymes. This fact is the basis for detection of these peptides by proteomic methods. These methods include matrixassisted laser desorption ionisation (MALDI-) time of flight(TOF)-profiling and imaging techniques. The latter allow the exact localization of the infection in various tissue compartments. If there is a high number of infections agents- discrimination of the germ may be possible. Additionally, various members of the S1oo family are elevated in infectious inflammatory conditions which could be detected by MALDI-TOF in the synovial membrane or fluid. The first step in the diagnosis of granulomatous inflammation is the exclusion of non-infectious conditions followed by molecular diagnosis of the infectious agents by PCR and sequencing procedures. Tuberculosis should always be ruled out or confirmed. A reliable method is PCR followed by in-situ hybridization to a chip to specify the mycobacterial subtype. If diagnosis was confirmed, resistance pattern can be evaluated by a similar method. Detection of fungal infections seen in PAS or Grocott-stains is performed by PCR and sequencing or by PCR and in-situ hybridization. In culture-negative joint infections, evaluation of $16 \mathrm{~s} \mathrm{rDNA}$ and $18 \mathrm{~s} \mathrm{rDNA}$ is recommended. To confirm and detect the specific germ PCR, sequencing and the search for blasts is recommended. Summarizing, molecular diagnostic methods allow a fast, reliable and precise diagnosis of joint and periprosthetic infections.

\section{European Session ESP/DGP New developments in the field of bone and soft tissue tumors}

\section{FR-K063}

\section{New classification of spindle/pleomorphic primary bone tumors}

\section{P. Picci ${ }^{* 1}$, P. Dei Tos ${ }^{2}$, D. Vanel ${ }^{3}$, M. Gambarotti ${ }^{3}$, A. Righi ${ }^{3}$, C. Ferrari ${ }^{3}$,} S. Benini ${ }^{3}$, S. Ferrari ${ }^{3}$

'Istituto Ortopedico Rizzoli, Laboratory of Experimental Oncology, Bologna, Italy, ${ }^{2}$ USSL ${ }^{9}$ Treviso, Treviso, Italy, ${ }^{3}$ stituto Ortopedico Rizzoli, Bologna, Italy

Due to a confirmed molecular diagnosis of a primary synovial sarcoma in the bone, we reviewed all cases treated at the Rizzoli Institute for a primary spindle/pleomorphic sarcoma of bone.

In 326 cases, with all pertinent histological, radiological and clinic information, TMAs were performed to re-classify the lesions. Undifferentiated Pleomorphic Sarcoma (UPS) and Fibrosarcoma that were the entities more frequently diagnosed in the past, dramatically decreased in number, whilst leiomyosarcoma greatly increased. Other entities were defined as myoepithelioma, synovial sarcoma, myofibroblastic sarcoma, solitary fibrous tumor, mixofibrosarcoma.

These multiple entities show different clinical, epidemiological and prognostic aspects, not evident with the old classification.

\section{FR-K064}

\section{Targeted treatment in soft tissue sarcomas}

\section{E. Wardelmann*, J. Zustin, S. Huss, I. Grünewald, M. Trautmann} W. Hartmann

Gerhard-Domagk-Institute for Pathology, University Hospital, Münster, Germany

Introduction. Because of the low incidence of soft tissue tumors (STS) the histopathologic diagnosis can be challenging for pathologists. Sarcomas account for approximately $1 \%$ of all malignancies. According to the new WHO classification from 2013 more than 80 different entities exist. The correct diagnosis is of major relevance for an appropriate therapy. Furthermore, molecular signatures may pinpoint potential therapeutic targets.

Genomic alterations and potential molecular targets in sarcomas. Two major genes often involved in sarcoma pathogenesis are the EWSR 1 or the FUS gene, both genes encoding RNA-binding proteins. The type of the DNA-binding domain originating from the fusion partners determines the tumor subtype. Sarcomas carrying EWSR 1 translocations are Ewing sarcomas/PNET, clear cell sarcomas, desmoplastic small round cell tumors, extraskeletal myxoid chondrosarcomas and rarely angiomatoid fibrous histiocytomas. The FUS gene is frequently involved in translocations of myxoid liposarcomas, angiomatoid fibrous histiocytomas and less often in Ewing sarcomas.The resulting fusion proteins act as aberrant transcription factors. The fusion product can also lead to the autocrine stimulation of an involved receptor tyrosine kinase, as for example in dermatofibrosarcoma protuberans where the growth factor PDGFB is placed under the control of the COL1A1 promoter. DFSP can be targeted very effectively with the tyrosine kinase inhibitor imatinib. A diagnostically specific gene fusion is NAB2-STAT6 in solitary fibrous tumors. For synovial sarcomas translocations involving the SYT gene are specific. Somatic mutations occur in gastrointestinal stromal tumors and point to the therapeutical target KIT or PDGFRA. MDM2/ $\mathrm{CDK}_{4}$ amplifications may be the key towards treatment of dedifferentiated liposarcomas. Another interesting potential target upregulated in different sarcoma subtypes could be the IGF/Akt/mTOR pathway. Akt/ mTOR inhibitors are effective in-vitro and in mouse models and are now introduced in clinical trials. Another interesting target could be the group of histone deacetylases (HDAC) which have shown to be involved in gene silencing for example in synovial sarcomas and endometrial stromal sarcomas.

Conclusions. Due to increasing molecular data from expression profiling and other molecular techniques differential diagnosis and subgrouping of sarcomas is facilitated. Novel targeted treatment regimens are developed on the basis of these results.

\section{Ausgewählte Vorträge zu den Hauptthemen}

\section{FR-065}

Different protein signatures of tyrosine kinases in soft tissue and bone sarcomas-high VEGFR2 is associated with shorter patients' survival

\section{E. Kampmann', A. Altendorf-Hofmann'², R. Duerr' ${ }^{3}$, L. Lindner', R. Issels', T. Kirchner', T. Knösel ${ }^{* 4,5}$}

'Department of Oncology, Munich, Germany, ${ }^{2}$ Department of General, Visceral and Vascular Surgery, Jena, Germany, ${ }^{3}$ Institute of Orthopedic Tumor Surgery, München, Germany, ${ }^{4}$ Institute of Pathology of the University of Munich, Munich, Germany, ${ }^{5}$ Institute of Pathology, Munich, Germany

Aims. Tyrosine kinases are promising targets for individual patients' therapy and new drugs are in phase 2 and phase 3 clinical trials. However the expression in different tumors is not evaluated before therapy as a predictive biomarker and only limited studies investigated the ex- 
pression on protein level. In this study we analyzed a well characterized cohort of soft tissue and bone tumors for different kinases and correlate the results with clinicopathological parameters including survival.

Methods. 170 soft tissue tumors and bone tumors of the Ludwig-Maximilians-University (LMU) were revisited and catagorized in the actual WHO classification system.

Results. High expression of VEGFR1 was detected immunohistochemically in $57 \%$, VEGFR2 (KDR) in $35 \%$, VEGFR 3 in $57 \%$, PDGFRA in $62 \%$ and PDGFRB in $59 \%$. High expression of VEGFR $1-3$ was significantly correlated with higher grading $\left(\mathrm{G}_{2} \mathrm{vs} \mathrm{G}_{3}, \mathrm{p}=0.020, \mathrm{p}=0.004, \mathrm{p}=0.021\right.$, respectively) and high VEGFR2 was significantly correlated with shorter patients survival $(\mathrm{p}<0.001)$. The tumor collective comprises undifferentiated pleomorphic sarcomas $(n=52)$, leiomyosarcomas $(n=30)$, synovialsarcomas $(n=16)$, liposarcomas $(n=21)$, angiosarcomas $(n=25)$ and other soft tissue sarcomas $(n=26)$.

Conclusions. Tyrosine kinases are differentially expressed in soft tissue and bone sarcomas and protein expression of tyrosine kinases should be evaluated before targeted therapy as predictive markers. High expression of VEGFR1-3 is significantly correlated with higher grading and VEGFR2 (KDR) is correlated significantly with shorter patients survival and might be a prognostic marker.

\section{FR-066}

Chordomas along the spine vary in morphology independent from localisation-presentation of a tissue bank from 43 patients including metastases, recurrences and 10 chordoma cell lines

A. von Witzleben ${ }^{* 1}$, T. Goerttler', S. Brüderlein', A. von Baer', M. Schultheiss', M. Wittau' ${ }^{2}$, S. Scheil-Bertram³ , L. Bullinger', R. Mayer-Steinacker', J. Lennerz', A. Flanagan', P. Möller', T.F.E. Barth'

IInstitute of Pathology, Ulm, Germany, ${ }^{2}$ Department of Surgery, UIm, Germany, ${ }^{3}$ Dr. Horst-Schmidt-Kliniken (HSK), Institute of Pathology and Cytology, Wiesbaden, Germany, ${ }^{4}$ Department of Internal Medicine III, University of Ulm, Ulm, Germany, Institute of Pathology, University College London, London, United Kingdom

Aims. Morphological and clinical characterization of a chordoma tissue bank including 63 paraffin archived chordoma samples from 43 patients. Establishment of chordoma cell lines.

Methods. Computer-based identification of chordoma samples using a search software on the tissue archive in the Institute of Pathology, Ulm, from 1986-2013. Histology, immunohistochemistry and in vitro cell culture.

Results. The tissue bank contains 63 tissue samples of 43 patients including 13 cryopreserved tissue probes. Patient data: female:male ratio $=1: 1.5$; age distribution: 17 to 84 years (median $=69$ years); survival data $(n=25): 16$ alive, 9 dead/2 due to chordoma related death; overall survival (0,5-234 months, median 47,6 months). Pathology: Size ranged from 0.4 to $30 \mathrm{~cm}$. The topographic distribution along the spine was: Os sacrum $(n=24)$; lumbar vertebrae $(n=6)$, clivus $(n=5)$, the cervical vertebrae $(n=3)$, thoracic vertebrae and transition of cervical and thoracic vertebrae $(\mathrm{n}=2$ each), and an extra-axial chordoma of the nasal septum $(n=1)$. All chordoma were brachyury positive. Ki-67 index ranged from $<1 \%$ up to $50 \%$. According to the WHO we identified 5 histological subtypes: not otherwise specified (NOS; $n=17$ ), NOS and partial polymorphic $(n=11)$, renal cell carcinoma like $(n=6)$, chondroid and polymorphic (each $n=3$ ), pleomorphic $(n=1)$. In general, polymorphism in clivus chordomas was less pronounced than in sacrum chordomas. Clivus chordomas had neither metastases nor recurrences. $10(23 \%)$ patients had distant metastases to lung $(\mathrm{n}=5)$, lymph node $(\mathrm{n}=3)$, liver $(\mathrm{n}=$ 2), and bone $(n=1) .21(39.50 \%)$ patients had recurrences (time range: 1 to 6 years, average 2.3 years). Metastases and recurrences were dependent from localization, size, and R-status but independent from histological subtype. Subtypes remained stable in recurrences and metastases in most cases. We meanwhile have established 1o stable chordoma-cell lines of various subtypes and various localizations, one being the first clivus chordoma cell line ever. In vitro the pleomorphic subtype had a higher proliferation index at initial passages.

Conclusions. Chordomas are morphological heterogeneous tumours of adults with a slight male predominance, a low proliferation-index, a non-random distribution along the spine, and without topographic histological clustering; metastases and recurrences are a function of localization and size; establishment of chordoma cell lines seems not to be influenced by the morphological subtype.

\section{FR-067}

Integrative DNA methylation and gene expression analysis in highgrade soft tissue sarcomas

M. Renner ${ }^{* 1}$, T. Wolf ${ }^{2}$, H. Meyer ${ }^{3}$, W. Hartmann ${ }^{4}$, R. Penzel ${ }^{2}$, A. Ulrich ${ }^{5}$, B. Lehner ${ }^{6}$, V. Hovestadt', E. Czwan', G. Egerer ${ }^{8}$, T. Schmitt ${ }^{8}$, I. Alldinger ${ }^{5}$, E.K. Renker ${ }^{6}$, V. Ehemann ${ }^{* 2}$, R. Eils ${ }^{3}$, E. Wardelmann ${ }^{4}$, R. Büttner ${ }^{9}$, P. Lichter ${ }^{7}$, B. Brors ${ }^{3}$, P. Schirmacher ${ }^{2}$, G. Mechtersheimer ${ }^{2}$

'Institute of Pathology, University Hospital Heidelberg, Heidelberg, Germany, ${ }^{2}$ Institute of Pathology, Ruprecht-Karls-University, Heidelberg, Germany, ${ }^{3}$ Theoretical Bioinformatics, German Cancer Research Center (DKFZ), ${ }^{69120}$ Heidelberg, Germany, Heidelberg, Germany, ${ }^{4}$ Institute of Pathology, University Medical Center, Münster, Germany, ${ }^{5}$ Department of General and Visceral Surgery, Ruprecht-Karls-University, Heidelberg, Germany, ${ }^{6}$ Division of Orthopaedic Oncology, Department of Orthopaedics, Trauma Surgery and Paraplegiology, University Hospital Heidelberg, Heidelberg, Germany, ${ }^{7}$ Division of Molecular Genetics, German Cancer Research Center (DKFZ), Heidelberg, Germany, ${ }^{8}$ Department of Hemathology, Ruprecht-Karls-University, Heidelberg, Germany, ${ }^{9}$ Institute of Pathology, University Hospital, Cologne, Germany

Aims. High-grade soft tissue sarcomas (STS) are a heterogeneous and complex group of aggressive malignant tumors showing mesenchymal differentiation. In the last years, STS have increasingly been classified on the basis of underlying genetic alterations. The role of aberrant DNA methylation in STS is not well understood and the usefulness of methylation-based classification of these tumors is unclear.

Methods. By using the Illumina Infinium HumanMethylation27 platform we performed genome-scale DNA methylation profiling of 80 primary, untreated high-grade STS representing eight relevant subtypes (dedifferentiated, pleomorphic and myxoid liposarcomas, leiomyosarcomas, synovial sarcomas, malignant peripheral nerve sheath tumors, myxofibrosarcomas, and undifferentiated high-grade pleomorphic sarcomas) two non-neoplastic fat samples, and 14 representative sarcoma cell lines.

Results. The primary samples were partioned into seven stable DNA methylation clusters. Within the sarcoma collection, a classification algorithm identified $216 \mathrm{CpG}$ sites, mapping to 246 genes, showing different degrees of DNA methylation between these seven groups. By integrating DNA methylation and mRNA expression data, we identified 27 genes showing negative and three genes showing positive correlation. The differences between the DNA methylation based sarcoma clusters were best represented by a set of eight $\mathrm{CpG}$ sites located in SPEG, NNAT, FBLN2, PYROXD2, ZNF217, COL14A1, DMRT2, and CDKN2A. Compared to non-neoplastic fat, neuronatin (NNAT) showed DNA hypomethylation and inverse gene expression in myxoid liposarcomas (MLS), and DNA hypermethylation and inverse gene expression in dedifferentiated and pleomorphic liposarcomas. Recovery of NNAT in the hypermethylated MLS cell line MLS-1765 decreased cell migration and viability.

Conclusions. Our analysis represents the first comprehensive DNA methylation study integrated with transcriptional data in primary highgrade STS. We discovered novel biomarkers, new genes relevant for pathogenesis of STS and NNAT as potential tumor suppressor gene in MLS. 


\section{FR-068}

Preoperative intensity-modulated radiation therapy (IMRT) for high-risk soft tissue sarcomas of extremities and retroperitoneum-histopathological response evaluation and molecular aspects

\section{Straub ${ }^{* 1}$, B. Röper ${ }^{2}$, H. Rech ${ }^{3}$, R. Eisenhart-Rothe, von ${ }^{4}$, W. Klaus ${ }^{5}$,} K. Specht ${ }^{1}$

'Institute of Pathology, Technical University of Munich, Munich, Germany, ${ }^{2}$ Klinik und Poliklinik für Strahlentherapie und Radiologische Onkologie Klinikum Rechts der Isar, Klinik und Poliklinik für Strahlentherapie und Radiologische Onkologie, TU München, München, Germany, ${ }^{3}$ Klinik für Orthopädie und Sportorthopädie, Klinikum Rechts der Isar, Technische Universität München, München, Germany, ${ }^{4}$ Department for Orthopedics, Technical Univerity of Munich, Klinikum rechts der Isar, Munich, Germany, ${ }^{5}$ Institut für diagnostische und interventionelle Radiologie Klinikum Rechts der Isar, Institut für diagnostische und interventionelle Radiologie, TU München, München, Germany

Aims. Neoadjuvant radiotherapy is a promising therapeutic option for high-grade soft tissue sarcomas (hSTS) of both extremitities and retroperitoneum. The aim of the current study was to evaluate histopathologic response following IMRT for hSTS and to determine factors predictive for therapy response (p53, p16 and genomic complexity).

Methods. Pretherapeutic biopsies and resection specimen of primary tumors and metastases or local recurrences of $44 \mathrm{hSTS}$ of extremities and 5 hSTS of retroperitoneum (18 pleomorphic sarcomas, 12 myxofibrosarcomas, 5 synovial sarcomas, 5 dedifferentiated liposarcomas (DLS), 3 myxoid/round cell liposarcomas, 2 leiomyosarcomas, 1 epitheloid sarcoma, 1 rhabdomyosarcoma, 1 alveolar soft part sarcoma and 1 sarcoma, NOS) after preoperative IMRT were included in the study. Percentage of vital tumor, peritumoral tissue, resection margins and tumor cytomorphology were evaluated histomorphologically. Mib1, $\mathrm{P}_{53}$ and p16 expression were analysed by immunohistochemistry. Molecular analysis of $\mathrm{p} 16$ and $\mathrm{p} 53$ is under way.

Results. Median age of patients was 57 yrs (range: $23-89$; M:F ratio 26:23). Metastasis occurred in 12 patients and local relapse in 4 . A clear resection margin (Ro) was achieved in 37 patients. In 7 patients (4 with retroperitoneal hSTS), tumor was found at the resection margin and in 5 patients, fibrosis was present at the resection margin $(\mathrm{Rx})$. Percentage of vital tumor ranged from $2-100 \%$ (median $42 \%$ ). In genomically highly complex sarcomas, median of vital tumor tissue was $30 \%$, as opposed to sarcomas with intermediate genomic complexity (median: $75 \%$ ) and translocation-associated sarcomas (median: 50\%). Comparison of biopsy and resection specimen showed increased cellular atypia after radiotherapy in sarcomas with complex and intermediate genotype whereas in translocation-associated sarcomas, cytomorphology remained unchanged. Tumor proliferation assessed by MIB1 immunohistochemistry was decreased in irradiated specimen, and increased in metastasis as compared to the primary biopsy. p 16 and 553 protein expression remained unchanged after RTX. Moreover, $\mathrm{p} 53$ and $\mathrm{p} 16$ expression status of primary tumors was not predictive for therapy response.

Conclusions. A high percentage of Ro resections can be achieved in hSTS of extremities after radiotherapy, whereas complete resection of retroperitoneal hSTS remains challenging. Response rates vary widely, with certain subgroups (genomically complex sarcomas) displaying a better response.
FR-069

Inhibition of Cathepsin $\mathrm{X}$ influences the immune response to Helicobacter pylori-infected mice

K. Frauenschläger*, J. Schlien, S. Schmidt, D. Jechorek, A. Roessner, S. Franke

Department of Pathology, Otto-von-Gericke University Magdeburg, Magdeburg, Germany

Aims. Helicobacter pylori is responsible for inducing chronic gastric inflammation that progresses to athrophy, metaplasia and gastric cancer. The immune response to $\mathrm{H}$. pylori significantly determines the outcome of infection. Our previous studies have shown an active role for Cathepsin X (Ctsz) in chronic inflammation and development of gastric metaplasia. The question of whether the higher scores of macrophages depending on Ctsz-deficiency are protective or risk factors for etiopathology was elucidated in a recently established gastric cancer model. Methods. Eight-week-old ctsz-/-/INS-GAS and corresponding wild-type (INS-GAS) mice were infected by oral gavage with H. pylori strain SS1. The mice were sacrified at 24,36 and 50 weeks post infection (wpi). The stomach was removed, and gastric strips were snap-frozen or embedded and stained with $\mathrm{H} \& \mathrm{E}$. Tissue sections were scored for epithelial lesions and inflammation. Ki-67, F4/80, $\mathrm{CD}_{3}$ and $\mathrm{CD}_{20} \mathrm{immunostai-}$ ning was used to measure epithelial proliferation and macrophage/lymphocyte migration. Cytokine upregulation was proven by Western blots and quantitative RT-PCR.

Results. SS1-infected INS-GAS and ctsz-/-/INS-GAS mice both harbored high numbers of bacteria and showed strong inflammation, foveolar hyperplasia, atrophy, cystica profunda and dysplasia. Importantly, expression of CCL2, MCP-1 and IL-6 increased significantly in the ctsz/- mouse stomachs as compared with wild-type INS-GAS mice. Consequently, ctsz-/- mice showed higher levels of macrophages, B- and T-lymphocytes, as well as dysplasia.

Conclusions. Bacterial infection and Ctsz signaling are required for gastric tumorigenesis in mice. They cooperate in regulation of CCL2, MCP1 and IL- 6 expression to recruit inflammatory cells, which contribute to gastric tumorigenesis.

\section{FR-070}

Characterization of pancreatic stellate cells in pancreatic ductal adenocarcinoma and cases of chronic pancreatitis of various etiologies

\section{Häberle*1, K. Steiger', S. Haneder', S. Berchtold', R.-T. Daraban',}

A. Segler', A.M. Schlitter', M. Erkan', I. Esposito'

'Institute of Pathology, Technical University of Munich, Munich, Germany, ${ }^{2}$ Department of Surgery, Klinikum rechts der Isar, Technische Universität München, Munich, Germany

Aims. An abundant stromal reaction is one of the hallmarks of pancreatic ductal adenocarcinoma and chronic pancreatitis. The cells mainly responsible for the stromal reaction are activated pancreatic stellate cells (PSC). Despite their crucial role in these two major pancreatic diseases, PSC are not well characterized regarding their origin, function and immunophenotype. However, it is known that PSC share numerous characteristics with hepatic stellate cells (HSC), which have been described and established as key effectors in liver fibrosis long before their pancreatic counterparts. The aim of this study was a detailed immunohistochemical analysis of PSC in pancreatic ductal adenocarcinoma and cases of chronic pancreatitis of various etiologies.

Methods. Resection specimens of pancreatic ductal adenocarcinoma $(\mathrm{n}=10)$ and chronic pancreatitis $(\mathrm{n}=12)$ were studied by means of histochemistry and immunohistochemistry. The composition of the stroma was characterized using Movat's pentachrome stain. PSC were then tested for a panel consisting of PSC markers (alpha-SMA, CD 34 , desmin, SPARC and NGFR), as well as established HSC markers (CD56, NGF, 
NT-3, TrkC and alpha-crystallin B). PSC freshly isolated from human pancreatic ductal adenocarcinoma and chronic pancreatitis tissue samples were evaluated by immunofluorescence.

Results. PSC expressed all tested markers in analogy to HSC. In cases of pancreatic ductal adenocarcinoma, perilesional staining patterns could be observed for alpha-SMA, CD34, desmin, SPARC, CD56, NT-3, TrkC and alpha-crystallin B. Cases of chronic pancreatitis showed a more focal expression of the tested markers and a heterogeneity of staining patterns among different etiologies. Generally, the overlapping staining pattern of alpha-SMA and SPARC seemed to correlate with collagenrich fibrosis, while the staining patterns of $\mathrm{CD}_{34}$ and NT-3 appeared to be associated with intense remodeling of the stroma.

Conclusions. The close similarities between PSC and HSC could be confirmed. Heterogeneous expression patterns of the tested markers might reflect different levels of activation or differentiation of PSC. Along with the heterogeneity of the stromal composition highlighted by Movat's stain, this strongly suggests an active role of the stroma in pancreatic ductal adenocarcinoma and chronic pancreatitis.

\section{Biobanking Workshop}

\section{FR-074}

\section{BIOBANQUES, a French infrastructure for biobanks}

\section{G. Dagher* \\ Inserm, Paris, Frankreich}

BIOBANQUES is a distributed infrastructure that builds on a landscape of 82 biobanks distributed all over France including disease-oriented studies and population-based cohorts. In addition 6 health oriented microorganism BRCs (mBRCs) are partners of the project.

It covers the whole spectrum of human diseases with more than 600 ongoing biological and clinical research programs, including 45 followup prospective surveys of population with 300,000 individuals included in the studies.

The ultimate aims of this project are to:

1) Improve academic and industry's access to biological resources collected in the French biobanks and mBRCs network and in European biobanks in coordination with BBMRI and MIRRI

2) Improve efficiency, reduce redundancy of biobanking related activities and reduce duplication of administrative processes

3) Build capacity that is cost-effective and sustainable

4) Identify the current investment in biobanking and strategies to optimise value for money

5) Improve biosecurity and biosafety according to national and international laws and recommendations.

BIOBANQUES will take French collection of biological resources to a new level of coordination and efficiency, by setting up a sustainable infrastructure that will provide new services, better access for users from public and private sector, develop public private partnership, interface with the pan European infrastructure BBMRI and EMbaRC/MIRRI and support French biobanks and mBRCs in increasing the value and the use of their collections.

\section{FR-075}

Cost calculations and adapted cost allocation in biobanking

A. Maier ${ }^{* 1,2}$, R. Kirsten ${ }^{1,2}$, P. Schirmacher ${ }^{1,2}$, E. Herpel $^{2,3}$

'BMBH am Pathologischen Institut, Universitätsklinikum Heidelberg, Heidelberg, Deutschland, ${ }^{2}$ Pathologisches Institut, Universitätsklinikum Heidelberg, Heidelberg, Deutschland, ${ }^{3} \mathrm{NCT}$ Gewebebank am Pathologischen Institut, Universitätsklinikum Heidelberg, Heidelberg, Deutschland

Depending on the general scope of services as well as the local and institutional framework, cost factors of scientific biobanks may have distinct characteristics. While generally labour costs are crucial, some biobanks experience high costs for equipment and associated service contracts. Even expenses for special consumables like freezer tubes can add up to significant factors. To cover these costs, biobanks with their delicate collectives rely more than other research facilities on long term funding strategies to ensure uninterrupted operation.

In order to achieve that, funding needs to be customized according to the local set-up and the focus of the biobank. Biobanking activities and the associated cost types should be separated and allocated to the sources available to the biobank.

Direct costs of operations like consumables and direct labour can be specified and attributed to their cause (usually research applications or larger biobanking projects) and charged using fee for service cost recovery models.

Indirect costs and expenses caused by the provision of services can, if at all, only with great difficulty and as a fraction of the total financial burden be attributed to the users of biobanks. Instead other sources like local site funding should be used to cover these costs. This can be justified by the central service infrastructure biobanks provide. They can offer services to local research facilities that otherwise would not be available. The portfolio of these services can go beyond the provision of material and lab services and include extensive areas like project management, personnel procurement as well as shared lab space and equipment.

Other infrequent and more substantial single cost items like expenses for special equipment and investment in the infrastructure which increase the service portfolio of the biobank can often be funded by applying to local or national sponsors.

In order to find the best allocation of cost, all biobanking expenses should be evaluated and separated into categories where the total amount of each type of cost can be calculated. The method used for cost accounting has to be adapted to insure reasonable results. In the case of research projects and single studies, direct costing can be a feasible alternative while for calculations on funding applications, models using full cost accounting will lead to acceptable results.

\section{Inflammation und Carcinogenesis |}

SA-021

\section{Protection and self-renewal of the gastric mucosa: implications for} carcinogenesis

\section{W. Hoffmann*}

Institut für Molekularbiologie und medizinische Chemie, Magdeburg, Germany

Background and aims. The gastric mucosa and its glands represent a tight barrier to the outside world. This barrier is protected by multilayered mucus which contains the mucins MUC5AC, MUC6, and the trefoil factor family peptide $\mathrm{TFF}_{2}$. Furthermore, two types of gastric glands form delicate homeostatic systems, i.e. fundic and antral units, which show continual bidirectional self-renewal via differentiation from stem and progenitor cells. It was the aim to analyze the protective gastric mucus as well as self-renewal of the gastric units. 
Methods. Gastric mucus was purified via FPLC and analyzed for its constituents. Furthermore, three characteristic regions (pit, proliferative, and lower neck regions) were isolated from fundic and antral units by the use of laser microdissection, and expression profiles concerning important marker genes were generated by RT-PCR analysis.

Results. TFF2 was shown to be associated mainly with MUC6. Furthermore, the surface mucous cells (SMCs) of fundic and antral units characteristically differed in the expression of certain secretory genes. The maturation of mucous neck cells (MNCs) and their trans-differentiation into chief cells as well as the maturation of antral SMCs and antral gland cells occurred stepwise.

Conclusion. The correct maturation particularly of MNCs and their trans-differentiation into chief cells is critical for homeostatic self-renewal of fundic units. Dysregulation of this multistep process can result in generation of the metaplastic SPEM lineage which is characterized by its strong ectopic TFF2 expression. The SPEM lineage is a well known pre-cancerous lesion which can further differentiate into intestinal metaplasia.

1. Kouznetsova I, Kalinski T, Meyer F, Hoffmann W (2011) Self-renewal of the human gastric epithelium: new insights from expression profiling using laser microdissection. Mol BioSyst 7:1105-1112

2. Hoffmann W (2012) Stem cells, self-renewal and cancer of the gastric epithelium. Curr Med Chem 19:5975-5983

3. Hoffmann W (2013) Self-renewal of the gastric epithelium from stem and progenitor cells. Front Biosci S5:720-731

\section{SA-K022}

\section{Death-associated kinase (DAPK) - a kinase with antagonistic func- tional duality in inflammation}

\section{R. Schneider-Stock* \\ Experimental Tumorpathology, Institute of Pathology, Friedrich-Alexander- University Erlangen-Nürnberg, Erlangen, Germany}

DAPK is a serine/threonine kinase involved in a considerable spectrum of cellular functions such as apoptosis, autophagy, migration, invasion, and inflammation. First described as a tumor suppressor DAPK may also act as an oncogene dependent on the molecular context and the experimental settings. Similarly it can have pro-inflammatory functions in mouse macrophages or it negatively regulates inflammation in U937 cells and human T cells. Interestingly, DAPK signaling is not only restricted to its kinase activity; instead DAPK acts as a scaffold protein to trigger and stabilize multi-protein complexes. We have shown that DAPK inhibits TNF-induced NFkB activation and pro-inflammatory cytokine expression through negative regulation of the pro-inflammatory transcription factor STAT3. Here DAPK deteriorates the STAT3 dimer building and NLS recognition. Vice versa DAPK has been identified as new inhibitory transcription target of STAT3 leading to attenuation of its anti-inflammatory role. Furthermore we have identified the pro-survival protein $\mathrm{HSF}_{1}$ as a new phosphorylation target of DAPK responsible for the induction of apoptotic cell death under mild inflammatory stress in colorectal cancer. Here, we unravelled a positive feedback loop with HSF1 in transcriptional regulation of DAPK.

Recently, we described for the first time an important role for DAPK in Ulcerative colitis and suggest a role in tumor transformation in inflammation-associated colorectal carcinogenesis. The overexpression of a new inflammation-associated miRNA has been identified to be a possible mechanism to stabilize the DAPK protein by targeting the $\mathrm{E}_{3}$ ubiquitin ligase DIP1. I will further present a new conditional DAPK ko mouse model to study colitis-associated carcinogenesis.

\section{Inflammation und Carcinogenesis II}

\section{SA-K023 \\ Carcinogenesis in inflammatory bowel disease}

\section{S. Leedham*}

Wellcome Trust Centre for Human Genetics, University of Oxford, Oxford, United Kingdom

Chronic intestinal inflammation is a significant risk factor for the development of intestinal cancer, however the carcinogenesis pathway of colitis associated colorectal cancer is far less well defined that its sporadic counterpart. This talk will compare and contrast the pathogenesis of colitis associated and sporadic colorectal cancer. We will explore the cell of origin, clonality, the accumulation of (epi)genetic mutations and lesion progression in these different cancer pathways. Several recent papers have demonstrated the presence of field cancerisation in colitisthe accumulation of oncogenic mutations in non-dysplastic crypts. As the decision to recommend prophylatic colectomy is usually based on the pathological detection of dysplasia, field cancerisation represents a difficult clinical problem for gastroenterologists and pathologists. We will explore the evidence for this phenomenon, how field cancerisation can be detected in clinical practice and the possible future combination of clinical and molecular medicine to address this issue.

\section{SA-024}

Inflammation-associated oxidative stress as initiator of colorectal neoplasia

\section{A. Pöhlmann*}

Department of Pathology, Otto-von-Gericke University Magdeburg, Germany, Magdeburg, Germany

Aims. The production of hydrogen peroxide $\left(\mathrm{H}_{2} \mathrm{O}_{2}\right)$ drives tumourigenesis in ulcerative colitis (UC). UC is characterised by periods of inflammatory recurrence and remission, events accompanied by cell death and regeneration of the colonic mucosa. However, much less is known about the function of cell cycle arrest in UC. We therefore aimed to answer the questions of whether (i) oxidative stress may serve to initiate tumourigenesis and (ii) cell cycle arrest links reparative and uncontrolled proliferative response to inflammation in our cellular model of UC.

Methods. We simulated inflammation-associated oxidative stress of the epithelium in UC by exposing human colonic epithelial cells (HCEC) to periods of ten $\mathrm{H}_{2} \mathrm{O}_{2}$ exposures, each followed by periods of cellular recovery, generating HCEC cycles (C)1 to C1o (C cell cultures).

Results. $\mathrm{H}_{2} \mathrm{O}_{2}$ activated the JNK-pathway, resulting in $\mathrm{S}$ - and $\mathrm{G}_{2} / \mathrm{M}$ arrest. Caspases, p21WAF1 and gamma- $\mathrm{H}_{2} \mathrm{AX}$ were identified as JNKregulated proteins. Importantly, caspases executed a non-apoptotic function as they mediated survival of oxidatively damaged HCEC via suppression of gamma-H2AX. This made the G1/S- and intra-S-checkpoint ineffective. A population of cells thereby survived. We showed that oxidative stress led to caspase-mediated proteolytic degradation of ATM that is upstream of gamma-H2AX. Our findings suggest that delayed arrest in the subsequent cell cycle phases via checkpoint override led to survival mediated by a targeting of the caspases by the MAPK/ JNK-signalling pathway. Furthermore, we hypothesise that cells surviving multiple $\mathrm{H}_{2} \mathrm{O}_{2}$ exposures directly pass over from cell cycle arrest to driven cell cycle progression, and that JNK plays a pivotal role in this process. Thereby, dysregulation of JNK seems to switch the signalling pathways from arrest to increased proliferation. The non-apoptotic function of caspases appears to initiate the neoplastic features as they suppress JNK activation and thus JNK-dependent DNA damage checkpoints. 
Conclusions. The cellular model presented here provides a unique in vitro system to investigate the molecular mechanisms that may underlie the early tumourigenic events in CAC, such as driven cell cycle progression and undetected DNA-damage, both hallmarks of cancer. This model further supports that chronic inflammation-associated oxidative stress is likely to initiate tumourigenesis.

\section{Inflammation und Carcinogenesis III}

\section{SO-057 \\ Inflammation, malignancy and immunology in gastrointestinal spindle cell tumors-beyond gastrointestinal stromal tumor (GIST) \\ E. Montgomery* \\ Johns Hopkins Medical Institutions, Department of Pathology, Baltimore, United States}

A host of mesenchymal tumors and tumefactions associated with the gastrointestinal tracts features prominent inflammatory cells and the mechanisms for the inflammation and the processes themselves remain poorly understood. Classic such lesions include Kaposi sarcoma, inflammatory fibroid polyp, sclerosing mesenteritis, and inflammatory myofibroblastic tumor but, more recently, the recognition of IgG4-related fibrosclerosing disease has resulted in modification of our views on pathogenesis and treatment of such inflammatory lesions in many anatomic sites. In some lesions, the inflammation may reflect viral influences (Kaposi sarcoma) or a bacterial infectious trigger (IgG4-related fibrosclerosing disease) whereas in others such an interaction is unclear and alterations in various genes have been detected (such as ALK rearrangements in inflammatory myofibroblastic tumor and PDGFRA mutations in inflammatory fibroid polyp and some GISTs). Even the inflammatory milieu of GIST may have an impact on outcome. We will discuss practical diagnostic considerations as well as some theoretical ones.

\section{SO-058 \\ DUOX2, inflammation and intestinal tumorigenesis in ulcerative colitis}

\section{R. Poulsom* \\ Blizard Institute, Digestive Diseases/ Queen Mary University of London, London, United Kingdom}

Background. Dual oxygenase 2 (DUOX 2 ) with its obligate protein partner DUOXA2 form an enzyme system in colorectal crypt epithelium capable of forming hydrogen peroxide $\left(\mathrm{H}_{2} \mathrm{O}_{2}\right)$, a powerful reactive oxygen species used in many species, from worm and fly through to mammals, to assist defence of the gut from pathogens. Some species utilise $\mathrm{H}_{2} \mathrm{O}_{2}$ to make strong biocides from halide anions or thiocyanate via lactoperoxidase thus reducing the levels of $\mathrm{H}_{2} \mathrm{O}_{2}$ that might otherwise damage host DNA.

Aims. We sought, first to determine which members of the NOX/DUOX family were expressed in normal colorectal tissues and whether the profile altered in response to inflammation in Ulcerative Colitis (UC), or in cancer. Secondly, to test in vitro whether their expression was affected by hypoxia or 5-ASA as these factors may relate to the increased risk of colorectal cancer in UC patients. Subsequently, we compared the responses of DUOX2/DUOXA2/LPO in human UC with homologues in mouse colitis.

Methods. We analysed from consented individuals fresh or formalinfixed paraffin-embedded biopsies with or without UC, and surgical resection specimens. mRNA levels in fresh materials were studied by qRT/PCR and/or expression microarrays. Localisation of mRNAs was by in situ hybridisation ( $35 \mathrm{~S}$ riboprobes or RNAscope 2.0 branched
DNA detection), and of proteins and damaged DNA nucleobase (8oHdG) by immunofluorescence.

Results and conclusion. Analysis of mRNA from fresh tissue revealed that the predominantly expressed enzyme system capable of making $\mathrm{H}_{2} \mathrm{O}_{2}$ in the large bowel is formed of DUOX2 and DUOXA2; expression was greater with inflammation, and in adenomas, but was lost in some cancers. Expression microarrays showed that inflammation and 5-ASA increased levels of DUOX 2 and DUOXA2 and DUOX 2 immunofluorescence within $6 \mathrm{~h}$ in vitro. Analysis of fixed materials showed $D_{U O X}$ mRNA normally confined to the apical surface of crypts, but extending deeper in association with inflammation; crypt abscesses expressed DUOX2 strongly. 8-oHdG was found in epithelial nuclei deep in crypts where ulceration and regeneration was present and together this supports the hypothesis that defensive production of $\mathrm{H}_{2} \mathrm{O}_{2}$ in this setting may also damage host DNA. Analysis of tissues from mice with dextran sulphate-induced colitis established the dynamics of Duox2, Duoxa2 and Lpo responses and revealed a fundamental difference between mouse and man that challenges the relevance of mouse models of human colitis.

\section{Aktuelle Habilitationen I}

\section{FR-055}

Molecular pathology and tumor biology of lobular breast cancer

M. Christgen ${ }^{* 1}$, R. Geffers' ${ }^{2}$, H. Christgen ${ }^{3}$, U. Lehmann', H. Kreipe ${ }^{1}$ 'Institute of Pathology, Hannover Medical School, Hannover, Germany, ${ }^{2}$ Helmholtz Centre Braunschweig, Department of Genome Analytics, Braunschweig, Germany, ${ }^{3}$ Hannover Medical School, Hannover, Germany

Aims. Invasive lobular breast cancer (ILBC) is the most common special type of breast cancer. ILBC represents a distinct entity in terms of histomorphology and biological behavior. Due to the lack of functional ILBC cell models, the tumor biology of lobular carcinomas remained partially unexplored.

Methods. The tumor biology of ILBC, in particular the progression of this tumor entity, was investigated by molecular analyses based on archival tissues and by functional studies based on new cell models.

Results. In addition to the inactivation of E-cadherin, mutational activation of the $\mathrm{PIK}_{3} \mathrm{CA}$ oncogene is involved in the carcinogenesis of ILBC. During the progression to local recurrence, $\mathrm{PIK}_{3} \mathrm{CA}$ mutations are positively selected for. As a prerequisite for functional analyses, the first ILBC cell line, named IPH-926, was established. IPH-926 ILBC cells harbor a homozygous E-cadherin frameshift mutation. However, ectopic re-expression of E-cadherin does not induce a pronounced transcriptional re-organization. The clonal evolutionary ancestry of IPH926 ILBC cells is characterized by a late $\mathrm{TP}_{53}$ mutation, acquisition of a secondary pleomorphic phenotype and dysregulation of the p53 target gene PHLDA3. Moreover, IPH-926 ILBC cells display a marked anticancer drug-resistance driven by an overexpression of the $A B C B 1 / M D R 1$ xenobiotica transporter. Interestingly, IPH-926 ILBC cells maintain a strictly luminal gene expression profile, although these tumor cells have undergone a progression-related conversion of the hormone receptor status in their clonal ancestry and exhibit a triple-negative immunophenotype.

Conclusions. In summary, the carcinogenesis and progression of ILBC is accompanied by distinct molecular alterations. For the first time, IPH926 ILBC cells allowed for studying the functional and cell biological consequences of such alterations in the correct cellular context. Our findings underscore the independence of a luminal molecular subtype from the hormone receptor status and underline the relevance of secondary mutational alterations, such as $\mathrm{PIK}_{3} \mathrm{CA}$ and $\mathrm{TP}_{53}$, for the behavior of ILBC. In contrast, it remains poorly-defined how inactivation of $\mathrm{E}$ cadherin promotes carcinogenesis in lobular neoplasia. 


\section{FR-056}

Evaluation of biomarkers in formalin-fixed, paraffin-embedded (FFPE) ovarian and breast cancer tissue samples for prediction of prognosis and therapy response

\section{S. Darb-Esfahani* \\ Institute of Pathology, Charité, Berlin, Germany}

Aims. The aim of our studies was the evaluation of biomarkers for a better definition of therapeutically relevant subgroups of breast and ovarian cancer.

Methods. We used the pre-therapeutic core biopsies from the neoadjuvant clinical study cohorts of the German Breast Group. Immunhistochemistry was performed on a tissue micro array and quantitative reverse transcriptase PCR (qRT-PCR) was performed after mRNA extraction from FFPE samples. We also used immunohistochemistry and qRT-PCR for the investigation of ovarian cancer samples from the Tumorbank Ovarian Cancer Network (TOC).

Results. Our results showed that in breast cancer a standardized classification based on the expression analysis of estrogen and progesterone receptors (ER, PR) as well as HER2 defines tumor subtypes with significantly different prognosis and response to neoadjuvant chemotherapy. The behavior and therapy response of HER2 positive carcinomas differ in dependence of the co-expression of hormone receptors. In a systematic screening, training, validation study we identified thymosin beta $15 \mathrm{~A}$ mRNA expression as a novel molecular marker for response to neoadjuvant chemotherapy in the aggressive subgroup of triple negative carcinomas. For ovarian carcinoma, one of our major aims was to establish structures that enable the evaluation of biomarkers within the setting of clinical studies, similarly to breast cancer. Here, we also investigated the expression of ER, and found that mRNA levels as determined by qRT-PCR had advantages over protein levels as determined by immunohistochemistry. We thereby established qRT-PCR as a method that was able to detect biologically relevant ER levels. Further markers that we analyzed on the mRNA and protein level in ovarian carcinoma were lysophosphatidic acid acyltransferase beta (LPAAT-beta), aldehyde dehydrogenase $1(\mathrm{ALDH})$, and components of the classical nuclear factor kappa B (NF-kB) pathway. For instance, we showed that ovarian carcinomas with co-expression of ALDH1 and epidermal growth factor receptor (EGFR) constitute a subgroup with a very unfavorable prognosis, suggesting that for those tumors EGFR inhibition might be a therapeutic option.

Conclusions. Our data constitute a basis for the further validation of the respective biomarkers in breast and ovarian carcinoma and provide a methodological basis for novel predictive tests.

\section{FR-057}

\section{Tissue-based biomarkers in cancer}

\section{J.K. Lennerz*}

Institute of Pathology, University Ulm, Ulm, Germany

Aims. Biomarkers are indicator molecules of health and disease and have evolved into critical tools with clinical relevance. They allow evaluation of a variety of aspects ranging from diagnosis to prediction of response to therapy. The overview provided here is composed of several biomarkers that cover diagnostic, prognostic and predictive tissue-biomarkers in cancer.

Methods. Briefly, the illustrated biomarkers are: (1) Mist1 as a diagnostic biomarker for normal gastric chief cell differentiation; (2) perinodular K19 pattern as a biomarker for intranodular pathology in the cirrhotic liver; (3) BRAF mutation status in the diagnosis of hairy cell leukemia; (4) microsatellite instability and mismatch repair protein detection as diagnostic biomarkers in colorectal cancer and Lynch syndrome; (5) SOCS1 gene mutations in diffuse-large-B cell lymphoma and (6) MET/ EGFR/HER2 gene amplification-status in gastroesophageal carcinoma

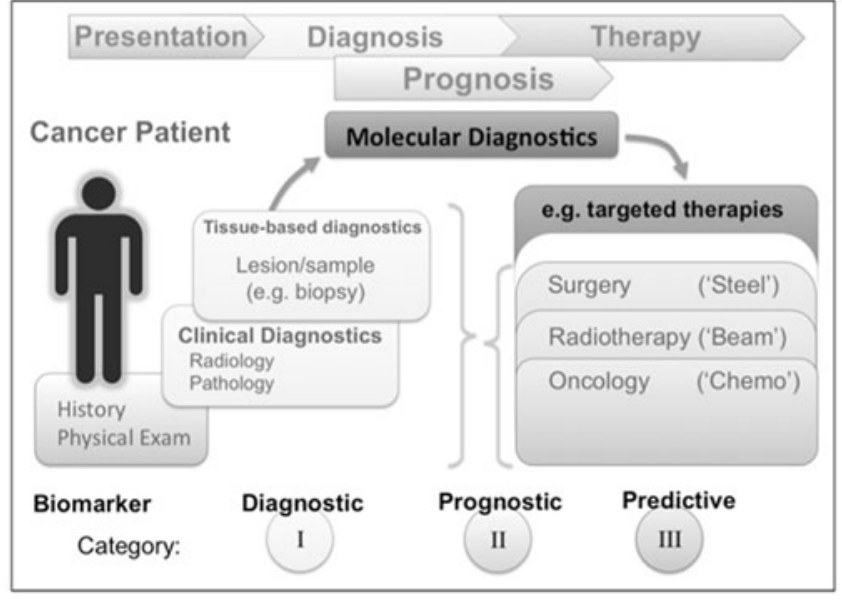

Fig. 1 | FR-057 $\triangle$ Biomarker in the clinical context. The three main categories of biomarkers are displayed as actionable components, exemplified in the workup of a cancer patient. Mod. from Lennerz JK, lafrate AJ, Pfeifer J, Möller P. Molekulardiagnostik in der Krankenversorgung von Krebspatienten - Teil I. Krebsmedizin 2012.21(2) 83-90

as prognostic biomarkers; and (7) MET gene amplification status as predictive for responsiveness to crizotinib in gastro-esophageal cancer. These cancer biomarkers are at different stages in the translational discovery- and implementation pipeline-aiming at integration into routine clinical practice.

Results. The overview provided here portrays the clinical relevance of these biomarkers and places them into the context of current prospective and future challenges of tissue-based biomarkers. Modalities of quick and reliable assessment as well as the identification of novel biomarkers of disease phenotype are expanding area of research that will extend diagnostic capabilities. When these assays are combined with stringent surrogate end-points, novel tissue-based biomarkers will accelerate the timeline of translating molecular understanding of disease biology into the clinic.

Conclusions. In summary, tissue-based biomarkers are one integral component of an expanded molecular toolkit in the diagnostic process and serve as indicators for the stratification of patients towards appropriate therapies (• Fig. 1).

\section{FR-058}

Integrative genomic and proteomic analysis of the functional relevance of mutational profiles in cancer: a systems biological approach

\section{F. Klauschen*}

Institute of Pathology, Charité, Berlin, Germany

Aims. The ability to study mutational profiles of individual tumors using next generation sequencing has raised hope to gain a better understanding of the mechanisms underlying cancer pathology. Recent analyses of data from cancer genome profiling projects have, for instance, revealed mutational similarities across major cancers supporting views that personalized therapies that target such actionable mutations may be efficacious independent of the classical organ-specific cancer types. Here, we study whether the genetic similarities observed across different cancer types are reflected on the more functional level of proteins by analysing available genomic and proteomic data and by studying cancer cell signaling using phosphoproteomics.

Methods. Our analysis is based on evaluating data on simple somatic mutations, copy number variations, gene methylation as well as gene and protein expression in more than 3,00o tumors from 12 major cancer 
entities from The Cancer Genome Atlas (TCGA). For the cancer cell signaling analysis we use EGFR-mutated and -wild type lung cancer cell culture models and perform targeted sequencing using the comprehensive cancer panel of the IonTorrent platform and mass-spectrometry based phosphoproteomics.

Results. Our analysis shows that the substantial genetic similarities present across all major cancers on the level of simple somatic mutations and copy number variations are reduced on the level of gene expression and are nearly completely absent on the proteomic level: While an average of $42 \%$ of the tumors are genetically more similar to tumors from a different organ or tissue than to their own, a comparison of the tumors based on a set of cancer-related proteins shows for nearly all cases that tumors from one organ/tissue are most similar to tumors from the same site, therefore largely confirming the current WHO classification. To show ways to further investigate the relationships between genomic and proteomic profiles, we perform targeted sequencing and integrate it with phosphoproteomic analyses of cancer cell signaling allowing us to simultaneously study the activity of about 1,00o proteins over time. Conclusions. To conclude, our work points to the differences between genomic and proteomic cancer profiling with respect to the established WHO classification of tumours and calls for an integration of genomic with proteomic approaches in efforts to gain functional insight into cancer pathology and ultimately to design novel personalized therapies.

\section{Aktuelle Habilitationen II}

\section{SA-011 \\ Macrophages in nodular lymphocyte predominant Hodgkin lym- phoma, T cell/histiocyte rich large B cell lymphoma and HIV-related classical Hodgkin lymphoma}

\section{S. Hartmann*}

Department of Pathology, Goethe University, Frankfurt, Germany

Aims. Abundant macrophage infiltration in tumors often correlates with a poor prognosis. T cell/histiocyte rich large B cell lymphoma (THRLBCL) is a distinct aggressive $\mathrm{B}$ cell lymphoma entity showing a high macrophage content.

Methods. To elucidate the role of tumor-associated macrophages in THRLBCL, gene expression profiling of microdissected histiocyte subsets of THRLBCL, nodular lymphocyte predominant Hodgkin lymphoma (NLPHL), Piringer lymphadenitis, sarcoidosis, nonspecific lymphadenitis, and monocytes from peripheral blood were performed. To compare quantitatively the composition of the microenvironment in NLPHL and THRLBCL, CD4+ T cells and CD163+ macrophages were counted and compared with cases of HIV-related classical Hodgkin lymphoma (cHL), usually showing a high macrophage content.

Results. In a supervised principal component analysis of the gene expression profiles obtained from histiocytes from THRLBCL, they were most closely related to epithelioid cells from NLPHL, with both types of cells expressing genes related to proinflammatory and regulatory macrophage activity. Moreover, histiocytes from THRLBCL strongly expressed metal-binding proteins like MT2A, by which histiocytes of THRLBCL can be distinguished from histiocyte subsets in NLPHL and HIV-related HL. Interestingly, the validation at the protein level showed a strong expression of TXN, CXCL9, MT2 A and SOD2 not only in macrophages of THRLBCL but also in the tumor cells of NLPHL and classical Hodgkin lymphoma (cHL). THRLBCL but also in the tumor cells of NLPHL and classical Hodgkin lymphoma (cHL). Quantification of the composition of the microenvironment revealed macrophage counts in THRLBCL in the range of HIV-associated cHL. Although the amounts of $\mathrm{CD}_{4}+\mathrm{T}$ cells in lymph nodes infiltrated by THRLBCL were not comparable to the setting of HIV-associated cHL, they were significantly decreased in THRLBCL compared to typical NLPHL.
Conclusions. Overall, the present findings indicate that macrophages occur in the microenvironment of THRLBCL in high numbers, similar to the setting observed in HIV-related cHL. They have acquired a distinct gene expression pattern, characterized by a mixed $\mathrm{M} 1 / \mathrm{M}_{2}$ phenotype and a strong expression of several metal binding proteins. The high expression of metal binding proteins in histiocytes of THRLBCL may be diagnostically useful, but a potential pathophysiological role remains to be identified.

\section{SA-012}

Prostaglandin E2 in cartilage-from the biological development to innovative approaches for tissue engineering of articular cartilage and its significance for modern pathology

\section{Brochhausen*}

Institute of Pathology, University Medical Centre of the Johannes Gutenberg-University, Mainz, Germany

Aims. Prostaglandin E2 represents an important mediator in cartilage metabolism and the pathophysiology of cartilage. To analyse systematically the effects of PGE2 and prostaglandin receptor ligands on growth plate and articular cartilage chondrocytes a three-dimensional cell culture-system was used mimicking articular cartilage architecture.

Methods. Rat growth plate chondrocytes were isolated and stimulated with PGE2 and prostaglandin receptor ligands. In a three dimensional cell culture system, mimicking the fibre arrangement of articular cartilage human articular chondrocytes were stimulated with equivalent concentrations of PGE2. For prolonged release PGE2 was immobilized in polylactide-co-glycolide-spheres. The release-kinetic was analysed by gas chromatography-tandem mass spectroscopy. The release system was integrated in the scaffold and seeded with chondrocytes. These constructs were assessed using molecular biological methods (PCR for collagen I, collagen II and collagen X), histology and synchrotron- $\mu \mathrm{CT}$ (SR- $\mu \mathrm{CT})$.

Results. Rat growth plate chondrocytes showed a dose dependent proliferation and increased collagen II expression after stimulation with low doses of PGE2. These effects were COX-II dependent and were mediated by the EP-1 receptor. The positive effects of PGE2 were also seen in human articular chondrocytes with a rapid phenotypic re-differentiation, cluster-formation and synthesis of collagen II, by depletion of collagen I and collagen X. By integrating PGE2 in the polylactide spheres a prolonged release was achieved. After integration of the release-system in the scaffold, cluster formation of cells and synthesis of extra-cellular matrix components were obvious already after 8 days in culture. Via SR- $\mu \mathrm{CT}$ the distribution of spheres and cells in the scaffold was detected without metal-labelling.

Conclusions. Low dose $\mathrm{PGE}_{2}$ is suitable to trigger cartilage regeneration. The used scaffold-release-constructs represent suitable devices for tissue engineering strategies. For the clinical use of these constructs pathological analyses of the regenerative potential of damaged cartilage will be obvious. To improve the understanding of the underlying mechanisms the three-dimensional cell-culture method could be modified by integrating further mediators for pathophysiological analyses. SR- $\mu \mathrm{CT}$ enables the analysis of the three-dimensionality which gives innovative perspectives for future analyses of morphogenesis during regeneration and tumour-formation.

\section{SA-013}

Aberrant reparative tissue remodelling

D. Jonigk*

Institute of Pathology, Hanover, Germany

Aims. Not only malignant changes can lead to the destruction of parenchymatous organs, but also the aberrant proliferation and matrix 
production by mesenchymal cells in a dysregulated reparative change. This fibrogenesis is the result of a complex pathogenesis which can be analyzed in animal models as well as in situ (in humans). The present study primarily follows the second approach and summarizes molecular pathological results of different diseases for aberrant and reparative tissue remodelling. Plexiform vasculopathy in $\mathrm{PAH}$, Quilty lesions after heart transplantation, BO, IL and EBV-induced smooth muscle proliferation are among the selected model diseases.

Methods. Using in situ molecular pathology, we could disprove a suspected participation of myofibroblastic cells in vascular remodelling in PAH lungs. For the first time, we could perform a comprehensive molecular characterization of the remodelling changes and prove, that plexiform vasculopathy represents a (complex regulated) epiphenomenon of PAH. Our methodical approach enabled us to describe miRNA expression compartment-specific for the first time and to draw conclusions from the disturbed regulatory mechanisms. We were also able to describe the chimeric character of the complex (lymph) neoangiogenesis in grafts following heart transplantation.

Results. After lung transplantation, we have identified a group of tissuebased molecular markers which can predict the occurrence of BO even in morphologically inconspicuous transbronchial biopsies. Furthermore, we could comprehensively document the molecular qualities of the remodelling process in the small airways in lung transplanted and nontransplanted patients. We could further elucidate the role of the MMPs and their antagonists, antiproteases, in the inflammatory changes and deduce therapeutic implications.

Conclusions. In summary, our work reveals that the in situ analysis can address the upcoming challenges and questions posed by the life sciences. It has to be expected that the technical possibilities will develop analogously to the increasing demands of patients, clinicians and researchers and the in situ approaches will gain more and more attention in the field of molecular pathology.

\section{SA-014}

\section{Endogenous lipids as modulators of inflammatory and toxic pro-} cesses

\section{S. Porubsky*1,2}

'Department of Pathology, University Medical Center Mannheim, University of Heidelberg, Mannheim, Germany, ${ }^{2}$ Cellular and Molecular Pathology, German Cancer Research Center, DKFZ, Heidelberg, Germany

Aims. The aim of the presented work was to explore the role of selected endogenous lipids in immunological and toxic processes. In particular, it was focused on the function of neutral glycosphingolipids globosides and isoglobosides in the biology of immune cells as well as on the role of globosides as receptors for Shigatoxins during an infection with enterohemorrhagic Escherichia coli (EHEC).

Methods. Mice with deficiency of globosides and isoglobosides were generated. Organs were analyzed by thin layer and high-performance liquid chromatography as well as by conventional and electron microscopy. Antigen presenting cells and T-cells were studies using flow cytometry, in vivo and ex vivo functional tests. Mechanisms of Shigatoxin 2-toxicity were explored on mice with tissue specific deficiency for globosides as well as on human kidney biopsies from the German 2011 EHEC epidemic.

Results. It could be shown that the previously implicated endogenous lipid ligand for iNKT-cells, isogloboside, can be produced in thymi in trace amounts and that isogloboside synthase $\left(\mathrm{iGb}_{3} \mathrm{~S}\right)$ is the exclusive enzyme responsible for its synthesis. However, as demonstrated by the iGb3S-deficient mouse, isogloboside is-in contrast to previous in vitro-based assumptions-dispensable for the development of iNKT-cells in vivo. It could be also shown that the interaction between iNKT-cells and antigen presenting cells is not mediated by isogloboside and that accumulation of globosides can interfere with this interaction. Furthermore it was documented in vivo, that globoside is the decisive receptor mediating the toxic effects of Shigatoxin 2. In addition to the previously recognized Shigatoxin 2-elicited thrombotic microangiopathy, we could now show that Shigatoxin 2 contributes to kidney failure also by exerting direct toxic effects to the globoside-expressing renal tubular cells.

Conclusions. These results enlighten the role of the endogenous lipids globosides and isoglobosides as modulators of immunological processes. Moreover, they pinpoint to the acute renal tubular damage as a new pathophysiologic mechanism responsible for kidney failure during the infection with EHEC.

\section{Hungarian-German Cooperation Meeting}

\section{SO-059}

A novel hyperplasia-neoplasia sequence in pancreatic endocrine tumors: glucagon cell adenomatosis

\section{B. Sipos ${ }^{* 1}$, J. Sperveslage', T. Henopp ${ }^{2}$, M. Anlauf 3 , G. Klöppel ${ }^{4}$}

'Department of Pathology, University Hospital, Eberhard Karls University, Tübingen, Germany, ${ }^{2}$ Institute of Pathology, Clinical Center, Traunstein, Germany, ${ }^{3}$ Institute of Pathology, Clinical Center, Limburg, Germany, ${ }^{4}$ Institute of Pathology, Technical University of Munich, Munich, Germany

Introduction. Glucagon cell adenomatosis (GCA) was recently recognized by us as a multifocal neoplastic disease of the endocrine pancreas unrelated to MEN1. Multiple micro- and a few macrotumors are found on the background of a hyperplasia of glucagon cells. The disease may cause unspecific abdominal symptoms and only rarely a glucagonoma syndrome. We aimed to investigate the genetic alterations of GCGR gene in patients with GCA.

Material and methods. FFPE pancreatic tissues from six patients showing multiple microadenomas and partly macroadenomas of glucagon cells were macro- or microdissected. Following DNA extraction all exons of the GCGR gene were analysed for mutations by Sanger and deep sequencing. Genotyping for all detected GCGR variants was performed by using Taqman Assay in 2560 healthy individuals.

Results. Sequencing of the GCGR gene revealed germ line mutations in three out of six patients. One patient showed two different heterozygous point mutations in the hyperplastic alpha cells as well as in the non-tumorous tissue leading to two premature stop codons. One patient harboured a homozygous stop mutation. The third patient showed two homozygous missense mutations of the GCGR gene that most likely also led to a dysfunction of the GCGR. These variants were not identified in healthy subjects. In the three other patients no germ line or somatic mutations of the GCGR gene were detected. GCA patients harbouring GCGR mutations showed a more advanced phenotype regarding alpha cell hyperplasia and exclusively developed macrotumors.

Conclusion. The finding of germ line "loss of function" mutations of the GCGR gene in three of six GCA cases suggests that deficiency in the GCGR signalling causes glucagon cell adenomatosis via glucagon cell hyperplasia. However, there is a second still unrecognized mechanism that leads to GCA in patients with wild type GCGR gene. 


\section{SO-060}

Tricellulin and microRNA expression might predict prognosis of epithelial hepatoblastoma

A. Kiss" ${ }^{* 1}$, K. Schlachter', M. Gyugos', B. Gyöngyösi', J. Halász', G. Lendvai',
I. Kenessey', Z. Jakab', M. Garami', Z. Schaff'
${ }^{12}$ nd Department of Pathology, Semmelweis University, Budapest, Hungary,
${ }^{22}$ nd Department of Pediatrics, Semmelweis University, Budapest, Hungary

Background and aims. The more differentiated fetal component of hepatoblastoma $(\mathrm{HB})$ is characterized by increased expression of tight junction (TJ) proteins claudin-1 and -2 when compared with embryonal component. MicroRNA (miRNA) expression patterns, as well as the recently identified TJ protein, tricellulin and epigenetic regulator enzyme $\mathrm{EZH}_{2}$ were investigated in epithelial subtypes of $\mathrm{HB}$ to relate them to survival.

Material and methods. 20 cases of epithelial HBs were subtyped as pure fetal components $(n=12)$ and embryonal components $(n=8)$, along with 15 non-tumorous surrounding liver samples were analysed by immunohistochemistry for tricellulin, $\beta$-catenin and $\mathrm{EZH}_{2}$ expression. Relative expressions of miR-17-5p, miR-18a, miR-21, miR-34a, miR-96, miR-122, miR-181a, miR-195, miR-210, miR-214, miR-221, miR-222, miR-223 and miR-224 were determined by TaqMan MicroRNA Assays applying miR-140 as reference.

Results. No significant differences were revealed in overall survival between fetal and embryonal/fetal types of HBs. Fetal component, however, showed considerably increased tricellulin expression while embryonal component displayed significantly increased nuclear $\mathrm{EZH}_{2}$ positivity in comparison to other epithelial subtype and non-tumorous surrounding hepatocytes. Strong nuclear $\beta$-catenin staining was notably more frequent in embryonal than in fetal type. High tricellulin expression was associated with significantly increased overall survival $(\mathrm{p}=\mathrm{0.03}$ ), while elevated $\mathrm{EZH} 2$ expression was linked to presence of distant metastases $(\mathrm{p}=0.013)$. Elevated level of miR-18a $(\mathrm{p}<0.01)$ was found in the embryonal component when compared with fetal component. Decreased miR-17-5p, miR-195, miR-210, miR-214 and increased miR221 levels were detected in the fetal component $(\mathrm{p}<0.02)$ in comparison with SL samples, whereas decreased miR-122 level was observed in the embryonal component $(\mathrm{p}<0.003)$. High miR-21, low miR-222 and low miR-224 levels proved to be independent prognostic factors for $\mathrm{HB}$ and were associated with significantly increased overall survival $(\mathrm{p}<0.03)$. Conclusions. Treated HBs showing high expression of tricellulin reveal significantly better overall survival independent of histological subtype. Increased nuclear expression of $\mathrm{EZH}_{2}$ was associated with the presence of distant metastases. Furthermore, miR-21, miR-222 and miR224 levels could serve as valuable tools to predict overall survival of $\mathrm{HB}$ patients regardless of epithelial subtype.

\section{SO-061}

\section{Frequent lipogenic phenotype in hepatocellular carcinoma}

B.K. Straub*1,2, L. Pawella', S. Singer', B. Gyöngyösi ${ }^{3}$, A. Kiss ${ }^{3}$, K. Breuhahn', P. Schirmacher

IInstitute of Pathology Heidelberg, Heidelberg, Germany, ${ }^{2}$ Institute of Pathology, University Hospital Heidelberg, Heidelberg, Germany, ${ }^{32} \mathrm{nd}$ Department of Pathology, Semmelweis University, Budapest, Hungary

Introduction. Hepatocellular carcinoma (HCC) accounts for the fifth most frequent cancer worldwide and the third most common cause of cancer mortality. Over $10 \%$ of HCCs show prominent fatty change with large size lipid droplets (LDs), yet, in own experiments, LD-accumulation was observed in over $90 \%$ of HCCs, suggesting an altered tumor metabolism/Warburg effect. LDs are decisively regulated by LD-associated proteins of the PAT/perilipin-family with its members perilipin, adipophilin, TIP47, S3-12 and MLDP (perilipins 1-5). We could previously show that many human cancers, especially HCC and carcinomas with clear cell phenotype, accumulate small LDs decorated by adipophilin, TIP47 and MLDP; yet the functional meaning of this finding is not yet clear.

Material and methods. LD-associated proteins of the PAT/perilipin family were analysed with immunohistochemistry in a tissue microarray comprising over $190 \mathrm{HCC}$, as well as with immunofluorescence microscopy, protein biochemical and molecular biological methods in over 20 cryopreserved HCCs. In addition, HCC cells of the line Huh7 were analysed under conditions of steatogenic treatment and downregulation by using si of shRNAs targeting adipophilin and/ or TIP47.

Results. Over 90\% of HCCs show accumulation of small LDs decorated by mainly adipophilin, but also TIP47 and MLDP. Whereas perilipin is positive in the vast majority of nontumorous liver specimens, as well as in focal nodular hyperplasia and hepatocellular adenoma, only about $30 \%$ of HCCs show focal positivity for perilipin. In HCCs in situ and in respective Huh7 cells in vitro, $\mathrm{LD}$-accumulation is regulated by both PPAR alpha and PPAR gamma. Interestingly, adipophilin staining intensity positively correlates with the (Ki67) proliferation rate. Untreated HCC cells of the lines Huh7, PLC, HepG2 and $\mathrm{Hep}_{3} \mathrm{~B}$ store lipids in LDs mainly covered by adipophilin and TIP47, only upon long-termtreatment with DMSO, perilipin is induced in non-proliferating, differentiated cells. In Huh7 cells with transient or stable downregulation of adipophilin and/ or TIP47 via si or shRNAs, not only LD amount and number decreased, but also cell vitality was decreased to about $80 \%$ (adipophilin) resp. 50-60\% (TIP47).

Conclusion. The majority of HCCs shows LD-accumulation and overexpression of associated proteins indicative of a lipogenic phenotype. PAT-proteins may therefore be interesting diagnostic and therapeutic targets in HCC.

\section{SO-062}

\section{Cancer-associated fibroblast, the key player in matrix remodeling}

\section{Kovalszky*, A. Fullar, K. Baghy}

Semmelweis University, First Department of Pathology \& Experimental Cancer Research, Budapest, Hungary

Recently increasing amount of evidence put the tumorous stroma into the focus of cancer research. The altered biological activity of tumor associated fibroblasts results in production of matrix proteins with altered structure or amount. Abnormal ECM can be responsible for disturbed regulation of cellular functions facilitating tumor growth. Furthermore these cells are implicated in the synthesis of regulatory factors responsible for the accelerated growth of tumor cells.

After the initiating observation revealing loss of syndecan-1 from the tumor cell surface, and detecting it on the tumor associated fibroblast it was decided to obtain more information about the phenotypic changes of stromal components of cervical cancer. To this end, surgical specimens as well as expression profile of normal and tumorous cervix derived fibroblast have been studied.

Increased immunostaining of fibronctin, laminin, vimentin, and smooth mucle actin indicated the presence of more activated fibroblasts with enhanced synthetic activity in the tumorous stroma of tumorous surgical specimens. Conversion of syndecan-1 expression could be witnessed, as well.

Fibroblasts explanted from cervical tissue supported the growth of primary as well as established cervical cancer cell lines. Microarray of fibroblasts from tumor free and tumorous tissue showed different expression profile where increased expression of fibroblast growth factor1, latent TGFB-binding protein 2, thrombospondin1, as well as the decreased expression of decorin, syndecan 4 , matrili2 and $\mathrm{SMAD}_{3}$ could be validated by PCR. dad

Increased production of MMP-1 and MMP-2 in fibroblasts and MMP-7 in tumor cells, as well as decreases in TIMP-1m RNA and protein in tumor associated fibroblast indicates their central role in invasion. The increased expression of laminin and its receptor $\mathrm{a} 6 \mathrm{~b} 4$ on cancer cells 
and fibronectin, as well as its receptors a5b1 on fibroblast suggests the regulatory effect of these matrix proteins on the behavior of cancer cells and fibroblasts. Certainly, migration of fibroblasts is facilitated by fibronectin, whereas tumor cells migrate for laminin. All these changes confer a proliferatory, migratory phenotype for the cervical cancer cells.

This work was supported by the National Science Found (OTKA) 100904.

\section{SO-K063 \\ Planning and design of tissue microarrays-application fields and data management}

\section{Tóth* ${ }^{*}$ R. Büttner}

Institute of Pathology, University Clinic Cologne, Cologne, Germany

Tissue microarray technology (TMA) refers to all procedures analysing multiple tissue samples on a single slide: hundreds of tissue samples can be assessed on a single microscopic slide. The main aim of TMA is to optimise the usefulness of paraffin tissue. TMA is widely used for formalin-fixed and parafffin-embedded tissue, but TMA can also be developed from fresh frozen tissue, fixed paraffinized, deparaffinized or needle biopsy material. The most frequently applied method is the immunohistochemistry (IHC) to evaluate protein expression as tissue biomarkers.

TMA provides a cost and time-efficient workflow to analyse a large number of patients. The pathologist has a critical part in the process: tumour or normal tissue will be encircled on HE stained sections, so in this step the quality of TMA is decided. IHC is very cost effective on TMA slides. Due to standardisation, further cooperation studies can be performed and more cohorts can be brought together.

However, TMA has some weaknesses and disadvantages. Technical skills and standardised laboratory reprocessing are critical for good TMA. Further, one of the quality limiting points is the quality of the tissue being arrayed. The major critical point of TMA is the problem of tissue heterogeneity and/or biomarker heterogeneity. This problem seems only theoretical, because many studies stated that there is a strong correlation between TMA cores and whole-tissue sections. The diameter of tissue core has a less pivotal role than the area that it covers. So far, there is no standardised operating protocol (SOP) or universal agreement for sampling and staining of TMA blocks and slides. The general consensus is that two $0.6 \mathrm{~mm}$ cores are adequately representative for immunohistochemical changes.

The role of TMA in translational research is indisputable. In one cohort validation process, studies can be performed and new potential markers can be tested and the correlation/relevance between known parameters and new information allows the rapid transfer to new therapy strategies. TMA technology has an important potential in retrospective studies based on formalin-fixed, paraffin embedded tissue, but it will also have an important role in prospective studies improving antibody research. TMA is a validated method of high-throughput analysis and used as a research tool for investigation of putative prognostic and predictive molecular targets in cancer research, especially in biobanking.

\section{SO-064}

Topo optical analysis of various amyloid deposits

\section{J. Makovitzky*}

Institute of Pathology, University Hospital Heidelberg, Heidelberg, Germany

Amyloid model is known to have a highly ordered structure: A glycoprotein core (AP) surrounded by helical structures of chondroitine and heparan sulfate. A filament network (AA, AL or others) constitutes the surface of the fibril. Here we studied paraffin sections of various mammalian tissues and ex vivo isolated fibrils. Sugar moieties in all amyloid deposits were visualized with the ABT-reaction, Sialic acid or O-acyl sialic acid. The glycosaminoglycan (GAG) components have been visualized in the amyloid deposits by the chemically intensified basophilic reaction (CIBR) and by the critical electrolyte concentration (CEC) method. The protein core was demonstrated with $0.1 \%$ aqueous solution of Congo red, Thiazine red, Eosin, Pinacyanol, Pinacyanol chloride, N,N'-Diethylspeudoisocyanine- chloride (PSI), Toluidine blue, 1,9-Dimethyl-methylene blue (1,9dmmb) and Rivanol. Unstained amyloid deposits showed an anisotropy. After specific staining we registered an additive topooptical reaction. After Toluidine blue and Rivanol staining, the sign of the anisotropy becomes linear negative, the dye molecules are oriented perpendicular to the surface. The latter is an inversive topo-optical staining reaction. Isolated fibrils show a similarly reaction. In mammalian amyloid we found strong acidic groups in linear order, but only weak acidic groups in avian amyloid (both AA). We observed a gradation of the topo-optical reaction intensity in murine and canine amyloid with changing polarization color from strong red orange at $0.1 \mathrm{M} \mathrm{MgCl} 2$ to hardly noticeable pink at 1.0 and $1.8 \mathrm{M} \mathrm{MgCl}_{2}$. Staining reaction appeared with stable strong positive results in cat, marten and cow, as well as in human amyloid. After enzyme e treatment we found an optimally oriented Congo red binding and more intensity compared to the controls. Differences in linearly ordered acidic groups indicate GAG components: Heparan and keratan sulphate are missing in avian amyloid. Mouse and dog exhibit all four components but with less intensity than cow, marten, cat and human amyloid. Polysaccharide chains are orientated parallel to the amyloid fibrils. Sialic- and O-acyl sialic acid was detectable with a perpendicular orientation of their $\mathrm{OH}$ groups in all samples. Sensitivity of AA amyloid for oxidation combined with enzymatic digestion varies between species.

\section{Sino-German Cooperation Meeting}

\section{SO-065}

An IL-6R/STAT3/miR-34a feedback loop controls EMT, invasion and metastasis of colorectal cancer

H. Hermeking ${ }^{* 1}$, M. Rokavec', M. Öner ${ }^{2}$, H. Li' ${ }^{2}$, R. Jackstadt ${ }^{2}$, J. Longchang', D. Lodygin ${ }^{3}$, M. Kaller', D. Horst' ${ }^{2}$, P. Ziegler', S. Schwitalla ${ }^{4}$, J. Slotta-Huspenina $^{5}$, F. Bader ${ }^{6}$, F. Greten 4

IInstitute of Pathology, Munich, Germany, ${ }^{2}$ Institute of Pathology, LudwigMaximilians-University, Munich, Germany, ${ }^{3}$ Institute for Multiple Sclerosis Research and Department of Neuroimmunology, Göttingen, Germany, ${ }^{4}$ Institute of Molecular Immunology, Klinikum rechts der Isar, Technical University Munich, Munich, Germany, ${ }^{5}$ Institute of Pathology, Technical University of Munich, Munich, Germany, ${ }^{6}$ Department of General Surgery, Klinikum rechts der Isar, Technical University Munich, Munich, Germany

Aims. The p53-induced microRNAs of the miR-34 family are known to inhibit epithelial-mesenchymal transition (EMT) and thereby presumably suppress the early steps of metastasis. Here we determined whether miR-34a is involved in the suppression of inflammation induced progression of intestinal tumors.

Methods. The functional studies were performed using colorectal cancer cell lines and induction of colitis in miR-34a knock-out mice. Expression analysis of components of the identified regulatory circuits was performed on tumor material obtained from cohorts of colorectal cancer (CRC) patients.

Results. Here, we show that exposure of CRC cells to the cytokine IL-6 activated the oncogenic $\mathrm{STAT}_{3}$ transcription factor, which then directly repressed the miR-34a gene via a conserved $\mathrm{STAT}_{3}$ binding site in the first intron. The repression of miR-34a was required for IL-6-induced EMT and invasion. Furthermore, we identified the IL-6 receptor (IL6R), which mediates STAT3 activation by IL-6, as a conserved, direct target of miR-34a. The resulting IL-6R/STAT $3 / \mathrm{miR}^{-34 a}$ feed-back loop 
was manifest in primary CRC as well as $\mathrm{CRC}$, breast and prostate cancer cell lines with a mesenchymal phenotype. An active IL-6R/STAT3/ miR-34a loop was necessary for EMT, invasion and metastasis of CRC cell lines and was associated with nodal and distant metastasis in CRC patient samples. Activation of p53 interfered with IL-6-induced invasion and migration by down-regulation of IL-6R expression in a miR34a-dependent manner. In miR-34a-deficient, colitis-associated intestinal tumors displayed up-regulation of p-STAT3, IL-6R, and SNAIL expression and progressed to invasive carcinomas, which could not be observed in wild type mice.

Conclusions. Collectively, the $\mathrm{p} 53 / \mathrm{miR}-34 \mathrm{a}$ axis suppresses tumor pro-

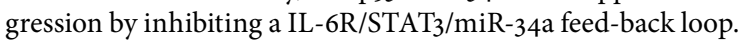

\section{SO-066}

\section{Chk1 circumvents checkpoint control and remodels chromatin,} promoting cell cycle progression in hydrogen peroxide-associated colitis

\section{K. Reissig ${ }^{* 1}$, T. Guenther ${ }^{2}$, A. Silver ${ }^{3}$, A. Schinlauer', R. Hartig', S. Boettcher', A. Roessner', A. Pöhlmann' \\ ${ }^{1}$ Institute of Pathology, University Hospital, Otto-von-Guericke-University, Magdeburg, Germany, ${ }^{2}$ Academic Department of Histopathology, St. Mark's Hospital, Harrow, Middlesex, United Kingdom, ${ }^{3}$ Colorectal Cancer Genetics, Queen Mary University, London, United Kingdom, ${ }^{4}$ Institute of Molecular and Clinical Immunology, University Hospital, Otto-von-Gueri- cke-University, Magdeburg, Germany}

Aims. Oxidative stress is a main risk factor of inflammatory bowel disease (IBD)-associated neoplasia, and the production of hydrogen peroxide $\left(\mathrm{H}_{2} \mathrm{O}_{2}\right)$ drives tumorigenesis in ulcerative colitis (UC). Our recently developed in vitro model of $\mathrm{H}_{2} \mathrm{O}_{2}$-associated colitis in human colonic epithelial cells (HCEC) showed phospho-JNK-dependent induction of DNA damage checkpoints following oxidative stress. We aimed to unravel the underlying mechanisms in changed DNA damage checkpoint control. Therefore, we focused on checkpoint kinase 1 (Chk1) to be responsible for survival and enhanced proliferation of developed HCEC due to its known role in DNA damage checkpoint control and chromatin remodeling.

Methods. Acute and chronic inflammation was mimicked by treating HCEC either with single (treated HCEC) or repeated boli (C cell cultures) of $\mathrm{H}_{2} \mathrm{O}_{2}$. DNA damage checkpoint proteins were investigated by Western blot analyses following Chkı siRNA transfection. Cell cycle progression and apoptosis were studied via FACS. To analyze chromatin remodeling in vitro (HCEC, $\mathrm{C}$ cell cultures) and in vivo (ulcerative colitis (UC)), samples were subjected to subcellular fractionation.

Results. Chk1 negatively regulates DNA damage checkpoint proteins via phospho-JNK suppression as we found JNK activation following Chk1 knockdown in treated HCEC and upregulation of p21WAF1 and $\gamma-\mathrm{H}_{2} \mathrm{AX}$, resulting in checkpoint activation. In $\mathrm{C}$ cell cultures, enhanced proliferation, despite DNA damage, was the consequence of checkpoint override, and could be attributed to Chk1. Concept proof by Chk1 knockdown in $\mathrm{C}$ cell cultures showed decreased proliferation. Investigation of Chkis role in chromatin remodeling showed accumulation of Chk1 and acetylated histones on chromatin, which would explain oncogene induction. Accumulated Chk1 on chromatin of UC samples suggested the importance of this pathway in vivo.

Conclusions. Enhanced proliferation, despite DNA damage, is a consequence of Chk1-dependent suppression of phospho-JNK and the resultant circumvention of checkpoint control. Furthermore, Chk1 remodels chromatin in $\mathrm{C}$ cell cultures through its increased binding. The resultant elevated association of acetylated histones may cause oncogene induction through enhanced binding of transcription factors. These results could be approved in vivo. In summary, the promotion of cell cycle progression improves survival and proliferation in $\mathrm{H}_{2} \mathrm{O}_{2}$ associated colitis.

\section{SO-067}

Major histocompatibility complex class I expression in biliary tract cancer-prognostic significance and correlation with tumor-infiltrating immune cells

\section{B. Goeppert ${ }^{* 1}$, L. Frauenschuh', M. Zucknick', S. Roessler', A. Stenzinger',} M. Andrulis', K. Joehrens' ${ }^{3}$, F. Klauschen ${ }^{3}$, A. Warth', M. Renner', A. Mehrabi ${ }^{4}$, M. Hafezi ${ }^{5}$, P. Schirmacher', W. Weichert ${ }^{\text {? }}$

IInstitute of Pathology, University Hospital Heidelberg, Heidelberg, Germany, ${ }^{2}$ Department of Biostatistics, Heidelberg, Germany, ${ }^{3}$ Institute of Pathology, Charité University Hospital, Berlin, Germany, ${ }^{4}$ Department of Surgery, Ruprecht-Karls-University, Heidelberg, Germany, ${ }^{5}$ Department of Surgery, University Hospital Heidelberg, Heidelberg, Germany

Aims. Biliary tract cancers (BTC) are relatively rare malignant tumors with poor prognosis. Previously, we have presented a detailed characterization of the inflammatory infiltrate in BTC. Here, we analyzed the expression of major histocompatibility complex class I (MHC I) on biliary tract cancer cells by immunohistochemistry and performed correlation analyses with tumor-infiltrating immune cells types, clinicopathological data, and patient survival.

Methods. MHC I expression was assessed by immunohistochemistry in a cohort of 375 BTC including extrahepatic (ECC; $n=157$ ), intrahepatic (ICC; $n=149$ ) and gallbladder (GBAC; $\mathrm{n}=69$ ) adenocarcinomas. MHC I expression on tumor cells was assessed by defining three expression categories (low-moderate-strong). Results were correlated with tumor-infiltrating T lymphocytes (CD4+, CD8+, Foxp3+), NK-cells (Perforin+), B lymphocytes (CD20+), macrophages (CD68+), and mast cells $(\mathrm{CD} 117+)$. Data were correlated with clinicopathological variables and patient survival.

Results. MHC I expression correlated significantly with survival of BTC patients. Patients with strong MHC I expression on tumor cells showed a significantly longer overall survival probability, while low MHC I expression correlated with a shorter patient survival $(\mathrm{p}=0.029)$. Correlation analyses with the quantity of specific tumor-infiltrating immune cells showed a positive correlation of MHC I expression and the total number of tumor-infiltrating T-lymphocytes (CD4+ and CD8+) and CD68+ macrophages, but not with CD2O+ B-lymphocytes.

Conclusions. Our results characterize the expression of MHC I and its prognostic impact on BTC patients. Correlation analyses with exact numbers of tumor-infiltrating immune cells indicate that tumoral expression of antigen presenting molecules and tumor associated inflammation might be of importance in the progression of BTC and ultimately have an influence on the outcome of BTC patients.

\section{SO-068}

KRAS, BRAF mutation and microsatellite instability status in chinese colorectal cancer patients

\section{J. Chen*, M. Ye, E. Xu, Y. Fu, Q. Huang, F. Chen, M. Lai}

Institute of Pathology, School of Medicine, Zhejiang University, Hangzhou, China

Aims. Colorectal cancer (CRC) has been one of the most common gastrointestinal malignancies in the world. The use of cetuximab and other target therapy drugs were more effective than before in the treatment of CRC. It will be of great clinical and social significance to find out the predictive biomarker and individual therapy. The KRAS and BRAF mutation play an important role in prediction of targeted therapy and microsatellite instability (MSI) is also an effectively predicted marker for chemotherapy outcome and post operative recurrence.

Methods. 717 sporadic Chinese colorectal cancer samples were selected and confirmed by clinical and pathological diagnosis from affiliated hospitals of Zhejiang University, the tumor area was located at HE slides from formalin-fixed paraffin-embedded (FFPE) blocks, the stroma and other non-cancer contents were removed by macrodissection, then 
tumor DNA was obtained by QIAGEN FFPE kit. 12,13 and 61 codon in KRAS and 600 codon in BRAF were determined by routine PCR and pyrosequencing. Meanwhile, isolated DNA from tumor and matched normal tissues were detected by five microsatellites sites named BAT25,BAT26, $\mathrm{D}_{2} \mathrm{~S}_{123}, \mathrm{D}_{5} \mathrm{~S}_{34} 6$ and $\mathrm{D}_{17} \mathrm{~S}_{25}$ o by FM-CE.

Results. In 717 CRC patients samples, 216 mutation cases of KRAS 12 codon were identified. Among them, 98 for $35 \mathrm{G}>\mathrm{A}$ mutation (13.7\%), 84 for $35 \mathrm{G}>\mathrm{T}$ mutation $(11.7 \%), 15$ for $34 \mathrm{G}>\mathrm{T}$ mutation $(2.1 \%), 14$ for were $34 \mathrm{G}>\mathrm{A}$ mutation (2.0\%), 4 for $35 \mathrm{G}>\mathrm{C}$ mutation (o.6\%) and 1 for $34 \mathrm{G}>\mathrm{C}$ mutation were detected in KRAS codon 12 mutation cases. Of the 70 KRAS 13 codon mutation cases, 57 for $38 \mathrm{G}>\mathrm{A}, 5$ for $37 \mathrm{G}>\mathrm{A}, 5$ for $37 \mathrm{G}>\mathrm{T}$ and $338 \mathrm{G}>\mathrm{T}$ were detected by pyrosequencing. Additionally, 6 for $182 \mathrm{~A}>\mathrm{T}$ and 1 for $183 \mathrm{~A}>\mathrm{T}$ were identified in KRAS 61 codon mutation status. In the same case group, 52 for $1799 \mathrm{~T}>\mathrm{A}$ were tested in BRAF 600 codon mutation. MSI status were detected in CRC patients from the same group, among 428 cases, 73 for MSI-H, 63 for MSI-L and 295 for MSS were identified by FM-CE. When clinical data were statistically analysed, KRAS mutation was significantly associated with age gender and cancer site, while it is no relationship between BRAF mutation and clinical features. MSI is associated with tumor sites, tumor size, lymph node metastasis status and TNM stage in CRC patients. There was no association between KRAS and BRAF gene mutations and MSI.

Conclusions. In our study, MSI status is correlated with prognosis in $\mathrm{CRC}$ patients. Based on the present data in our research, the prognostic evaluation of KRAS mutation is uncertain.

\section{SO-069 \\ Modulation of tumor-infiltrating immune cells in hepatocellular carcinoma}

\section{N. Waldburger*, B. Straub, P. Schirmacher, S. Roessler} Institute of Pathology, Heidelberg, Germany

Aims. Hepatocellular carcinoma (HCC) is the third leading cause of cancer death worldwide. Due to high genomic instability, HCC is very heterogeneous in its clinical presentation and genetic makeup. An integrative genomic and transcriptomic approach identified two novel tumor suppressor genes, $\mathrm{SORBS}_{3}$ (Vinexin) and $\mathrm{SH}_{2} \mathrm{D}_{4} \mathrm{~A}$ on chromosome $8 p$, which are preferentially deleted in patients with poor outcome. In addition, both genes are associated with cytokine immune signaling suggesting their influence on the tumor microenvironment. In multiple solid tumors including cancers of colon, ovary, kidney and bladder, it has been shown that the antitumor inflammatory response has an impact on tumor behavior and patient outcome. In this study, we aimed at linking $\mathrm{SORBS}_{3}$ and $\mathrm{SH}_{2} \mathrm{D}_{4} \mathrm{~A}$ expression with tumor-infiltrating immune cells.

Methods. $\mathrm{CD}_{3}$ and $\mathrm{CD} 8$ expressing tumor-infiltrating immune cells were immunohistochemically determined on tissue microarrays including 127 HCC specimens with comprehensive pathological and clinical data including survival. Additionally, $\mathrm{SORBS}_{3}$ and $\mathrm{SH}_{2} \mathrm{D}_{4} \mathrm{~A}$ expression was assessed on the same tissue microarrays. Correlations were analyzed using Pearson's correlation coefficients. Prognostic impact was evaluated using the Kaplan-Meier method and Cox regression model.

Results. We found that patients with SORBS 3 -negative HCC had a higher risk to die compared to the population with SORBS3-positive tumor cells [Hazard ratio (HR) 1.89, 95\% CI 1.07-4.16). In contrast, $\mathrm{SH}_{2} \mathrm{D}_{4} \mathrm{~A}$ was not significantly associated with outcome in our collective and the median density of $\mathrm{CD}_{3}+$ or $\mathrm{CD} 8+$ infiltrating T-lymphocytes did not correlate with patient outcome. In addition, $\mathrm{SH}_{2} \mathrm{D}_{4} \mathrm{~A}$ was positively correlated with the presence of $\mathrm{CD}_{3}+$ and $\mathrm{CD} 8+$ tumor infiltrating immune cells confirming a potential role of $\mathrm{SH}_{2} \mathrm{D}_{4} \mathrm{~A}$ in immune cell infiltration.

Conclusions. The density of tumor infiltrating CD8+ T-lymphocytes in HCC in situ did not show an impact on patient outcome in our patient collective. HCCs with loss of $\mathrm{SH}_{2} \mathrm{D}_{4} \mathrm{~A}$ expression exhibited decreased numbers of $\mathrm{CD} 8+$ cytotoxic T-lymphocytes which have been indica- ted in anti-tumor activity. This suggests that HCC patients without $\mathrm{SH}_{2} \mathrm{D}_{4} \mathrm{~A}$ expression demonstrate a modified tumor immune status. Therefore, further characterization of the tumor-infiltrating immune cell populations may add to our understanding of the HCC tumor immune cell interaction.

\section{SO-070}

Low expression of miR-150 in pediatric intestinal Burkitt lymphoma

\section{Wang ${ }^{* 1}$, W. Yang ${ }^{2}$, M. Li $i^{3}$, Y. $L i^{i}$}

'Department of Pathology, Basic Medical College, Capital Medical University, Beijing, China, ${ }^{2}$ Department of Pathology, Jiangxi Children's Hospital, Jiangxi, China, ${ }^{3}$ Department of Pathology, Peking University Health Science Center, Beijing, China

Aims. Burkitt lymphoma (BL) is a highly aggressive B-cell lymphoma with rapid proliferation. It has become evident that miRNAs are involved in hematopoietic malignancies. This study was undertaken to investigate the miRNA expression patterns of pediatric intestinal BL tissues.

Methods. We collected 28 BL patients and 8 reactive lymphoid hyperplasia (RLH) samples. miRNA expression profiling was performed in BL and RLH tissues to identify BL-related miRNAs, which was further analyzed by qRT-PCR and miRNA-ISH. In addition, immunohistochemistry (IHC) was used to define the protein targets of the BL-related miRNAs. Furthermore, we evaluated cell growth status by using Methylthiazolyldiphenyl-tetrazolium bromide (MTT) assay in Raji cell line, which was transected with the BL-related miRNA mimics or inhibitors. Results. MiRNA expression profiling showed that miR-15o had an extremely decrease expression levels in pediatric intestinal BL patients. By using both miRNA in situ hybridization (ISH) and quantitative realtime PCR (qRT-PCR) analysis, pediatric intestinal BL had significantly reduced levels of miR-15o expression compared with RLH ( $\mathrm{p}<0.05)$. However, there is no significant correlation of miR-15o expression and EBV status in pediatric intestinal BL cases ( $p>0.05)$. Moreover, immunohistochemistry (IHC) defined that c-myb and survivin are the protein targets of miR-15o. Furthermore, we evaluated cell growth status by using Methylthiazolyldiphenyl-tetrazolium bromide (MTT) assay in Raji cell line, which was transected with the miR-150 mimics. Re-expression of miR-15o reduced proliferation of Raji cells.

Conclusions. Taken together, pediatric intestinal BL showed an extremely low expression of miR-150 compared with RLH. Re-expression of miR-15o reduced proliferation of Raji cells, which suggest that miR-150 could provide new insights into novel targeted therapeutic strategies in pediatric intestinal BL.

\section{SO-071}

Prostatitis related mitogenic stimuli cause loss of NKX3.1: increased risk for prostate cancer?

\section{J. Decker*, P. Harazim, G. Jain, T. Kießling, P. Möller, R. Marienfeld}

\section{Institute of Pathology, University Ulm, Ulm, Germany}

Aims. $\mathrm{NKX}_{3.1}$ is a prostate cancer suppressor shown to be reduced in the majority of primary human prostate cancers and prostate intraepithelial neoplasia (PIN). Further, a $\mathrm{NKX}_{3.1}$ knock out mouse model displayed a higher incidence of PIN formation. Thus, it is hypothesized that the $\mathrm{NKX}_{3.1}$ reduction might be involved in the initiation of prostate cancer. However, the molecular basis of the initial $\mathrm{NKX}_{3.1}$ loss is still a matter of debate. The aim of this study was to clarify whether prostatitis might be a cause for this $\mathrm{NKX}_{3.1}$ loss and therefore represent a risk for prostate cancer.

Methods. $\mathrm{NKX}_{3.1}$ expression in prostatitis, prostate cancer and normal prostate tissue was analyzed by immunohistochemistry. PCa cell lines were stimulated with inflammation-linked cytokines and growth fac- 
tors and $\mathrm{NKX}_{3.1}$ or AR expression was determined by western blot and qPCR. Expression levels of $\mathrm{NKX}_{3.1}$ and AR target genes were estimated by qPCR.

Results. We observed a reduction of $\mathrm{NKX}_{3.1}$ in areas of prostates which are positive for B cell and MPS markers $\mathrm{CD}_{20}$ and CD68. Stimulation of PCa cell lines with the epidermal growth factor (EGF) caused a distinct reduction of $\mathrm{NKX}_{3.1}$ protein and mRNA expression. Similar to EGF stimulation a treatment with phorbol-12-myristate-13-acetate (P) and calcium ionophor Ionomycin (I) caused a dramatic reduction of $\mathrm{NKX}_{3.1}$ protein as well as mRNA level. $\mathrm{NKX}_{3.1}$ target genes were found to be derepressed upon EGF or $\mathrm{P}+\mathrm{I}$ stimulation while androgen receptor mRNA expression was found to be decreased. EGF or P+I induced $\mathrm{NKX}_{3.1}$ loss in PCa cell lines appears to depend on two different mechanisms. While the decrease of $\mathrm{NKX}_{3.1}$ mRNA levels largely remained unaffected upon pretreatment with MG132 or Bortezomib (Bo), the stabilization of $\mathrm{NKX}_{3.1}$ protein after co-treatment with $\mathrm{EGF} / \mathrm{P}+\mathrm{I}$ and MG132 or Bo suggests the participation of the proteasomal pathway in this process. In addition, the decline in $\mathrm{NKX}_{3.1} \mathrm{mRNA}$ levels appears to be independent from a reduced $\mathrm{NKX}_{3.1}$ promoter activity as determined by luciferase reporter assays.

Conclusions. Taken together our results imply that mitogenic stimuli originated during an inflammatory process might be the cause for reduced $\mathrm{NKX}_{3.1}$ levels in luminal prostate epithelial cells and thus could be the connection between prostatitis and prostate cancer initiation.

\section{SO-072}

\section{Double CD19/CD79a positive t-cell acute lymphoblastic leukemia}

\author{
W. Zhang ${ }^{* 1}, K . F U^{2}$, J. YUAN' ${ }^{2}$ L. LIU' ${ }^{2}$, W. LIU', S.J. Pirruccello ${ }^{2}$ \\ 'West China Hospital, Department of Pathology, Sichuan University, \\ Chengdu, China, ${ }^{2}$ Department of Pathology and Microbiology, University \\ of Nebraska Medical Center, Omaha, United States
}

Aims. We report a rare case of double CD19/CD79a positive $\mathrm{T}$-cell acute lymphoblastic leukemia (T-ALL).

Methods. Morphology, immunophenotype and antigen receptor gene rearrangement were observed.

Results. A 32-year-old man presented with a 2-month history of difficulty swallowing and weight loss. Bilateral supraclavicular lymphadenopathy, a large mediastinal mass, bilateral pleural effusion and pericardial effusion were noted on physical examination and chest X-ray. Cells isolated from the mediastinal mass, left subclavicular lymph node and bone marrow $(B M)$ were positive for cytoplasmic $C_{3}$ and surface $C_{5}$, $\mathrm{CD}_{7}, \mathrm{CD}_{10}, \mathrm{CD}_{19}, \mathrm{CD}_{34}, \mathrm{CD}_{3} 8$ and $\mathrm{CD}_{79}$ a by immunostaining and/or flow cytometry. The cells were also positive for nuclear TdT but were negative for $\mathrm{CD}_{2}, \mathrm{CD}_{4}, \mathrm{CD} 8$ and $\mathrm{CD}_{2} \mathrm{O}$. Surface immunoglobulin light chain expression was absent and the immunoglobulin gene rearrangement study was negative. However, the cells were positive for oligoclonal biallelic T-cell receptor gamma locus (TCRG) gene rearrangements. Conclusions. CD79a-positive T-ALL has been reported often, but CD19positive T-ALL is extremely rare. In this case, antigen receptor gene rearrangement studies were highly informative for correct lineage assignment.

\section{SO-073}

Redoxregulation as a risk factor of carcinogenesis in chronic inflammation

\section{F. Lasitschka*1, C. Leowardi-Bauer², M. Kadmon'2, P. Schirmacher'}

'Institute of Pathology, University Hospital Heidelberg, Heidelberg, Germany, ${ }^{2}$ Department of Surgery, University Hospital Heidelberg, Heidelberg, Germany

Ziel. Die individuelle Prognoseabschätzung der malignen Entartung bei Patienten mit Colitis ulcerosa bzw. Morbus Crohn ist bislang nur in sehr begrenztem Rahmen möglich. Daher ist die Etablierung von zuverlässigen und in der Praxis leicht bestimmbaren prädiktiven Tumormarkern eine vordringliche Aufgabe der gastroenterologischen Pathologie. Es gibt Hinweise aus Tiermodellen, dass gewebsassoziierte Entzündungsmediatoren die Entstehung und Progression solcher Tumoren wesentlich beeinflussen.

Methoden. Im Rahmen des hier durchgeführten Projektes wurden sowohl mittels kompartimentspezifischer Expressionsanalyse molekularen Mechanismen der chronischen Entzündung, die zur Malignomentstehung beitragen identifiziert und diese mit bereits vorhandenen Genexpressionsdaten von chronisch entzündlichen Darmerkrankungen korreliert, als auch die prognostische Relevanz dieser entzündungsassoziierten Mediatoren und insbesondere des Transporterproteins xCT bezüglich Tumorwachstum, Metastasierung und Überlebensdauer analysiert.

Ergebnisse. Die immunhistochemisch bestimmbare xCT-Expression in kolorektalen Karzinomen zeigt einen Unterschied zwischen sporadischen und entzündungsassoziierten kolorektalen Karzinomen. Die xCT-Expressionsmuster lassen sich mit etablierten molekularen prognostischen Faktoren bei kolorektalen Karzinomen korrelieren.

Schlussfolgerung. Die differenzielle Expression von xCT in der entzündungsvermittelten Karzinogenese ist ein wichtiger Faktor der Tumorprogression.

\section{Dialog zur Zytopathologie}

\section{FR-K071}

\section{Cytology and histology: competitors or dream team?}

\section{Bubendorf*}

Institute for Pathology, University Hospital Basel, Basel, Switzerland

It is undisputed that both histology and cytology serve the same purpose, which is achieving an accurate diagnosis to guide further treatment or patient management. Cytology material is obtained in a non-invasive or less invasive manner than histological material and reveals more cellular and nuclear details than histology. On the other hand, a definitive diagnosis of a disease that relies mainly on histoarchitectural features may not be achieved by cytology alone including follicular carcinoma of the thyroid, some lymphomas and sarcomas. In many diagnostic procedures, both cytological and histological specimens are obtained. Considering both cytological and matched histological findings can greatly improve the diagnostic yield both in a neoplastic and non-neoplastic context, as illustrated by the following examples: bronchoalveolar lavage in conjunction with transbronchial biopsies gives a clearer picture than either one alone in interstitial lung diseases, and allows to better diagnose or exclude opportunistic infections if combined with special stains and techniques. In case of lung cancer, ultrasound-guided transbronchial fine-needle aspirates are superior to transbronchial for staging of mediastinal lymph nodes. Similarly, urothelial carcinoma in situ that may be invisible by cystoscopy can easily be detected by bladder or pelvic washes. Voided urines complement cystoscopy and bladder washings in patients under surveillance by covering the upper urinary tract. Unfortunately, cytology and histology laboratories are often physically separated, which makes such added diagnostic value difficult if not impossible. Ideally, not only the laboratories but also the individual pathologists and cytopathologists tightly interact on a daily basis. In the field of lung cancer, it has been recommended that even the same person should evaluate both biopsies and cytologies whenever possible, not only to optimize the diagnostic yield, but also to prioritize all available material for predictive marker testing. It is now broadly accepted that almost all molecular analyses used on histological material are also applicable to cytological specimens provided that appropriate validation and quality control is in place. Importantly, continuous feedback 
by histology continuously increases the diagnostic skills in cytology. In summary, historical fences can safely be thorn down to unify the dream team of cytology and histology where they have not already met.

\section{FR-072}

\section{Scope of molecular diagnostics on cytological specimens}

\author{
L. Heukamp ${ }^{* 1,2,3}$ \\ 'Lung Cancer Group Cologne, University Cologne, Cologne, Germany, \\ ${ }^{2}$ Center for Integrated Oncology Cologne/ Bonn, University Cologne, \\ Cologne, Germany, ${ }^{3}$ Institute of Pathology, University Hospital Cologne, \\ Cologne, Germany
}

Personalized medicine based on patient stratification for targeted therapies using molecular analyses has proven to be of clinical benefit in hematological as well as solid tumors. The discovery of targetable mutations in the epidermal growth factor receptor (EGFR) gene and rearrangements the anaplastic lymphoma kinase (ALK) has led to a remarkable improvement in overall survival in lung cancer patients.

So far mutation analysis for solid tumors from FFPE tissue was based mainly on single PCR followed by Sanger sequencing which is both labor and cost intensive and limited by the amount of available DNA and sensitivity of Sanger sequencing.

These techniques are also available to cytological specimens, however limited tissue and sensitivity has been a challenge.

With the advent of bench top devices for next generation sequencing (NGS) we have reduced the time required for comprehensive molecular diagnostics and to minimize the amount of required input DNA while at the same time increasing the number of target regions analyzed in routine diagnostics. This technology is very well suited to analyze cytological specimens. Scope and limitations of NGS analysis on cytology specimens will be discussed.

\section{FR-073}

\section{Molecular diagnostics without tissues and cells? Liquid biopsy}

E. Dahl*

Institute of Pathology, University Hospital, RWTH Aachen University, Aachen, Germany

Aims. Liquid biopsy refers to the molecular analysis of DNA usually drawn from blood for the early detection, diagnosis or treatment stratification of diseases such as cancer. An important future application of liquid biopsy may be the early detection of drug resistance associated mutations emerging after targeted therapy of cancer patients.

Methods. Cancer patients exhibit increased numbers of circulating tumor cells (CTCs) and cell free DNA (cfDNA) of tumor origin (also known as ctDNA) in their blood. Modern detection procedures allow exact measurement of these rare blood analytes. The two main procedures used are beaming PCR and next generation sequencing (NGS). Both techniques are capable of detecting mutations with frequencies far below 1 in 1000 molecules.

Results. Counting of CTCs has been established as a surrogate marker for survival prognosis in patients with metastatic tumors. However, molecular examination of CTC-DNA to adapt personalized cancer therapy is still in its infancy. Though ctDNA can be detected in most tumors, its frequency varies considerably between different tumor entities and tumor stages. Some studies have detected known driver mutation in cfDNA with high specificity (>95\%) and acceptable sensitivity (>85\%). However, false-positive ctDNA results (i.e. a known tissue mutation was not detectable in cfDNA) may be associated with histopathological parameters such as mucinous tumor histology.

Conclusion. Some of the recent studies suggest that analysis of cfDNA may be a useful biomarker in cancer surveillance. Whether tumor recurrence or resistance is more efficiently detectable by cfDNA analysis than by current imaging techniques will be settled after this potential application has been validated in prospective studies. Currently a major problem is the lack of knowledge on the origin, stability and degradation of cfDNA in different clinicopathological conditions and how these parameters may affect analysis. CTCs on the other hand have clear limitations due to their rareness. Furthermore, the number of methods used for CTC isolation are numerous and not sufficiently standardized. Thus currently, a representative molecular analysis of CTCs does not appear possible for the majority of patients. Quality management of tissue based molecular diagnostics is well established in Germany. Due to the current limitations of cfDNA and CTC analysis, it will be a real challenge to reach a similar level of reproducibility in liquid biopsy diagnostics.

\section{AG Gastroenteropathologie - Oberer GI-Trakt}

\section{DO-001}

Tumor regression grading of esophageal adenocarcinomas after neoadjuvant treatment-comparison of two commonly used histopathologic grading systems

\section{E. Diamantis-Karamitopoulou', S. Thies', I. Zlobec', K. Ott' ${ }^{2}$,M. Feith ${ }^{3}$ J. Slotta-Huspenina ${ }^{4}$, K. Becker', R. Langer*1}

IInstitute of Pathology, University of Bern, Bern, Switzerland, ${ }^{2}$ Department of Surgery, Ruprecht-Karls-University, Heidelberg, Germany, ${ }^{3}$ Department of Surgery, Technical University of Munich, Munich, Germany, ${ }^{4}$ Institute of Pathology, Technical University of Munich, Munich, Germany

Aims. Multimodal treatment has been successfully implemented for the therapy of locally advanced gastroesophageal carcinomas. The determination of tumor regression by histopathology provides important prognostic information. Tumor regression grading (TRG) systems which categorize the degree of regressive changes mostly refer onto the amount of therapy induced fibrosis in relation to residual tumor (e.g. Mandard system) or the estimated percentage of residual tumor in relation to the former tumor site (e.g. Becker, Schneider or Chirieac System). Currently there is no common standard for reporting tumor regression in gastrointestinal cancers and it is a matter of debate, which system may provide better results in terms of interobserver agreement or prognostic value. In this study we compared the application of these two major principles for assessment of TRG on esophageal adenocarcinomas after neoadjuvant chemotherapy.

Methods. Haematoxylin-Eosin stained slides from 89 resection specimens were independently investigated by three pathologists from different institutions. TRG was determined by the Mandard ( 5 TRGs; relation fibrosis/tumor) and the Becker system (4 TRGs; residual tumor in \%).

Results. Interobserver agreement (weighted kappa) for the Becker system was 0.78 and 0.62 for the Mandard system. Evaluation of the whole embedded tumor site/bed showed improved results (Becker: o.83; Mandard: 0.73) as compared with only one representative slide (Becker: 0.68; Mandard: 0.71). Both systems had prognostic impact for overall survival (log rank Becker: $\mathrm{p}=0.047$; Mandard: $\mathrm{p}=0.053$ ). Modification into a three-tiered system showed comparable interobserver agreement but better prognostic stratification for both systems (log rank Becker: $\mathrm{p}=0.015$; Mandard $\mathrm{p}=0.03$ ). This modified TRG was also an independent prognostic factor for overall survival adjusting for lymph node involvement and resection status [modified Becker: $\mathrm{p}=0.007, \mathrm{HR}=2.87$ (95\% CI 1.3-6.4); modified Mandard: $\mathrm{p}=0.016, \mathrm{HR}=2.58$ (95\% CI 1.2-5.8)]. Conclusions. Both TRG systems provide substantial to excellent interobserver agreement for estimation of tumor regression after neoadjuvant chemotherapy. A simplified three-tiered system (complete regression $/ 1-50 \%$ residual tumor $/>50 \%$ residual tumor) reveals the best reproducibility and prognostic value. In line with the results of other 
studies tumor regression grading according to this system can be recommended for every standard pathology report of esophageal adenocarcinomas following neoadjuvant therapy.

\section{DO-002 \\ Mechanisms of HDAC inhibitors and Azacytidine for cancer cell selective therapy of the esophagus}

T.D. Ahrens ${ }^{* 1}$, S. Timme', J. Hoeppner ${ }^{2}$, J. Ostendorp' ${ }^{1}$, M. Follo ${ }^{3}$, U.T. Hopt ${ }^{2}$, M. Werner', S. Lassmann

'Department Pathology, University Medical Center Freiburg, Freiburg, Germany, ${ }^{2}$ Clinic for General and Visceral Surgery, University Medical Center, Freiburg, Germany, ${ }^{3}$ Clinic for Hematology, Oncology and Stem Cell Transplantation, Freiburg, Germany

Aims. Epigenetic alterations influence carcinogenesis and progression of esophageal squamous-cell carcinomas (ESCC) and Barrett's adenocarcinomas (BAC). Here, we analyzed the efficacy of targeting epigenetic modifiers in esophageal cancer (EC) cells in vitro and expression of epigenetic modifiers in EC tissues.

Methods. FFPE tissue specimens of ESCCs and BACs $(n=20)$ were examined by immunohistochemistry for HDAC1-3, histone $\mathrm{H}_{3}$ acetylation, DNMT1 and DNA methylation (DNA-me). In vitro, OE21 (ESCC) and $\mathrm{OE}_{33}$ (BAC) cells were compared to non-neoplastic Het-1A cells. Three different HDAC inhibitors (HDACi;SAHA/MS-275/FK228) and Azacytidine (AZA) were tested alone and in combination with examination of HDAC inhibition ( $\mathrm{H}_{3}$-acetylation/activity assay), cell viability (MTS-assay) and cell cycle (p21 and Ki-67levels/S-phase).

Results. Compared to normal esophageal squamous epithelium (NESE), HDAC1-3 expression was maintained in $80 \%$ of ECs. However, histone acetylation was reduced in $60 \%$ of all cases, especially in ESCCs (80\%), suggesting HDAC hyper-activity. DNMT1 levels were variable in NESE as well as in ECs. But ECs tended to show a complete loss of DNMT1 (40\%) compared to NESE (15\%). DNA-me was frequently reduced (6o\%) or lost (40\%) in ECs compared to NESE (reduced: $40 \%$; lost: $0 \%)$. In vitro, all three cell lines showed inhibition of HDAC activity and subsequent histone $\mathrm{H}_{3}$ hyper-acetylation as well as p21 upregulation (mRNA/ protein). However, only EC cell lines ( $\left.\mathrm{OE}_{21} / \mathrm{OE}_{33}\right)$ exhibited reduced cell viability, especially for combination of MS-275/AZA. In addition, analysis of S-Phase revealed accumulation of S-phase cells in Het-1A, but reduction in $\mathrm{OE}_{21}$ cells for all $\mathrm{HDACi} / \mathrm{AZA}$ combinations. In $\mathrm{OE}_{33}$ cells, a decrease of S-Phase cells was detectable only for MS-275/AZA. This was in line with cell proliferation (=Ki-67 levels): Het-1A cells displayed no changes after HDACi/AZA, whereas $60 \%$ of OE21 cells showed a loss. For OE 33 cells, Ki-67 reduction (47\%) was only observed for MS-275/AZA.

Conclusions. Analysis of FFPE tissues indicated hyper-activity of HDACs and disturbed DNA-me in ECs tissues, suggesting HDACi/ AZA as attractive novel therapeutic approach. This is underlined by our in vitro data: non-neoplastic and cancer cells showed basic responses (e.g. histone hyper-acetylation) to HDACi/AZA, but only cancer cell lines showed reduced cell viability. Thus, HDACi/AZA act cancer-cell selective and are attractive for further comprehensive investigations in EC therapy.

Funded by DFG SFB992/C3 Project.

\section{DO-003 \\ Putative tumor biological and prognostic value of integrins in gastric cancer}

\section{Böger*, V.S. Warneke, H.-M. Behrens, C. Röcken}

Department of Pathology, Christian-Albrechts-University, Kiel, Germany

Aims. We investigated the expression of two $\alpha v$-integrins, $\alpha v \beta_{3}$ and $\alpha v \beta_{5}$, in gastric cancer (GC) testing the following hypotheses: are these molecules expressed in GC, are they putatively involved in GC biology and are they prognostically relevant.

Methods. Tissue micro arrays were generated from formalin-fixed and paraffin embedded tissue samples of 482 GCs. Immunostaining was done with antibodies directed against $\alpha v \beta_{3}$ (EM22703) and $\alpha v \beta_{5}$ (EMo9902). Immunostaining of tumor, stroma and endothelial cells was evaluated separately by quantity and intensity (tumor and stroma cells) or intensity (endothelial cells), generating an immunoreactivity score (IRS). The IRS of each antibody was dichotomized by the median, and patients were classified as "negative“ or „positive“. The expression of both antibodies was correlated with clinicopathological patient characteristics (gender, tumor localisation, Laurén phenotype, TNM-classification, tumor differentiation) and patient survival.

Results. Both integrins, i.e. $\alpha v \beta_{3}$ and $\operatorname{\alpha v} \beta_{5}$, were expressed in at least one tumor component in all GCs. There were significant correlations between a positive $\alpha v \beta_{3}$-status and clinicopathological patient characteristics, such as a minor pT-category, a minor UICC-/ „Kiel“-stage and a better tumor grading $\left(\mathrm{Gl}_{1} / \mathrm{G}_{2}\right)$. Furthermore, the difference regarding the expression of $\alpha v \beta_{3}$ and $\alpha v \beta_{5}$ within the tumor subtypes according to Laurén was striking: A positive $\alpha v \beta_{3}$ - and $\alpha v \beta 5$-status was significantly more often observed in tumors of the intestinal subtype, especially in the tumor stroma. Moreover, patients which were grouped as "positive" regarding the expression of $\alpha v \beta_{3}$ in endothelial cells showed a significantly longer survival. A positive $\alpha v \beta 5$-status was observed significantly more often in male patients and correlated significantly with proximal tumor localization and better tumor differentiation $\left(\mathrm{G} 1 / \mathrm{G}_{2}\right)$. Conclusions. Integrins $\alpha v \beta_{3}$ and $\alpha v \beta_{5}$ are expressed in GC and are involved in tumor biology. Our results form a basis for further investigations in consideration of integrins ' potential as putative predictive prognostic biomarkers, especially regarding their potential of differentiating between the Laurén phenotypes.

\section{DO-004}

\section{Histology-based prediction of lymph node metastases in early} gastric cancer

\section{Lippert ${ }^{* 1}$, U. Ronellenfitsch², R. Grobholz ${ }^{3}$, A. Marx', G. Kähler' ${ }^{2}$, T. Gaiser ${ }^{1}$} ${ }^{1}$ Department of Pathology, University Medical Centre Mannheim, University of Heidelberg, Mannheim, Germany, ${ }^{2}$ Department of Surgery, University Medical Centre Mannheim, University of Heidelberg, Mannheim, Germany, ${ }^{3}$ Department of Pathology, Kantonsspital Aarau, Aarau, Switzerland

Aims. In selected cases, early gastric cancer (EGC) can be successfully treated by endoscopic therapy (endoscopic mucosal resection/endoscopic submucosal dissection) as an organ-sparing option of treatment. However, endoscopic resection alone is only indicated in patients with negligible risk of concurrent lymph node metastases (LNM). Therefore, the aim of the study was to determine predictive factors for LN involvement.

Methods. We histologically reviewed patients with pT1 gastric adenocarcinoma who had undergone surgical therapy from 1972-2005, and checked for parameters including LNM, local depth of infiltration and Lauren classification. A total of 275 patients were enrolled into this study. Histologically, intestinal tumours according to Lauren classification were predominant $(53.6 \%)$ compared to diffuse subtype (30.8\%), and mixed-type (15.6\%), respectively. $13.1 \%$ of all cases had LNM.

Results. At a submucosal invasion level of 1 (upper third of the submucosal level, sm1), the odds ratio was 4.9 for LNM compared with mucosal invasion levels $\left(\mathrm{m}_{1}, \mathrm{~m}_{2}, \mathrm{~m}_{3}\right.$; p-value: 0.0331$)$. The prevalence of LNM was $16.7 \%$ in $\mathrm{sm} 1,14.0 \%$ in $\mathrm{sm} 2$, and $23.4 \%$ in $\mathrm{sm}_{3}$, while the prevalence of LNM in $\mathrm{m} 1$ was $0.0 \%, 2.1 \%$ in $\mathrm{m}_{2}$, and $7.4 \%$ in $\mathrm{m}_{3}$. The level of infiltration into the submucosa, the presence of signet ring cells, and mixed-type gastric carcinomas according to Lauren classification were significantly correlated with the risk of LNM.

Conclusions. As the prevalence of LNM has been demonstrated to rise from $7.4 \%$ in $\mathrm{m}_{3}$ to $16.7 \%$ in $\mathrm{sm} 1$ invasion levels, gastric resection appe- 
ars to be appropriate in tumours $\geq \mathrm{pT} 1$ ( $\mathrm{sm} 1)$. A detailed risk factor analysis for pT1 (sm1) subgroup treated sufficiently with an organ-sparing option, will be performed and presented at the conference. Our study identified several histopathological risk factors for the presence of LNM in EGC. From an oncological standpoint, endoscopic resection alone might not be sufficient for tumours which show one ore more of these factors.

\section{DO-005}

\section{Expression of lincRNA and snoRNA species is associated with ag-} gressive behaviour of gastrointestinal stromal tumours (GISTs)

\section{E. Moskalev*1, I. Bure', E. Braginskaja', A. Gholami', I.-M. Schaefer ${ }^{3,}$ A. Agaimy', A. Hartmann', F. Haller \\ 'Institute of Pathology, Friedrich-Alexander-University, Erlangen-Nurem- berg, Germany, ${ }^{2}$ Technical University of Munich, Freising, Germany, ${ }^{3}$ Institute of Pathology, Göttingen, Germany}

Aims. Different types of non-coding transcripts are crucial components of the regulatory machinery of the cell. Their deregulation is functionally involved in tumourigenesis. For example, high expression levels of one of most prominent long non-coding transcripts, the HOTAIR, endow tumours of different origin with aggressive behaviour through epigenetic reprogramming of multiple genomic sites that are relevant for cancer development. However, the role of most non-coding transcripts-miRNAs being an exception-just begins to be elucidated. GISTs are the most common mesenchymal tumours of the gastrointestinal tract and are characterised by heterogeneous clinical behaviour. These tumours arise in different portions of the gastrointestinal tract, and the anatomic location correlates with aggressive behaviour suggests suggesting the influence of positional information. Although the majority of GISTs harbour activating mutations in either KIT or PDGFRA receptors, underlying mechanisms of the clinical heterogeneity are poorly understood. Here, we explored the possibility that different classes of non-coding RNAs may be involved in different clinicopathological traits of GISTs.

Methods. The genome wide transcription profiling was performed by using Affymetrix GeneChip 2.0 microarrays. A total of 42 GIST samples were analysed including GISTs of (1) low vs. high risk, (2) KIT vs. PDGFRA mutations, (3) stomach vs. small intestine, both KIT mutant. qPCR was employed for validation of expression data.

Results. Differentially expressed transcripts were identified between all the groups-significant differences of more than 2 fold were considered-that included lincRNAs, sn- or snoRNA species and miRNA precursor forms. We next focused on GISTs of low and high risk and performed validation of differentially expressed transcripts that are of both mechanistic and biomarker interest for the disease. Differential expression of seven lincRNAs and eight sn-/snoRNAs was confirmed by using qPCR.

Conclusions. Analysis of a large independent cohort of clinically characterised GISTs revealed that the transcripts were strongly associated with aggressive behaviour and can be used as prognostic factors of the disease. Functional analysis of most prominent transcripts is underway, which will show the mechanistic details of their functions.

E.A.M. is supported by the Interdisciplinary Centre for Clinical Research (IZKF). I.M.S. is supported by the Dr. Mildred Scheel Stiftung für Krebsforschung (No. 110822).

\section{DO-006}

Molecular analysis of mixed adenoneuroendocrine carcinomas (MANEC) indicates a common clonal origin of adeno and neuroendocrine components

\author{
C. Schaaf*1, M. Vieth ${ }^{2}$, L. Veits', G. Heinemann', A. Jung', T. Kirchner', \\ D. Horst ${ }^{7}$ \\ 'Institute of Pathology of the University of Munich, Munich, Germany, \\ ${ }^{2}$ Institute of Pathology, Bayreuth, Germany
}

Aims. Neuroendocrine carcinomas (NECs) of the gastrointestinal tract are rare, but highly aggressive tumors. Little is known about the genetic alterations of these tumors and the origin of their tumor cells. Half of the NECs are mixed with adenocarcinoma/adenoma components (MANECs). In this study we evaluated whether the adenocarcinoma/ adenoma and neuroendocrine carcinoma components of MANECs are clonal, suggesting that NECs may originate from adenocarcinomas or adenomas.

Methods. H\&E-, CD56, synaptophysin and NSE stainings were used to identify neuroendocrine and adenocarcinoma/adenoma components of MANECs. These components then were microdissected and DNA was isolated. Subsequently, they were used for mutation analysis in KRAS and BRAF genes using pyrosequencing.

Results. We identified identical mutation status in the adenocarcinoma/ adeno and neuroendocrine carcinoma components of 13 analyzed MANECs. In specific, 6 cases showed mutations in KRAS exon 2 or BRAF, while 7 cases were KRAS wild type.

Conclusions. The same mutation status in the adenocarcinoma/adeno and neuroendocrine carcinoma component strongly suggests clonality of both components. Neuroendocrine carcinomas of the gastrointestinal tract therefore may develop out of gastrointestinal adenocarcinomas or adenomas.

\section{AG Gastroenteropathologie - Pankreas/Leber}

\section{DO-007 Integrin subunits in pancreatic ductal adenocarcinoma and its precursor lesions - an immunohistochemical study}

K. Steiger ${ }^{* 1}$, S. Haneder', S. Berchtold', M. Trajkovic-Arsic ${ }^{2}$, I. Esposito ${ }^{7}$ IInstitute of Pathology, Technical University of Munich, Munich, Germany, 2II. Medizinische Klinik und Poliklinik, Klinikum rechts der Isar, Munich, Germany

Aims. Integrin-mediated tumor cell interactions with the extracellular matrix may play an active and crucial role in directing the malignant phenotype in pancreatic ductal adenocarcinoma (PDAC). Therefore, we aimed at identifying relevant integrin subunits in epithelial-mesenchymal interactions in PDAC and its precursor lesions.

Methods. We investigated the expression of different integrin subunits $\left(\alpha_{1}, \alpha \mathrm{V}, \beta_{1}, \beta_{3}, \beta 6\right)$ in human PDAC $(\mathrm{n}=34)$ and its precursor lesions $(n=26)$ as well as in different mouse models (LSL-KrasG12D/+Ptfia+/ Cre(ex1) n=3; LSL-KrasG12D/+Ptfia+/Cre(ex1)P 53 LoxP/LoxP n=2; LSL$\mathrm{KrasG12D/+Ptf1a+/Cre(ex1)TNC-/-} \mathrm{n}=2$ ) by immunohistochemistry. Results. In transgenic mouse models, a diffuse expression of $\alpha \mathrm{V} \beta_{3}$-integrin in single epithelial cells in the majority $(>90 \%)$ of mouse PanIN and an increasing expression frequency during PDAC progression occurs. In contrast, $\alpha \mathrm{V} \beta_{3}$-integrin is expressed in only one third of human PDAC and could not be detected in human PanINs. $\alpha V \beta 6$-integrin is expressed with increased intensity during progression from precursor lesions to PDAC in humans and, dependent on the presence of Tenascin $\mathrm{C}$ (TNC), in transgenic mouse models. More than $90 \%$ of human PDAC express $\alpha \mathrm{V} \beta 6$-integrin. Concerning all other tested subunits, no specific correlation between PDAC progression and expression was found. 
Conclusions. $\alpha \mathrm{V} \beta 6$-integrin is widely expressed in human PDAC and its precursor lesions. First findings in transgenic mouse models reveal a strong interaction of this heterodimer with TNC in pancreatic adenocarcinoma. $\alpha \mathrm{V} \beta 6$-integrin might thus play an important role in epithelial-mesenchymal interaction in PDAC and its precursor lesions. In addition, it might serve as a diagnostic marker in multimodal molecular imaging of early PDAC.

\section{DO-008}

\section{C-Met, HER-family protein expression and patient survival in pri-} mary pancreatic ductal adenocarcinoma and corresponding lymph node metastasis

\section{A. Muckenhuber*1, A. Stenzinger', F. Bergmann', J. Werner' ${ }^{2}$, M. Thomas ${ }^{3}$, W. Wilko? \\ 'Institute of Pathology, University Hospital Heidelberg, Heidelberg, Germany, ${ }^{2}$ Department of Surgery, Ruprecht-Karls-University, Heidelberg, Germany, ${ }^{3}$ Roche Pharma Research and Early Development (pRED), Patho- logy, Penzberg, Germany}

Aims. Agents targeting tyrosine kinase receptors of the HER family including the EGF-receptor and the HER2 receptor are the best established and most widely used targeted therapies in cancer. Due to the dismal prognosis and high mortality rate pancreatic ductal adenocarcinoma (PDAC) patients are in dire need of novel therapeutic approaches that allow a significant improvement in survival times. While some clinical trials of agents targeting HER receptors have been initiated in PDAC, data on expression levels of tyrosine kinase receptors involved in potentially oncogenic signaling pathways like the HER-family and c-Met has been scarce, inconsistent and restricted to primary cancers. Methods. Primary cancers and lymph node metastasis of 56 patients with pancreatic ductal adenocarcinoma were included in this study. Immunohistochemical staining of HER1 (EGFR), HER2, HER3, HER4 and c-Met and a chromogenic in situ hybridization (CISH) with a cMet specific DNA probe were performed. Expression levels and gene copy numbers were correlated with clinicopathological data and patient survival.

Results. While expression levels of HER-family proteins were low in the majority of cases, c-Met showed a broad range of expression levels with medium and marked membranous expression in almost half of the cases. C-Met showed an upregulation in lymph node metastasis although the trend was not strong enough to be statistically significant while the HER-family proteins showed consistent expression levels between primary tumors and lymph node metastasis. Membranous expression of cMet in the primary tumors showed a significant correlation with poorer patient survival $(\mathrm{p}=0.049)$. Most cases with marked expression of $\mathrm{c}-\mathrm{Met}$ did not show an amplification of the Met gene.

Conclusions. C-Met is a commonly expressed tyrosine kinase receptor in pancreatic ductal adenocarcinoma and should be considered a valid target for future therapeutic approaches as it is associated with poorer patient survival. As most of the cases with high c-Met expression did not show an amplification of the Met-gene other mechanisms of overexpression, like mutations of the CBL-binding site rendering the receptor resistant to degradation recently described in gastric cancer, should be considered. Tyrosine kinase receptors of the HER-family are expressed in some pancreatic ductal adenocarcinomas but larger patient cohorts are needed to assess a possible biological relevance due to low expression frequency and levels.

\section{DO-009}

Prognostic significance of ZEB1 expression in cancer cells and cancer associated fibroblasts in pancreatic head cancer

P. Bronsert ${ }^{* 1,2}$, I. Kohler', S. Timme', M. Werner', O. Schilling ${ }^{3}$, T. Brabletz', U.T. Hopt ${ }^{4}$, D. Bausch', B. Kulemann ${ }^{4}$, T. Keck', U. Wellner ${ }^{5}$

'Department of Pathology, University Medical Center Freiburg, Freiburg, Germany, ${ }^{2}$ Department Pathology, University Medical Center Freiburg, Freiburg, Germany, ${ }^{3}$ Institute of Molecular Medicine and Cell Research, AlbertLudwigs-University Freiburg, Freiburg, Germany, ${ }^{4}$ Department of Surgery, Clinic for General and Visceral Surgery, University Medical Center Freiburg, Freiburg, Germany, ${ }^{5} \mathrm{Clinic}$ for Surgery, University Clinic of Schleswig-Holstein Campus Lübeck, Lübeck, Germany

Aims. Pancreatic ductal adenocarcinoma (PDAC) is characterized by an aggressive biology and poor prognosis. Experimental evidence has suggested a role for the transcriptional repressor ZEB1 in epithelialmesenchymal transition, invasion and metastasis in PDAC. ZEB1 expression has been observed in cancer cells as well as stromal fibroblasts. Our study aimed to evaluate the prognostic value of $\mathrm{ZEB} 1$ expression in PDAC tissue.

Methods. $\mathrm{N}=117$ cases of PDAC were included in the study. Patient baseline and follow-up data was extracted from a prospectively maintained database. After clinicopathological re-review, serial sliced tissue slides were immunostained for ZEB1 and Pan-Cytokeratin. ZEB1 expression in cancer cells and adjacent stromal fibroblasts was graded separately and correlated to routine histopathological parameters and survival after resection.

Results. High ZEB1 expression in cancer cells and in stromal cancer associated fibroblasts (CAF) was significantly associated with poor prognosis. There was also a trend for poor prognosis with a lymph node ratio of over o.10. In multivariate analysis, stromal ZEB1 expression grade was the only independent factor of survival after resection

Conclusions. Our data demonstrate that ZEB1 expression in cancer cells as well as in stromal fibroblasts are strong prognostic factors in PDAC. Stromal ZEB1 expression is identified for the first time as an independent predictor of survival after resection of PDAC. This observation suggests that therapies targeting ZEB1 and its downstream pathways could hit both cancer cells and supporting CAF.

\section{DO-010}

Deregulation of DNA-dependent protein kinase catalytic subunit contributes to human hepatocarcinogenesis development and has a putative prognostic value

\section{Calvisi*1, M. Frau' ${ }^{2}$, F. Feo ${ }^{2}$, R. Pascale², F. Dombrowski', M. Evert}

Institut für Pathologie, Greifswald, Germany, ${ }^{2}$ Department of Clinical and Experimental Medicine, University of Sassari, Laboratory of Molecular Pathology and Oncology, Sassari, Italy

Aims. DNA-dependent protein kinase catalytic subunit (DNA-PKcs) plays a pivotal role in the non-homologous end-joining pathway for DNA double-strand break repair and is required for cellular resistance to ionizing radiation. Alterations in DNA-PKcs favour or inhibit carcinogenesis, depending on the cancer type. Its role in human hepatocellular carcinoma (HCC) remains unknown.

Methods. Levels of DNA-PKcs and its targets, including $\mathrm{H}_{2} \mathrm{~A}$ histone family member X ( $\left.\mathrm{H}_{2} \mathrm{AFX}\right)$, and heat shock transcription factor-1 (HSF1) were assessed by immunohistochemistry and/or immunoblotting and quantitative real-time RT-PCR in a collection of human HCC. Proliferation, apoptosis, microvessel density, and genomic instability were also determined. HSF1 cDNA or DNA-PKcs-specific siRNA as well as administration of the DNA-PKcs inhibitor NU7441 were used to explore the role of both genes in HCC cell lines. Activator protein-1 (AP-1) binding to DNA-PKcs promoter was evaluated by chromatin immunoprecipi- 
tation. Kaplan-Meier curves and multivariate Cox model were used to study the impact on clinical outcome.

Results. Total and phosphorylated DNA-PKcs and $\mathrm{H}_{2} \mathrm{AFX}$ were upregulated in HCC. Activated DNA-PKcs positively correlated with HCC proliferation, genomic instability, and microvessel density, and negatively with apoptosis and patient's survival. Proliferation decline and massive apoptosis followed DNA-PKcs silencing in HCC cell lines. Total and phosphorylated HSF1 protein, mRNA, and activity were upregulated in HCC. Mechanistically, we demonstrated that HSF1 induces DNAPKcs upregulation through the activation of the MAPK/JNK/AP-1 axis. Conclusions. DNA-PKcs transduces HSF1 effects in HCC cells, and might represent a novel target and prognostic factor in human HCC.

\section{DO-011}

Dissecting the molecular and cellular mechanisms driving NAFLDand NASH-induced HCC

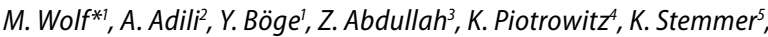 M. Ringelhan ${ }^{2}$, C. Thiele ${ }^{4}$, M. Tschöp ${ }^{5}$, K. Unger ${ }^{6}$, P. Knolle ${ }^{3}$, A. Weber', M. Heikenwälder ${ }^{2}$}

'Institute of Surgical Pathology, University Hospital, Zurich, Switzerland, ${ }^{2}$ Institute of Virology, Technical University of Munich, Munich, Germany, ${ }^{3}$ Institutes of Molecular Medicine and Experimental Immunology, University of Bonn, Bonn, Germany, ${ }^{4}$ LIMES Life Sciences Institute, University of Bonn, Bonn, Germany, ${ }^{5}$ Institute for Diabetes and Obesity, Technical University of Munich, Munich, Germany, ${ }^{6}$ Research Unit of Radiation Cytogenetics, Helmholtz Zentrum München, Munich, Germany

Aims. Hepatocellular carcinoma (HCC) is the third most prevalent type of cancer with an annual death rate exceeding 500,000 deaths. Risk factors for developing HCC are chronic HBV or HCV infection, chronic alcohol consumption and obesity. Increased hepatic fat accumulation due to excessive calorie and fat uptake causes nonalcoholic fatty liver disease (NAFLD), ranging from mild steatosis to steatohepatitis, cirrhosis and potentially HCC. The major co-founding etiology for HCC in industrialized countries has recently switched from viral infections to dietary-induced etiology, making HCC the most rapidly increasing type of cancer in the U.S. The precise chain of events on molecular level that ultimately leads to HCC development in livers of obese people are largely unknown and appropriate mouse models for studying NASH- or NAFLD-induced development of HCC development as a consequence of chronic fat uptake are lacking.

Methods. Long-term feeding of $\mathrm{C}_{57} \mathrm{BL} / 6$ and selected knockout mice with choline-deficient high fat diet (CD-HFD) to cause steatosis and liver damage. Hepatic fat accumulation (triglycerides, Sudan staining, MRI), serum parameters (ALT, AST and cholesterol) and other metabolic parameters (GTT, PTT) were quantified at several time-points. Livers were further analyzed on molecular (RNA and protein expression, immunohistochemistry) and cellular level (flow cytometry).

Results. Long-term CD-HFD feeding of $\mathrm{C}_{57} \mathrm{BL} / 6$ mice led to severe liver steatosis with features of nonalcoholic steatohepatitis (NASH) accompanied by elevated serum cholesterol and transaminase levels as well as enhanced presence of activated intrahepatic immune cells. Interestingly, HCC development was found in approximately $25 \%$ of mice. HCC were characterized by the expression of $\mathrm{GP}_{73}, \mathrm{AFP}$ and $\mathrm{A} 6$ and were negative for GS expression. Steatosis development depended on the presence of activated lymphocytes and HCC development necessitated LT $\beta$ R signaling within hepatocytes.

Conclusions. Long-term supply of CD-HFD is a suitable model to study $\mathrm{NASH}$ and dietary-induced HCC development recapitulating features of human liver disease.

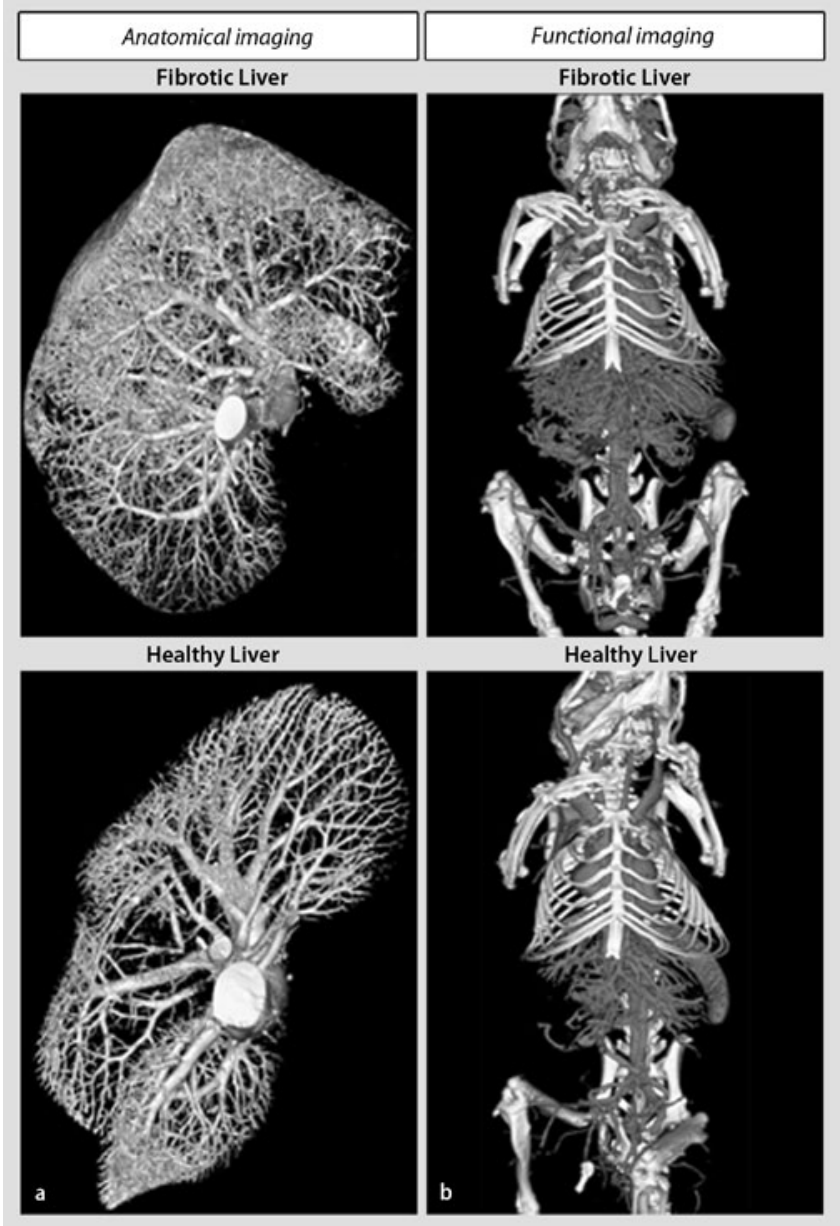

Fig. 1 | D0-012 $\Delta$ a) High resolution $\mu C T$ imaging after casting of hepatic blood vessels. b) In vivo $\mu \mathrm{CT}$ imaging of fibrosis-associated angiogenesis in fibrotic livers.

\section{DO-012}

Anatomical, functional, and molecular imaging of liver fibrosis

J. Ehling ${ }^{* 1,2}$, M. Bartneck ${ }^{3}$, F. Gremse', F. Kiessling', R. Knuechel', F. Tacke ${ }^{3}$, T. Lammers'

${ }^{1}$ Helmholtz-Institute for Biomedical Engineering, Department of Experimental Molecular Imaging, RWTH Aachen, Aachen, Germany, ${ }^{2}$ Institute of Pathology, RWTH University, Aachen, Germany, ${ }^{3}$ Department of Medicine III, RWTH Aachen, Aachen, Germany

Aims. Millions of people suffer from early- to late-stage liver fibrosis, which often progresses to cirrhosis and hepatocellular carcinoma. However, hardly any imaging agents and protocols are available for the non-invasive diagnosis and staging of liver fibrosis. The aim of our efforts was to establish novel CT- and MR-based approaches for visualizing fibrosis and for non-invasive treatment monitoring.

Methods. Two different animal models were used [carbon tetrachloride $\left(\mathrm{CCl}_{4}\right)$-induced fibrosis and bile duct ligation (BDL)]. Contrast-enhanced microCT $(\mu \mathrm{CT})$ was performed for imaging of blood vessels in fibrotic livers, providing highly detailed information on the $3 \mathrm{D}$ morphology of microvessels, as well as on the relative blood volume (rBV). rBV's were determined at 2, 4, 6 and 8 weeks of continuous $\mathrm{CCl}_{4}$ exposure, and at 3 weeks after BDL. The potential of imaging for therapy monitoring in liver fibrosis was demonstrated upon inhibition of the chemokine CCL2, a key factor for recruiting inflammatory monocytes. Additionally, the potential of an elastin-specific contrast agent (ESMA) 


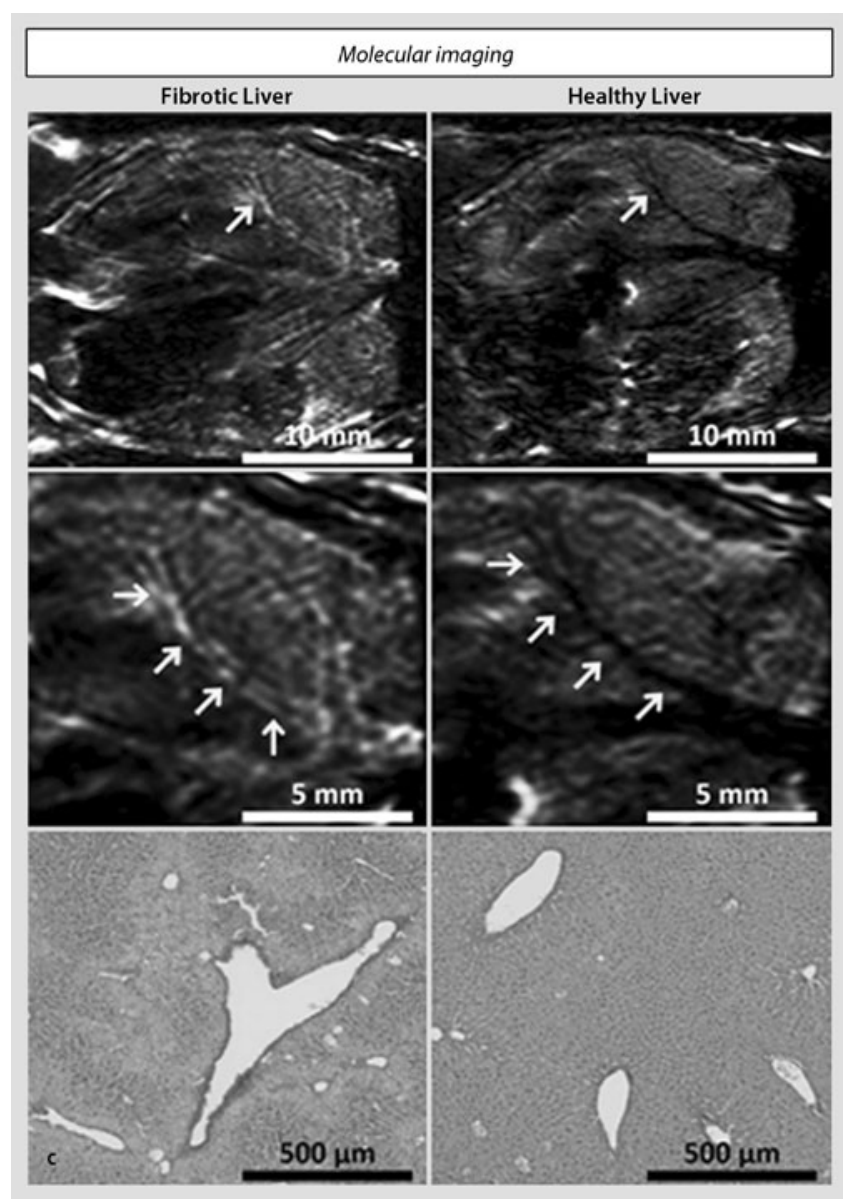

Fig. 1 | DO-012 $\Delta$ c) Elastin-based molecular MRI of liver fibrosis and validation via Elastica Van Gieson-staining

was assessed for molecular T1-weighted MRI purposes. Findings were validated by FACS, qPCR and histology.

Results. In fibrotic livers, areas of sprouting angiogenesis were observed (- Fig. 1a,b) and the rBV increased from early- to late-stage disease (from $+11 \%$ at 2 weeks to $+40 \%$ at 8 weeks of $\mathrm{CCl}_{4}$ exposure; $\mathrm{n}=4$ ). Inflammatory monocytes expressed higher amounts of VEGF in fibrotic livers, and the pharmacological inhibition of monocyte infiltration, by anti- $\mathrm{CCl}_{2}$ directed agents, inhibited fibrosis-associated angiogenesis $\left(\mathrm{rBV}=25 \%\right.$ vs. $\mathrm{rBV}=18 \%$ at 8 weeks of $\mathrm{CCl}_{4}$ exposure; $\mathrm{n}=4$ ). When performing ECM-targeted molecular MRI using ESMA, a specific perivascular T1 signal enhancement was observed in fibrotic livers (• Fig. 1c). Conclusions. We here describe novel anatomical, functional and molecular imaging approaches for visualizing and staging liver fibrosis, as well as for treatment monitoring. The findings contribute to a better understanding of the molecular mechanisms of fibrosis progression, showing e.g. that the infiltration of inflammatory monocytes promote angiogenesis and perivascular elastin deposition in fibrotic livers.

\section{AG Gastroenteropathologie - Unterer GI-Trakt}

DO-013

Differentiation of primary and metastatic tumours in synchronous multifocal colonic and bronchopulmonary adenocarcinoma by targeted next generation sequencing

K. Kunze ${ }^{* 1}$, M. Frank', J. Bodner', W. Blau ${ }^{3}$, U. Sibelius ${ }^{3}$, M. Rummel ${ }^{3}$, R. Hörbelt'², W. Padberg' ${ }^{2}$, R. Engenhart-Cabillic', A. Bräuninger', S. Gattenlöhner ${ }^{1}$ ${ }^{1}$ Institute of Pathology, Justus-Liebig-University, Gießen, Germany, ${ }^{2} \mathrm{Clinic}$ of Surgery, Justus-Liebig-University, Gießen, Germany, ${ }^{3}$ Clinic of Internal Medicine, Justus-Liebig-University, Gießen, Germany, ${ }^{4}$ Clinic of Radiation Therapy, Philipps-University, Marburg, Germany

Aims. We report the case of a 67-year-old female patient with a multifocal colonic adenocarcinoma ( $\mathrm{pT}_{3}$ No Lo Vo G2 Ro and pT1 No Lo Vo G2 Ro) with classical histological and immunohistochemical morphology. During staging two tumor-suspicious lesions were detected in both sides of the lung, and after right-sided upper lobectomy an additional bronchopulmonary adenocarcinoma (pT1a N1a L1 Vo G2 Ro) with expression of "lung-specific" immunomarkers was identified. By contrast, histological examination of the left-sided lung tumor revealed a biphenotypic adenocarcinoma with tubular and mucinous differentiation with a "lung-specific" profile in the tubular areas $\left(\mathrm{CK}_{7}+, \mathrm{CK}_{2} \mathrm{O}-\right.$, $\mathrm{CDX}_{2}-$, $\mathrm{TTF}_{1+}+$ ), and a pattern compatible with bronchopulmonary mucinous adenocarcinoma $\left(\mathrm{CK}_{7}+, \mathrm{CK}_{20} \pm, \mathrm{TTF}_{1}-\right)$ in the mucinous tumor parts. However, since the immunomarker CDX2 was moderately positive and its expression was proposed to be highly suggestive for a lung metastasis of a primary colonic adenocarcinoma, further molecular genetic approaches were applied.

Methods. To differentiate the clinical setting between primary and metastatic carcinoma, we analysed the KRAS mutation status first, and then performed further extended genetic studies using targeted next generation sequencing (Comprehensive Cancer Panel of Ion Torrent). Results. The bronchopulmonary adenocarcinoma of the right lung showed a KRAS G12S mutation, the $\mathrm{pT}_{3}$ colonic adenocarcinoma a KRAS G12D mutation and the pT1 colonic adenocarcinoma an unmutated KRAS wild type (KRAS WT) status. In the heterogeneously differentiated adenocarcinoma of the left lung a KRAS G12D mutation identical to that found in the $\mathrm{pT}_{3}$ colonic carcinoma was detected in both the tubular and the mucinous tumor parts. The analysis with targeted next generation sequencing revealed completely different mutations in all four carcinomas, indicating the presence of four individual primary carcinomas of the colon and the lung.

Conclusions. We therefore conclude that targeted next generation sequencing has the capacity to differentiate in a clinical setting between primary and metastatic carcinoma with high impact on tumor classification, prognosis and therapy.

\section{D0-014}

Expression of cancer stem cell markers correlates with haematogeneous liver metastases but not with peritoneal carcinomatosis in colorectal cancer

\section{J. Neumann*1, L. Löhrs², M. Albertsmeier', S. Reu', C. Bruns'², T. Kirchner? M. Angele ${ }^{2}$ \\ 'Institute of Pathology of the University of Munich, Munich, Germany, ${ }^{2}$ Department of General- Visceral-, Transplantation-, Vascular- and Thora- cic-Surgery, Medical Center of the University of Munich, LMU München, Munich, Germany}

Aims. Metastatic spread is mainly driven by stem cell features of tumor cells. Although peritoneal carcinomatosis (PC) displays a late stage in colorectal cancer (CRC) most patients present without synchronous haematogeneous distant metastases. However, thus far expression of 
cancer stem cell markers has not been analyzed in the context of PC and haematogeneous spread to the liver. Therefore, it was our intention to analyze the expression of markers associated with cancer stem cell features in CRC in correlation with different patterns of distant spread in CRC.

Methods. Immunohistochemistry for $\mathrm{CD}_{133}, \mathrm{CD}_{44}$ and $\beta$-catenin was applied to a collection of CRC specimen with exclusive PC $(n=18)$, exclusive hepatic metastasis $(n=37)$ as well as combined PC and hepatic spread $(n=13)$. A cohort of CRC patients $(n=56)$ who did not develop any metastases within 5 years served as controls.

Results. CD133 was most frequently expressed in CRC with hepatic metastases (45.9\%), whereas in patients with exclusive PC (11.1\%), patients without any metastases (12.5\%) and patients showing combined PC and hepatic spread $(15.4 \%)$ lower expression rates of this stem cell marker were detected $(\mathrm{p}=0.001)$. For $\mathrm{CD}_{44}$, the highest rates of expression were detected in patients with exclusive hepatic metastases (70.3\%) as well as in patients with combined PC and hepatic spread (76.9\%) compared to patients with exclusive PC $(55.6 \%)$ and the control group $(23.2 \%$; $\mathrm{p}<0.001)$. Nuclear expression of $\beta$-catenin was correlated with the occurrence of liver metastases (73.0\%), exclusive PC (66.7\%) and combined PC with hepatic spread (69.2\%) but was expressed less frequently in the control group $(16.6 \%$; $\mathrm{p}<0.001)$. Combined expression of $\beta$-catenin with $\mathrm{CD}_{44}$ and/or CD133 was most frequently found in CRC with hepatic metastases (67.6\%) and in CRC with combined PC and hepatic spread (53.8\%). In contrast, in CRC with exclusive PC (27.8\%) and in the control group (3.8\%) significantly lower levels of this marker combination could be detected $(\mathrm{p}<0.001)$.

Conclusions. We were able to demonstrate that the expression of markers associated with stem cell features of tumor cells correlates with different patterns of metastatic spread in CRC. These data indicate that CRC developing solely PC but no hematogenous metastases lack the stem cell features needed for dissemination. Therefore, in concordance with the clinical course of the disease exclusive PC from CRC should be viewed as an advanced stage of locally aggressive tumor growth without stem cell features.

\section{D0-015}

\section{Colonic crypt like structural organization in colorectal cancers}

\section{Cernat ${ }^{1,2}$, R. Jackstadt' ', L. Kriegl', H. Hermeking', A. Jung ', T. Kirchner ${ }^{\text {1, }}$} D. Horst*1

'Institute of Pathology, Ludwig-Maximilians-University, Munich, Germany, ${ }^{2}$ Victor Babes University of Medicine and Pharmacy, Timisoara, Romania

Aims. Normal colonic crypts are stereotypical structures with distinct stem cell, proliferating, and differentiating compartments. Colon cancers derive from normal colonic mucosa by a stepwise accumulation of mutations but, in contrast to normal mucosa, form morphologically disarrayed glands. We aimed to understand whether colon cancer glands preserve a compartmental organization, similar to normal colonic crypts and assessed its relevance for disease outcome.

Methods. Normal colonic mucosa, colon cancer xenografts, spheroid cultures, and regular colon cancer cell cultures were analyzed for expression of beta-Catenin, Ki67, and CK2o by triple immune fluorescence staining and confocal microscopy. Additionally, we characterized a cohort of more than 220 colon cancer cases with clinical follow up data for preserved structural organization using conventional immune staining.

Results. A subset of colon cancers formed glands with a structural organization similar to normal colonic crypts and putative stem cell, proliferating, and differentiating compartments. In these tumors, we found nuclear beta-Catenin at the infiltrative tumor edge, $\mathrm{CK}_{2} \mathrm{O}$ in glandular areas of the tumor center and Ki67 predominantly in between. This organization could be observed in full in primary colon cancers and xenograft tumors, while spheroid cultures and cultured colon cancer cell lines lacked complete structural organization and tumor cell hete- rogeneity. In a colon cancer case collection, tumors with a high degree of preserved crypt like architecture showed better clinical outcome. Conclusions. When grown in tissue contexts, colon cancers preserve some degree of the structural organization of normal colonic crypts, indicating that intestinal morphogenetic programs are still active in these tumors. Since colon cancers with a preserved crypt like architecture were clinically less aggressive, we propose this finding may be considered to improve colon cancer grading systems.

\section{DO-016}

Fluorescence Endoscopy using a fluorescently labeled MMP inhibitor ligand to detect early colorectal cancer

\section{P. Lenz ${ }^{* 1}$, D. Bettenworth ${ }^{1}$, K. Schwegmann ${ }^{2}$, A. Faust ${ }^{2}$, M. Schäfers ${ }^{2}$ \\ D. Domagk', C. Poremba ${ }^{3}$}

'Department of Medicine B, University Hospital of Muenster, University of Muenster, Muenster, Germany, ${ }^{2}$ European Institute for Molecular Imaging, University Hospital of Muenster, University of Muenster, Muenster, Germany, ${ }^{3}$ Institute of Pathology Munich-North, Munich, Germany

Aims. Colorectal cancer (CRC) is one of the most frequent malignancies in humans; especially patients with ulcerative colitis are at increased risk due to the chronic colonic inflammation. Matrix metalloproteinases (MMPs) are key enzymes in metastatic processes and elevated expression levels are correlated with poor prognosis in humans. The aim of the study was to evaluate MMP-2/-9 guided fluorescence endoscopy (FE) for early tumor detection in a murine model of CRC.

Methods. Colorectal cancer was induced in $\mathrm{C} 57 \mathrm{Bl} / 6$ WT mice $(\mathrm{n}=15)$ by single i.p. injection of azoxymethane (AOM) and cyclic administration of dextran sodiumsulfate (DSS). A fluorescently labeled MMP-selective tracer was intravenously injected $24 \mathrm{~h}$ before endoscopic examination. Tumor development was assessed in vivo by white light endoscopy (WLE) and FE. Ex vivo, tracer uptake was confirmed by fluorescence reflectance imaging system (FRI) of explanted murine colons. Tumors were graded in HE staining by an experienced pathologist and evaluated with $\beta$-catenin and Ki67 immunohistochemistry. Finally, the expression and activity of MMP- 2 and 9 in colorectal tumors was assessed by immunohistochemistry, western blot analysis and zymography.

Results. The MMP-specific tracer provided a significant higher contrast of colonic adenomas as compared to the adjacent non-malignant mucosa during FE ( $75.9 \pm 8.7$ vs. $66.5 \pm 7.4$, mean gray values, $p<0.001)$. FRI detected significantly higher tracer uptake in colorectal adenoma as compared to healthy mucosa (average radiant efficiency: 1.41 E+12 \pm 0.75 $\mathrm{E}+12$ vs. $0.62 \mathrm{E}+12 \pm 0.44 \mathrm{E}+12[\mathrm{p} / \mathrm{s} / \mathrm{cm} 2 / \mathrm{sr}] /[\mu \mathrm{W} / \mathrm{cm} 2], \mathrm{p}<0.001)$ and revealed a tumor size dependent increase (tumors $<2 \mathrm{mm:} 2.55 \pm 1.49 \mathrm{vs}$. tumors $2-4 \mathrm{~mm}: 3.23 \pm 1.72$ vs. tumors $>4 \mathrm{~mm} 4.99 \pm 1.50$, $\mathrm{p}<0.01$ ). Standard histology showed colorectal adenomas up to high-grade dysplasia and pT1 carcinomas. IHC proved elevated expression levels of MMP-9 in the above mentioned lesions. Correspondingly, western blot analysis and zymography indicated higher levels of active MMP-2/-9 and its pro-enzymes in colorectal dysplasia.

Conclusions. Mucosal MMP-2 and -9 expression was significantly increased in colorectal high-grade dysplasia and $\mathrm{pT} 1$ tumors. Elevated MMP-expression of colorectal dysplasia could be detected in vivo by FE, which might help to improve adenoma detection in the future. Additionally, a prognostic parameter could be visualized directly by FE. 


\section{DO-017}

\section{Histone deacetylases and histone marks in colorectal carcinomas}

L. Lutz*1, I. Coutino Fitzner', A.-L. Geßler', B. Riedel', F. Makowiec ${ }^{1,2,}$

M. Werner', S. Lassmann ${ }^{1}$

'Department of Pathology, University Medical Center Freiburg, Freiburg, Germany, ${ }^{2}$ Department of Surgery, University Medical Center Freiburg, Freiburg, Germany

Aims. Epigenetic regulation of colorectal cancers (CRC) has become evident at the level of DNA methylation in the carcinogenesis of $\mathrm{CpG}$ Island Methylator Phenotype (CIMP-type) CRCs. Little is known about the role of histone modifications in CRCs, especially in view of potential therapeutic targeting by Histone Deacetylase inhibitors (HDACi). This study therefore examined class I HDACs expression and levels of active and repressive histone marks.

Methods. Serial sections of formalin-fixed and paraffin-embedded tissue specimens of 49 primary CRCs (no neoadjuvant treatment) were stained for the enzymes HDAC1, HDAC2 and $\mathrm{HDAC}_{3}(41 / 49)$ as well as the histone marks $\mathrm{H}_{3} \mathrm{~K} 9 \mathrm{ac}, \mathrm{H}_{3} \mathrm{~K}_{4} \mathrm{me}_{3}$ and $\mathrm{H}_{3} \mathrm{~K}_{9} \mathrm{me}_{3}$ by immunohistochemistry. IHC was scored by evaluation of intensity and percent of stained tumor cells, followed by statistical evaluation regarding clinicpathological parameters (patient age and gender, tumor localisation, tumor stage, nodal status, grading, histotype).

Results. Strong and frequent $\mathrm{HDAC}_{1}, \mathrm{HDAC}_{2}$ and $\mathrm{HDAC}_{3}$ protein expression was found in $33 / 49(67 \%), 49 / 49(100 \%)$ and $33 / 41$ (80\%) cases, respectively. Whilst HDAC2 was distributed homogeneously throughout cases, HDAC1 showed intratumoral heterogeneity in 10/49 $(20 \%)$ cases, in few cases even involving the same tumor gland. High levels of histone marks were detected in 36/49 (73\%), 49/49 (100\%) and 38/49 (78\%) cases for $\mathrm{H}_{3} \mathrm{~K} 9 \mathrm{ac}, \mathrm{H}_{3} \mathrm{~K}_{4} \mathrm{me}_{3}$ and $\mathrm{H}_{3} \mathrm{~K}_{9} \mathrm{me}_{3}$, respectively. Protein expression of $\mathrm{HDAC}_{3}$ was inversely correlated with tumor stage ( $\mathrm{p}<\mathrm{o.01}$ ). High levels of $\mathrm{H}_{3} \mathrm{~K}_{9} \mathrm{me}_{3}$ and $\mathrm{H}_{3} \mathrm{~K} 9 \mathrm{ac}$ were associated with rectal tumor localization $(\mathrm{p}=0.04 ; \mathrm{p}=0.03)$, whilst high levels of $\mathrm{H}_{3} \mathrm{~K}_{4} \mathrm{me}_{3}$ were linked with lower tumor grading $(\mathrm{p}=0.002)$.

Conclusions. This study demonstrates that individual members of class I HDACs are not co-ordinately regulated within CRCs, whereby 1) $\mathrm{HDAC}_{1}$ is variably regulated within individual CRCs and 2) $\mathrm{HDAC}_{3}$ is down-regulated in more advanced CRCs. Nevertheless, the frequent class I HDAC expression may represent additional targets for intervention in future. Furthermore, distinct levels of histone marks in rectal CRCs and of the active $\mathrm{H}_{3} \mathrm{~K}_{4} \mathrm{me}_{3}$ mark in poorly differentiated CRCS may suggest a functional role of epigenetic drivers for CRC phenotypes. *This study is part of the Deutsches Konsortium für Translationale Krebsforschung (DKTK) and was also partially funded by the Mushett Family Foundation, Chester, NJ, USA.

\section{DO-018}

Improved molecular classification of serrated lesions of the colon by immunohistochemical detection of BRAF V600E

I. Mesteri', G. Bayer', J. Meyerer, , D. Capper, ${ }^{2,3}$, A. von Deimling ${ }^{2,3}$, P. Birner ${ }^{* 1,3}$ 'Deparment of Pathology, Medical University of Vienna, Vienna, Austria, ${ }^{2}$ German Cancer Research Center, DKFZ, Neuropathology, Heidelberg, Germany, ${ }^{3}$ Institute of Pathology, Ruprecht-Karls-University, Neuropathology, Heidelberg, Germany

Aims. The V6ooE mutation of the BRAF gene has been implicated as an important oncogenic driver in serrated lesions of the colon and as a specific marker for the serrated carcinogenic pathway. Recent findings point to microvesicular hyperplastic polyps that have similar histologic and molecular features to sessile serrated adenomas/polyps, as potential colorectal carcinoma precursors. Aim of this study was to evaluate BRAF V6ooE mutation status by immunohistochemistry in serrated lesions of the colon with regard to histomorphology.
Methods. We investigated 194 serrated lesions of the colon, comprising 42 sessile serrated adenomas/polyps, 16 traditional serrated adenomas, 136 hyperplastic polyps and 20 tubular/tubulovillous adenomas (conventional adenomas) with the novel BRAF V6ooE mutation-specific antibody VE1. In addition, BRAF exon 15 and KRAS exon 2 status was investigated by capillary sequencing in selected cases.

Results. All sessile serrated adenomas/polyps (42/42, 100\%), 15/16 (94\%) traditional serrated adenomas and 84/136 (62\%) hyperplastic polyps were VE1 positive. None of the VE1 negative serrated lesions showed a BRAF V6ooE mutation. Forty out of 42 (95\%) sessile serrated adenomas/polyps displayed areas with microvesicular hyperplastic polyp-like features. In microvesicular hyperplastic polyps, VE1 positivity was significantly associated with nuclear atypia $(\mathrm{p}=0.003)$; however, nuclear atypia was also present in VE1 negative cases.

Conclusions. Immunostaining with VE1 allows not only the detection of BRAF V6ooE mutation but also the correlation with histomorphology on a cellular level in serrated lesions. VE1 enables a subclassification of microvesicular hyperplastic polyps according to the mutation status. This improved classification of serrated lesions including immunohistochemical evaluation of BRAF V6ooE mutation may help identifying lesions with higher potential to progression into sessile serrated adenoma/polyp, and further to BRAF V6ooE-mutated colorectal cancer. 1. Mod Pathol 2013 Jul 26. doi: 10.1038/modpathol.2013.126

\section{AG Hämatopathologie I}

\section{DO-019}

Standardisation of preanalytic steps in processing of bone marrow trephines

\section{Anagnostopoulos*, K. Jöhrens, D. Lenze, A. Ullrich, M. Hummel Institute of Pathology, Charité University Hospital, Berlin, Germany}

Aims. There is a general lack of standardisation in processing of bone marrow trephines. Our established protocol delivers good morphology and immunostaining results, however, molecular studies fail in a significant proportion of cases. We tried to improve and standardize the fixation and decalcification steps of bone marrow trephine processing. Methods. 45 bone marrow trephines were cut into two parts and processed in parallel using either the in-house established protocol or the MolDecal solution from Milestone, Bergamo, Italy and the BoneStation device of the same company which allows fixation and decalcification under constant stirring at controlled temperature levels. We then evaluated the conventional morphology, histochemistry (detection of Napthol AS-D chloroacetatesterase/NASDCL), immunohistology (detection of $\mathrm{CD}_{20}, \mathrm{CD}_{3}, \mathrm{CD}_{117}, \mathrm{CD}_{13} 8$ and $\mathrm{Ki} 67$ ) as well as the performance of $\mathrm{FISH}$, the quality of extracted DNA and the results of immunoglobulin gene rearrangement.

Results. The combination of shortened fixation time combined with decalcification in MolDecal at $37^{\circ} \mathrm{C}$ or $47^{\circ} \mathrm{C}$ led in the majority of cases to various degrees of cell shrinkage. Detection of NASDCL was in contrast greatly improved in $>90 \%$ of the cases. The results of immunohistology were variable: while Ki67 detection was usually similar to the in-house protocol, membrane-bound and cytoplasmic antigens showed frequent deterioration in particular in specimens decalcified at $47^{\circ} \mathrm{C}$. In $>90 \%$ of the cases MolDecal solution led to high quality DNA allowing assessment of immunoglobulin gene rearrangements and delivering excellent FISH results, while the in house protocol led to almost opposite results. Conclusions. Manipulation of preanalytic steps greatly affects morphology, histochemistry, immunohistology and molecular analysis of bone trephines. We are currently optimizing our protocol to reach an optimal morphology, antigen and nucleic acid preservation. 


\section{DO-020}

Comparative analysis of protocols and hybridization efficiency of six commercially available BCR/ABL-FISH fusion probes on formalinfixed and paraffin-embedded bone marrow trephines

\section{T. Knas ${ }^{* 1}$, M. Buck', F. Stegelmann², P. Möller', T. Barth ${ }^{7}$ \\ ${ }^{1}$ Institute of Pathology, University Ulm, Ulm, Germany, ${ }^{2}$ Department of Internal Medicine, University Hospital of Ulm, Ulm, Germany}

Aims. The detection of the translocation $t(9,22)$ is the diagnostic hallmark for chronic myelogenous leukemia (CML). FISH analysis on bone marrow trephines is technically challenging. The aim was to investigate the reproducibility of FISH analysis in CML diagnostics and to identify the most efficient BCR/ABL-FISH fusion probe and protocol for the detection of the BCR/ABL fusion on formalin-fixed and paraffin-embedded bone marrow trephines.

Methods. Pilot studies of 17 bone marrow trephines with six commercial BCR/ABL-FISH fusion probes identified two probes with highest hybridization efficiency. These two probes were used for FISH analysis of 35 (22 CML and 13 non-CML) bone marrow trephines. Gold standard was classical karyotyping and/or detection of the BCR/ABL fusion transcript by RT-PCR performed simultaneously on bone marrow blood.

Results. The comparison of the six commercial BCR/ABL-FISH fusion probes identified two probes as most promising to detect the BCR/ABL fusion. The four main selection criteria for good hybridization efficiency were: 1 . Low background staining, 2. Low autofluorescence, 3 . Low non-specific hybridization signals, 4 . Specific and easily evaluable fluorescent hybridization signals. With one of these probes we achieved a sensitivity and specificity of $100 \%$ in 34 trephines: One trephine had to be excluded from analysis due to low hybridization efficiency. Best results were achieved with a slide thickness of $2 \mu \mathrm{m}$ and with a protease digestion time of 25 minutes and a concentration of $25 \mathrm{mg} / 100 \mathrm{ml}$. Conclusions. We have identified one commercial BCR/ABL-FISH fusion probe with good hybridization efficiency showing a high diagnostic sensitivity and specificity. Therefore, FISH analysis for the detection of the BCR/ABL fusion is a powerful tool in the diagnostic algorithm of CML on bone marrow trephines.

\section{DO-021}

\section{Genetic diversity in the development of SM-CMML as revealed by} NGS

M. Rechsteiner*1,2, R. Mueller ${ }^{3}$, T. Reineke', J. Goede ${ }^{3}$, A. Bohnert' ${ }^{1}$, Q. Zhong', M.G. Manz ${ }^{3}$, H. Moch', P. Wild', D. Zimmermann', M. Tinguely'

${ }^{1}$ Institute of Surgical Pathology, University Hospital, Zurich, Switzerland, ${ }^{2}$ Institute for Surgical Pathology, University Hospital Zurich, Zurich, Switzerland, ${ }^{3}$ Clinicis for Hematology, University Hospital Zurich, Zurich, Switzerland

Aims. Systemic mastocytosis (SM) associated with chronic myelomonocytic leukemia (CMML) is a rare disease of SM associated with clonal haemtological non-mast cell lineage disease (AHNMD). KITD816V was recently found in SM and CMML suggesting a common tumor precursor. Whole PB, single cell-fractions, bone marrow trephine (BMT) and normal tissue were investigated with different molecular techniques including NGS in search of a more detailed pathogenesis of SM-CMML.

Methods. PB of a patient with SM-CMML was investigated with two different NGS approaches including 1) NimbleGen Sequence Capture followed by sequencing with the GS Junior System (Roche, Switzerland) and 2) the Ion AmpliSeqTM Comprehensive Cancer Panel (CCP, Life Technologies) followed by sequencing with the Ion Proton (Ion Torrent, Life Technologies). Identified variants were verified by Sanger sequencing.

Results. Low coverage deep-sequencing (10x) on a GS 454 Junior revealed two as yet unreported SNVs (CBFA2T3 and CLTCL1) and one 1-bp deletion (BLM) which were verified in $\mathrm{PB}$ and the SM part of BMT by
Sanger sequencing. Since, the same variants were detected in normal heart tissue, they were classified as germ-line mutations. High coverage (>100ox) targeted re-sequencing on a Ion Proton revealed 177 variants in coding regions. Excluding SNPs, thefinal list comprised 11 variants. Among these 11 variants, genes implicated in SM-AHNMD pathogenesis were detected, such as TET2 (p.Thr1027fs, p.Cys1263Ser) and RUNX1 (p.AsniogSer). These variants were verified in PB and the SM part of BMT by Sanger sequencing whereas no mutation was detected in normal heart tissue. To investigate whether these mutations occurred at different checkpoints of lineage differentiation, FACS-sorted PB cell populations were analysed. Sanger sequencing revealed no signals in Band T-cells, whereas similar signal intensities for both TET2 mutations were seen in $\mathrm{CD}_{34}+$ precursor cells and $\mathrm{CD}_{16}+$ granulocytes comparable to the signals in the SM part of BMT. In contrast, RUNX1 exhibited a double intensity of the variant in $\mathrm{CD}_{34}+$ cells compared to the SM part of BMT and a homozygous variant signal in granulocytes.

Conclusions. This is one of the first in depth-analysis showing triple (KITD816V, TET2, RUNX1) mutations in the SM compartment of SMAHNMD. A hypothetical disease-model confirms a multistep pathogenesis of SM-CMML and adds further evidence that SM-CMML represents a spectrum of the same disease with common tumor progenitors.

\section{DO-022}

Coexistence of JAK-2 V617F mutated and splice factor mutated clones in patients with primary myelofibrosis (PMF) displaying different dynamics during and after allogenic bone marrow transplantation

S. Bartels ${ }^{* 1}$, G. Büsche', J. Schlue', B. Schlegelberger ${ }^{2}$, N. Kröger ${ }^{3}$, H. Kreipe', U. Lehmann'

'Institute of Pathology, Hannover Medical School, Hannover, Germany, ${ }^{2}$ Institute of Cell and Molecular Pathology, Medical School Hannover, Hannover, Germany, ${ }^{3}$ Department of Stem Cell Transplantation, University Medical Center Hamburg-Eppendorf, Hamburg, Germany

Aims. This project addresses the question whether patients with primary myelofibrosis (PMF) harbour more than one malignant clone and whether the occurrence and persistence of different mutations in different clones is related to the course of disease.

Methods. Genomic DNA was isolated from decalcified and formalinfixed paraffin-embedded bone marrow trephines from three patients with PMF taken before and after allogenic bone marrow transplantation. Standard histological and immunohistochemical work-up (CD3, $5,20,23,33,34,42,71,117$, MPO, Lysozym) was also performed. JAK-2, $\mathrm{SRSF}_{2}, \mathrm{U}_{2} \mathrm{AF} 1$ hot spots of mutation in exon 14, exon 1, and exon 2 and 6 , respectively, were analyzed by bidirectional pyrosequencing and Sanger sequencing. Quantification of allele frequencies and absence of mutated clones was independently validated by ultra-deep amplicon 454-sequencing. Morphological and molecular findings were correlated with clinical data and course of disease.

Results. Patient 1 showed a JAK-2 V617F (30\% allele frequency) and $\mathrm{U}_{2} \mathrm{AF} 1 \mathrm{Q} 157 \mathrm{P}$ (40\%) mutation-positive unclassifiable myeloproliferative disease with osteomyelofibrosis. After bone marrow transplantation the morphological picture changed completely to a JAK-2-negative RAEB-II whereas the $\mathrm{U}_{2} \mathrm{AF}_{1}$ mutation remained at a constant level. Patient 2 showed initially a JAK-2 V617F (80\%) and SRSF2 $\mathrm{P}_{95} \mathrm{H}$ (50\%) mutation in an unclassifiable myeloproliferative disease with osteomyelofibrosis. The SRSF2 mutation level remained at the same level after bone marrow transplantation for more than four years whereas the JAK-2 mutation stayed after complete loss directly after the bone marrow transplantation at a much lower level (approx. 15\%). Patient 3 showed a myelofibrosis and displayed after bone marrow transplantation initially a complete loss of the SRSF2 and the JAK-2 clone. However, the SRSF2 clone reappeared 9 months after transplantation and was not affected by a second transplantation. By contrast, the JAK-2 clone did not reappear. 
Conclusions. Combined mutations in JAK-2 and splice factor genes in patients with fibrosing MPN affect different clones which are independently affected by bone marrow transplantation and are associated with a distinct morphological appearance and clinical course. Ultra-deep amplicon sequencing enables highly sensitive and quantitative detection of minor clones, also in routinely processed bone marrow trephines.

\section{DO-023}

Fingolimod, PTP1B and PTPIP51-three promising molecules against chronic myeloid leukemia

\section{A. Brobeil ${ }^{* 1}$, W. Blau' ${ }^{2}$, A. Bräuninger', A. Schwarz', M. Rummel', S. Gatten- löhner? \\ 'Institute of Pathology, Justus Liebig University Giessen, Giessen, Germany, ${ }^{2}$ Department of Internal Medicine IV/V, Justus-Liebig University Giessen, Giessen, Germany}

Aims. In clinical practice chronic myeloid leukemia (CML) is specifically treated with tyrosine kinase inhibitors (TKI) targeting the BCRAbl fusion kinase. Yet, some patients develop resistance to the applied TKI necessitating a change of the TKI to another generation. Of note, a recent study showed that Fingolimod (FTY720), a sphingosine-1 analogon, is able to induce an antileukemic effect in CML cells due to an activation of the protein phosphatase (PP2a). Subseqently, a BCR-Abl degradation and an inactivation of AKT, as well as a suppression of the Wnt signaling pathway was observed [1] . Yet, the exact underlying molecular mechanism remains still unclear.

Methods. Therefore, we investigated the two CML cell lines, namely $\mathrm{K}_{562}$ and KCL-22, in regard to the signaling modulation of the rare described signaling molecules protein tyrosine phosphatase $1 \mathrm{~B}(\mathrm{PTP} 1 \mathrm{~B})$ and protein tyrosine phosphatase interacting protein 51 (PTPIP51) under Fingolimod application. Both proteins are described to play a role in the pathogenesis of acute myeloid leukemia [2]. Protein-protein interactions were investigated by in situ proximity ligation assays. Furthermore, the mitochondria were isolated and tested for changes in the PTPIP $_{51}$ binding. All cells were evaluated by flow cytometry in regard to the viability of mitochondria and apoptosis induction.

Results. Here we could show that higher amounts of PTPIP51 are localized at the mitochondria under Fingolimod treatment as compared to the untreated control group. This effect was associated with induction of apoptosis of the CML cells. Moreover, we could show that PTP1B interacts with BCR-Abl fusion protein in situ and that the interaction is decreased after Fingolimod application. In consequence, more BCRAbl was found to be associated with the proteasomal apparatus driving the degradation of BCR-Abl protein.

Conclusions. Fingolimod resembles a new antileukemic drug in the context of TKI failure, which acts through the $\mathrm{PTP}_{1} \mathrm{~B}$ and $\mathrm{PTPIP}_{51}$ axis to degrate BCR-Abl protein and to induce apoptosis in the malignant cells 1. Neviani P, Harb JG, Oaks JJ, Santhanam R, Walker CJ, Ellis JJ, Ferenchak G, Dorrance AM, Paisie CA, Eiring AM, Ma Y, Mao HC, Zhang B, Wunderlich M, May PC, Sun C, Saddoughi SA, Bielawski J, Blum W, Klisovic RB, Solt JA, Byrd JC, Volinia S, Cortes J, Huettner CS, Koschmieder S, Holyoake TL, Devine S, Caligiuri MA, Croce CM, Garzon R, Ogretmen B, Arlinghaus RB, Chen CS, Bittman R, Hokland P, Roy DC, Milojkovic D, Apperley J, Goldman JM, Reid A, Mulloy JC, Bhatia R, Marcucci G, Perrotti D (2013) PP2A-activating drugs selectively eradicate TKI-resistant chronic myeloid leukemic stem cells 2. Brobeil A, Bobrich M, Graf M, Kruchten A, Blau W, Rummel M, Oeschger S, Steger K, Wimmer M, (2010) PTPIP51 is phosphorylated by Lyn and c-Src kinases lacking dephosphorylation by PTP1B in acute myeloid leukemia

\section{DO-024}

Casein kinase 1a1 haploinsufficiency contributes to the hematopoietic stem cell expansion in a murine model of del(5q) myelodysplastic syndrome

R.K. Schneider ${ }^{* 1,2}$, M. McConkey ${ }^{2}$, M. Jaeras ${ }^{2}$, B. Ebert ${ }^{2}$

IInstitute of Pathology, RWTH University, Aachen, Germany, ${ }^{2}$ Department of Hematology, Harvard Medical School, Boston, United States

Aims. Casein kinase 1a $\left(\mathrm{CSNK}_{1} \mathrm{~A}_{1}\right)$ is a serine/threonine kinase and putative tumor suppressor gene located in the common deleted region (5q32) in del(5q) myelodysplastic syndrome (MDS). CSNK1 $\mathrm{A} 1$ is a regulator of the canonical WNT signaling pathway and may play a role in the clonal advantage of del(5q) hematopoietic stem cells (HSC).

Methods. To explore the biology and therapeutic potential of CSNK1A1 in MDS, we generated a conditional Csnk1a1 knockout mouse model in which exon 3-critical for the kinase function-is flanked by two loxP sites. To determine whether Csnk1a1 is required for hematopoiesis in vivo, the Cre-loxP system was used to inactive Csnk1a1 in hematopoietic cells in vivo. Experiments were performed in bone marrow transplantation assays in lethally irradiated recipient mice (Csnkıa1-/MxiCre+, Csnk1a1-/+MxiCre+ and $\mathrm{Mx} 1 \mathrm{Cre}+)$ to exclude extrinsic effects of Csnk1a1 deletion in the bone marrow stroma.

Results. Conditional homozygous inactivation of Csnk1a1 (Csnk1a1-/$\mathrm{Mx} 1 \mathrm{Cre}+)$ resulted in bone marrow failure, ablation of HSC, 553 induction, severe anemia and rapid lethality within 7-12 days, confirming that Csnk1a1 is essential for hematopoiesis. In striking contrast, the survival of Csnk1a1 haploinsufficient mice (Csnkı1a1-/+MxıCre+) was not impaired and a significant expansion of HSC, in particular long-term (LT) HSC (lin low/ckit+/Sca1+/CD48-/CD150+), repopulating hematopoiesis for a life-time, was confirmed in comparison to $\mathrm{Mx} 1 \mathrm{Cre}+$ controls. Further Csnk1a1 haploinsufficiency induced intranuclear $\beta$-Catenin accumulation, intrinsic HSC expansion with enhanced proliferation and cell cycle progression as well as increased stem cell renewal assessed by competitive transplantation assay, but no malignant transformation. Conclusions. Taken together, our results indicate that Csnk1a1 is essential for hematopoietic stem and progenitor cell survival. Csnkıa haploinsufficiency leads to increased HSC expansion and gain of function and might play an important role in the clonal advantage of the HSC in del(5q) MDS. This finding highlights the potential of preferential elimination of the del(5q) HSC sensitized by Csnk1aı haploinsufficiency and thus provides a potential therapeutic window. Consistent with this hypothesis, targeting the haploinsufficient kinase activity with the CSNK1 small molecule inhibitor D4476, selectively targets Csnk1a1 haploinsufficient HSC in vitro and in vivo.

\section{DO-025}

p16ink4a is expressed in neoplastic mast cells in systemic mastocytosis and may indicate a possible role of oncogene-induced senescence in the pathogenesis of the disease

\section{Walz*1, K. Petat-Dutter', S. Berezowska², F. Rueff ${ }^{3}$, A. Reiter', T. Kirchner',} H.-P. Horny', K. Sotlar ${ }^{1}$

'Instute of Pathology, University of Munich, Munich, Germany, ${ }^{2}$ Instute of Pathology, University of Bern, Bern, Switzerland, ${ }^{3}$ Department of Dermatology and Allergology, University of Munich, Munich, Germany, ${ }^{43} \mathrm{rd}$ Medical Clinic, University Medicine Mannheim, Mannheim, Germany

Aims. According to the current WHO classification, mastocytosis is defined as a myeloproliferative neoplasm. However, not proliferation but abnormal accumulation of mast cells (MC) is central in pathogenesis in this disease. Here, we evaluated the expression of pi6ink4a in lowgrade [i.e., indolent systemic mastocytosis (ISM)] and high-grade [i.e., aggressive systemic mastocytosis (ASM) and mast cell leukemia (MCL)] mastocytosis and tested whether p16ink4a acts as an indirect marker of senescence. 
Methods. Bone marrow biopsies from 72 patients with various subtypes of SM (ISM, n=46; ASM, n=20; MCL, n=6) and 10 controls of various hematologic disorders with mast cell hyperplasia $(\mathrm{MCH})$ were investigated by immunohistochemistry for the expression of pi6ink4a. The percentage of (tryptase-positive) MC and the staining intensity for p16ink4a were assessed according to the immunoreactive score (IRS) for breast cancer published by Remmele and Stegner (Remmele, Pathologe 1987).

Results. p16ink4a immunoreactivity was absent in $\mathrm{MC}$ of $\mathrm{MCH}(\mathrm{n}=10)$. However, MC of 44/46 (96\%) ISM and of $15 / 16$ (94\%) of ASM/MCL cases expressed p16ink4a with variable IRS scores. While the mean percentage of stained MC in ISM was $51 \%$, the percentage for ASM/MCL was $19 \%$. Accordingly, the mean IRS was 6.8 in ISM and 2.8 in ASM/MCL. Conclusions. p16ink4a represents a new IHC marker for neoplastic MC in SM. Its reduced expression may be indicative for the presence of a high-grade variant of the disease. In addition, the expression of p16ink4a in ISM may indicate the possibility of oncogene (KIT-D816V)induced senescence in the pathogenesis of ISM.

\section{DO-026}

Lineage specific distribution of TET2 mutations in systemic mastocytosis

C. Walz ${ }^{* 1}$, D. Hofmann ${ }^{1,2}$, L. Butschek', T. Haferlach ${ }^{3}$, A. Reiter', J. Schwaab ${ }^{4}$, S. Schnittger ${ }^{3}$, H.-P. Horny', K. Sotlar

${ }^{1}$ Instute of Pathology, University of Munich, Munich, Germany, ${ }^{2}$ Instute of Pathology, Munich, Germany, ${ }^{3}$ MLL Münchner Leukämielabor GmbH, Munich, Germany, ${ }^{43}$ rd Medical Clinic, University Medicine Mannheim, Mannheim, Germany

Aims. Systemic mastocytosis (SM) is a clonal myeloid neoplasm characterized by proliferation and accumulation of neoplastic mast cells in different organs which usually harbor the D816V-mutated variant of KIT. In approximately $20-30 \%$ of patients with SM, additional heterogeneous mutations within the tumor suppressor gene "The Ten-Eleven Translocation-2" (TET2) are identified which are believed to precede the KIT mutation in tumorigenesis. However, the specific distribution of TET2 mutations in different hematopoietic lineages remains unknown. Methods. Twelve patients suffering from SM with an associated clonal hematological non-mast cell lineage (SM-AHNMD) disease and harboring both, mutant KIT and TET2 in whole genomic DNA, were identified by next-generation-sequencing using peripheral blood. Sections of paraffin-embedded bone marrow biopsies were stained with antibodies against $\mathrm{CD}_{3}, \mathrm{CD}_{15}$ and mast cell tryptase. Single stained cell were then laser-microdissected using the technique of laser pressure catapulting. Pooled DNA samples of about 200 cells were used to amplify the previously identified mutational loci of KIT and TET2 followed by Sanger sequencing.

Results. Interestingly, mutated KIT and one or two TET2 mutations were invariably present in the microdissected mast cell population in all cases (12/12). In contrast, $67 \%$ of cases displayed KIT D816V within the CD15-positive granulocytic lineage (8/12), while mutated TET2 was detected in $83 \%$ of patients (10/12). Within the lymphoid lineage, KIT D816V was only identified in one case $(1 / 12,8 \%)$, while mutated TET2 was found in four patients $(4 / 12,33 \%)$. If two different TET2 mutations were present in the same patient $(n=7)$, they invariably showed a congeneric lineage distribution.

Conclusions. Here we show that TET2 mutations in SM-AHNMD are consistently present in mast cells, are typically present also in the granulocytic lineage and are rarely present in the lymphocytic lineage. Furthermore, mutation frequency suggests that TET2 mutations occur in a more immature progenitor than KIT D816V.

\section{AG Hämatopathologie II}

D0-027

Expression of Mucin-1 in multiple myeloma and its precursors: correlation with glycosylation and subcellular localisation

E. Ellert ${ }^{* 1}$, U. Mandel', H. Clausen ${ }^{2}$, N. Lehners ${ }^{3}$, M.S. Raab ${ }^{3}$, H. Gold-

schmidt ${ }^{3}$, R. Schwartz-Albiez', M. Andrulis ${ }^{1}$

'Institute of Pathology, Ruprecht-Karls-University, Heidelberg, Germany, ${ }^{2}$ Copenhagen Center for Glycomics, Copenhagen, Denmark, ${ }^{3}$ Department of Hemathology, Ruprecht-Karls-University, Heidelberg, Germany, ${ }^{4}$ German Cancer Research Center, DKFZ, Functional Genome Analysis, Heidelberg, Germany

Aims. MUC1 is a heterodimer which consists of an extracellular N-terminal subunit (MUCiN) and the transmembrane C-terminal subunit (MUC1C). MUC1N is among the best characterized tumour-associated antigens that is up-regulated and aberrantly glycosylated in multiple myeloma (MM). MUC1C is involved in activation of oncogenic signalling pathways, aberrantly expressed and translocated to the nucleus in MM cell lines. Taken together, these observations indicate that both MUC 1 subunits may have an oncogenic role in MM. However, the expression of MUC1C has not been studied in primary MM samples yet. The aim of the study was to analyse expression of both MUC1 subunits in different clinical stages of MM and correlate these data with the disease outcome.

Methods. The study cohort $(\mathrm{n}=131)$ included bone marrow biopsies without plasma cell neoplasia $(n=10)$, with MGUS $(n=14)$, smoldering MM $(\mathrm{n}=24), M M(\mathrm{n}=60$; including biopsies taken before treatment $\mathrm{n}=28$ and at relapse/progress $n=32$ ) and extramedullary plasmacytoma $(n=23)$. The differential expression of MUC1 subunits was investigated by immunohistochemistry with monoclonal antibodies against $\mathrm{MUC1N}$ (EMA and 5 E10), tumor associated-glycoforms of MUC1N (5E5) and MUC1C subunit.

Results. Clonal plasma cells showed reduced MUCiN expression as detected by EMA and ${ }_{5}$ E10 antibodies. The tumor-associated MUC1N epitope ${ }_{5} \mathrm{E}_{5}$ was found in neoplastic plasma cells but not in normal controls. Nuclear localization of MUC1C was frequently observed in normal plasma cells and was equally frequent in all diseases stages. In extramedullary infiltrates the absence of nuclear MUC1C was significantly associated with increased proliferation rate $(\mathrm{p}=0.05)$. Loss of both MUC1 subunits in $M M(n=12)$ was associated with significantly shorter overall survival and was more frequent in pre-treated MM samples.

Conclusions. In summary, our findings indicate that aberrant MUC1N glycosylation is an early event in the pathogenesis of multiple myeloma. MUC1C nuclear localization is not an abnormal finding and is unlikely to be a major driver of tumor progression in MM. Complete loss of both MUC1 subunits is frequently observed in relapsed disease and is associated with adverse outcome.

\section{DO-028}

Detectability of MYD88 L265P mutations in formalin fixed and decalcified bone marrow biopsies from patients with Waldenström's macroglobulinemia

\section{A.M.May ${ }^{* 1}$, I.B. Capaldi',2, A. Schmitt-Gräff', G. Kayser', J.C. Perazzo²,}

M. Werner', P. Fisch'

'Department of Pathology, Universtity Medical Center, Freiburg, Germany, ${ }^{2}$ Laboratory of Hepatic Encephalopathy and Portal Hypertension, University of Buenos Aires, Buenos Aires, Argentina

Aims. Diagnosis of bone marrow infiltration by Waldenström's macroglobulinemia (WM)/lymphoplasmacytic lymphoma poses a diagnostic challenge in hematopathology. There is no definitive morphology or immunophenotype that can reliably distinguish between infiltration 
of paraffin-embedded bone marrow sections by WM/LPL and other indolent lymphomas, in particular that of splenic marginal zone type (SMZL) that also may show plasmacytic maturation. Recently, a new oncogenic gain of function mutation in the human MYD88 gene has been described that is present in most WM/LPL cases, but only seldomly detectable in other B-cell chronic lymphoproliferative disorders, including SMZL. Here we describe two newly developed diagnostic protocols for detection of this $\mathrm{L}_{265} \mathrm{P}$ mutation in paraffin-embedded archival tissues that are particularly applicable to decalcified bone marrow biopsies.

Methods. We use regular Sanger sequencing and an allele-specific MYD88 L256P PCR.

Results. Sanger sequencing showed reproducibly good results for patient's samples with a bone marrow infiltration down to approximately $10 \%$ B-cell lymphoma cells. When using the allele specific PCR we were able to detect the presence of the MYD88 L265P mutation down to approximately $1 \%$ of lymphoma cells in the bone marrow.

Conclusions. We show that these methods are easily applicable to archival bone marrow specimens and markedly improve the diagnostic accuracy in bone marrow infiltration by indolent B-cell lymphomas.

\section{DO-029}

\section{Frequency of the hallmark translocation $t(14 ; 18)$ and secondary genetic events in follicular lymphoma patients treated within the German Low-grade Lymphoma Study Group (GLSG)}

\section{E. Leich ${ }^{* 1}$, H. Horn ${ }^{* 2}$, M. Wartenberg' ${ }^{1}$ A.M. Staiger ${ }^{* 3}$, E. Hostert, M.} Unterhalt, W. Klapper ${ }^{5}$, K. Koch ${ }^{5}$, H.-W. Bernd ${ }^{6}$, A.C. Feller ${ }^{6}$, M. Hummel', H. Stein 7 , M.-L. Hansmann ${ }^{8}$, T.F. Barth ${ }^{9}$, P. Möller ${ }^{9}$, M. Dreyling ${ }^{4}$, W. Hidde$\mathrm{mann}^{4}$, G. Ott ${ }^{10}$, A. Rosenwald

'Institute of Pathology, Universität Würzburg, Würzburg, Germany, ${ }^{2} \mathrm{Dr}$. Margarete Fischer-Bosch-Institute of Clinical Pharmacology, Stuttgart, Germany, ${ }^{3}$ Dr. Margarete Fischer-Bosch Institute of Clinical Pharmacology, Stuttgart, Germany, ${ }^{4}$ GLSG Study Center, Department of Internal Medicine III, University of Munich, München, Germany, ${ }^{5}$ Institute of Pathology, Hematopathology Section and Lymph Node Registry, Universitätsklinikum Schleswig-Holstein, Campus Kiel, Kiel, Germany, ${ }^{6}$ Institute of Pathology, Universitätsklinikum Schleswig-Holstein, Campus Lübeck, Lübeck, Germany, ${ }^{7}$ Institute of Pathology, Campus Benjamin Franklin, Charité Universitätsmedizin, Berlin, Germany, ${ }^{8}$ Institute of Pathology, Universitätsklinikum Frankfurt, Frankfurt, Germany, ${ }^{9}$ Institute of Pathology, University Ulm, Ulm, Germany, ${ }^{10}$ Department of Clinical Pathology, Robert-Bosch-Krankenhaus, Stuttgart, Germany

Aims. The hallmark BCL2 translocation $\mathrm{t}(14 ; 18)(\mathrm{q} 32 ; \mathrm{q} 21)$ constitutes the primary genetic event in $85 \%$ of follicular lymphoma (FL). However, it is not sufficient for neoplastic transformation. Numerous studies were recently performed, aiming at the elucidation of the prognostic impact of secondary genetic alterations in FL. Among those, rearrangements of MYC and BCL6, as well as recurrent losses in chromosomes 1p, 6q and $17 \mathrm{p}$ were detected that were found to occur more frequently in patients with an adverse clinical course. Owing to the fact that previously published data were generated almost exclusively in small patient cohorts, it is of considerable interest to validate the impact of genetic alterations in a large study cohort of homogenously treated FL patients.

Methods. Paraffin-embedded tumor samples of 539 FL patients treated with MCP, CHOP, or R-CHOP within GLSG trials were investigated by fluorescence in situ hybridization (FISH) on tissue microarray (TMA) format to detect breaks at BCL2, BCL6 and MYC loci, as well as genomic deletions in chromosomes $1 \mathrm{p}$ (1p36.3), 6q (6q21 and 6q25) and 17p (17p13.1 and 17p13.3), respectively.

Results. An extensive primary correlative study of TMA vs. full-slide samples yielded highly satisfactory results, thus forming the proof of principle for a large investigative TMA FISH study. Interpretable results were obtained in $65-82 \%$ of tumor samples, depending on the probe

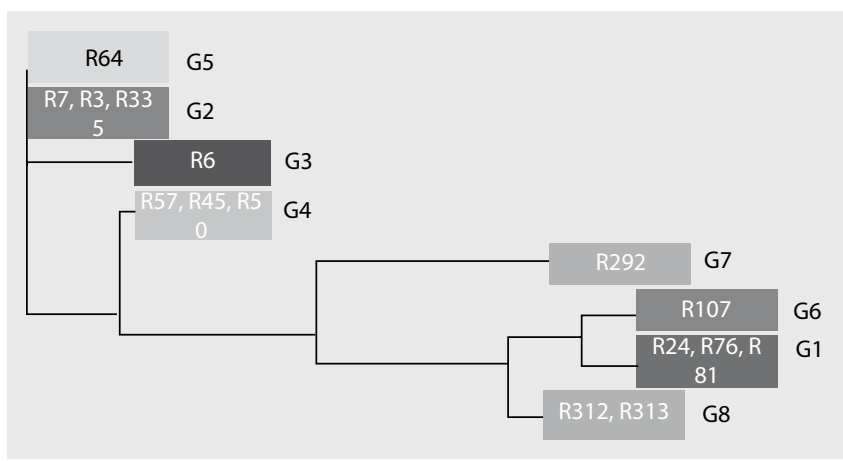

Fig. 1 | D0-030 $\triangle$ Ongoing mutations: Phylogenetic tree of the eight sequence groups with the most frequent representatives. Spatial distance of the groups corresponds to the number of diverging base pairs

used (deletion probes: $65-82 \%$; translocation probes: $78-82 \%$ ). BCL2 was found to be translocated in $86 \%(361 / 421)$ of cases, which is in line with previous findings. Breaks in BCL6 were observed in 10\% (45/441) and the MYC breaks in $2 \%(10 / 420)$. Signal constellations indicative for deletions of $1 \mathrm{p}_{3} 6.3,6 \mathrm{q} 21,6 \mathrm{q} 25,17 \mathrm{p} 13.1$ and $17 \mathrm{p} 13.3$ were observed in $20 \%$ (59/293), 30\% (111/367), 33\% (105/322), 20\% (74/362) and $24 \%$ (89/371), respectively.

Conclusions. Correlation of these genetic events with the outcome of FL patients is still ongoing. However, upon completion of this project we might have genetically identified prognostic subgroups of FL, a prerequisite for risk stratification in this tumor that may allow for a more individualized treatment of patients. Finally, we will be able to answer the question whether there is a prognostic difference between FL patients with and without the translocation $\mathrm{t}(14 ; 18)$.

\section{DO-030}

Ultra deep sequencing detects ongoing mutations and interfollicular trafficking in follicular lymphoma in situ

\section{P. Kosmidis*1, I. Bonzheim', C. Dufke², S. Colak', T. Hentrich ${ }^{2}$, C. Schroeder ${ }^{2}$, P. Bauer', P. Adam', F. Fend'}

'Institute of Pathology and Neuropathology, General Pathology and Pathological Anatomy, Eberhard Karls University, Tuebingen, Germany, ${ }^{2}$ Institute of Human Genetics, Eberhard Karls University, Tuebingen, Germany

Aims. Follicular Lymphoma (FL) is characterized genetically by significant ongoing hypermutations and interfollicular trafficking. Follicular lymphoma in situ (FLIS) is defined as a population of $\mathrm{t}(14 ; 18)+$, and strongly BCL2 expressing B cells confined to reactive germinal centers structures [1] . Only part of the FLIS lesions is considered to progress to an overt FL. The presence of the genetic characteristics of manifest FL in FLIS lesions has not yet been analyzed. In this study we analyzed a unique case of FLIS lesions in multiple lymph nodes in a patient without history or evidence of a manifest FL.

Methods. We used laser-microdissection and next generation sequencing (NGS) of the immunoglobulin heavy chain gene from DNA samples of pooled follicles and single germinal centers colonized by the FLIS. Subsequently phylogenetic trees and topographic maps of the allel distribution were calculated.

Results. We monitored significant intraclonal diversity (ongoing mutations) and interfollicular migration patterns of the FLIS cells with the representatives of the different sequence cluster groups being detected with varying frequency not only in adjacent follicular structures but also in disparate lymph nodes.

Conclusions. Our analyses for the first time monitored extensive ongoing mutations and significant interfollicular migration patterns between germinal centers of the same lymph node as well as between different lymph nodes of a FLIS lesion. Of note, the patient did not have 


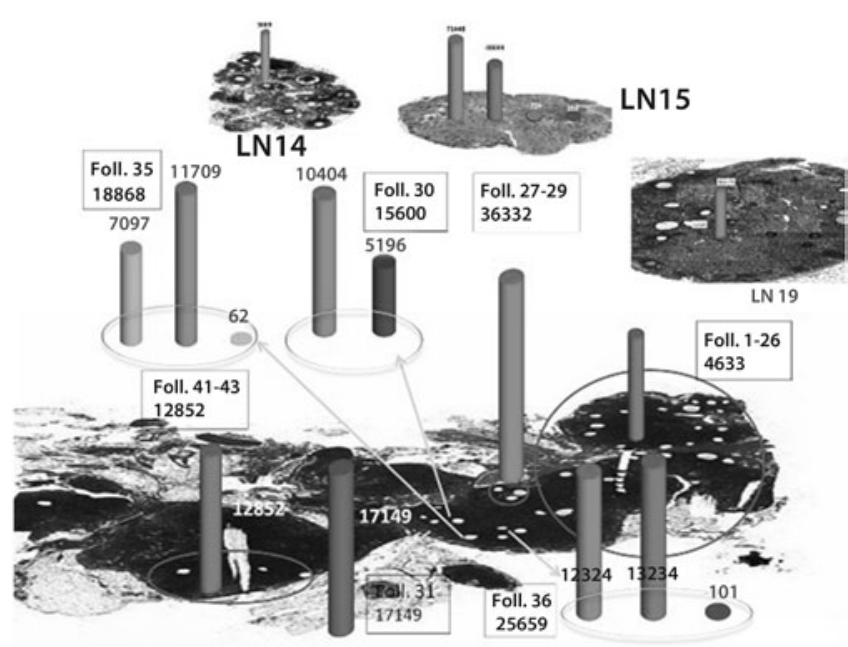

Fig. 2 | DO-030 $\Delta$ Topographic migration and somatic hypermutation map of FLIS

a history nor any evidence of a manifest FL in the follow-up time of now four years. Further studies are warranted to elucidate the precise interconnection of FLIS with manifest FL on a genetical and immunological level (• Fig. 1, 2).

\section{Literatur}

1. Swerdlow SH (2008) World Health Organization Classification of Tumours. WHO Classification of Tumours of Haematopoietic and Lymphoid Tissues

\section{DO-031 \\ MYC status in diffuse large B-cell lymphoma with immunoblastic morphology}

\section{A.M. Staiger ${ }^{* 1}$, H. Horn ${ }^{2}$, A. Rosenwald ${ }^{3}$, G. Ott ${ }^{4}$, M.M. Ott ${ }^{5}$}

'Dr. Margarete Fischer-Bosch Institute of Clinical Pharmacology, Stuttgart, Germany, ${ }^{2}$ Dr. Margarete Fischer-Bosch-Institute of Clinical Pharmacology, Stuttgart, Germany, ${ }^{3}$ Institute of Pathology, Universität Würzburg, Würzburg, Germany, ${ }^{4}$ Department of Clinical Pathology, Robert-Bosch-Krankenhaus, Stuttgart, Germany, ${ }^{5}$ Institute of Pathology, Caritas-Hospital, Bad Mergentheim, Germany

Aims. We have previously shown that immunoblastic (IB) morphology of tumors constitutes a significant risk in patients with diffuse large Bcell lymphoma (DLBCL) (Ott et al. 2010, Blood). In our daily diagnostic experience, we repeatedly encountered the presence of a MYC gene rearrangement in DLBCL with IB morphology. We therefore undertook a systematic study to determine the frequency of 8q24/MYC rearrangements in DLBCL of immunoblastic type and compared the data obtained to a group of non-IB tumors.

Methods. Paraffin-embedded tumor samples of 39 DLBCL with IB morphology and 69 non-IB DLBCL were investigated by fluorescence in situ hybridization (FISH) with break-apart probes to detect translocations of the MYC-, BCL2- and BCL6-loci and immunohistochemistry for protein expression of $\mathrm{CD} 10, \mathrm{MUM} 1 / \mathrm{IRF}_{4}, \mathrm{BCL} 2, \mathrm{BCL} 6, \mathrm{Ki} 67$, and MYC, respectively.

Results. Signal constellations indicative of a translocated MYC gene were found in $13 / 39$ IB DLBCL (33.3\%). In contrast, only 5/69 tumor specimens (7.2\%) were MYC-positive in the non-IB DLBCL samples. All 12 MYC-break-positive IB-DLBCL analyzed harbored an juxtaposition of MYC to the IGH-locus (MYC-IGH fusion), while one sample was not interpretable. MYC translocations were observed in association with a BCL6 rearrangement in two IB-DLBCL $(2 / 13,15.4 \%)$ and with a dual BCL2 and BCL6 translocation in one tumor sample (1/13, 7.7\%). An amplification of the BCL2 gene was detected in one MYC-positive IB$\operatorname{DLBCL}(1 / 13,7.7 \%)$. IB-DLBCL specimens with MYC translocations were more often CD1o positive and IRF4/MUM1 negative than their MYC non-rearranged counterparts [ $7 / 13(53.8 \%)$ vs. $3 / 26(11.5 \%), p=0.0035$ and $8 / 13$ (61.5\%) vs. $20 / 23$ (86.9\%), $\mathrm{p}=0.08$, respectively]. A higher proportion of MYC break positive IB had a Ki67 index $>80 \%$ in comparison to MYC break-negative tumors [11/13 (84.6\%) vs. $13 / 26$ (50\%), $\mathrm{p}=0.037]$ ]. 22 tumors (22/39, 56.4\% showed overexpression of MYC protein (MYChigh) with samples harboring between $41 \%$ and $100 \%$ stained nuclei. $11 / 13$ (84.6\%) cases were MYChigh in the MYC translocation-positive group and 11/26 $(42 \%)$ in the translocation-negative group $(\mathrm{p}=0.01)$.

Conclusions. These results confirm that IB are a major constituent of DLBCL with MYC rearrangements and clearly delineate the importance of MYC testing in this tumor subtype.

\section{DO-032}

The prognostic impact of gene rearrangements and protein expression of MYC, BCL2 and BCL6 in elderly and young high-risk DLBCL patients enrolled in prospective studies of the DSHNHL (for the DSHNHL and MMML-MYC-SYS)

H. Horn ${ }^{* 1}$, M. Ziepert ${ }^{2}$, C. Becher ${ }^{3}$, A.M. Staiger', T.F. Barth ${ }^{5}$, H.-W. Bernd ${ }^{6}$, M. Wartenberg ${ }^{7}$, A.C. Feller ${ }^{6}$, W. Klapper ${ }^{8}$, C. Stuhlmann-Laeisz ${ }^{8}$

M. Hummel', H. Stein ${ }^{9}$, D. Lenze ${ }^{9}$, S. Hartmann ${ }^{10}$, M.-L. Hansmann ${ }^{10}$, C. Schmelter, P. Möller ${ }^{5}$, S. Cogliatti ${ }^{11}$, M. Pfreundschuh ${ }^{12}$, L. Trümper ${ }^{13,}$ R. Siebert ${ }^{3}$, M. Loeffler ${ }^{2}$, N. Schmitz ${ }^{14}$, G. Ott ${ }^{15}$, A. Rosenwald ${ }^{7}$

${ }^{1}$ Dr. Margarete Fischer-Bosch-Institute of Clinical Pharmacology, Stuttgart, Germany, ${ }^{2}$ Institute for Medical Informatics, Statistics, and Epidemiology, Universität Leipzig, Leipzig, Germany, ${ }^{3}$ Institute of Human Genetics, Christian-Albrechts-University Kiel and University Hospital Schleswig- Holstein, Campus Kiel, Kiel, Germany, ${ }^{4}$ Dr. Margarete Fischer-Bosch Institute of Clinical Pharmacology, Stuttgart, Germany, ${ }^{5}$ Institute of Pathology, University Ulm, Ulm, Germany, ${ }^{6}$ Institute of Pathology, Universitätsklinikum Schleswig-Holstein, Campus Lübeck, Lübeck, Germany, ${ }^{7}$ Institute of Pathology, Universität Würzburg, Würzburg, Germany, ${ }^{8}$ Institute of Pathology, Hematopathology Section and Lymph Node Registry, Universitätsklinikum Schleswig-Holstein, Campus Kiel, Kiel, Germany, ${ }^{9}$ Institute of Pathology, Campus Benjamin Franklin, Charité Universitätsmedizin, Berlin, Germany, ${ }^{10}$ Institute of Pathology, Universitätsklinikum Frankfurt, Frankfurt, Germany, ${ }^{11}$ Institute of Pathology, Kantonal Hospital St.Gallen, St. Gallen, Switzerland, ${ }^{12}$ Medizinische Klinik I, Saarland University Medical School, Homburg/ Saar, Germany, ${ }^{13}$ Department of Hematology and Oncology, Georg-August Universität, Göttingen, Göttingen, Germany, ${ }^{14}$ Department of Hematology, Asklepios Klinik St. Georg, Hamburg, Germany, ${ }^{15}$ Department of Clinical Pathology, Robert-Bosch-Krankenhaus, Stuttgart, Germany

Aims. MYC rearrangements occur in 5-10\% of diffuse large B-cell lymphomas (DLBCL) and confer an increased risk to CHOP and R-CHOP treated patients. However, many studies harbour only small patient cohorts with relatively high median patient age. The prognostic relevance of MYC translocations and protein expression was, therefore, investigated in two prospectively randomized trials of the DSHNHL.

Methods. Tumor samples from 442 and 112 patients with de novo DLBCL enrolled in the RICOVER-60 (patients >60 years; Pfreundschuh et al. 2008, Lancet Oncol) and in the MegaCHOEP trials (young high-risk patients 18-6o years, aaIPI 2 or 3; Schmitz et al. 2012, Lancet Oncol) of the DSHNHL, respectively, were stained for the expression of MYC, BCL2, and BCL6, and fluorescence in-situ hybridisation (FISH) was performed to detect MYC-, BCL2- and BCL6-breaks.

Results. Rearrangements of MYC, BCL2 and BCL6 were detected in $8.8 \%, 13.5 \%$ and $28.7 \%$ in DLBCL of elderly patients, respectively and in $13.6 \%, 20.7 \%$ and $30.9 \%$ in DLBCL of young high-risk patients, respectively. The presence of a MYC translocation, and MYChigh, BCL2high and BCL6low protein expression were associated with inferior survival (EFS, OS) in elderly DLBCL patients in uni- and multivariate analy- 
ses. Most important, an immunohistochemical (IHC) and a FISH/IHC prognostic risk score could be defined that identifies a group of $15 \%$ of elderly patients with extremely poor prognosis within the high-risk IPI group. In contrast to elderly patients (also within IPI group 3-5), presence of a BCL2 break ( $R R=4.7,95 \%$ CI 1.8-12.2) were associated with inferior overall survival in univariate and multivariate analyses in young high-risk DLBCL patients. MYC breakage $(\mathrm{RR}=2.4,95 \% \mathrm{CI}$ 0.8-7.5) and protein overexpression of MYC $(\mathrm{RR}=2.4,95 \% \mathrm{CI}$ 0.9-6.5), but not $\mathrm{BCL} 2$ or BCL6 indicated inferior overall survival. BCL2 overexpression was associated with inferior EFS ( $\mathrm{RR}=2.2,95 \% \mathrm{CI}$ o.9-5.5) and $\mathrm{PFS}(\mathrm{RR}=2.8$, 95\% CI 1.0-8.2).

Conclusions. MYC status with BCL2 and BCL6 expression predicts outcome in elderly DLBCL patients and identifies a subset of patients with dismal prognosis. Analysis of prognostic risk factors in tumors of younger high-risk patients confirmed the importance of MYC testing in DLBCL. Remarkably, breaks at BCL2 emerged as a strong negative prognostic indicator in younger high-risk patients in contrast to elderly and elderly high-risk patients. Therefore, modifications of the spectrum of biological risk factor testing across patient age groups are advised.

\section{DO-033}

TP53 mutations are frequent events in double-hit B-cell lymphomas with MYC and BCL2 but not MYC and BCL6 translocations

\section{N. Gebauer', V. Bernard ${ }^{* 1}$, W. Gebauer ${ }^{2}$, A.C. Feller', C. Thorns', H. Merz' 'Department of Pathology, Reference Centre for Lymph Node Pathology and Hematopathology, University Hospital of Schleswig-Holstein, Campus Luebeck, Lübeck, Germany, ${ }^{2}$ German Red Cross Blood Transfusion Service, Institute Oldenburg, Germany, Oldenburg, Germany}

Aims. Double-hit lymphomas (DHL) with MYC and either BCL2 or BCL6 rearrangements are rare neoplasms with an aggressive clinical presentation and unfavourable prognosis [2]. Despite recent advances, molecular characterisation of DHL remains insufficient especially the role of $\mathrm{TP}_{53}$ pathway disruption is unknown $[1,3]$.

Methods. We employed a next-generation sequencing approach in order to investigate the mutational status of $\mathrm{TP}_{53}$ in DHL ( $=37 ; 17 \mathrm{MYC}+/$ $\mathrm{BCL} 2+; 16 \mathrm{MYC}+/ \mathrm{BCL} 6+; 4$ triple hit) and performed capillary sequencing of all detected variants. 18 cases of sporadic Burkitt lymphoma and 20 cases of diffuse large B-cell lymphoma were analyzed in comparison. Subsequently, we correlated genomic data with immunohistochemical reactivity for $\mathrm{p} 53$.

Results. We identified TP53 mutations in $\mathrm{MYC}+/ \mathrm{BCL}_{2}+$ lymphomas at a frequency intermediate between DLBCL and Burkitt lymphoma ( $35 \%$ vs $25 \% / 55 \%)$. The majority of sequence variations $(27 / 33 ; 82 \%)$ was detected within the evolutionarily conserved DNA binding domain. Remarkably, $\mathrm{TP}_{53}$ mutations were particularly rare in MYC+/BCL6+ lymphomas $(6.25 \%)$. Most tumours with $\mathrm{TP}_{53}$ mutations were shown to express the protein at an elevated level $(17 / 18 ; 93,4 \%)$ with more than $50 \%$ positive staining cells, however we also detected an elevated $\mathrm{TP}_{53}$ expression in tumours with wild type TP53 (10/30; 33,3\%).

Conclusions. Our findings indicate a significant difference between these two types of DHL at a molecular level with pathogenetic implications, as arguably, $\mathrm{TP}_{53}$ mutations inhibiting $\mathrm{p}_{53}$ mediated promotion of apoptosis pose a synergistic advantage in clonal evolution of cells with malignantly enforced overexpression of BCL2. Immunohistochemical staining appears to be a sensitive surrogate of $\mathrm{TP}_{53}$ mutation status with moderate specificity.

\section{Literatur}

1. Aukema SM, Kreuz M, Kohler CW, Rosolowski M, Hasenclever D, Hummel M, Kuppers R, Lenze D, Ott G, Pott C, Richter J, Rosenwald A, Szczepanowski M, Schwaenen C, Stein H, Trautmann H, Wessendorf S, Trumper L, Loeffler M, Spang R, Kluin PM, Klapper W, Siebert R (2013) Biologic characterization of adult MYC-translocation positive mature B-cell lymphomas other than molecular Burkitt lymphoma. Haematologica
2. Aukema SM, Siebert R, Schuuring E, van Imhoff GW, Kluin-Nelemans HC, Boerma EJ, Kluin PM (2011) Double-hit B-cell lymphomas. Blood 3. Gebauer N, Bernard V, Feller AC, Merz H (2013) ID3 Mutations Are Recurrent Events in Double-hit B-Cell Lymphomas. Anticancer Research

\section{DO-034}

\section{Autophagy promotes survival and growth of Hodgkin's lymphoma} cells

\section{K. Birkenmeier*1, K. Moll', T. Wenz' ${ }^{2}$, S. Dröse ${ }^{3}$, M.-L. Hansmann ${ }^{1}$}

'Dr. Senckenberg Institute of Pathology, Medical School, Goethe-University, Frankfurt am Main, Germany, ${ }^{2}$ Institute for Genetics, Köln, Germany, ${ }^{3}$ Clinic of Anesthesiology, Intensive-Care Medicine and Pain Therapy, Goethe-University Hospital, Frankfurt am Main, Germany

Aims. Autophagy, a catabolic pathway to degrade cellular components, promotes cell survival in response to various stressors, but can also induce cell death. The effect of autophagy on cellular metabolism is celltype dependent, linked with both, tumor suppression or promotion. A number of drugs that inhibit autophagy are available. The finding that autophagy enhances tumor cell survival would thus lead to strategies for novel therapies. Therefore we studied the status and the pathogenic role of autophagy in classical Hodgkin's lymphoma (cHL).

Methods. To investigate the autophagy status in cHL we analyzed expression of autophagy-relevant genes by Western Blot and immunohistochemistry of a total of $6 \mathrm{cHL}$ cell lines, as compared to normal B cells (isolated by MACS from tonsillar cell suspension of healthy donors) and primary cases and investigated autophagic flux by fluorescence microscopy. We furthermore performed functional experiments to determine the role of autophagy in survival, growth and metabolism of cHL cells. Results. We found increased expression of key autophagy-relevant genes in $\mathrm{cHL}$ cell lines and primary cases. $\mathrm{LC}_{3} \mathrm{I}$ processing to $\mathrm{LC}_{3} \mathrm{II}$, the letter protein being specifically localized to autophagic structures, was increased in cHL cells, as revealed by Western Blot analysis of cHL cell lines and immunohistochemistry of lymphoma cases. The tumor cells of 13 of totally 17 tested cases were strongly LC 3 II-positive (at least $75 \%$ positive tumor cells per case). Only 1 case demonstrated negative staining (less than $25 \%$ positive tumor cells per case). Consistently with these findings, fluorescence staining revealed elevated numbers of autophagic vacuoles in cHL cells. We found that the treatment of $\mathrm{cHL}$ cells with chloroquine (CQ), which blocks autophagosome degradation, further increased the number of $\mathrm{LC}_{3} \mathrm{II}$ puncta and the expression of $\mathrm{LC}_{3} \mathrm{II}$ in $\mathrm{cHL}$ cell lines suggesting intact autophagic flux. Fluorescence staining revealed co-localization of $\mathrm{LC}_{3} \mathrm{II}$ with the lysosomal proteins EEA1, Rab7A and LAMP-1 in all cHL cell lines analyzed. Treatment of cHL cells with CQ induced apoptosis and reduced proliferation rates. Moreover, we observed changes in cellular energy metabolism, as evidenced by decreased mitochondrial oxygen consumption rates and elevated levels of glucose consumption and lactate production.

Conclusions. We conclude that autophagy is constitutively activated in cHL. Moreover, cHL cells require autophagy for growth and survival making them sensitive to autophagy inhibition. 


\section{Abstracts}

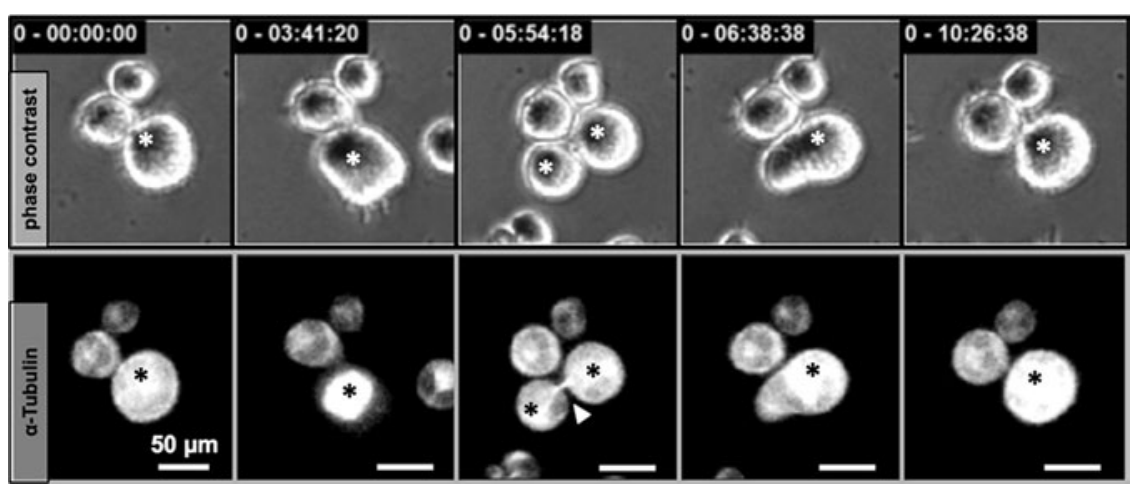

Fig. 1 | DO-036 $<$ Incomplete cytokinesis precedes re-fusion of daughter cells

\section{DO-035}

Nodular lymphocyte predominant Hodgkin lymphoma and T cell/ histiocyte rich large B cell lymphoma-endpoints of a spectrum of one disease?

\section{S. Hartmann ${ }^{* 1}$, C. Döring', C. Jakobus', B. Rengstl', S. Newrzela',}

T. Tousseyn ${ }^{2}$, X. Sagaert ${ }^{2}$, M. Ponzoni ${ }^{3}$, F. Facchetti ${ }^{4}$, C. de Wolf-Peeters ${ }^{2}$

\section{Steidl', R. Gascoyne ${ }^{5}$, R. Küppers ${ }^{6}$, M.-L. Hansmann}

'Department of Pathology, Goethe University, Frankfurt, Germany, ${ }^{2}$ Institute of Pathology, Catholic University Leuwen, Leuwen, Belgium, ${ }^{3}$ San Raffaele, Milan, Italy, ${ }^{4}$ University of Brescia, Brescia, Italy, ${ }^{5}$ Department of Pathology and Laboratory Medicine, University of British Columbia, Vancouver, Canada, ${ }^{6}$ Institute of Cell Biology, Essen, Germany

Aims. In contrast to the commonly indolent clinical behavior of nodular lymphocyte predominant Hodgkin lymphoma (NLPHL), T cell/histiocyte rich large B cell lymphoma (THRLBCL) is frequently diagnosed in advanced clinical stages and has a poor prognosis. Besides the different clinical presentations of these lymphoma entities, there are variants of NLPHL with considerable histopathologic overlap compared to THRLBCL. Especially THRLBCL-like NLPHL, a diffuse form of NLPHL, often presents a histopathologic pattern similar to THRLBCL. The present study therefore originally aimed to find markers, which can be applied in the differential diagnosis between NLPHL and THRLBCL. Methods. Gene expression profiling of microdissected tumor cells of NLPHL, THRLBCL-like NLPHL and THRLBCL was performed. Immunohistochemical stainings of the tumor microenvironment were quantified in a blinded fashion.

Results. In unsupervised analyses, the lymphomas did not cluster according to their entity. Moreover, even in supervised analyses, very few consistently differentially expressed transcripts were found, and for these genes the extent of differential expression was only moderate. Hence, there are no clear and consistent differences in the gene expression of the tumor cells of NLPHL, THRLBCL-like NLPHL and THRLBCL. Characterization of the tumor microenvironment for infiltrating $\mathrm{T}$ cells and histiocytes revealed significant differences in the cellular composition between typical NLPHL and THRLBCL cases. However, THRLBCL-like NLPHL was more closely related to THRLBCL than NLPHL.

Conclusions. In conclusion, NLPHL and THRLBCL may represent a spectrum of the same disease. The different clinical behavior of these lymphomas may be strongly influenced by differences in the lymphoma microenvironment, possibly related to the immune status of the patient at the timepoint of diagnosis.
DO-036

Incomplete cytokinesis and re-fusion of small mononucleated Hodgkin cells leads to multinucleated Reed-Sternberg cells

B. Rengst ${ }^{* 1}$, S. Newrzela', R. Küppers' ${ }^{2}$, M.A. Rieger ${ }^{3}$, M.-L. Hansmann ${ }^{1}$ 'Dr. Senckenberg Institute of Pathology, Medical School, Goethe-University, Frankfurt am Main, Germany, ${ }^{2}$ Institute of Cell Biology (Cancer Research), Medical School, University of Duisburg-Essen, Essen, Germany, ${ }^{3}$ Department of Hematology/Onkology (LOEWE Center for Cell and Gene Therapy), Medical School, Goethe-University, Frankfurt am Main, Germany

Aims. The multinucleated Reed-Sternberg (RS) cells are pathognomonic for classical Hodgkin lymphoma (HL) and their presence is essential for diagnosis. However, the development of these giant tumor cells is controversially discussed.

Methods. Therefore, HL cell lines were monitored by long-term timelapse microscopy for up to 12 days with focus on the rare giant cell compartment to investigate their origin and fate.

Results. It was postulated that RS cells arise from mononucleated Hodgkin cells via endomitosis. Conversely, continuous single cell tracking of HL cell lines showed that cell fusion is the main route of RS cell formation [1]. In contrast to growth-induced formation of giant Hodgkin cells, fusion of small mononuclear cells followed by size increase gives rise to giant RS cells. Importantly, we nearly exclusively observed fusion of cells originating from the same ancestor, termed re-fusion. In the majority of cases, re-fusion of daughter cells was preceded by an incomplete cytokinesis, visualized by a microtubule bond between the cells. We confirm at the level of individual tracked cells that giant Hodgkin and RS cells have little proliferative capacity, further specifying small mononuclear Hodgkin cells as the proliferative compartment of the HL tumor clone. In addition, sister cells showed a shared propensity for re-fusion, which provides evidence of early RS cell fate commitment. Conclusions. The presented study shows that RS cell generation is neither due to cell fusion of unrelated Hodgkin cells nor to endomitosis, but is mediated by re-fusion of daughter cells that underwent mitosis. This surprising finding indicates the existence of a novel mechanism for the generation of multinuclear RS cells, which might have implications beyond HL, as RS-like cells are frequently observed in several other lymphoproliferative diseases (• Fig. 1).

\section{Literatur}

1. Rengstl B (2013) Incomplete cytokinesis and re-fusion of small mononucleated Hodgkin cells lead to giant multinucleated Reed-Sternberg cells. PNAS 10(51):20729-20734, doi: 10.1073/pnas.1312509110 


\section{AG Informatik, innovative Bildgebung und Biobanking}

\section{DO-037 \\ Virtual slide telepathology with scanner systems for intraoperative frozen-section consultation}

\section{S. Ribback ${ }^{* 1,2}$, M. Evert ${ }^{2}$, K. Gromoll-Bergmann ${ }^{3}$, F. Dombrowski ${ }^{2}$ \\ ${ }^{1}$ Institut für Pathologie, Greifswald, Germany, ${ }^{2}$ Institut für Pathologie, Universitätsmedizin Greifswald, Greifswald, Germany, ${ }^{3}$ Klinik für Urologie, Asklepios Klinik Pasewalk, Pasewalk, Germany}

Aims. Telepathology facilitates pathology services over a distance using digital imaging and telecommunication for primary diagnostic practice, including intraoperative frozen sections. Virtual Slide (VS) technology provides digitizing of histological slides by scanner systems and improves remote assessment substantially.

Methods. In this retrospective investigation we determined diagnostic accuracy of intraoperative frozen sections that were primarily assessed as VS. Afterwards they were compared to the final diagnosis at the processed paraffin embedded tissue and evaluated as concordant or discordant. Validity (sensitivity, specificity, positive and negative predictive value) was determined. Discordant diagnoses were reviewed and revalidated. The scanner was installed at the laboratory (client site) affiliated to the surgical suite and is connected to the telepathology scan and viewer software. After operative biopsy by the surgeon, the medical assistant processes and scans the frozen slides. The pathologist assesses the VS via the internet and makes his diagnosis from the remote site. Tissue assessment was mainly requested for urological, gynecological and dermatological resections.

Results. 1204 intraoperative frozen sections of 293 patients were conducted in the course of this study at our department over a period of $2.5 \mathrm{ye}-$ ars. 97.1\% of cases handled issues of tumor free margins. Primary tumor diagnosis was much less inquired. $98.59 \%$ of all intraoperative frozen sections were accurately diagnosed in the initial telepathological assessment, surpassing data of $94-97 \%$ from the literature. Tumor affection was present in $15.6 \%$ of frozen sections, in 174 instances already assessed in the initial slides (sensitivity 92.6\%). Discrepant diagnoses compared to the final diagnosis occurred in $1.41 \%$. Time between tissue arrival in the laboratory and diagnosis announcement to the surgeon averages 10.58 \pm 8.19 minutes per frozen section.

Conclusions. As in many peripheral hospitals a local pathologist is not available telepathology is a necessity to ensure professional pathology services for intraoperative frozen sections in a short time. The quality of services by Virtual Slide warrants this technology as a highly acceptable solution for these diagnostic approaches.

\section{DO-038}

\section{Comparative visual assessment of conventional and virtual slides}

G. Kayser ${ }^{* 1}$, A. Oser', N. Hörter', A. Csanadi', C. Otto', B. Passlick ${ }^{2}$

M. Werner

'Department Pathology, Universtity Medical Center Freiburg, Freiburg, Germany, ${ }^{2}$ Department of Thoracic Surgery, University Medical Center, Freiburg, Germany

Aims. Virtual microscopy (VM) is on the verge to step into daily routine pathology. Powerful scanning machines are available which are capable to digitize the daily load of a medium sized pathology laboratory. Despite missing standards, guidelines and comparative studies of conventional and virtual diagnostics, vendors advertise various software suites. The aim of this study was therefore to compare visual assessment of conventional (CS) and virtual immunohistochemically stained slides (VS) with focus on reproducibility.

Methods. TMAs of lymph node metastases included 151 patients with non-small cell lung cancer. Of these expression of several metabolic en- zymes was investigated. Immunohistochemical protocols consisted of a double staining procedure against ME in combination with SLC1A5, and a second assay directed against GLUT1 and PKM2. Stained slides were digitized using a Panoramic Scan ${ }_{3}$ CCD Hitachi camera, Zeiss 2ox objective NA 0.8 ) with extended focus settings ( 5 planes, $0.2 \mu \mathrm{m}$ distance). Each assay was evaluated by conventional bright field microscopy (Olympus BX51) and by VM by the same investigator to avoid inter-observer variability. Both, CS and VS were assessed for specific immunohistochemical staining of both antibodies of each protocol. Staining intensity and fraction of positive tumor cells were multiplied to an $\mathrm{H}$-score. All three values obtained for each antibody in conventional and digital slides were compared using Spearman's Rho.

Results. Visual analyses of CS were regarded as gold standard for evaluation of immunohistochemical stains. Constraints in the evaluation of VS were loss of sharpness in high resolution and single out of focus tiles. Despite these obstacles, for all four markers highly significant correlation coefficients of $>0.9$ were calculated $(p<0.001)$. Thus, we could prove that visual evaluation of VS delivers results highly similar to conventional analysis. Focusing on faint intensities assessment of specific features appeared clearer in VS. This is most probably due to harmonized illumination, shading corrections and standardized color perception in slide scanners. These features result in more standardized display of VS compared to conventional bright field microscopy.

Conclusions. Visual analysis of CS and VS delivers analogous results independent from immunohistochemical assay and investigator. Specific details may be assessed in VM more accurately due to standardized acquisition and display.

\section{D0-039}

Integration of multimodal images opens new horizons in histopathology

J. Berger ${ }^{* 1}$, S. Heldmann', D. Trede ${ }^{2}$, F. von Eggeling ${ }^{3}$, J. Oetjen ${ }^{4}$, M. Becker ${ }^{5}$, G. Ernst ${ }^{3}$, O. Guntinas-Lichius ${ }^{6}$, T. Alexandrov ${ }^{2,4,7,8}, H$. Thiele ${ }^{1,7}$

${ }^{1}$ Fraunhofer MEVIS Project Group Image Registration, Lübeck, Germany, ${ }^{2} \mathrm{SCiLS}$ GmbH, Bremen, Germany, ${ }^{3}$ Institute of Human Genetics, Jena University Hospital, Jena, Germany, ${ }^{4}$ MALDI Imaging Lab, University Bremen, Bremen, Germany, ${ }^{5}$ Bruker Daltonic GmbH, Bremen, Germany, ${ }^{6}$ Department of Otolaryngology, Friedrich-Schiller University, Jena, Germany, ${ }^{7}$ Steinbeis Innovation Center SCiLS, Bremen, Germany, ${ }^{8}$ Center for Industrial Mathematics, University Bremen, Bremen, Germany

Aims. Aim of the presented strategy is the generation of a multimodal, $3 \mathrm{D}$ reconstruction of a tumor sample by use of high-resolution microscopic H\&E image scans and matrix-assisted laser desorption/ionization mass spectrometry imaging (MALDI MSI) data to investigate the spatially resolved molecular content of tissue within its morphological context.

Methods. Serial sections of an oral squamous-cell carcinoma (OSCC) were analyzed by MALDI MSI, H\&E-stained and digitized with a highresolution scanner. For $3 \mathrm{D}$ reconstruction of the tumor based on $\mathrm{H} \& \mathrm{E}$ images we recaptured the spatial correspondence between neighboring slides by means of rigid image registration. This was followed by an elastic image registration to correct nonlinear deformations. For $3 \mathrm{D}$ MALDI MSI data processing a spatial segmentation of the entire ${ }_{3} \mathrm{D}$ MALDI MSI data was obtained by performing a clustering analysis, to detect different morphological regions in the tissue. Co-registration of the high resolution H\&E-stained microscopic images and the MALDI MSI data united anatomical, histological and molecular information in $3 \mathrm{D}$.

Results. We developed an interactive tool that links ${ }_{3} \mathrm{D}$ volume rendering, orthogonal slicing in axial, coronal, and sagittal direction, and clipping along a free plane with arbitrary angle and offset. MALDI MSI cluster results representing defined regions of functional difference can in this way be rendered independently of the whole volume and allow a better understanding of tissue borders and the spatial context. The 


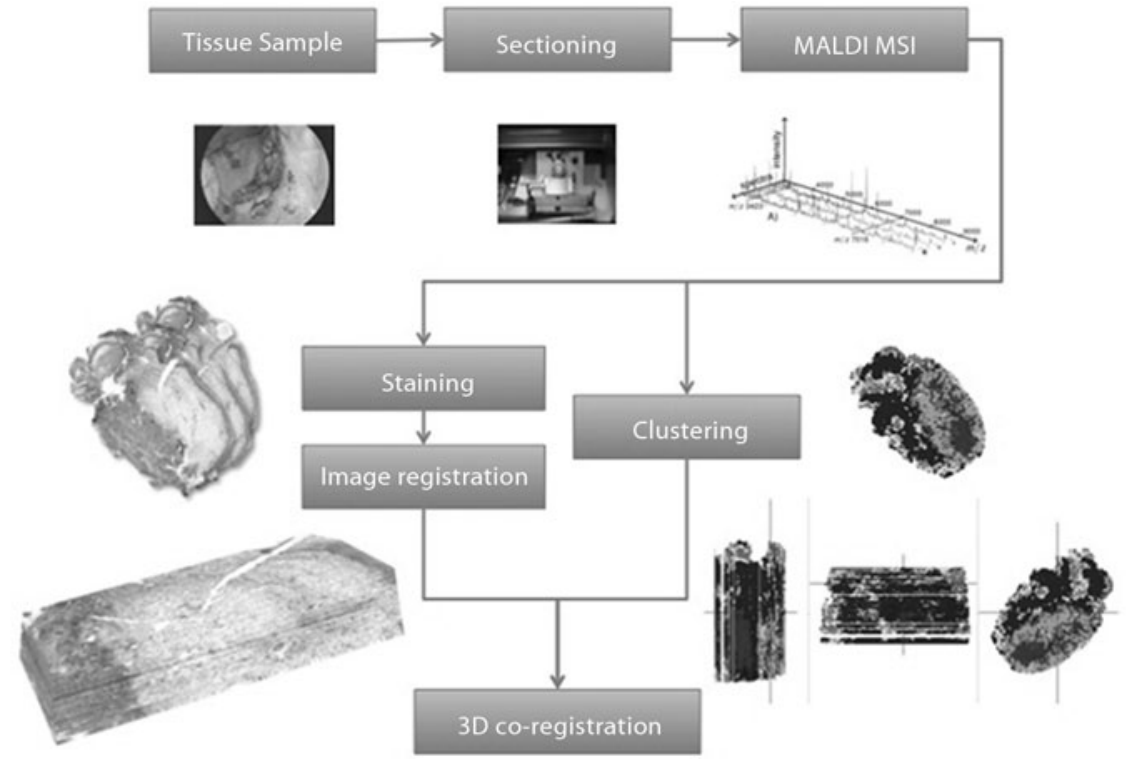

Fig. 1 | D0-039 $<$ Serial sections were measured by MALDI MSI and H\&E stained. Rigid and elastic registration was used for the $3 \mathrm{D}$ reconstruction. Spatial segmentation was performed on the entire 3D MALDI MSI data cluster analysis will as well be presented on a $2 \mathrm{D}$ dataset of a larynx carcinoma in comparison to the human annotation of a pathologist. In addition we present a new MALDI MSI data analysis approach that clusters $\mathrm{m} / \mathrm{z}$-images according to their spatial similarity. In summary, our approach gives new capabilities for understanding and analysis of the complex course of tumorigenesis and tumor progression.

Conclusions. The integration of 3 D MALDI MSI data to 3 D-reconstructed H\&E scans allows a correlation between histological and molecular information. Moreover, this model is open for additional image modalities (e.g. IHC) which will further enhance the information content (- Fig. 1).

\section{DO-040}

Platform independent telemicroscopy for ultrastructural diagnostic and research

\section{J.A. Schröder*, H. Siegmund, C. Grafe, F. Hofstädter \\ Institute of Pathologie, University of Regensburg, Regensburg, Germany}

Aims. Transmission electron microscopy (TEM) is still used as an ancillary tool or gold standard to complement some histopathological diagnoses. For "second opinion" consultation of difficult cases we used over years an interactive remote TEM operation via Internet, unfortunately the software maintenance by the supplier has expired. This contribution focuses on the presentation of an alternative approach.

Methods. Ultrathin resin sections are examined in the LEO-912/Zeiss TEM equipped with a customized side-entry digital camera image acquisition system (iTEM). We applied for remote camera operation and online image sharing with external experts the software called TeamViewer running on a WIN7 server.

Results. The TeamViewer software (business licence) allows cross platform (Windows, Mac, Android) global connections with other computers. It was used in the "Meeting Mode" for online sharing of the TEM images captured by the microscope camera, in the "Remote Mode" to remote control of the CCD-camera and the TEM using the GUI of the iTEM software from external computers via Internet. We experienced a fast and high quality image transfer to the remote expert, the remote camera and microscope operation was performed with negligible delay depending on the available bandwidth and daytime of the link. Connections breaks were not noticed. Additional discussion and communications tools like annotations, object measurement, databank access combined with optional parallel live video and voice transfer of the consultation participants increases significantly the telepresence cooperation and networking. Our centralized EM unit is integrated into the diagnostic service of the Pathology Department of the Uniklinikum Regensburg as well as for external responders and research institutions. The received samples cover a broad spectrum of diseases including rapid virus detection, a number of neoplastic and especially non-neoplastic conditions of the kidney, muscle, nervous system, skin, cilia defects, storage diseases, liver biopsies, respiratory diseases, toxic lesions, and more. The necessary expert consultations of some difficult TEM diagnoses of above mentioned samples showed a good time and efficiency performance in the TeamViewer mediated telepathologic networking. Conclusions. The alternative telemicroscopy approach with the low-cost and platform independent TeamViewer software for a consultation network in ultrastructural pathological diagnostic and research projects revealed an encouraging performance.

\section{D0-041}

Advanced visualization of consecutive serial sections to reveal new insights in histological architecture

N. Zerbe ${ }^{* 1,2}$, D. Heim', J. Kantonen ${ }^{1,3}$, F. Klauschen ${ }^{* 1}$, P. Hufnagl', H. Bläker ${ }^{1}$ 'Institute of Pathology, Charité - Universitätsmedizin Berlin, Berlin, Germany, ${ }^{2}$ Central Biomaterialbank Charité, Charité - Universitätsmedizin Berlin, Berlin, Germany, ${ }^{3}$ Department of Pathology, Haartman Institute, Faculty of Medicine, University of Helsinki, Helsinki, Finland

Aims. Histological slides provide very limited 3-dimensional information. However there are histological structures with larger extent that can never be fully displayed within one slide. Shape and relative position of those structures can't be evaluated with traditional microscopy or macroscopy. The approach presented here aims at a highly automated reconstruction of large-scale 3-dimensional information from a series of slides and displaying results in an intuitive way. The introduced approach was initially developed to easily retrace growth and distribution in an intermediate range of tubular and serrated adenomas in colorectal mucosa.

Methods. Different series of histological slides of colorectal mucosa with 40 to 230 slides per series were digitized. In the preparation process of those slides attention has been paid especially to minimize tissue deformation and gaps in the slide sequence. After digitization consecutive slides are registered pairwise using SIFT and subsequent geometric validation. For each sequence regions of $3000 \times 3000$ pixels at the mag- 
nification of $5 \times$ containing the structures that should be visualized in $3 \mathrm{~d}$ are selected. Afterwards the corresponding regions in all slides of a series are computed based on registration information. A single image for each region and each slide is extracted. Further a rigid transformation based on registration information is applied to each image. One possibility for visualisation is to create a "stack video" from a series of transformed consecutive single images of one region that displays the images with variable speed one after another. To create a $3 \mathrm{~d}$ model, contours of objects that shall be rendered have to be determined. Therefore a semi -automatic algorithm based on "snakes" is applied. Contours are used to create mask images that are input to a program based on VTK framework that creates and displays the $3 \mathrm{~d}$ model.

Results. From 4 series of $\sim 400$ virtual slides overall, stack videos of 9 regions and $3 \mathrm{~d}$ models of 3 regions were created. Using that $3 \mathrm{~d}$ visualisation enables pathologists to review relative positions of crypts, adenomas and muscularis mucosa at a glance. This illustration allows identification of different types of adenomas.

Conclusions. The presented method provides a good efficiency to retrieve spatial information from serial sections of histological slides. The most important challenges are time consuming scanning process as well as the creation of contours. The method described can be applied to many contexts of pathology.

\section{DO-042}

Classification of thymic neuroendocrine carcinomas by a ki67-based digital image processing approach

\section{C.-A. Weis" ${ }^{* 1}$, B. Griessmann', M. Deligiani', P. Ströbel', A. Marx ${ }^{1}$} 'Institute of Pathology, University Medical Center, Mannheim, Germany, ${ }^{2}$ Institute of Cellular and Molecular Immunology, Georg August University of Göttingen, Göttingen, Germany

Aims. The 2003 WHO-classification of neuroendocrine carcinomas (NEC) in the thymus is based on the nomenclature of neuroendocrine tumour occurring in the lung distinguishing typical carcinoid, atypical carcinoid, large cell neuroendocrine carcinoma (LCNEC) and small cell carcinoma (SCC). The classification of NEC especially pulmonary ones further developed in the last years. Meanwhile, the ki67-index has been suggested as an alternative approach to classify pulmonary NEC. In the thymus respective data are missing. In the present study we describe a method to automatically measure the ki67-/ $\mathrm{H}_{3}$-index in immunostained sections and correlate it to the classification of thymic NEC.

Methods. The patient collective comprises 35 cases of confirmed thymic NEC, assigned according to the actual WHO-criteria (morphology, necrosis, mitoses) to typical carcinoid, atypical carcinoid, LCNEC and SCC. Determination of average and hot-spot-related ki67- $/ \mathrm{H}_{3}$-indeces is achieved using MATLAB-based image processing of immunostained sections that were fully digitalized by an Aperio ScanScope.

Results. Digital image processing based calculation of the ki6 $7 / \mathrm{H}_{3}$-index in thymic NEC is possible and leads to similar results as by-hand evaluation (good reliability, good inter- and intraobserver reproducibility). For overall image analysis, the indices show to each other due to extreme outlier a weak correlation (0.57). Furthermore, they weakly correlate to the classification of NEC.

Conclusions. The ki67-/ $\mathrm{H}_{3}$ index can be calculated in a fully automatic way and leads to reliable results. In analogy to the recent development in pulmonary NEC, we are mapping the classification by individual $\mathrm{ki} 67 / \mathrm{H}_{3}$-indeces. In this context, the overall indices only show a weak correlation. The next step is to map the classification by the hotspots (• Fig. 1).

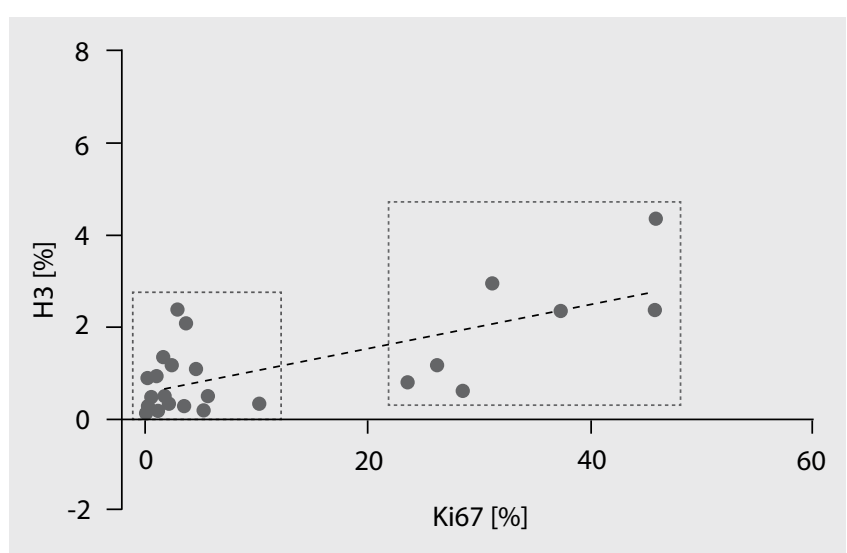

Fig. 1 | DO-042 $\Delta$ Ki67-plotted against $\mathrm{H3}$-index for one image per case. Within the blue box ( 7 cases) are atypical carcinoids $(n=2), \operatorname{LNEC}(n=3)$ and SCC $(n=2)$; within the red box ( 16 cases) are typical $(n=7)$ and atypical carcinoids $(n=6)$, LNEC $(n=1)$ and SCC $(n=2)$

\section{DO-043}

Analysing MicroRNA and transcription factor co-regulatory networks in high and low proliferating osteosarcoma cells

\section{E. Korsching*1, J. Smida², D. Maugg ${ }^{2}$, D. Baumhoer ${ }^{3}$, M. Nathrath ${ }^{2}$, K. Poos ${ }^{1}$} ${ }^{1}$ Institute of Bioinformatics, University Hospital Muenster, Muenster, Germany, ${ }^{2}$ Clinical Cooperation Group Osteosarcoma, Helmholtz Center Munichhen, TU Munich, Munich, Germany, ${ }^{3}$ Bone Tumor Reference Center at the Institute of Pathology, University Hospital Basel, Basel, Switzerland

Aims. Osteosarcomas (OS) are primarily characterized that they show a complex variety of genomic alterations. There are a lot of speculations how these alterations might form either, the typical phenotype of OS, and the perfect conservation of a deregulated and strong proliferative cellular state. On the molecular level less is known on the function of gene networks and their regulatory states which are creating this phenotype. Our objective in this situation was to get insight in the systems biology of OS by focusing on microRNA and transcription factor (TF) co-regulatory networks.

Methods. The process of tumor occurrence and the impact in the clinical situation is heavily bound to (deregulated) cell proliferation. So we created a case-control design based on expression data of OS cell lines according to their high or low proliferative activity (mRNA and microRNA). On that basis we applied a theoretical analysis workflow additionally embedding third party informational resources from e.g. TargetScan. Finally a model representing a work hypothesis for further wet lab validation was formed.

Results. The network core we obtained is based on 12 proliferation-related microRNAs and their corresponding target genes. On this basis their functional influence was examined. The key co-regulators are the microRNAs miR-9-5p, miR-138, and miR-214 and the TFs SP1 and MYC. The factors are components in the NFKB- and RB1-signaling and focal adhesion processes. The linkers are the target genes, amongst others, CDK6, CTNNB1, E2 F4, HES1, ITGA6, NFKB1, NOTCH1, and SIN3A. The resulting (in several aspects still hypothetical) model explains possible core interactions by (de-)regulatory phenomena in OS cell proliferation based on the mentioned microRNA and TF combinations. Conclusions. We identified a stable set of microRNA and TF regulators that cooperatively control growth-related pathways in OS. Those players might be also valuable therapeutic targets. The presented study underlines the benefit to study complex diseases from a network perspective. The microRNA and TF co-regulatory networks can be viewed online (http://complex-systems.uni-muenster.de/co_networks.html) or can be sent for offline usage. 


\section{DO-044}

\section{Computational approaches to panel sequencing: obstacles and} perspectives in translating a marker panel to the clinic

T. Wolf*1,2,3, J. Budczies ${ }^{3,4,5}$, S. Gade ${ }^{6}$, A. Stenzinger' ${ }^{1}$ S. Loib/6 ${ }^{6}$, G. von Minckwitz $^{6}$, C. Denkert ${ }^{3,5}$, W. Weichert ${ }^{1,3}$

'Institute of Pathology, University Hospital Heidelberg, Heidelberg, Germany, ${ }^{2}$ German Cancer Research Center, DKFZ, Heidelberg, Germany, ${ }^{3}$ German Cancer Consortium (DKTK), Heidelberg, Germany, ${ }^{4}$ German Cancer Research Center, DKFZ, Cancer Genome Research, Heidelberg, Germany, ${ }^{5}$ Institute of Pathology, Charité University Hospital, Berlin, Germany, ${ }^{6}$ German Breast Group, Neu-Isenburg, Germany

Aims. Next generation sequencing (NGS) has enabled researchers to address questions from all aspects of medical and biological research. It has also led to a paradigm shift from single gene analysis to whole genome or exome approaches. While these approaches are interesting in a research setting, they are not always feasible in the area of clinical diagnostics and other aspects of medical care. In these cases panel sequencing, using the Ion Torrent PGM sequencer, offers a better balance between therapeutical relevance and costs as well as storage and computational burden.

Methods. A wide selection of bioinformatics applications is available for next generation sequencing data analysis. However, most of these tools were not designed with a clear focus on clinical data analysis. Thus, we developed a software package that integrates all steps of the analysis, including the direct integration of clinical data. It thereby builds on tools specifically designed for the processing of Ion Torrent data. Our main goal was to make the results interpretable to clinical researchers. This encompasses the use of a novel clustering approach suited perfectly for NGS data visualization as well as the possibility to use decision tree models to prioritize detected mutations.

Results. The resulting software is based on the freely available R environment for statistical computing and graphics. So far no R application specifically tailored to all aspects of the Ion Torrent analysis workflow has been available.

Conclusions. The reliance on the command line often prevents the most novel approaches from being used in everyday applications. Thus, we make use of the R Shiny technology, enabling us to present the user with an easy to use graphical interface.

\section{DO-045}

\section{Computational profiling of gene deletions in CISH assays}

Q. Zhong ${ }^{* 1,2}$, J.H. Rüschoff ${ }^{2}$, C.D. Fankhauser ${ }^{2}$, H. Moch ${ }^{1,2}$, W. Jochum ${ }^{3}$, P.J. Wild ${ }^{1,2}$

'Institute of Surgical Pathology, University of Zurich, Zurich, Switzerland, ${ }^{2}$ Institute of Surgical Pathology, University Hospital, Zurich, Switzerland, ${ }^{3}$ Institute of Pathology, Cantonal Hospital St. Gallen, St. Gallen, Switzerland

Aims. Chromogenic in situ hybridization (CISH) is a bright-field alternative to fluorescence in situ hybridization (FISH). By establishing a dual-color assay using a combined chromogenic in situ hybridization for the gene of interest and its matching centromeric region, $\mathrm{CISH}$ can be used for detection of gene deletions. However, statistical quantification of gene deletions is based on manual counting of CISH signals under a microscope, which is time-consuming and subjective. This study aims to implement an automatic workflow that will facilitate large-scale CISH analysis.

Methods. We developed a computational framework that uses computer vision algorithm to detect CISH point signals, and generates models of gene deletions based on color signal classification by supervised machine learning.

Results. We applied our method to a tissue microarray with 65 prostate tissue cores for estimating loss of the tumor suppressor gene PTEN (phosphatase and tensin homologue). The resulting tri-modal (homo- zygous gene deletion, heterozygous gene deletion/heterogeneity, and no deletion) distribution was validated by manual labeling of three pathologists, PTEN FISH analysis, and immunohistochemistry, respectively. Conclusions. Our computational framework demonstrates accurate profiling of PTEN deletions in FFPE (Formalin-Fixed, Paraffin-Embedded) tissues and may enable objective detection of signal gains and losses of any genes in image-based computational pathology.

\section{DO-046}

OntoPathLex - a systematic approach for a terminology in pathology

\section{T. Schrader ${ }^{* 1}$, C. Jacquelinet ${ }^{2}$, C. Daniel ${ }^{3}$, G. Haroske ${ }^{4}$}

'University of Applied Sciences Brandenburg, Department of Informatics and Media, Brandenburg, Germany, ${ }^{2}$ Agence de la Biomédecine, Paris, France, ${ }^{3} \mathrm{AP}-\mathrm{HP}$ - INSERM, Paris, France, ${ }^{4}$ Institute of Pathology, DresdenFriedrichstadt General Hospital, Dresden, Germany

Aims. PathLex was published 2011 by the IHE working group for Anatomical Pathology as to close the gap between SNOMED and Pathology. The establishment of a terminology was a prerequisite for the development of structured reports in Pathology. The structure of PathLex was based on the document structure of structured reports. Standard terms were created for a series of tumour locations and entities. The management of these terms was done in Excel sheets. This strategy was successfully until the list became longer and longer. At last, it was impossible to control PathLex terms related to consistency and completeness. The idea of ontology for pathology terms arose from the concept of SNOMED CT. Although both in SNOMED and in PathLex the existing terms and the terminology system has a hierarchical structure, but the list was created by usage, not by a systematic approach. The current work presented here follows another concept: for the description of any type of lesions a set of generic terms is used. This set is now the basis for a group of terms with the same term structure.

Methods. The structure of PathLex was analysed related to depth of hierarchical structure and meaning. The IHE proposal for structured reports determined the essential terms for each type of lesion. A Prolog script was written to create a simple hierarchical list of ontological tree with its terms, and a second file as an $\mathrm{OBO}$-ontology file. This file can be read by the OBO Ontology Editor and the hierarchical structure can be graphically viewed.

Results. Two different files were created by the Prolog: a simple hierarchical list for review and control and an OBO-file which can read by various ontology editors. An application can use the simple list and get information about the hierarchical structure of each term. Using the OBO-file all meta-information about the term are available especially the ID and the source of the ID such as SNOMED, LOINC or ICD. All generic terms were used for each location and disease entity, this ensure a completeness of terms for disease entity in each location. However, some regions or diseases have specific terms and/or the generic terms for e.g. tumour description cannot be used. In such case constraints expressed in Prolog creates a specific terminology tree.

Conclusions. This systematic approach to create an ontology for terminology ensures a better completeness than terminology creation by usage. In this new ontology, you will find a useful term in all meaningful locations. 


\section{D0-047}

\section{Development of an android based interactive guide of the Berliner} Medizinhistorisches Museum der Charité

\author{
I. Klempert ${ }^{* 1}$, N. Zerbe ${ }^{1,2}$, T. Arndt ${ }^{3}$, T. Schnalke ${ }^{4}$, P. Hufnagl' \\ ${ }^{1}$ Institute of Pathology, Charité, Berlin, Germany, ${ }^{2}$ Institute of Pathology, \\ Charité University Hospital, Berlin, Germany, ${ }^{3}$ University of Applied Scien- \\ ces Berlin, Applied Computer Science, Berlin, Germany, ${ }^{4}$ Berliner Medizin- \\ historisches Museum der Charité, Charité, Berlin, Germany
}

Aims. Pathology is the science of diseases that ranges from macroscopic to histologic, and of course molecular changes. To offer a holistic education we wanted to involve portable electronic devices to combine information of diseases with microscopic changes and formalin fixed organs (macroscopic preparation).

Methods. We combined our virtual microscope "AndroScope" with a new developed user-interface of the "Berliner Medizinhistorisches $\mathrm{Mu}$ seum" (BMM) for android based mobile devices such as smartphones and tablet-PCs. As content we used images of the exhibition samples, information on the respective organ and disease, as well as the epidemiology and whole slide images for visualization of histological changes. Linkage of digital content and samples is realized using QR-codes to assure valid and user-friendly recognition.

Results. The "BMM Guide" is available for all visitors of the museum on lendable devices or for students (professional audience) using their personal devices via the web-access eduroam. The guide is connected to the internet. It is designed to easily expand, change or transfer the content catalogue data.

Conclusions. The museum guide is an easy handling, self-explanatory blended learning tool that will be embedded in the general education. This guide for the exhibition of the Berliner Medizinhistorisches $\mathrm{Mu}-$ seum opens a new branch for self-study of students. Nevertheless he still has a potential to be integrated in curricular lectures in the future.

\section{DO-048}

\section{Pathologic-anatomical collections in Germany-results of a nation- wide survey}

\section{F. Lasitschka*, P. Schirmacher \\ Institute of Pathology, University Hospital Heidelberg, Heidelberg, Germany}

Ziele. Im Rahmen einer bundesweiten Umfrage in universitären Instituten für Pathologie wurde ein Status quo der derzeitigen Situation pathologisch-anatomischer Sammlungen erhoben.

Methoden. Schriftliche Umfrage über die Geschäftsstelle der DGP.

Ergebnisse. Noch bis in die 1960er Jahre war die Arbeit in pathologischanatomischen Sammlungen ein Kernstück in der Ausbildung angehender Mediziner. Der dann eingeleitete Trend zum Diapositiv führte bis heute zu einer kontinuierlichen Erweiterung audiovisueller Lehrmittel zum Studium und Selbststudium, die die Sammlung um den Preis der Virtualität verdrängte. Insbesondere der Unterricht in der Pathologie leidet vermehrt unter dem Rückgang an Obduktionen in den vergangenen Jahren. Daher müssen Dozenten auf den Operationseingang zurückgreifen. Dies erfordert - aufgrund der zeitnahen Aufarbeitungspflicht - eine gewisse Spontaneität von den Lehrenden und kann daher nicht dauerhaft in die Lehre integriert werden. So besteht die Gefahr, dass sich die Studierenden gerade am Anfang ihrer Ausbildung immer weiter vom eigentlichen Lerngegenstand - dem menschlichen Organismus - entfernen. Das Sammlungskonzept vieler pathologischer Sammlungen beruhte in der Vergangenheit weniger auf einer zielgerichteten Sammlung spezifischer Präparate, als vielmehr auf einem universellen Sammlungsanspruch aller bei Obduktionen und in der täglichen Diagnostik gewonnenen Präparate von möglicher Relevanz für didaktische Zwecke oder zur Dokumentation (bei seltenen Erkrankungen).
Schlussfolgerung. Für die Zukunft sind umfangreiche Neukonzeption der Sammlungspräsentation, auch in Hinblick auf den Aufgabenwandel innerhalb des Fachs Pathologie (z. B. verstärkte Bedeutung der Molekularpathologie), sowie Aufarbeitung der Sammlungsgeschichte und Herstellung neuer, im bisherigen Bestand fehlender Krankheitsentitäten, bzw. Restaurierung älterer Präparate außerordentlich wichtig, um die wachsende Bedeutung des Fachs und die Vermittlung seiner Inhalte zu gewährleisten.

\section{DO-049}

\section{Clinical autopsies-unpopular, expensive and dying?}

\section{W.D. Schmitt*1 , S. Fittkau' ${ }^{2}$, A. Stang ${ }^{3}$, S. Hauptmann ${ }^{4}$}

${ }^{1}$ Institute of Pathology, Charité - University medicine of Berlin, Berlin, Germany, ${ }^{2}$ Department of Anesthesiology and Intensive Care Medicine, BG-Kliniken Bergmannstrost, Halle (Saale), Germany, ${ }^{3}$ Institute of Clinical Epidemiology, Martin-Luther-University Halle-Wittenberg, Halle (Saale), Germany, ${ }^{4}$ Institute of Pathology, Düren, Germany

Aims. The number of clinical autopsies in Germany is decreasing continuously. Many reasons for this development are in discussion, especially improved possibilities in clinical diagnostics as well as growing concerns of relatives, but usually these are generalized impressions of individual cases. To get a more systematic view of pros and cons, we performed a survey among clinicians asking multiple aspects that might influence the decision about performing clinical autopsies.

Methods. A questionnaire had been sent out to 531 clinical colleagues of all hospitals in Halle (Saale), namely BG-Kliniken Bergmannstrost, Diakonie-Krankenhaus, Krankenhaus St. Elisabeth und St. Barbara, Krankenhaus Martha-Maria Halle-Dölau, and Universitätsklinikum Halle (Saale). The return rate was about $52 \%$ with most questionnaires completely answered.

Results. The topic of the first part was the interaction between clinicians and relatives. In most cases, relatives are interested in the cause of death and the option of a clinical autopsy seems to be addressed quite often. Interestingly, autopsies are more frequently offered by younger and female clinicians, respectively. Most doctors are especially interested in an autopsy in cases of unexpected death, unusual course of disease and rare diseases, whereas estimated criticism of therapy was only an equivocal reason. The second part of the survey focused on economics and law. Remarkably there was a high variation in estimations of the costs of one single autopsy, and different opinions which amount would be appropriate. Furthermore, most doctors seem to be well-informed about the legal situation but the majority would prefer a veto-based legal situation (as it is in other federal states but not in Saxony-Anhalt) expecting more clinical autopsies. About one third of clinicians would support a general outside postmortem examination by an independent pathologist or coroner. Finally we asked about personal thoughts. Only single participants were concerned about ethic aspects of autopsies or had personal aversions. On the other side, most clinicians accept autopsies as quality assurance and expect scientific proceedings. The majority noted that their education was not sufficient about details of autopsies and the legal situation, and one of two remarked education shortcomings concerning death certificates.

Conclusions. Our survey provides an interesting insight into the topic of clinical autopsies from the view of clinicians. 


\section{Abstracts}

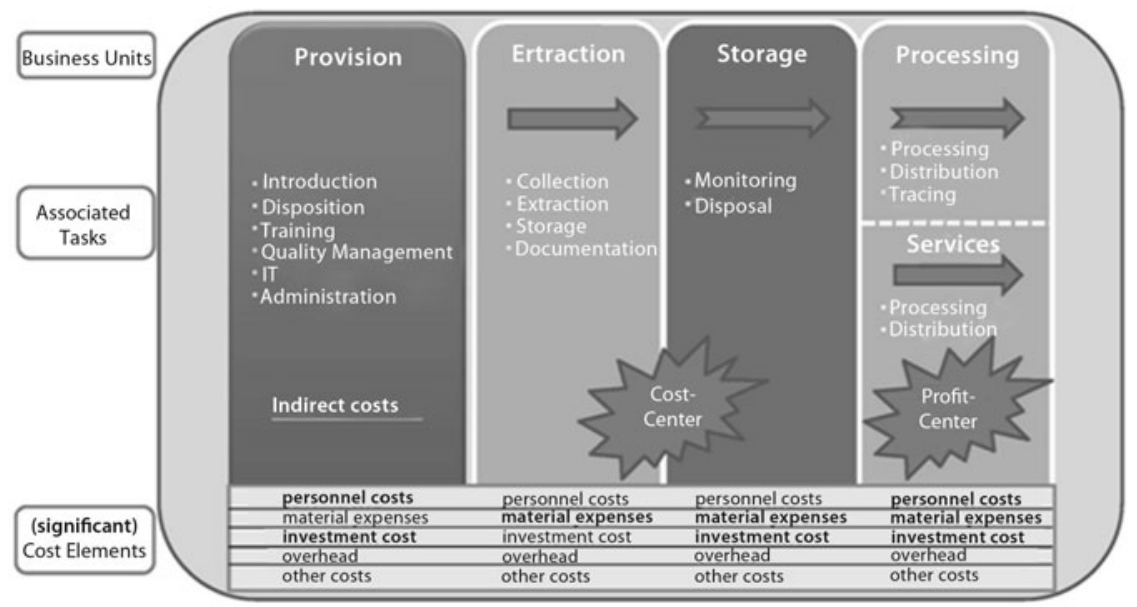

Fig. 1 | DO-051 $<$ Organisational structures of a biobank and respective cost elements

\section{DO-050}

\section{Cost analysis within a scientific biobank-example from the NCT-} Tissue Bank, Heidelberg

\section{A. Maier ${ }^{* 1,2}$, R. Kirsten ${ }^{1,2}$, P. Schirmacher ${ }^{1,2}$, E. Herpe ${ }^{2,3}$ \\ ${ }^{1} \mathrm{BMBH}$ at the Institute of Pathology, University Clinics Heidelberg, Heidel- berg, Germany, ${ }^{2}$ Institute of Pathology, University Hospital Heidelberg, Heidelberg, Germany, ${ }^{3} \mathrm{NCT}$ Tissue Bank at the Institute of Pathology, University Clinics Heidelberg, Heidelberg, Germany}

Aims. One of the goals of the BioMaterialBank Heidelberg (BMBH), an infrastructure project funded by the BMBF, is to support participating biobanks to operate efficiently and to increase their long-term economic viability. As part of these efforts cost analysis and calculations for biobanking activities are performed. Due to the heterogeneous processes and products as well as the prevailing organisational settings of biobanks, cost information is normally not available and difficult to obtain. Based on the cost analysis project of the Tissue Bank of the National Center for Tumor Diseases (NCT) at the Institute of Pathology of the University Hospital Heidelberg which is a member of the BMBH we will show how this information can be ascertained in a laboratory environment.

Methods. At the start of the project an elaborate analysis of the relevant cost drivers in the tissue bank was performed. The results revealed that cost of personnel, equipment and consumables as well as expenses for services such as maintenance and repairs were representing central factors. In addition, it was found that especially for labour cost it was difficult to attribute the expenses to the various products and services offered by the biobank. In order to solve this problem all activities performed by laboratory personnel were monitored and categorized into more than 130 individual tasks. For the identified tasks repeated time measurements were performed and the data was evaluated using statistical methods. Finally, a matrix containing measured tasks and offered products/services was prepared for all products and services offered by the biobank.

Results. Together with other cost parameters, the results describe the basis for an accurate estimation of direct costs for products and services prepared by the laboratory. It also provides the distribution coefficients for indirect costs accrued in the biobank. This information is used to determine the cost intensity of operational areas and individual projects. It can furthermore help to support cost efficiency measures in specific areas.

Conclusions. In the future it is intended to transfer the generated information on cost distributions into a database that will be used for automated cost evaluation of requested samples and services of research applications. This will make cost planning of internal projects more reliable and also support a fee-for-service funding model where specific costs of biobanking operations will be charged directly to liable external parties.

\section{DO-051}

Organisational structures and cost allocation with a business units approach for biobanks

A. Maier*1,2, R. Kirsten ${ }^{1,2}$, P. Schirmacher ${ }^{1,2}$, E. Herpel ${ }^{2,3}$

'BMBH at the Institute of Pathology, University Clinics Heidelberg, Heidelberg, Germany, ${ }^{2}$ Institute of Pathology, University Hospital Heidelberg, Heidelberg, Germany, ${ }^{3} \mathrm{NCT}$ Tissue Bank at the Institute of Pathology, University Clinics Heidelberg, Heidelberg, Germany

Aims. The structure of a research biobank can become complex as it grows in size and as it simultaneously handles multiple projects. Thus, developments of cost trends can easily be overlooked and achieving adequate cost allocation can become challenging. To counter this and to increase visibility of internal processes biobanks can make use of the centre-concept that separates parts of the operations into smaller units. Methods. To use this approach, processes within the facility have to be analysed and grouped into homogeneous or at least similar organisational areas. In the case of a biobank four areas can be identified: provision, extraction of material, storage and processing of collected samples. In each of these areas a typical distribution of cost elements can be determined. The characteristics of this cost pattern and the significance of single cost elements will vary from biobank to biobank depending on their individual setting and scope of service.

Results. With the definition of these areas and the allocation of the inherent expenses, cost transparency is greatly increased and management control is simplified. Each area can be operated as a single business unit with individual responsibility and budget. Controlling instruments such as operating numbers can be used to manage and monitor the units and the relationships among them. The business units approach can also help to select the best cost allocation in case of multiple funding sources. As with the cost pattern, the appropriate allocation depends on the individual setup of the biobank and the corresponding institutional framework. In the long term even cost behaviour patterns inside the units can be identified through adapted monitoring.

Conclusions. Operating biobanks by using business units can improve cost control and visibility. Complex structures are simplified and available funds can be utilized for the most appropriate expenses (- Fig. 1, 2). 


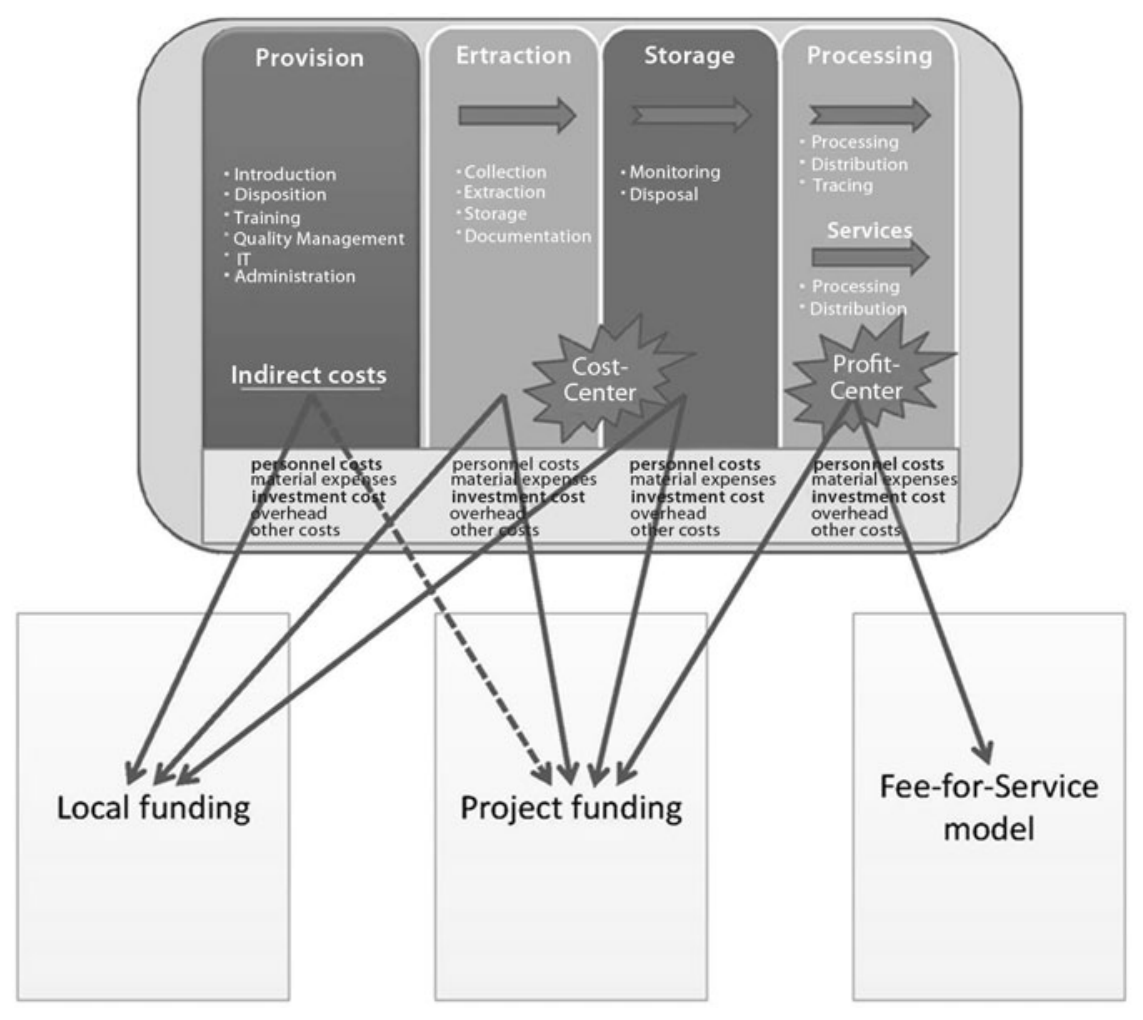

Fig. 2 | DO-051 $<$ Possible cost allocation in a business units setup

\section{DO-052}

New workflows and STARLIMS ${ }^{\circledR}$ modules for managing tissue samples with rack scanners

\section{Döllinger ${ }^{* 1,2}$, M. Wieland ${ }^{1,3}$, E. Herpe ${ }^{2,4}$, R. Kirsten ${ }^{1,2}$}

'BMBH at the Institute of Pathology, Heidelberg University Hospital, Heidelberg, Germany, ${ }^{2}$ Institute of Pathology, University Hospital Heidelberg, Heidelberg, Germany, ${ }^{3}$ German Cancer Consortium (DKTK), German Cancer Research Center, Heidelberg, Germany, ${ }^{4}$ NCT Tissue Bank at the Institute of Pathology, Heidelberg University Hospital, Heidelberg, Germany

Aims. Due to the enormous sample throughput of centralized biobanks, a manual registration and drawing of biosamples is no longer applicable. Therefore, many biobanks use laboratory information management systems (LIMS) in combination with rack scanners, which allow to store, move and draw large numbers of samples more efficiently and securely. By using rack scanners, empty storage positions can be restocked to save space in freezers. Here, we present workflows and appropriate STARLIMS $^{\star}$ modules for the registration and drawing of tissue samples using rack scanners as already implemented at the NCT Tissue Bank Heidelberg.

Methods. For the registration and drawing of samples with rack scanners, the following operational sequences have been designed:

Sample Registration Process

1. initial registration and aliquotation:

1.1 aliquot source sample into data matrix labeled tubes

1.2 link tube ids to submission number

1.3 assign specimen type and fill amount

2. rack scanning and storage

3. pseudonymization and documentation of metadata

To support this workflow, we developed the STARLIMS ${ }^{\star}$ module "ScanReception". To save space and time, this module can be controlled without keyboard or mouse by either using touchscreen input or by scanning specifically designed QR codes with a handheld scanner. Sample Drawing Process

After a project proposal has been approved, the sample drawing process consists of the following steps:
1. project registration

2. database query to find appropriate samples

3. sample drawing to empty picking rack

4. pseudonymization of submission numbers and pre-printing slides

5. tissue sectioning and mounting onto slides

6. re-scanning of picking rack and replacement of remainders

7. exit control and sample transfer

For the management of tissue requests, we implemented a module called "ProposalManager". Furthermore, the "RackScan" module, originally designed for liquid biobanking, has been adapted to the workflows.

Results. The implementation of the presented workflows and STARLIMS $^{\oplus}$ modules at the NCT Tissue Bank Heidelberg resulted in a significantly improved overall performance and higher data quality. Additionally, the usage of rack scanners allows a more compact storage by restocking empty positions, thereby reducing the overall costs of biobanking.

Conclusions. The rollout of the described IT solutions to further participating tissue biobanks of the BioMaterialBank Heidelberg (BMBH) is scheduled to be finished in 2014. If desired, our expertise can also be provided to other biobanks outside Heidelberg.

\section{Literatur}

[BO] E. Herpel, N. Koleganova, B. Schreiber, B. Walter, v. Kalle C, P. Schirmacher (2012) Structural requirements of research tissue banks derived from standardized project surveillance, Springer-Verlag, Virchows Archiv, Berlin/Heidelberg, 79-86, 461, Pathologisches Institut, Universität Heidelberg

[B1] Esther Herpel, Michael Hummel (2013) Research biobanks—development and structure. Georg Thieme Verlag KG, Deutsche medizinische Wochenschrift, Stuttgart, 1069-1072, http://www.ncbi.nlm.nih.gov/pubmed/23670264, Pathologisches Institut, Universität Heidelberg 
[B2] Esther Herpel, Peter Schirmacher (2013) Gewebebanken - die Beteilung der außeruniversitären Pathologie ist wichtig! Bundesverband Deutscher Pathologen e.V., patho. (Ausgabe 4.2013), Berlin, 8-9, Pathologisches Institut, Universität Heidelberg

[B3] E. Herpel, M. Hummel, M. Dietel, P. Schirmacher (2012) Gewebebanken in der Tumorforschung. Springer-Verlag, FORUM 2012-27, Berlin/Heidelberg, 47-53, Pathologisches Institut, Universität Heidelberg

\section{AG Oralpathologie}

\section{DO-K053}

Different effects of zolendronate on mandibular osteoblasts and long bone osteoblasts and osteoclasts in the development of BRONJ

\section{S.J. Hwang ${ }^{* 1,2}$, S.S. Lim ', I.S. Kim}

'Dental Research Institute, School of Dentistry, Seoul National University, Seoul, South Korea, ${ }^{2}$ Department of Oral and Maxillofacial Surgery, School of Dentistry, Seoul National University, Seoul, South Korea

Aims. Bisphosphonates (BPs) are widely used for the treatment of osteoporosis and Paget's diesease, and for the management of metabolic bone disease. Bisphosphonate related osteonecrosis of jaw (BRONJ) is a serious side effect, because of difficult management, even though its incidence is low. One of most potent BPs, zoledronic acid (ZA) can cause BP-related osteonecrosis of the jaws (BRONJ) with a poorly understood pathophysiology. The aim of this study was to find a clue for the development of BRONJ by evaluating the cytotoxic effects of ZA on osteoblasts and examining the action mechanism of ZA on osteoclast differentiation.

Methods. Jaw bone osteoblasts (JB-OBs), long bone osteoblasts (LB$\mathrm{OBs}$ ) and bone marrow macrophages (BMMs) were isolated from $\mathrm{Balb} / \mathrm{C}$ mouse. Osteoblasts were treated with $\mathrm{ZA}$ at increasing doses (o.01, 0.1, 1, 5, 10, 50 and 100 micromole) for 7 days. The cytotoxic effects of ZA appeared from day 3 at high dose of 50 and 100 micromole with increase of apoptosis in both types of osteoblasts, of which JB-OBs were more sensitive than LB-OBs.

Results. Western blotting showed increased expression of apoptotic markers, $\mathrm{p}_{21}$ and $\mathrm{p}_{53}$ at 50 micromole of ZA. BMMs were pretreated with various concentrations (o, o.01, 0.1, 1 micromole) of $Z A$ for $1 \mathrm{~h}$ before stimulating with receptor activator of nuclear factor kappaB ligand, the osteoclast differentiation factor. ZA attenuates osteoclast differentiation by suppressing the development of multinuclear cells. ZA decreased the transcriptional expression of osteoclast transcription factor, NFATc1 as well as ATP6vod2 gene which was involved in cell-cell fusion during osteoclastogenesis.

Conclusions. These findings revealed that $\mathrm{ZA}$ at high dose induces apoptosis, more apparently in JB-OBs than LB-OBs, and attenuates osteoclast differentiation, suggesting that chronic administration of $\mathrm{ZA}$ might exert adverse effect on bone metabolism.

\section{DO-054}

Membrane connexin 43 acts as an independent prognostic marker in oral squamous cell carcinoma

\section{P. Brockmeyer ${ }^{* 1}$, K. Jung ${ }^{2}$, C. Perske ${ }^{3}$, H. Schliephake ${ }^{4}$, B. Hemmerlein ${ }^{5}$}

${ }^{1} \mathrm{Clinic}$ of oral and facial surgery, University of Goettingen, Goettingen, Germany, ${ }^{2}$ Institute of medical statistics, University of Goettingen, Goettingen, Germany, ${ }^{3}$ Institute of Pathology, University of Goettingen, Goettingen, Germany, ${ }^{4}$ Clinic of oral and facial surgery, University of Goettingen, Goettingen, Germany, ${ }^{5}$ Institute of Pathology, HELIOS Hospital, Krefeld, Germany

Aims. The aim of the present study was to evaluate the expression and localization of connexin (Cx) $26,-43$ and -45 in a group of 35 patients with primary oral squamous cell carcinoma (OSCC) with the objective of making a more accurate disease prognosis.

Methods. We analysed the expression of connexins in tissue samples of primary OSCC, matching oral mucosa free of dysplasia, and its associated lymph node metastases (LNM) by semiquantitative immunohistochemistry of membraneous, cytoplasmic and nuclear connexin expression using an immunoreactive score. The influence of general clinical parameters, such as gender or age was analysed using Cox proportional hazard regressions. The strength of the influence was described by the hazard ratio. Connexin expression was correlated with survival time using the Kruskal-Wallis test and the associated baseline characteristics described above. The influence of the type of tissue (oral mucosa, primary cancer, lymph node metastasis and the subcellular localization (nucleus, cytoplasm, plasma membrane) and their interaction with the expression of each protein was analysed by nonparametric analysis of variance for data with repeated measurements. If significant interactions were observed, all tissue types and subcellular localization were also compared pairwise with each other using non-parametric analysis of variance. All tests were performed at a significance level of alpha $=5 \%$ using the statistical software R (version 2.15, www.rproject.org).

Results. Cx43 was overexpressed in tumour cells compared to epithelia in dysplasia-free mucosa. High membrane expression of $\mathrm{Cx} 43$ on tumour cells was the only statistically significant and independent prognostic factor of short OS ( $\mathrm{p}=0.0088$ ). Membrane expression of $\mathrm{Cx} 43$ in matching dysplasia-free mucosa acted similarly, but did not reach statistical significance $(p=0.059)$. No correlation was found between the Cx26, Cx45 expression and OS.

Conclusions. Cx43 expression in dysplasia-free mucosa may indicate a very early stage of tumour promotion. Although overexpression of $\mathrm{Cx} 43$ is found in invasive tumours we only found membrane $\mathrm{Cx} 43$ expression to correlate with OS. This observation suggests that cytoplasmic $\mathrm{Cx}_{43}$ serves as storage and only membrane translocation may promote the formation of gap junctions and gap junctional intercellular communication (GIIC) with prognostic relevance.

\section{DO-055}

Focused deep genomic of profiling of a phase III HNSCC study cohort identifies recurrent predictive and prognostic mutations and mutation clusters in stage IV oro- and hypopharynx carcinomas

\author{
A. Stenzinger*1, T. Wolf', V. Endris', N. Pfarr', V. Budach², U. Keilholz ${ }^{3,}$ \\ I. Tinhofer ${ }^{2}$, W. Weichert ${ }^{1,4}$ \\ 'Institute of Pathology, University Hospital Heidelberg, Heidelberg, \\ Germany, ${ }^{2}$ Department of Radiooncology, Berlin, Germany, ${ }^{3}$ Department \\ Medicine III, Berlin, Germany, ${ }^{4}$ Nationales Zentrum für Tumorerkrankun- \\ gen, Heidelberg, Germany
}

Aims. During the recent years genomic profiling studies led to a basic genetic characterization of head and neck squamous cell carcinomas. However, due to i) low coverage of these exome-wide studies, ii)the clinical and pathological heterogeneity of HNSCC and iii) small case se- 
ries, the prevalence and particularly clinical significance of the hitherto uncovered molecular landscape remains elusive.

Methods. We designed an HNSCC-specific gene panel for highly sensitive focussed deep sequencing (av coverage $\times 1.500$ ) and sequenced 100 cases of locally advanced hypo- and oropharynx squamous cell carcinomas treated with definite radiochemotherapy from a multicentric phase III study (AROo401).

Results. Deep sequencing revealed recurrent and frequent mutations in $\mathrm{TP}_{53}$, FAT1, VEGFR2, $\mathrm{PCDH} 15$ and $\mathrm{NOTCH}_{1}$ as well as rare mutations in MET, CASP8, MYC, FGFR 3 and $\mathrm{PIK}_{3} \mathrm{CA}$. Moreover, we identified 4 mutation clusters, which show a distinct mutation profile. Both, single mutations and mutation clusters stratified patients into prognostic and predictive subgroups and showed associations with the HPV-status and smoking habits.

Conclusions. Our study is the current largest study on genomic profiling of HNSCC worldwide. Focused deep sequencing of a phase III HNSCC study cohort shows a) the specific prevalence of mutations in stage IV oro- and hypopharynx squamous cell carcinomas and b) prognostic and predictive mutations and mutation clusters which may also have clinical relevance with respect to targeted therapy.

\section{DO-056}

Radiosensitizing effects of MEK inhibitor PD-0325901 in squamous cell carcinoma cell lines of the head and neck with wild-type and mutated p53

\section{A. Affolter ${ }^{* 1,2}$, A. Stenzinger ${ }^{2}$, K. Lorenz ${ }^{2}$, F. Kirner ${ }^{2}$, J. Wolf ${ }^{2}$, K. Sommer $^{2}$, J. Heß ${ }^{3}$, P. Schirmacher ${ }^{2}$, W. Weichert ${ }^{1}$ \\ 'Institute of Pathology, University Hospital Heidelberg, Heidelberg, Germany, ${ }^{2}$ Institute of Pathology, Ruprecht-Karls-University, Heidelberg, Germany, ${ }^{3}$ Department of Otorhinolaryngology, Heidelberg, Germany}

Aims. Kopf-Hals-Plattenepithelkarzinome (HNSCC) weisen häufig eine hohe Strahlenresistenz auf. Wir konnten in Vorarbeiten zeigen, dass der durch Bestrahlung (IR) aktivierte MAPK-Signaltransduktionsweg eine wichtige Rolle bei der Resistenzentwicklung spielt. Der Einfluss von p53-Mutationen auf das Bestrahlungsansprechen von HNSCC wird hingegen kontrovers diskutiert. Zur Überwindung des Nichtansprechens auf IR haben wir den MEK-Inhibitor PD-0325901 auf sein Potential als Radiosensitizer in Plattenepithelkarzinom-Zelllinien des Kopf-Hals-Bereiches mit (FaDu, Detroit 562) und ohne (UM-SCC-17B) p53-Mutation und in vitalen kultivierten Gewebeschnitten (Ex vivoModell) von Plattenepithelkarzinomen untersucht.

Methods. Die Tumorzelllinien wurden mit PD-0325901 in unterschiedlichen Konzentrationen behandelt und nach $24 \mathrm{~h}$ mit o, 1 und 8 Gy bestrahlt. Mittels Western-Blot wurde die Expression von aktiviertem ERK untersucht. Als Maß für das Ansprechen auf Bestrahlung und Inhibition wurde in MTT-Assays die Zellproliferation unter Behandlung analysiert. Analog erfolgte eine Inhibitorbehandlung und Bestrahlung von vitalen HNSCC-Gewebeschnitten.

Results. Wir konnten unabhängig vom p53-Status eine ausgeprägte Phosphorylierung von ERK unter Bestrahlung und somit die Aktivierung von Überlebensmechanismen der Zellen beobachten. Konsequenterweise führte eine MEK-Inhibiton in allen Zelllinien zu einer verminderten Strahlenresistenz. Die in den Zellkulturen erhobenen Befunde ließen sich teilweise durch das Ex-vivo-Modell stützen.

Conclusions. Unsere Daten belegen, dass der derzeit klinisch getestete Inhibitor PD-0325901 in Plattenepithelkarzinomen des Kopf-Hals-Bereiches eine radiosensibilisierende Wirkung in Kombination mit IR ausübt. Ein unterschiedliches Ansprechen von p53-mutierten und nicht-mutierten Zelllinien konnte nicht beobachtet werden.

\section{DO-057}

Multiple effects of ASMA positive activated fibroblasts on EGFR signalling in OSCC cells

\section{A. Berndt ${ }^{* 1}$, R. Büttner ${ }^{2}$, P. Richter', M. Franz ${ }^{3}$, I. Petersen', C. Liebmann ${ }^{4}$}

${ }^{1}$ Institute of Pathology, University Hospital Jena, Friedrich Schiller University Jena, Jena, Germany, ${ }^{2}$ Institute of Biochemistry, Center of Molecular Biomedicine, Jena, Germany, ${ }^{3}$ Department of Internal Medicine I, University Hospital Jena, Friedrich Schiller University Jena, Jena, Germany, ${ }^{4}$ Institute of Biochemistry, Center of Molecular Biomedicine, Friedrich Schiller University Jena, Jena, Germany

Aims. The reciprocal interaction of cancer associated fibroblasts (CAF's) with oral squamous carcinoma cells (OSCC) is suggested to influence EGFR signalling. We could recently show that the secretome of a smooth muscle actin (ASMA) positive model CAF's is able to induce upregulation of EGFR associated with an activation of Erk and Akt as well as a down regulation of BIM. Here we investigate the molecular background of these changes in more detail.

Methods. As a model for CAF's, hTERT-BJ1 fibroblasts were stimulated with TGF- $\beta_{1}$ to generate a myofibroblast phenotype. Conditioned medium (FCMunstim, FCMTGF) were used to stimulate PE/CA-PJ15 OSCC cells up to 4 days. The underlying molecular signalling pathways of EGFR upregulation and the decrease in BIM expression was assessed by application of inhibitors of matrix metalloproteinases (MMP's), EGFR, MEK, PI3K, and Stat3 (GM6001, Gefitinib, PD98059, LY29400, and STA-21). Effects were detected by Western Blotting. Results were compared to OSCC cells stimulated with conditioned medium of untreated fibroblasts.

Results. Our results revealed a) that the up-regulation of EGFR mediated by the secretome of model CAF's depends on an activation of MMP's in the OSCC cells, b) that this EGFR up-regulation is mediated via the Stat3 pathway, and c) that the anti-apoptotic effect (BIM downregulation) is dependent on the $\mathrm{PI}_{3} \mathrm{~K}$ pathway.

Conclusions. In summary, soluble factors of activated myofibroblasts are capable to induce multiple effects on EGFR signalling including different molecular pathways. Shedding of cellular EGFR ligands and/or the proteolytic liberation of matrikines may play a critical role in this process. Detailed knowledge on these pathways will help to understand the potential role of CAF's in cancer progression and modulation of therapeutic response.

\section{DO-058}

Salivary and adnexal tumours: pathogenetic, histological, and terminological similarities

\section{S. Ihrler*1, M. Mollenhauer ${ }^{2}$, A. Jung ${ }^{3}$, C. Weiler', F. Eckert ${ }^{1}$}

'Labor für Dermatohistologie und Oralpathologie, Munich, Germany, ${ }^{2}$ Institute of Pathology, München, Germany, ${ }^{3}$ Institute of Pathology, Ludwig Maximilians Universität, München, Germany, ${ }^{4}$ Praxis für Pathologie, KarlOlga-Krankenhaus, Stuttgart, Germany

Aims. There are remarkable similarities between different types of salivary and cutaneous adnexal tumours, despite major discrepancies in basic microscopic anatomy. Scientific publications focusing on this relationship are almost lacking.

Methods. Identification and investigation of those types of salivary and adnexal tumours with similarities concerning histology and terminology, based on own archival material and on literature review.

Results. The most striking similarity (both histologically and genetically) is with salivary pleomorphic adenoma/myoepithelioma and cutaneous mixed tumour/myoepithelioma. Multistep carcinogenesis comprising intraductal carcinoma, as recently identified in carcinoma ex pleomorphic adenoma, is similarly shown in cutaneous carcinoma ex spiradenoma, only rarely in carcinoma ex mixed tumour. Major histological analogies are manifest for mucinous/mucoepidermoid carci- 


\section{Abstracts}
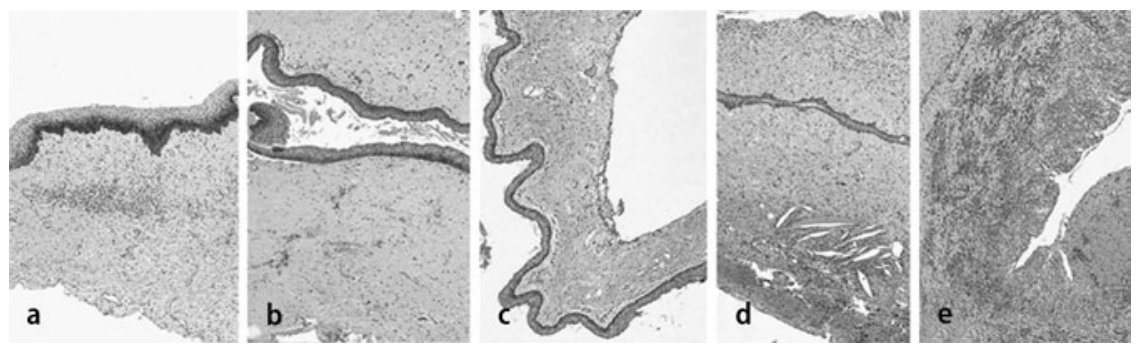

Fig. 1 | D0-060 4

noma, for lymphadenoma/lymphoepithelial carcinoma, for sebaceous adenoma/carcinoma, and for adenoid-cystic carcinoma of salivary and cutaneous adnexal origin, as well as for salivary basal cell adenoma and cutaneous spiradenoma/cylindroma.

Conclusions. Histological and presumably pathogenetic similarities of certain salivary tumour types relate to eccrine, apocrine, and sebaceous (but not to tricho-follicular) types of adnexal tumours. Multistep carcinogenesis including intraductal carcinoma, originating from benign tumours - analogous to salivary carcinoma ex pleomorphic adenomais likewise demonstrated in few types of adnexal tumours, especially in spiradenoma. Despite histomorphologic similarities, differential diagnostic problems in routine practice are rather rare, as they are topographically restricted to those lesions located in the parotid and perioral regions.

\section{DO-059}

\section{No detection of the amplicon 12q13.2 in keratozystic odontogenic tumors by fluorescence in situ hybridization}

\section{J.A. Fröhlich, D. Straub, B. Mankel, U.F. Vogel* Institute of Pathology, University Hospital, Eberhard-Karls-University, Tuebingen, Germany}

Aims. Despite characteristic histopathological features like parakeratinization of the stratified squamous epithelium and palisading of basal cells the keratocystic odontogenic tumor (KCOT) is sometimes difficult to diagnose correctly. Therefore, additional testing is urgently needed. In 2007 an amplicon on $12 q 13.2(12: 54,734,054-58,508,538)$ with 15 consecutive amplified genes (4,0- to 6,2-fold change) was described in KCOTs by array-comparative genomic hybridization. The detection of this amplicon by fluorescence in situ hybridization (FISH) could help in the differential diagnosis of KCOT.

Methods. Four FISH probes were constructed out of bacterial artificial chromosome (BAC) clones (2171N14, RPCI-11 HS 79906, RPCI-11 HS ${ }_{123} \mathrm{~K}_{3}$, RPCI-11 HS258J5) which cover most of the amplicon at 12q13.2. FISH was performed on a total of 50 KCOTs, 25 follicular cysts and 25 radicular cysts which were installed in tissue microarrays with a core diameter of $4 \mathrm{~mm}$.

Results. No amplification at 12q13.2 could be detected by FISH either in the KCOTs nor in the follicular and radicular cysts.

Conclusions. FISH does not help in the differential diagnosis of KCOT when using our probes.

\section{DO-060 \\ EpCAM is a useful marker in the diagnosis of keratocystic odonto- genic tumor}

D. Straub, J.A. Fröhlich, U.F. Vogel*

Institute of Pathology, University Hospital, Eberhard-Karls-University, Tuebingen, Germany

Aims. Keratocystic odontogenic tumor (KCOT) is a benign uni- or multicystic, intraosseous tumor of odontogenic origin with a potential infiltrative behavior. Therefore, a correct histopathological diagnosis with the exclusion of a radicular or follicular cyst is mandatory to justify the more aggressive surgical treatment. Despite characteristic histopathological features (e.g. palisading basal cells, parakeratosis) the differential diagnosis of KCOT is sometimes difficult and raises the need of ancillary testing. Due to the fact that KCOT is in some cases part of the inherited naevoid basal cell carcinoma syndrome (NBCCS) with a strong expression of EpCAM in basal cell carcinoma, the expression of EpCAM in KCOTs, radicular and follicular cysts was examined. Methods. Diagnosed between 1993 and 2010, 128 KCOTs, 147 follicular cysts and 115 radicular cysts were retrieved from the archives of our institute and installed in 35 paraffin tissue microarrays (PTMAs) (core diameter: $4 \mathrm{~mm}$ ). The PTMAs were stained immunohistochemically with a monoclonal mouse antibody (anti-EpCAM; clone Ber-EP4, dilution: 1:10o; Dako) using an automated stainer (Benchmark, Ventana). Results. 89\% (114 cases) of the KCOTs expressed EpCAM in the squamous epithelium in a heterogeneous fashion: $35 \%$ (46 cases) displayed a weak, $42 \%$ ( 54 cases) a moderate and $9.4 \%$ (12 cases) a strong basal and parabasal staining. In squamous epithelium with underlying inflammation, EpCAM expression was reduced or lost. 1.6\% (2 cases) showed only a superficial staining pattern. $7 \%$ (14 cases) of the KCOTs were negative for EpCAM in contrast to $70 \%$ (103 cases) of the follicular cysts and $99 \%$ (114 cases) of the radicular cysts. Two follicular cysts (2\%) displayed a weak to moderate expression in the basal/parabasal layer whereas $28 \%$ ( 42 cases) showed a weak to strong EpCAM expression in the superficial epithelial layers. Only one case of the radicular cysts expressed EpCAM in a few cells in the superficial epithelial layers.

Conclusions. The immunohistologically detected expression of EpCAM in the basal and parabasal layers of the squamous epithelium may be helpful in the diagnosis of KCOTs (• Fig. 1).

\section{D0-061}

Distinct patterns of NAB2-STAT6 gene fusions in solitary fibrous tumors (SFTs)/haemangiopericytomas (HPCs) of the head and neck region

\section{A. Agaimy ${ }^{* 1}$, s. Barthelmess', H. Geddert ${ }^{2}$, C. Boltze ${ }^{3}$, A. Hartmann ${ }^{1,}$ \\ F. Haller}

'Institute of Pathology, Friedrich-Alexander-University, Erlangen-Nuremberg, Germany, ${ }^{2}$ Institute of Pathology, St. Vincent's Hospital, Karlsruhe, Karlsuhe, Germany, ${ }^{3}$ Institute of Pathology, SRH-Klinikum, Gera, Gera, Germany

Aims. Recently, recurrent gene fusions of the genes NAB2 and STAT6 have been identified in the majority of tumors from the solitary fibrous tumor (SFTs)/haemangiopericytoma (HPCs) spectrum. For the current study, we evaluated whether tumors from this spectrum located in the head and neck region have similar features.

Methods. A cohort of nine SFTs/HPCs from the head and neck region was identified in the archives of our institutions. Additionally, eight sinonasal tumors with features of sinonasal hemangio-/glomangiopericytoma were found. All tumors were analysed for the presence of a NAB2-STAT6 fusion by RT-PCR, and in positive cases the breakpoints were identified by Sanger sequencing of PCR products. 
Results. Regarding the SFTs/HPCs, there were two cases in the orbita, two cases in the parotid gland, one case each in the cheek and in the tongue, and one case each from the sublingual, the parapharyngeal and the nuchal region. Notably, four (44\%) of the tumors including both tumors located in the orbita, one case in the parotid gland and one case in the cheek harbored NAB2-STAT6 fusions involving NAB2 exon 3 with fusion to STAT6 exons 17, 18 and 19, which is a rather uncommon fusion variant among SFTs/HPCs from other sites. Two of the remaining cases were positive for the classical 6-16 fusion variants, while the remaining three cases were of wild type status. In contrast, none of the eight (100\%) of the classical sinonasal HPCs/glomangiopericytomas demonstrated a NAB2-STAT6 gene fusion.

Conclusions. In conclusion, tumors of the SFT/HPC spectrum arising in the head and neck region are characterized by distinct fusion variants of the NAB2-STAT6 fusion gene that differ from their counterparts in the peripheral soft tissue and retroperitoneum. On the other hand, classical sinonasal HPCs do not harbor NAB2-STAT6 gene fusions indicating different molecular pathogenesis unrelated to soft tissue-type SFT/HPCs.

\section{DO-062}

MED15 is overexpressed at high frequency in head and neck squamous cell carcinoma and is implicated in TGF $\beta$ signaling

\section{A. Offermann ${ }^{* 1}$, Z. Shaikhibraim², M. Braun', D. Böhm', W. Vogel" G. Kristiansen ${ }^{3}$, A. Schröck ${ }^{1,4}$, S. Perner}

IInstitute of Pathology, Department of Prostate Cancer Research, University hospital Bonn, Bonn, Germany, ${ }^{2}$ Department of Prostate Cancer Research, Pathology, University of Bonn, University of Bonn, Bonn, Germany, ${ }^{3}$ Department of Pathology, University Medical Center, Molecular Pathology, Bonn, Germany, ${ }^{4}$ Otorhinolaryngology, University Medical Center, Bonn, Germany

Aims. The Mediator Complex is an evolutionarily conserved multi protein complex which is essential for the transcription of protein encoding genes and the regulation of diverse signaling pathways. The subunit MED15 is essential for the TGF $\beta$ pathway, which plays a major role in the pathogenesis of head and neck squamous cell carcinoma (HNSCC). In this study, we investigated the relevance of the Mediator subunit MED15 in tissues as well as in HNSCC cell lines. Furthermore we examined the relationship of MED15 with the TGF $\beta$ pathway.

Methods. MED15 expression level was determined by immunohistochemistry (IHC) and gene amplification/rearrangement status by Fluorescence In-situ hybridization (FISH) on tissue microarrays (TMAs) comprising 719 HNSCC patients. For correlation studies, TMAs were assessed for the proliferation marker Ki67 as well as for $\mathrm{pSMAD}_{3}$ as an activation marker of the TGF $\beta$ signaling by IHC. In several HNSCC cell lines, we performed MED15 knockdown by siRNA as well as overexpression by a plasmid followed by proliferation assay. To investigate the role of MED15 upon TGF $\beta$ signaling, we treated MED15 knockdown cells with recombinant TGF $\beta_{1}$ and evaluated the expression of $\mathrm{pSMAD}_{3}$ and TGF $\beta$ target genes level by western blot. Furthermore, we determined whether TGF $\beta$ signaling leads to increased expression of MED15 by treating cells with TGF $\beta 1$

Results. We found MED15 to be overexpressed at high frequency in HNSCC tissues compared to benign tissue. MED15 overexpression correlates with $\mathrm{Ki} 67$ as a proliferation marker as well as with $\mathrm{PSMAD}_{3}$ as a marker of the TGF $\beta$ signaling activation. Additionally, we found MED15 to be amplified in HNSCC tissues as well as in two HNSCC cell lines. Knockdown or overexpression of MED15 in the cell lines affects cell proliferation as well as TGF $\beta$ signaling. Furthermore, we show that TGF $\beta$ signaling activation leads to increased expression of MED15 at protein level in HNSCC cell lines.

Conclusions. Our findings reveal a potential role of MED15 in HNSCC, and a direct implication with TGF $\beta$ signaling which is known to play an important role in the pathogenesis of HNSCC.

\section{AG Herz-, Gefäß-, Nephro- und Transplantatpathologie}

\section{DO-063}

International guidelines and recent developments in monitoring of donor-specific antibodies after organ transplantation

C. Süsal*

Department of Transplantation Immunology, Institute of Immunology, University of Heidelberg, Heidelberg, Germany

In organ transplantation, antibody-mediated allograft injury caused by donor-specific HLA antibodies (DSA) has recently been identified as one of the major causes of late graft loss. I will summarize the recent findings on the impact of DSA development on graft outcome in organ transplantation with a focus on risk factors for de novo alloantibody induction and recently published guidelines for monitoring of DSA during the post-transplant phase.

\section{DO-064}

The Fischer to Lewis model of rat transplant glomerulopathy is characterised by subendothelial immune complexes similar to a subset of human transplant glomerulopathy

P. Zeuschner"1, S. Zell', H. Höflich', K. Säuberlich', J. Wittig', C.L. Bockmeyer', V. Grau'2, J.U. Becker*1

'Institute of Pathology, Hannover Medical School, Hannover, Germany, ${ }^{2}$ Sektion Experimentelle Chirurgie, Klinik für Allgemein-, Viszeral-, Thorax-, Transplantations- und Kinderchirurgie, Universitätsklinikum Gießen, Gießen, Germany

Aims. The life sustaining Fischer to Lewis rat model of renal transplantation is a well established model of chronic humoral rejection and transplant glomerulopathy. Despite its widespread use in pathophysiologic and therapeutic research, thorough and comprehensive histologic descriptions have never been published.

Methods. Six months after life-sustaining transplantation without immunosuppression 8 allografts were harvested for histologic examination including immunohistochemistry and electron microscopy and compared to 4 isografts. Findings were compared to a prospective cohort of 4 patients with transplant glomerulopathy (membranoproliferative glomerulonephritis and thrombotic microangiopathy as primary diseases excluded).

Results. Besides the classic findings of transplant glomerulopathy with double-contours of glomerular basement membranes and focal and segmental glomerulosclerosis we found subendothelial and mesangial electron dense immune complexes only in the rat allografts, not in the isografts. On immunostains, these deposits were found to contain IgG and IgM. In a subset of human transplant biopsies with transplant glomerulopathy ( 2 out of 4 cases examined so far), similar subendothelial immune complex deposits were found on electron microscopy. All were also positive for immunoglobulin and complement.

Conclusions. Immune complex formation seems to occur in a subset of human transplant glomerulopathy and is faithfully reproduced in the Fischer to Lewis rat model. Further studies in human biopsies and correlation with the rat model will show the serologic, pathophysiologic and prognostic implications of immune complex formation in transplant glomerulopathy. 


\section{DO-065}

\section{Pre-transplant liver evaluation: results of the DGP/DTG/DSO con-} sensus

\section{Flechtenmacher*1, H.A. Baba', W.O. Bechstein ${ }^{3}$, A. Pascher ${ }^{4}$ \\ H.-H. Kreipe ${ }^{5}$, P. Schirmacher', C. Schleicher ${ }^{6}$}

'Institute of Pathology, University Hospital Heidelberg, Heidelberg, Germany, ${ }^{2}$ Institute of Pathology and Neuropathology, University Hospital, Essen, Germany, ${ }^{3}$ Department of General and Visceral Surgery, University Hospital, Frankfurt, Germany, ${ }^{4}$ Department of General, Visceral and Transplantation Surgery, University Hospital Charite, Berlin, Germany, ${ }^{5}$ Institute of Pathology, University Hospital, Hannover, Germany, ${ }^{6}$ Deutsche Stiftung Organtransplantation, Stuttgart, Germany

Aims. As demand for organs increases, marginal donor livers are increasingly considered for transplantation. Pathologists are more and more asked to evaluate these livers histopathologically using frozen sections at high urgency for acceptability.

Methods. On 23 October 2013 a workshop was held in Frankfurt with representatives from the DGP (Deutsche Gesellschaft für Pathologie), DTG (Deutsche Transplantationsgesellschaft) and DSO (Deutsche Stiftung Organtransplantation) for exchange of information, to discuss the prerequisites and conditions for proper pre-transplant evaluation of donor liver tissue and to provide guidelines for frozen section evaluation and reporting.

Results. Histopathological reports should give information about the extent of macrovesicular (and microvesicular) steatosis, as well as potential fibrosis, inflammation, necrosis and any other relevant finding. To evaluate transplant suitability the correlation of the histopathological findings with the clinical conditions is essential and requires excellent communication between pathologists, surgeons and all the other members of the transplantation team. Structured procedures support this process. Expert-driven consent over workflow, biopsy indications, tissue acquisition, required clinical information, tissue evaluation and reporting as well as structure and coverage for nationwide service has been reached.

Conclusions. Pre-transplant histopathological evaluation is of value in the selection of donor livers for transplantation and should be performed according to the consented regulations in order to avoid unnecessary loss of precious organs and to prevent inappropriate organs from being transplanted. Consent over workflow, tissue evaluation and reporting as well as structure and coverage for nationwide service has been reached and will be presented.

\section{D0-066 \\ Direct acute tubular damage contributes to Shigatoxin-mediated kidney failure}

S. Porubsky*1,2, G. Federico ${ }^{2}$, R. Jennemann ${ }^{2}$, J. Müthing ${ }^{3}$, E. Gröne ${ }^{2}$, N. Gretz', N. Obermüller ${ }^{5}$, O. Jung ${ }^{5}$, I. Hauser ${ }^{5}$, H. Geiger ${ }^{5}$, H.-J. Gröne ${ }^{2}$, C. Betz ${ }^{5}$

'Institute of Pathology, University Medical Centre, University of Heidelberg, Mannheim, Germany, ${ }^{2}$ Cellular and Molecular Pathology, German Cancer Research Center, DKFZ, Heidelberg, Germany, ${ }^{3}$ Institute for Hygiene, Münster, Germany, ${ }^{4}$ Metabolic Center Heidelberg, Ruprecht-Karls-University, Heidelberg, Germany, ${ }^{5}$ Department of Nephrology, University Hospital Frankfurt/Main, Frankfurt/Main, Germany

Aims. In 2011, a Shigatoxin-producing Escherichia coli (STEC) $\mathrm{O}_{104}: \mathrm{H}_{4}$ infection spread through Germany affecting 3816 patients. The pathogenesis and optimal therapy of Shigatoxin-mediated kidney failure remain controversial. Our aim was to explore the pathophysiology of Shigatoxin-mediated kidney failure.

Methods. Long-term outcome was investigated in patients with STECassociated hemolytic uremic syndrome at the University Hospital in Frankfurt/Main, Germany. Patients ( $n=11$, aged 22 to 44 years) had kid- ney biopsies and were treated with supportive therapy (without complement inhibiting antibodies). To corroborate clinical and histological data in vivo, murine models with global and tissue specific (renal tubular cells, endothelial cells and platelets) deficiencies for the Shigatoxinreceptor globotrihexosylceramide $\left(\mathrm{Gb}_{3}\right)$ were analyzed.

Results. Despite a severe affection [LDH 1944U/1 (753-2792), platelets 33/ $\mathrm{nl}(19-124)$ and hemoglobin $6.2 \mathrm{~g} / \mathrm{dl}$ (5.2-7.8); median (range)], all patients were discharged after 33 days (19-43) with no neurological symptoms and no dialysis requirement [creatinine $1.39 \mathrm{mg} / \mathrm{dl}$ (o.84-2.86)]. After a 12-month follow-up, the creatinine decreased to $0.90 \mathrm{mg} / \mathrm{dl}$ (o.82-1.52). Analysis of kidney biopsies (available in 10 patients) revealed a dominant acute tubular damage but not thrombotic microangiopathy as the leading cause of the renal failure. To identify the tubular compartment as the determinant of the acute renal damage, mouse models were implemented. In wild-type mice, which -like humans- express $\mathrm{Gb}_{3}$ in renal tubular epithelium, Shigatoxin2-mediated tubular damage led to lethal electrolyte disturbance. Tubule-specific depletion of Gb3 protected mice from acute renal failure, but favored development of cerebral purpura.

Conclusions. Acute tubular damage represents an additional and currently possibly underestimated mechanism of Stx2-mediated acute kidney failure. An excellent renal outcome can be achieved by supportive therapy only.

\section{DO-067}

Co-localization of proteins with amyloid plaques in renal biopsies: data gernerated by MALDI imaging mass spectrometry

R. Casadonte ${ }^{* 1}$, M. Kriegsmann ${ }^{2}$, M. Otto ${ }^{1,3,4}$, R. Paape ${ }^{5}$, D. Suckau ${ }^{5}$, S.-O. Deininger ${ }^{5}, K_{\text {. Amann }}{ }^{6}$, J. Kriegsmann ${ }^{1,3,4}$

${ }^{1}$ Proteopath GbR, Trier, Germany, ${ }^{2}$ University of Heidelberg, Trier, Germany, ${ }^{3}$ Center for Histology, Cytology and Molecular Diagnostics, Trier, Germany, ${ }^{4}$ Institute of Molecular Pathology, Trier, Germany, ${ }^{5}$ Bruker Daltonik GmbH, Bremen, Germany, ${ }^{6}$ Institute of pathology University Erlangen-Nürnberg," Erlangen, Germany

Aims. The main aim of this study is to identify amyloid proteins and establish their distribution in amyloidosis tissue sections using imaging mass spectrometry (IMS) and liquid chromatography-tandem mass spectrometry (LC-MS/MS) analysis. A secondary aim was to investigate protein signals associated with amyloidosis disease.

Methods. Renal FFPE biopsy specimens with AA-amyloidosis were cut $(\sim 10 \mu \mathrm{m})$ and mounted onto regular slide for Congo red staining. Serial sections were cut $(\sim 2 \mu \mathrm{m})$ and mounted onto ITO-coated conductive slides for IMS analysis. After deparaffination and antigen retrieval procedures, sections were on-tissue trypsin digested, sprayed with matrix using the ImagePrep device (Bruker Daltonik, Bremen), and subsequently analyzed by MALDI MS using a Bruker Autoflex Speed mass spectrometer (Bruker Daltonik, Bremen). Mass spectra data were loaded to ClinProTools software allowing profiles and statistical analysis to be directly linked to the histological image and subsequently compared to the IMS images for correlation study. Consecutive tissue sections were processed in parallel to provide both protein distribution and identification simultaneously. Peptides were extracted from tissues with $20 \mu \mathrm{L}$ of buffer solution (10\% ACN, 1.1\% TFA) and subjected to LCMS/MS analysis for protein identification.

Results. Three peptides for serum amyloid A (SAA) (1456.7, 1612.8, 1670.7), and four peptides for serum amyloid P-component (SAMP) proteins $(1156.6,1166.6,1406.7$, and 1811.8) were identified that matched the masses assigned by IMS analysis. MS images were compared with optical images of the Congo-red serial tissue section resembling the amyloid deposition. Two proteins, vitronectin and apolipoprotein E, were identified and found to correlate with amyloid regions.

Conclusions. IMS with LC-MS/MS analysis using on-tissue tryptic digestion allowed the identification of amyloidosis specific peptides and the precursor proteins providing information on both protein distribution 
and identification simultaneously. The association of vitronectin and apolipoprotein-E proteins with renal amyloid shown in this study suggests a role for these proteins in the process of amyloidogenesis. Previous studies demonstrated the contribution of vitronectin and apoliporpotein E proteins with the formation of amyloid protein $[1,2]$.

1. Shin TM et al (2008) Formation of soluble amyloid oligomers and amyloid fibrils by the multifunctional protein vitronectin

2. Kim J et al (2012) Anti-apoE immunotherapy inhibits amyloid accumulation in a transgenic mouse model of $A \beta$ amyloidosis

\section{DO-068}

\section{Widespread expression of the lipid droplet-associated protein} perilipin 5 in normal and diseased human tissues

\section{Hashani', L.M. Pawella', J. Lehmann-Koch', J. Schumacher², G. Mechtersheimer', P. Schirmacher', B.K. Straub ${ }^{* 1,3}$ \\ ${ }^{1}$ Institute of Pathology Heidelberg, Heidelberg, Germany, ${ }^{2}$ Department of Internal Medicine III, University Hospital Mainz, Mainz, Germany, Institute of Pathology, University Hospital Heidelberg, Heidelberg, Germany}

Aims. Diseases associated with the accumulation of lipid droplets (LDs) are steadily increasing in western countries. LDs are decisively regulated by LD-associated proteins of the PAT/perilipin-family with its members perilipin, adipophilin, TIP47, S3-12 and MLDP (perilipin 1-5). Perilipin 5 (synonyms: myocardial lipid droplet protein/ MLDP, OXPAT) has been reported to be specific for the oxidative tissues heart, skeletal muscle and liver by its function in coupling LDs to mitochondria. Mice deficient in perilipin 5, lacked detectable LDs in the heart, in contrast overexpression of perilipin 5 in the heart resulted in cardiac steatosis; yet both deletion and overexpression of perilipin 5 in mice lead to cardiac dysfunction. Nevertheless, perilipin 5 expression in human tissues and in disease especially liver disease has not been analysed in detail.

Methods. Antibodies specific for human perilipin 5 were produced in rabbits. Perilipin 5 was analysed on the mRNA and protein level in human tissues and in disease in situ as well as in cell culture models.

Results. Perilipin 5 was detected near-ubiquitously in liver, skeletal muscle, heart, adipose tissue, and epithelia of gastrointestinal and urogenital tract with particular abundance in mitochondria-rich cell types. In cardiomyopathies, skeletal muscle diseases, and in hepatic steatogenesis, perilipin 5 was upregulated and localized to larger and more numerous LDs. In adipogenesis as well as in hepatocyte steatogenesis, LD maturation in situ (plurivacuolar to univacuolar adipocytes; acute to chronic hepatocyte steatosis) and in vitro (steatosis models) generally involves the sequential involvement of perilipins 3, 5, 2 and finally perilipin 1. Liver diseases implicated in alterations of mitochondria such as mitochondriopathies, Wilson's disease, acute liver injury and ballooned cells in steatohepatitis showed upregulation and recruitment of perilipin 5 from the cytoplasm to LDs. In bland steatosis, perilipin 5 protein amount partly correlated with lipid content in situ and in vitro and perilipin 5 colocalized with perilipins 1 and 2, but virtually not 3 , at LDs suggesting mutually exclusive functions of perilipins 3 and 5 . In tumorigenesis, perilipin 5 was especially upregulated in lipo-, leio-, rhabdomyosarcoma, hepatocellular carcinoma and clear cell renal cell carcinoma.

Conclusions. Our data link perilipin 5 to general functions in oxidative energy supply in normal and diseased tissues independent of cell-type and of the other perilipins.

\section{AG Molekularpathologie I}

\section{DO-069}

\section{Concomitant mutation and amplification of the ERBB2 (HER2) gene} in human tumors

T. Grob*, M. Sandmann, A. Quaas, A. Marx, G. Sauter

Institute of Pathology, University Medical Center Hamburg Eppendorf, Hamburg, Germany

Aims. Amplification of the ERBB2 (HER2) gene is a well-known genetic aberration in many tumor entities. Up to $20 \%$ of breast and gastric cancer show ERBB2 gene amplification and anti-HER2 targeting therapies are well established for these tumors. In other tumor entities such as lung cancer, colorectal cancer and bladder cancer the fraction of ERBB2 amplified tumors is considerably lower. In contrast to breast cancer, these tumors show heterogeneity of ERBB2 amplification in a substantial proportion. Oncogenic EGFR mutation with subsequent EGFR amplification is a common phenomenon in lung cancer. In analogy, primary ERBB2 mutation could explain the observed heterogeneity of ERBB2 amplification in some tumors.

Methods. 100 tumors showing ERBB2 amplification by fluorescence in situ hybridization were examined for $\mathrm{ERBB}_{2}$ mutation: 50 breast cancer samples, including 10 cases with borderline gene amplification, 10 cases with heterogeneous gene amplification and 11 samples of metastatic lesions as well as 15 colorectal carcinomas, 12 gastric carcinomas, 12 nonsmall cell lung cancers and 10 urothelial carcinomas. Tumor DNA was extracted and examined for mutations in the tyrosine kinase domain (exons 18-23) of the ERBB2 gene by Sanger sequencing.

Results. ERBB2 mutation was found in two metastatic lesions of breast cancer, two lung cancers and two colorectal cancers. In five of these cases short in-frame insertions in exon 20 were found. In one case of a breast cancer lung metastasis a missense mutation in exon 19 (p.L755S) was detected. The semi-quantitative chromatograms show an overrepresentation of the mutations indicating a selective amplification of the mutated allele.

Conclusions. In most breast and gastric cancers homogeneity of ERBB2 amplification within a certain tumor suggests an early genetic alteration in carcinogenesis. However in other tumor entities the heterogeneity of $\mathrm{ERBB}_{2}$ amplification indicates a late genetic event. The finding of a concomitant mutation in ERBB2 amplified tumors might explain the heterogeneity in some of these tumors. The effect of a primary oncogenic ERBB2 mutation might be increased by selective amplification of the mutated allele resulting in an additional growth advantage. If $\mathrm{ERBB} 2$ amplified tumors with a primary ERBB2 mutation also benefit from an HER2 targeted therapy needs to be elucidated. 
DO-070

Interim analysis of the non-interventional monitoring program "biomarkers of excellence" on HER2-testing in breast cancer (January to September 2013)

J. Rüschoff*1,2, P. Middel', ${ }^{1,2}$, K. Tiemann ${ }^{3}$, U. Diergardt ${ }^{4}$, R. Weimann ${ }^{5}$, W. Haedicke ${ }^{6}$, C.-D. Gerharz', A. Neuber ${ }^{8}$, R. Bollmann ${ }^{9}$, C. TennstedtSchenk ${ }^{10}$

${ }^{1}$ Institut für Pathologie Nordhessen, Kassel, Germany, ${ }^{2}$ TargosMolecularPathology $\mathrm{GmbH}$, Kassel, Germany, ${ }^{3}$ Hämatopathologie Hamburg, Hamburg, Germany, ${ }^{4}$ Praxis Dr. Markus Ruwe und Kollegen, Hagen, Germany, ${ }^{5}$ Klinikum Saarbrücken Pathologisches Institut, Saarbrücken, Germany, ${ }^{6}$ Dres. Claus Minck Wolfgang Haedicke und Susanne Reyher-Klein, Berlin, Germany, ${ }^{7}$ Bethesda-Krankenhaus Institut für Pathologie, Duisburg, Germany, ${ }^{8} \mathrm{Dr}$. Angelika Neuber und Kollegen, Wesel, Germany, ${ }^{9}$ Dres. Magdolna Bollmann, Reinhard Bollmann, Hildegard Heller, u. w., Bonn, Germany, ${ }^{10}$ Gemeinschaftspraxis für Pathologie, Mühlhausen, Germany

Aims. Due to the use of HER2-targeted drugs, the prognosis for patients with HER2-positive breast cancer has improved significantly. To obtain an up-to-date picture of the quality of HER 2 testing in Germany, a noninterventional, prospective monitoring program was started in January 2013. In this program, up to 160 pathology institutes are planned to take part in. The aim is a non-selective documentation of about 16,000 breast cancer cases from routine diagnostics.

Methods. In this first interim analysis data from 3603 breast cancer cases from 22 institutes, which documented at minimum 50 cases, were included.

Results. 21 of 22 participating institutes used immunohistochemistry (IHC) as primary testing method; only one institute used in-situ-hybridisation (ISH) primarily. Half of the institutes used for IHC analysis a commercially available testing kit and about $80 \%$ of the institutes referred to a commercially available solution for tissue fixation. Almost all institutes based their IHC grading on the ASCO/CAP-Guidelines published in 2007 (cut-off defined as $30 \%$ of tumour cells). Only one institute used the FDA cut-off-threshold for IHC (10\% of tumour cells). For classification of ISH testing results, half of the institutes referred to the ASCO/CAP threshold (ratio >2.2).

Of the breast cancers documented $12.6 \%$ were classified as $\mathrm{IHC}_{3}+$ HER2-positive and $13.5 \%$ as borderline (IHC 2+). Among the latter, not all cases were reassessed by ISH. Overall, the average HER2-positivity was $15.4 \%$ with a moderate variation. Only two institutes tested on average outside the range of $10-25 \%$. Most of the HER2-positive tumours documented (93.2\%) were ductal carcinomas of grade 2 or 3 (>95\%). Lobular carcinomas showed, in contrast to ductal carcinomas, a lower HER2-positivity rate (5.8\% vs. $17.0 \%$ ). Mammary carcinomas with an extensive lymph node infestation ( $\left.\mathrm{pN}_{2}, \mathrm{pN}_{3}\right)$ exhibited a $10 \%$ higher HER2-positivity compared to mammary carcinomas where $\leq_{3}$ lymph nodes were affected (pNo, pN1; $23 \%$ vs. $13 \%$ ). In 190 cases HER2-positivity was assessed at an earlier stage of the disease. Retesting revealed that the HER 2 status had changed on average in 1 of 8 cases.

Conclusions. The results of the interim analysis on HER2 testing in Germany confirm a tight correlation of HER2 status with grade of tumour differentiation and nodal status. The HER 2 positivity rates from the $\mathbf{2 2}$ institutes analysed may indicate testing problems at two individual institutes but suggest an in general high overall HER2 testing quality in Germany.
D0-071

Mass spectrometry-based proteomic profiling reveals new biomarkers for primary lung cancers and lung metastases

H. Bohnenberger*1, H. Serve ${ }^{2}$, C. Lenz ${ }^{3}$, F. Bremmer', H. Henric-Petri', A. Emmert $^{4}$, S. Wagner', J. Corso', J. Strecker', S. Küffer', R. Holland ${ }^{4}$,

M. Sebastian', M. Hinterthaner', F.A. Schöndube ${ }^{4}$, H.-U.Schildhaus', B. Danner', H. Urlaub ${ }^{3}$, P. Ströbel', T. Oellerich ${ }^{2}$

'Institute of Pathology, University Medical Center Göttingen, Göttingen, Germany, ${ }^{2}$ Department of Hematology/Oncology, Johann Wolfgang Goethe University, Frankfurt, Germany, ${ }^{3}$ Bioanalytical Mass Spectrometry Group, Max Planck Institute for Biophysical Chemistry, Göttingen, Germany, ${ }^{4}$ Department of Thoracic and Cardiovascular Surgery, University Medical Center Göttingen, Göttingen, Germany

Aims. Lung cancer is the primary reason of cancer-related mortality worldwide. Neoplasia of the lung can be divided into two main groups consisting of small cell and non-small cell lung cancer (NSCLC) with a further subdivision of NSCLC in adenocarcinoma and squamous cell carcinoma. Furthermore, the lung is a common site of metastasis of various neoplasms. The distinction of primary neoplasms from lung metastases has far-reaching clinical and therapeutic implications; hence a pathological differentiation of these entities is of great importance. However, in particular the distinction of carcinomas with squamous cell morphology remains difficult due to a lack of appropriate immunohistochemical biomarkers.

Methods. High resolution mass spectrometry allows to quantitatively determine protein expression patterns in a comprehensive and unbiased manner. We established a work-flow to extract large amounts of proteins from formalin fixed paraffin embedded (FFPE) tissues by using a filter aided sample preparation method. Purified proteins were subsequently subjected to a Super-SILAC-based experimental set-up that allows for identification and quantification of thousands of proteins by high-end mass spectrometry.

Results. In this study we characterized the proteome of 80 FFPE samples including tumor free lung tissues, squamous cell carcinomas, adenocarcinomas and small cell carcinomas of the lung. Moreover, lung metastases derived from squamous cell carcinoma from head-neck tumors and colorectal adenocarcinomas as well as the corresponding primary tumors were included in the analysis. Using a spike-in Super-SILAC quantification standard we were able to identify and quantify more than 2000 proteins per sample. Unsupervised clustering analysis clearly separated the histological subtypes of lung cancer and furthermore, also metastases could be distinguished from primary lung cancers with similar histological morphology using their protein expression profiles. Conclusions. Collectively, this study provides a comprehensive database of proteomic biomarkers of lung cancer that can be used to improve lung cancer diagnostics including the discrimination of metastases from primary lung cancer. Moreover, pathway analysis of proteomic data revealed mechanistic insights into pathophysiological features of lung cancer subtypes. The experimental approach presented here can be adapted for investigation of other tumor types and opens up the opportunity to characterize pathological archives by large-scale proteomics. 


\section{DO-072}

\section{PAK1: a new target of Thymoquinone}

\section{El-Baba*1, F. Fahlbusch', M. Vijayalakshmi ${ }^{3}$ H. Gali-Muhtasib ${ }^{4}$, R. Schneider-Stock ${ }^{* 5}$}

'Experimental Tumorpathology, Institute of Pathology, Erlangen, Germany, ${ }^{2}$ Department of Pediatrics and Adolescent Medicine, Friedrich-AlexanderUniversity of Erlangen-Nürnberg, Erlangen, Germany, ${ }^{3}$ Faculty of School of Chemistry and Biotechnology, Sastra University, Thanjavur, India, ${ }^{4}$ Institute of Biology, American University, Beirut, Lebanon, ${ }^{5}$ Experimental Tumorpathology, Institute of Pathology, Friedrich-Alexander-University ErlangenNürnberg, Erlangen, Germany

Aims. Thymoquinone (TQ) was reported to possess anti-inflammatory, anti-neoplastic and anti-/ pro-oxidant properties. The ability of TQ to induce apoptosis in tumor cells makes it a promising chemotherapeutic candidate. The mechanism by which TQ acts on tumors is not fully understood. It is well known that post-transcriptional modification and more precisely protein phosphorylation controls most, if not all cellular events. To identify new TQ targets in colorectal cancer (CRC), we conducted a phospho-peptide analysis and performed in vitro and in silico analysis.

Methods. Using a peptide chip array, we have identified approximately fifty candidates (among them 11 kinases) showing a phosphorylation fold change CHR: greaterlike\# ${ }^{* * *} 2$ in colorectal HCT116 tumor cells after a $24 \mathrm{~h}$ TQ treatment. Using in silico methods (pathway analysis software, docking algorithms and phosphorylation signature patterns), deregulated proteins of interest were classified according to their relevance in cancer networks and biofunctions. To confirm the in silico data, we performed transfections, co-immunoprecipitation, western blotting and flow cytometry for several candidates on different CRC cell lines.

Results. The analysis revealed that top TQ-regulated pathways and biological functions were cell cycle control and cancer pathways. The verification of p21-activating kinase (PAK1)phosphorylation sites by western blot revealed a time-dependent change in the expression patterns of two phosphorylation sites (PAKThr212, PAK1Thr423) creating a specific kinase profile at early and late time points after TQ treatment. PAK1 is a key molecule in the AKT-MEK-ERK pathway (a central signaling network for current targeted therapy) and has been never described as a TQ target. We further studied the role of PAK1 in TQ-induced apoptosis. In the presence of IPA-3, an allosteric inhibitor of PAK1, we observed a significant increase in the phosphorylation level of PAKThr212 and pERK together with a remarkable induction of apoptosis in the combination treatment in comparison to TQ alone. Structural modeling suggests a direct binding of TQ near to the Thr212 site influencing the PAK1 conformation possibly strengthening a direct interaction of ERK and PAK1. Conclusions. This study shows for the first time that a small molecule like TQ might bind directly to a kinase, PAK1. Because TQ seems to affect the central network of the RAF/MEK/ERK pathway, combination of targeted therapies with TQ might produce sustained antitumor efficacy.

\section{DO-073}

\section{Expression of the stem cell marker ecotropic viral integration site-1 in prostate cancer}

\section{S. Hagedorn ${ }^{* 1,2}$, A. Queisser ${ }^{1,2}$, W. Vogel ${ }^{1,2}$, D. Böhm ${ }^{1,2}$, C. Lengerke $^{3,}$ \\ N. Wernert', G. Kristiansen ${ }^{2}$, S. Perner ${ }^{1,2}$}

'Institut of Pathology, Department of Prostate Cancer Research, University hospital, Bonn, Germany, ${ }^{2}$ Department of Pathology, University Medical Center, Bonn, Germany, ${ }^{3}$ Department of Biomedicine, University Hospital, Division of Medical Genetics, Basel, Switzerland

Aims. Prostate cancer (PCa) is the most common cancer in men in the western world. It is known that the accumulation of molecular changes is essential for prostate carcinogenesis. For example, mutations in tumor suppressorgenes like PTEN and $\mathrm{TP}_{53}$ as well as in the oncogenes MYC and KRAS are important for PCa progression. However, the role of transcription factors with stem cell marker properties during prostate carcinogenesis is insufficiently examined. Due to gene rearrangements the stem cell marker ecotropic viral integration site-1 (EVI-1) is often overexpressed in leukaemia but also in solid tumours. The aim of this study was to investigate the protein expression pattern of EVI-1 as well as the rearrangement/amplification rate in a PCa progression cohort and PCa cell lines.

Methods. Rearrangement/amplification status as well as the expression levels of EVI-1 were investigated on paraffin-embedded tissues of a PCa progression cohort (benign prostate tissue $n=11$, primary $P C a n=121$, localized lymph nods $n=11$, distant metastasis $n=31$ ). Staining against EVI-1 was done with immunohistochemistry (IHC) and analysed by two independent pathologists. We determined the intensity $(\mathrm{o}=$ negative, $1=$ low, $2=$ medium, $3=$ high) of the staining as well as the quantity of positively stained cells. PCa cell lines were also screened for EVI-1 protein expression by western blot and for RNA expression by qRT-PCR. To determine the rearrangement/amplification rate of EVI-1 fluorescence in-situ hybridisations (FISH) were performed using BAC-clones flanking the gene or spanning the whole gene, respectively.

Results. We could show that in benign prostate tissue EVI-1 reveals low expression in $100 \%$ of the basal cell layer. In primary PCa samples we could detect a stronger but heterogeneous expression level of EVI-1. The number of stained cells as well as the intensity of the stained cells increases with the progression to lymph node and distant metastasis. Western blot and qRT-PCR analysis revealed that the PCa cell line PC-3 also expresses EVI-1. FISH analysis showed no EVI-1 rearrangement and amplification.

Conclusions. Our data indicate that EVI-1 plays an important role during prostate cancer progression. The EVI-1 expressing PCa cell line $\mathrm{PC}-3$ provides a good basis for further functional studies.

\section{DO-074}

Relationship between 18F-Fluorodeoxyglucose accumulation, BRAF-mutation and GLUT1-expression in the metastases of cutaneous malignant melanoma

\section{B. Schwindenhammer*1, H.S. Bachmann², F. Mairinger', J. Nagarajah ${ }^{3}$, K. Griewank', S.-Y. Sheu', K.W. Schmid', F. Grabellus'}

'Institute for Pathology, University Hospital of Essen, University of Duisburg-Essen, Essen, Germany, ${ }^{2}$ Institute for Pharmacogenetics, University Hospital of Essen, University of Duisburg-Essen, Essen, Germany, ${ }^{3}$ Department of Nuclear Medicine, University Hospital of Essen, University of Duisburg-Essen, Essen, Germany, ${ }^{4}$ Department of Dermatology, University Hospital of Essen, University of Duisburg-Essen, Essen, Germany

Aims. Malignant tumors exhibit higher energy requirements compared with healthy tissues, which is accomplished by a switch to so-called "aerobic glycolysis". This general increase in glucoses metabolism in tumor cells is responsible for the accumulation of [18F]-fluorodeoxyglucose (18F-FDG) in PET/CT. One effect of mutations of the BRAF gene seems to be GLUT1 over-expression. We reported supporting evidence of this relationship in papillary thyroid carcinomas. Furthermore, a positive correlation between BRAF or KRAS mutations and 18F-FDG uptake in colorectal cancer has been demonstrated, and the potential use of PET/ $\mathrm{CT}$ to predict BRAF or KARS mutations has been discussed. Compared with colorectal cancer, BRAF mutations are much more common in malignant melanomas (MMs). The aim of this study was to investigate whether BRAF mutations have the potential to induce GLUT1 overexpression in MM metastases, which may be clinically reflected by increased glucose uptake in tumor tissue as measured by $18 \mathrm{~F}-\mathrm{FDG}$ PET/CT.

Methods. DNA from the resected MM metastases of 47 patients was analyzed for BRAF V6oo mutations by pyrosequencing. Expression of GLUT1 and the proliferation antigen Ki- 67 was defined by immuno- 
histochemistry. The staining intensity of GLUT1 was assessed with the Remmele immune reactive score (IRS). Glucose uptake in the same lesions was assessed by $18 \mathrm{~F}-\mathrm{FDG}$ PET/CT and was calculated as maximum standardized uptake values (SUVmax).

Results. In our sample population of 47 patients, the incidence of BRAF V6oo mutations was $26(23 \times \mathrm{V} 600 \mathrm{E}, 3 \times \mathrm{V} 600 \mathrm{~K})$. The average age of the patients with BRAF V6oo mutations $(53 \mathrm{y})$ was significantly lower than that of patients exhibiting the wild-type (wt) genotype (67 y) at the time of metastasis $(\mathrm{p}=0.001)$. The GLUT1-IRS exhibited a positive correlation with the extent of $18 \mathrm{~F}-\mathrm{FDG}$ uptake $(\mathrm{p}=0.039)$ as well as a trend toward correlation with the Ki-67 proliferation index $(\mathrm{p}=0.071)$. There was no correlation between SUVmax and BRAF mutation status or between GLUT1 expression and BRAF mutation status.

Conclusions. This patient-based study of melanoma metastases confirmed the previously well-documented relationship between GLUT1 overexpression and 18 F-FDG uptake in human malignancies. The hypothesis that GLUT1 overexpression and enhanced 18F-FDG uptake are the result of a constitutively activated RAF/MEK/ERK pathway via BRAF V6oo mutations could not be confirmed. These findings point to a more complex regulation of GLUT1 expression in melanomas.

\section{DO-075}

\section{Notch inhibition improves caspase-8 knockout-related intestinal inflammation}

\section{M.K. Jeon*, C. Klaus, U. Schneider, E. Kaemmerer, R. Knuechel-Clarke, N. Gassler Institute of Pathology, Aachen, Germany}

Aims. Loss of caspase- 8 in enterocytes is associated with severe intestinal inflammation. Notch signaling plays an important role in the maintenance of epithelial integrity by regulating the balance of absorptive and secretory cell lines. We hypothesized that inhibition of Notch signaling could improve the outcome of caspase- 8 knockout mice by promotion of the intestinal mucosal barrier.

Methods. Caspase- 8 knockout mice and special cell culture systems were used to analyze effects of intestinal caspase- 8 deficiency on molecular pathways using molecular assays and functional approaches. Functionality of the Notch pathway was pharmacologically modified using a gamma-secretase inhibitor.

Results. Intestinal depletion of caspase-8 was crucial for the development of reduced secretory activity of the mucosal cells with barrier leakage and severe inflammatory response. Inactivation of Notch was able to overcome the caspase- 8 knockout-related disorders. Despite caspase-8 deletion Notch inhibition was associated with development of a secretory phenotype, diminishing of inflammatory activity, and improved outcome.

Conclusions. In the intestine, Notch signaling is probably able to modify caspase-8-related functions. This association could be of relevance in the pathogenesis of inflammation-related intestinal disorders and carcinogenesis.

\section{DO-076 \\ CDK4 is expressed in leiomyosarcomas as a potential target for inhibition by PD-0332991 (Palbociclib)}

M. Böhm ${ }^{* 1}$, R. Marienfeld', S. Brüderlein', M. Wittau' ${ }^{2}$, M. Schultheiß ${ }^{3,}$ A. von Baer ${ }^{3}$, R. Mayer-Steinacker', L. Bullinger', P. Möller', T. Barth ${ }^{1}$ 'Institute of Pathology, University Hospital, Ulm, Germany, ${ }^{2}$ Department of General and Visceral Surgery, Ulm, Germany, ${ }^{3}$ Department of Trauma Surgery, Ulm, Germany, ${ }^{4}$ Department of Internal Medicine III, Ulm, Germany

Aims. Leiomyosarcoma (LMS) is characterized by a high genomic complexity. Current treatment is based on complete resection and non-spe- cific chemotherapy. Our aim was to identify potential structures for targeted therapy.

Methods. In a whole genome approach we scanned 26 samples from 14 patients by SNP-array-analysis using DNA of primary tumor tissue and at least one metastasis. In a candidate gene approach we performed fluorescence-in-situ-hybridization (FISH), and immunohistochemistry (IHC) for CDK4, MDM2 and Rb. The two LMS cell lines SK-UT-1 and SK-LMS-1 were used for cell cycle inhibition with PD-0332991(Palbociclib) as a selective Inhibitor of $\mathrm{CDK}_{4} / 6$. For in vitro analysis we performed Western blotting for $\mathrm{Rb}$ and Phospho-Rb (Ser78o), as well as cell cycle analysis (FACS) and proliferation assays (Alamar Blue).

Results. SNP-Array-Analysis indicated CDK4 amplification as a common feature in LMS: observed in 4 of 24 cases $(16 \%)$, whereas MDM2 amplification was co-amplified with $\mathrm{CDK}_{4}$ only once (4\%). FISH analysis showed $\mathrm{CDK}_{4}$ amplification in 6 of 35 cases (17\%), whereas MDM2 was co-amplified in 2 of 18 cases (11\%). Immunohistochemistry for $\mathrm{CDK}_{4}$ was positive in 38 of 83 LMS (46\%), for MDM2 in 1 of 20 LMS (5\%). Rb was positive in 9 of 19 LMS (49\%). Western Blot analysis showed that SK-UT-1 and SK-LMS-1 both express high levels of CDK4, while $\mathrm{Rb}$ was expressed in SK-LMS-1 but not in SK-UT-1. Incubation of cells with Palbociclib led to a strong decrease in protein levels of Phospho- $\mathrm{Rb}$ in SK-LMS-1 and a decreased cell proliferation and Go/G1-Phase arrest with decreased S/G2 fractions, while the same treatment of SK-UT-1 showed no effect.

Conclusions. Abnormalities in the cell cycle signaling, especially CDK4amplification and/or loss of RB1 are common findings in LMS. LMS cell line SK-LMS-1 responds well to specific inhibition of this pathway. Therefore, our data suggest that LMS patients characterized by CDK 4 and $\mathrm{Rb}$ co-expression might benefit from the treatment with $\mathrm{CDK}_{4}$ inhibitors such as Palbociclib, and respective therapeutic strategies should be tested in the future.

\section{DO-077}

\section{Chimeric read-through RNAs in renal cell carcinoma}

\section{Pflüger ${ }^{* 1}$, P. Schraml', M.A. Rubin' ${ }^{2}$, H. Moch}

1 Institute of Surgical Pathology, University Hospital, Zurich, Switzerland, 2Department of Pathology and Laboratory Medicine, Weill Cornell Medical College of Cornell University, New York, United States

Aims. A growing body of literature on RNA chimeras in prostate cancer clearly indicates that chimeric read-through transripts have implications in cancer. RNA chimeras are transcripts that are composed of sequences transcribed from two or more individual genes. The best known mechanism underlying the formation of RNA chimeras are gene fusions, which imply structural rearrangement of the genome. Chimeric read-through RNAs are defined as transcripts originating from two directly adjacent genes located close to each other $(<10 \mathrm{~kb})$ on the same DNA strand. Hence, they supposedly arise through mechanisms not a priori requiring genomic rearrangement. Their expression pattern and their putative physiological or disease-associated functions have never been investigated in human kidney tumors.

Methods. We used paired-end whole transcriptome sequencing (RNASeq) and a computational data filtering pipeline to nominate readthrough events in seven frozen human renal cell carcinomas (RCC) and verified their expression in malignant and benign renal tissues using PCR.

Results. 324 read-through events were called in total; 22/27 (81\%) nominees passed validation with conventional PCR and were sequenced at the junction region. Often, we identified various isoforms of a given read-through event. The most obvious splicing pattern ( $53 \%$ of isoforms) is the exclusion of terminal exons from the upstream parent gene and initial exons from the downstream parent gene, using known exonintron boundaries. Some isoforms (39\%) used new GT-AG splice sites in introns or exons to lengthen or shorten an exon or to introduce a new exon from intergenic sequence; some $(8 \%)$ even contained the ent- 
ire intergenic sequence. In $12 \%$ of the isoforms the exon junction was in-frame which might fuse both parent ORFs forming an intact fusion ORF. We selected 14 read-throughs for differential expression analysis by TaqMan PCR. Most were broadly expressed in both RCC and matched adjacent benign kidney tissue. Three read-throughs were differentially expressed in cancer tissue; BCo39389-GATM has significantly elevated levels in cancer, KLK4-KRSP1 is expressed in only $16 \%(15 / 96)$ of RCCs and TMED6-COG8 expression was significantly higher in $\mathrm{TFE}_{3}$ translocated and $\mathrm{TFE}_{3}$-expressing/non-translocated RCCs.

Conclusions. Chimeric read-through RNAs are potential new stakeholders in renal carcinogenesis identifiable by whole transcriptome sequencing.

\section{DO-078}

Solitary fibrous tumors (SFTs)/haemangiopericytomas (HPCs) with different variants of the NAB2-STAT6 gene fusion are characterized by specific histomorphology and distinct clinicopathological features

\section{S. Barthelmeß*}

Institute of Pathology, University Hospital Erlangen, Erlangen, Germany

Aims. Solitary fibrous tumors (SFTs) were originally described in the pleura as tumors with spindle-shaped tumor cells interlaced with connective tissue fibers. In contrast, haemangiopericytomas (HPCs) are characterized by ramifying vessels with perivascular fibrosis bordered by round tumor cells. Despite these distinct histological features, SFTs and HPCs are merged together in the 4 th edition of the WHO classification of tumors of the soft tissue. Recently, gene fusions between $\mathrm{NAB}_{2}$ and STAT6 have been identified to presumably be the leading events in the tumorigenesis of SFTs/HPCs. This common mechanism reinforced the unifying concept of the two entities. Interestingly, the functional relevance of different breaking points between the two genes has not been considered to date. The aim of this study was to determine the impact of the distinct fusion variants on histological and clinicopathological features.

Methods. We analyzed a cohort of 52 patients aged between 31 and 84 years diagnosed with SFT/HPC of different localizations. We established a multiplex assay and performed RT-PCR to identify the different fusion variants. Exact breaking points were detected by means of Sanger sequencing.

Results. We discovered 12 different NAB2-STAT6 fusion variants in 48 of 52 samples (92\%). Separating the variants into categories concerning the potential functional effects of the predicted fusion protein, we established two groups. Variant I, featuring fusions of $\mathrm{NAB}_{2}$ exon 4 with STAT6 exon $2 / 3$, was found in 27 cases (56\%). Variant II, presenting fusions of NAB2 exon 6 with STAT6 exon 16/17 was observed in 11 cases $(23 \%)$. Interestingly, $92 \%$ of the cases with variant I were located at pleuropulmonary sites, whereas $100 \%$ of the tumors with variant II were from extrathoracic localizations. Furthermore, tumors with variant I occurred at significantly higher age, displayed the morphology of classical fibrous SFTs and had generally a favorable clinical behavior. In contrast, patients with tumors of the variant II were significantly younger and their tumors showed the histology of typical HPCs with frequent recurrences.

Conclusions. Taken together, the genetic data presented in our study suggest that SFT and HPC are indeed two related but separate entities with distinct morphological and clinical features. [1] We show that even if the same genes are affected, the different breaking points lead to specific chimeric fusion proteins with distinct functional impact.

1. Barthelmeß S, Geddert H, Boltze C, Moskalev EA, Bieg M, Sirbu H, Brors B, Wiemann S, Hartmann A, Agaimy A, Haller F (2014) Solitary fibrous tumors (SFTs)/haemangiopericytomas (HPCs) with different variants of the NAB2-STAT6 gene fusion are characterized by specific histomorphology and distinct clinicopathological features. Am J Pathol, accepted for publication

\section{DO-079}

\section{DAPK as an adverse prognostic factor in colorectal cancer}

J. Ivanovska*1, I. Zlobec ${ }^{2}$, E. Diamantis-Karamitopoulou', H. Dawson'2, V.H. Koelzer ${ }^{2}$, A. Agaimy ${ }^{3}$, F. Garreis', A. Lugli ${ }^{2}$, A. Hartmann ${ }^{3}$, R. SchneiderStock ${ }^{* 1}$

'Experimental Tumorpathology, Institute of Pathology, Friedrich-Alexander-University Erlangen-Nürnberg, Erlangen, Germany, ${ }^{2}$ Institute of Pathology, University of Bern, Bern, Switzerland, ${ }^{3}$ Institute of Pathology, Friedrich-Alexander-University, Erlangen-Nuremberg, Germany, ${ }^{4}$ Institute of Anatomy Chair II, Friedrich-Alexander-University, Erlangen-Nuremberg, Germany

Aims. Death-associated protein kinase (DAPK) is a cytoskeleton-associated serine/threonine kinase. It functions as a potent inhibitor of cell polarization and migration and was described as a metastasis suppressor. DAPK loss has been shown to be associated with advanced tumor stages and unfavorable prognosis in many tumor types. However, the prognostic relevance of DAPK in patients with colorectal cancer is only poorly understood. Thus, we aimed to investigate the impact of DAPK protein expression on clinical outcome in patients with colorectal cancer.

Methods. Samples from 220 colorectal cancer patients with a median follow-up of 60 months were analyzed. The formalin-fixed paraffin embedded samples were spotted in quadruplicates ( 2 spots from the tumor center, 2 spots from the invasion front) on tissue microarrays and were immunohistochemically stained for DAPK. Furthermore, the effect of endogenous DAPK protein expression on cell migration was analyzed applying the Electric Cell-Substrate Impedance Sensing (ECIS) system with stable DAPK knockdown (shRNA) and DAPK overexpressing cell lines generated from HCT116 wild type colon cancer cells.

Results. In Kaplan-Meier curve and log-rank analysis DAPK loss was associated with longer survival time ( $\mathrm{p}<0.001)$. The 5 -year survival rates were $52.3 \%$ and $25.6 \%$ in patients with DAPK positive and DAPK negative tumors, respectively. Additionally, DAPK negative tumors showed significantly higher numbers of tumor infiltrating lymphocytes than DAPK positive tumors. In $76.1 \%$ of DAPK positive tumors, the presence of high-grade tumor budding was observed. After adjusting for TNM stage in multivariate Cox regression analysis, DAPK retained its strong independent prognostic value. Patients with DAPK overexpressing tumors had a 1.9 time increased risk of death from the tumor than those with DAPK negative tumors demonstrating an adverse correlation with prognosis. Furthermore, the mean expression of DAPK in tumor center versus invasion front showed a loss of expression toward the front $(p=0.0126)$. This fact is in agreement with ECIS migration analysis results indicating higher migration rate for DAPK shRNA cells.

Conclusions. In contrast to the well-known function of DAPK as a tumor suppressor, our data showed DAPK overexpression to be associated with aggressiveness of tumors and an unfavorable outcome. This interesting and new finding has to be further evaluated.

\section{AG Molekularpathologie II}

\section{DO-080}

Implementation of NGS in lung cancer diagnostics at the University Hospital Zurich

M. Rechsteiner*, Q. Zhong, A. Bohnert, A. Soltermann, V. Tischler, H. Moch, P. Wild

\section{Surgical Pathology, University Hospital Zurich, Zurich, Switzerland}

Aims. To predict the response of patients with lung adenocarcinoma to targeted therapies, a sequential approach in molecular diagnostics is currently pursued. This includes in a first step the determination of the mutation status of EGFR by Sanger sequencing. In a second step, 
rearrangements of ALK and ROS 1 are determined by fluorescence in situ hybridization (FISH) in case of wild-type EGFR. Finally, if no alterations can be found, KRAS, BRAF and/or HER2 are sequenced.

Methods. To reduce costs and time, a next-generation sequencing (NGS) approach was set up to determine the mutation status of EGFR, KRAS, BRAF, and HER2 in parallel. Fifty-four patients were included in the study, comprising tissue from resections, biopsies, and cytological smears. All patients were previously characterized either by whole exome sequencing and/or Sanger sequencing for their EGFR status.

Results. Using the multiplexed 454 Junior workflow (Roche), eight patients could be analysed in a single sequencing run including nine target regions for each patient (EGFR exon 18-21, KRAS exon 2-3, BRAF exon 15, and HER2 exon 19-20). This approach resulted in enough coverage of each amplicon to detect all the previously identified mutations by Sanger sequencing in EGFR including large insertions and deletions. Moreover, low frequency mutations $(<10 \%)$ were detected by the NGS approach and validated with an independent PCR and deep-sequencing run. As a quality control, cell line DNA was integrated in each PCR as positive control and run in parallel to the eight patient samples. This inter- and intra-run controls revealed the background error rate (o.38\%) and hotspot target regions where increased errors may be expected, i.e., homopolymers or bases next to the primer site. This quality control step in our workflow and the positive validation results, led us to the launch of this target panel for routine diagnostics at the University Hospital Zurich.

Conclusions. As the number of predictive mutations are continuously increasing, we are currently cross-validating a subset of the above mentioned patients with our PGM system (Ion Torrent, Life Technologies), using different AmpliSeq panels (Life Technologies). In summary, NGS is a reliable and fast approach which decreases hands-on working time and is cost effective in routine diagnostics.

\section{DO-081}

\section{Implementation of parallel sequencing of FFPE derived DNA in} routine diagnostics of lung cancer

K. König ${ }^{* 1,2,3}$, M. Peifer ${ }^{1,4}$, J. Fassunke ${ }^{2,5,6}$, M. Ihle $e^{2,5,6}$, H. Künstlinger ${ }^{2,3,5}$, C. Heydt ${ }^{2,3,5}$, K. Stamm 7 , F. Ueckeroth ${ }^{2,3,5}$, C. Vollbrecht ${ }^{3}$, M. Bos ${ }^{1,2,4}$, M. Gardizi $^{1,2}$, M. Scheffler ${ }^{1,2}$, L. Nogova $a^{1,2}$, F. Leenders' ${ }^{4}$, K. Albus ${ }^{2,3,5}$, L. Meder ${ }^{1,2,6}$, K. Becker ${ }^{8}$, J. Altmüller ${ }^{8}$, M. Kloth ${ }^{6}$, P. Nürnberg ${ }^{8}$, T. Henkel', M. Odenthal ${ }^{6}$, T. Zander ${ }^{1,2}$, R. Thomas ${ }^{1,2,4}$, J. Wolf f, , S. Merkelbach-Bruse ${ }^{1,2,6}$, R. Büttner ${ }^{2,3,5}$, L. Heukamp 23,5

'Lung Cancer Group Cologne, University Cologne, Cologne, Germany, ${ }^{2} \mathrm{Cen}$ ter for Integrated Oncology Cologne/ Bonn, University Cologne, Cologne, Germany, ${ }^{3}$ Institute of Pathology, University Cologne, Cologne, Germany, ${ }^{4}$ Department of Translational Genomics, University Cologne, Cologne, Germany, ${ }^{5}$ Lung Cancer Group Cologne, University Hospital Cologne, Cologne, Germany, ${ }^{6}$ Institute of Pathology, University Hospital, Cologne, Germany, ${ }^{7}$ Targos Molecular Pathology GmbH, Kassel, Germany, ${ }^{8}$ Cologne Center for Genomics, University Hospital, Cologne, Germany

Aims. The Network Genomic Medicine Lung Cancer, a molecular lung cancer screening network was set-up by the Lung Cancer Group Cologne to stratify patients for early clinical trials of novel targeted therapies in lung cancer. Since genomic analysis of tumors for therapy stratification is becoming a challenge in terms of work load, tissue availability as well as cost, we established parallel sequencing of multiplexed target amplicons generated by a multiplex PCR in routine diagnostics.

Methods. We established, validated and implemented a diagnostic approach based on a 102 amplicon multiplex PCR followed by sequencing on a bench top Illumina sequencer (MiSeq) in routine diagnostics of formalin-fixed paraffin-embedded (FFPE) tissue.

Results. Analyzing a validation cohort of 181 samples, previously sequenced by Sanger technology we found the novel sequencing approach to require significantly less input material and to be more relia- ble, robust and cost effective than Sanger sequencing. We found that comprehensive biomarker testing by our multiplex approach provided significant novel information adding to histological diagnosis and clinical staging. Results of the first 965 consecutively analyzed lung cancer samples identified driver mutations in the expected published frequencies. Furthermore we show that potentially targetable DDR2 mutations occurred at a frequency of $6 \%$ in both adenocarcinomas and squamous cell carcinoma patients.

Conclusions. Multiplex biomarker testing using parallel sequencing provides the possibility to analyze thousands of NSCLC samples per annum for 102 amplicons in a high through put fashion, while reducing turnaround time and amount of input DNA significantly compared to previously implemented Sanger sequencing. In addition, combined histological and genomics-based diagnostics adds valuable clinical information and more precise staging in the case of multiple tumors.

\section{DO-082}

\section{Establishment of multiplexed amplicon sequencing to assess hot} spot and pathway related variants in chronic lymphocytic leukemia

\section{Vollbrecht" ${ }^{* 1}$, C. Schweighofer ${ }^{2}$, U. Koitzsch', L. Wilden², K. König L. Heukamp ${ }^{1}$, R. Büttner', M. Odenthal' \\ 'Institute of Pathology, University Hospital, Cologne, Germany, ${ }^{2}$ Medical Clinic I, University Hospital, Cologne, Germany}

Aims. Recurrent mutations in tumor relevant genes have been identified and proposed as new indicators of prognostic subsets in patients with chronic lymphocytic leukemia (CLL). Next generation sequencing (NGS) technologies allow the simultaneous analysis of genomic mutations in numerous target regions in one single experiment and are able to reach a higher sensitivity than conventional sequencing technologies. We aimed to establish and validate a NGS panel of distinct genes, known to be potentially mutated and/or targeted in CLL, which can be run as a diagnostic screening assay in a high-throughput fashion for clinical research purposes and potential routine applications in the future. Methods. 2 CLL gene panels targeting the most disease related genes in CLL, i.e. ATM, MYD88, $\mathrm{NOTCH}_{1}, \mathrm{SF}_{3} \mathrm{~B}_{1}$, and $\mathrm{TP}_{53}$, were designed and specific primers obtained for complete coding exon or hot spot regions of each gene. Using 4 primer pools resulting in 338 amplicons, a PCR-based multiplex technology was applied to prepare target-enriched DNA libraries from purified B-cell DNA from 136 CLL patients. Samples were sequenced using a MiSeq instrument (Illumina). Conventional Sanger sequencing served as a reference technology. Additionally, 2 dilution series of cell line DNA with known $\mathrm{TP}_{53}$ or ATM mutation were used as a positive control and for sensitivity determination. Alignment and variant calling was performed using an internal algorithm. Data was visually verified with integrative genomic viewer.

Results. We obtained $>10$ million reads per run covering 338 amplicons from 15 genes of interest. The median coverage per amplicon was approximately 2,00o reads (range: 15 to $>25,000$ ). 3 genes (ATM, NOTCH1, $\mathrm{SF}_{3} \mathrm{~B}_{1}$ ) demonstrated regions of low amplicon representation, which had to be discarded from evaluation during quality assessment. $\mathrm{Mu}$ tation hot spots were detected in ATM, NOTCH1, TP $53, \mathrm{SF}_{3} \mathrm{~B} 1, \mathrm{XPO} 1$ and $\mathrm{FBXW}_{7}$, and are currently undergoing verification by Sanger sequencing. Series of mutated cell line DNA followed a linear relationship with increasing amounts of tumor DNA and sensitivity between 5-10\%. Conclusions. We successfully established a CLL-dedicated sequencing panel based on one-step multiplexed amplification chemistry and applicable to primary CLL sample material and an Illumina MiSeq sequencing platform. Current sequencing results demonstrate overall acceptable gene coverage for diagnostic purposes in the majority of selected target regions, even though underrepresented amplicons/gene regions occur. 


\section{DO-083}

\section{A novel NOTCH and ASCL1 dependent pathway underlying the pathology of small cell lung cancer}

\section{Meder ${ }^{* 1,2}$, K. König ${ }^{2,3,4}$, M. Peifer, ${ }^{2,5}$, L. Ozretic', D. Böhm ${ }^{6}$, C. Ade', K. Albus ${ }^{2,8}$, W. Hartmann ${ }^{9}$, A. Florin ${ }^{8}$, U. Rommerscheidt-Fuss ${ }^{8}$, S. Perner 6 , M. Eilers, , R.K. Thomas ${ }^{2,3,5,10}$, R. Büttner ${ }^{2,3,8}$, L. Heukamp ${ }^{* 1,2,3}$ \\ 'Institute of Pathology, University Hospital Cologne, Cologne, Germany, ${ }^{2}$ Lung Cancer Group Cologne, University Cologne, Cologne, Germany, ${ }^{3} \mathrm{Cen}$ - ter for Integrated Oncology Cologne/ Bonn, University Cologne, Cologne, Germany, ${ }^{4}$ Institute of Pathology, University Cologne, Cologne, Germany, ${ }^{5}$ Department of Translational Genomics, University Cologne, Cologne, Germany, ${ }^{6}$ Institute of Pathology, University Hospital Bonn, Bonn, Germa- ny, ${ }^{7}$ Biocenter, University of Würzburg, Würzburg, Germany, ${ }^{8}$ Lung Cancer Group Cologne, University Hospital Cologne, Cologne, Germany, 'Institute of Pathology, University Hospital Münster, Münster, Germany, ${ }^{10}$ Cologne Center for Genomics, University Hospital, Cologne, Germany}

Aims. Small cell lung cancer (SCLC) is the most aggressive form of lung cancer, characterized by early metastasis, neuroendocrine differentiation, a very high mitotic rate, frequent areas of necrosis, and early relapse following therapy. The development of a small cell phenotype is a paradigm for tumor plasticity as mixed tumors with non-small cell and SCLC components are frequently seen and adenocarcinomas have been shown to switch to a small cell phenotype to evade targeted therapy by EGFR inhibitors. Loss of heterozygosity $(\mathrm{LOH})$ in $\mathrm{RB}_{1}$ and $\mathrm{TP}_{53}$ are the oncogenic driver lesions in SCLC. Furthermore the NOTCH target acheate-scute homologue 1 (ASCL1), is supposed to be pivotal in the NE differentiation in SCLC. The aim of this study was to elucidate the mechanism underlying the development of a small cell phenotype.

Methods. Based on Affimetrix expression array data of 50 NSCLC and 50 SCLC cell lines, integrative genome analysis of SCLC patients and a human whole genome wide shRNA lentiviral screen, we propose a pathway dependent on NOTCH and ASCL1 that explains the features of 'small cell-ness' occurring in SCLC and in addition serves as a hypothesis how mixed tumor phenotypes may develop.

Results. We could show that upon expression of ASCL1, a NOTCH target, the morphology of NSCLC cells with a p53 mutation can be switched into a small cell phenotype. Furthermore ASCL1 expression induced canonical WNT-signaling and inactivation of RB. These data generated so far led us to the conclusion that SCLC can not only arise from stem-cells out of a NE niche but also from NSCLC with p53 mutation that acquire $\mathrm{LOH}$ in $\mathrm{RB}$ and an inactivating NOTCH mutations. Conclusions. We propose a comprehensive pathway that explains the development of a small cell phenotype in mixed SCLC and NSCLC tumors.

\section{DO-084 \\ Functional analysis of FGFR inhibition and differentially expressed microRNAs in myxoid liposarcoma cells}

H. Künstlinger ${ }^{* 1}$, S. Dümcke ${ }^{2}$, J. Fassunke ${ }^{1}$, H.-U. Schildhaus ${ }^{3}$, C. Jonas' M.A. Ihle', C. Heydt', E. Wardelmann ${ }^{4}$, R. Büttner', S. Merkelbach-Bruse ${ }^{1}$ 'Institute of Pathology, University Hospital Cologne, Cologne, Germany, ${ }^{2}$ Computational Biology and Regulatory Networks, Max Planck Institute for Plant Breeding Research, Cologne, Germany, ${ }^{3}$ Institute of Pathology, University Hospital Göttingen, Göttingen, Germany, ${ }^{4}$ Gerhard Domagk Insitute of Pathology, University Hospital Münster, Münster, Germany

Aims. Myxoid liposarcomas account for more than one third of liposarcomas and about $10 \%$ of all adult soft tissue sarcomas. The tumours are characterised by a specific chromosomal translocation leading to the chimeric oncogene FUS-DDIT3 or EWS-DDIT3. These fusion proteins act as aberrant transcription factors therefore we implemented comparative expression analyses using cDNA and microRNA microarrays in tumour and fat samples. We aimed to identify differentially expressed genes and microRNAs which are involved in tumourigenesis of myxoid liposarcoma and thus may serve as diagnostic or prognostic biomarkers or as therapeutic targets.

Methods. The microarray analyses revealed differentially expressed genes and microRNAs. Overexpression of the identified candidate gene FGFR 2 was validated by qPCR and immunohistochemistry in primary tumour samples. Myxoid liposarcoma cells were treated with the FGFR inhibitors PD173074, TKI258 and BGJ398 as well as specific siRNAs. Additionally, a combined treatment of FGFR inhibitors and Trabectedin was implemented. Functional effects were examined using MTT assay, apoptosis/cytotoxicity assay and scratch assay. Additionally, a bioinformatical analysis of cDNA and microRNA microarrays using BIRTA (Bayesian Inference of Regulation of Transcriptional Activity) revealed several microRNAs with differential activity in myxoid liposarcoma. A selection of these microRNAs was analysed using qPCR in primary tumours as well as functional experiments in cell lines.

Results. FGFR2, together with other members of the FGF/FGFR family, was detected to be overexpressed in myxoid liposarcomas compared to fat tissue. Treatment of liposarcoma cells with FGFR inhibitors reduced cell proliferation and induced apoptosis. Significant effects on the migration of the cells could be seen. Combination of the FGFR inhibitors with Trabectedin further increased the impact. Among other selected microRNAs, tumour-suppressive miR-145 which is involved in adipocyte differentiation showed an inhibitory effect on proliferation and induced apoptosis after transient transfection in myxoid liposarcoma cells.

Conclusions. Our study revealed a potential role of FGFR signalling in the pathogenesis of myxoid liposarcoma. FGFR inhibition showed effects on proliferation and cell migration and induced apoptosis in vitro. Differentially expressed microRNAs, like miR-145, were identified and their functional role could be verified in vitro.

\section{DO-085}

\section{HSP90 inhibition-a promising therapeutic strategy in lipo-} sarcoma?

S. Steinmann ${ }^{* 1}$, A. Agaimy², P. Aman ${ }^{3}$, H. Gali-Muhtasib ${ }^{4}$, F. Haller ${ }^{5}$, R. Schneider-Stock ${ }^{* 6}$

'Experimental Tumorpathology, Institute of Pathology, Erlangen, Germany, ${ }^{2}$ Institute of Pathology, Friedrich-Alexander-University, Erlangen-Nuremberg, Germany, ${ }^{3}$ Sahlgrenska Academy at Göteborg University, Göteborg, Sweden, ${ }^{4}$ Institute of Biology, American University, Beirut, Lebanon, ${ }^{5}$ Institute of Pathology, University Hospital Erlangen, Erlangen, Germany, ${ }^{6}$ Experimental Tumorpathology, Institute of Pathology, Friedrich-Alexander-University Erlangen-Nürnberg, Erlangen, Germany

Aims. Liposarcomas represents the commonest subgroup of mesenchymal malignancies (sarcomas) of soft tissues in adults. Genetic and histopathological heterogeneity diminishes the efficacy of currently available therapeutic strategies. Within therapeutic advances, NVPAUY922 (AUY), a small molecule heat shock protein 9o (Hsp9o) inhibitor, emerged to be a novel promising drug with potent antitumor activity. The Hsp9o-chaperone is a reasonable therapeutic target because it maintains the signaling of multiple oncogenic pathways. The aim of this study was to determine in vitro functional effects of AUY in liposarcoma cell lines.

Methods. The biological impact of the new synthetic Hsp9o inhibitor AUY was evaluated in vitro in the human myxoid liposarcoma cell lines MLS402 and MLS1765 and the dedifferentiated liposarcoma cell line SW872. Cell viability studies were undertaken using crystal violet staining. Cells were analyzed for apoptosis and cell cycle arrest by use of flow cytometry and Western Blot analysis. Dysregulation of components of the $\mathrm{PI}_{3} \mathrm{~K} / \mathrm{AKT}$ and RAS/MEK/ERK signaling pathways were assayed by Western blotting. mRNA expression levels were examined using real-time polymerase chain reaction (RT-PCR). Immunohistochemical expression of HSP9o protein was determined in 58 liposarco- 
mas for immunohistochemical expression of HSP9o protein 21 atypical lipomatous tumor/well differentiated liposarcoma (ALT/WDL), $18 \mathrm{de}-$ differentiated liposarcoma (DDL), and 19 myxoid/round cell liposarcoma (MRL).

Results. Treatment of the cell lines MLS402, MLS1765 and SW872 with AUY led to dose-dependent decrease in cell viability with IC50 values ranging from 30 to $70 \mathrm{nM}$ which were surprisingly lower compared to the Hsp9o-inhibitor 17-allyamino-17-demethoxy-geldanamycin (17AAG). AUY induced changes in cell cycle accompanied by induction of apoptotic cell death. Increased levels of Cyclin B1 and Histone 3 were evidence for an enhanced aberrant mitotic entry. Total loss of Chk1 protein kinase and increased levels of p21 mRNA confirmed a G2/M-phase arrest. Elevated levels of apoptosis markers p 53 and cleaved Caspase 3 correlated with the pro-apoptotic effect of AUY. Furthermore, AUY induced depletion of phosphorylated AKT, MEK and ERK.

Conclusions. Taken together, our data indicate that the Hsp9o inhibitor AUY induces the desired cytotoxicity in liposarcoma cells and may therefore serve as a new effective strategy for antitumor treatment of liposarcomas.

\section{AG Urologische Pathologie I}

\section{DO-086}

\section{FoxF1 is a potential oncogene in prostate cancer}

M. Nowak ${ }^{* 1}$, R. Menon', A. von Maessenhausen', C. Merz', M.A. Svensson', N. Wernert ${ }^{3}$, G. Kristiansen ${ }^{3}$, O. Andrén ${ }^{2}$, S. Perner ${ }^{1}$

${ }^{1}$ Institute of Pathology, Department of Prostate Cancer Research, University hospital Bonn, Bonn, Germany, ${ }^{2}$ Department of Urology, University Hospital of Örebrö, Örebrö, Sweden, ${ }^{3}$ Department of Pathology, University Medical Center, Bonn, Germany

Aims. Castration resistant prostate cancer (CRPC) is the most aggressive form of prostate cancer (PCa) with a poor prognosis, and remains a significant therapeutic challenge. Key to the development of novel therapeutic strategies is to identify molecular targets of this lethal disease. FoxF1 belongs to the family of forkhead transcription factors. However, the exact function of FoxF1 remains unclear. The aim of the study was the analysis of FOXF1 within prostate cancer.

Methods. Here, using tissuemicroarrays of a prostate cancer progression cohort we analyzed the protein expression of FoxF1. FoxF1 in prostate tumor cell lines was analyzed by Western Blot and real-time PCR.

Results. Primary tumors and distant metastases exhibited a significantly higher FoxF1 expression compared to benign tissue. mRNA and Western Blot analysis revealed a considerable expression range amongst CRPC-derived cell lines, with $\mathrm{PC}_{3}$ cells showing the lowest FoxF1 expression. Overexpression of FoxF1 in prostate tumor cells stably transfected with FoxF1 resulted in loss of E-Cadherin and gain of Vimentin expression, indicative of epithelial-to-mesenchymal transition (EMT). Furthermore, overexpression of FoxF1 led to an increased migration rate in vitro without affecting the proliferation rate.

Conclusions. In summary, our results point to role of FoxF1 as a potential oncogene in prostate cancer.

\section{DO-087}

Role of trefoil factor-3 peptide (TFF3) in prostate cancer progression

M. Nowak ${ }^{* 1}$, C. Merz ${ }^{1}$, M.A. Svensson ${ }^{2}$, N. Wernert ${ }^{3}$, G. Kristiansen ${ }^{3,}$

O. Andrén ${ }^{2}$, S. Perner

${ }^{1}$ Institute of Pathology, Department of Prostate Cancer Research, University hospital Bonn, Bonn, Germany, ${ }^{2}$ Department of Urology, University Hospital of Örebrö, Örebrö, Sweden, ${ }^{3}$ Department of Pathology, University Medical Center, Bonn, Germany

Aims. Prostate cancer ( $\mathrm{PCa}$ ) is the second most abundant cancer diagnosis in men and accounts for about $10 \%$ of cancer-related deaths. Whilst standard therapeutic options for primary tumors exist, longterm remissions inevitably lead to the development of castration-resistant prostate cancer (CRPC) and development of lymph node and distant metastasis. One of the most frequent mutations in CRPC is the gene-fusion of the Ets-family transcription factor ERG with the promoter region of the serine protease TMPRSS2. This aberrant expression of ERG in prostate cells perturbs normal gene expression. One of the genes regulated by ERG is $\mathrm{TFF}_{3}$, which is over-expressed in prostate cancer. The ability of tumors to metastasize depends on the coordinated expression of extracellular matrix degrading enzymes (MMPs) and adhesion molecules. TFF 3 (ITF, intestinal trefoil factor) is a $18 \mathrm{kDa}$ protein with a characteristic trefoil structure containing three conserved cysteine residues. To date, neither the exact function nor its receptors are well defined. Aim of this study was to identify the receptor for $\mathrm{TFF}_{3}$ and its role in prostate cancer progression.

Methods. Protein expression of $\mathrm{TFF}_{3}$ in prostate (tumor) cell lines $\mathrm{BPH}-$ 1, DU145, PC-3, LnCaP was determined by ELISA. Migration towards $\mathrm{TFF}_{3}$ was determined by transwell migration assay, involving antibodymediated blockade. Intracellular calcium mobilization was analyzed by flow cytometry.

Results. Among the tested cell lines BPH-1 cells showed the highest expression. LnCap cells were $\mathrm{TFF}_{3}$ negative, $\mathrm{PC}_{3}$ and $\mathrm{DU}_{145}$ showed intermediate concentrations within this spectrum. Which soluble factors regulate the expression of $\mathrm{TFF}_{3}$ in prostate cancer cells is not known. Using antibody-mediated blockage of the interleukin-6 receptor (IL$6 \mathrm{R})$, we show that $\mathrm{TFF}_{3}$ expression is driven by autocrine IL-6. Incubation with $\mathrm{TFF}_{3}$ mobilized calcium from intracellular stores and led to phosphorylation of $\mathrm{STAT}_{3}$, independent of the EGFR, which has been discussed as a potential $\mathrm{TFF}_{3}$ receptor. Furthermore, we show that $\mathrm{TFF}_{3}$ is a chemokine, which is mediated by the chemokine receptor CXCR4. Conclusions. In summary, our results point to a complex autocrine loop between IL- 6 and $\mathrm{TFF}_{3} \mathrm{CXCR}_{4}$ as a receptor driving the expression of $\mathrm{TFF}_{3}$.

\section{DO-088 \\ VEGF-C induces radioresistance in prostate cancer independent of autophagy}

S. Haberlau', P. Hönscheid ${ }^{2}$, R. Jimenez ${ }^{3}$, G. Baretton ${ }^{2}$, D. Tindall', K. Datta ${ }^{5}$, M. Krause ${ }^{1}$, M. Muders ${ }^{* 2}$

'Radiation Oncology/Oncoray, University Hospital Carl Gustav Carus, Dresden, Germany, ${ }^{2}$ Institute of Pathology of University Hospital Carl Gustav Carus, Medical Center TU Dresden, Dresden, Germany, ${ }^{3}$ Mayo Clinic Foundation, Department of Pathology, Rochester, United States, ${ }^{4}$ Mayo Clinic Foundation, Department of Urology, Rochester, United States, ${ }^{5}$ Department of Biochemistry, University of Nebraska Medical Center, Omaha, United States

Aims. VEGF-C is frequently overexpressed in prostate carcinoma and has been associated with therapy resistance and autophagy induction. This work evaluates the role of VEGF-C and VEGF-C induced autophagy in radioresistance of prostate cancer.

Methods. In vitro-experiments were performed using the human prostate carcinoma cell lines PC-3, LNCaP and DU145. Clonogenic sur- 
vival was examined by colony formation assays after knock down of VEGF-C, ATG5, and after addition of rhVEGF-C. gammaH2AX foci analysis was executed to detect radiation induced DNA damage. Autophagic flux was tested after radiation using a Light-Chain-3 II immunoblot. Human tissue was evaluated by standard immunohistochemistry for VEGF-C. All patients have undergone radical prostatectomy and adjuvant radiotherapy. The quantity and intensity of the staining was evaluated by two pathologists independently (R.J. and M.M.) and a staining score was established. This score was correlated with biochemical relapse up to 23 years after radiotherapy. Multivariate Cox analysis was performed.

Results. VEGF-C levels correlate with a significant higher radioresistance in colony formation assays and human tissue samples in multivariate analysis. The risk of biochemical recurrence increases 2.8 -fold (95\% confidence interval: 1.109-7.237; $\mathrm{p}=0.03$ ) when VEGF-C is highly expressed in prostatectomy patients with adjuvant radiotherapy. Although autophagic flux was reduced after VEGF-C depletion during radiation RNA interference for the autophagy related gene 5 (ATG5) showed no effect on radiosensitivity. Phosphorylation of gammaH2AX as one indicator for DNA double strand breaks during radiation is not affected by VEGF-C.

Conclusions. Although involved in chemotherapy resistance of prostate cancer, autophagy is not involved in VEGF-C mediated radiation resistance. Other mechanisms of VEGF-C mediated radioresistance are under investigation.

\section{DO-089 \\ MED12 overexpression is a frequent event in castration-resistant prostate cancer}

A. Offermann ${ }^{* 1}$, Z. Shaikhibraim', M. Braun', R. Menon', I. Syring ${ }^{1,2}$, W. Vogel', C. Ruiz', T. Zellweger', C. Rentsch ${ }^{5}$, M. Svensson ${ }^{6}$, O. Andren ${ }^{6,7}$, L. Bubendorf', S. Biskup ${ }^{8}$, S. Duensing ${ }^{9}$, J. Kirfel' ${ }^{10}$, S. Perner ${ }^{1}$

'Institute of Pathology, Department of Prostate Cancer Research, University hospital Bonn, Bonn, Germany, ${ }^{2}$ Department of Urology, University Medical Center, Bonn, Germany, ${ }^{3}$ Institute of Pathology, University Hospital Basel, Basel, Switzerland, ${ }^{4}$ Department of Urology, St. Clara Hospital, Basel, Switzerland, ${ }^{5}$ Department of Urology, University Hospital Basel, Basel, Switzerland, ${ }^{6}$ Department of Urology, University Hospital of Örebrö, Örebrö, Sweden, ${ }^{7}$ School of Health and Medical Science, University of Örebro, Örebro, Sweden, ${ }^{8}$ Center for Genomics and Transcriptomics, Tuebingen, Germany, ${ }^{9}$ Section of Molecular Urooncology, Department of Urology, University Heidelberg, Heidelberg, Germany, ${ }^{10}$ Department of Pathology, University Medical Center, Molecular Pathology, Bonn, Germany

Aims. Castration resistant prostate cancer (CRPC) is the most aggressive form of prostate cancer ( $\mathrm{PCa}$ ) and remains a significant therapeutic challenge. The Mediator Complex is an evolutionarily conserved multi protein complex which is essential for the transcription of protein encoding genes and the regulation of diverse signaling pathways. The subunit MED12 is involved in the Wnt/ $\beta$-Catenin pathway, which is increased or altered in advanced, hormone refractory PCa. The gene encoding MED12 has recently been reported to be mutated in PCa. Therefore, the aim of our study was to investigate the role of MED12 and its relationship with $\beta$-Catenin in PCa and whether MED12 may serve as a novel target for therapeutic intervention for patients with CRPC.

Methods. We have assessed the protein expression status of MED12 by immunohistochemistry (IHC) on a PCa progression cohort consisting of 110 locally defined $\mathrm{PCa}, 89$ patients with primary $\mathrm{PCa}$ and 92 corresponding lymph node metastases, and 42 CRPC. In PCa cell lines, we performed MED12 knockdown by siRNA followed by MTT proliferation assay. To investigate the effect of $\mathrm{Wnt} / \beta$-Catenin signaling on the expression of MED12, the Wnt pathway was activated by treating cells with Lithium Chloride following western blot and qRT-PCR analysis for MED12.
Results. We found MED12 to show a strong nuclear overexpression in $40 \%$ of the CRPC, compared to a nuclear overexpression in only $10 \%$ of primary non-metastasized $\mathrm{PCa}, \mathrm{O} \%$ of primary lymph node metastasized PCa, and $9 \%$ of lymph node metastases. Further, MED12 nuclear overexpression was significantly correlated with increased proliferative activity. In PCa cell lines, we showed that MED12 knockdown decreases proliferation and that activation of Wnt $/ \beta$-Catenin pathway leads to increased MED12 expression.

Conclusions. Our findings suggest that MED12 may be involved in promoting PCa cancer cell proliferation and survival in absence of androgens, and may serve as a novel target for therapeutic intervention for patients with CRPC.

\section{DO-090 \\ The genomic evolution of prostate cancer under the selective pressure of anti-androgen therapy}

J. Gsponer*1, T. Dietsche', A. Rufle', T. Lorber', D. Juscevicius', V. Perrina', E. Lenkiewicz' ${ }^{2}$, T. Zellweger ${ }^{3}$, A. Bachmann ${ }^{4}$, M.T. Barrett ${ }^{2}$, C.A. Rentsch ${ }^{4}$, C. Ruiz' L. Bubendorf'

${ }^{1}$ Institute of Pathology, University Hospital, Basel, Switzerland, ${ }^{2}$ The Translational Genomics Research Institute (TGen), Phoenix, United States, ${ }^{3}$ Department of Urology, St. Clara Hospital, Basel, Switzerland, ${ }^{4}$ Department of Urology, University Hospital, Basel, Switzerland

Aims. The implementation of novel technologies such as array comparative genomic hybridization $(\mathrm{aCGH})$ and next-generation sequencing has led to a deeper understanding of the genomic nature of cancer. However, these analyses have classically been done without respecting intra-tumor heterogeneity. Here, we applied a methodology that allows us analyzing the genomic profile of distinct tumor populations from individual tumors and their clonal evolution during the progression to castration-resistant disease and therefore we can infer novel genetic alterations in the course of the development of therapy resistance.

Methods. Matched pre- and post- hormone treated fresh frozen and/or formalin fixed prostate cancer samples were selected from our biobank. Clonal tumor populations were flow-sorted according to their nuclear DNA content. Sorted tumor populations were subjected to whole genome CGH and to full exome sequencing analyses by the use of Agilent SurePrint $2 \times 400 k$ microarrays and the SureSelect All Exon Kit, respectively. Target aberrations were evaluated using a prostate cancer tissue micro array (TMA) and fluorescence in situ hybridization as well as immunohistochemistry.

Results. The de-convolution of the genomic evolution in the investigated tumors shows that genetic aberrations and mutations are newly occurring under the selective pressure of anti-androgen therapy, as already shown previously. More importantly, judging on the temporal dynamics of changes in allelic frequencies, we cannot only observe events that become more abundant, but also cancer associated mutations that are negatively selected for. We are currently following up several of these targets, of which at least one shows significant association with overall survival and differences in its expression pattern between hormone naïve and castration resistant cancer.

Conclusions. Genomic profiling of distinct clonal tumor populations during prostate cancer progression allows for analysis of intra-tumoral heterogeneity and the underlying clonal evolution. Importantly, this approach identifies genomic aberrations that were selected for under the pressure of hormone ablation therapy. 


\section{DO-091}

\section{The value of prostate cancer reference pathology in the PREFERE} trial

G. Kristiansen ${ }^{* 1,2}$, T. Thiesler ${ }^{3}$, G. Baretton ${ }^{4}$, M. Toma ${ }^{4}$, M. Dietel $^{5}$, E. Kilic ${ }^{5}$, A. Erbersdobler ${ }^{6}$, S. Bertz', A. Hartmann ${ }^{7}$

'Institute of Pathology, University Bonn, Bonn, Germany, ${ }^{2}$ Institute of Pathology, University Hospital Bonn, Bonn, Germany, ${ }^{3}$ Department of Pathology, University Medical Center, Department of Prostate Cancer Research, Bonn, Germany, ${ }^{4}$ Institute of Pathology, University Hospital Carl Gustav Carus, Dresden, Germany, ${ }^{5}$ Institute of Pathology, Charité University Hospital, Berlin, Germany, ${ }^{6}$ Institute of Pathology, University Hospital, Rostock, Germany, ${ }^{7}$ Institute of Pathology, Friedrich-Alexander-University, Erlangen-Nuremberg, Germany

Aims. The PREFERE-trial, a nation-wide study of to compare the outcomes of different treatment modalities (surgery, external beam radiation, brachytherapy and active surveillance) in low to intermediate-risk prostate cancer, has commenced in 2013. Since the study inclusion criteria depend significantly on histological parameters, a mandatory reference pathology was implemented in the study protocol. We aim to report on the procedure, the progress and pitfalls of reference pathology in the PREFERE trial and to provide first preliminary data of its results. Methods. Study cases sent in for re-evaluation (November 2013) to the five reference centers were considered. The histopathological results of the reference pathology was compared to the primary histology reports. Results. Biopsies of 48 patients were re-analysed, the median reporting time was five calendary days. The diagnosis of cancer could be confirmed in each case. However, marked differences in the correct identification of the number of positive cores, the tumor extent or the Gleason scores were noted, which in consequence led to the study exclusion of 10 patients $(21 \%)$.

Conclusions. Although it is too early to estimate the full value of the central reference pathology in the PREFERE trial, this preliminary data already indicates its importance.

\section{DO-092}

\section{Myoglobin expression in clear renal cell cancer is an independent} marker for survival

\section{C.L. Behnes ${ }^{* 1}$, M. Scharpf ${ }^{2}$, F. Bremmer', H.-J. Radzun', P. Ströbel', F. Fend', J. Bedke}

'Institute of Pathology, University Medical Center Göttingen, Göttingen, Germany, Institute of Pathology and Neuropathology, University Hospital, Tübingen, Germany, ${ }^{3}$ Institute of Urology, University Hospital, Tübingen, Germany

Aims. Myoglobin (MB) is a member of the hemoprotein superfamily and well known as an oxygen reservoir in myocytes of skeletal and myocardial muscles. Recently an ectopic expression of MB in different cancer tissues as well as an increase of expression under hypoxia has been demonstrated. In an earlier investigation we could show this effect in different renal cell carcinoma (RCC) cell lines. Furthermore MB expressing breast cancer does have a better prognosis. In this study we investigated whether MB also plays a role in clear cell RCC and a potential influence of the prognosis.

Methods. In the present study the expression of MB, VEGF and $\mathrm{CD}_{31}$ were examined by immunohistochemistry in 158 low risk (pTia and pTib) and 107 high risk clear cell RCC (pT3a and higher tumor stage; $\mathrm{n}=265$ ). The immunohistochemical data was statistically analysed for differences of expression, correlation between $\mathrm{MB}$ and $\mathrm{CD}_{31}$ expression as well as prognostic significance.

Results. The statistical examination demonstrated a significant higher expression of $\mathrm{MB}$ in low risk clear cell RCC compared to high risk clear cell RCC $(\mathrm{p}<0.001)$. On the contrary the capillary density in the $\mathrm{CD}_{31}$ staining was significant higher in the high risk clear cell RCC $(\mathrm{p}<0.001)$.
As might be expected, there was a negative correlation between MB expression and capillary density $(\mathrm{r}=-0.84)$. In addition the Kaplan-Meier survival analysis revealed a strong positive effect of $\mathrm{MB}$ and a negative effect of $\mathrm{CD}_{31}$ on overall survival.

Conclusions. The enrichment of MB expression in clear cell RCC was correlated with lower tumor stage, lower capillary density and higher overall survival. The current results indicate that MB plays a critical role in clear cell RCC as an independent prognostic marker for survival.

\section{DO-093 \\ PBRM1 is functionally regulated in renal cell carcinomas by p53 induced protein degradation}

S. Macher-Göppinger ${ }^{* 1,2}$, K.E. Tagscherer ${ }^{1,2}$, J. Kopitz ${ }^{3}$, S. Singer ${ }^{1}$, M. Hohenfellner', P. Schirmacher', W. Roth ${ }^{1,2}$

'Institute of Pathology, University Hospital Heidelberg, Heidelberg, Germany, ${ }^{2}$ Clinical Cooperation Unit Molecular Tumor Pathology, German Cancer Research Center (DKFZ), Heidelberg, Germany, ${ }^{3}$ Department of Applied Tumor Biology, Institute of Pathology, University Heidelberg, Heidelberg, Germany, ${ }^{4}$ Department of Urology, University Hospital Heidelberg, Heidelberg, Germany

Aims. About $40 \%$ of clear-cell renal cell carcinomas (ccRCC) have been shown to harbor mutations in polybromo-1 (PBRM1), encoding the BAF18o subunit of a SWI/SNF chromatin remodelling complex, which qualifies PBRM1 as a major cancer gene in ccRCC. PBRM1 protein alters chromatin structure and known functions include transcriptional regulation by controlling the accessibility of DNA and influencing 553 transcriptional activity. In our study we investigated the possible interaction of $\mathrm{p} 53$ and PBRM1 and underlying mechanisms.

Methods. In vitro experiments using different cell lines have been performed. Methods included adenoviral transduction, colony forming assays, quantitative PCR analysis, gene knock down by short interfering RNA (siRNA), immunoblot analysis and radioactive pulse-chase analysis. Furthermore PBRM1 expression was analyzed by immunohistochemistry in tumor tissue samples.

Results. Adenoviral transduction of PBRM1 in a RCC cell line devoid of PBRM1 resulted in diminished colony formation. Gene knockdown of PBMR1 revealed an influence on $\mathrm{p} 53$ function, indicated by changes of mRNA levels of $\mathrm{p}_{53}$ target genes. Moreover, a marked decrease of PBRM1 protein levels upon p53 activation was observed. This effect was abolished by siRNA mediated down regulation of $\mathrm{p} 53$, but was independent of p21 or transcriptional regulation of PBRM1. Pulse-chase experiments determined post-translational protein degradation as the underlying mechanism of p53-dependent PBRM1 regulation which was accordingly inhibited by proteasome inhibitors. However, protein degradation of PBMR1 was independent of GSK $3-\beta$ and MDM-2, as indicated by RNA interference experiments.

Conclusions. In summary our results confirm the influence of PBMR1 on $\mathrm{p}_{53}$ function in RCC derived cell lines, and show that PBRM1 is a target of $\mathrm{p}_{53}$ induced proteasomal protein degradation 


\section{DO-094}

New biomarkers to differentiate malignant germ cell tumours of the testis established by the SILAC-method (stable isotope labelling by/with amino acids in cell culture) in combination with high-resolution mass spectrometry

F. Bremmer*1, H. Bohnenberger', S. Kueffer', T. Oellerich ${ }^{2}$, H. Urlaub ${ }^{3}$ H. Serve', Y. Maatoug', A. Strauss', H.J. Radzun', P. Ströbel', C.L. Behnes' ${ }^{1}$ Institut für Pathologie, University Medical Center, Göttingen, Germany, 2Department of Hematology/Oncology, Goethe University, Frankfurt, Germany, ${ }^{3}$ Bioanalytical Mass Spectrometry Group, Max Planck Institute for Biophysical Chemistry, Göttingen, Germany, ${ }^{4}$ Department of Urology, University Medical Center, Göttingen, Germany

Aims. Malignant germ cell tumours of the testis are the most common malignant tumours in young men between 18 to 35 years. For the therapeutic management it is important to differentiate the histological subtypes. Thus it is crucial to find new biomarkers for the different histological tumour entities. In addition new biomarkers may help to understand pathophysiological processes in these tumour types.

Methods. For quantitative proteomic studies we used high-resolution mass spectrometry in combination with the SILAC method (stable isotope labeling by amino acid in cell culture).The two germ cell tumour cell lines NTERA-2 (representing embryonic carcinomas) and TCAM-2 (representing seminomas) were cultured in the presence of amino acids of different mass and subsequently analyzed by mass spectrometry. Results were further verified by western blot and immunohistochemical analysis.

Results. We detected a total number of 342 differential expressed proteins. After intensive research in literature- and protein-data bases we chose antibodies against the microfilament Destrin, the tetraspanin CD81, and PHF6 for western blot and immunohistochemical analysis ( $n=148$ formalin-fixed and paraffin-embedded testis tumour tissue). The results confirmed the findings of mass spectrometry analysis and allowed to distinguish different germ cell tumours subtypes, especially seminomas and embryonic carcinomas.

Conclusions. (I) High-resolution mass spectrometry in combination with the SILAC method is suitable to differentiate between different tumour cell lines on the protein level. (II) The results of SILAC measures are reproducible in western blot analysis. (III) The detected proteins can be applied for immunohistochemical analysis on formalin-fixed and paraffin-embedded tumour tissue samples especially to distinguish seminomas from embryonic carcinomas. (IV) The method is helpful to establish new biomarkers differentiating histological subtypes of tumours.

\section{AG Urologische Pathologie II}

\section{DO-095}

Results of a retrospective study in bladder cancer patients: Neuropilin-2 and its ligand VEGF-C as independent predictors of treatment response after transurethral resection and radiochemotherapy

B. Keck', S. Wach', H. Taubert', O. Ott' ${ }^{2}$, S. Zeiler', F. Kunath', S. Bertz', C. Weiss', P. Hönscheid ${ }^{6}$, C. Rödel', R. Sauer ${ }^{2}$, R. Fietkau' ${ }^{2}$, B. Wullich", F. Krause , K. Datta ${ }^{8}$, G. Baretton ${ }^{6}$, A. Hartmann ${ }^{4}$, M. Muders*6

${ }^{1}$ Department of Urology, Friedrich-Alexander-University, Erlangen-Nuremberg, Germany, ${ }^{2}$ Department of Radiation Oncology, Erlangen-Nuremberg, Germany, ${ }^{3}$ Institute of Pathology, University Hospital Carl Gustav Carus, Dresden, Germany, ${ }^{4}$ Institute of Pathology, Friedrich-Alexander-University, Erlangen-Nuremberg, Germany, ${ }^{5}$ Department of Radiotherapy and Oncology, Goethe University, Frankfurt, Germany, ${ }^{6}$ nstitute of Pathology of University Hospital Carl Gustav Carus, Medical Center TU Dresden, Dresden, Germany, ${ }^{7}$ Department of Urology, Medical Faculty, Vienna, Austria, ${ }^{8}$ Department of Biochemistry, University of Nebraska Medical Center, Omaha, United States

Aims. Bladder sparing therapy of urothelial carcinoma (UC) can be performed by transurethral resection (TURBT) and subsequent radio(RT) or radiochemotherapy (RCT). In selected patients comparable oncologic outcomes to radical surgery have been reported. However, prognostic markers that can be used to assign bladder cancer patients to the most appropriate treatment option are urgently needed. Our laboratory has already published in vitro data that Neuropilin-2 ( $\left.\mathrm{NRP}_{2}\right)$ and its ligand VEGF-C play an important role in therapy resistance. We therefore analyzed the prognostic impact of these proteins in bladder cancer patients treated with TURBT and adjuvant RT or RCT.

Methods. Tumor specimens of 247 UC patients treated with TURBT and RT or RCT and a follow of 15 years were analyzed on a tissue micro array by immunohistochemistry for their VEGF-C and NRP2 protein expression. An immunoreactive score (IRS) was calculated ranging from 0-12. An IRS less or equal 4 was defined as low- and an IRS more than 4 as strong protein expression. Overall (OS) and cancer specific survival (CSS) was calculated by the Kaplan Meier method. Univariate and multivariate Cox's proportional regression analyses were performed to analyze $\mathrm{NRP}_{2}$ and VEGF-C protein expression as possible prognostic factors.

Results. Mean CSS dropped from 170 months to 68.7 months when $\mathrm{NRP}_{2}$ is highly expressed $(\mathrm{p}=0.007) . \mathrm{NRP}_{2}$ and VEGF-C co-expression showed an even stronger effect on CSS, which decreased from 178.5 months to 18.3 months when both proteins are co-expressed $(\mathrm{p}=0.003)$. In univariate Cox's regression analyses $\mathrm{NRP}_{2}$ expression and $\mathrm{NRP}_{2} /$ VEGF-C co-expression emerged as prognostic factors for CSS (NRP2: HR 3.34; 95\% CI 1.32-8.44; $\mathrm{p}=0.011$; $\mathrm{NRP}_{2} /$ VEGF-C co-expression: HR 8.70; 95\% CI 1.79-42.20; $\mathrm{p}=0.007$ ). In multivariate statistical models $\mathrm{NRP}_{2}$ expression proved to be an independent prognostic factor for overall survival (OS; HR: 3.42; 95\% CI 1.48-7.86; $\mathrm{p}=0.004$ ) and was associated with a 3.85 -fold increased risk of cancer specific death $(95 \% \mathrm{CI}$ 0.91-16.24, $\mathrm{p}=0.066$ ) in patients treated with RCT.

Conclusions. $\mathrm{NRP}_{2}$ protein expression in FFPE samples is an independent prognostic maker for OS and tends to be an independent prognostic marker for CSS in bladder cancer patients treated with TURBT and RCT. It can predict treatment outcome alone or in combination with VEGF-C expression. 


\section{D0-096}

\section{Surgical pathology dissection of cystectomy specimens with} bladder cancer-more is not always more

\section{N.T. Gaisa*1, H. Wilms', P.J. Wild ${ }^{2}$, A. Heidenreich ${ }^{3}$, R. Knüchel-Clarke ${ }^{1}$ Institute of Pathology, RWTH University, Aachen, Germany, ${ }^{2}$ Institute of Surgical Pathology, University Hospital Zuerich, Zürich, Switzerland, ${ }^{3}$ Department of Urology, University Hospital, RWTH Aachen University, Aachen, Germany}

Aims. Histological tumour subtyping, staging and grading are of utmost importance to stratify the treatment of patients with bladder cancer and should be as precise as possible. The aim of this study was to investigate the prognostic impact of standard clinicopathological parameters in cystectomy patients, and to compare a standard and pragmatic approach in specimen handling (partial embedding) with an advanced method (embedding of the entire mucosa).

Methods. We conducted a study of $n=121$ completely embedded cystectomy specimens and clinico-histopathological data with median follow-up data of 21.5 months (range 1-67 months). A comparison of the embedding schemes was possible for 88 patients. Two-dimensional tumour maps (macro-photographs and histology-based maps) visualised the data. Kaplan-Meier plots, Cox regression estimators, Chi-square and $\mathrm{McNemar}$ tests were used.

Results. In a univariate analysis tumour stage (pTsum), positive lymph node status ( $\left.\mathrm{pN}_{1}-3\right)$, increased number of positive lymph nodes $(\geq 3)$, lymph (L1) and blood ( $\left.\mathrm{V}_{1}\right)$ vessel invasion, positive surgical margin status (R1-2), increased tumour size $(\geq 3 \mathrm{~cm})$, smoking, and prolonged time intervals between first diagnosis and cystectomy were associated with a reduced overall survival time (all $\mathrm{p}<0.05$ ). In a multivariate Cox regression model for overall survival only blood vessel invasion $(\mathrm{p}=0.008$, $\mathrm{HR}=3.35,95 \% \mathrm{CI} 1.375-8.161$ ) and organ-confined (pTis-pT2) versus nonorgan-confined disease ( $\mathrm{pT}_{3}-\mathrm{pT} 4$; $\left.\mathrm{p}=0.021, \mathrm{HR} 2.669,95 \% \mathrm{CI} 1.157-6.159\right)$ were found significant. Advanced versus standard embedding revealed significant improvement for the detection of carcinoma in situ (50 vs 61, $\mathrm{p}=0.003$ ) and lymph vessel invasion ( 18 vs $24, \mathrm{p}=0.041$ ), but no significant advances for the detection of maximum tumour stage, tumour multifocality or blood vessel invasion (all p>0.05).

Conclusions. TNM classification including blood vessel invasion, is of highest importance to stratify patients with advanced invasive bladder cancer. Histopathological details are detected more reliably by whole organ embeddings, but the advanced approach showed no significant improvement for outcome related parameters (max. tumour stage, blood vessel invasion) in our cohort. Pragmatic surgical pathology dissection and embedding protocols are sufficient to obtain valid diagnoses. In spite of important insights into bladder tumourigenesis and spread, a tedious whole organ embedding approach does not seem to be crucial for treatment decisions.

\section{DO-097}

Transmembrane transport protein aquaporin 3 is an independent prognostic parameter for invasive bladder carcinoma

\section{W. Otto ${ }^{* 1}$, N. Georgopoulos ${ }^{2}$, S. Denzinger ${ }^{3}$, M. Burger ${ }^{3}$, F. Hofstädter', A. Hartmann 1, P. Rubenwolf ${ }^{5}$}

'Institute of Pathology, University Hospital Erlangen, Erlangen, Germany, ${ }^{2}$ Department of Chemical and Biological Sciences, University of Huddersfield, Huddersfield, United Kingdom, ${ }^{3}$ Department of Urology, University of Regensburg, Regensburg, Germany, ${ }^{4}$ Institute of Pathology, University of Regensburg, Regensburg, Germany, ${ }^{5}$ Department of Urology, University of Mainz, Mainz, Germany

Ziele. Lange Zeit wurde davon ausgegangen, dass das ubiquitär vorkommende transmembrane Transportprotein Aquaporin (AQP) im Urothel nicht existiert. Grund für diese Annahme war die physiologische Funktion der Harnblase als Reservoir, die ein Transportprotein für Wasser und andere Moleküle in Urothelzellen nicht sinnvoll erscheinen ließ. Rubenwolf konnte im Jahr 2009 verschiedene der 13 AQP-Isoformen auch im Urothel auf Gen- und Proteinebene nachweisen. Dabei war $\mathrm{AQP}_{13}$ im normalen Urothel besonders gut exprimiert. In einer Pilotstudie konnten wir an wenigen Fällen von Urothelkarzinomen der Stadien $\mathrm{pTa}$, $\mathrm{pT} 1$ und $\mathrm{pT} 2$ zeigen, dass ein $\mathrm{AQP}_{3}$-Verlust mit zunehmender Invasion und schlechter Tumordifferenzierung assoziiert war. Wir stellen nun aktuelle Ergebnisse zur prognostischen Bedeutung der $\mathrm{AQP}_{3}$ Expression für früh- und muskelinvasive Urothelkarzinome vor.

Methoden. Die beiden untersuchten Kollektive wurden an einem deutschen Einzelzentrum behandelt: A. 87 pT1-Harnblasenkarzinome der Jahre 2002-2009 per TUR-Blase und anschließender Bacillus Calmette-Guérin-Instillationsbehandlung. B. 100 muskelinvasive Tumoren der Stadien $\mathrm{pT}_{2}$ (28\%), $\mathrm{pT}_{3}$ (55\%) und $\mathrm{pT}_{4}(17 \%)$, die 2001-2006 einer radikalen Zystektomie zugeführt wurden. Wir asservierten die entsprechenden Präparate und sammelten retrospektiv klinische Parameter und Follow-up-Daten der Patienten. Immunhistochemische Färbungen erfolgten an $4 \gamma$ m dicken Schnitten nach der Biotin-Avidin-Methode mit dem $\mathrm{AQP}_{3}$-Antikörper (polyclonal anti- $\mathrm{AQP}_{3}$, host: rabbit, antigen: human $\mathrm{AQP}_{3}$, dilution 1:2000, Abcam, USA).

Ergebnisse. $\mathrm{A}$. $\mathrm{AQP}_{3}$-Expressionsverlust war verbunden mit einem statistisch signifikant schlechteren progressionsfreien Überleben (PFS) von $20 \%$ gegenüber $72 \%(\mathrm{p}=0,020)$, was sich auch in der multivariaten Cox-Regressionsanalyse als unabhängiger Prädiktor niederschlug: HR 7,$58 ; 95 \%$ CI 1,29-44,68; $p=0,025$. B. Expressionsverlust war nicht nur für PFS ( $19 \%$ vs. $75 \% ; \mathrm{p}=0,043)$, sondern auch das tumorspezifische Überleben (CSS, $18 \%$ vs. $75 \%$; $p=0,030$ ) ungünstig. In der multivariaten CoxRegressionsanalyse war $\mathrm{AQP}_{3}$-Expressionsverlust ein unabhängiger Prognoseparameter für PFS (HR 2,87; 95\% CI 1,06-7,80; $\mathrm{p}=0,038$ ) und CSS (HR 3,33; 95\% CI 1,22-9,10; $\mathrm{p}=0,019$ ).

Schlussfolgerung. Verlust von Aquaporin 3 ist ein unabhängiger Prognoseparameter des invasiven Urothelkarzinoms der Harnblase. Weitere multizentrische Studien sind erforderlich, die insbesondere abschließend die Frage klären müssen, nach welchen Kriterien die Expression von $\mathrm{AQP}_{3}$ im Urothelkarzinom beurteilt werden sollte.

\section{D0-098}

ITIH5 is a novel candidate tumor suppressor gene and putative prognostic biomarker for relapse risk stratification in bladder cancer

M. Rose*1, N.T. Gaisa', P. Antony' ', D. Fiedler', A. Heidenreich' ${ }^{2}$, W. Otto ${ }^{3}$, S. Denzinger ${ }^{3}$, S. Bertz', A. Hartmann ${ }^{4}$, A. Karl' ${ }^{5}$, R. Knüchel', E. Dahl' 'Institute of Pathology, RWTH University, Aachen, Germany, ${ }^{2}$ Department of Urology, RWTH Aachen University, Aachen, Germany, ${ }^{3}$ Department of Urology, Caritas St. Josef Medical Centre, University of Regensburg, Regensburg, Germany, ${ }^{4}$ Institute of Pathology, University Hospital Erlangen, Erlangen, Germany, ${ }^{5}$ Department of Urology, LMU Munich, Munich, Germany

Aims. The extracellular matrix protein inter-alpha-trypsin inhibitory heavy chain 5 ( ITIH $_{5}$ ) has been associated with tumor suppression in several tumor entities including breast cancer. However, its putative role in bladder cancer is unknown and thus we initiated a study to decipher ITIH5 expression and its prognostic or biological impact on development of human urothelial cancers (UC).

Methods. Epigenetic gene regulation was studied in cell lines (UROtsa, RT112, J82) and normal/cancerous bladder tissues by realtime PCR, methylation-specific PCR, and pyrosequencing. Using immunohistochemistry, loss of ITIH5 protein expression was analyzed on a large tissue microarray $(n=120)$ which included comprehensive clinical patient data. In vitro demethylation analysis was performed in human bladder cell lines. In order to address the functional role of $\mathrm{ITIH}_{5}$, an in vitro tumor model was generated that reflects a papillary high grade UC subtype based on RT112 cancer cells. Afterwards both mock and ITIH5 reexpressing RT112 cells were assayed in cell biological experiments such 
as wound healing assays. Statistical evaluations were performed with SPSS 17.0.

Results. Expression analysis showed a significant loss of ITIH 5 mRNA in $61 \%(n=45)$ of UC. ITIH 5 downregulation in UC was further evident on protein level $(65.5 \%, \mathrm{n}=55)$. Tumor-specific ITIH 5 promoter methylation was demonstrated in $50 \%$ of papillary none-invasive pTa and $68 \%$ of invasive UC. Aberrant ITIH 5 promoter methylation in bladder tumors was tightly linked with loss of $\mathrm{ITIH}_{5}$ mRNA expression, which was functionally confirmed by in vitro demethylation experiments. Furthermore pyrosequencing based analysis revealed that $\mathrm{ITIH}_{5}$ promoter hypermethylation was significantly associated with progressive bladder cancers. In light of that, a distinct cohort of clinically challenging $\mathrm{pT} 1$ high grade UC was analyzed for ITIH5 expression. We found an association between loss of ITIH5 expression and unfavorable prognosis of UC patients without distant metastasis (recurrence-free survival; hazard ratio: 4.35, $\mathrm{p}=0.048$ ). In vitro, $\mathrm{ITIH}_{5}$ re-expression in high grade RT112 bladder cancer cells led to a suppression of cell migration and colony spreading.

Conclusions. We provide evidence that downregulation of ITIH 5 by aberrant DNA hypermethylation may provoke invasive phenotypes in human bladder cancer. Moreover, ITIH 5 protein might become a prognostic biomarker for relapse risk stratification in high grade bladder cancer patients.

\section{DO-099 \\ Identification of genomic changes in clonal tumor populations of bladder cancer recurrences}

\section{Rämö̈*1, V. Perrina', J. Gsponer', C. Ruiz', M. Barrett², C. Rentch ${ }^{3}$ L. Bubendorf'}

${ }^{1}$ Institute of Pathology, University Hospital, Basel, Switzerland, ${ }^{2}$ The Translational Genomics Research Institute (TGen), Phoenix, United States, ${ }^{3}$ Department of Urology, University Hospital, Basel, Switzerland

Aims. Bladder cancer is the fifth most common cancer in the world and has a major socioeconomic impact due to its very high recurrence rate. It is known that genomic aberrations accumulate during cancer progression and provide a growth advantage to the tumor cell. We interrogated the clonal composition of bladder cancer and genomically analyzed the clonal populations within these tumors with the aim to assess the dynamics of these cancer populations and their mutations over time.

Methods. A sample cohort consisting of formalin fixed and paraffin embedded (FFPE) tumor specimens from 14 patients with bladder cancer was selected; each of these patients with at least two time points and a minimal time interval of one year between the two biopsies. Nuclei were extracted from the tissues and stained with DAPI or Draq7. Tumor populations were sorted according to their DNA content (ploidy) by usage of the BD Influx or ARIA III FACS. DNA from sorted populations was subjected to whole genome high resolution CGH microarrays. Results. Most of the bladder cancers analyzed so far are composed of multiple clonal tumor populations. The availability of pure sorted bladder cancer material allowed the detection of high and focal amplicons, as well as of homozygous deletions at an unprecedented resolution. Distinct clonal tumor populations from the same biopsies revealed shared as well as population-specific genomic aberrations. Further, the inclusion of additional time points (recurrences) from the same patients allowed determining which clonal populations were able to recur and which genomic aberrations were required for this process.

Conclusions. Genomic profiling of sorted tumor populations allowed for the dissection of intratumoral genomic heterogeneity in bladder cancer. The further bioinformatic analysis and the application of next-generation sequencing data of these sorted populations will provide us with the possibility of inferring the clonal evolution of each bladder cancer in this study. Our results will help to better understand the molecular and genomic mechanisms of bladder cancer recurrence.

\section{AG Dermatopathologie}

\section{SO-004}

\section{Adverse drug reactions of the skin}

\section{E. Bierhoff*}

Heinz-Werner-Seifert-Institut für Dermatopathologie, Bonn, Germany

Aims. A drug reaction of the skin is defined as an undesirable response of the skin provoked by a medical substance. The skin is the most frequent organ of the manifestation of an adverse drug reaction. In the meanwhile any drug is supposed to be able to cause an adverse drug reaction. Adverse drug reactions of the skin are most frequently due to antibiotics, non-steroidal anti-inflammatory drugs, psychotropic agents and heart medication like beta-blockers, calcium-channel blockers. New agents like EGFR-inhibitors, multikinase-inhibitors or immunotherapeutical agents often show adverse skin reactions. An important fact is the drug interaction in which one drug affects the action of another. The spectrum of the reaction pattern is different between the different drugs and classes of drugs. The reaction pattern may mimic many common inflammatory dermatoses clinically as well as histologically. The most frequent clinical reaction patterns are exanthematous, urticarial, photosenisitive, fixed drug, erythema multiforme up to toxic epidermal necrolysis. Histomorphological clues of an adverse drug reaction are so called strange patterns which do not correlate with defined patterns of an inflammatory skin reaction. Further clues are single or collective necrois of keratinocytes in all levels of the epidermis, atypi$\mathrm{cal} /$ regenerative basal keratinocytes, eosinophils and plasmacells in the predominant superfical infalmmatory infiltrate, neutrophils in ectatic capillaries, strong extravasation of erythrocytes, vasculitis-like changes in the upper plexus combined with endothelial swelling. The clinical and histomorphological diagnosis of an adverse drug reaction of the skin should be a diagnosis of exclusion.

Methods. A drug reaction of the skin is defined as an undesirable response of the skin provoked by a medical substance. The skin is the most frequent organ of the manifestation of an adverse drug reaction.

Results. In the meantime any drug is supposed to be able to cause an adverse drug reaction. Adverse drug reactions of the skin are most frequently due to antibiotics, non-steroidal anti-inflammatory drugs, psychotropic agents and heart medication like beta-blockers, calciumchannel blockers. New agents like EGFR-inhibitors, multikinase-inhibitors or immunotherapeutical agents often show adverse skin reactions.

Conclusions. The clinical and histomorphological diagnosis of an adverse drug reaction of the skin should be a diagnosis of exclusion.

\section{SO-005}

\section{The many faces of dedifferentiated malignant melanoma}

\section{A. Agaimy ${ }^{* 1}$, R. Stoehr', L. Heinzerling ${ }^{2}$, G. Niedobitek ${ }^{3}$, A. Hartmann',} R. Schneider-Stock', T. Mentzel ${ }^{4}$

Institute of Pathology, Friedrich-Alexander-University, Erlangen-Nuremberg, Germany, ${ }^{2}$ Department of Dermatology, University Hospital, Erlangen, Erlangen, Germany, ${ }^{3}$ Institute of Pathology, Unfallkrankenhaus, Berlin, Berlin, Germany, ${ }^{4}$ Dermatopathologische Gemeinschaftspraxis, Friedrichshafen, Friedrichshafen, Germany

Aims. Malignant melanoma represents a common highly aggressive skin cancer. Diagnosis is mainly based on a combination of topographical, histomorphological and immunohistochemical features. On occasion, melanoma may undergo extensive divergent or metaplastic differentiation so that the melanocytic nature of the neoplasm is not recognizable any more. The term "dedifferentiated melanoma" has been applied recently for this uncommon variant. 
Methods. We herein describe the histomorphological, immunohistochemical and molecular features of 6 dedifferentiated melanomas at metastatic sites (3 of unknown primary).

Results. Histology showed unclassified spindle cell sarcoma (3), adenocarcinoma with sarcomatoid dedifferentiation and metaplastic bone formation (1), and undifferentiated pleomorphic sarcoma with rhabdoid cells (1) or osteoclastic giant cells (1). All tumors lacked expression of protein S10o, Pan-Melanoma, HMB45, Melan A and SOX10. Two sarcomatous cases showed focal desmin and pancytokeratin expression. Dedifferentiated metastases occurred 3, 43, and 45 months after diagnosis of conventionally differentiated melanoma. In one case, the primary tumor was detected only one year after occurrence of the dedifferentiated metastasis. In the 3 cases with known primary, the skin of back and shoulder and the nasal orifice were the sites of primary tumor. Molecular analysis of dedifferentiated metastatic melanoma revealed BRAF V6ooE mutation in 2 of 3 cases with known skin primary as opposed to a wild-type in all 3 cases of unknown primary.

Conclusions. In conclusion, dedifferentiated metastatic melanoma should be considered in any case of unusual or undifferentiated neoplasm, especially in patients with a history of melanoma. BRAF mutation testing is helpful in further characterizing some of the cases. Thus, history of melanoma and molecular analysis (that might demonstrate the characteristic BRAFV6oo mutation and thus exclude clear cell sarcoma) are the mainstay for diagnosis. Recognition of this rare but highly aggressive presentation of melanoma is mandatory for appropriate patient treatment in the light of available therapies specifically targeting BRAF.

\section{SO-006}

Performance testing of RREB1, MYB and CCND1 fluorescent in situ hybridisation (FISH) in spindle cell- and desmoplastic melanoma argues for a step-by-step testing algorithm

S.E. Weissinger ${ }^{* 1}$, M. Frick', D.N. Silvers ${ }^{3}$, P. Möller', B.A. Horst ${ }^{3}$, J.K. Lennerz' IInstitute of Pathology, University Ulm, Ulm, Germany, ${ }^{2}$ Institute of Physiology, Ulm, Germany, ${ }^{3}$ Department of Dermatology, Columbia University Medical Center, Section Dermatopathology, New York, United States

Aims. Diagnostic identification of spindle-cell (SM) and desmoplastic melanoma (DM) as a melanoma can be challenging. A recently established triple-probe FISH assay (RREB1/MYB/CCND1) can be helpful in the diagnosis of conventional melanoma (CM). The diagnostic sensitivity of this assay in SM/DM has not been ascertained. Here, we determined the frequency of RREB1, MYB, and CCND1 abnormalities in an SM/DM cohort.

Methods. We identified $33 \mathrm{SM} / \mathrm{mixed} / \mathrm{DM}$ cases from two institutions and hybridized with commercially available RREB1/MYB/CCND1 probe-sets. Applying diagnostic cut-offs from CM, we assessed test performance measures in SM/DM and compared the results between histotypes and with literature values for CM.

Results. Individual probes showed the following sensitivities: RREB1 $(24 / 32=75 \%)$, MYB $(10 / 27=37 \%)$, and CCND1 $(6 / 29=21 \%)$ with an overall sensitivity of $88 \%$ ( $n=29 / 33$ true positives). Because of the relatively high sensitivity of RREB1, we modelled a consecutive FISH testing algorithm (- Fig. 1; RREB1 $>\mathrm{MYB}>\mathrm{CCND}$ 1), whereby the number of hybridizations can be drastically reduced (from $n=123$ to $n=57$ ). While the overall sensitivity in DM was considerably lower $(10 / 14=71 \%)$, in comparison to $\mathrm{SM}(18 / 19=95 \%, \mathrm{p}=0.14)$ or the reported sensitivity in $\mathrm{CM}(304 / 369=82 \%$, $\mathrm{p}=0.29$ ), these differences did not reach statistical significance.

Conclusions. The (RREB1/MYB/CCND1) triple melanoma FISH assay demonstrates an overall sensitivity of $88 \%$ in SM/DM, and we propose a practically efficient step-by-step testing algorithm. The relatively high false negative rate in DM (29\%) underlines the biological differences and the need for additional reliable diagnostic assays for this specific melanoma subtype.

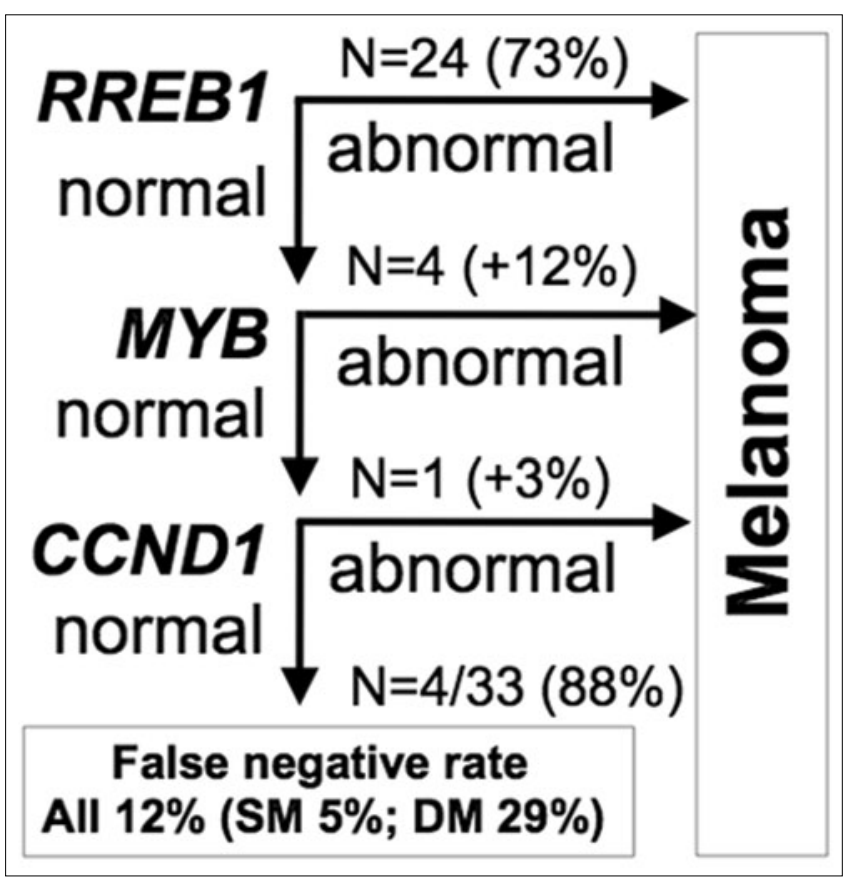

Fig. 1 |SO-006

SO-007

Immunohistochemical screening identifies a set of targetable molecular tissue-based biomarkers in mucosal melanoma of the head and neck

\section{Grünmüller*1, S.E. Weissinger', J. Thierauf', J. Veit', P. Möller', J.K. Lennerz ${ }^{1}$ \\ ${ }^{1}$ Institute of Pathology, University Ulm, Ulm, Germany, ${ }^{2}$ Department of Otorhinolaryngology Ulm University, Ulm, Germany}

Aims. Therapy of mucosal melanoma in the head and neck region is difficult due to location and frequent distant metastasis or advanced stage at presentation. In cutaneous and other mucosal melanoma, a significant fraction of cases express targetable proteins. Screening in mucosal melanoma of the head and neck (MMHN) has, to our knowledge, not been performed and here we evaluated the immunohistochemical labeling pattern in a case-series of MMHN.

Methods. We assembled archived tissue samples into a tissue microarray and stained for KIT, TP 53 , MYC, MDM2, HER2, EGFR, MET, ALK, FLI-1, VEGFR and PDGFRA. For comparison with prior reports, LG/ SEW/JKL performed a meta-review of the literature regarding marker positivity and diagnostic cutoffs for each marker. Statistical analysis was performed using Graphpad Prism and R.

Results. Of the 10 patients with mucosal melanoma, seven were female and the median age at diagnosis was 71 ; range: $42-88$ years. By location, $40 \%$ of the tumors presented in the paranasal sinuses, whereas the nasal cavity, septum and orbita/conjunctiva were affected in $20 \%$, respectively. We found positivity for PDGFRA (100\%), MYC (80\%), MET (40\%), EGFR (30\%), HER2 (10\%) and FLI1 (o\%), which has, to our knowledge, previously not been reported in MMHN. While most markers showed no significant differences to general mucosal melanoma $(\mathrm{p}=0.12-0.28)$, PDGFRA $(\mathrm{p}=0.0003)$ and MYC displayed higher- $(\mathrm{p}=0.04)$, and FLI1 lower fractions of positive cases $(\mathrm{p}=0.002)$. Positivity for KIT $(60 \%)$, TP 53 $(50 \%), \mathrm{MDM}_{2}(\mathrm{o} \%)$ and ALK (o\%) in our series, were in the range of prior published values in MMHN ( $\mathrm{p}=0.32-1.0)$; however, we found a smaller fraction of VEGFR-positivity in our series ( $60 \%$ vs. $100 \%$; $\mathrm{p}=0.01)$. There was no correlation of the expression pattern with location. 
Conclusions. We specify biomarkers with recurrent expression in mucosal melanoma of the head and neck. Given that inhibitors are clinically available, our data can be used for screening and target identification.

\section{SO-008}

\section{GNAQ and GNA11 mutations in uveal melanomas}

\author{
A. Zimpfer*1, B. Schneider', K. Wolff ${ }^{2}$, A. Zhivov' ${ }^{2}$, R. Guthoff ${ }^{2}$, A. Erbers- \\ dobler \\ Institute of Pathology, University of Rostock, Rostock, Germany, \\ ${ }^{2}$ Department of Ophthalmology, University of Rostock, Rostock, Germany
}

Aims. Malignant melanoma of the uvea is the most common primary malignant intraocular neoplasm in adults worldwide. Different from skin melanomas, uveal melanoma does not harbour mutations in BRAF, NRAS or KIT, but instead shows mutations in GNA11 or GNAQ We aimed to analyse GNAQ or GNA11 mutations in a subset of previously immunohistochemically characterized uveal melanomas.

Methods. 67 cases of 36 male and 31 female patients (age range 41-88, median 67) were immunohistochemically characterized on tissue microarrays. The cases comprise 57 choroideal melanomas, 8 melanomas of the iris and 2 melanomas of the ciliary body. Tumour tissue of 21 cases was analyzed by amplification of GNA11 exon 4 and 5 and GNAQ exon 4 and 5 , respectively, and Sanger sequencing.

Results. 61/62 (98.4\%), 52/64 (81.3\%), 54/64 (84.4\%), 34/61 (55.7\%), 37/61 (60.7\%), 35/61 (57.4\%) cases were immunreactive for Melan A, HMB45 S10o, cyclin D1, c-kit (CD117), PTEN, respectively. By sequence analysis 1/21 GNA11 exon 4 mutation, 7/21 GNA11 exon 5 mutations, 3/21 GNAQ exon 4 mutations and 6/21 GNAQ exon 5 mutations were found. 2 cases haboured 2 and 3 simultanous mutations.

Conclusions. By Sanger sequencing 6 mutations of GNAQ exon 4 or 5 and 1 mutation of GNA11 exon 4 was found, which have not been described in the literature yet. One case with liver and brain metastases in the follow-up showed 3 mutations of GNAQ in exons 4 and 5. Up to know, there are no known effective treatment options in patients with metastatic uveal melanomas, but recent studies showed protein kinase $\mathrm{C}$ inhibition as a rational therapeutic target for melanoma patients with GNAQ or GNA11 mutations.

\section{AG Zytopathologie}

\section{SO-001 \\ Immuncytochemical detection of HPV L1 core protein in negative ThinPrep Pap test with negative HPV HC II test}

\section{G. Richter ${ }^{* 1}$, U. Hahlbohm², K. Fels², N. Speich ${ }^{3}$ \\ ${ }^{1}$ Institute of Pathology Dr. Richter, Hameln, Germany, ${ }^{2}$ Institute of Patholo- gy, Dr. Richter, Hameln, Germany, ${ }^{3}$ Genopath GbR, Bonn, Germany}

Aims. Sexually transmitted carcinogenic human papillomavirus (HPV) infections are extraordinarily prevalent worldwide. However, most incident HPV infections clear within a few years, whereas a small minority leads to invasive cancer. In clarification of before suspect Pap tests or colposcopies we detect HPV L1 core protein in cytological and molecular negative cases.

Methods. For clarification of cytological or colposcopic suspect findings of the cervix uteri, we performed computer assisted ThinPrep Pap test. In addition, we perfomed immunocytochemistry against p16 (Roche) and HPV L1 (Cytoimmun) by using the Roche Benchmark GX. Moreover we investigated the liquid based cytology by using the Hybrid Capture Test II to detect high risk HPV. In the case of positive detection of HPV L1 in negative conventional staining and negative HC II test we performed the Papillocheck test and in addition a PCR/direct sequen- cing approach using the consensus primers $\mathrm{GP}_{5}+/ \mathrm{GP} 6+$ and MYo9/ My11.

Results. In three cases of mild cervical dysplasia we could only detect the HPV L1 core protein by using immunocytochemistry, while computer assisted ThinPrep Pap test and the HC II Test (only high risk) were negative. A further molecular investigation using the Papillocheck test and the consensus PCR/direct sequencing approach we could detect in this cases the following HPV types: in 1 case HPV type 34 (unknown risk) and low risk type 42 , in another case the low risk type 42 and in the third case we could detect HPV high risk type 18 and low risk type 43. Conclusions. These data seems to demonstrate that there is no specific HPV type detectable in lesions of negative computer assisted ThinPrep Pap test and negative HC II test (only high risk HPV), whereas HPV L1 core protein could be verified by immunocytochemistry. In 2 of 3 cases we detected a low risk type. However there is still not enough information about the clinical follow up of these lesions and corresponding studies to this direct comparison on a larger group of patients will be necessary to proof the importance of this observation.

\section{SO-002}

DNA image cytometry as adjuvant method in gynecological cytology-a follow-up study of Pap III cases

\section{G. Schumann, N. Pomjanski, M. Schramm, S. Biesterfeld*}

Department of Cytopathology, Heinrich Heine University, Düsseldorf, Germany

Aims. Group III (according to Munich nomenclature II) or Pap III specimens comprise a small group of gynecological smears with heterogenous, but suspicious cytological changes, representing $0.7 \%$ of all Pap smears in our institution. In this study, the follow-up of 155 consecutive patients with a Pap III diagnosis was evaluated. In 72 cases DNA image cytometry had been performed additionally as adjuvant diagnostic method.

Methods. Clinical follow-up data was collected using a questionnaire. Additionally, the files of the respective departments of pathology involved during the follow-up period were considered.

Results. Only in 26 cases, no conclusive follow-up data could be obtained. In 129 cases $(83.2 \%)$, Pap smears $(n=57)$ or histology $(n=72)$ had been performed during the follow-up period. In these specimens, 69 patients were free of relevant changes, 44 revealed precancerous lesions, and 15 were diagnosed with invasive carcinoma (uterine cervix: 5; endometrium: 7; others: 3 ). One patient constantly showed cytologic changes consistent with Pap III. DNA aneuploidy was present in 33 of 72 cases investigated; only five of them (15.1\%) showed a negative Pap control smear. All cases with histological follow-up and the remaining ones with cytological follow-up were at least preneoplastic retrospectively. Out of the 39 cases diagnosed as non-aneuploid, four showed precancerous conditions in control specimens (10.3\%), while the remaining 35 cases had a negative follow-up.

Conclusions. Adjuvant methods are applied to come to a more precise diagnosis in equivocal cases. Our study implies that DNA image cytometry represents such a suitable tool in the difficult group of Pap III smears. 


\section{SO-003}

The use of patient derived malignant effusions as in vitro models for a personalized healthcare-approach in anticancer therapy

T. Vlajnic ${ }^{* 1}$, C. Ruiz', L. Arabi', S. Wyttenbach', B. Baschiera', S. Rothschild', S. Kustermann ${ }^{3}$, A. Zippelius' ${ }^{2}$, A. Roth' ${ }^{3}$, L. Bubendorf ${ }^{\prime}$

'Institute of Pathology, University Hospital Basel, Basel, Switzerland, ${ }^{2}$ Department of Medical Oncology, University Hospital Basel, Basel, Switzerland, ${ }^{3}$ Non-Clinical Safety, Pharma Research, Hoffmann-La Roche Ltd, Basel, Switzerland

Aims. The use of patients' own cancer cells for in vitro selection of the most promising treatment is an attractive concept in personalized medicine. Human carcinoma cells from malignant effusions (MEs) are well suited for this purpose since they have already adapted to the liquid environment in the patient and are not enclosed by stromal cell compartments. Aim of this study was the establishment of a consistent approach for the in-vitro ex-vivo culture of MEs and the comprehensive analysis of the effect of chemotherapeutic as well as targeted drugs.

Methods. MEs from patients with lung or breast cancer were selected for this study. After morphological and molecular characterization, epithelial cells were cultured in cell culture medium supplemented with $30 \%$ of patient derived sterile filtered effusion supernatant. Growth characteristics were monitored in real-time using the xCelligence system (Roche Applied Science). MEs were treated with targeted therapeutics (Crizotinib, Erlotinib) according to their genomic profile or with chemotherapeutics based on the recommendation by the oncologist.

Results. We have developed a reliable system for the ex-vivo culture of MEs and the application of drug tests in-vitro. The use of an enrichment system for epithelial cells (such as the MACS system) allowed for culturing of effusions with only moderate tumor cell content. Experiments using drugs revealed dose-dependent effects and specific growth inhibitory effects of targeted therapeutics, such as Crizotinib and Erlotinib. Conclusions. In this study, we show that cancer cells from patient derived MEs can be cultured and treated in-vitro. Based on our developed setup, these MEs can be genomically characterized by whole genome approaches in order to identify genomic vulnerabilities and then subjected to growth tests by using the respective targeted drugs. This approach could be an important step towards personalized healthcare, at least for patients with malignant effusions.

\section{AG Paidopathologie I}

\section{SO-009}

Vascular maldevelopment of the placenta in a diabetic mouse model

E. Nusshold', K. Koller', V. Stiegelbauer', I. Halbwedl', H. Popper',

S. Povoden², D. Kratky', G. Höfler', B. Gürtl ${ }^{* 1}$

Institute of Pathology, Medical University, Graz, Austria, ${ }^{2}$ Institute of Molecular Biology and Biochemistry, Medical University of Graz, Graz, Austria

Aims. Despite strict glycemic control diabetic pregnancies lead to an increased incidence of intrauterine fetal death and macrosomia. The mechanisms causing these conditions have not been identified in detail. We therefore established a mouse model of diabetes and investigated the placentas of late pregnancies.

Methods. Mice were injected with streptozotocin and after 2 weeks tested for their metabolic state. Diabetic animals were mated and placentas retrieved at the 18 th day of gestation. Detailed examination of formalin-fixed, paraffin-embedded placentas was performed. Vascular development was investigated by digital analysis of immunohistochemical stains with antibodies against $\mathrm{CD}_{31}$.

Results. Vessel density in most of the diabetic placentas analysed so far was lower in comparison to control using Tissue Studio Digital Patho- logy Image Analysis Software (Definiens, Germany). In addition the width of the capillary membrane was increased in diabetic placentas measured by ImageJ software.

Conclusions. In our mouse model a deficient vascular development of the placenta appears to contribute to fetal demise.

\section{SO-010}

Does insulin therapy influence the placental expression of vascular endothelial cadherin and $\beta$-catenin in diabetic patients?

\section{A.M. Müller*1, U. Gembruch ${ }^{2}$, R. Fimmers ${ }^{3}$, S. Vokshory-Makoi ${ }^{4}$}

${ }^{1}$ Center of Pediatric Pathology, MVZ Venusberg, University of Bonn, Bonn, Germany, ${ }^{2}$ Dept. of Obstetrics and prenatal Medicine, Center of Obstetrics and Gynecology, University Clinic Bonn, Bonn, Germany, ${ }^{3} \mathrm{IMBIE}$, University Clinic Bonn, Bonn, Germany, ${ }^{4}$ Center of Pediatric Pathology and Pathology, MVZ Venusberg, University Clinic Bonn, Bonn, Germany

Aims. To investigate the expression of vascular endothelial cadherin and $\beta$-catenin in fetoplacental vessels of pregnancies complicated by diabetes mellitus.

Methods. Placenta specimen from pregnancies complicated by Type I diabetes $(n=10)$, by Type II diabetes $(n=5)$, by gestational diabetes treated with insulin $(\mathrm{n}=2 \mathrm{O})$ and without insulin $(\mathrm{n}=21)$ and from normal term pregnancies $(n=30)$ were subjected to immunohistochemistry and microscopy. Image analysis tools were used to quantify immunoreactivity. Results. The expression of vascular endothelial cadherin and ${ }^{* * *}$-catenin was significantly reduced in those patients, who had been treated with insulin regardless of underlying diabetic subtype. Patients with gestational diabetes, who had not been treated with insulin, showed similar levels of vascular endothelial cadherin and $\beta$-catenin as the control group. No correlation was found regarding smoking, nulliparous, birthweight, HbA1c, duration of pregnancy, and maternal body mass index.

Conclusions. In pregnancies complicated by diabetes hyperinsulinemia appears to a major factor influencing VE-cadherin and $\beta$-catenin expression. Hence, diabetes per se might be compatible with normal levels of vascular endothelial cadherin and $\beta$-catenin in fetoplacental vessels as long as insulin treatment can be avoided e.g by a diet.

SO-011

Expression of nicotinic acetylcholine receptor alpha7 (nAChRalpha7) in placentas of smokers and of patients with preeclampsia

\section{E. Hilbert ${ }^{* 1}$, A. Schneider ${ }^{2}$, W. Coerdt ${ }^{3}$, A.M. Müller ${ }^{4}$}

${ }^{1}$ Center of pediatric pathology and pathology, MVZ Venusberg, Uniklinik Bonn, Bonn, Germany, ${ }^{2}$ Institute of Medical Biometry, Epidemiology and Computer Science (IMBEI), University Clinic Mainz, Mainz, Germany, ${ }^{3}$ Institute of Genetics, University Clinic Mainz, University of Mainz, Mainz, Germany, ${ }^{4}$ Center of Pediatric Pathology, MVZ Venusberg, University of Bonn, Bonn, Germany

Aims. Although placentas of women with preeclampsia show pathomorphological changes comparabel to placentas of women who smoke during pregnancy, smoking seems to prevent the onset of preeclampsia. alpha7 nicotinic acetylcholine receptor (nAChRalpha7) in placenta is mainly expressed by trophoblast cells. Acetylcholine via nAChRalpha7 exerts an antiinflammatory and proangiogenetic effect. Up to now there are no studies comparing placental expression of nAChRv7 in placentas of smokers and of patients with preeclampsia.

Methods. By immunolocalisation trophoblastic nAChRalpha7-expression was studied in placentas of 35 smokers, 31 women with preeclampsia and 39 healthy nonsmokers (control group). Antigen-expression was evaluated by computer-assisted image analysis.

Results. Trophoblastic nAChRalpha7-expression was significantly higher in placentas of smokers than in placentas of women with preeclam- 
psia and placentas of the control group ( $\mathrm{p}<\mathrm{o.001)}$. In preeclamptic placentas nAChRalpha7 was slightly but not significantly elevated $(\mathrm{p}=1)$. In placentas of preeclamptic smokers trophoblastic nAChRalpha7 was reduced.

Conclusions. We present the first study on human placental trophoblastic nAChRalpha7 immunolocalisation demonstrating a significantly elevated nAChRalpha7-expression in placentas of smokers but not in patients with preeclampsia. Considering the antiinflammatory and proangiogenetic effect of $\mathrm{nAChRalpha7}$, it has to be discussed whether the paradoxic ("protective") effect of smoking-at least partially-on the onset of preeclampsia is caused by nAChRalphay induced angiogenesis antagonizing preeclamptic induced placental hypoxia. The proangiogenetic and antiinflammatory effect of the increased expression of nAChRalphay in smokers as a protective influence in developing preeclampsia should be studied further.

\section{SO-012 \\ Placental VE-Cadherin expression in smokers and patients with preeclampsia}

\section{A.M. Müller*1, A. Egger 1,2, R. Fimmers ${ }^{3}$, U. Gembruch ${ }^{4}$ \\ ${ }^{1}$ Center of Pediatric Pathology, MVZ Venusberg, University of Bonn, Bonn, Germany, ${ }^{2}$ Center of pediatric Pathology, MVZ Venusberg, University Clinic Bonn, Bonn, Germany, ${ }^{3} \mathrm{IMBIE}$, University Clinic Bonn, Bonn, Germany, ${ }^{4}$ Dept. of Obstetrics and prenatal Medicine, Center of Obstetrics and Gyne- cology, University Clinic Bonn, Bonn, Germany}

Aims. Vascular endothelial (VE)-Cadherin is involved in the maintenance of the endothelial cell-cell adhesion and thereby the integrity of the endothelial barrier. Up to now it is unknown whether cigarette smoke or preeclampsia has an effect on the endothelial barrier density. Methods. VE-Cadherin expression in placentas of smokers $(n=26)$, patients with preeclampsia $(n=26)$, smoking patients with preeclampsia $(\mathrm{n}=4)$ and healthy pregnant woman without pregnancy complications $(n=32)$ was investigated by immunolocalisation. The staining intensity was evaluated by digital image analysis.

Results. Placentas of patients with preeclampsia showed a statistically significantly increased VE-Cadherin expression while VE-Cadherin expression in placentas from smokers was similar to that of healthy patients.

Conclusions. While the ingredients of tobacco smoke obviously do not influence placental VE-Cadherin expression, the significantly elevated VE-Cadherin expression in preeclamptic placentas indicate an increased placental endothelial density. It has to be discussed whether this tightened blood-vessel-barrier contributes to fetal growth restriction in preeclampsia.

\section{SO-013}

\section{Gestational diabetes-a clinicopathological study from Oslo}

\section{G. Turowski*1, B. Roald ${ }^{2}$}

'Oslo University Hospital (OUS), Department of pathology, Center for pediatric and pregnancy related pathology, Faculty of Medicine, University of Oslo, Oslo, Norway, ${ }^{2}$ Oslo University Hospital, Department of pathology, Center for pediatric and pregnancy related pathology, University of Oslo, Faculty of Medicine, Oslo, Norway

Aims. Gestational diabetes can cause fetal macrosomia, fetal growth restriction and fetal death. It can also result in clinical normal pregnancies. In line with this placental microscopy can reveal combinations of maternal circulatory disorders and maturation defects and also normal morphology. Our hypothesis is that the maturation defects relate to metabolic dysfunctions in diabetes. The aim of this study is to investigate correlations between placental histopathology related to villous and maternal circulatory disorders, immun protein profile related to a panel of relevant protein markers and clinical biochemical information on gestational diabetes and obesity.

Methods. Placentas from 80 women from a population based study (STORK-Groruddalen), of a total of 823 pregnant Oslo multiethnic women from 65 countries were studied. The main focus of the research project was to improve prenatal care and to increase knowledge about gestational diabetes. The 80 placentas (gestational week 37-41) were selected in three groups: 40 women with gestational diabetes and 40 without gestational diabetes, 20 obese (high BMI) and 20 with normal weight. Placenta parenchyma was selected from areas without focal lesions, formalin fixed and embedded in paraffin blocks. Tissue microarrays (TMAs) were made from this material, and the rutin stained (Hematoxylin-Eosin) sections and immunostains for lipid- and glucose metabolism (AGE, Glut 1, 3, 4 and 12, Insulin-like growth factor $1, \mathrm{Li}$ pase, Leptin, PLA2, HGF, HAI-1, VEGF, CD 34 , FGF-2, TNF- ${ }^{* * *}$, CD14, Interleukin-1 $\beta$, eNOS, Caspase 3) were examined for histopathological changes according to international published criteria with special focus on maturation disorders and maternal circulatory disorders.

Results. The pathology study and the clinicopathological correlations will be presented.

Conclusions. The conclusion will be presented.

\section{SO-014}

\section{Expression of AP-2y in placentas of patients with preeclampsia and} of smokers

\section{T. Hager**1, H.A. Schneider', U. Gembruch ${ }^{3}$, J. Schmitz', R. Fimmers ${ }^{5}$,}

\section{A.M. Müller ${ }^{6}$}

'Institute for Pathology, University Hospital of Essen, University of Duisburg-Essen, Essen, Germany, ${ }^{2}$ Deptartment of Pediatric Pathology, University Clinic Bonn, Bonn, Germany, ${ }^{3}$ Department of Obstetrics and Prenatal Medicine, University Clinic Bonn, Bonn, Germany, ${ }^{4}$ Institute of Pathology, Grevenbroich, Germany, ${ }^{5}$ Institute for Medical Biometry, Informatics, and Epidemiology, University of Bonn, Bonn, Germany, ${ }^{6}$ Department of Pediatric Pathology, University Clinic Bonn, Bonn, Germany

Aims. Transcription factor activator protein-2 y (AP-2y) belongs to the family of DNA binding proteins. The AP-2 family plays an important role in embryonic development inducing cell-type specific stimulation (proliferation and suppression) of terminal differentiation. AP-2y is an essential factor for forming tropho-ectodermal cells following implantation. There is evidence for a possible key role of the AP-2y in placental development, in fact this study aimed to investigate whether AP-2y expression may be influenced under condition of preeclampsia (being a serious pregnancy complication) and by smoking (having deleterious effects on trophoblastic development).

Methods. The expression of AP-2y was studied in trophoblastic epithelium and endothelium of placentas from patients with preeclampsia $(n=43)$ and smokers $(n=45)$ and compared with the expression in placentas of a control group of healthy pregnant women $(n=33)$ at gestational ages of 9 to 43 weeks. AP-2y expression was determined by immunohistochemistry allowing differential expression in primary, secondary and tertiary villi.

Results. In comparison with control placentas trophoblastic as well as endothelial AP-2y expression was significantly elevated in preeclamptic placentas. Endothelial AP-2y expression in placentas of smokers was similar to those of healthy women, while trophoblastic AP-2y expression in smokers' placenta was only insignificantly higher. Expression rates of AP-2y did not differ between primary, secondary and tertiary villi in all three groups of patients.

Conclusions. AP-2y expression is enhanced in preeclamptic trophoblastic epithelium and placental endothelium. A possible correlation between increased trophoblastic and endothelial AP-2y expression in patients with preeclampsia and reduced trophoblastic invasion and migration in preeclampsia has to be discussed. Increased AP-2y expression may be a protective placental capacity against hypertonia in preec- 
lampsia. Enhanced trophoblastic AP-2y expression in smokers' placentas may indicate a compensatory response to the disturbed balance between proliferation and differentiation of villi induced by smoking

\section{AG Paidopathologie II}

\section{SO-015}

\section{Regulation of the TGF- $\beta$ signalling pathway in nephroblastomas}

M. Pincus ${ }^{* 1}$, V. Stiegelbauer', I. Leuschner' ${ }^{2}$, G. Höfler', B. Gürt ${ }^{\prime}$ 'Institute of Pathology, Medical University, Graz, Austria, '2Institute of Pathology, Christian-Albrechts-University, Kiel, Germany

Aims. Activation of the TGF- $\beta$ pathway plays a pivotal role in human tumorigenesis, acting either as tumor suppressor or showing oncogenic characteristics. Micro RNAs (miRNAs) are small non-coding RNAs, which regulate gene expression by post-transcriptional mechanisms. Activation of the TGF- $\beta$ signalling pathway by deregulation of miRNAs has already been described in nephroblastomas. In our study we therefore investigated several miRNAs that influenced gene activation of the TGF- $\beta$ signalling pathway in an in-silico analysis.

Methods. Target genes and miRNAs of the TGF- $\beta$ pathway were analysed by the DIANA-mirPath programme, which is a tool to identify molecular pathways targeted by single or multiple miRNAs. Expression levels of miRNAs were investigated in different subtypes of nephroblastomas by quantitative real-time PCR.

Results. The in-silico analysis demonstrated miR-27a, miR-145 and miR-193-5p to target several genes within the TGF- $\beta$ signalling pathway. Samples analysed so far showed a lower expression of miR-27a. Some of the samples had expression levels of miR-145 comparable to normal renal parenchyma.

Conclusions. Our results suggest that a deregulation of the TGF- $\beta$ pathway in nephroblastomas is modulated by several miRNAs.

\section{SO-016}

\section{WBSCR17 and its role in tumor pathogenesis}

V. Stiegelbauer ${ }^{* 1}$, J. Huang', E. Bonyadirad ${ }^{2}$, U. Senanayake', H. Schaider ${ }^{3}$, M. Speicher', I. Leuschner ${ }^{5}$, G. Höfler', B. Gürtl'

IInstitute of Pathology, Medical University, Graz, Austria, ${ }^{2}$ Department of Orthopedic Surgery, Medical University of Graz, Graz, Austria, ${ }^{3}$ Department of Dermatology, Medical University, Graz, Austria, ${ }^{4}$ Institute of Human Genetics, Medical University of Graz, Graz, Austria, Institute of Pathology, Christian-Albrechts-University, Kiel, Germany

Aims. Williams-Beuren syndrome is characterized by different cardiovascular disorders and is associated with gene deletions in the WilliamsBeuren syndrome critical region. A re-analysation of the William-Beuren syndrome (WBS) critical region on 7 q11.23 showed the mapping of 8 new transcripts, among them WBSCR17. The protein encoded by this gene belongs to the polypeptide $\mathrm{N}$-acetylgalactosaminyltransferase family. In our own previous investigations overexpressing HOXC6 in a nephroblastoma cell-line we observed a statistically significant increase in expression of WBSCR17. We therefore investigated the role of WBSCR 17 in tumorigenesis further.

Methods. WBSCR 17 was overexpressed in commercially available HEK293 cells using a lentiviral system. A colony forming assay was performed, and in addition, cells were subcutaneously transplanted into nude mice.

Results. WBSCR 17 was successfully overexpressed in the HEK 293 cells. In the colony forming assay we identified a statistically higher number of colonies in the cells overexpressing WBSCR17 in comparison to control cells. 1 million of cells were subcutaneously transplanted into nude mice. In all but one mouse tumors developed at the site of injection, as the cell line is tumorigenic in nude mice. Histopathologic examination of the tumors so far revealed larger areas of necrosis in some of the control tumors compared to the ones overexpressing WBSCR 17 .

Conclusions. Overexpression of WBSCR17 might contribute to the development of malignant tumors.

\section{SO-017}

Autopsy findings in fetuses with trisomy 18-a multitude of abnormalities

U. Bauer ${ }^{* 1,2}$, H. Stepan ${ }^{3}$, R. Faber', L.-C. Horn ${ }^{5,6}$

${ }^{1}$ Institut of Pathology, Leipzig, Germany, ${ }^{2}$ University of Leipzig, Institute of Pathology, Leipzig, Germany, ${ }^{32}$ Department of Obstetric \& Gynecology, Division of Praenatal Diagnostics, Leipzig University Hospital, Leipzig, Germany, Leipzig, Germany, ${ }^{4}$ Centre of Perinatal Medicine,, Leipzig, Germany, Institute of Pathology, Dept. of Breast, Gynecologic and Perinatal Pathology, University Hospital, Dept. of Breast, Gynecologic and Perinatal Pathology, Leipzig, Germany, ${ }^{6}$ Institute of Pathology, Leipzig, Germany

Aims. Trisomy 18 is a chromosomal disorder characterized by a broad clinical picture and a very reserved prognosis. There are descriptions of more than 130 different anomalies, which can involve virtually all organs and systems. The most frequent malformations are heart and kidney anomalies and characteristic craniofacial features as well as limb deformities.

Methods. A review of our institutional database enclosed 755 fetal autopsy cases ( 382 male, 358 female, 22 not clarified) between January 2006 and November 2013. Overall, we counted 154 number of fetuses with chromosomal aberration, including 40 fetuses with trisomy 18 (26\%). In these trisomy 18 fetuses, we analysed the frequency of different malformations that were found during clinical autopsy. Fetal malformations were grouped as: abnormalities at the heart and great vessels, craniofacial, limb, other internal malformations and central nerve systems. In the cases with denied autopsy, only an external examination of the fetuses was performed for the recognition of malformations.

Results. The most common abnormalities found in our study group were placental dysmaturity $(87.5 \%, \mathrm{n}=35 / 40)$ and craniofacial malformations $(72.5 \%, n=29 / 40)$. The majority of fetuses with craniofacial alterations $(n=25 / 40 ; 62.5 \%)$ showed limb malformations with different severity. $17.5 \%$ of the fetuses presented with cardiac malformations $(n=7 / 40)$, $15 \%$ showed other internal organ abnormalities $(n=6 / 40)$ and $17.5 \%$ had defects of the central nervous system $(n=7 / 40)$.

Conclusions. Our data confirms that the most frequent malformations in trisomy 18 are primary limb and craniofacial ones, followed by abnormalities of the heart and the central nervous system. Furthermore the majority of the examined placentas with trisomy 18 showed villous dysmaturity. 


\section{SO-018}

Minimally invasive, imaging guided virtual autopsy compared to conventional autopsy in foetal, newborn and infant cases: study protocol for the paediatric virtual autopsy trial

C. Rüegger ${ }^{* 1}$, C. Bartsch' ${ }^{2}$, R.M. Martinez ${ }^{2}$, S. Ross' ${ }^{2}$, S.A. Bolliger ${ }^{2}$, B. Koller', L. Held ${ }^{3}$, E. Bruder', P.K. Bode', R. Caduff', B. Frey ${ }^{6}$, L. Schäffer', H.U. Bucher' 'Department of Neonatology, University Hospital, Zurich, Switzerland, ${ }^{2}$ Institute of Forensic Medicine, University of Zurich, Zurich, Switzerland, ${ }^{3}$ Division of Biostatistics, University of Zurich, Zurich, Switzerland, ${ }^{4}$ Institute for Pathology, University Hospital, Basel, Switzerland, ${ }^{5}$ Department of Pathology, University Hospital, Zurich, Switzerland, ${ }^{\circ}$ University Children's Hospital, Zurich, Switzerland, 'Division of Obstetrics, University Hospital, Zurich, Switzerland

Aims. In light of declining autopsy rates around the world, post-mortem MR imaging is a promising alternative to conventional autopsy in the investigation of infant death. A major drawback of this non-invasive autopsy approach is the fact that histopathological and microbiological examination of the tissue is not possible. The objective of this prospective study is to compare the performance of minimally invasive, virtual autopsy, including CT-guided biopsy, with conventional autopsy procedures in a paediatric population.

Methods. Foetuses, newborns and infants that are referred for autopsy at three different institutions associated with the University of Zurich will be eligible for recruitment. All bodies will be examined with a commercial CT and a 3 Tesla MRI scanner, and investigators will be blinded to the results of conventional autopsy. After cross-sectional imaging, CT-guided tissue sampling will be performed by a multifunctional robotic system (Virtobot) allowing for automated post-mortem biopsies. Virtual autopsy results will be classified with regards to the likely final diagnosis and major pathological findings and compared to the results of conventional autopsy, which remains the diagnostic gold standard.

Results. There is an urgent need for the development of alternative post-mortem examination methods, not only as a counselling tool for families and as a quality control measure for clinical diagnosis and treatment but also as an instrument to advance medical knowledge and clinical practice.

Conclusions. This interdisciplinary study will determine whether virtual autopsy will narrow the gap in information between non-invasive and traditional autopsy procedures.

\section{SO-019 \\ Mosaic tetrasomy 12p-Pallister-Killian syndrome, a diagnostic challenge for the fetal pathologist}

\section{K. Schoner ${ }^{* 1}$, B. Fritz ${ }^{2}$, H. Rehder ${ }^{3}$, H. Rehder}

'Institute of Pathology, Fetalpathology, University of Giessen and Marburg, Philipps-University Marburg, Marburg, Germany, ${ }^{2}$ Institute of Human Genetics, Philipps-University Marburg, University of Giessen and Marburg, Marburg, Germany, ${ }^{3}$ Institute of Pathology, Fetalpathology, University of Giessen and Marburg, Philipps-University Marburg, Marburg, Germany, ${ }^{4}$ Institute of Medical Genetics, Medical University Vienna, Vienna, Austria

Aims. Pallister-Killian syndrome (PKS) is caused by chromosomal mosaicism with one cell line carrying an additional isochromosome $\mathrm{i}(12 \mathrm{p})$. The rate of normal to tetrasomic cells may vary, as does the fetal phenotype. Characteristic features may include distinguished craniofacial dysmorphisms and organ malformations, primarily polydactyly and diaphragmatic hernia. We report on 10 PKS fetuses and compare their chromosomal and morphological profiles in order to find out whether the characteristic phenotype differs with the rate and distribution of normal and tetrasomic cells.

Methods. Autopsy comprised thorough documentation, and radiological and histological examination. Cytogenetic analysis was performed prenatally on chorionic or amniotic cells and postnatally on fetal fibro- blasts. FISH, using a 12p specific probe, was applied to metaphases and interphase nuclei.

Results. Fetal age ranged from 18 to 24 gestational weeks. The rate of cells, tetrasomic for $12 \mathrm{p}$ varied with tissue related origin and ranged for example from $0 \%$ to $100 \%$ in chorionic villus cells. The severity of malformations was most pronounced in fetuses with at least $50 \%$ tetrasomic cells in fetal fibroblasts, but was independent from the tetrasomic cell rate within the malformed organ. Also facial features seemed to be rather independent from the all-over rate of tetrasomic cells.

Conclusions. Characteristic features enable fetopathological diagnosis of PKS, even in cases, in which prenatal detection of the tetrasomic cell line had failed. The role of the rate and distribution of tetrasomic cells on the fetal phenotype appears irritatingly ambiguous, suggesting a general rather than a local impairment. It is best demonstrated in affected homozygous twins discordant for PKS.

\section{SO-020}

\section{What's new in cholestasis?}

\section{A. Knisely*}

Institute of Liver Studies, Histopathology, King's College Hospital, London, United Kingdom

Aims. Cholestasis in children and infants, as in adults, takes three forms: obstruction, non-specific injury to hepatobiliary cells, and specific inborn errors in hepatobiliary cells, sometimes unmasked by ingestions or exposures. Only the mix is different. Obstruction in adults (gallstones, tumours) is common, in children rare; inborn errors of function in adults are rare, in children common.

Methods. The role of histopathologists in assessing cholestasis in children is both augmented and diminished by genetics. When an identifiable phenotype accompanies cholestasis (coarsely granular bile, Byler disease; lymphoedema, Aagenaes syndrome), analysis of patients with that phenotype may permit linking phenotype to chromosomal locus. And histopathologists establish aspects of phenotype: Gene errors have proteome counterparts, and histopathologists interrogate the proteome. Results. Cheap, rapid sequencing, whether targeted or full-genomic, may sometimes supplant histopathologic diagnosis. What sequencing takes with one hand, however, it gives with the other. Absent lesions consonant with disruption of gene $\mathrm{X}$, who is to say, when sequence variation in gene $\mathrm{X}$ is found, that hepatobiliary disease is to be ascribed to that variation? To find such lesions histopathologically is easier in infants, whose disease often is due to absence of a gene product, than in older children or adults, in whom not absence but dysfunction is the rule, dysfunction that may be intermittent. But to define these lesions, to find them, and to use them in diagnosis will be our task.

Conclusions. Approaches to histopathologic diagnosis, in liver, of newly identified forms of intrahepatic cholestasis and of cholangiopathy-not limited to those mentioned above-will be discussed, as will be to what extent such diagnosis is feasible for uncommon disorders outside centralised or reference laboratories. 


\section{AG Gynäko- und Mammapathologie I}

\section{SO-021 \\ Evidence of neuroendocrine cells in type 2 ovarian carcinoma and prognostic implication}

\section{E.T. Taube ${ }^{* 1,2}$, C. Denkert ${ }^{3}$, J. Sehouli ${ }^{4}$, K. Pietzner,, S. Darb-Esfahani ${ }^{2,3}$ ${ }^{1}$ Institute of Pathology, Charité, Berlin, Germany, ${ }^{2}{ }_{1}$, Germany, ${ }^{3}$ Institute of Pathology, Charité University Hospital, Berlin, Germany, ${ }^{4}$ Department of Gynecology, European Competence Center for Ovarian Cancer (EKZE), Charité, Berlin, Germany}

Aims. Neuroendocrine differentiation in type 2 carcinomas has only seldom been described. However, in our consultancy experience we have been pointed at a case of neuroendocrine relapse in a patient with type 2 ovarian carcinoma where retrospectively a minor neuroendocrine component could be detected. Therefore we hypothesized that neuroendocrine differentiation might be more frequent in type 2 ovarian carcinoma than suspected by morphology.

Methods. We immunophenotyped a cohort of 180 primary type 2 carcinomas for chromogranin and synaptophysin expression.

Results. Synaptophysin expression with $>20 \%$ positive cells was found in $4(2.2 \%)$ out of 174 patients and chromogranin A expression was seen in $36(20 \%)$ out of 176 patients. Kaplan-Meier analysis revealed that carcinomas with synaptophysin expression of $>20 \%$ of positive cells had a significantly shorter survival time than those with $0-20 \%$ of positive cells $(\mathrm{p}<0.0001)$. Synaptophysin expression remained a significant prognostic factor in multivariate analysis $(\mathrm{HR}=10.8 \mathrm{o}, 95 \%$ confidence interval 3.11-37.57, p<0.0001), independently of age, FIGO stage, and residual tumor after surgery. A trend towards shorter survival was seen in patients with tumors that expressed chromogranin, irrespective of the amount of positive cells $(\mathrm{p}=0.165)$.

Conclusions. A neuroendocrine differentiation is important to keep in mind when a neuroendocrine tumour of unknown primary is detected in regional or temporal connection with an ovarian carcinoma. A minor neuroendocrine component in ovarian type 2 carcinomas might imply a dismal prognosis.

\section{SO-022}

\section{Gonadotropin receptor expression determines the prognostic} significance of Her2 and GPER in ovarian cancer cases-a potential diagnostic algorithm

\section{S. Heublein ${ }^{* 1}$, M. Lenhard ${ }^{2}$, T. Kirchner ${ }^{3}$, U. Jeschke ${ }^{4}$, D. Mayr ${ }^{3}$}

'Department of Obstetrics and Gynaecology, Campus Innenstadt, Munich, Germany, 2Department of Obstetrics and Gynaecology, Campus Großhadern, LMU, München, Germany, ${ }^{3}$ Institute of Pathology, Ludwig-Maximilians-University, Munich, Germany, ${ }^{4}$ Department of Obstetrics and Gynaecology, Campus Innenstadt, LMU, Munich, Germany

Aims. Trastuzumab and tamoxifen have been evaluated for their efficacy in ovarian cancer in clinical trials. Though their efficacy has been shown to be rather moderate, several studies observed enhanced therapeutic potency of those treatment approaches in a small, so far not further characterized subgroup of patients. We recently highlighted gonadotropin receptors (GnRs) such as FSHR, LH/CG-R to be widely expressed in EOC tissue and to be of opposing prognostic significance. As G-protein coupled receptors like FSHR, LH/CG-R might crosstalk with each other as well as with receptor tyrosine kinases; we investigated whether the prognostic impact of Her2 and of the G-protein coupled estrogen receptor (GPER) depends on the patients' GnR profile. Since Her2 and GPER are effectively targeted by trastuzumab and tamoxifen, respectively, the algorithm proposed herein might be of clinical interest. Methods. The GnR status of ovarian cancer patients as well as their Her2 $(n=153)$ and GPER $(n=151)$ immunophenotype was determined by immunohistochemistry. Receptor positivity was correlated with clinicopathological variables and Kaplan-Meier survival analysis was performed prior and post stratification.

Results. Her2 as well as GPER failed to be of prognostic significance in EOC prior to stratification. Interestingly, after the panel had been stratified according to GnR expression, we found that Her2 positivity significantly predicted shortened overall survival only in FSHR negative cases. Further GPER was related to more favorable outcome in FSHR negative, LH/CG-R negative or in double negative patients. Strengthening this observation the GPER activating agents tamoxifen and G1 reduced ovarian cancer cell proliferation only in case the FSHR/LH/ CG-R system was not co-activated.

Conclusions. This study found Her2 and GPER to be of prognostic significance only in FSHR and in FSHR/LH/CG-R negative cases, respectively. Hence we propose that stratification of EOC patients according to their GnR status followed by subgroup analysis for HER2 and GPER might help to identify those patients most likely to respond to treatment strategies targeting either Her2 or GPER. If proven true, immunostaining for GnRs followed by subgroup analysis for Her 2 and GPER during pathological workup might assist to choose the therapy most likely to be effective. Needless to say that properly powered clinical trials are needed in order to prove our hypothesis.

\section{SO-023}

Results of the Retrospective multicenter Outcome survey in Borderline Ovarian Tumors of the AGO Study Group

S. Hauptmann*1, F. Kommoss' ${ }^{2}$, N. Ewald-Riegler ${ }^{3}$, A. Reuss ${ }^{4,}$

N. de Gregorio ${ }^{5}$, S. Mahner ${ }^{6}$, C. Fotopoulou', B. Schmalfeldt ${ }^{8}$, A. Burges ${ }^{9}$,

F. Hilpert ${ }^{10}$, T. Fehm ${ }^{11}$, W. Meier' ${ }^{12}$, P. Hillemanns ${ }^{13}$, L. Hanker ${ }^{14}$,

A. Hasenburg ${ }^{15}$, H.-G. Strauss ${ }^{16}$, M. Hellriege ${ }^{17}$, P. Wimberger ${ }^{18,}$

K. Baumann ${ }^{19}$, M.-D. Keyver-Paik ${ }^{20}$, U. Canzler ${ }^{18}$, K. Wollschlaeger ${ }^{21,}$

D. Forner ${ }^{22}$, J. Pfisterer ${ }^{23}$, W. Schröder ${ }^{24}$, K. Münstedt ${ }^{25}$, B. Richter ${ }^{26}$, A. du Bois ${ }^{27}$

'MVZ für Histologie, Zytologie und molekulare Diagnostik Trier, Trier, Germany, ${ }^{2}$ Institute of Pathology, Mannheim, Germany, ${ }^{3}$ Dr. Horst Schmidt Klinik GmbH, Department for Gynecology and gynecological Oncology, Wiesbaden, Germany, ${ }^{4}$ Coordination Center for Clinical Studies, Mannheim, Germany, ${ }^{5}$ Dptm for Gynecology, University Hospital Ulm, Ulm, Germany, ${ }^{6}$ Dptm for Gynecology, University Hospital Hamburg, Hamburg, Germany, ${ }^{7}$ Dptm for Gynecology, Charité Hospital CVK, Berlin, Germany, ${ }^{8}$ Dptm for Gynecology, Technical University Munich, Munich, Germany, ${ }^{9} \mathrm{Dptm}$ for Gynecology, University Hospital Munich-Großhadern, Munich, Germany, ${ }^{10}$ Dptm for Gynecology, University Hospital Schleswig-Holstein, Kiel, Germany, "Dptm for Gynecology, University Hospital Duesseldorf, Duesseldorf, Germany, ${ }^{12} \mathrm{Dptm}$ for Gynecology, Lutheran Hospital Duesseldorf, Duesseldorf, Germany, ${ }^{13}$ Department for Gynecology, Hanover Medical School, Hanover, Germany, ${ }^{14} \mathrm{Dptm}$ for Gynecology, University Hospital Frankfurt, Frankfurt, Germany, ${ }^{15} \mathrm{Dptm}$ for Gynecology, University Hospital Freiburg, Freiburg, Germany, ${ }^{16} \mathrm{Dptm}$ for Gynecology, University Hospital Halle, Halle (Saale), Germany, ${ }^{17} \mathrm{Dptm}$ for Gynecology, University Hospital Goettingen, Goettingen, Germany, ${ }^{18}$ Dptm for Gynecology, University Hospital Dresden, Dresden, Germany, ${ }^{19}$ Dptm for Gynecology, University Hospital Gießen-Marburg, Marburg, Germany, ${ }^{20}$ Dptm for Gynecology, University Hospital Bonn, Bonn, Germany, ${ }^{21}$ Dptm for Gynecology, University Hospital Magdeburg, Magdeburg, Germany, ${ }^{22}$ Dptm for Gynecology, Sana Hospital, Remscheid, Germany, ${ }^{23}$ Center for Gynecological Oncology, Kiel, Germany, ${ }^{24}$ Gynaecologicum, Bremen, Germany, ${ }^{25}$ Dptm for Gynecology, University Hospital Marburg-Gießen, Gießen, Germany, ${ }^{26}$ Dptm for Gynecology, Elbeland Hospital, Radebeul, Germany, ${ }^{27}$ Dptm for Gynecological Oncology, KEM, Essen, Germany

Aims. Although most patients with ovarian borderline tumors (BOT) are cured by surgery some experience recurrent disease. Risk factors for recurrent disease are poorly defined, mainly due to scarcity of stu- 
dies with large and well defined populations. Therefore, the AGO Study Group performed a systematic retrospective/prospective pattern of care study including central pathology review.

Methods. All consecutive patients diagnosed with BOT 1998- 2008 in 27 institutions were included. Tumor samples were sent for central histopathological review to 3 gynecological pathologists, clinical data were collected, and patient follow-up was updated by phone and/or questionnaire.

Results. For 1,042 of 1,236 pts a pathological review was obtained, resulting in the 950 confirmed BOT cases analyzed here. Median age was 49 years; $82 \%$ of patients had FIGO stage I disease; 152 pts had implants including 26 pts with invasive implants. Serous type was diagnosed in $68 \%$ and mucinous in $31 \%$; micropapillary serous BOT, microinvasion (serous and mucinous BOT), and intraepithelial carcinoma (mucinous BOT) were observed in 99, 49, and 24 pts, respectively. Complete debulking was achieved in $92 \% .166$ (17\%) underwent fertility preserving surgery. Overall, 74 (7.8\%) pts experienced relapse and 43 (4.5\%) died of disease. In young patients ( $<40 y)$ recurrent disease was more frequently observed (17.9\% vs. $3.6 \%$ ), and $2 / 3$ recurrences with SBOT histology were localized in the preserved ovary, whereas in older patients the recurrences occurred in the peritoneum as serous carcinoma. Of note, in 8 of the 22 patients (36\%) the recurring invasive carcinoma was high grade [1]. Conclusions. This is the world's largest series of BOT with central pathology review. Prognosis of BOT is good even without adjuvant therapy. Inadequate staging, residual tumor, fertility sparing surgery and higher FIGO stage were associated with shorter progression-free survival. Unexpectedly, a significant number of recurring lesions were high grade serous carcinomas.

\section{Literatur}

1. du Bois A et al (2013) Borderline tumours of the ovary: A cohort study of the Arbeitsgemeinschaft Gynäkologische Onkologie (AGO) Study Group. Eur J Cancer 49:1905-14

\section{SO-024}

\section{St. Gallen 2011 and 2013 clinicopathological subtyping of breast cancer: impact of different proliferation assessment methods on luminal $a$ and $b$ subtype distribution}

\section{Focke*, D. Gläser, K. Finsterbusch, T. Decker \\ Dietrich Bonhoeffer Medical Centre, Department of Pathology, Neubrandenburg, Germany}

Aims. The St Gallen consensus 2011/2013 strongly recommends subtyping of breast cancer (BC), and introduced BC clinicopathological subtypes (cpBCST) defined as subsets of risks and predictive biomarkers established worldwide. To differentiate Luminal A and the Her2 negative Luminal B BCs the St Gallen Consensus advises Ki67-labelling index (Ki67-LI) for defining proliferation. Since no standardized methodology for Ki67-LI is available so far, some 'alternative measure of proliferation' was also suggested by the expert panel. We analysed the influence of different proliferation assessment methods on the Luminal A and B BC subtype distribution.

Methods. CpBCST was performed according to the St. Gallen criteria on 494 BCs. We assessed proliferation by different variants of the Ki67Labeling-Index (KI67LI) estimation. As additional alternative method we used the Mitotic Activity Index (MAI), the only prospectively evaluated and most well reproducible proliferation measurement method in BC (Baak et al 2008). The Ki67-LI calculation was based on counting any nuclear staining of distinctly defined target areas: (1) 100 tumor cells within the hot spot (Ki67-100), (2) 1020 tumor cells in 3 HPF in the tumor periphery including the hot spot (Ki67-102operiphery), and (3) 1020 tumor cells in 3 HPF including hot spot, cold spot and an intermediate area (Ki67-102ospectrum). Ki67 staining quality and counting accuracy was tested as excellent by the 2011 circulation test of the German Quality Initiative in Pathology. According to the St Gallen recommen- dations 2011 and 2013, both respective cut-offs $(<14 \%$ and $<20 \%)$ were used for this analysis. The MAI was measured in 10 microscopic fields with a total area of $1.59 \mathrm{~mm} 2$ using a cut-off $<10$ as recommended by Baak et al. 2008.

Results. Of 494 BCs, $11 \%$ were Triple-negative, 3\% Her2+, 6\% Luminal B Her2+, and 10\% Special Type. The rates of Luminal A vs. Luminal B-Her2neg BCs using the $<14 \%$ Ki67-LI cutoff for the different proliferation assessment methods were: MAI 50\% vs. 20\%; Ki67-1020spectrum: $31 \%$ vs. $39 \%$, Ki67-102operiphery: $22 \%$ vs. $48 \%$, and Ki67-10o: $11 \%$ vs. $59 \%$, respectively. The rates of Luminal A vs. Luminal B-Her2neg BCs using the $<20 \%$ Ki67-LI cutoff came out with: MAI $50 \%$ vs. $20 \%$; Ki67-1020spectrum: $45 \%$ vs. $25 \%$, Ki67-1020periphery: $35 \%$ vs. $35 \%$, and Ki67-100: $18 \%$ vs. $52 \%$, respectively.

Conclusions. Subtyping of Luminal A and Luminal B Herneneg BCs according to the St. Gallen Consensus 2011/2013 is highly influenced by the method of proliferation assessment.

\section{SO-025}

Factors on breast core biopsy associated with intermediate and low risk by Oncotype $\mathrm{DX}$ testing on excision specimen. A retrospective analysis of 55 cases

\section{Z. Varga ${ }^{* 1}$, A. Lebeau ${ }^{2,3}$, P. Went ${ }^{4}$, P. Sinn ${ }^{5}$}

'Institute of Surgical Pathology, University Hospital Zurich, Zurich, Switzerland, ${ }^{2}$ Institute of Pathology, University Medical Center Hamburg Eppendorf, Hamburg, Germany, ${ }^{3}$ Pathology Group Practice, Lübeck, Germany, Lübeck, Germany, ${ }^{4}$ Institute of Pathology Enge, Zurich, Switzerland, Institute of Pathology, University Hospital Heidelberg, Heidelberg, Germany

Aims. Genomic grades and risk scores based on conventional histopathological parameters are determined usually on breast excision specimens after completing axillary and breast surgery. High risk Oncotype DX recurrence scores (RS) implicate a higher benefit for the administration of chemotherapy, but in case of intermediate risk RS, the decision for or against chemotherapy mostly depends upon conventional histological parameters. We have addressed the question, if it is possible to determine factors on breast core biopsies predicting non-high risk (intermediate and low low-risk) RS on the excision specimen.

Methods. Retrospectively, we have analyzed 55 breast core biopsies and compared this to known Oncotype DX test results of the excision specimens. Histological subtype, tumor grading, hormone receptor and HER2 status were available. Molecular subtypes were derived from these results. Luminal A/B subtypes was defined as HER2 negative and ER/PR high positive (Luminal A) or ER high/PR low positive (Luminal B). Results were statistically analyzed by Fisher's exact test.

Results. Lobular histological type was seen only with low (58\%) and intermediate risk RS (42\%), whereas ductal carcinomas showed a high risk RS in $23 \%$ of cases $(\mathrm{p}=0.025)$. Luminal A phenotype was associated with non-high risk RS (96\%) rather than Luminal B phenotype $(52 \%$, $\mathrm{p}=0.0037)$. G1 and $\mathrm{G} 2$ carcinomas had mainly non-high risk RS (91\%, $88 \%$ resp.), G3 carcinomas were all high risk RS (100\%, $p=0.03)$. There was non-significant difference between $\mathrm{G}_{1}$ and $\mathrm{G}_{2}$ cancers on core biopsies and RS in excision specimens.

Conclusions. We could not identify any single factor on core biopsies predicting intermediate risk RS. Lobular histology, non-high grade differentiation ( $\mathrm{G}_{1}$ and $\mathrm{G}_{2}$ ) and Luminal A subtype on core biopsy were significantly associated with non-high risk (low and intermediate) RS. Poorly differentiated carcinomas and Luminal B phenotype were significantly associated with high risk RS. These factors may be considered when planning therapy schemata based on breast core biopsies. 


\section{SO-026}

New roads in breast cancer. The role of gene expression assays for classification, prognosis, and prediction

\section{P. Sinn* \\ Institute of Pathology, University Hospital Heidelberg, Heidelberg, Germany}

Aims. The indication for chemotherapy in breast cancer has traditionally been based on prognostic factors, such as histopathology, (p)TNM stage, and clinical tumor characteristics, as well as combined approaches, prognostic scores, and clinical algorithms, such as the St. Gallen Consensus. It has repeatedly been shown that these prognosis-based approaches lead to an overtreatment of patients, and a majority of patients may not benefit from adjuvant cytotoxic chemotherapy. Therefore, classic prognostic and predictive criteria are not specific enough for decision making regarding adjuvant chemotherapy.

Methods. Multigene assays have provided a new approach to breast cancer subtyping and also to prognostic and predictive tumor classification. The molecular phenotypic classification scheme that divides breast cancer into four main classes (luminal A, luminal B, HER2-enriched, basal-like) was verified by different approaches, including gene expression analysis, genetic and epigenetic studies, and can be considered as established. Open questions remain regarding the reproducibility between different methodological approaches, the definition of each subtype, and the way in which heterogeneity within these subclasses should be addressed. Any further information and clinical usefulness above that is provided by the intrinsic subclassification, in addition to ER, PR, HER 2 and proliferation as determined by IHC, has only been partially explored yet.

Results. Commercially available gene profiling assays assays (e.g. Oncotype DX ${ }^{\oplus}$, Endopredict ${ }^{\oplus}$, Mammaprint $^{\oplus}$, Prosigna ${ }^{\circ}$, MammaTyper ${ }^{\circ}$ ) were created to reduce overtreatment and to guide treatment selection, especially the decision for or against chemotherapy in the (neo)adjuvant setting in hormone-receptor positive patients, when conventional clinicopathologic features are indeterminate or would have suggested otherwise. Statistically, the higher the individual risk of recurrence, the greater the likelihood that a patient will benefit from chemotherapy. Patients with a low risk score have only a small absolute benefit from cytotoxic chemotherapy. Multigene tests can be combined to a molecular-clinical risk score (such as EPclin) in order to improve the prognostic assessment of a given patient.

Conclusions. Therefore, it can be said, that as of today, no signature can replace the classical clinicopathologic parameters, but molecular assays may add additional information when there is no clear indication for chemotherapy otherwise.

\section{SO-027}

RANK expression as a prognostic and predictive factor in GeparTrio-a neoadjuvant breast cancer study

B.M. Pfitzner ${ }^{* 1}$, D. Branstetter ${ }^{2}$, B. Lederer', S. Loib/ ${ }^{3}$, C. Denkert ${ }^{1}$,

P. Fasching ${ }^{4}$, A. Fisseler-Eckhoff ${ }^{5}$, S. Kümmel ${ }^{6}$, I. Baumann ${ }^{7}$, C. Hanusch ${ }^{8}$, C. Jackisch ${ }^{9}$, W.C. Dougall' ${ }^{10}$, G. von Minckwitz ${ }^{3,11}$

'Institute of Pathology, Charité-University medicine of Berlin, Berlin, Germany, ${ }^{2}$ Amgen Inc., Departement of Pathology, Seattle, United States, ${ }^{3}$ German Breast Group, Neu-Isenburg, Germany, ${ }^{4}$ University gynecological hospital, University Erlangen, Erlangen, Germany, ${ }^{5}$ Institute of Pathology, Dr. Horst Schmidt Kliniken, Wiesbaden, Germany, ${ }^{6}$ Hospital Essen Mitte, Breast Care Center, Essen, Germany, ${ }^{7}$ Institute of Pathology, health care center (MVZ), Böblingen, Germany, ${ }^{8}$ Rotkreuzklinikum München, Gynecological Hospital, Munich, Germany, ${ }^{9}$ Sana Klinikum Offenbach, Gynecological Hospital, Offenbach, Germany, ${ }^{10} \mathrm{Amgen}$ Inc., Therapeutic Innovation Unit, Seattle, United States, "University Women's Hospital Frankfurt, Frankfurt, Germany

Aims. RANK ligand (RANKL), a key factor for bone remodeling and metastasis, is crucial for the development of mouse mammary glands during pregnancy. RANKL functions as a major paracrine effector of the mitogenic action of progesterone in mouse and human mammary epithelium via its receptor RANK and has a role in ovarian hormonedependent expansion and regenerative potential of mammary stem cells. Pharmacologic inhibition of RANKL attenuates the development of mammary carcinoma and inhibits metastatic progression in multiple mouse models. This study analyzed primary breast carcinoma samples from the GeparTrio study to correlate the expression of human RANK and RANKL (using specific, monoclonal antibodies validated and optimized for IHC) with pathological complete response (pCR: ypTo ypNo), disease-free (DFS), and overall (OS) survival after anthracycline-taxane-based neoadjuvant chemotherapy.

Methods. Pre-treatment FFPE core biopsies $(n=601)$ from participants of the neoadjuvant GeparTrio study were analyzed for percentage and intensity of immunohistochemical RANK and RANKL expression. Antibodies against human RANK (N-1H8; Amgen) and human RANKL (M366; Amgen) were used for IHC staining. The overall expression measured by IHC was calculated using the $\mathrm{H}$ scoring method.

Results. RANK protein was expressed within the tumor epithelium in $160(27 \%)$ patients. A cutoff of an $\mathrm{H}$-score of $>8.5$ was identified as optimal for prediction of pCR, DFS, and OS. High RANK expression $(>8.5$ $\mathrm{H}$-Score) was observed in $14.5 \%$ of patients and was strongly correlated with high tumor grade $(\mathrm{p}<0.023)$ and negative hormone-receptor status $(\mathrm{p}<0.001)$. Patients with high RANK expression showed a higher $\mathrm{pCR}$ rate $(23.0 \%$ vs $12.6 \%, p=0.010)$, shorter DFS $(p=0.038)$ and OS $(p=0.011)$ compared to patients with low RANK expression. However, prognostic and predictive information was not independent from other baseline parameters in multivariate analyses. Only $6 \%$ of samples expressed RANKL within the tumor epithelium, which was not correlated with any clinical features.

Conclusions. Higher RANK expression in the primary tumor is associated with a higher sensitivity to chemotherapy, but also a higher risk of relapse and death. However, due to its strong correlation with grade and hormone-receptor status, RANK does not provide independent predictive and prognostic information. 


\section{AG Gynäko- und Mammapathologie II}

\section{SO-028 \\ Identification and validation of novel T cell epitopes for Mamma- globin A}

S. Münst ${ }^{* 1}$, S.D. Soysal ${ }^{2}$, J. Kan-Mitchell, X. Zhang ${ }^{4}$, T. Fleming ${ }^{4}$,

V. Tiriveedhi ${ }^{4}$, T. Mohanakumar ${ }^{4}$, J. Herndon ${ }^{4}$, D. Oertli ${ }^{2}$, W. Gillanders ${ }^{4}$,

P. Goedegebuure ${ }^{4}$

${ }^{1}$ University Hospital Basel, Institute of Pathology, Basel, Switzerland, ${ }^{2}$ Department of Surgery, University Hospital, Basel, Switzerland, ${ }^{3}$ Department of Biological Sciences and Border Biomedical Research Institute, University of Texas at El Paso,, El Paso, United States, ${ }^{4}$ Washington University School of Medicine, Department of Surgery, St. Louis, United States

Aims. Mammaglobin-A (Mam-A) is a secretory protein that is overexpressed in $80 \%$ of human breast cancers. Its broad expression in breast cancer tissue as well as its specificity for breast renders it an attractive target for a vaccination therapy against breast cancer. Several CD8 T cell candidate epitopes of Mam-A have been identified, but so far none of them has been validated in breast cancer patients. Our aim was to identify new epitopes of Mammaglobin-A (Mam-A) using a systematic epitope mapping.

Methods. We conducted a systematic epitope mapping with antigenspecific human T-cells primed in vitro by autologous dendritic cells transduced to express Mam-A. We identified two immunodominant as well as two subdominant epitopes of Mam-A, which were used to generate specific CD8 $\mathrm{T}$ cells in vitro. The reactivity of these specific T cells against the epitopes was assessed using an IFN-gamma ELISPOT assay. Results. We could show that the CD8 T cells are able to recognize the specific epitopes presented by antigen presenting cells, and that they are able to specifically lyse human breast cancer cells in a Mam-A specific, HLA-A2 dependent manner. In addition, we also validated all our CD8 $\mathrm{T}$ cell epitopes in Mam-A+ breast cancer patients and are thus the first to show that these epitopes are spontaneously immunogenic in vivo. Additionally we found that the preexisting CD8 T cell immunity against these epitopes is significantly enhanced by Mam-A cDNA vaccination. Conclusions. We present a novel and broadly applicable method to identify Mam-A T cell epitopes and show they can be used to generate CD8 $\mathrm{T}$ cells in vitro. Our results in Mam-A+ breast cancer patients indicate for the first time that Mam-A cDNA vaccination effectively enhances specific CD8 T cell immunity and support Mam-A cDNA vaccination as a novel therapeutic strategy for breast cancer patients.

\section{SO-029}

Evaluation of the impact of preanalytical tissue handling on the results of the EndoPredict breast cancer prognosis test

\section{Poremba ${ }^{* 1}$, J. Uhlendorff ${ }^{2}$, B.M. Pfitzner ${ }^{3}$, G. Hennig ${ }^{4}$, K. Bohmann ${ }^{2}$, H. Bojar ${ }^{5}$, V. Krenn ${ }^{5}$, F. Haufe ${ }^{2}$, M. Averdick ${ }^{2}$, M. Dietel ${ }^{3}$, R. Kronenwett ${ }^{2}$, C. Denkert ${ }^{3}$}

${ }^{1}$ Institute of Pathology Munich North, Munich, Germany, ${ }^{2}$ Sividon Diagnostics $\mathrm{GmbH}$, Cologne, Germany, ${ }^{3}$ Institute of Pathology, Charité University Hospital, Berlin, Germany, ${ }^{4}$ Siemens Healthcare Diagnostics GmbH, Eschborn, Germany, ${ }^{5}$ Center for Molecular Oncology und Molecular Pathology CMOMP, Düsseldorf, Germany

Aims. EndoPredict is a quantitative reverse transcription PCR assay that predicts the likelihood of distant recurrence in patients with primary ER-positive/HER2-negative breast cancer. The test is performed on RNA isolated from formalin-fixed, paraffin-embedded tissue sections. In this study, we analyzed the impact of the time to fixation, the fixation duration, the tissue section storage time and the tumor cell content (TCC) on the EndoPredict test results.
Methods. To study the impact of the time to fixation and the fixation duration, two tumors were cut into several pieces of identical size. Tissue pieces of the same tumor were stored for up to 24 hours prior to fixation or for up to 10 days in formalin. The influence of the sample storage time was assessed using tissue sections of 10 breast tumors. At the beginning of the study one section of each tumor was used for EndoPredict analysis. The remaining two sections were stored at $+4^{\circ} \mathrm{C}$ or at $+20^{\circ} \mathrm{C}$ for 12 months before being analyzed by EndoPredict. To study the impact of the TCC on the EndoPredict result whole tissue sections with varying TCC and paired macrodissected, tumor enriched samples (100\% TCC) where used.

Results. A time to fixation of up to $12 \mathrm{~h}$ and a fixation time of up to 5 days did not affect the EndoPredict test result. EP scores of these specimens were similar to the reference value with a mean deviation of $0.37 \mathrm{EP}$ score units (time to fixation study) or $0.02 \mathrm{EP}$ score units (time in formalin). Only the specimens with a storage time of $24 \mathrm{~h}$ prior to fixation or 10 days in formalin showed a remarkable decrease in the EP score. The EP scores of tissue sections that have been stored for 12 months at $+4^{\circ} \mathrm{C}$ or $+20^{\circ} \mathrm{C}$ showed a correlation of 0.99 when compared to results of non-stored sections. Mean deviation between stored and non-stored sections was <0.04 EP score units. Studies regarding the impact of the tumor cell content are currently performed. Results will be presented and discussed.

Conclusions. Tissue handling parameters that have been analyzed in this study do not influence EndoPredict test results.

\section{SO-030}

Interlaboratory variability of Ki67 labelling index in breast cancer tissue microarrays

\section{Focke ${ }^{* 1}$, D. Gläser', K. Finsterbusch', H. Bürger' ${ }^{2}$, E. Korsching ${ }^{3}$, M.o.t.} German Breast Screening Pathology Initiative ${ }^{4}$, T. Decker ${ }^{1}$

'Dietrich Bonhoeffer Medical Centre, Department of Pathology, Neubrandenburg, Germany, ${ }^{2}$ Department of Pathology, Paderborn, Germany, ${ }^{3}$ University of Münster, Institute for Bioinformatics, Münster, Germany, ${ }^{4}$ German Breast Screening Pathology Initiative, Neubrandenburg, Germany

Aims. Estimation of tumour proliferation by assessment of the Ki67-labelling index (Ki67-LI) is increasingly recommended for prognostication and adjuvant chemotherapy decisions in breast cancer. Although many national and international guidelines recommend specific cutoffs to define "high proliferation", recently the need for a more standardized approach has been recognized. Our aim was to investigate interlaboratory variance of Ki67-LI results using TMA and centralized assessment to exclude preanalytic influences and postanalytic variance, respectively.

Methods. 25 pathology laboratories (19 German, 3 Austrian, 1 Dutch, 1 Hungarian and 1 Suisse) performed Ki67 staining of a TMA slide according to their daily practice in house protocol (including internal and external quality assurance). 38 samples per lab were centrally analysed. The Ki67-LI was calculated after counting first all tumour cells and subsequently all Ki67 positive tumour cells of each sample regardless of staining intensity. For each tissue sample we evaluated the range of Ki67-LIs between different labs.

Results. The range of Ki67-LIs between the labs was: $<5 \%$ in 1 (\%), $5-10 \%$ in $5(13 \%), 10-15 \%$ in $2(5 \%), 16-20 \%$ in $5(13 \%), 20-25 \%$ in $4(10 \%), 25-30 \%$ in $6(16 \%), 30-35 \%$ in $1(3 \%), 35-40 \%$ in $3(8 \%), 40-45 \%$ in $2(5 \%), 45-50$ in $1(3 \%)$ and $>50 \%$ in 8 samples $(21 \%)$, respectively. Thus, in $55 \%$ of results of the 25 labs (21 of the 38 TMA specimens) the Ki67-LI differed by more than $25 \%$. The analysis of variance (ANOVA) came out with $\mathrm{F}=5.0$ which is much larger than the critical $F$ value of 1.53 for these study results $(\mathrm{p}=1.5996 \mathrm{E} 13)$. This means that the observed interlaboratory variance of 5.0 is systematic and not due to sampling error. The respective standard deviation is $18.4 \%$.

Conclusions. In a setting strictly standardised in terms of preanalytic influences by using TMA and postanalytic variance by centralised 
quantification, Ki67-LI seems to be heavily influenced by laboratoryspecific analytic variables. Taking this into consideration, there may be a risk of prognostic or predictive misclassification in some breast cancer patients in daily practice.

\section{SO-031 \\ Computer-assisted Ki67 scoring in the GeparTrio breast cancer study cohort}

F. Klauschen ${ }^{* 1}$, S. Wienert', J. Blohmer' ${ }^{2}$, B. Pfitzner', H. Eidtmann ${ }^{3,}$ B. Gerber', H. Tesch', J. Hilfrich ${ }^{6}$, J. Huober, T. Fehm ${ }^{8}$, J. Barinoff', C. Jackisch ${ }^{10}$, W. Eiermann ${ }^{11}$, T. Rüdiger ${ }^{12}$, E. Erbstößer ${ }^{13}$, P. Hufnagl", S. Loib/14, M. Dietel', G. von Minckwitz ${ }^{14}$, C. Denkert ${ }^{1}$

'Institute of Pathology, Charité, Berlin, Germany, ${ }^{2}$ Gertrauden Hospital, Berlin, Germany, ${ }^{3}$ Universitätsfrauenklinik Kiel, Kiel, Germany, ${ }^{4}$ Universitätsfrauenklinik Rostock, Rostock, Germany, ${ }^{5}$ Onkologie Bethanien, Frankfurt, Germany, ${ }^{6}$ Henrietten-Stiftung, Hannover, Germany, ${ }^{7}$ Kantonspital St. Gallen, St. Gallen, Switzerland, ${ }^{8}$ Universitätsfrauenklinik Tübingen, Tübingen, Germany, ${ }^{9}$ Dr. Horst Schmidt Klinik, Wiesbaden, Germany, ${ }^{10}$ Städtische Kliniken, Offenbach, Germany, "'Klinikum zum Roten Kreuz, München, Germany, ${ }^{12}$ Städtisches Klinikum, Karlsruhe, Germany, ${ }^{13}$ AMEOS Klinikum St. Salvator, Halberstadt, Germany, ${ }^{14}$ German Breast Group, Neu-Isenburg, Germany

Aims. Recent studies have cast some doubt on the reliability of "visual" Ki67 scoring by pathologists, especially within the lower-yet clinically important-proliferation range. Here, we present and apply a novel automated image analysis approach for Ki67-quantification in breast cancer tissue and compare automated and manual Ki67 scoring and survival analysis results in the GeparTrio breast cancer study cohort. Moreover, we perform an error analysis of post-laboratory observer-independent aspects of Ki67 scoring.

Methods. Study cohort: The GeparTrio study (NCToo544765) is a multicenter, randomized, prospective neoadjuvant, phase III trial. $1166 \mathrm{pa}$ tients with primary breast cancer ( $\mathrm{T}_{2}-4, \mathrm{No}-3, \mathrm{Mo}$ ) for whom survival times were available. Histology, immunohistochemistry, slide digitization: Tissue preparation and Ki67 staining were performed according to standard routine laboratory procedures. Tissue regions used for Ki67 scoring were selected by trained pathologists and digitized with a microscope camera at 40ox. Automated and manual Ki67 quantification: Manual Ki67 scoring was performed by trained pathologists according to routine guidelines. The automated Ki67 scoring is based on a contour-based cell detection approach which was integrated into the CognitionMaster software platform.

Results. We compared computational Ki67 scoring results with manual scoring performed by pathologists. The results show deviations of $10 \%$ on average. The Ki67 scores show Pearson correlations between automated and manual scoring of $\mathrm{r}>0.8(\mathrm{p}<0.001)$. We performed KaplanMeier survival analysis for the $3 \mathrm{Ki} 67$ score groups $0-15 \%, 15.1-35 \%$ and $35.1-100 \%$. Overall significances are $\mathrm{p}<0.001$ for automated as well as for manual scoring. Moreover, the error analysis we performed demonstrates statistical sampling effects as a major source of inter-observer variation.

Conclusions. We present and clinically validate a novel computer-assisted Ki67 scoring approach and show how application of this approach may not only assist pathologists in their daily routine but also help understand recently reported inter-observer variability of Ki67 diagnostics.

\section{SO-032}

\section{Prognostic relevance of MIB-1-expression in lymph node metasta-} ses of breast cancer

\section{S. Awan, M. Schramm, S. Biesterfeld* \\ Department of Cytopathology, Heinrich Heine University, Düsseldorf, Germany}

Aims. The expression of MIB-1 has been frequently evaluated as prognostically useful in primary breast cancer. Lymph node metastases, however, have been rarely investigated, and the prognostic impact of their MIB-1-expression is unclear. In our study, we present prognostic data on a series of $39 \mathrm{N1}$-breast cancer cases with MIB-1 analysed both in primary and secondary tumour with a follow-up period of up to 11.5 years. Methods. MIB-1-immunohistochemistry was performed using a standard protocol. The percentage of immunoreactive cells was evaluated by manual counting in 20 high-power-fields. The results were correlated with clinical and morphological data.

Results. The mean values (11.1\% vs. $20.7 \%)$ and the median values $(7.9 \%$ vs. $18.4 \%$ ) for MIB-1 were quite different between primary tumours and lymph node metastases and only weakly correlated $(r=0.57)$. In univariate survival analysis (threshold: the respective median value), the MIB-1 expression in the lymph nodes was of higher prognostic significance $(\mathrm{p}=0.0001)$ compared to that in primary tumours $(\mathrm{p}=0.0029)$. In a multivariate Cox regression model, the MIB-1 expression in the lymph nodes and the progesterone receptor status (PR) were entered as prognostically independent factors while the MIB-1 expression of the primary tumour, the tumour grading and the estrogen receptor status were rejected.

Conclusions. The analysis of the MIB-1 expression in lymph node metastases proved to be of higher prognostic significance than in primary breast cancer possibly due to the expansion of a distinct tumour clone during lymph node metastasizing. Thus, quantitative MIB-1 evaluation of tumour-positive lymph nodes can be recommended for breast cancer.

\section{SO-033}

Response of MCF-7 mamma carcinoma cells to glyoxal and methylglyoxal

\section{N. Nass*, A. Roessner, T. Kalinski}

Department of Pathology, Otto-von-Gericke University Magdeburg, Germany, Magdeburg, Germany

Aims. The alpha-oxo-aldehydes glyoxal and methylglyoxal are byproducts of fatty acid oxidation and glycolysis. Due to the high glycolytic activity caused by the Warburg effect, cancer cells produce significantly more aldehydes than other cells. These substances are highly reactive and modify amino groups on proteins, often resulting in loss of function. These modifications also result in stable accumulating products, the so called advanced glycation end products (AGEs). In this study we analysed the responses of the estrogen receptor positive mamma carcinoma cell line MCF-7 to glyoxal and methylglyoxal to evaluate if dialdehyde defence might be a target for breast cancer treatment.

Methods. Cell vitality was determined by the resazurin assay. Activation of MAP-kinases and NF- $\kappa \mathrm{B}$ was determined by Western blotting, using specific antibodies against the phosphorylated proteins. Oxidative stress was measured by loading the cells with the oxidation-sensitive fluorochrome dihydrofluorescein-diacetate. Mode of cell death was analysed by caspase activity assay and annexin-V flow cytometry.

Results. Dialdehydes resulted in cell death in low millimolar concentrations. Cells exposed to the aldehydes responded by a rapid activation of MAP-kinase signalling especially $\mathrm{p} 38$-MAPK and production of reactive oxygen species as well as activation of caspase $3 / 7$ activity, finally leading to cell death. Interestingly, a tamoxifen resistant derivative of MCF-7 showed an additional activation of the NF- $\kappa$ B transcription factor by methylglyoxal only and was altogether more sensitive to the alde- 
hydes. Addition of the antioxidant $\mathrm{N}$-acetyl cystein protected the cells, demonstrating that oxidative stress was a major cause of cell death. Conclusions. These data demonstrate that exogenous aldehyde stress is cytotoxic to mamma carcinoma cells by causing severe oxidative stress. Inhibition of aldehyde defence enzymes such as glyoxalases might therefore be a promising therapeutic option against breast cancer.

\section{SO-034}

Expression of folate receptor alpha as potential therapy target in invasive breast cancer

\section{K. Friedrich ${ }^{* 1}$, F. Goerl', A. Gatzweiler², B. Richter ${ }^{3}$, A. Werner', P. Wimber- ger ${ }^{5}$ G. Baretton}

'Institute of Pathology, University Hospital Carl Gustav Carus Dresden, TU Dresden, Dresden, Germany, ${ }^{2}$ Department of Gynecology and Obstetrics, St. Joseph-Stift, Dresden, Germany, ${ }^{3}$ Department of Gynecology and Obstetrics, Elblandkliniken Radebeul, Radebeul, Germany, ${ }^{4}$ Department of Gynecology and Obstetrics, Diakonissenkrankenhaus Dresden, Dresden, Germany, ${ }^{5}$ Department of Gynecology and Obstetrics, University Hospital Carl Gustav Carus Dresden, TU Dresden, Dresden, Germany

Aims. Folate plays an essential role in DNA-synthesis and DNA repair mechanisms. Thus the folate pathway may be a target for cancer therapy in the future. Folate receptor alpha (FRA) is overexpressed in lung adenocarcinomas, ovarian and endometrial cancers as well as in some breast cancers. The purpose of the study was to analyse the FRA expression in invasive breast cancers in correlation to clinicopathological features and biomarkers including hormone receptors, HER-2, Ki67 p53 and EGFR.

Methods. The expression of FRA, estrogen and progesterone receptor HER-2, Ki67, p53 and EGFR was analysed in 409 formalin fixed, paraffin-embedded specimens of invasive breast carcinoma using tissue microarrays. All staining results were scored semiquantitatively. The statistical analysis was done by Chi-squared test and multivariate discriminant analysis.

Results. Membranous expression of FRA was observed in 95 cases, ranging from $1 \%$ to $100 \%$ of tumor cells with different staining intensities. FRA expression was correlated with histopathological grade 3 , hormone receptor negativity, high proliferation activity, expression of $\mathrm{p} 53$ and EGFR in univariate analyses. The comparison of triple negative breast cancers with HER-2 positive breast cancers revealed a significant higher frequency of FRA positive cases in the triple negative group than in HER-2 positive group. The multivariate analysis revealed estrogen receptor expression as independent marker for discrimination between FRA positive and negative breast cancers. The results of outcome analysis will be reported.

Conclusions. The expression of FRA is associated with basal like subtype features. The FRA may be an attractive therapy target especially for triple negative breast cancers. The predictive value of FRA expression for FRA targeted therapies like Farletuzumab remains to be evaluated in clinical trials.

\section{SO-035}

Loss of secreted frizzled-related protein-1 expression in breast cancer is associated with a characteristic lipid profile, lymph node positivity, hormone receptor negative status and a worse prognosis of the patients

\section{S.F. Brockmoeller*1,2, J. Budczies ${ }^{3}$, M. Hilvo ${ }^{4}$, B. Mueller ${ }^{3}$, M. Dietel M. Oresic', J. Griffin ${ }^{2}$, C. Denkert ${ }^{3}$}

'Leeds University, Pathology, Leeds, United Kingdom, ${ }^{2}$ Department of Biochemistry and Cambridge Systems Biology Centre, Department of Biochemistry and Cambridge Systems Biology Centre, University of Cambridge, Cambridge, United Kingdom, ${ }^{3}$ Institute of Pathology, Charité University Hospital, Berlin, Germany, ${ }^{4} \mathrm{VTT}$ Technical Research Centre of Finland, Espoo, Finland

Aims. Breast cancer is the most common cancer in women (Jemal et al. 2011). The lipidome of breast cancer can be used to define subtypes of breast cancer with varying patient prognoses (Brockmoller et al. 2012, Denkert et al. 2012, Hilvo et al. 2011). These changes in metabolism were transferred to the level of the regulatory proteins where secreted frizzled-related protein-1 (SFRP-1) a regulator of lipid metabolism showed promising results (Caldwell et al. 2004). The aim of this study was to further investigate the SFRP-1

Methods. SFRP-1 staining (SFRP-1: Abcam, catalogue no. ab4193) was performed for 231 patients with primary breast cancer. The stains were digitised (Mirax, Scan; Zeiss, Jena, Germany), blind-evaluated (VMscope, Berlin, Germany) and the results were statistically analysed (version 18.o; SPSS, Inc., Chicago, IL, R). A weak (IRS of o-4) or strong SFRP-1 (IRS of 6-12) expression group was different. The RNA expression of SFRP-1 was evaluated with a second independent dataset. The results of the immunohistochemical analysis were correlated with the lipidomic (UPLC-MS, Hilvo et al. 2011) data of corresponding fresh-frozen samples.

Results. Low cytoplasmatic expression SFRP-1 showed a significant correlation with nodal status $(\mathrm{p}=0.054)$, hormone receptor $(\mathrm{p}=0.00023)$ and the molecular subtypes $(\mathrm{p}=0.0027)$. A low expression of SFRP-1 in the RNA data (DASL Illumina method) showed a significantly worse prognosis for the patients ( $\mathrm{p}=0.031, \mathrm{n}=514$; www.kmplot.com). All together 22 lipids were significantly altered lipids with respect to SFRP-1 low and high expression. From the identified lipids PC (18:2/18:0) was the only lipid which was up-regulated, and the following lipids were down regulated: TG(42:0), TG(56:5), TG(56:7), TG(56:8), TG(56:9), TG(58:10), $\mathrm{TG}(58: 8)$ and $\mathrm{TG}(58: 9)$.

Conclusions. Low SFRP-1 expression has a worse patient prognosis (mRNA) and is significantly correlated with nodal positive status, hormone receptor negative status, and molecular subtype (Ugolini et al. 2001, Klopocki et al. 2004). An increase in one of the de novo synthesise lipids PC (18:2/18:0) could mean that SFRP-1 down regulation effects the regulation of de novo lipid synthesis in breast cancer. It has been shown that an increase of leioleic acid in serum correlates with an increased risk of developing breast cancer (Chajes et al. 1999).

\section{SO-036}

Structured reporting increases the essential data content of pathology reports of oncological breast resection specimens

\section{K. Aumann ${ }^{* 1}$, K. Niermann', P. Bronsert' ${ }^{1}$, D. Hauschke ${ }^{2}$, E. Stickeler, M. Werner}

'Department of Pathology, University Medical Center Freiburg, AlbertLudwigs-University, Freiburg, Germany, ${ }^{2}$ Department of Medical Biometry and Medical Informatics, University Medical Center, Freiburg, Germany, ${ }^{3}$ Gynecological Hospital, University Medical Center Freiburg, Freiburg, Germany

Aims. There is increasing evidence that not only the way of data acquisition but also the design of data visualization (i.e. the format of the 
report) has impact on the quality of pathology reports. Therefore, we investigated the correlation between the amount of transmitted data and the format of pathology reports. Here, we describe a TNM-adapted toolset including a Pathology Information System-integrated structured template that contributes to improving pathology reports of oncological breast resection specimens.

Methods. All reports of oncological breast resection specimens of the Department of Pathology, University Medical Center Freiburg, between 2003 and $2011(n=4181)$ were classified into descriptive reports (DR, $\mathrm{n}=856$ ), structured reports arranged in TNM relevant tumor spread, lymph node and resection status (SR, $\mathrm{n}=2455)$, or template based synoptic reports (TBSR, $n=870$ ). They were compared regarding the content of nine organ specific essential data (ED) proposed by the College of American Pathologists, the Royal College of Pathology and the Association of German Pathologists. The amount of recorded ED was summarized and an essential data score (EDS) was calculated. Statistical analyses were performed by using exact Fisher Test or Mann-Whitney Test. Results. Median EDS of the DRs was 7, of SRs 8, and of TBSRs 9. In total, only $28.4 \%$ of the reports had an EDS of 9 ; divided into the report types $4 \%$ of DRs, $21.4 \%$ of SRs, and $72.3 \%$ of TBSRs obtained an EDS of 9. In detail, the most striking and statistically highly significant differences could be seen comparing the report types regarding the content of tumor size, tumor location, appropriate tumor grading, and additional pathological findings (pairwise comparison each $\mathrm{p}<0.0001$ ), as well as angio-invasion (SR vs TBSR $\mathrm{p}<0.043$; DR vs SR and TBSR $\mathrm{p}<0.0001$ ). Conclusions. Due to structured reporting and in particular by reporting based on a checklist pathology reporting of oncological breast specimens is improved regarding the content of ED and the clarity of the data layout. The use of TBSRs led to a reduction in failed data transfer and therefore to an increase in the quality of pathology reports. Accordingly, reporting in a standardized format or checklist revealed the highest quality of pathology reports. This data clearly shows that it is recommended to use a structured format of pathology reports.

\section{AG Pneumopathologie}

\section{SO-037}

Screening for ROS1 translocations in non-small cell lung cancer: clinicopathological analysis of 1478 cases

\section{A. Warth ${ }^{* 1}$, T. Muley ${ }^{2}$, H. Dienemann ${ }^{2}$, B. Goeppert' ${ }^{1}$ A. Stenzinger', P.A. Schnabel', P. Schirmacher', R. Penzel', W. Weichert ${ }^{1}$ 'Institute of Pathology, University Hospital Heidelberg, Heidelberg, Germany, ${ }^{2}$ Thoracic Hospital Heidelberg, Heidelberg, Germany}

Ziele. Die molekulare Charakterisierung des nichtkleinzelligen Lungenkarzinoms (NSCLC) führte zur Entdeckung zahlreicher, potentiell durch spezifische Therapeutika angehbarer Mutationen. Jüngst wurden chromosomale Translokationen unter Beteiligung von ROS 1 (c-ros oncogene 1, receptor tyrosine kinase) entdeckt, welche in NSCLCs mit spezifischen Charakteristika aufzutreten scheinen. Nach ersten Daten profitieren entsprechende Patienten von einer Therapie mit Crizotinib. Ziel der Studie war es, klinisch-pathologische Merkmale von Patienten mit ROS1-Translokation zu spezifizieren und den Stellenwert einer immunhistochemischen Präselektion zu bestimmen.

Methoden. Es wurde eine große NSCLC-Kohorte von 1478 Fällen immunhistochemisch mit einem spezifischen ROS1-Antikörper analysiert, positive Fälle wurden weiter mittels Fluoreszenz in situ Hybridisierungen (FISH) auf ROS1-Translokationen untersucht. Sowohl immunhistochemisch als auch FISH-positive Fälle wurden systematisch auf assoziierte klinische oder pathologische Merkmale sowie dem Überleben analysiert.

Ergebnisse. Insgesamt wiesen 68 Fälle eine positive Immunreaktivität gegen ROS 1 auf, in 9 dieser Fälle konnte eine Translokation des ROS 1
Genlokus mittels FISH bestätigt werden. Eine ROS 1 Expression fand sich vorwiegend in Adenokarzinomen weiblicher Patienten, in niedrigen Tumorstadien, sowie in Fällen mit Expression von TTF1 und Napsin. Weiter zeigte sich eine Assoziation zu lepidischen, azinären und soliden Wuchsmustern. Die Expression von ROS1 konnte zudem als stadienunabhängiger Prädiktor eines besseren Überlebens identifiziert werden.

Schlussfolgerung. Zusammenfassend konnte gezeigt werden, dass ROS 1 Translokationen seltene molekulare Alterationen im NSCLC sind. Sowohl eine auf Immunhistochemie als auch auf klinisch-pathologischen Charakteristika basierende Präselektion erscheint hilfreich, um eine Anreicherung entsprechender Fälle zu erreichen.

\section{SO-038}

Detection of ALK rearranged non-small cell lung carcinomas by immunohistochemistry: comparison of different antibodies (D5F3 and 5A4), detection systems and automated immunostainers

\section{T. Menter ${ }^{* 1}$, J. Diebold², A.-K. Zimmermann ${ }^{3}$, W. Jochum ${ }^{4}$, B. Baschiera',} R. Epper', S. Grieshaber', L. Tornillo', K. Kerr' ', L. Bubendorf', S. Savic' 'Institute of Pathology, University Hospital Basel, Basel, Switzerland, ${ }^{2}$ Institute of Pathology, Luzerner Kantonsspital, Luzern, Switzerland, ${ }^{3}$ Institute of Surgical Pathology, University Hospital, Zurich, Switzerland, ${ }^{4}$ Institute of Pathology, St. Gallen, Switzerland, ${ }^{5}$ Department of Pathology, Aberdeen, United Kingdom

Aims. Immunohistochemistry (IHC) has become a promising method for prescreening predictive ALK-rearrangements in non-small cell lung carcinomas (NSCLC). Various ALK antibodies, enhanced detection systems and automated immunostainers are available. We therefore aimed to compare the performance of: 1. the $\mathrm{D}_{5} \mathrm{~F}_{3}$ (Cell Signaling, Ventana) and the $5 \mathrm{~A}_{4}$ (Novocastra, Leica) monoclonal antibodies (mAb) and 2. the $5 \mathrm{~A} 4 \mathrm{mAb}$ on two different automated immunostainers (BenchMark, Ventana and BOND-MAX, Leica)

Methods. Sixtyfive formalin-fixed and paraffin-embedded non-squamous NSCLC with available ALK FISH results were retrospectively analysed. The specimens were enriched for ALK FISH-positive NSCLC $(24 / 65,36.9 \%)$. IHC was performed with the OptiView Amplification Kit (Ventana) on BenchMark using the $\mathrm{D}_{5} \mathrm{~F}_{3}$ and the ${ }_{5} \mathrm{~A}_{4} \mathrm{mAb}$, respectively. Additionally IHC with the $5 \mathrm{~A} 4 \mathrm{mAb}$ and the Bond Polymer Refine Detection Kit (Leica) on Bond-MAX were performed. For $\mathrm{D}_{5} \mathrm{~F}_{3}$ any strong IHC-staining $(3+)$ was regarded as a positive result, as suggested by the manufacturer. For $5 \mathrm{~A} 4$ any IHC-staining $(1+, 2+, 3+)$ was regarded as a positive result. The IHC results of all three protocols were compared to the FISH results. In case of a discrepancy the IHC-stained slide and the FISH signals were reviewed.

Results. Fifty-seven out of 65 NSCLC (87.7\%) were evaluable and all showed congruent results for all three IHC protocols used. Twenty-three out of 24 ALK FISH positive NSCLC (95.8\%) were ALK IHC-positive. Only one ALK FISH positive NSCLC (3.2\%) was false negative by IHC. The sensitivity, specificity and positive and negative predictive values for ALK IHC compared to ALK FISH were $96 \%, 100 \%, 100 \%$ and $97 \%$, respectively, irrespective of antibody, detection system and immunostainer used.

Conclusions. $\mathrm{D}_{5} \mathrm{~F}_{3}$ and ${ }_{5} \mathrm{~A}_{4}$ are equally well suited and BenchMark and BOND-MAX immunostainers can both be used for prescreening ALKrearrangements in NSCLC. 


\section{SO-039}

A novel method for simultaneous detection of ALK, RET and ROS-1 rearrangements in non-small cell lung cancer (NSCLC) specimens by means of multiplex amplicon RNA massive parallel sequencing

E. Moskalev ${ }^{* 1}$, S. Merkelbach-Bruse ${ }^{2}$, H.-U. Schildhaus' ${ }^{2}$, J. Frohnauer ${ }^{1}$, C. Boltze ${ }^{3}$, T. Schubert', A. Dimmler ${ }^{5}$, H. König ${ }^{6}$, F. Fuchs ${ }^{7}$, H. Sirbu ${ }^{8}$, R. Rieker', A. Hartmann', F. Haller

'Institute of Pathology, Friedrich-Alexander-University, Erlangen-Nuremberg, Germany, ${ }^{2}$ Institute of Pathology, Cologne, Germany, ${ }^{3}$ Institute of Pathology, Gera, Germany, ${ }^{4}$ Institute of Pathology, Speyer, Germany, ${ }^{5}$ Institute of Pathology, Karlsruhe, Germany, ${ }^{6}$ Practice for Pathology, Ingolstadt, Germany, ${ }^{7}$ Midical Clinic ${ }^{1}$, University of Erlangen-Nuremberg, Erlangen, Germany, ${ }^{8}$ Surgical Clinic, University of Erlangen-Nuremberg, Erlangen, Germany

Aims. Clear benefit from application of tyrosine kinase inhibitors for treatment of non-small cell lung cancer necessitates introduction of robust methods for detection of tyrosine kinase fusions to molecular diagnostics of NSCLC. Most common genetic rearrangements include tyrosine kinase domains of ALK, RET and ROS-1. Given the fact that amplicon based massive parallel sequencing is being increasingly implemented in the routine molecular diagnostic work-up of NSCLCs, we aimed at establishing a multiplex amplicon based next-generation sequencing assay for detection of ALK, RET and ROS-1 fusions, which is feasible for formalin-fixed paraffin-embedded (FFPE) tumour samples. The assay takes advantage of the unbalanced expression of $3^{\prime}-$ and 5 '-portions of the tyrosine kinase mRNAs in rearranged NSCLC cases. Methods. We analysed a total of 52 patient samples that included 22 ALK rearranged cases, 4 RET transclocations, 4 ROS1 translocations, 8 EGFR mutated cases, 6 KRAS mutated samples and 8 cases that harboured no mutations in these genes. NSCLC specimens were obtained from the Institutes of Pathology in Cologne, Erlangen, Gera, Ingolstadt, Karlsruhe and Speyer. Additionally, lung cancer cell lines with defined ALK and ROS1 rearrangements ( $\mathrm{H}_{2228}$ and $\mathrm{HCC}_{7} 8$, respectively) as well as wild type cells $\mathrm{H}_{1299}, \mathrm{H}_{1703}, \mathrm{H}_{1975}, \mathrm{H}_{4} 60$ were included in the study. Plasmids that contain the cloned full length cDNAs of ALK, RET and ROS-1 were employed as a wild type controls for multiplex PCR. Fragments of ALK exons 15-16 (5'-region), ALK exons 24-26 (3'-region), RET exons 3-4 (5'-region), RET exons 17-19 (3'-region), ROS-1 exons 1718 (5'-region), ROS-1 exons 41-42 (3'-region) and hypoxanthine ribosyl tranferase 1 (HPRT1) were amplified in a multiplex PCR. Massive parallel sequencing was performed using the GS Junior Titanium chemistry according to the standard protocols.

Results. The assay could reliably detect the ALK rearranged cases. In contrast, our assay demonstrated generally less specificity regarding RET and ROS1 expression, which could be observed both within cases with and without RET or ROS1 rearrangements. Nevertheless, three of four RET rearranged cases could be clearly detected by our assay. There was no unbalanced expression of ROS-1 3'- and 5'-portions observed, which was most likely due to endogenous expression of the gene.

Conclusions. We established and characterised the performance of multiplex massive parallel sequencing assay for detection of ALK, RET and ROS-1 rearrangements.

\section{SO-040}

European Harmonization Study for the immunohistochemical detection of ALK-gene-rearrangements in non-small cell lung cancer (NSCLC)

M. von Laffert ${ }^{* 1}$, A. Warth ${ }^{2}$, R. Penzel', P. Schirmacher ${ }^{2}$, K. Kerr ${ }^{3}$, G. Elmberger', H.-U. Schildhaus ${ }^{5}$, R. Büttner ${ }^{5}$, F. Lopez-Rios 6 , S. Reu', T. Kirchner', P. Pauwels ${ }^{8}$, K. Specht ${ }^{9}$, E. Drecoll ${ }^{9}$, H. Höfler ${ }^{9}$, D. Aust ${ }^{10}$, G. Baretton $^{10}$, L. Bubendorf ${ }^{11}$, S. Stallmann ${ }^{12}$, A. Fisseler-Eckhoff', A. Soltermann ${ }^{13}$, V. Tischler ${ }^{13}$, H. Moch ${ }^{13}$, F. Penault-Llorca ${ }^{14}$, H. Hager ${ }^{15}$, F. Schäper ${ }^{16}$, D. Lenze', M. Hummel', M. Dietel'

'Pathology, Charité, Berlin, Germany, ${ }^{2}$ Pathology, Heidelberg, Germany, ${ }^{3}$ Pathology, Aberdeen, United Kingdom, ${ }^{4}$ Pathology, Stockholm, Sweden, ${ }^{5}$ Pathology, Cologne, Germany, ${ }^{6}$ Pathology, Madrid, Spain, ${ }^{7}$ Institute of Pathology, Ludwig-Maximilians-University, Munich, Germany, ${ }^{8}$ Pathology, Edegem, Belgium, ${ }^{9}$ Institute of Pathology, Technical University of Munich, Munich, Germany, ${ }^{10}$ Pathology, Dresden, Germany, ${ }^{11}$ Pathology, Basel, Switzerland, ${ }^{12}$ Pathology, Wiesbaden, Germany, ${ }^{13}$ Pathology, Zurich, Switzerland, ${ }^{14}$ Pathology, Clermont-Ferrand, France, ${ }^{15}$ Pathology, Århus, Denmark, ${ }^{16}$ Pathology, Berlin-Buch, Germany

Aims. The reliable identification of non-small cell lung cancer (NSCLC) patients with ALK-gene activation is crucial for the treatment with ALK-TKIs. The studies leading to the approval of this treatment modality were based on fluorescence in-situ hybridization (FISH), which is therefore the current diagnostic gold standard. Since ALK-FISH is labour-intensive, needs experienced personnel and recent studies based on immunohistochemistry (IHC) showed promising results, ALK-FISH might perceptively be replaced by IHC in a reliable and reproducible manner across different laboratories.

Methods. To test an ALK IHC assay in a multi-centre setting (16 European institutes), two tissue microarrays (TMA) consisting of 15 NSCLC cases were independently tested for ALK-protein expression (binary interpretation: positive vs. negative) by each laboratory using the Ventana ALK $\left(\mathrm{D}_{5} \mathrm{~F}_{3}\right)$ Antibody combined with OptiView DAB IHC detection and OptiView Amplification kits. The included cases were unequivocal ALK break-positive (6x), negative (7x) and ALK positive-"borderline" (2x) as demonstrated by FISH. The latter (RT-PCR:EML4-ALK Variant 1 and $3 \mathrm{a} / \mathrm{b})$ showed a low percentage of ALK break-positive cells $(\sim 15 \%)$ being challenging for unequivocal FISH-diagnosis. Prior to TMA-based analysis, each instrument was calibrated and qualified using the VENTANA ALK 2inı Control Slides. Furthermore a webinar-based training was given to all observers.

Results. All 7 ALK FISH-negative cases were homogenously scored as ALK-IHC negative. All 16 participants scored the 2 ALK positive-"borderline" samples as positive according to their ALK protein expression. Concordant IHC interpretation was also noticed in 4 of the 6 unequivocal ALK break positive cases. In the remaining two cases, a weak-heterogeneous ALK-IHC staining was described by some observers, which would have resulted in a subsequent FISH (or PCR) testing in a routine diagnostic setting.

Conclusions. After harmonization of the staining instruments and training of the observers, the ALK- $\mathrm{D}_{5} \mathrm{~F}_{3}$ IHC assay can be regarded as a reliable technique for the detection of ALK protein expression and has the sensitivity to replace FISH. At the moment, it might help as an alternative predictive test in samples with reduced FISH interpretability (minimal tumor content, decalcified/altered tissue, ALK-"borderline" cases). Further experience is needed to implement this test as a standalone pre-screening tool leading to a broadly used, rapid, less costly and reliable ALK-testing. 


\section{SO-041}

\section{The influence of autophagy inhibition on EGFR-TKI resistant non-} small cell lung cancer cells

\section{Y. Chen*, C. Polasky, I. Petersen \\ Institute of Pathology, Friedrich Schiller University, Jena, Germany}

Aims. Epidermal growth factor receptor (EGFR) targeted therapy using EGFR-tyrosine kinase inhibitors (TKIs) such as gefitinib and erlotinib has been recognized as a promising strategy to treatment of patients with advanced non-small cell lung cancer (NSCLC) harbouring activated EGFR mutations. However, the efficacy of EGFR-TKI is significantly decreased by natural and acquired resistance. Evidence showed that upregulation of autophagy could be one of the mechanisms underlying EGFR-TKI resistance. Therefore, the aim of the study was to investigate the influence of autophagy inhibitor chloroquine on gefitinib-resistant lung cancer cell lines.

Methods. Three NSCLC cell lines including H2170 (EGFR Wild type), H1975 (EGFR exon 21 L858R and exon 20 T79oM), and H1650 (EGFR exon 19 E746_A75odel and loss of PTEN expression) were treated with gefitinib, chloroquine, or drug combination. The effects of drug treatment on cellular survival and proliferation were determined by cell viability and colony formation assay as well as apoptotic assay. Western blotting was performed to analyse the alteration of pathways involved. Results. It turned out that chloroquine alone or in combination with gefitinib inhibited cell proliferation rate, the ability to form colony, and caused apoptosis in the NSCLC cell lines tested. Moreover, we found that in the EGFR-TKI sensitive cell line $\mathrm{H}_{2170}$, combined treatment led to inactivation of Akt. In the EGFR-TKI resistant cell line H1975, mTOR was activated after gefitinib, chloroquine, or combined treatment. However, in $\mathrm{H}_{16} 60$ cells, resistant to EGFR-TKI, no clear alteration was observed, indicating other mechanisms/pathways might be involved in enhanced drug toxicity.

Conclusions. Taken together, the results indicate that chloroquine is a potential drug in cancer treatment and a combination therapy of chloroquine with gefitinib might be able to overcome the EGFR-TKI resistance via induction of apoptosis in NSCLC cells.

\section{SO-042}

\section{Molecular analysis of different growth patterns of pulmonary adenocarcinoma}

\section{F. Lasitschka*1, T. Muley' ${ }^{2}$, M. Meister ${ }^{3}$, A. Warth', P. Schnabel', R. Kuner', J. Pfannschmidt ${ }^{2}$, H. Dienemann ${ }^{2}$, H. Zabeck ${ }^{2}$}

${ }^{1}$ Institute of Pathology, University Hospital Heidelberg, Heidelberg, Germany, ${ }^{2}$ Thoraxklinik Heidelberg, Heidelberg, Germany, ${ }^{3}$ Thoraxclinic, University Hospital, Heidelberg, Germany, ${ }^{4}$ German Cancer Research Center, DKFZ, Division of Cancer Epidemiology, Heidelberg, Germany

Ziele. Pulmonale Adenokarzinome der neu definierten Subtypen: lepidisch, azinär, papillär, mikropapillär und solide weisen charakteristische Wachstumseigenschaften und damit auch unterschiedliche klinische Überlebensprognosen auf. Bei Patienten mit lepidischen Adenokarzinomen finden sich hierbei besonders günstige Verläufe, während das solide Wachstumsmuster mit einer besonders schlechten Prognose assoziiert ist. Die zu unterstellenden Unterschiede in den $\mathrm{Ge}$ nexpressionssignaturen zwischen lepidischer und solider Adenokarzinomarchitektur sollen im Rahmen dieser Studie identifiziert und bewertet werden.

Methoden. Aus Kryoschnitten operierter Adenokarzinom-Patienten wurde mittels Lasermikrodissektion Gesamt-RNA von je 10 reinen Proben lepidischer und solider Adenokarzinomanteile isoliert und nach Quantifizierung und Hybridisierung einer Genexpressionsanalyse zugeführt. Aus den Microarraydaten wurden mittels LIMMA-Analyse differenziell exprimierte Gene identifiziert. Die Genontologie-Statistik erfolgte mittels GOstat.
Ergebnisse. Der Vergleich der Genaktivität zwischen den beiden Mustern zeigte 730 hoch- und 113 herunterregulierte Gene. Hieraus wurden zur weiteren immunhistochemischen Validierung zunächst 4 Kandidatengene ausgewählt, die möglicherweise die prognostischen Unterschiede erklären könnten: Hochreguliert im soliden Muster fanden sich u.a. ERRF1, ein Inhibitor des EGFR-Pathways, PFKP und ITPKA, assoziiert mit Tumorprogression; hochreguliert im lepidischen Subtyp ANG (Angiogenin), assoziiert mit Gefäßneubildung, Apoptose und Überleben.

Schlussfolgerung. Die Analyse konnte signifikante Unterschiede in der Aktivität von vorbekannten Karzinom-assoziierten Genen zwischen soliden und lepidischen Adenokarzinomanteilen aufzeigen. Diese und weitere Forschungen zur Validierung könnten helfen, die Unterschiede in Biologie und Prognose der einzelnen Subtypen zu erklären sowie Ansatzpunkte für die weitere Entwicklung neuer Methoden zur immunhistochemischen Identifizierung und ggf. Therapie zu liefern.

\section{SO-043}

Lung neuroendocrine tumors: correlation of ubiquitylation and sumoylation with nucleocytosolic distribution of PTEN

\section{A. Soltermann ${ }^{* 1}$, S. Collaud ${ }^{2}$, V. Tischler', A. Atanassoff', A. Perren ${ }^{3}$,} P. Komminoth ${ }^{4}$, C. Oehlschlegel ${ }^{5}$, W. Weder ${ }^{2}$, H. Moch ${ }^{1}$

'Institute of Surgical Pathology, University Hospital, Zurich, Switzerland, 2Division of Thoracic Surgery, University Hospital, Zurich, Switzerland, ${ }^{3}$ Institut of Pathology, University, Bern, Switzerland, ${ }^{4}$ Institute of Pathology, Triemli Hospital, Zurich, Switzerland, Institut of Pathology, Cantonal Hospital, St. Gallen, Switzerland

Aims. The tumor suppressor phosphatase and tensin homolog (PTEN) is a pleiotropic enzyme, inhibiting $\mathrm{PI}_{3} \mathrm{~K}-\mathrm{Akt}$ signaling in the cytosol and stabilizing the genome in the nucleus. Nucleo-cytosolic shuttling is dependent on reversible post-translational modifications like ubiquitylation and sumoylation. This differential compartmentalization was investigated in lung neuroendocrine tumors (lung NET).

Methods. A multicenter lung NET cohort of 297 patients (314 tissue specimens from 192 surgeries and 122 biopsies) was investigated on two tissue microarrays (TMA). PTEN immunoreactivity was $\mathrm{H}$-scored by two investigators in both nucleus and cytosol using monoclonal antibody $6 \mathrm{H}_{2}$.1. PTEN and 553 gene status was assessed by FISH. Results were correlated with immunoreactivity for $\mathrm{SUMO}_{2} / 3$ (small ubiquitin-related modifier protein 2/3, nucleus and cytosol), USP-7 (herpes virus-associated ubiquitin-specific protease 7 , only nucleus) and overall survival.

Results. The surgery TMA included 58 typical carcinoids (TC), 42 atypical carcinoids (AC), 32 large cell neuroendocrine carcinomas (LCNEC) and 60 small cell lung carcinomas (SCLC). In comparison to the carcinoids, LCNEC and SCLC presented a loss of nuclear and cytosolic PTEN (inter-observer kappa value o.7 for both compartments) together with a loss of PTEN and p53. Concomitantly, a loss of nuclear USP-7 but increase of nuclear and cytosolic $\mathrm{SUMO}_{2} / 3$ was found in the high grade tumors. In the biopsy TMA, PTEN was able to distinguish AC from SCLC. All lung NET showed higher PTEN immunoreactivity in the cytosol than in the nucleus, whereas the greatest nucleo-cytosolic difference due to marked loss of nuclear protein was found for SCLC. Loss of PTEN and USP-7 but increase of $\mathrm{SUMO}_{2} / 3$ correlated with poorer survival (univariate). PTEN was not significant against categorized histology (multivariate). Among carcinoids, loss of cytosolic PTEN (both scores) delimitated a subgroup with poorer survival (all p-values $<0.05$ ). Conclusions. Loss of PTEN on both gene and protein level is associated with the high grade neuroendocrine tumors SCLC and LCNEC but may also define a subgroup of carcinoids with poorer prognosis. The protein loss occurs in both nucleus and cytosol and is possibly influenced by the concomitant decrease of nuclear USP-7. In contrast, sumoylation increases in high grade lung NET, favoring a sequestration mechanism. 

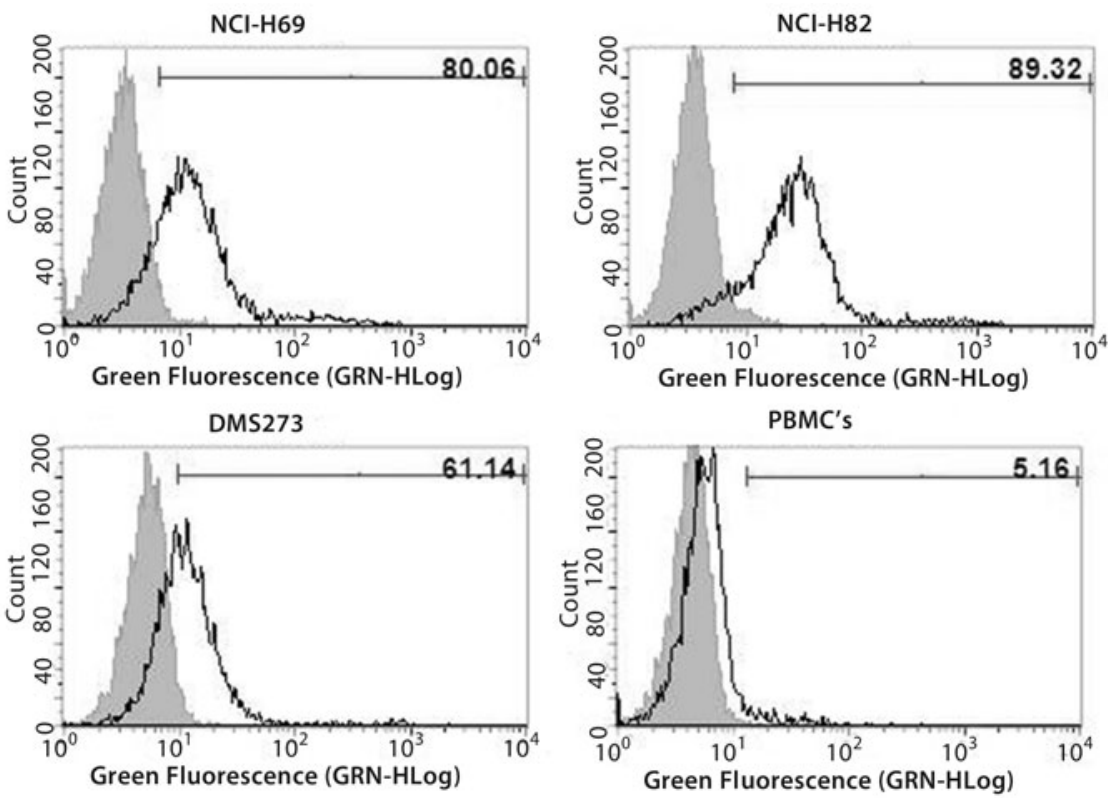

Fig. 1 | SO-044 $<$ Analysis of selected scFv antibody by flow cytometry on different SCLC cell lines. Histograms of different SCLC-cell lines and PBMCs (control) with (black) and without (gray) SCLC specific antibody. The selected antibody bind $61-89 \%$ of SCLC cells compared to $5 \%$ of PBMCs, thereby proofing to be specific

\section{SO-044}

On slide selection on patient SCLC tissue (FFPE) for generation of specific antibodies

\section{A. ten Haaf* ${ }^{*}$ K. Fries, S. Pscherer, S. Gattenlöhner, M.K. Tur Institut für Pathology, Giessen, Germany}

Aims. Because of their high specificity monoclonal antibodies play a major role in immunohistochemistry and targeted cancer therapy. Based on these features the process of production and the detection of new antibodies are very important. An effective method for the generation of new specific antibodies is the phage display technology, in which a repertoire of antibodies displayed on a bacteriophage is used for selection of monoclonal antibodies against a given target. Standard phage display methods use purified proteins, transfected cells or cell lines as target structure. However, the production of recombinant proteins could be very challenging and a suitable target cell line is not always available. Therefore, we wanted to test whether FFPE patient tissue slides, which are typically used in histopathology, can be used for generation of specific antibodies.

Methods. The human Tomlinson phage library was applied on malignant tissue, while healthy tissue was used for subtraction. Exemplarily we use tissue slides of a patient with small cellular lung cancer for selection and normal lung tissue for the depletion of unspecific antibodies. The specificity of the selected antibodies was proven by ELISA on immobilized SCLC cell membranes and by flow cytometry on human SCLC cell lines NCI-H69, NCI-H82 and DMS273. Individual scFv antibodies were sequenced and analyzed using the KABAT database.

Results. 89 out of 176 tested scFv antibodies (49.7\%) showed specific binding activity by ELISA. After sequencing 18 positive antibodies, 9 individual $\mathrm{scFv}$ could be identified. 5 of 6 isolated antibodies proved to be specific for SCLC cells in flow cytometry.

Conclusions. We could demonstrate the generation of specific antibodies against SCLC directly isolated on patient tissue using phage display. This facilitates to bypass the known difficulties with target antigen provision for antibody selection. In addition, this method may also be used to develop targeted drugs for personalized therapy. In a next step the selected $\mathrm{scFv}$ will be verified in immunohistochemistry on different SCLC tissue. For therapeutic use isolated antibodies will be linked to an immunotoxin and their efficacy will be tested in vitro and in vivo (• Fig. 1).

\section{SO-045}

Integrative molecular and anatomical characterization of idiopathic pulmonary fibrosis

J. Wehling ${ }^{* 1}$, P. Braubach', M. Kellner', N. Izykowski', L. Knudsen², A. Haverich ${ }^{3}$, G. Warnecke ${ }^{3}$, J. Vogel-Claussen ${ }^{4}$, H. Kreipe', F. Länger', M. Kühnel', D. Jonigk'

'Institute of Pathology, Hannover Medical School, Hannover, Germany, ${ }^{2}$ Institute for Functional and Applied Anatomy, Hannover Medical School, Hannover, Germany, ${ }^{3}$ Department of Cardiothoracic, Transplantation and Vascular Surgery, Hannover Medical School, Hannover, Germany, ${ }^{4}$ Institute for Radiology, Hannover Medical School, Hannover, Germany

Aims. Idiopathic pulmonary fibrosis (IPF) is a progressive, debilitating disease which leads to respiratory failure due to excessive collagen deposition and destruction of lung architecture. Since the etiology of IPF is poorly understood and no adequate medicinal treatment is available, lung transplantation still remains the only therapeutic option. In IPF there appears to be a correlation between the spatial localization of fibrotic changes and disease progression. The present study characterizes histopathological changes seen in IPF lungs with molecular changes and anatomical/three-dimensional patterns.

Methods. Freshly explanted IPF lungs $(\mathrm{n}=10)$ from patients undergoing lung transplantation were inflated and scanned by computer tomography. Here, representative areas of fibrotic remodeling were identified and sampled. The internal structure of the samples was analyzed in three-dimensional datasets by using scanning laser optical tomography (SLOT). Subsequently, areas of interest were laser-microdissected and a set of fibrosis-associated genes and corresponding endogenous controls were analyzed by real-time PCR and immunohistochemistry. The results were correlated with the individual clinical findings.

Results. The three-dimensional position of fibroblastic foci was visualized properly by using SLOT. By correlating the ${ }_{3} \mathrm{D}$ change patterns with the molecular microenvironment, an integrative model of IPF could be generated.

Conclusions. Correlation of the techniques outlined above (CT, SLOT, microscopy, 3D reconstruction, laser-microdissection, RT-PCR and immunohistochemistry) with clinical changes enables us to characterize IPF on a macroscopic, microscopic and molecular level for the first time. 


\section{Abstracts}
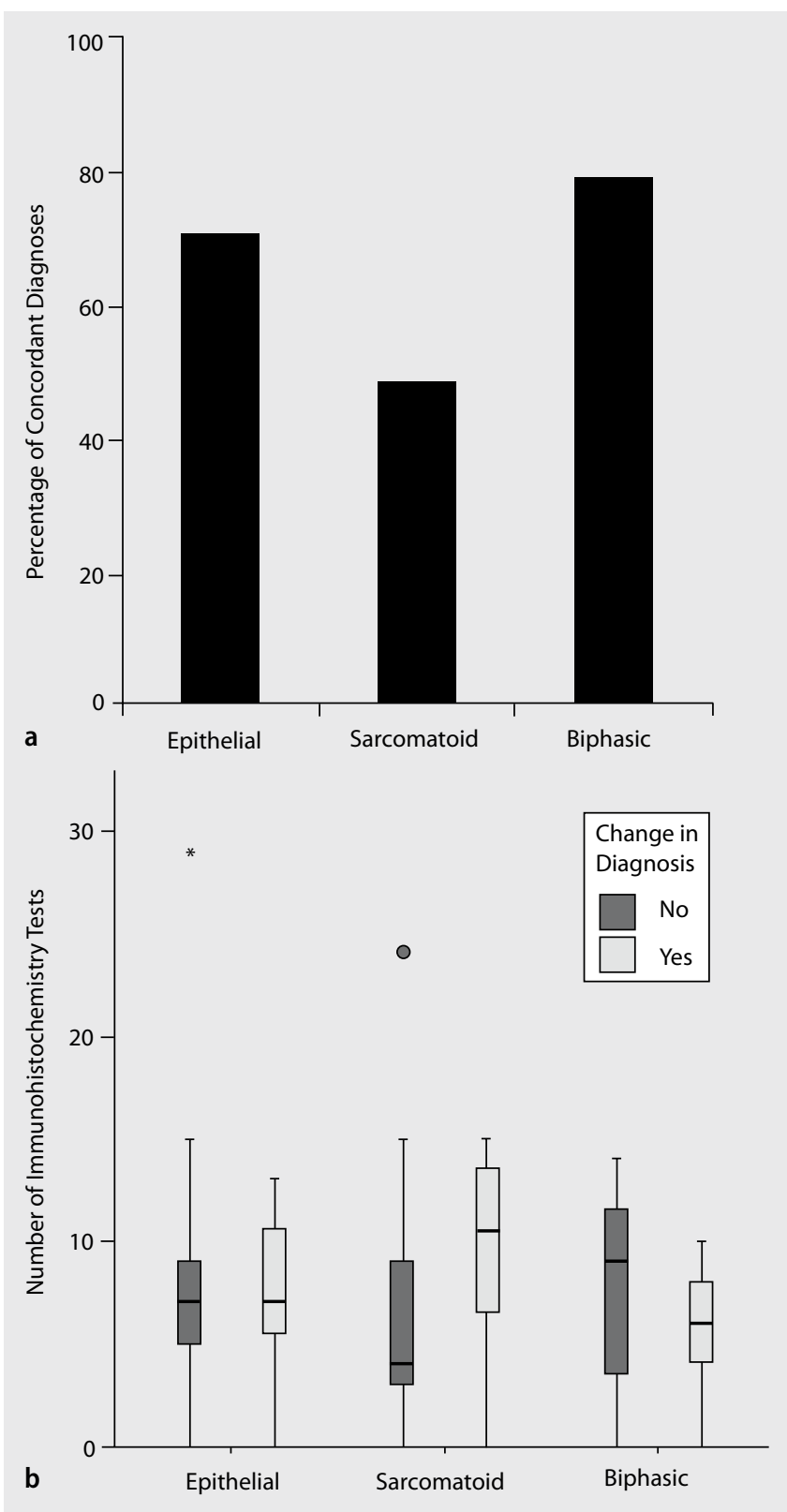

Fig. 1 | S0-046 $\Delta$ a) Sarcomatoid mesothelioma (MM) was more problematic than other MM types $(p=0.016)$. b) Immunohistochemistry performed by the referring laboratory did not improve diagnosis. Mann Whitney $\mathrm{U}$ test

\section{SO-046}

\section{Difficulties and controversies in mesothelioma diagnosis-} the Australian Experience

\section{S. Klebe ${ }^{* 1}$, D. Henderson ${ }^{1,2}$}

'Department of Anatomical Pathology, SA Pathology, Flinders University, Adelaide, Australia, ${ }^{2}$ Department of Anatomical Pathology, Flinders University, Adelaide, Australia

Aims. Pleural neoplasms may be difficult to diagnose because they must be distinguished from metastatic malignancy involving the pleura, and from benign reactive processes causing pleural thickening, as well as unusual other (non-mesothelial) primary neoplasms. Primary pleural neoplasms are uncommon, with secondary involvement being more frequent. Australia has the highest incidence of mesothelioma in the world. Flinders Medical Centre is a recognised expert referral centre for mesothelioma. We discuss the role of expert opinion, and consider current topics and controversies in the diagnosis of mesothelioma, including the usefulness of immunohistochemical markers, new prognostic markers, the role of molecular biology in diagnosis and cytology-only diagnosis. We also consider those other pleural lesions that must be differentiated from malignant mesothelioma.

Methods. A total of 275 consecutive referred cases were retrieved from the files of the Department of Anatomical Pathology at Flinders Medical Centre.

Results. Overall, there was only fair agreement $(\kappa=0.3155)$ between the original diagnosis and the „expert“ opinion. Sarcomatoid mesotheliomas presented particular difficulty, and immunohistochemistry is only of limited usefulness in this instance.

Conclusions. The diagnosis of mesothelioma is difficult, especially in sarcomatoid subtypes or in cases with other unusual histologies. Immunohistochemistry is of limited usefulness, but molecular techniques may be helpful in selected cases (• Fig. 1).

\section{SO-047}

Inhibition of Aquaporin 1 in malignant mesothelioma: in vitro effects and findings in a heterotopic mouse model

\section{S. Klebe ${ }^{* 1}$, J. Driml', K. Griggs', Y.Y. Cheng' ${ }^{2}$, G. Reid ${ }^{2}$}

'Department of Anatomical Pathology, SA Pathology, Flinders University, Adelaide, Australia, ${ }^{2}$ Asbestos Disease Research Institute, Bernie Banton Centre, Sydney, Australia

Aims. Aquaporin $1(\mathrm{AQP} 1)$ is an independent prognostic marker for malignant pleural mesothelioma (MPM). AQP1 is a trans-membrane protein involved in fluid homeostasis, cell proliferation and motility. AQP1 has been implicated in various aspects of tumour development. The aim of the current study was to determine the functional role of AQP1 in MPM, using in vitro and in vivo models, and to ascertain whether the specific inhibition of AQP1 had potential for treatment of mesothelioma.

Methods. Primary MPM cells obtained from patients' pleural effusions and a MPM cell line (NCI-H226) were subjected to a specific AQP1 blocker $\left(\mathrm{AqBO}_{50}\right)$ and $\mathrm{AQP1}$ SiRNA knockdown. Cell proliferation and colony formation using MTS and anchorage independent assays were assessed. Levels of AQP1 expression were determined by immunohistochemistry and RT-PCR. The influence of AQP1 on tumour growth in vivo was studied using a heterotopic mouse model. Briefly, $1 \times 106$ NCI-H226 cells were injected subcutaneously in the hind flank of $\mathrm{BALB} / \mathrm{C}$ nude mice and allowed to develop before daily intra-tumour injections of AQP1 blocker. Tumour size was measured daily.

Results. AQP1 expression correlates with cell proliferation in primary MPM cells $\left(\mathrm{R}_{2}=0.69\right)$. Inhibition of $\mathrm{AQP} 1$ with the specific blocker or SiRNA in MPM cells (both NCI-H226 and primary, $\mathrm{p}<0.05$ ) significantly decreased cell proliferation. AQP1 blockade also decreased number and size of colonies $(\mathrm{p}<0.05)$ formed after NCI-226 cells were subjected to anchorage independent growth. However, whilst there was initial reduction in tumour size in vivo, in the heterotopic mouse model the size of tumours was not significantly altered over 2 weeks using $20 \mu \mathrm{M}$ of AQP1 blocker daily locally compared to an untreated control $(n=6$ for each group).

Conclusions. Our results suggest that $\mathrm{AQP} 1$ plays a functional role in MPM. Modulation of AQP1 decreases cell proliferation and colony formation in vitro, but there was no significant alteration in the growth of MPM tumours in a heterotopic mouse model. Further dose-finding studies, different types of application and combinatorial therapies are required to further investigate the role of $\mathrm{AQP}_{1}$ in $\mathrm{MPM}$ and the potential clinical benefits of a specific AQP1 blocker in the treatment of MPM. 


\section{SO-048}

Inflammatory pseudotumor of the lung revisited

\section{A. Agaimy ${ }^{* 1}$, P. Ströbel', A. Morresi-Hauf', R.J. Rieker', A. Hartmann', I.-M. Schaefer ${ }^{2,4}$}

IInstitute of Pathology, Friedrich-Alexander-University, Erlangen-Nuremberg, Germany, ${ }^{2}$ Institute of Cellular and Molecular Immunology, Georg August University of Göttingen, Göttingen, Germany, ${ }^{3}$ Institute of Pathology, Asklepios Fachkliniken München-Gauting, Gauting, Germany, ${ }^{4}$ Department of Pathology, Brigham and Women's Hospital, Harvard Medical School, Boston, United States

Aims. Inflammatory pseudotumor (IPT) of the lung has long been considered the prototype of inflammatory myofibroblastic tumor (IMT), based on histological features and expression of alpha smooth muscle actin. Over the years, several names have been used for these lesions including IPT, IMT, plasma cell granuloma, and others. Although several etiologic and genetic aspects of different tumors included under "IPT" have been clarified during the last two decades, the term pulmonary IPT still remains in use until now.

Methods. We reviewed our files for lesions diagnosed as IPT in the lung over the last 10 years. Diagnosis was revised to IgG4-related IPT if unequivocal histologic, immunohistochemical, and clinical features were present. Diagnosis of IMT was based on absence of features of IgG4-related disease and presence of features characteristic of IMT of soft tissue including ALK expression.

Results. Ten cases were retrieved ( 6 men and 4 women at a mean age of 42 years). Two children (age 4 and 10 yrs) were included. All lesions fulfilled criteria for diagnosis of IgG4-related IPT and none was true IMT (all ALK negative). Three patients had other organ manifestations of IgG4-related disease. Lung manifestations preceded those in other organs in one patient and occurred years after extrapulmonary manifestation in the other two patients. Two tumors recurred several times over 5 years. Six cases were initially diagnosed as IMT although ALK immunostaining was not performed at that time. One tumor was considered as being of infectious etiology. Only three recent cases were correctly diagnosed as IgG4-related on initial evaluation. Histologic examination showed strikingly variable hyaline fine reticular (storiform) fibrosclerosis with occasionally keloid-like quality. Obliterative angiitis was observed in several cases. IgG4 immunostaining revealed clusters of positive plasma cells ranging from $>10$ to $>100 / \mathrm{HPF}$ with a $\operatorname{IgG}_{4}: \operatorname{IgG}$ ratio of $>40 \%$ in all cases.

Conclusions. This study demonstrates that the majority of lung lesions diagnosed in the past as IMT or IPT represents IgG4-related IPT with or without other organ manifestations of this systemic disorder. ALK positive IMT similar to those occurring in soft tissue, mesentery and urinary tract are probably very rare in the lung. Immunostaining with ALK, IgG4, and IgG are mandatory for appropriate classification of pulmonary IPT. Potentially misleading designations, like plasma cell granuloma, should be avoided.

\section{SO-049}

Use and misuse of the TNM classification of lung tumours

\section{Wittekind*}

\section{Institut für Pathologie, Leipzig, Germany}

The TNM classification of lung tumours has undergone some changes in the 2010 published 7 th edition of the TNM Classification of malignant tumours. The changes are based on findings of the IASLC and concern definitions of $\mathrm{T}$ and $\mathrm{M}$ categories as well as stage groups. They do include a better description of regional lymph nodes base on a uniformly accepted definition as edited by the IASLC. The changes may offer problems in the use of the definitions and these will be discussed. In addition questions and answers from the UICC TNM Help Desk showing typical examples of use and misuse will be presented.

\section{AG Knochen-, Gelenk- und Weichgewebspathologie}

\section{SO-050 \\ Endoglin expression differentiates between septic and aseptic endoprosthesis loosening}

\section{B. Hermanns-Sachweh ${ }^{* 1}$, T. Mumme'2, S. Gravius ${ }^{3}$, P. Jansen \\ 'Institute of Pathology, RWTH University, Aachen, Germany, ${ }^{2}$ Orthopedic Klinic Neuss, Neuss, Germany, ${ }^{3}$ Department of Orthopedics, University Hospital Bonn, Bonn, Germany}

Aims. Loosening of endoprostheses, either septic or aseptic, is a serious complication. A better understanding of pathogenesis may help to prevent or treat this complication. Previous investigations revealed increased vascularization in the periprosthetic membrane in septic endoprosthesis loosening. Based on these findings we sought to investigate angiogenesis in septic and aseptic endoprosthesis loosening.

Methods. Tissue samples from endoprosthetic redo surgery were investigated histologically for inflammation, wear particles, vascularization and fibrosis. To identify vascular structures antibodies against CD 31, CD 34, factor VIII and CD 105 (endoglin) were applied for immunohistochemical investigations. According to a consensus classification of Morawietz the tissue samples were divided into four types: type I (wear particle induced, $\mathrm{n}=11$ ) type II (infectious type, $\mathrm{n}=7$ ), type III (combined type, $\mathrm{n}=7$ ) and type IV (indeterminate type, $\mathrm{n}=7$ ).

Results. Patients with septic endoprosthesis loosening (type II) showed a pronounced infiltration of neutrophil granulocytes in the periprosthetic membrane and an enhanced neovascularization indicated by positive immunoreaction with antibodies against CD 105 (endoglin). Tissue samples classified as type I, type III and type IV showed significantly less immune reaction for $\mathrm{CD} 105$ (endoglin).

Conclusions. In septic and aseptic periprosthetic membranes inflammation and vascularization are found in different expression. However, in aseptic loosening, there is nearly no neovascularization with CD 105 positive immune reaction. Therefore, Endoglin (CD 105) expression allows for differentiation between septic and aseptic endoprosthesis loosening.

\section{SO-051}

\section{TERT promoter mutations in soft tissue sarcomas are recurrent in myxoid/round cell liposarcomas}

G. Mechtersheimer ${ }^{* 1}$, M. Renner', C. Kölsche ${ }^{2}$, R. Penzel', A. von Deimling², W. Hartmann ${ }^{3}$, E. Wardelmann ${ }^{3}$, P. Schirmacher

IInstitute of Pathology, Heidelberg, Germany, ${ }^{2}$ Department of Neuropathology, Heidelberg, Germany, ${ }^{3}$ Gerhard-Domagk Institute of Pathology, Münster, Germany

Aims. Telomeres are structures at the physical ends of chromosomes that maintain chromosomal integrity intact. In normal somatic cells, the telomere length progressively shortens with cell division, finally leading to replicative senescence. The vast majority of cancer cells maintain their telomeres due to up-regulation of the enzyme telomerase reverse transcriptase (TERT), allowing their durable capacity to divide. Recently, two recurrent point mutations in the TERT promoter region (C228T and $\mathrm{C} 250 \mathrm{~T}$ ) have been found in human cancers, leading to a new transcription binding site for ETS transcription factors, increased induction of TERT, and subsequently to immortalization. This study was conducted to investigate the prevalence of TERT promoter mutations in a comprehensive series of over 300 soft tissue sarcomas, covering 13 (sub)types.

Methods. 315 sarcoma samples, which were carefully subtyped according to the current WHO classification by means of histopathology in conjunction with immunohistochemistry and molecular analysis, were collected from the archives of the Institute of Pathology, University of 
Heidelberg. A $193 \mathrm{bp}$ fragment of the TERT promoter region covering the hot-spot mutations at positions 1,295,228 (C228T) and 1,295,2560 (C250T) was amplified, and direct sequencing of the PCR products was performed for the forward and reverse strand on an ABI 3500 genetic analyzer.

Results. TERT promoter mutations were detected in 32/315 tumors, and comprised of 29 cases with C228T mutation and 3 cases with C250T mutation. TERT promoter mutations were highly recurrent in myxoid/ round cell liposarcomas $(25 / 36 ; 69.5 \%)$, and were more frequently found in myxoid as compared to round cell subtypes $(p=0.0485)$. Yet, the TERT promoter mutation status was not associated with tumor grading or staging. TERT promoter mutations were also found in 4/32 solitary fibrous tumors. Among the other 247 sarcoma samples which e.g. included 61 dedifferentiated and 15 pleomorphic liposarcomas, the presence of TERT promoter hot spot mutations was confined to $2 / 35$ malignant peripheral nerve sheath tumors and $1 / 30$ synovial sarcomas.

Conclusions. TERT promoter hot spot mutations are recurrently found in myxoid/round cell liposarcomas, especially in the myxoid subtype, and in a subset of solitary fibrous tumors, while the prevalence of these mutations is generally low in sarcomas.

\section{SO-052}

Hemophilic arthropathy: MALDI (matrix-assisted laser desorption/ ionization) imaging mass spectrometry (IMS) data

\section{Kriegsmann ${ }^{* 1}$, R. Casadonte ${ }^{2}$, T. Randau 3 , S. Gravius ${ }^{3}$, S.-O. Deininger ${ }^{4}$, M. Otto ${ }^{2,5,6}$, J. Kriegsmann ${ }^{2,5,6}$ \\ 'University of Heidelberg, Trier, Germany, ${ }^{2}$ Proteopath GbR, Trier, Germany, ${ }^{3}$ Department of Orthopedics and Joint surgery/University Bonn, Bonn, Germany, ${ }^{4}$ Bruker Daltonik GmbH, Bremen, Germany, ${ }^{5}$ Center for Histology, Cytology and Molecular Diagnostics, Trier, Germany, ${ }^{6}$ Institute of Molecular Pathology, Trier, Germany}

Aims. Arthropathy as a result of repeated joint bleeding is a severe complication in patients with hemophilia. In the evaluation of synovial tissue specimens, histology alone is non-specific and there is a considerable morphological overlap with other joint diseases. There are several biomarkers found to be involved in the disease process such as iron, ferritin and fibrinogen molecules that are abundant in high concentration in blood $[1,2]$. We applied MALDI IMS as a new technique to show the distribution of various proteins, at the peptide level, that have been suggested to play a key role in the pathogenesis of the disease.

Methods. Synovial tissue of 6 patients with hemophilic arthropathy were sectioned and stained with iron staining (Berlin-blue). A serial section of each sample was subjected to in situ trypsin digestion at $37^{\circ} \mathrm{C}$ for $1.5 \mathrm{~h}$ in a humidity chamber after deparaffination and antigen retrieval. Trypsin solution $(0.1 \mu \mathrm{g} / \mu \mathrm{l})$ was deposited on the tissue sections by vibrational spraying (ImagePrep, Bruker Daltonik $\mathrm{GmbH}$ ). Matrix solution ( $7 \mathrm{mg} / \mathrm{ml}$ in 50/50 acetonitrile $/ 0.5 \%$ TFA) was deposited onto digested sections using the same devise. MALDI imaging data were acquired at a spatial resolution of $200 \mu \mathrm{m}$ using a Bruker Autoflex Speed TOF/TOF system and visualized with FlexImaging software. Spectral analysis was performed with FlexAnalysis 3.3 and ClinProTools 3.3 software programs (Bruker Daltonik $\mathrm{GmbH}$ ).

Results. We detected several peaks predictive in mass to charge ratio with ferritin light (m/z 1608) and heavy chain (m/z 1345), alpha- (m/z 1071) and beta- ( $\mathrm{m} / \mathrm{z} 1274)$ hemoglobin subunits, truncated coagulation factor VIII peptide ( $\mathrm{m} / \mathrm{z} 1502,1176)$, beta- and gamma fibrinogen peptides (m/z 980, 1032, 1117 and 1683), and annexin A2 (m/z 1111, 1268, 146o, 2164). Additionally, the distribution of these proteins in synovial tissue sections was demonstrated.

Conclusions. MALDI IMS detected and mapped specific proteins in the synovial membrane of patients with hemophilic arthropathy that are probable drivers in the pathogenesis of the disease.

Literatur
1. Roosendaal G et al (1999) Pathology of synovitis and hemophilic arthropathy 2. Valentino LA et al (2008) Blood-induced joint disease: the confluence of dysregulated oncogenes, inflammatory signals, and angiogenic cues

\section{SO-053}

STAT6 immunohistochemistry in the differential diagnosis of tumors with haemangiopericytoma-like growth pattern

A. Agaimy*1, H. Taubert ${ }^{2}$, s. Barthelmess' ${ }^{1}$ H. Geddert ${ }^{3}$, C. Boltze ${ }^{4}$, A. Hartmann', F. Haller

'Institute of Pathology, Friedrich-Alexander-University, Erlangen-Nuremberg, Germany, ${ }^{2}$ Department of Urology, Friedrich-Alexander-University, Erlangen-Nuremberg, Germany, ${ }^{3}$ Institute of Pathology, St. Vincent's Hospital, Karlsruhe, Karlsuhe, Germany, ${ }^{4}$ Institute of Pathology, SRH-Klinikum, Gera, Gera, Germany

Aims. Originally, "haemangiopericytoma" (HPC) was defined as a tumor showing endothelial-lined tubes and sprouts and a pattern of welldeveloped branching "staghorn" thick-walled vessels with perivascular fibrosis. However, it has been found that this pattern is not specific for HPCs. Instead, the repeated observation of this feature in other neoplasms has challenged the originality of this tumor entity, which has been merged with tumor from the solitary fibrous tumor (SFT) spectrum in the recent WHO Soft Tissue Tumor classification editions. Only recently, recurrent gene fusions involving the genes $\mathrm{NAB}_{2}$ and STAT6 have been identified in tumors from the SFT/HPC spectrum. Notably, the fusion event leads to nuclear translocation and accumulation of the resulting chimeric fusion protein. In contrast, the wild type form of STAT6 protein is localized in the cytoplasm with relatively low abundance.

Methods. A series of 20 tumors including fourteen SFTs/HPCs and six other neoplasms with HPC-like pattern was collected from the archives from our institutes. Immunohistochemistry for STAT6 was performed, and both the staining intensity and the localization were noted. Additionally, a series of 78 soft tissue neoplasms with well-established diagnoses including liposarcomas, leiomyosarcomas, rhabdomyosarcomas and synovial sarcomas was stained using tissue micro arrays.

Results. All fourteen SFTs/HPCs showed a strong nuclear staining for STAT6, while none of the other tumors did. Some liposarcomas showed a faint cytoplasmic staining, which was clearly different from the nuclear signal in the SFTs/HPCs. Notably, six neoplasm with haemangiopericytoma-like growth patterns were negative for STAT6 immunohistochemistry.

Conclusions. STAT6 immunohistochemistry is a useful and specific marker to establish the diagnosis of SFT/HPC, and is especially helpful in other neoplasms with haemangiopericytoma-like growth pattern.

\section{SO-054}

Histopathologic evaluation of passive structures in shoulder instability

\section{Morawietz ${ }^{* 1}$, S. Pauly ${ }^{2}$, M. Scheibel ${ }^{2}$}

'Diagnostik Ernst von Bergmann, Department of Pathology, Potsdam, Germany, ${ }^{2}$ Center for Musculoskeletal Surgery, Charité University Hospital, Humboldt University Berlin, Berlin, Germany

Aims. This study aims to determine different histopathologic stages of shoulder instability by assessing biopsies of passive stabilizing structures. Possible correlations between histopathologic findings and clinical parameters were investigated.

Methods. Thirty consecutive patients ( 4 female/26 male, mean age 33 years) with clinically evident anterior shoulder instability received arthroscopic procedures, during which the passive stabilizing structures of the glenohumeral joint (labrum, anterior band of the inferior glenohumeral ligament) were sampled. Biopsies were examined using routine 
stains as well as immunohistochemistry and in-situ-hybridization for Decorin. Clinical data such as age, total number of dislocations and period since initial dislocation were tested for statistical correlation with the following histopathological parameters: 1) inflammatory infiltrate, 2) lipomatous change, 3) vascular proliferation, 4) tissue fragmentation and 5) cellularity.

Results. The histopathologic evaluation showed only little variation between all samples. Some changes regarding cellularity, matrix swelling and collagen fiber disruptions could be observed, but these were not significant. Immunohistochemical analysis revealed a net-like expression of Decorin. Clinical parameters were statistically independent from all five histopathological parameters. Especially, no significant differences were found in patients with one dislocation vs. more than one dislocations.

Conclusions. In contrast to macroscopic findings among respective grades of shoulder instability, the present study did neither detect different histopathological stages of shoulder damage nor a correlation between clinical items and histopathological findings. Therefore, the histopathologic interpretation of samples from passive stabilizing structures of the shoulder, especially regarding insurance questions, has to be done with appropriate care.

\section{SO-055}

Sclerosing epithelioid fibrosarcoma/low-grade fibromyxoid sarcoma: two difficult-to-diagnose cases confirmed by MUC4 immunostaining and FUS-FISH

\section{A. Agaimy*, J. Frohnauer, A. Hartmann, F. Haller Institute of Pathology, Friedrich-Alexander-University, Erlangen-Nurem- berg, Germany}

Aims. Low-grade fibromyxoid sarcoma (LGFMS; synonym: hyalinizing spindle cell tumor with giant rosettes) is rare special subtype of fibrosarcoma characterized by alternating collagenized fascicles of spindle cells and hypocellular myxoid areas lacking significant atypia. Giant collagen rosettes surrounded by cellular aggregates are infrequent but if present represent a strong clue to diagnosis. Sclerosing epithelioid fibrosarcoma (SEF) on the other hand is a less frequent fibrosarcoma subtype that features rounded carcinoma-like epithelioid cells within sclerotic stroma. To date, ca. 100 cases of SEF were reported. Occurrence of tumors with hybrid features of LGFMS and SEF suggested histogenetic relationship between the two which could be confirmed by detection of FUS-translocation in both entities.

Methods. We describe clinicopathological and molecular features of two challenging cases.

Results. The first patient was a 23 years female with $22 \mathrm{~cm}$ kidney tumor diagnosed as "adult nephroblastoma", "anaplastic malignant tumor of kidney" and "highly malignant mesenchymal tumor, NOS" in different institutions. She developed skeletal and pulmonary metastases and died of disease 6 years later. Histological examination at our institution showed characteristic features of SEF on H\&E stained slides warranting immunostaining for $\mathrm{MUC}_{4}(+)$. Diagnosis of SEF was then confirmed by FISH. The other patient was a 78 -year-old male with clear cell renal cell carcinoma (RCC) resected in 1995 followed by pancreatic metastasis (resected in 2013). As incidental radiological finding during examination for RCC metastasis, a nodular lesion was seen near the major trochanter of the femur. A core needle biopsy showed bland looking hypocellular fibrous tissue lacking any atypia or mitoses but with focal myxoid changes. Immunostaining for $\mathrm{MUC}_{4}$ again was strongly positive as well as FUS FISH. Tumor resection with clear margins was conducted. Histological examination confirmed LGFMS without giant rosettes or SEF-like areas.

Conclusions. These cases illustrate that: 1) familiarity with the characteristic histomorphology of rare sarcoma types facilitates diagnosis even on core needle biopsy; 2) lack of familiarity with these uncommon but distinctive patterns precludes diagnosis even after tumor resection; 3 ) available novel diagnostic tools can help to establish diagnosis in difficult cases, and 4) fully bland histology does not exclude malignancy as biological assessment is often only possible if the entity is defined.

\section{Inflammation und Karzinogenese - Postersession}

\section{FR.2-049}

Pathogenesis of pouchitis-analysis of adherent junction proteins

\section{F. Lasitschka ${ }^{* 1}$, M. Tossing ${ }^{1}$, M. Kadmon ${ }^{2}$, C. Leowardi-Bauer 2}

'Institute of Pathology, University Hospital Heidelberg, Heidelberg, Germany, ${ }^{2}$ Department of Surgery, University Hospital Heidelberg, Heidelberg, Germany

Ziele. Die Pouchitis, als Entzündung der Ileummukosa des Pouches, ist eine der Hauptkomplikationen nach restaurativer Proktokolektomie und ileoanaler Pouchanlage (IAP) bei Colitis-ulcerosa(CU)-Patienten. Die Ausprägung der Symptomatik variiert und kann den Patienten jedoch stark in seiner Lebensqualität beeinträchtigen. Bei langdauernder Entzündung der Ileummucosa des Pouches wurde in Einzelfällen eine Entwicklung von prämalignen Epitheldysplasien und manifesten Karzinomen, analog zur Kolonmukosa beschrieben. In der Literatur finden sich Hinweise darauf, dass pathogenetische Mechanismen der Grunderkrankung Colitis ulcerosa in Zusammenhang mit einer gestörten Reaktion auf bakterielle Antigene in der Pathogenese der Pouchitis zugrunde liegen. Einen wichtigen Mechanismus stellt hierbei eine Störung der intestinalen Barriere dar, welche aus einer Reihe von antimikrobiellen Peptiden der intestinalen Mukosa, dem Mukus selbst, „tight junctions" zwischen den intestinalen Epithelzellen sowie dem mukosalen Immunsystem gebildet wird.

Methoden. Screening mittels RTqPCR-Profiler-Arrays auf eine Dysregulation adhärenter Junktionsproteine in einem definierten Kollektiv von Pouchitispatienten. Validierung von Zielgenen mittels qPCR an einem unabhängigen Kollektiv. Analyse der Zielantigene mittels Immunhistochemie.

Ergebnisse. Pouchitispatienten mit Colitis ulcerosa zeigen eine deutliche Dysregulation verschiedenster adhärenter Junktionsproteine bei der schwergradigen Pouchitis im Vergleich zum unauffälligen Pouch und zu Pouchbiopsaten von FAP-Patienten.

Schlussfolgerung. Die Pouchitis bei Colitis ulcerosa Patienten ist im Vergleich zu FAP-Patienten durch eine Dysregulation adhärenter Junktionsproteine gestört, die sich schon im unauffälligen Pouch manifestiert und damit eine fundamentalen Unterschied zwischen den beiden Krankheitsentitäten darstellt.

\section{FR.2-050}

The stimulating effect of Interleukin- 6 in TMPRSS2-ERG positive prostate cancer

C. Merz ${ }^{* 1}$, M. Nowak', W. Vogel', M.A. Svensson' ${ }^{2}$ J. Carlson², O. Andrén', N. Wernert ${ }^{3}$, G. Kristiansen ${ }^{3}$, S. Perner ${ }^{1}$

'Institute of Pathology, Department of Prostate Cancer Research, University hospital Bonn, Bonn, Germany, ${ }^{2}$ University Hospital of Örebro, Örebro, Sweden, ${ }^{3}$ Institute for Pathology, Bonn, Germany

Aims. Prostate cancer is the most frequently diagnosed cancer in men in the western world. About $50 \%$ of all cases are associated with the TMPRSS 2 -ERG gene fusion resulting in an overexpression of the ERG transcription factor. The clinical significance of the gene fusion remains still unclear. The pleiotropic cytokine interleukin-6 (IL-6) however is frequently overexpressed with advanced and metastatic prostate cancer. In this study we addressed the role of ERG in IL- 6 production. Moreover, we aimed at understanding interactions between signaling 
pathways downstream of the IL-6 receptor (CD126) and the androgen receptor (AR).

Methods. Using prostatectomy in a tissue microarray format of $116 \mathrm{pa}-$ tients ( $n=90$, prostate cancer; $n=26$, benign prostate tissue) from Cohort of Swedish Men we analyzed the co-expression of ERG and IL-6 using immunohistochemical double staining.

Results. Expression of ERG is strongly correlated with IL-6 expression $(\mathrm{r} 2=0.7656 ; \mathrm{p}<0.0001)$. Forced expression of ERG in the prostate tumor cell lines PC-3 and LNCaP resulted in significantly increased secretion of IL-6. Antibody-mediated blockade of CD126 in PC-3 cells significantly reduced the production of IL-6, suggesting a feed-forward mechanism driving the IL- 6 production. Treatment with dihydrotestosterone (DHT) in hormone-sensitive LNCaP cells increased the secretion of IL-6, conversely treatment with IL- 6 exhibited a translocation of the AR to the nucleus. These data point to a cross-talk between the signaling pathways elicited by IL- 6 and DHT.

Conclusions. In summary, we determined the strong co-expression of ERG and IL- 6 in a large patient cohort and demonstrated at which point the IL- 6 and AR receptor pathway interact.

\section{FR.2-051}

The exportin-2/importin- $a-1$ transport cycle is linked to p53 in liver cancer

J. Winkler ${ }^{* 1}$, A. Ori ${ }^{2}$, C. Sticht ${ }^{3}$, E. Eiteneuer ${ }^{1}$, K. Holzer ${ }^{1}$, A. Andres-Pons' ${ }^{2}$, R. Geffers ${ }^{4}$, M. Beck², V. Ehemann', N. Gretz ${ }^{3}$, K. Breuhahn', T. Longerich', P. Schirmacher', S. Singer ${ }^{1}$

${ }^{1}$ Institute of Pathology, Heidelberg, Germany, ${ }^{2}$ European Molecular Biology Laboratory, Heidelberg, Germany, ${ }^{3}$ Medical Research Centre, Mannheim, Germany, ${ }^{4}$ Helmholtz Centre for Infection Research, Braunschweig, Germany

Aims. Nucleocytoplasmic transport is an essential, highly efficient, and selective process in eukaryotic cells. The exchange of macromolecules between the nucleus and the cytoplasm occurs exclusively through the nuclear pore complex and is mediated by nuclear transport receptors of the karyopherin family (including exportins and importins). Nuclear transport factors have been linked to malignant transformation previously, however, their role in hepatic tumorigenesis remains poorly understood. Here, we studied the functional relevance and regulation of exportin-2 [= Cellular Apoptosis susceptibility (CAS)] and its transport substrate importin- $\alpha-1$ in liver cancer.

Methods. We combined gene expression array and tissue microarray (TMA) data derived from HCC patient samples to identify significantly deregulated transport factors. As the most promising candidates exportin-2/CAS and importin- $\alpha-1$ were functionally characterized in HLE and HLF cells by using a variety of in vitro assays combined with RNAi. Identification of downstream targets and upstream regulators involved gene expression arrays and quantitative mass spectrometry.

Results. The expression profiling of nuclear transport factors revealed that exportin-2/CAS and importin- $\alpha-1$ are significantly overexpressed in HCC patient samples compared to non-tumorous liver samples. Immunohistochemical analyses of TMAs confirmed high expression levels of exportin-2/CAS in the majority of HCCs and indicated a correlation with tumor grading $(\mathrm{r}=0.4 ; \mathrm{p}<0.01)$ and nuclear accumulated $\mathrm{p} 53(\mathrm{r}=0.4 ; \mathrm{p}<0.01)$. Functional analyses in vitro showed a decrease in tumor cell growth paralleled by an increase in apoptotic cell death after siRNA-mediated depletion either of exportin-2/CAS or importin- $\alpha-1$. Gene expression arrays upon exportin-2/CAS knockdown and further validation experiments identified the $\mathrm{X}$-linked inhibitor of apoptosis (XIAP) as a mediator of the pro-survival function of exportin-2/CAS. Finally, by using shotgun proteomics we observed distinct expression changes of karyopherins upon induction of wild-type p 53 with exportin-2/CAS and importin- $\alpha-1$ being significantly repressed.

Conclusions. Our data indicates that the CAS/importin- $\alpha-1$ transport cycle plays a pro-survival role in liver cancer cell lines that is at least partially linked to XIAP. Furthermore, we found that CAS and importin- $\alpha-1$ are targets of p53-mediated repression, which may represent a novel aspect of $\mathrm{p} 53^{\text {s }}$ s ability to control tumor cell growth in hepatocarcinogenesis.

\section{FR.2-052}

\section{Loss of prolyl hydroxylase- 2 in myeloid cells and T-lymphocytes impairs tumor development}

\section{B. Wielockx*, S. Mamlouk, C. Jakob, G. Baretton}

Institute of Pathology, Dresden, Germany

Aims. The tumor microenvironment plays a pivotal role during cancer development and progression. The balance between suppressive and cytotoxic responses of the tumor immune microenvironment has been shown to have a direct effect on the final outcome in various human and experimental tumors. Recently, we demonstrated that the oxygen sensor HIF-prolyl hydroxylase 2 ( $\mathrm{PHD}_{2}$ ) plays a detrimental role in tumor cells, driving systemic growth and metastasis in mice. In the current study, we investigated whether the conditional ablation of $\mathrm{PHD}_{2}$ in the hematopoietic system changes tumor development/proliferation of tumor cells.

Methods. We used an in vivo model of tumor development in selfmade conditional hematopoietic $\mathrm{PHD}_{2}$ deficient mouse lines including (LysM:cre-PHD2 f/f, CD4:cre-PHD2 f/f). Analyses were based on tumor growth, tumor associated hematopoietic cell sorting (FACS), and gene profiling

Results. We demonstrated that the conditional ablation of $\mathrm{PHD}_{2}$ in the hematopoietic system leads to reduced tumor volume, intriguingly generated by an imbalance between enhanced cell death and improved proliferation of tumor cells. This effect seems to rely on the overall down regulation of pro- as well as anti-tumoral cytokines. Using different genetic approaches, we were able to confine this complex phenotype to the crosstalk of $\mathrm{PHD}_{2}$-deficient myeloid cells and T-lymphocytes. Conclusions. Taken together, our findings reveal a multifaceted role for $\mathrm{PHD}_{2}$ in several hematopoietic lineages during tumor development and might have important implications for the development of tumor therapies in the future.

\section{FR.2-053}

TNFa-enhanced pancreatic cancer progression is blocked by selective inhibition of T cell alternatively activated p38

\section{M.M. Gaida*1,2, M.S. Alam², F. Bergmann', F. Lasitschka', S.P. Hussain 3 ,} J.D. Ashwel/2

'Institute of Pathology, Ruprecht-Karls-University, Heidelberg, Germany, ${ }^{2}$ National Cancer Institute, National Institutes of Health, Laboratory of Immune Cell Biology, Bethesda, United States, ${ }^{3}$ National Cancer Institute, National Institutes of Health, Laboratory of Human Carcinogenesis, Bethesda, United States

Aims. Pancreatic ductal adenocarcinomas (PDAC) have a tumor-promoting inflammatory microenvironment, and manipulation of local cytokine production is an attractive, if unrealized, therapeutic approach. The tumor microenvironment contains a large number of tumor infiltrating T cells (TIL), which are a major source of cytokines, many of which are regulated by the MAPK p38. In antigen-activated T cells, $\mathrm{p}_{3} 8$ is exclusively activated via a MAPK cascade independent mechanism that can be blocked by Gadd $45 \alpha$.

Methods. A p38-binding fragment of Gadd 45 a comprising residues $71-85$ was synthesized as a fusion protein with $11 \mathrm{~N}$-terminal arginines to make it cell-permeable. KPC mice, which are genetically engineered to develop K-ras-mediated pancreatic cancer were lethally irradiated and then transplanted with bone marrow from mice that can or cannot activate $\mathrm{p} 38$ via the alternative pathway. In some experiments, KPC 
mice were given Gadd 45 a (11R) 71-85 i.v. A tissue microarray containing pancreatic carcinoma biopsies from 193 patients was stained for infiltrating $\mathrm{T}$ cells that expressed alternatively-activated $\mathrm{p} 38$ and $\mathrm{TNF} \alpha$ the results of which were correlated with clinical and pathological parameters.

Results. KPC mice displayed alternatively activated p38 TIL, and ablation of this pathway in bone marrow chimeric mice statistically significantly prolonged their survival. The cell-permeable Gadd 45 a (11R) 71-85 prevented downstream effects of alternatively activated p38 such as $\mathrm{T}$ cell proliferation and TNF $\alpha$ production in vitro. Mice treated i.v. with (11R) $71-85$ had reduced TNFa expression in CD4+ TIL as well as reduced levels of TNFa-inducible pro-tumorigenic chemokines (CCL2, CCL5, VEGF, TGF $\beta$ ) and delayed PanIN and cancer formation. The presence of a high percentage of $\mathrm{p} 38$ alternatively activated TIL in human pancreatic tumors defined a subgroup of patients with particularly aggressive disease.

Conclusions. Alternative 338 activation in CD4+ TIL leads to TNFa production and enhanced pancreatic tumor growth, and can be targeted for therapeutic benefit.

\section{FR.2-054}

Two major pathways of vulvar carcinogenesis: HPV-induced vulvar cancers overexpress p16ink4a, HPV-negative cancers associated with dermatoses lack p16ink4a-overexpression

\section{S. Regauer ${ }^{* 1}$, O. Reich ${ }^{2}$, B. Eberz ${ }^{3}$ \\ Institute of Pathology, Medical University, Graz, Austria, ${ }^{2}$ Department of Gynecology, Graz, Austria, ${ }^{3}$ Gynäkologische Praxis Mürzzuschlag, Mürzzu- schlag, Austria}

Aims. Vulvar squamous cell carcinomas (SCC) are rare cancers. They arise either through transforming infections with Human Papilloma Virus (HPV) or independent of HPV. HPV-induced cancers arise through slowly progressing vulvar intraepithelial neoplasia (VIN). This allows for several treatment options, including a watchful waiting approach for an eventual regression, ablative therapies and time-intense medical treatments. HPV-negative SCC often arise in inflammatory skin diseases, in particular in the background of lichen sclerosus (LS) and lichen planus (LP). These cancers show occasional p53 mutation and develop through a rapidly progressing precursor lesion called "differentiated VIN" (d-VIN), which needs immediate treatment. Stratification of SCC and precursor lesions into these two etiologies can not be achieved reliably by histology alone, but requires additional analysis of overexpression of p16ink4a and p53.

Methods. Archival formalin-fixed material of 90 invasive vulvar SCC and 50 pre-invasive vulvar lesions were evaluated for the expression of p53 and p16ink4a, an indirect marker for a transforming infection with HPV-HR genotypes, and correlated with the presence of LS, LP and DNA of 28 HPV-genotypes.

Results. 40/9o invasive vulvar SCC (18pT1a, 22 mostly small pTib, 5 with regional lymph node (LN) metastases) and 40/50 precursors showed p53-negativity and p16ink4a-over expression, which was independent of the HPV-HR genotype involved. 35/40 HPV-induced SCC and 32/40 HPV-induced VIN revealed a single HPV-HR-genotype (HPV-HR16 in 79\%), the remainder showed multiple HPV-HR-genotypes. 50/90 SCC (42 mostly large pT1b, 8 pT2, 21/50 with regional LN metastases at presentation) and 10/50 precursor lesions lacked HPV-DNA and pi6ink4aexpression and revealed peritumoral LS or LP. LP-associated SCC arose in non-hair bearing vulvar mucosa (hymenal ring, vestibulum, labia minora, periclitoral and urethral mucosa). Only $50 \%$ of SCC and d-VIN showed nuclear p53 expression. HPV-negative d-VIN showed various histologies, including hyperkeratotic, verrucous, atrophic, flat and basaloid lesions.

Conclusions. p16ink4a-overexpression identifies HPV-HR-induced VIN and SCC and pr6ink4a-negativity identifies HPV-negative d-VIN and SCC. Histological features and p53 expression alone are not helpful for etiologic stratification. Correct etiologic classification, particularly of pre-invasive lesions, allows optimal therapy decisions and prevents undue delays and possibly progression to invasive cancer.

\section{FR.2-055}

\section{An apoptosis-driven pathway to hepatocarcinogenesis}

Y. Böge ${ }^{* 1}$, F. Böhm', J. Friemel', L. Frick', J. Mertens'ㄹ, R. Maire', R. Boger', H. Moch', B. Müllhaupt ${ }^{2}$, H. Schulze-Bergkamen ${ }^{3}$, M. Heikenwälder ${ }^{1,4}$, A. Weber

'Institute of Surgical Pathology, University Hospital, Zurich, Switzerland, ${ }^{2}$ Hepatology and Gastroenterology, University Hospital, Zurich, Switzerland, ${ }^{3}$ German Cancer Research Center, DKFZ, Functional Genome Analysis, Functional Genome Analysis, Heidelberg, Germany, ${ }^{4}$ Institute of Virology, Technical University of Munich, Munich, Germany

Aims. Chronic liver diseases are characterized by a constant loss of hepatocytes. Apoptosis is a hallmark in liver tissue from patients with chronic liver diseases, e.g. HBV/HCV infection, autoimmune disorders or chronic alcohol intake. Apoptosis is regulated by a counterbalancing network of pro- and anti-apoptotic proteins. Mcl-1 is a crucial pro-survival factor, preventing cells to undergo apoptosis. Recently, we have shown that mice with hepatocyte-specific deletion of Mcl-1 (Mcl-1 $\Delta$ hep) reveal increased hepatocyte apoptosis, constant liver regeneration and spontaneous development of hepatocellular carcinoma (HCC).

Methods. To better understand apoptosis- and proliferation-driven tumorigenesis, we analyzed Mcl-1 $\Delta$ hep and Mcl-1 $\Delta$ hep-TNFR1-/- mice for gene expression, apoptosis, proliferation and DNA damage and compared our findings to human liver tissues including HCC of various etiologies.

Results. Remarkably, those Mcl-1 $\Delta$ hep mice which eventually developed liver tumors at 12 months had displayed significantly higher serum transaminase levels early in life compared to those Mcl-1 $\Delta$ hep mice which subsequently did not develop liver tumors. Deletion of TNFR1 leading to reduced hepatocytes apoptosis in Mcl-1 $\Delta$ hep-TNFR1-/- mice also caused reduction of liver damage, proliferation and finally reduced tumorigenesis. RNA microarray analysis uncovered well-described oncogenes as well as novel, putative oncogenes, significantly deregulated in liver tissue of Mcl-1 $\Delta$ hep mice. Genes deregulated in Mcl-1 $\Delta$ hep hepatocarcinogenesis were validated in human HCC with various etiologies and could be confirmed in two different patient cohorts.

Conclusions. These data indicates that liver tumor development is determined by the (accumulated) amount of liver damage in mice and men. These findings suggest that a vicious circle of chronic liver damage, apoptotic hepatocyte death and consecutive regenerative proliferation may be considered as the decisive event generally underlying human chronic liver disease-related HCC development, irrespective of the underlying etiology.

\section{FR.2-056}

Fast food diet in Mcl-1delhep mice for the study of NAFLD, NASH and hepatocarcinogenesis

\section{F. Böhm*1, Y. Böge', P. Hirsova ${ }^{2}$, M. Heikenwälder ${ }^{3}$, G. Gores ${ }^{2}$, A. Weber ${ }^{1}$} 'Institute of Surgical Pathology, University Hospital, Zurich, Switzerland, ${ }^{2}$ Institute of Gastroenterology and Hepatology, Mayo Clinic, Rochester, United States, ${ }^{3}$ Institute of Virology, Technical University of Munich, Munich, Germany

Aims. Non-alcoholic fatty liver disease (NAFLD) is an increasing burden in western countries due to life style and obesity. NAFLD can progress to non-alcoholic steatohepatitis (NASH), and may further progress to liver cirrhosis and hepatocellular carcinoma (HCC). Treatment of wild type mice for 4 months with FFD (fast food diet) (high energy diet with high levels of saturated fatty acids, cholesterol and fructose) 
recapitulates features of NASH and NAFLD such as steatosis, hepatocellular injury, inflammation and fibrosis. We recently established a mouse model by hepatocyte-specific depletion of Mcl-1 (Mcl-1delhep), which closely resembles human chronic liver disease. Mcl-1delhep mice reveal constant apoptosis of hepatocytes, compensatory proliferation, and spontaneous development of HCC. Feeding FFD for long term allows us to study molecular mechanisms of NAFLD/NASH-related tumorigenesis in wild type as well as in Mcl-1delhep mice (as a model of concurrent liver damage).

Methods. After $6 \mathrm{M}$ on FFD liver pathophysiology proceeding tumor development was evaluated, with the later time-point we studied tumorigenesis compared to mice kept on normal diet. The liver tissue was analyzed by histochemical stainings and immunohistochemistry as well as by molecular biological methods (RT-PCR, WB). Serum was tested for transaminases, bilirubin, cholesterol and triglycerides.

Results. At 6 months of age Mcl-1delhep mice showed more fibrosis on FFD compared to wild type mice and higher expression levels of proinflammatory cytokines such as TNFa and IL6. Transaminase levels (ALT, AST) and AP were significantly higher in Mcl-1 delhep mice compared to wild type animals. Wildtype mice showed fatty liver development but no tumor development after 1 year on FFD. At the age of 12 months FFD Mcl-1delhep mice had a tumor incidence of $80 \%$ compared to $40 \%$ on normal chow. In addition the tumor multiplicity was increased on FFD in Mcl-1delhep mice. In contrast to Mcl-1 delhep tumors on normal chow the tumors on FFD showed a different growth pattern with no distinct borders and nodule in nodule pattern.

Conclusions. Feeding Mcl-1delhep mice with FFD induced NASH let to a higher tumor incidence compared to normal diet. Gene expression analysis and immunohistochemistry staining will allow us the identification of markers important in HCC development under NASH conditions.

\section{FR.2-057}

\section{The lymphocytic infiltrate is reduced in metastases compared to} corresponding primary tumors of breast cancer patients

\section{B. Sobottka-Brillout*, H. Moch, Z. Varga Institute of Surgical Pathology, University Hospital, Zurich, Switzerland}

Aims. Metastasis to distant sites is the most common cause of death in cancer patients. The role of the immune system in metastasis is recently receiving increasing attention. While immune cells are commonly regarded as surveillants protecting against tumor cells, experimental evidence suggests that they may be misled by the tumor itself, at worst clearing the way for metastasis. To study the role of lymphocytes in the process of metastasis, we semi-quantitatively investigated and further characterized the lymphocytic infiltrate within primary tumors and their corresponding metastases in 115 patients diagnosed with breast cancer.

Methods. Formalin fixed and in paraffin embedded tumor tissues were available from 115 patients diagnosed with breast cancer with metastastic disease. Metastases occurred in brain, bone, liver or soft tissue. The primary tumor was invasive ductal or lobular carcinoma. Lymphocytic infiltrates were seminquantitatively (percentage of lymphocytes per high power field) investigated on the primary tumor and in the metastases. Lymphocytic infiltration was further characterized by CD2O, CD4 and CD8 immunohistochemistry on both sites. Additionally, clinicopathological parameter including predictive factors were correlated with the findings.

Results. Our data demonstrated that the group of breast cancer patients with brain metastasis was significantly younger at the age of primary diagnosis (mean age 48 years; $p<0.05$ ) and diagnosis of metastasis (mean age 52 years; $\mathrm{p}<0.05$ ) onset. This group also showed significantly worse recurrence free survival time (median recurrence free survival 3 years; $\mathrm{p}<0.001)$ when compared with the remaining patients. The primary tumors in this brain metastasis group were significantly more frequently estrogen $(\mathrm{p}<0.01)$ and progesterone $(\mathrm{p}<0.01)$ receptor negative but more frequent HER2 $(\mathrm{p}<0.01)$ positive. The overall lymphocyte infiltrate was denser within primary tumors compared to the corresponding metastasis. This finding was irrespective of metastatic sites. CD2O + B lymphocytes were significantly less dense in the primary tumors with brain metastasis $(\mathrm{p}<0.05)$. Their CD4+ and CD8+ T lymphocyte infiltrate was reduced when compared to patients with other metastatic sites.

Conclusions. Our findings are consistent with a hypothesis that the immune system of patients with metastatic cancer is thought to reside in an immunosuppressive state. An immunmodulatory therapy in metastatic cancer could be the translational clinical relevance.

\section{FR.2-058}

Turning NF-кB into a pro-death factor in the chemotherapy of colorectal cancer

M. Metzig ${ }^{* 1,2}$, D. Fuchs ${ }^{1,2}$, P. Schirmacher ${ }^{2}$, W. Roth ${ }^{1,2}$

${ }^{1}$ German Cancer Research Center, Heidelberg, Germany, ${ }^{2}$ Institute of Pathology, University Hospital Heidelberg, Heidelberg, Germany

Aims. NF- $\kappa B$ is a central transcription factor with key functions for tissue homeostasis and immune responses. Constitutively active NF- $\kappa \mathrm{B}$ signaling is an important link between chronic inflammation and cancer. NF- $\mathrm{kB}$ is mostly described as an anti-apoptotic factor contributing to carcinogenesis and chemoresistance. However, there is growing evidence that NF- $\kappa \mathrm{B}$ can also promote cell death, especially in the case of alternative forms of programmed cell death. Necroptosis or programmed necrosis is a recently described form of RIP1-dependent cell death, which occurs under caspase-deficient conditions and may play a role in chronic inflammatory bowel disease and intestinal carcinogenesis. The relationship between chemotherapeutic agents and necroptosis is largely unknown. The aim of our project was to study the molecular principles of 5-Fluoruracil (5-FU)-induced cell death and the regulatory function of NF- $\kappa B$ in colorectal cancer cells. We investigated the hypothesis whether targeted induction of necroptosis could represent a novel therapeutic approach for otherwise therapy-resistant colorectal cancer. Methods. Cytotoxicity and apoptosis assays, FACS analysis, siRNA knockdown, Western blot, co-immuoprecipitation, quantitative realtime PCR.

Results. In our experiments we tested several colorectal cancer cell lines for their resistance towards 5-FU. In HCT116 cells, treatment with 5-FU resulted in cell death which was rescued by the addition of the pan-caspase inhibitor Z-VAD, indicating the induction of apoptosis in these cells. Surprisingly, the 5-FU-resistant HT29 cell line was sensitized towards 5-FU-induced cell death when co-treated with Z-VAD. This effect was rescued by the use of necrostatin, a RIP1-specific kinase inhibitor, indicating the induction of necroptosis in these cells. Interestingly, HT29 cells undergoing necroptosis showed a strong up-regulation of NF- $\kappa B$ signaling indicated by elevated levels of phosphorylated IkB protein as well as NF- $\kappa \mathrm{B}$ dependent target genes. Inhibition of NF- $\kappa \mathrm{B}$ via knockdown of RelA blocked necroptosis in these cells.

Conclusions. Our experiments identify NF- $\kappa B$ as a promoter of cell death under certain circumstances, namely upon triggering of RIP1-dependent necroptosis after combined treatment with 5-FU and caspase inhibitors. Taken together, these findings suggest that specific induction of necroptosis could represent a highly promising novel therapeutic strategy for patients with colorectal cancer. 


\section{FR.2-059}

CD56 (140kD) up-regulation plays a pivotal role in the pathogenesis of ischemic cardiomyopathy

\section{M.K. Tur*, A. ten Haaf, A. Bräuninger, S. Gattenlöhner \\ Institute of Pathology, University Hospital of Giessen, Justus Liebig Uni- versity of Giessen, Giessen, Germany}

Aims. CD56 (NCAM1) is a cell adhesion molecule that induces cell-tocell interactions during embryonic development, cell migration, organogenesis and interactions between neural cells. Three main isoforms with molecular weights of 120,140 , and $180 \mathrm{kDa}$ are known. We have previously shown that $\mathrm{CD}_{5} 6(140 \mathrm{kDa})$ is specifically overexpressed in ischemic cardiomyopathy (ICM). Therefore, the aim of this study was to elucidate the role of $\mathrm{CD}_{5} 6$ in the pathogenesis of human ICM.

Methods. We used quantitative real-time PCR and immunohistochemistry (IHC) in human ICM and a rat model of coronary obstruction to clarify which isoform is responsible for the up-regulation of CD56 in ICM. We performed differential whole transcriptome expression analyses and functional in vitro cardiomyocyte cell culture assays to analyse the cellular effect of CD56 $(140 \mathrm{kDa})$ overexpression in the development of ICM. The detection of CD56 (140 kD)-dependent gene expression changes in human ICM was analysed at the protein level using semiquantitative IHC. We performed functional assays (Fluorescence-based calcium assay, MTT, Annexin V and TUNEL) to test the influence of CD56 (140 kDa)-overexpression in the cardiac muscle cell line HL-1.

Results. We could show that the up-regulation of CD56 ( $140 \mathrm{kD})$ is associated with profound gene expression changes, increased apoptosis, and reduced $\mathrm{Ca}(2+)$ signalling in failing human cardiomyocytes. Because apoptosis and $\mathrm{Ca}(2+)$-related sarcomeric dysfunction are molecular hallmarks of ICM in humans, our results provide strong evidence that CD56 (140 kD) up-regulation plays a pivotal role in the pathogenesis of ICM.

Conclusions. The results of this study provide strong evidence that the $140-\mathrm{kD}$ isoform of $\mathrm{CD}_{5} 6$ directs the molecular pathogenesis of ischemic cardiomyopathy and may be a target for future immunotherapeutic strategies in the treatment of this common and often fatal disease [1].

1. Tur MK, Etschmann B, Benz A, Leich E, Waller C, Schuh K, Rosenwald A, Ertl G, Kienitz A, ten Haaf A, Bräuninger A, Gattenlöhner S (2013) The $140-\mathrm{kD}$ Isoform of CD56 (NCAM1) Directs the molecular pathogenesis of ischemic cardiomyopathy, Elsevier, The American Journal of Pathology, 1205-1218

\section{FR.2-060}

\section{Differences in the proteomic pattern of colonic and pancreatic} carcinoma in multi tissue assays by MALDI imaging mass spectrometry (IMS)

J. Kriegsmann ${ }^{* 1,2,3}$, R. Casadonte ${ }^{2}$, M. Kriegsmann ${ }^{4}$, V. Schommer ${ }^{3}$, D. Aust ${ }^{5}$, G. Baretton ${ }^{5}$, S.-O. Deininger ${ }^{6}$, D. Suckau ${ }^{6}$, M. Otto ${ }^{1,2,3}$

${ }^{1}$ Center for Histology, Cytology and Molecular Diagnostics, Trier, Germany, ${ }^{2}$ Proteopath GbR, Trier, Germany, ${ }^{3}$ Institute of Molecular Pathology, Trier, Germany, ${ }^{4}$ University of Heidelberg, Trier, Germany, ${ }^{5}$ University of Dresden, Department of Pathology, Dresden, Germany, ${ }^{6}$ Bruker Daltonik GmbH, Bremen, Germany

Aims. Adenocarcinomas represent the most common metastatic tumors of unknown primary site. Among these, liver metastases of colonic and pancreatic adenocarcinoma are of special importance. Classification of the metastatic tumor concerning tissue of origin is required. The goal of this study was to identify a proteomic signature in FFPE tissue to discriminate between colon and pancreatic carcinoma using MALDI IMS.

Methods. A total of 85 needle core biopsies of colonic and 86 core biopsies of pancreatic carcinomas collected in multi tissue assays were on-tissue trypsin digested and subsequently analyzed by MALDI imaging mass spectrometry. Data collected from each tissue section were correlated with a serial hematoxylin and eosin-stained sections. A training set, including 39 colon and 32 pancreas primary carcinoma biopsies, was used by a support vector machine (SVM) algorithm to select a group of candidate peptide signals to discriminate carcinomas of the two different tissue origins. A classification model was generated and validated with two testing sets, one including 23 colonic and 35 pancreatic carcinomas, and one including 23 different colonic and 19 pancreatic carcinoma core biopsies. Statistical analysis was performed with ClinProTools 3.o (Bruker Daltonik GmbH, Bremen).

Results. From the training set, twenty-five ion peptides were selected (through the statistical criteria of a Wilcoxon/Kruskal-Wallis test) by the SVM classification model discerning colonic from pancreatic primary carcinoma. These discriminatory features correctly classified the first test set with a sensitivity of $88.8 \%$ and a specificity of $91.5 \%$, and the second test set with a sensitivity of $83.9 \%$ and a specificity of $98.3 \%$. Six $\mathrm{m} / \mathrm{z}$ discriminant signatures $(530.1,543.2,788.4,836.3,852.4,1562.8$ ) exhibited a statistical significance with a PWKW $<10-6$ and a minimum 2-fold intensity difference threshold.

Conclusions. A proteomic pattern to distinguish colonic from pancreatic carcinoma was established using MALDI IMS technology. We have demonstrated that a histological classification of colonic and pancreatic carcinoma is possible by applying MALD-Imaging on FFPE-tissues. This proof-of-principle study has shown that proteomic classification by MALDI represent a valuable approach for histopathological diagnostics without applying immunohistochemistry or further molecular techniques.

\section{FR.2-061}

\section{Immune dyregulation in lichen planus associated HPV-negative} vulvar cancers

\section{S. Regauer*, C. Beham-Schmid Institute of Pathology, Medical University, Graz, Austria}

Aims. Lichen planus (LP) is a rare chronic dermatosis believed to represent an autoimmune dysregulation that affects hair bearing skin and mucosal surfaces. At least $25 \%$ of patients have disease manifestation in the anogenital skin and mucosa. In mucosal sites, LP has a risk for development of a squamous cell carcinoma (SCC) of up to $5 \%$. In the vulva, LP-associated SCC arise independently of human papilloma virus (HPV) via the rapidly progressing precursor lesion differentiated vulvar intraepithelial neoplasia (d-VIN). LP as well as d-VIN and LPassociated SCC typically feature dense inflammatory infiltrates with a dominance of T-lymphocytes. We evaluated the inflammatory infiltrate of all LP-associated SCC diagnosed in 2013 for T-lymphocytes with monoclonally rearranged T-Zell-receptor-Gamma-Locus (TCR@).

Methods. LP-associated, HPV-negative vulvar pTib SCC of 10 women (average age at presentation $60 \mathrm{yrs}$, range $37-85 \mathrm{yrs}$ ) were analyzed. Two SCC revealed only scant inflammatory infiltrates insufficient for analysis; 8/10 SCCs with sufficient inflammatory infiltrates were analyzed with consensus primers for variables $\mathrm{V}_{1}-11$ and adjacent regions of the TCR@ (T-gamma-A, T-gamma-B; iMVivoScribe technologies).

Results. Clonal accumulation of T-lymphocytes was demonstrated in 5/8 SCC: pt.\#1 (47 yrs; 1 recurrent SCC, regional LN metastasis, dead of disease within 11 months) had a monoclonal peak within T-Gamma-A, region Vg10+Jg1.3/2.3, [154 nucleotides (nt)]. pt.\#2 (67 yrs) had a monoclonal peak within T-Gamma-A, region Vg10+Jg1.1/2 (183 nt). Pt.\#3 (71yrs) revealed 3 biclonal peaks within T-Gamma-A: region Vg10+Jg1.3/2.3 (154 and $160 \mathrm{nt}$ ); region Vg10+Jg1.1/2.1 (183 and 190 nt); region $\mathrm{Vg} 1.8+\mathrm{Jg} 1.1 / 2.1$ (236 and $242 \mathrm{nt})$, and a monoclonal peak within T-Gamma B in the region Vg9+Jg1.3/2.3 (168 nt). pt.\#4 (77 yrs; 1 recurrent SCC in the residual LP within 20 month) had a monoclonal peak at T-Gamma A in the region Vg1-8+JG1.3/2.3 (199 nt) und at T-Gamma B: Vg9+JG1.1/2.1 (211 nt). pt.\#5 (85 yrs) revealed a monoclonal peak within T-Gamma-B in the region Vg9+, Jg1.3/2.3 (178 nt). 
Conclusions. $60 \%$ of LP-associated vulvar SCC revealed T-lymphocytes with TCR@. The resulting reduced T-cell diversity within tumor infiltrating lymphocytes may create a permissive environment for multifocal development of d-VIN and immunological escape of invasive tumor cells. This may explain the rapid evolution of d-VIN to invasive SCC, and early escape of single metastatic tumor cells to lymph nodes.

\section{FR.2-062}

Proinflammatory human 6-sulfo lacnac+ dendritic cells accumulate in intestinal acute graft versus host disease

U. Sommer*1, S. Tuve', B. Larsson'2, R. Wehner ${ }^{3}$, N. Zimmermann ${ }^{4}$, M. Kramer', A. Kloß ${ }^{3}$, C. Günther', J. Babatz'2 R. Schmelz'2, S. Brückner², J. Schetelig', M. Bornhäuser ${ }^{2}$, K. Schäkel ${ }^{5}$, M. Bachmann ${ }^{3}$, D.E. Aust', M. Schmitz ${ }^{3}$, G.B. Baretton

'Institute of Pathology of University Hospital Carl Gustav Carus, Medical Center TU Dresden, Dresden, Germany, ${ }^{2}$ Medizinische Klinik und Poliklinik

1, University Hospital Carl Gustav Carus, Dresden, Germany, ${ }^{3}$ Insitute of Immunology, Medical Faculty, TU Dresden, Dresden, Germany, ${ }^{4}$ Department of Dermatology, University Hospital of Dresden, TU Dresden, Dresden, Germany, ${ }^{5}$ Department of Dermatology, Ruprecht-Karls-University, Heidelberg, Germany

Aims. Dendritic cells (DCs) are professional antigen-presenting cells, which display a unique capacity to induce and expand proinflammatory CD8+ CTLs and CD4+ T-cells. Preclinical studies have suggested that DCs play a critical role in Tcell-mediated inflammation in acute graft-versus-host disease (aGVHD) following allogenic stem cell transplantation (HSCT). However, little is known about the involvement of human DCs in the pathogenesis of aGVHD. Human 6-sulfo LacNAc+ (slan) DCs are known to display pronounced proinflammatory properties and are detectable in affected tissues of patients with various inflammatory diseases. In this study, we explored the tissue infiltration and cytokine expression of slanDCs in colorectal biopsies of patients suffering from intestinal aGVHD.

Methods. The present study includes 65 patients who underwent HSCT at the University Hospital of Dresden. Patients underwent diagnostic colonoscopy whenever symptoms of lower GI-GvHD occurred at a median time of 32 days after HSCT. Histopathological grading was performed as described by Lerner et al. None of the patients had evidence of viral or bacterial infection of the GI-tract. Two colorectal biopsies per patient (total $n=124$ ) were formalin-fixed, paraffin-embedded, sectioned and stained for the slan molecule, TNF- $\alpha$ and interleukin-23. For quantification of slanDCs in tissues, stained cells were counted in three high power fields (HPF) of a section and the mean value was determined. The significance of the results was determined by using the statistical software R Version 2.15.1 and the package 'nlme' Version 3.1-108. Values of $\mathrm{p}<0.05$ were considered significant.

Results. SlanDCs were found preferentially in the stroma in 119 of 124 biopsies with varying frequencies. Remarkably, slanDCs locally expressed the proinflammatory cytokines TNF- $\alpha$ and IL-23, which are implicated in the pathogenesis of aGVHD. Significantly higher numbers of slanDCs were detected in histologically confirmed aGVHD grade 1-4 tissues compared to grade o tissues $(\mathrm{p}<0.05)$. The density of slanDCs was comparable between the different aGVHD grades. Steroid treatment neither influenced the number of slanDCs in aGVHD tissues nor their potential to express TNF- $\alpha$ and IL-23.

Conclusions. The accumulation of slanDCs expressing proinflammatory cytokines in colon biopsies with aGVHD indicate that slanDCs may contribute to the immunopathogenesis of this disease.

\section{FR.2-063}

Next-generation sequencing for the characterization of triplenegative breast cancer

M. Kriegsmann ${ }^{* 1}$, P. Sinn', T. Wolf', A. Stenzinger', C. Denkert ${ }^{2}$, J. Budczies², N. Pfarr' ${ }^{1}$ V. Endris', W. Weichert ${ }^{1}$

'Institute of Pathology, University of Heidelberg, Heidelberg, Germany, ${ }^{2}$ Institute of Pathology, Charité University Hospital, Berlin, Germany

Aims. Triple negative breast cancer (TNBC) accounts for approximately $15 \%$ of all breast cancers and is a heterogeneous group as indicated by recent molecular studies. To characterize the genomic landscape of triple negative breast cancer with respect to different TNBC subtypes defined by immunohistochemistry and with respect to patient outcome we used a next-generation panel sequencing approach on a large cohort of well characterized TNBC tumors.

Methods. We performed next-generation panel sequencing (44 genes, 137 amplicons) on formalin-fixed paraffin embedded triple negative breast cancer specimens $(n=142)$. The detected mutations were subsequently tested for associations with immunohistochemically defined TNBC subtypes assessed by a panel of 13 markers including $\mathrm{CK}_{7}, \mathrm{CK}_{5} / 6$, CK14, CK18, CK19, VIM, EGFR BCL2, Ki-67, p53, p16, WT1, CD117 and survival.

Results. We identified frequent mutations in several genes involved in cell survival, apoptosis and proliferation. p53 mutations and exceptionally high rates of $\mathrm{PIK}_{3} \mathrm{CA}$ mutations were the most prevalent detectable genomic aberrations. Further, we found some striking differences in mutation prevalences when we compared our data to results obtained by exome sequencing. Mutational profiles clusted with molecular subtypes defined by immunohistochemistry and had impact on patient survival.

Conclusions. Our data underline the molecular heterogeneity of TNBC and the necessity for molecular stratification in order to provide the basis for a personalized therapy in the future.

\section{FR.2-064}

\section{Constitutively activated SHP2/ERK/c-Myc signaling in germinal} center $B$ cell derived lymphoma

X. Jiang 1,2, J. Wu', H. Guo', Y. Shi', J. Wang', H. Pan', R. Zhou* ${ }^{* 1,2}$

'Zhejiang University School of Medicine, Department of Pathology and Pathophysiology, Hangzhou, China, ${ }^{2}$ Institute of Pathology and Forensic Medicine, Zhejiang University, Hangzhou, China

Aims. The entry of $\mathrm{B}$ cells into germinal center undergoes proliferation, somatic hypermutation (SHM) and class switch recombination (CSR) to generate high-affinity antibodies. However, CSR and SHM both induce double strand DNA breaks resulting in aberrant chromosome translocations that are associated with $\mathrm{B}$ cell lymphomagenesis such as GC B cell derived lymphomas (B-GCCLs). The first identified ubiquitous protein tyrosine phosphatase (PTP) proto-oncogene $\mathrm{SHP}_{2}$ has a critical but poorly defined function in the regulation of immune responses. $\mathrm{SHP}_{2}$ deregulation is frequently observed in solid cancers and hematologic malignancies. Here we report that constitutive activity of $\mathrm{SHP}_{2}$ and its downstream kinase ERK are essential for the viability and progression of B-GCCL cells.

Methods. In this study, using conditional reverse genetics and in vitro cell culture complemented by bioinformatics analyses, we assess the activated status and effects of $\mathrm{SHP}_{2} / \mathrm{ERK}$ signaling in $4 \mathrm{~B}$-GCCL lines (Raji, OCI-LY8, SU-DHL5 and Pfeiffer) and 2 non-B-GCCL lines (OCILY3, OCI-LY10).

Results. Loss of $\mathrm{SHP}_{2}$ or inhibition of ERK activation kills all 4 B-GCCL lines but not 2 non-B-GCCL lines. Mechanistically, SHP2/ERK signaling blockade impedes c-Myc transcriptional activity, which results in the repression of B-GCCL proliferative phenotype signatures, including Bcl6 and AP-1, and increased expression of the cell cycle inhibitor 
p27kip1. Furthermore, $\mathrm{SHP}_{2} / \mathrm{ERK}$ signaling are required for maintaining CD19/c-Myc loop, which preferentially promotes survival of distinct subtype of B-GCCL cells carrying MYC/IGH translocation.

Conclusions. These findings demonstrate a critical function of $\mathrm{SHP}_{2} /$ ERK signaling upstream of c-Myc in B-GCCL cells and provide a rationale for targeting SHP2 in B-GCCL therapy.

\section{FR.2-065}

Detection of epidermal growth factor receptor mutation in pleural effusion and biopsy samples from non-small cell lung cancer patients by high resolution melting analysis and sequencing

\section{J. Lin*, Y. Gu, M. Deng, Y. Lu, Y. Ding}

Southern Medical University, School of Basic Medical Sciences, Department of Pathology, GuangZhou, China

Aims. To detect epidermal growth factor receptor (EGFR) mutations in pleural effusion, including supernatant and cell pellets, and paraffin embedded biopsy tissue samples from non-small cell lung cancer (NSCLC) patients. And to compare the sensitivity of high resolution melting (HRM) analysis and direct sequencing.

Methods. 36 pleural effusion samples and 22 matched biopsy tumor samples from NSCLC patients were collected. Mutations of EGFR gene in exons 18 to 21 were detected using both HRM analysis and direct sequencing. EGFR mutations in the supernatant and the cell pellets are examined separately for each pleural effusion sample.

Results. EGFR mutations were identified in 19 cases out of the total 36 pleural effusion samples by HRM analysis, including 13 cases in exon 19,5 cases in exon 21 and 1 case in exon 20 . No mutation in exon 18 was found in these samples. The mutation rate of exon 19 and exon 21 was $50 \%$ (18/36). Among them, 15 mutations were identified by both HRM and sequencing, while other 4 mutations were detected only by HRM analysis. Among 36 pleural effusion samples, 18 mutations were found in cell-free supernatant while 13 mutations found the cell pellets as detected by HRM analysis. EGFR mutations were identified in 12 cases of the matched 22 cases of biopsy tissue samples. Furthermore all the 12 mutations were detected in both pleural effusion samples and biopsy tissue samples.

Conclusions. This study suggested the exploitability of pleural effusion for detecting EGFR mutations in late NSCLC patients, furthermore the cell-free supernatant of pleural effusion might be a better resource for mutation detection. Meanwhile, HRM analyses would be a reliable and sensitive method to determine EGFR mutation status in both pleural effusion samples and biopsy tissue samples clinically, which shed novel insights into the EGFR tyrosine kinase inhibitor therapy.

\section{FR.2-066}

Selective disruption of tyrosine phosphatase Shp2 promotes alternative activation of macrophages and predisposes pulmonary fibrosis in mice

Y. Ke ${ }^{*}$, B. Tao

Zhejiang University School of Medicine, Department of Pathology and Pathophysiology, Hangzhou, China

Aims. Alternative activation of macrophages (M2) in lungs has been implicated as a causative agent in pulmonary fibrosis. However, the mechanisms underlying M2 polarization are poorly characterized. Shp2 is a ubiquitously expressed cytoplasmic tyrosine phosphatase, acting a central role in the regulation of a variety of immune functions. In this work, we aim to understand the function of Shp2 in regulation activation of macrophages.

Methods. To further characterize the functions of Shp2 in macrophage in vivo, we generated monocyte/macrophage-specific Shp2 knockout mice under the control of LysM-Cre promoter (Shp2flox/flox, LysM-
Cre). Combined with biochemistry and pathological studies, this work has been foucsed on in vivo physiological roles of Shp2 in M2-associated pulmonary fibrosis.

Results. In this study, first we found that inactivation of Shp2 augments IL-4-mediated $\mathrm{M}_{2}$ in vitro; suggesting a negative regulation of Shp2 in controlling macrophages polarized toward the M2 phenotype. The Shp2-deficient mice exhibited a marked shift of polarized balance in favor of IL-4-mediated M2. Additionally, there was a notable increase in arginase 1 activity in Shp2 $\mathrm{KO}$ following intraperitoneal injection of chitin. Additionally, these mutant mice are more sensitive to bleomycin-induced inflammation and pulmonary fibrosis in comparison with control. Mechanistically, Shp2 associates with IL-4Ralpha and inhibits JAK1-STAT6 signaling via its catalytic domain. Shp2 lost increases JAK1 binding with IL-4Ralpha, which augments the activation of IL-4-regulated JAK1/Stat 6 activation, leading to the observed M2 plasticity.

Conclusions. In summary, on the basis of these findings, we defined a novel function of Shp2 in regulation of $\mathrm{M}_{2}$ polarization, which may provide valuable insight into $\mathrm{M} 2$ participation in the pathogenesis of pulmonary fibrosis and uncover a promising therapeutic option for this currently untreatable disease.

\section{FR.2-067}

High expression of IGFBP7 in fibroblasts induced by colorectal cancer cells is co-regulated by TGF-beta and Wnt signaling in a Smad2/3-Dvl2/3-dependent manner

\section{H. Wen, S. Lin, C. Rao, H. Deng*}

Zhejiang University School of Medicine, Department of Pathology and Pathophysiology, Hangzhou, China

Aims. Fibroblasts in the tumor microenvironment are a key determinant in cancer progression and may be a promising target for cancer therapy. Insulin-like growth factor binding protein 7 (IGFBP7) is known as a tumor suppressor in colorectal cancer (CRC). The present study investigated the inductive mechanism of IGFBP 7 expression in fibroblasts by supernatant from the CRC cell line, SW62O.

Methods. Fibroblasts (HELFs) were cultured in SW620 supernatant, treated by TGF- $\beta_{1}$, TGF- $\beta_{1}$ receptor antagonist (SB431542), TGF- $\beta_{1}$ specific antibody (AF), Wnt signaling pathway inhibitor (DKK-1) respectively. Q-PCR, and Western Blot were used to detect the expression of IGFBP7 and related genes and proteins of TGF- $\beta$ and Wnt signaling pathways.

Results. The results showed that the expression of IGFBP7 was up-regulated in the fibroblasts when treated with SW620 supernatant and exogenous TGF- $\beta_{1}$. The IGFBP 7 induced by SW620 supernatant or TGF- $\beta_{1}$ was partially inhibited by the TGF- $\beta_{1}$ specific antibody AF and TGF- $\beta_{1}$ receptor antagonist SB431542. The Wnt signaling-targeted genes, c-Myc, CCND1 and the proteins Dvl2/3, were all up-regulated in fibroblasts expressing high levels of IGFBP7, and the up-regulation could be inhibited both by the Wnt signaling antagonist $\mathrm{DKK} 1$ and by the TGF- $\beta 1$ receptor antagonist $\mathrm{SB} 431542$.

Conclusions. CRC cells promote the high expression of IGFBP7 in $\mathrm{fi}^{-}$ broblasts, most likely through the co-regulation of TGF- $\beta$ and Wnt signaling in a Smad2/3-Dvl2/3 dependent manner. Taken together, these data suggest that the fibroblasts could be a novel therapeutic target in tumor therapy. 


\section{AG Gastroenteropathologie \\ Postersession - Oberer GI-Trakt}

\section{FR-001 \\ Multilayered epithelium at the gastroesophageal junction is a marker of gastroesophageal reflux disease: data from a prospective central European multicenter study (histoGERD Trial)}

\section{N. Schneider*1, E.-M. Wolf', W. Plieschnegger ${ }^{2}$, M. Geppert ${ }^{3}$, B. Wigging- haus $^{4}$, G. Höss', A. Eherer ${ }^{6}$, P. Rehak ${ }^{7}$, M. Vieth ${ }^{8}$, C. Langner ${ }^{1}$ \\ ${ }^{1}$ Institute of Pathology, Medical University, Graz, Austria, ${ }^{2}$ Krankenhaus der Barmherzigen Brüder, Department of Internal Medicine, St. Veit/Glan, Austria, ${ }^{3}$ Private Practice, Internal medicine, Bayreuth, Germany, ${ }^{4}$ Private Practice, Internal medicine, Osnabrück, Germany, ${ }^{5}$ Department of Surgery, Medical University of Graz, Graz, Austria, 'Department of Internal Medicine, Medical University of Graz, Graz, Austria, ${ }^{7}$ Unit for Biomedical Engineering \& Computing, Medical University of Graz, Graz, Austria, ${ }^{8}$ Institute of Patho- logy, Klinikum Bayreuth, Bayreuth, Germany}

Aims. Multilayered epithelium is defined as hybrid epithelium with characteristics of both squamous and columnar epithelium. Our aim was to evaluate the clinicopathological significance of the lesion by relating its presence to various histologic and clinical and/or endoscopic features indicating gastroesophageal reflux disease (GERD).

Methods. A total of 1,071 individuals undergoing gastroscopy for various non-selected reasons participated in a prospective cross-sectional study ( 576 females and 495 males; median age 53 years). Biopsy material was systematically sampled from the gastroesophageal junction. The histologic diagnosis of esophagitis was made according to the Esohisto consensus guidelines. The columnar epithelium in the biopsy samples was classified according to the type of underlying glands: pure mucinous (cardiac mucosa), pure oxyntic (oxyntic mucosa), or mixed mucinous/oxyntic (oxyntocardiac mucosa). The endoscopic diagnosis of esophagitis was made according to the modified Los Angeles classification. The diagnosis of Barrett's esophagus was made according to Prague's C\&M criteria.

Results. Multilayered epithelium was identified in 103 (9.6\%) individuals, frequently within or adjacent to the ducts of proper esophageal glands. Its presence was associated with increasing age $(\mathrm{p}<0.001)$, high BMI $(p=0.026)$, hiatal hernia $(p<0.001)$, and the endoscopic diagnoses of esophagitis $(\mathrm{p}=0.002)$ and Barrett's esophagus $(\mathrm{p}<0.001)$. Upon histology, multilayered epithelium was associated with features of the squamous epithelium indicating GERD, particularly intercellular space dilation $(\mathrm{p}=0.005)$, and presence of cardiac mucosa $(<0.001)$. For intestinal metaplasia a trend was noted $(\mathrm{p}=0.094)$

Conclusions. In conclusion, multilayered epithelium was observed in about every tenth individual undergoing upper gastrointestinal endoscopy. The association with histologic and clinical features indicating GERD advocates the lesion as promising new marker for reflux esophagitis. The association with cardiac mucosa and Barrett's esophagus suggests multilayered epithelium to be an intermediate step in the development of columnar metaplasia and, ultimately, Barrett's esophagus.

\section{FR-002}

Microsatellite instability is uncommon in gastric cancer and correlates with patient survival

\section{Mathiak*, V. Warneke, H.-M. Behrens, J. Haag, S. Krüger, C. Röcken Institute of Pathology, Christian-Albrechts-University, Kiel, Germany}

Aims. We investigated microsatellite instability (MSI) in gastric cancer (GC) testing the following hypotheses: (1) is the identification of GC subtypes suitable to tailor MSI-analysis, (2) is MSI of prognostic signifi- cance, (3) should MSI-status be considered in patient treatment and (4) do MSI GCs harbor EpCAM-mutations.

Methods. Out study cohort consisted of 611 patients who underwent surgery between 1997 and 2009. Screening was carried out by immunohistochemistry using tissue micro arrays and antibodies directed against $\mathrm{MSH}_{2}, \mathrm{PMS}_{2}, \mathrm{MLH} 1$ and MSH6. GCs lacking expression of any of the four repair proteins and cases with unequivocal immunostaining were studied through PCR-assay for the MSI-status. Since no consensus panel has been established for GC, we used a five mononucleotid marker panel less vulnerable to storage dependent DNA damage. 451 patients were eligible for immunohistochemical and potential molecular pathologic analysis.

Results. 34 GCs were MSI-high ( $\geq 2 / 5$ markers, 7.5\%). Patients with MSI-H GCs were significantly older, either intestinal or unclassifiable phenotype, had less lymph node metastases and showed a better mean overall survival. Median patient survival differed by nearly 2 years, i.e. 14 months vs. 35 months between MSI-H and microsatellite stable GCs. EPCam has been shown to be involved in cancer biology. Since the gene is mapped to chromosome $2 \mathrm{p}_{2} 1$ upstream of $\mathrm{MSH}_{2}$ we analyzed a subgroup of MSI-H tumors for EPCAM mutations. None of the MSI-H GCs harboured an EpCAM gene-mutation.

Conclusions. Our data provide evidence that MSI-H identifies a distinct subgroup of GCs, with favourable prognosis. MSI-status should be assessed in GC.

\section{FR-003}

Frequency and clinicopathological features of fibroelastotic changes in the gastrointestinal tract

I. Lichtmannegger ${ }^{* 1}$, S. Gölder'2, A. Probst'², G. Dönmez ${ }^{2}$, A. Agaimy³, E. Langer', W. Müller', J. Spatz' ${ }^{6}$, B. Märkl'

'Institute of Pathology, Klinikum Augsburg, Augsburg, Germany, ${ }^{2}$ III. Medical Clinic, Klinikum Augsburg, Augsburg, Germany, ${ }^{3}$ Institute of Pathology, University Hospital Erlangen, Erlangen, Germany, ${ }^{4}$ Practice for Pathology, Augsburg, Germany, ${ }^{5}$ Practice for Pathology, Starnberg, Germany, ${ }^{6}$ Visceral Surgery, Klinikum Augsburg, Augsburg, Germany

Aims. Fibroelastotic changes (FEC) in the gastrointestinal tract (GIT) were subject of only a few studies in the recent years and are still thought to be quite rare. In contrast, we saw a large number of these elastic fiber accumulations within the mucosal, submucosal or muscular layer of all sites of the GIT and therefore performed a study, collecting these cases in order to find out more about frequency as well as most common location and association with corresponding clinical features.

Methods. Within a 2-year period, we found 162 cases with FEC in daily routine biopsies of the upper and lower GIT and correlated the histopathological results with clinical data and endoscopic findings.

Results. We identified three different histological patterns of FEC that we classified into three groups (fibroelastosis, angioelastosis and elastofibromas). The elastotic accumulations often appear as polyp-forming lesions, especially in the colon ( 23 cases/14\%). The remainders were histological findings without identifiable gross mass and microscopically diffuse non-polyp forming elastotic deposits. The positive samples were composed of 9 esophageal, 117 gastric and 36 intestinal biopsies. Histologically, the lesions displayed the following characteristics: granular and/ or fibrillar, eosinophilic, amorphous accumulations within connective submucosal tissue with scattered fibroblasts, small blood vessels and in some cases serrated outlines. Frequently related findings were gastritis, mucosal ulcer/erosion, post-interventional status, previous irradiation and history of GI lymphoma. Eight cases presented endoscopically as highly suspicious areas and lead to surgical resection. On 39 colon cases of our series (including surgical specimens) we additionally performed immunohistochemical staining with $\mathrm{CD}_{34}$ and saw an increase of positive cells. These results are consistent with previous studies and support the hypothesis of a potential role for $\mathrm{CD}_{34}$ positive mesenchymal cells in the development of elastotic fiber accumulation. 
Conclusions. In conclusion, fibroelastotic lesions are more common in the GIT than previously recognized. They often present as benign polyps or diffuse deposits, most commonly in the upper GIT ( $78 \%$ vs. $22 \%$ lower GIT). The FEC accompany other diseases like ulcers and atrophic gastritis or represent residual findings after certain interventions. Attention has to be paid to the fact that the diagnosis of FEC within a GIT biopsy does not exclude an underlying or adjacent malignant process.

\section{FR-004}

Comparison of Her2/neu scoring between microscopic and computer assisted methods assessing the 10 percent cut-off rule in gastric cancer

H.-M. Behrens ${ }^{* 1,2}$, V. Warneke ${ }^{3}$, C. Böger ${ }^{2}$, N. Garbrecht ${ }^{2}$, E. Jüttner' ${ }^{2}$, W. Klapper $^{2}$, M. Mathiak ${ }^{2}$, I. Oschlies' ${ }^{2}$ U. Rudolph ${ }^{2}$, C. Stuhlmann-Laeisz ${ }^{2}$, D. Trick', P. Hufnagl', C. Röcken ${ }^{3}$

'Institute of Pathology, Charité - University medicine of Berlin, Berlin, Germany, ${ }^{2}$ Institute of Pathology, Christian-Albrechts-University, Kiel, Germany, ${ }^{3}$ Department of Pathology, Christian-Albrechts-University, Kiel, Germany

Aims. Our goal was to test the degree of reproducibility of Her2/neu scoring of gastric cancer (GC) between pathologists while paying special attention to the $10 \%$ cut-off rule. Further we tried to find out if reproducibility could be improved using virtual microscopy.

Methods. Ten pathologists rated 12 Her2/neu immunohistochemically stained large sections of GC. Rating was done according to Rüschoff's scoring scheme, except that we queried the highest identifiable staining intensity separately from the corresponding positive tumor ratio. Thus, we gained "raw data" as seen by the pathologists, so that we could identify those cases where a downgrade due to the $10 \%$ cut-off rule would occur. The experiment was carried out repeatedly first using the microscope, then by unassisted virtual microscopy, and finally by virtual microscopy augmented by a manual outline drawing function as a simple assisting method.

Results. Highest identified staining intensities as well as positive tumor ratios vary between pathologists within the same method (inter-observer) and for the same pathologist between methods (intra-observer). In all our comparisons, kappa tests indicate only a moderate agreement for both parameters. Using plain or assisted virtual microscopy could not improve reproducibility of either parameter, nor could it reduce the number of slides rated discordantly concerning the $10 \%$ cut-off limit. In contrast, the positive tumor ratios estimated from the drawn outlines of tumor tissue and positive tumor tissue were in nearly perfect agreement with the values we afterwards calculated from the drawings. We further observed that the variation of estimated positive tumor ratios depends on the geometric complexity of each specimen. A subgroup analysis showed that board certified pathologists more reliably reproduce staining intensities than residents, while there is no such trend in estimating positive tumor ratios.

Conclusions. We identified the heterogeneous distribution of Her2/neu positive tumor areas in GC along with the gradients of staining intensities as causes for only moderate reproducibility of scoring. Both make it difficult to determine a clear, definite border around positive tumor areas, either by unassisted visual inspection or by explicit drawing. We propose to explicitly mention in the pathology report if the Her2/neupositive tumor area is near $10 \%$ to make clear there is a risk of misinterpretation of the staining results.

\section{FR-005}

The Thioredoxin system in esophageal cancers and its impact on epigenetic therapy

T.D. Ahrens ${ }^{* 1}$, S. Timme', J. Ostendorp', L. Bogatyreva², J. Hoeppner ${ }^{3}$, U.T. Hopt ${ }^{3}$, D. Hauschke', M. Werner', S. Lassmann'

'Department Pathology, University Medical Center Freiburg, Freiburg, Germany, ${ }^{2}$ Department of Medical Biometry and Medical Informatics, University Medical Center, Freiburg, Germany, ${ }^{3} \mathrm{Clinic}$ for General and Visceral Surgery, University Medical Center, Freiburg, Germany

Aims. The thioredoxin system physiologically maintains the cellular redox-balance and may be involved in cancer cell responses to epigenetic drugs. One component is the multi-functional protein thioredoxin (Trx), involved in DNA metabolism, transcription factor activity and antioxidant function. Trx activity is regulated by thioredoxin reductase (TXNRD) and its endogenous inhibitor Thioredoxin-interacting protein (TXNIP). So far, the thioredoxin system has not been evaluated in esophageal cancers (ECs). Thus, we investigated the thioredoxin system in EC in situ and its potential involvement in esophageal cell responses to epigenetic-acting drugs in vitro.

Methods. FFPE tissue specimens $(n=37)$ of esophageal squamous cell carcinoma (ESCC) and Barrett's adenocarcinoma (BAC) were examined by semi-quantitative immunohistochemistry for Trx, TXNIP and TXNRD. In vitro, $\mathrm{OE}_{21}$ (ESCC), $\mathrm{OE}_{33}$ (BAC) and non-neoplastic Het1A cells were characterised for Trx, TXNIP and TXNRD. Moreover, changes in the thioredoxin system (western blot) and reactive oxygen species (flow cytometry) were analysed after the histone deacetylase inhibitor (HDACi) MS-275, Azacytidine (AZA) or their combination.

Results. Normal esophageal epithelia (NESE) showed cytoplasmic and nuclear staining for Trx. However, nuclear Trx expression was lost or reduced in $90 \%$ of all ECs. TXNIP showed cytoplasmic localisation in the basal layer of NESE and tended to be reduced in ECs, especially in BACs. This nuclear shift of Trx and reduced TXNIP expression in situ was reflected in $\mathrm{OE}_{21}$ and $\mathrm{OE}_{33}$ compared to non-neoplastic Het-1A cells, thus providing an in vitro-model to analyse the thioredoxin system. Quantification of Trx activity revealed an increase in both EC cell lines. After HDACi/AZA treatment only EC cell lines showed elevated reactive oxygen species production and induction of apoptosis. In OE21 cells an increase of TXNIP was detectable after HDACi/AZA, suggesting its association with reduced Trx activity and thus less antioxidant function.

Conclusions. ECs show impairment of the thioredoxin system, with loss of nuclear Trx and reduced TXNIP levels. In vitro, only EC cell lines react by increased ROS and apoptosis induction after HDACi/AZA, supporting an aberrant and/or non-functional thioredoxin system in EC cells. Thus, HDACi/AZA may induce oxidative stress and subsequent cell death selectively in EC cells, whereas non-neoplastic cells are secured due to their functional thioredoxin system.

Funded by DFG SFB992/C3 Project. 


\section{FR-006}

Inhibition of migration in esophageal cancer cells by ErbB targeting tyrosine kinase inhibitors via reduced Akt, STAT3 and RhoA activity

\section{C.D. Fichter ${ }^{* 1,2}$, V. Gudernatsch ${ }^{2}$, M. Follo ${ }^{3}$, G. Schmidt ${ }^{4}$, K. Aktories ${ }^{4}$, M. Werner ${ }^{2,5}$, S. Lassmann ${ }^{2,5,6}$}

${ }^{1}$ Faculty of Biology, University of Freiburg, Freiburg, Germany, ${ }^{2}$ Department Pathology, University Medical Center Freiburg, Freiburg, Germany, ${ }^{3}$ Department of Internal Medicine I (Hematology/Oncology), Core Facility, University Medical Center Freiburg, Freiburg, Germany, ${ }^{4}$ Institute of Experimental and Clinical Pharmacology and Toxicology, University of Freiburg, Freiburg, Germany, ${ }^{5}$ Comprehensive Cancer Center Freiburg, University Medical Center Freiburg, Freiburg, Germany, ${ }^{6} \mathrm{BIOSS}$ Centre for Biological Signalling Studies, University of Freiburg, Freiburg, Germany

Aims. Receptor tyrosine kinases of the ErbB-family (ErbBs) play a role in cell adhesion and migration, are often overexpressed in esophageal cancers and represent therapeutic targets. Here, we studied the impact of ErbB targeting tyrosine kinase inhibitors (TKIs) on cell migration and underlying cell signalling pathways in esophageal squamous cel carcinoma (ESCC) and esophageal adenocarcinoma (EAC) cell lines. Methods. Effects of TKIs on cell morphology, cell migration, actin cytoskeleton organization and cell adhesions were assessed by H\&E staining, ORIS migration assay and phalloidin+FAK-staining in OE21 (ESCC), $\mathrm{OE}_{33}$ (EAC) and Het-1A (non-neoplastic esophageal epithelium) cell lines. Signalling pathways were analyzed by antibody arrays (R\&D Systems) and Rho-GTPase effector pull-down assays.

Results. The TKIs Erlotinib, Gefitinib and Lapatinib reduced cell migration ( $\mathrm{p} \leq \mathrm{0.01})$, induced focal adhesions and reorganization of the actin cytoskeleton in OE21 cells, with EGFR overexpression and EGFR homodimers. This was accompanied by decreased Akt ( $\mathrm{p} \leq \mathrm{0.01}), \mathrm{STAT}_{3}$ and RhoA ( $\mathrm{p} \leq 0.05)$ activity. In contrast, only Lapatinib impeded cell migration $(\mathrm{p}=0.06)$ and induced morphological changes in $\mathrm{OE}_{33}$ cells, with HER2 overexpression, HER2 homodimers and EGFR/HER2 heterodimers. To further study the impact of specific ErbB heterodimers on cell morphology and migration, Het-1A cells were lentivirally transduced with DNA constructs for fusion proteins allowing controlled activation of EGFR/HER2 or HER2/HER 3 heterodimers. Induction of EGFR/HER2 or HER2/HER3 heterodimerization in these cells caused receptor phosphorylation and morphological changes reflected in reorganization of the actin cytoskeleton.

Conclusions. In conclusion, this study indicates that the ErbB signaling network plays a role in the organization of the actin cytoskeleton and focal adhesions. Its inhibition by TKIs reduces migration of esophageal cancer cells, suggesting TKIs as promising therapeutic approach. Our newly established model system for controlled activation of individua ErbB-family dimers may be used to further study their role in initial invasion and/or migration of esophageal cancer cells.

This study is part of the DFG-funded SFB850, C5 project and was also partially funded by the Mushett Family Foundation, Chester, NJ, USA.

\section{FR-007}

Diagnosing gastritis: imaging mass spectrometry to discern normal gastric mucosa from Helicobacter pylori-associated B-gastritis

P. Matheis ${ }^{* 1}$, R. Casadonte ${ }^{2}$, V. Schommer ${ }^{3}$, M. Otto ${ }^{2,3,4}$, J. Kriegsmann ${ }^{2,3,4}$ 'University Medical Center of the Johannes Gutenberg University Mainz, Mainz, Germany, ${ }^{2}$ Proteopath GbR, Trier, Germany, ${ }^{3}$ Institute of Molecular Pathology, Trier, Germany, ${ }^{4}$ Center for Histology, Cytology and Molecular Diagnostics, Trier, Germany

Aims. Current diagnosis of Helicobacter pylori gastritis relies on the in dividual histopathological examination of the gastric biopsy specimen. The aim of our study is to establish imaging mass spectrometry (IMS) technology as a new screening tool for gastric disease associated with
Helicobacter pylori infection, to support pathological diagnosis. An alternative working flow for diagnosing gastritis is proposed.

Methods. We investigated 55 infected and 26 non-infected bioptic samples of gastric mucosa of the antrum and corpus regions. FFPE samples were deparaffinized, antigen retrieved and on-tissue digested with trypsin. Subsequently, MALDI analysis was performed using an Autoflex Speed mass spectrometer. Mass spectra from regions of interest were exported for classification analysis. Four sample groups, antrum infected (cases $=28$ ), corpus infected (cases $=27$ ), antrum normal (cases $=13$ ), and corpus normal (cases $=13$ ), were created to produce an average spectrum representative of each group. Mass spectra were exported and loaded into ClinProTools software for classification analysis.

Results. We first investigated whether it was possible to detect specific signals discriminating infected and normal samples. Four $\mathrm{m} / \mathrm{z}$ values $(896,940,1213,1836)$ showed differential expression in the Helicobacter pylori infected samples. We then investigated whether it was possible to detect specific signals correlated to bacterial peptides in the antrum comparing infected with non-infected antrum tissues to build a classification model using a Support Vector Machine (SVM) algorithm. SVM incorporated eight ion peptides $(896,1213,1615,1629,1693$ 1836, 310o, 3101) through statistical comparison (Wilcoxon/Kruskal-Wallis test). The class-prediction model generated was then applied to a test set of nine infected samples which could classify the gastritis subgroup with an accuracy of $93 \%$.

Conclusions. IMS can be used to detect Helicobacter pylori in bioptic gastric samples to differentiate infected from non-infected tissues. Single mass-to-charge ratios can be attributed to specific proteins to identify the protein structure of the analyzed tissues. This methodology is able to analyze multiple samples simultaneously. Cost and time effective diagnosis of B-gastritis is possible by MALDI mass spectrometry. Histopathological investigation may be the second step of the diagnostic procedure after exclusion of bacterial infection by MALDI mass spectrometry.

\section{FR-008}

Identification and functional analysis of miRNA/mRNA target pairs in gastrointestinal stromal tumours (GISTs)

I. Bure ${ }^{* 1}$, I.-M. Schaefer', A. Agaimy', A. Hartmann', F. Haller', E. Moskalev' IInstitute of Pathology, Friedrich-Alexander-University, Erlangen-Nuremberg, Germany, 'Institute of Pathology, Göttingen, Germany

Aims. Gastrointestinal stromal tumours (GISTs) are the most common mesenchymal tumours of the gastrointestinal tract with a heterogeneous clinical behaviour. The key genetic alterations and tumour initiating events in GISTs are gain-of-function mutations of the receptor tyrosine kinases KIT and PDGFRA, rendering these kinases and downstream intracellular signalling cascades $\mathrm{PI}_{3} \mathrm{~K} / \mathrm{AKT}_{1}$ and RAS/ RAF/ERK constitutively active. Notably, GISTs from different anatomical localisations display characteristic histological growth patterns, and distinct phenotypic features that are associated with KIT and PDGFRA mutations have been documented. We have earlier reported on differential miRNA and mRNA expression patterns in GISTs according to anatomical localisation and genotype. Aberrant expression of miRNAs may contribute to malignant behaviour and tumour progression by stimulating cell proliferation and eventually evasion of apoptosis. Therefore, the aim of the current study was to decipher the functional role of the novel miRNA species that are differentially expressed in GISTs with regard to clinicopathologic features.

Methods. An in silico analysis using several computational prediction algorithms was applied to genome wide mRNA and microRNA expression data sets in order to identify potential microRNA-mRNA pairs. Cell lines GISTT1 and GIST48b were transfected with respective miRNAs by using lipofectamin reagent. Cell viability was monitored by resazurin assay. The expression of the chosen targets in GIST cell lines was quantified by qRT-PCR and confirmed by Western blot. 
Results. Specific combinatorial miRNA-mRNA expression signatures were revealed in our sample set characteristic of clinicopathological groups of GIST. An in silico analysis revealed significant inverse correlations between miR-665/CD34 and miR-409-3p/PRKCA. The inverse correlation of expression was confirmed by qRT-PCR and Western blot for predicted target pairs. The miRNAs/mRNAs pairs were mapped to pathways of relevance in GISTs ( $\mathrm{PI}_{3} \mathrm{~K} / \mathrm{AKT}$ and RAS/RAF/ERK) as well as to regulatory hubs of cell proliferation and apoptosis. Using transfected GIST cell lines GISTT1 and GIST48b, we could validate the functional interaction between the two miRNA-mRNA pairs.

Conclusions. Using a combined approach of bioinformatic prediction and experimental validation, novel interactions between miR-665/ $\mathrm{CD}_{34}$ and miR-409-3p/PRKCA were identified in GISTs that have potential regulatory effects on major signalling pathways and cell cycle control.

\section{FR-009}

Inactivating mutations of the cell cycle regulators TP53, RB1 and CDKN2A correlate with increased mitotic counts and a phenotypic shift to round cell/epithelioid morphology in gastrointestinal stromal tumors (GIST)

\section{Merten*, A. Agaimy, E.A. Moskalev, A. Hartmann, F. Haller}

Institute of Pathology, Friedrich-Alexander-University, Erlangen-Nuremberg, Germany

Aims. Gastrointestinal stromal tumors (GIST) are the most common mesenchymal tumors of the gastrointestinal tract. Approximately $30 \%$ of GISTs metastasize. Tumors with high risk for malignant behavior are treated adjuvantly with receptor tyrosine kinase inhibitors. While the morphologic appearance is not regularly included in the estimation of malignant behavior, we reported recently that a shift from spindled to round cell/epithelioid morphology in GISTs with KIT mutations is associated with increased mitotic counts and a high risk for malignancy. The aim of the current study was to evaluate the mutation status of the important cell cycle regulators $\mathrm{TP}_{53}, \mathrm{RB}_{1}$ and $\mathrm{CDKN}_{2} \mathrm{~A}$ by massive parallel sequencing in a large cohort of GISTs with clinical follow-up. Methods. A cohort of 90 primary GISTs with clinical follow-up was analyzed for mutations of the cell cycle regulators $\mathrm{TP}_{53}, \mathrm{RB}_{1}$ and $\mathrm{CDKN}_{2} \mathrm{~A}$ by massive parallel sequencing. We designed a custom sequencing panel including 117 amplicons to cover $>90 \%$ of the coding sequences of the three genes. After library preparation, the tumor samples were sequenced on a MiSeq machine.

Results. The mean coverage of the different amplicons among all 90 tumors was 6382 , with $>90 \%$ of the samples and amplicons having a coverage $>$ 10oox. Six tumors $(7 \%)$ harbored mutations in one of the three genes, including three cases with $\mathrm{RB}_{1}$ mutations, two cases with $\mathrm{TP}_{53}$ mutations, and 1 case with a CDKN2A mutation. All six tumors were of the high-risk category, and four of the cases were clinically malignant $(66 \%)$. Notably, four of the tumors with $\mathrm{TP}_{53}, \mathrm{RB}_{1}$ or $\mathrm{CDKN}_{2} \mathrm{~A}$ mutation were of the hypercellular round cell/epithelioid morphology.

Conclusions. Mutations within the genes $\mathrm{TP}_{53}, \mathrm{RB}_{1}$ and $\mathrm{CDKN}_{2} \mathrm{~A}$ are relatively infrequent events in GISTs. However, tumors with a mutation within one of these important cell cycle regulators are characterized by high proliferation and clinically malignant behavior. Inactivation of one of these cell cycle regulators by somatic mutation is an important step in GIST tumor progression, and correlates to a phenotypic shift from spindled to round cell/epithelioid morphology.

\section{FR-010}

Periampullary and duodenal adenocarcinoma in patients with cystic fibrosis (mucoviscidosis)

\section{A. Agaimy ${ }^{* 1}$, F. Haller', R. Stoehr', J. Siebler ${ }^{2}$, A. Hartmann', S. Zirlik ${ }^{2}$}

${ }^{1}$ Institute of Pathology, Friedrich-Alexander-University, Erlangen-Nuremberg, Germany, 2Department of Gastroenterology, Pneumology and Endocrinology, Friedrich-Alexander-University, Erlangen-Nuremberg, Germany

Aims. Cystic fibrosis (CF; mucoviscidosis) is an autosomal recessive inherited disease caused by mutations in the gene encoding for cystic fibrosis transmembrane conductance regulator (CFTR) located at chromosome 7. An increased risk for gastrointestinal malignancy in affected patients has been suggested recently as several cases of adenocarcinoma arising in the esophagus, stomach, small intestine, colon, pancreas and pancreatobiliary system have been recorded. Remarkably, these carcinomas affected predominantly young patients (under 40 years), thus arguing for a CF-related increased risk and against a mere coincidence. To our knowledge, duodenal/ampullary carcinomas have not been reported in CF patients.

Methods. We reviewed our patients attending a specialized CF outpatient clinic service and those recorded in the files of the Institute of Pathology, University of Erlangen for occurrence of gastrointestinal neoplasms.

Results. Three patients with CF and duodenal adenocarcinoma were identified. Tumors originated from the bulbus duodeni, periampullary duodenum and papilla of Vater. Patients were two men and one woman with age at the time of carcinoma diagnosis of 33,42 and 42 years. Histological examination showed poorly differentiated intestinal type adenocarcinoma in all cases, one with prominent mucinous component. Two patients developed peritoneal carcinomatosis soon after diagnosis; one patient died of disease a few years after diagnosis. High-grade intraepithelial neoplasia was seen at the periphery of the carcinoma in all cases. None of our patients had evidence of malignancy in the remainder of the gastrointestinal tract.

Conclusions. We conclude that duodenal carcinoma is overrepresented in our CF patients. These cases add to the accumulating evidence that patients with CF carry an increased risk for gastrointestinal malignancies. Confirmation of these results, identification of specific CF patient subset at higher risk, and characterization of possible precursor lesions are necessary to develop specific screening methods for these patients.

\section{FR-011}

Massive parallel sequencing of succinate dehydrogenase (SDH) subunits in gastrointestinal stromal tumors (GIST) distinguishes between patients with heritable Carney-Stratakis syndrome and inheritable Carney Triad

F. Haller*1, A. Agaimy', E.A. Moskalev', C. Otto ${ }^{2}$, H. Geddert ${ }^{3}$, E. Geissinger', M. Werner', A. Hartmann ${ }^{1}$

IInstitute of Pathology, Friedrich-Alexander-University, Erlangen-Nuremberg, Germany, ${ }^{2}$ Institute of Pathology, University Medical Center, Freiburg, Germany, ${ }^{3}$ Institute of Pathology, St. Vincent Hospital, Karlsruhe, Germany, ${ }^{4}$ Institute of Pathology, University of Würzburg, Würzburg, Germany

Aims. The majority of GISTs that occur in children and young adults under the age of 18 have a wild type status of the receptor tyrosine kinases KIT and PDGFRA. In contrast, these tumors are characterized by a deficient function of the complex II of the mitochondrial respiratory chain (succinate dehydrogenase subunits A, B, C, D). Noteworthy, approximately $5 \%$ of gastric GISTs that occur in adults are SDH-deficient and KIT/PDGFRA wild type. Taken together, SDH-deficient GISTs of all ages arise either in context of the heritable (autosomal dominant) Carney-Stratakis-Syndrom, or as a part of the non-heritable (idiopathic) Carney Triad. Patients with the Carney-Stratakis-Syndrom harbor inactivating germline mutations in one of the four SDH subunits, with 
concurrent somatic inactivation of the second allele by either genetic loss or additional mutations. In contrast, no recurrent genetic event has been reported in patients with Carney Triad so far. The aim of the current study was to establish a diagnostic molecular pathology assay for detection of SDH mutations.

Methods. A cohort of $>300$ GISTs was evaluated by immunohistochemical staining for SDHB and SDHA. Twelve tumors showed loss of SDHB with two cases showing a concurrent loss of SDHA. In four cases, amplicon-based massive parallel sequencing of all four SDH subunits was performed. Additionally, in two of the four cases whole exome sequencing was performed both for DNA from the tumor and from blood.

Results. One patient harboured a germline mutation in SDHB, with concurrent somatic loss of the wild type allele in the tumor. This was seen both by amplicon-based sequencing and whole exome sequencing. Another patient harboured a germline mutation in SDHA, with a different second somatic mutation in the tumor. While the tumor of the first patient showed only a loss of SDHB but intact SDHA staining, the tumor from the second patient was negative for both SDHA and SDHB. The remaining two patients that were sequenced had a wild type status for all four SDH subunits; one patient had clear-cut Carney Triad.

Conclusions. SDH-deficient GISTs can occur at any age, and can be identified by loss of SDHB immunostaining. These tumors are typically wild type for KIT and PDGFRA, and do not respond to tyrosine kinase inhibitors. The mutational analysis of the four subunits of SDH by amplicon-based massive parallel sequencing helps to distinguish patients with heritable Carney-Stratakis syndrome vs. inheritable Carney Triad.

\section{FR-012}

\section{Frequency of unsuspected malignant tumours in autopsy}

\section{K. Schierle ${ }^{* 1}$, M. Wagner' ${ }^{2}$, T. Gradistanac', J. Bertolini', C. Wittekind' 'Institute of Pathology, Leipzig, Germany, '2Diakonissenkrankenhaus, Leipzig, Germany}

Aims. To determine the incidence of clinically unknown malignant tumours in a larger series of autopsies.

Methods. A retrospective 10-year review of 4592 autopsies from university and hospitals autopsy service.

Results. In recent decades the multiple tasks of autopsies have focused on an important role in quality management. This includes comparisons between pre-mortem a post-mortem diagnoses. A special focus has been the detection of pre-mortem undiagnosed malignant tumours. Unsuspected neoplasia rate was $5.7 \%$ (263 of 4592 patients). This figure corresponds to $20.2 \%$ of all detected malignant tumours. Cancer was the cause of death in $23.2 \%$ of these individuals. More than $50 \%$ of the patients with clinically undetected tumours were older than 70 years. The most frequently detected tumours were prostatic carcinomas (23.9\%), colorectal carcinomas (16.3\%), kidney carcinomas (13\%), lung carcinomas (12.7\%) and liver carcinomas (6.5\%), respectively.

Conclusions. The $5.7 \%$ incidence of clinically unsuspected cancer seems high in view of imaging and other diagnostic clinical techniques. The rate emphasizes the necessity for a higher rate of autopsies than practiced today. In correlation to the mortality rate in Germany there are consequences for the health care policy of the future to concern.

\section{FR-013}

Origin, histology and endoscopic findings of secondary tumours in the gastrointestinal tract

\section{M.M. Gilg ${ }^{* 1}$, C. Langner', H.-P. Gröchenig ${ }^{2}$}

'Institute of Pathology, Medical University, Graz, Austria, ${ }^{2}$ Hospital of Barmherzige Brüder, Department of Internal Medicine, St. Veit/ Glan, Austria

Aims. Secondary tumours of the gastrointestinal tract (GIT) are a rare finding during endoscopy and a diagnostic challenge both for the pa- thologist and the endoscopist. The incidence has been described with one metastasis amongst 3847 gastroscopies and one metastasis amongst 1872 colonoscopies. The aim of this study was to conduct a systematic analysis of secondary tumours in the GIT.

Methods. This retrospective study consists of 96 patients diagnosed with a secondary tumour in the GIT between 1984 and 2013 at our institute. To obtain clinical and pathological data a local database review was conducted. Inclusion criterion was the endoscopic detection of haematogenous metastasis confirmed by histopathology. Patients with malignant lymphoma, local tumour recurrence or direct invasion by cancer tissue were excluded.

Results. 96 patients, with a male to female ratio of 2:3 and a mean age of $64.3 \pm 12.9$ [range $33-87$ ] years at diagnosis, were identified in the database. The mean interval between the diagnosis of the primary and secondary tumour was $52.4 \pm 54.1$ [0-251] months. Longest intervals were seen in renal cell carcinoma (mean 67.1 669.8 [o-251] months) and breast cancer (65.3 \pm 47.7 [o-178] months) patients, shortest intervals in lung cancer (mean $11.8 \pm 11.3$ [0-26] months). In $90 \%$ of patients the primary tumour was known at time of endoscopy, with breast cancer $(33 \%)$ and malignant melanoma $(31 \%)$ being the most common primary site, followed by renal cell carcinoma (15\%) and lung cancer (5\%). There was no significant difference $(\mathrm{p}=\mathrm{0.442})$ between the localization of metastases in the upper ( $83 \%$ of secondary tumours) versus the lower GIT ( $17 \%$ of secondary tumours) regarding primary tumour entity. Multiple lesions $(>2)$ were present in 29 (31\%) out of 93 patients. In $60 \%$ of cases, the endoscopists did suspect a secondary tumour. In only $54 \%$ of patients the clinically known primary tumour was communicated to the pathologist.

Conclusions. Breast cancer and malignant melanoma were the most common primary sites of secondary gastrointestinal tumours, whereas lung cancer, in contrast to previously reported studies, was rare in our dataset. In $40 \%$ of cases endoscopists did not suspect metastasis to the GIT, but considered a primary lesion. This may be the reason why knowledge about history of malignant disease was not shared with the pathologist in almost every second patient.

\section{FR-014}

Raman-spectroscopic characterization of the adenoma-carcinoma sequence in a mouse model

N. Vogler ${ }^{* 1}$, T. Bocklitz ${ }^{2}$, C. Schmidt', M. Schmitt' ${ }^{2}$, R. Bräuer ${ }^{3}$, F. Greten ${ }^{4}$, A. Stallmach', J. Popp ${ }^{2,5}$, I. Petersen ${ }^{3}$

${ }^{1}$ Clinic of Internal Medicine IV, University Hospital, Jena, Germany, ${ }^{2}$ Institute of Physical Chemistry and Abbe School of Photonics, Friedrich-Schiller University Jena, Jena, Germany, ${ }^{3}$ Institute of Pathology, Friedrich-Schiller-University, Jena, Germany, ${ }^{4}$ Georg-Speyer-Haus, Frankfurt, Germany, ${ }^{5}$ LeibnizInstitute of Photonic Technology, Spectroscopy/Imaging, Jena, Germany

Aims. In colorectal cancer development, usually referred to as the adenoma-carcinoma sequence, it is highly desired to recognize suspicious regions or obtain a reliable tumor diagnosis directly during a colonoscopy. One such technique, proven to be a powerful optical tool for tissue diagnostics, is Raman micro-spectroscopy. Raman spectra provide a molecular "fingerprint" of the sample. This can be used to highlight significant changes in the metabolism or of the cytoskeleton of cells during the cancer development. Thus, the studies reported here aim for a qualitative and quantitative identification of specific Raman signatures for different tumor stages via a tailored chemometric analysis and, hence, the classification of healthy and altered tissue.

Methods. Mice with an intestinal epithelial cell (IEC)-specific deletion of the tumor suppressor gene p $53_{3}$ were generated. These mice were treated intraperitoneally once a week for 6 weeks with the carcinogen azoxymethane (AOM; $10 \mathrm{mg} / \mathrm{kg}$ ). Mini endoscopy was performed on anesthetized mice. For histological and Raman spectroscopic investigations mice were sacrificed at different time points after the AOM application. $20 \mu \mathrm{m}$ thick cryosections of colon and rectum of Cre+ and Cre- 
Tp53 $\triangle$ IEC mice both treated with AOM were Raman spectroscopically investigated (CRM 300 , WITec GmbH, Germany; Deep Depletion CCD camera (DU401 BR-DD, ANDOR)). The sections were excited with a $785 \mathrm{~nm}$ diode laser focused through a 5ox objective (NA 0.7). Raman images were obtained in scanning mode with a step size of $5 \mu \mathrm{m}$ and an integration time of $2 \mathrm{~s}$ per spectrum. The selection of the areas to be measured was carried out in coordination with pathologists. Subsequently, preprocessing and statistical modeling were performed using the software package R.

Results. A dataset containing Raman spectra of both colon and rectum of 55 mice ( $27 \mathrm{Cre}+/ 28$ Cre- individuals) was created followed by a twostep modeling. Selecting the epithelium first, the mathematical model for classification is built upon the resulting set of epithelial spectra. Based on a subset of the measured mice the classification of normal epithelial and tumor tissue is obtained with an accuracy of $94 \%$.

Conclusions. It could be shown that the different stages of the adenoma-carcinoma sequence could successfully be distinguished using Raman micro-spectroscopy on cryosections. Further studies are needed to transfer these results to in vivo endoscopic measurement conditions.

Funded by DFG (PO 563/13-1, STA 295/9-1, PE 602/6-1).

\section{FR-015}

\section{Histology, immunohistochemistry and ultrastructure to evaluate} tissue-material interaction of different peritoneal adhesion barriers

\section{V.H. Schmitt ${ }^{* 1}$, C. Brochhausen', A. Mamilos', C.N.E. Planck', T.K. Rajab ${ }^{3}$, R. Zehbe ${ }^{4}$, M. Wallwiener ${ }^{5}$, H. Hierlemann ${ }^{6}$, H. Planck ${ }^{6}$, C.J. Kirkpatrick' IInstitute of Pathology, University Medical Centre of the Johannes Guten- berg-University, Mainz, Germany, ${ }^{2}$ Department of Gynaecology and Obstetrics, Eberhard Karls University, Tuebingen, Germany, ${ }^{3}$ Brigham and Womens Hospital, Harvard Medical School, Boston, United States, ${ }^{4}$ Institute for Material Sciences and Technologies, Technical University Berlin, Berlin, Germany, ${ }^{5}$ Department of Obstetrics and Gynecology, Ruprecht-Karls-Uni- versity, Heidelberg, Germany, ${ }^{6}$ Institute of Textile Technology and Process Engineering, Denkendorf, Germany}

Aims. Adhesion barriers are widely used to prevent serosal adhesion formation. Since the pathophysiology of adhesion formation is not clearly understood and an optimal barrier material has not yet been found, it is essential to analyse the cellular mechanisms and tissue reaction within peritoneal wound healing and adhesion formation. This study represents the first systematic histomorphological, immunohistochemical and ultrastructural investigation of clinically used adhesion barriers. Methods. Wistar rats were treated with 5 different adhesion barriers (Adept $^{\oplus}$, Intercoat ${ }^{\oplus}$, Spraygel ${ }^{\oplus}$, Seprafilm ${ }^{\oplus}$, SupraSeal ${ }^{\star}$ ) or remained untreated after standardized peritoneal damage.14 days postoperatively, the extent of adhesions was scored macroscopically. Treated areas were explanted and processed according to standardized methods for histological (HE,EvG,ASD), immunohistological (anti-CD68) and ultrastructural (SEM,TEM) analysis. Inflammation, foreign body reaction and extent of fibrosis were assessed according to the ISO score for biological evaluation of medical devices. Statistical analyses were performed using Mann-Whitney-U and Kruskal-Wallis test. Using SEM, TEM and exemplary synchrotron- $\mu \mathrm{CT}$ the extent of remesothelialization was assessed.

Results. All materials revealed a good overall biocompatibility. However, considerable differences were established with respect to the individual parameters inflammation, foreign body reaction, macrophage infiltration and fibrosis. As seen macroscopically, the most effective barrier revealed moderate inflammation and no fibrosis. No correlation was found for the number of CD68-positive macrophages and the tissue response to the barriers. Ultrastructurally, the macroscopically most effective barrier $\left(\right.$ SupraSeal $\left.{ }^{\circledR}\right)$ revealed the highest level of remesothelialization, which could be confirmed by synchrotron- $\mu \mathrm{CT}$.
Conclusions. Relevant differences between the barriers were apparent. Most importantly, inflammation, giving a negative impact in the ISO score,was seen to be necessary for a healing process with minimal fibrosis. Interestingly, CD68-positive macrophages did not correlate with the tissue reaction, underlying the recent understanding of macrophages as a dynamic regulative cell-type with various functions and phenotypes. Our results suggest that current scores for biomaterial evaluation should evolve in parallel with new pathophysiological knowledge. In these processes pathologists should be actively engaged as the experts on histopathological changes in tissue damage and disease.

\section{FR-016 \\ Migration of enterocytes is diminished in intestinal caspase-8 knockout mice}

\section{N. Gassler, M.K. Jeon*, C. Klaus, U. Schneider, E. Kaemmerer, M. Schiffer, P. Kuhn}

Institute of Pathology, Aachen, Germany

Aims. Caspase- 8 is essential for establishment and integrity of the intestinal barrier. In caspase- 8 knockout animals, necroptosis is dramatically increased in crypt-lining epithelia associated with severe inflammation. However, maturation of enterocytes and villus forming morphogenesis is found. The study was designed to investigate caspase-8 dependent effects on migration of enterocytes.

Methods. In vivo tracing of enterocytes in intestinal caspase- 8 knockout as well as control mice were performed using the BrdU approach. Anti-Ki67 immunostainings and statistical analysis of morphometric data were done.

Results. In small intestinal mucosa, migration of caspase- 8 deleted enterocytes was diminished when compared with controls. Individual parts of the small intestine were differentially affected.

Conclusions. Aberration in caspase- 8 functions could be involved in the pathogenesis of inflammation-related intestinal disorders.

\section{FR-017}

\section{Endoplasmatic reticulum stress in pancreatic neuroendocrine} tumors

E. Klieser' ${ }^{1}$, R. Illig', S. Stättner ${ }^{2}$, F. Primavesi ${ }^{2}$, T. Jäger ${ }^{2}$, S. Swierczynski², T. Kiesslich ${ }^{3}$, R. Kemmerling ${ }^{4}$, P. DiFazio ${ }^{5}$, D. Neureiter ${ }^{* 1}$

'Institute of Pathology, Salzburger Landeskliniken/Paracelsus Medizinische Privatuniversität, Salzburg, Austria, ${ }^{2}$ Universitätsklinik für Chirurgie, Salzburger Landeskliniken, Paracelsus Medizinische Privatuniversität, Salzburg, Austria, ${ }^{3}$ Universitätsklinik für Innere Medizin I, Salzburger Landeskliniken, Paracelsus Medizinische Privatuniversität, Salzburg, Austria, ${ }^{4}$ Medizinisches Versorgungszentrum für Histologie, Zytologie und Molekulare Diagnostik, Trier, Germany, ${ }^{5}$ Institut für Chirurgische Forschung, Philipps-Universität Marburg, Marburg, Germany

Aims. Endoplasmic Reticulum (ER) stress is a highly conserved cellular defence mechanism that responds to perturbations of ER function. Regulative mechanisms of ER stress-related apoptotic cell death in human cancer are new promising targets for anticancer drug development. The role of ER-stress in pancreatic neuroendocrine tumors (PNET) still remains unclear.

Methods. We analyzed the expression pattern of three key players of ER-stress $\mathrm{CHOP} / \mathrm{GADD} 153, \mathrm{ATF}_{4}$ and $\mathrm{BIP} / \mathrm{GRP}_{78}$ in a human tissue microarray (TMA) of PNET resected between 1997 and 2013. All cases were extensively characterized clinically and pathologically according to published guidelines.

Results. 49 cases $(29$ (59.2\%) female versus 20 (40.8\%) male) with PNET (40.8\% immunohistochemically endocrine positive) showed following detailed clinicopathological characteristics: G1-3 56.3-29.2-14.5\%; pT1-4 38.8-22.4-32.7-6.1\%; pNo-1 67.3-32.7\%; pMo-1 79.6-20.4\%. Immunohis- 
tochemical profiling revealed a significant up-regulation of $\mathrm{CHOP}$, $\mathrm{ATF}_{4}$ and BIP in all PNET cases compared to normal pancreatic control tissue. Additionally, a partial significant shift of CHOP and ATF4 was observed between the cytosol and the nuclei, where they could act as transcription factors. Correlation analysis generally indicated a tendential association of clinicopathological data with localized expression patterns of ER-stress associated proteins, whereby BIP and CHOP were significantly associated with grading and pathological T-categories. Conclusions. The up-regulation of these three ER-stress members $\mathrm{CHOP}, \mathrm{ATF}_{4}$ and BIP in PNETs indicates the presence and engagement of the ER-stress pathway in this tumor entity. Once ER-stress is capable of supporting continuous proliferation and survival even under adverse micro environmental conditions in human cancerogenesis, the current data demonstrate another possible anticancer therapy option in PNET.

\section{FR-018 \\ Novel prognostic markers revealed by a proteomic approach separating benign from malignant insulinomas}

J. Friemel', I. Alkatout ${ }^{2}$, B. Sitek ${ }^{3}$, M. Anlauf', A. Scarpa ${ }^{5}$, A. Perren $^{6}$, G. Klöppel', B. Sipos*8

'Institute of Surgical Pathology, University Hospital, Zurich, Switzerland, 2Department of Gynecology and Obstetrics, University Hospital Schleswig-Holstein, Kiel, Germany, ${ }^{3}$ Medical Proteom-Center, Ruhr-University, Bochum, Germany, ${ }^{4}$ Group Practice of Pathology, Limburg, Germany, ${ }^{5}$ Institute of Pathology, University of Verona, Verona, Italy, ${ }^{6}$ Institute of Pathology, University of Bern, Bern, Switzerland, 'Institute of Pathology, Technical University of Munich, Munich, Germany, ${ }^{8}$ Department of Pathology, University Hospital, Eberhard Karls University, Tübingen, Germany

Aims. The prognosis of pancreatic neuroendocrine tumors (PanNETs) is related to size, histology and proliferation rate. However, this stratification needs to be refined further. We conducted a proteome study on insulinomas, a well-defined PanNET tumor entity, in order to identify proteins that can be used as biomarkers for malignancy.

Methods. Based on a long follow-up, insulinomas were divided into those without metastases (benign) and those with metastases (malignant). Microdissected cells from six benign and six malignant insulinomas were subjected to a procedure combining fluorescence dye saturation labeling with high-resolution two-dimensional gel electrophoresis. Differentially expressed proteins were identified using nanoLC-ESI-MS/ MS and validated by immunohistochemistry on tissue microarrays containing 62 insulinomas.

Results. Sixteen differentially regulated proteins were identified among 3000 protein spots. Immunohistochemical validation revealed that aldehyde dehydrogenase 1 (ALDH1) and voltage-dependent anion-selective channel protein $1(\mathrm{VDAC})$ ) showed significantly stronger expression in malignant insulinomas than in benign insulinomas, whereas Tumor Protein $\mathrm{D}_{52}$ (TPD52) binding protein was expressed less strongly in malignant insulinomas than in benign insulinomas. Using multivariate analysis, low TPD52 expression was identified as a strong independent prognostic factor for both recurrence-free and overall disease-related survival.

Conclusions. In conclusion, the three proteins ALDH1, VDAC1 and TPD52 fulfil the requirements for biologic markers that can be used beyond ENETS and WHO criteria for prognostic stratification of resected insulinomas. To our knowledge, these are the first protein markers that have been described in a well-defined group of PanNETs. In the light of these results, it seems promising to further validate the identified proteins and associated processes in PanNET as well as to extend proteome expression studies to other functioning and non-functioning PanNETs in order to find new biological markers for their prognostic assessment.

\section{AG Gastroenteropathologie Postersession - Pankreas/Leber}

\section{FR.1-001}

p53-dependent Nestin regulation couples tumor suppressive functions and cell fate decisions in liver cancer

D.F. Tschaharganeh ${ }^{* 1}$, W. Xue ${ }^{2}$, D.F. Calvisi ${ }^{3}$, A. Banito', L.E. Dow ${ }^{1}$, M. Evert ${ }^{3}$, D. Capper', A. Lujambio', G. Enikolopov' ${ }^{2}$, T. Longerich ${ }^{5}$, S.W. Lowe'

${ }^{1}$ Memorial Sloan-Kettering Cancer Center, Department of Cancer Biology \& Genetics, New York, United States, ${ }^{2} \mathrm{CSHL}$, Cold Spring Habor, United States, ${ }^{3}$ Institute of Pathology, University Medicine, Greifswald, Germany, ${ }^{4}$ Institute of Pathology, Ruprecht-Karls-University, Heidelberg, Germany, ${ }^{5}$ Institute of Pathology, University Hospital Heidelberg, Heidelberg, Germany

Aims. The p53 tumor suppressor coordinates a series of anti-proliferative responses that restrict the expansion of malignant cells and, as a consequence, is lost or mutated in the majority of human cancers. One poorly understood $\mathrm{p} 53$ response that may play a critical role in restricting tumors relates to its ability to limit the self-renewal of stem and progenitor cells. Here we investigated the ability of $\mathrm{p}_{53}$ to restrict progenitor cells in liver cancer and the functional consequence.

Methods. Murine liver tumors with regulable $\mathrm{p}_{53}$ expression were generated and transcriptome profiling after $\mathrm{p} 53$ restoration was performed. Nestin promoter-GFP mice were used to visualize Nestin promoter activity in adult livers and murine liver tumors in vivo. A novel transgenic liver tumor model was generated and consequence of Nestin suppression was functionally evaluated in vivo and in vitro using RNA interference. Finally, p53 status and Nestin expression were determined by immunohistochemistry, Sanger sequencing, and array-CGH in a large cohort of human hepatocellular carcinomas (HCCs) and cholangiocarcinomas (CCs).

Results. P53-deficient murine liver tumors exhibited Nestin-positive tumor cells independent of the driving oncogene. Reactivation of p53 in murine liver tumors and human HCC cells with dysfunctional p53 abolished Nestin promoter activity and expression on mRNA and protein levels. Promoter analysis identified the transcription factors Sp1 and Sp3 as crucial mediators for p53-induced Nestin repression. Inhibition of Nestin in a transgenic mouse model led to complete blockade of tumor initiation in vivo as well as significantly reduced colony formation and subcutaneous tumor growth of human and murine liver cancer cell lines. Nestin expression was restricted to liver progenitor cells in adult livers and Nestin-positive tumor cells retained a progenitor-like phenotype capable to differentiate to HCCs or CCs in vivo depending on additional oncogenic hits. Human HCCs and CCs showed elevated Nestin expression, which was significantly associated with decreased patient overall survival, and correlated with p53 deletion and mutation. Conclusions. Our results suggest that p53-dependent repression of the stem cell marker Nestin plays an important tumor-suppressive role in liver cancer. Furthermore, our results highlight the regulatory role of p53 in maintenance of progenitor cells in liver tumors and shed new light on a common cellular origin of both, hepatocellular carcinomas and cholangiocarcinomas. 


\section{FR.1-002}

Clear cell foci of altered hepatocytes after intraportal pancreatic islet transplantation-implementation of the model from rat to mouse

\section{S. Ribback ${ }^{* 1,2}$, J. Sonke ${ }^{2}$, A. Hensel' ${ }^{2}$, F. Dombrowski \\ IInstitut für Pathologie, Greifswald, Germany, ${ }^{2}$ Institut für Pathologie, Universitätsmedizin Greifswald, Greifswald, Germany}

Aims. Low number intraportal transplantation of pancreatic islets in diabetic rats is a model of hormonal induced hepatocarcinogenesis. The resulting local hyperinsulinism in the liver acini downstream of the transplanted islets leads to clear cell preneoplastic liver foci which progress to hepatocellular adenomas and carcinomas. Metabolic alterations at the posttranslational level have been characterized in the rat model, i.e. up-regulation of glycolysis and de-novo-lipogenesis as well as down-regulation of gluconeogenesis and glycogenolysis. To further consider regulation of gene expression we implemented this model in the mouse.

Methods. 40 of 80 streptocotozin diabetic (single intraperitoneal dosis of $240 \mathrm{mg} / \mathrm{kg}$ body weight; blood glucose level $>20 \mathrm{mmol} / \mathrm{l}$ ) $\mathrm{C}_{57} \mathrm{Bl} / 6$ J mice received an intraportal transplantation of $60-70$ isolated, isologous pancreatic islets into the liver. 40 diabetic mice remained without transplantation. Another 40 mice were transplantated without previous streptozotocin injection and were therefore normoglycemic. 40 animals remained untreated. Mice were sacrificed after 1 week or 4 weeks, respectively.

Results. As intended, the diabetic mice remained hyperglycemic despite islet transplantation, because the number of transplanted islets was low. Continuous hyperglycemia is necessary in this model, so that the islets are stimulated to permanent maximal insulin synthesis and secretion. As a result, local hyperinsulinism and simultaneous hyperglycemia is obtained at the downstream liver acini. Liver tissue was examined after perfusion fixation using histological, immunohistochemical and electron microscopic methods. Engraftment of the transplanted islets is located in peripheral portal vein branches. As early as after 1 week, hepatocytes at the downstream region of the transplants reveal clear cell morphology, which corresponds to an increased storage of glycogen and lipids in consequence of the local hyperinsulinism and simultaneous hyperglycemia. After 4 weeks, glycogen storing acini show an increased proliferative activity compared to extrafocal liver tissue and were larger than after 1 week.

Conclusions. Morphological alterations of murine hepatocellular clear cell foci after intraportal pancreatic islet transplantation resemble preneoplastic liver lesions of other hepatocarcinogenesis mouse models. The islet transplantation model is applicable to the mouse and constitutes the basis for future knockout models and long time experiments of hepatocarcinogenesis.

\section{FR.1-003}

\section{$\beta$-catenin and YAP signaling synergizes to promote hepato-} blastoma in mice and men

D. Calvisi*1, J. Tao ${ }^{2}$, S. Ranganathan' ${ }^{3}$, A. Cigliano', L. Jang², B. Fan², S. Armeanu-Ebinger', S. Delogu', S. Ribback', F. Dombrowski', S. Monga ${ }^{3}$, X. Chen ${ }^{2}$, M. Evert ${ }^{1}$

'Institut für Pathologie, Universitätsmedizin Greifswald, Greifswald, Germany, ${ }^{2}$ Department of Bioengineering and Therapeutic Sciences, University of California, San Francisco, United States, ${ }^{3}$ Department of Pathology, University of Pittsburgh, Pittsburgh, United States, ${ }^{4}$ Department of Pediatric Surgery and Pediatric Urology, Children's University Hospital, Tübingen, Germany

Aims. Aberrant activation of Wnt/ $\beta$-catenin and Yap signaling pathways has been independently observed in the major liver tumor types, including hepatocellular carcinoma (HCC), intrahepatic cholangiocarcino- ma (ICC), and hepatoblastoma (HB). However, the functional interplay between these two pathways in liver cancer remains poorly understood. Methods. We evaluated the activation of Wnt/ $\beta$-catenin and Yap pathways in a collection of human HCC, ICC, and HB by immunohistochemistry. Effects of both protooncogenes on the growth of human $\mathrm{HB}$ cell lines were assessed using silencing approaches, including specific siRNAs against $\beta$-catenin and Yap genes and inhibitors of $\beta$-catenin and Yap transcriptional activity. Levels of $\beta$-catenin, Yap, and related downstream targets (c-Myc Axin2, DKK1, Cyclin D1, for $\beta$-catenin, and CTGF, JAG1, CYR61, and Survivin for Yap, respectively) were assessed by western blotting or real-time RT-PCR. Furthermore, the crosstalk between Yap and $\mathrm{Wnt} / \beta$-catenin cascades was investigated in vivo by overexpressing activated forms of $\beta$-catenin and Yap genes in the mouse liver via hydrodynamic gene delivery.

Results. $\beta$-catenin and Yap were concomitantly activated in $79 \%$ of human HB specimens, while no such association was evident in human HCC or ICC samples. Silencing of Yap or $\beta$-catenin gene alone resulted in decreased proliferation and increased apoptosis, whereas concomitant suppression of Yap and $\beta$-catenin genes was highly detrimental for the growth of $\mathrm{HB}$ cell lines in vitro. At the molecular level, suppression of Yap led to decreased transcriptional activity of $\beta$-catenin, whereas knockdown of $\beta$-catenin triggered downregulation of Yap. Combined hydrodynamic delivery of activated forms of $\beta$-catenin and Yap genes in the mouse liver led to rapid tumorigenesis, with $100 \%$ mortality 11 weeks after injection of Yap/ $\beta$-catenin mice. In this mouse model, Yap oncogenic effects were fully dependent on its interaction with TEAD transcription factors. Histologically, most tumors developed in Yap/ $\beta$ catenin mice were reminiscent of human fetal $\mathrm{HB}$ and expressed elevated levels of Yap and $\beta$-catenin canonical targets.

Conclusions. Coordinated deregulation of Wnt/ $\beta$-catenin and Yap pathways may be a pivotal oncogenic mechanism in human HB. The Yap/ $\beta$-catenin mouse model might be highly useful to unravel the molecular mechanisms responsible for $\mathrm{HB}$ development in humans and as well as to generate innovative therapies against this deadly disease.

\section{FR.1-004}

Genomic profiling of clonal tumor populations from hepatocellular carcinomas in non-cirrhotic liver reveals undetected populationspecific genomic aberrations

\section{Schlageter*, V. Perrina, T. Lorber, L. Qualiata, L. Tornillo, M. Kovac, C. Ruiz, L. Terracciano}

Institute of Pathology, University Hospital Basel, Basel, Switzerland

Aims. Hepatocellular carcinoma (HCC) is the most common type of liver cancer and $15-20 \%$ of cases occur in the non-cirrhotic liver. Similar to other tumor types, HCC is caused and driven by a sequential accumulation of genomic aberrations in cancer-relevant genes. In current genomic studies, intratumoral heterogeneity is usually not taken into consideration. Further, contamination with normal cells can dramatically reduce the resolution and thus the detection of focal genomic aberrations. Aim of this study was to obtain pure clonal tumor populations from HCC occurring in non-cirrhotic liver, subject them to high resolution genomic profiling and to discover population specific genomic aberrations that could serve as predictive markers or drug targets in the near future.

Methods. We selected 8 surgically resected tissue samples from patients with HCC without underlying cirrhosis. We applied DNA content based flow-sorting by usage of a BD Influx flow sorter and DAPI as staining reagent. Populations with distinct DNA content (diploid and aneuploid) were sorted and DNA was processed. Briefly, DNA was extracted, whole genome amplified, digested, labeled and hybridized to high resolution whole genome CGH microarrays. Bioinformatic analysis of the resulting data was thoroughly analyzed by usage of the software DNA Analytics 
Results. Aneuploid populations were detected (and sorted) in about $42 \%$ of the patients. Genomic profiling of the distinct sorted clonal populations revealed tumor populations with specific aberrations as well as populations with almost "flat" genomes. We detected larger aberrations (amplifications and deletions) that had previously been reported in the context of HCC, such as 1q gain, $6 \mathrm{p}$ gain or loss of $9 \mathrm{p}$. Interestingly, we also observed homozygous deletions and focal amplifications that have not been reported in the context of HCC occurring in non-cirrhotic liver so far, such as a deletion only covering the IRF2 gene. Furthermore a deletion of the putative tumor suppressor gene GSTT1 was found in several samples.

Conclusions. Genomic profiling of sorted clonal tumor populations is a reliable approach for the detection of genomic aberrations that were not discovered until now due to intratumoral heterogeneity and contamination with normal cells. We are convinced that the here presented approach will help us to elucidate the context of vulnerabilities of patients with hepatocellular carcinoma and to develop novel tailored and personalized treatment strategies for these patients.

\section{FR.1-005}

Molecular characterization of primary hepatic malignant epitheloid hemangioendothelioma

\section{Mogler*1, A. Böhnisch'², A. Brüntgens', V. Endris', R. Penzel', P. Schirmacher', N. Pfarr ${ }^{1}$ \\ IInstitute of Pathology, Heidelberg, Germany, ${ }^{2}$ Institute of pathology, Heidelberg, Germany}

Aims. Epitheloid hemangioendothelioma (EHE) is a rare malignant vascular tumor of unknown etiology and molecular pathogenesis. It may arise at many different sites, but liver is among the more frequent manifestations. Course of the disease is highly variable and so far only limited therapeutic options are available such as resection and liver transplantation. Our aim is to molecularly characterize this tumor entity in order to find new potential therapeutical targets.

Methods. EHEs were histologically classified by two expert liver pathologists and further characterized by immunohistology. Twelve formaline fixed and paraffin embedded primary hepatic EHE were immunohistochemically stained for potential predictive markers (Vascular Endothelial Growth Factor Receptor 2, KIT, HER2, Estrogen- and Progesterone Receptors). To test for genetic alterations, all samples were Sanger sequenced for c-kit, epidermal growth factor receptor (EGFR) and braf. Eight samples were analyzed by next generation sequencing using the Ion Personal Genome Machine System and the AmpliSeq Cancer Hotspot Panel v2 comprising 207 amplicons from frequently mutated genomic "hot spot" regions of 50 known human cancer genes. Results. All EHEs were negative for the markers tested by immunohistochemistry and wild type for EGFR, c-kit and braf. Interestingly, all eight NGS-sequences EHEs showed genetic polymorphisms in the $\mathrm{p} 53$ gene locus and three out of the eight samples genetic polymorphism in the KIT gene locus. One sample showed a mutation in the platelet derived growth factor receptor (PDGFR) alpha gene locus and one sample in the p53 gene locus. For all other tested markers wild type sequences were found.

Conclusions. Hepatic EHEs seem to represent a genetically quite homogenous group of malignant vascular tumors with only few mutations of potential therapeutical relevance.

\section{FR.1-006}

MALDI imaging mass spectrometry to characterize a hepatocellular carcinoma arising in a dysplastic nodule

M. Kriegsmann ${ }^{* 1}$, R. Casadonte ${ }^{2}$, T. Longerich ${ }^{1}$, P. Schirmacher ${ }^{1}$, M. Otto ${ }^{2,3,4}$, J. Kriegsmann ${ }^{2,3,4}$

'University of Heidelberg, Trier, Germany, ${ }^{2}$ Proteopath GbR, Trier, Germany, ${ }^{3}$ Center for Histology, Cytology and Molecular Diagnostics, Trier, Germany, ${ }^{4}$ Institute of Molecular Pathology, Trier, Germany

Aims. We present the case of a 6o-year-old male patient, who was transplanted for alcohol-induced liver cirrhosis. During pathological work-up a $1.4 \mathrm{~cm}$ measuring lesion was detected. Histology revealed a high-grade dysplastic nodule with nodule-in-nodule progression to hepatocellular carcinoma (HCC). We aimed at the identification of differentially expressed peptides during stepwise hepatocarcinogenesis in this patient using MALDI imaging mass spectrometry.

Methods. The tumor-containing liver tissue was sectioned and stained with hematoxylin and eosin. A serial section was subjected to in situ trypsin digestion at $37^{\circ} \mathrm{C}$ for $1.5 \mathrm{~h}$ in a humidity chamber, following deparaffination and antigen retrieval. Trypsin $(0.5 \mu \mathrm{g} / \mu \mathrm{l})$ was applied onto the sections by vibrational spraying (ImagePrep, Bruker Daltonik $\mathrm{GmbH}$ ). Matrix solution ( $7 \mathrm{mg} / \mathrm{ml}$ in $50 / 50$ acetonitrile/o.5\% TFA) was deposited onto digested sections using the same devise. MALDI imaging data were acquired at a spatial resolution of $200 \mu \mathrm{m}$ using an Autoflex Speed TOF/TOF system and visualized with FlexImaging software. Spectral analysis was performed with FlexAnalysis 3.3 and ClinProTools 3.3 software programs (Bruker Daltonik $\mathrm{GmbH}$ ).

Results. We detected several differentially expressed $\mathrm{m} / \mathrm{z}$ ion peptides between non-tumorous surrounding liver, dysplastic nodule, and HCC. While some $\mathrm{m} / \mathrm{z}$ values were unique for the HCC, but could not be identified in the area of the dysplastic nodule or the surrounding liver tissue, other $\mathrm{m} / \mathrm{z}$ values were exclusively present in the dysplastic nodule and/or in the surrounding liver tissue but not in the area containing the HCC.

Conclusions. Using MALDI imaging we successfully identified peptides differentially expressed between non-tumorous surrounding liver, dysplastic nodule, and HCC. Larger series are needed to evaluate whether these peptides or the corresponding proteins might serve as diagnostic markers in daily practice.

\section{FR.1-007}

Serum reponse factor (SRF) is involved in the transcriptional upregulation of the functional p53 inhibitor MDM4 in hepatocellular carcinoma

\section{R. Pellegrino ${ }^{* 1}$, A. Thavamani ${ }^{2}$, R. Geffers ${ }^{3}$, P. Schirmacher ${ }^{1}$, A. Nordheim ${ }^{2}$,} T. Longerich

'Institute of Pathology, University Hospital Heidelberg, Heidelberg, Germany, ${ }^{2}$ Department for Molecular Biology, Interfaculty Institute of Cell Biology, University of Tübingen, Tübingen, Germany, ${ }^{3} \mathrm{Helmholtz}$ Center for Infection Research, Braunschweig, Germany

Aims. MDM4 represents a p53-binding protein, which negatively regulates its transcriptional activity. We have previously demonstrated that $\mathrm{MDM}_{4}$ is upregulated in about $50 \%$ of human hepatocellular carcinomas (HCC), both at messenger RNA (mRNA) and protein level. Although we identified 1932 gains as a mechanism leading to $\mathrm{MDM}_{4}$ overexpression in human HCC, many liver tumors show a balanced $\mathrm{MDM}_{4}$ gene locus. Thus, we hypothesized that additional mechanisms are responsible for aberrant $\mathrm{MDM}_{4}$ expression. The transcriptional regulation of $\mathrm{MDM}_{4}$ remains largely elusive. Here, we show that overexpression of SRF is involved in the transcriptional upregulation of MDM4 in HCC thereby supporting its protumorigenic function.

Methods. To identify potential transcription factors (TFs) binding sites in the MDM4 promoter, we took advantage of the MAPPER database. 
Expression profiling of human HCCs was used to analyze a potential association between $\mathrm{MDM}_{4}$, SRF, and co-acting factors. Transfection of gene-specific siRNAs was used to knockdown SRF in HCC cell lines. In addition, MDM4 protein expression was analyzed in SRF-VP16 transgenic mice.

Results. Using in silico analysis we identified a putative SRF binding site in the MDM4 promoter region. Overexpression of SRF mRNA compared to normal liver tissue was observed in a collection of human HCCs. In addition, a strong positive correlation between MDM4 and SRF expression was found in these samples, which was paralleled by overexpression of MKL1-2 and ELK1, which are transcriptional co-activators acting in complex with SRF. The knockdown of SRF by gene-specific siRNAs decreased MDM4 mRNA and protein levels in HCC cell lines, which resulted in re-activation of $\mathrm{p}_{53}$ target genes and reduction of cell viability in vitro. Finally, upregulation of $\mathrm{MDM}_{4}$ was observed in SRFVP16 transgenic mice in vivo.

Conclusions. Taken together, our data demonstrate a crucial role for SRF in the transcriptional activation of $\mathrm{MDM}_{4}$ expression, thereby supporting the oncogenic $\mathrm{MDM}_{4}$ activity in HCC in vitro and in vivo.

\section{FR.1-008 \\ New insights in HOXA13 and HOTTIP role as prognostic factors in hepatocellular carcinoma}

L. Quagliata*1, M. Matter', L. Tornillo', M. Heim², C. Cillo³, L. Terracciano IInstitute of Pathology, University Hospital, Molecular Pathology, Basel, Switzerland, ${ }^{2}$ Department of Biomedicine, University Hospital, Division of Medical Genetics, Basel, Switzerland, ${ }^{3}$ Medical School,, Federico II University, Naples, Italy

Aims. Previously, we observed that among the transcriptional factors family of HOX-genes, HOXA13 is highly deregulated in hepatocellular carcinoma (HCC). HOTTIP, a recently described long non-coding RNA, is located at the 5'HOXA locus (in contiguity with HOXA13) and binds WDR5/MLL-complexes driving transcription along the entire HOXA locus. Lately, we reported HOTTIP/HOXA13 deregulation as a key feature in HCC development, controlling liver-cancer cells apoptosis. Finally, we outlined HOTTIP/HOXA13 as predictive markers of HCC patients' outcome and disease progression. Here, driven by Chromatin-Immunoprecipitation (ChiP) data, we aimed to corroborate our findings by screening a large cohort of HCCs evaluating HOXA13 levels and selected metastasis-associated genes directly regulated by HOXA13. Methods. A TMA (tissue-microarray), comprises a total of $\mathrm{n}=305$ specimens, $\mathrm{n}=82$ from normal liver, $\mathrm{n}=108$ from cirrhotic patients and $\mathrm{n}=115$ from HCCs, has been immunohistochemically stained for HOXA13, CK-7, CK-19, E-Cad, Galectin-3, Prune and Nm23-H2. Staining scores have been correlated with clinical-pathological data.

Results. HOXA13 is altered in $41 \%$ of analysed samples, confirming our previous reports. Corroborating our gene expression data, we observed that higher HOXA13 levels are associated with poorer patients' outcome and grading (Edmondson and BCLC). HOXA13 expression is accompanied by high liver progenitor markers levels, CK-7 and CK19. Furthermore, high HOXA13 expression is coupled with low E-Cad and increased Gal-3, Prune and $\mathrm{Nm}_{2} 3-\mathrm{H}_{2}$, providing a molecular basis for its clinical association with metastasis formation.

Conclusions. HOXA13 IHC-protein levels predict HCC disease outcome, confirming our gene-expression data and making HOXA13 a suitable marker for liver carcinogenesis evaluation. Additional experiments aiming to elucidate HOXA13 role in promoting metastasis are urgently needed.

\section{FR.1-009}

Imaging mass spectrometry to distinguish hepatocellular carcinoma from normal liver tissues

M. Kriegsmann ${ }^{* 1}$, R. Casadonte ${ }^{2}$, N. Waldburger ${ }^{1}$, M. Otto ${ }^{2,3,4}$, S.-O. Deininger ${ }^{5}$, D. Suckau ${ }^{5}$, J. Kriegsmann ${ }^{2,3,4}$

'University of Heidelberg, Trier, Germany, ${ }^{2}$ Proteopath GbR, Trier, Germany, ${ }^{3}$ Center for Histology, Cytology and Molecular Diagnostics, Trier, Germany, ${ }^{4}$ Institute of Molecular Pathology, Trier, Germany, ${ }^{5}$ Bruker Daltonik GmbH, Bremen, Germany

Aims. Recent advances in radiographic imaging allow the detection of smaller liver lesions. However, the distinction of benign lesions from well differentiated hepatocellular carcinoma (HCC) remains a challenge. In order to characterize the molecular peptide pattern of hepatocellular carcinoma, MALDI imaging mass spectrometry had been performed.

Methods. Fourteen HCC and eleven normal liver biopsies from different patients were included in our study. All samples were subjected to trypsin and matrix deposition using the ImagePrep device (Bruker, Bremen), and were subsequently analyzed by MALDI IMS using a Bruker Autoflex Speed mass spectrometer. Mass spectra profiles were extracted from tissue samples, and two sample groups including HCC and benign liver were then created and subjected to spectral and statistical analysis using ClinProTools 3.o software.

Results. Statistical comparison revealed a nearly 2 -fold change alteration of eleven $\mathrm{m} / \mathrm{z}$ signals $(551,582,617,632,710,829,1314,1483,1607$, $1705,1706)$ in the benign liver tissue and a 2 -fold change of three $\mathrm{m} / \mathrm{z}$ signals $(660,944,1325)$ in the HCC patients on the basis of $\mathrm{p}<0.05$ and with a 99.9 confidence level.

Conclusions. Our results indicate that MALDI Imaging mass spectrometry can be a useful ancillary technique for the differentiation of HCC from normal liver tissue. Further studies will involve a larger number of cases in order to provide greater statistical power and to build a classification model that can differentiate HCC from benign lesions.

\section{FR.1-010}

Primary neuroendocrine carcinoma of the extrahepatic bile ducts-case report of a truly orphan entity

\section{F. Primavesi', S. Stättner', T. Jäger', R. Illig², E. Klieser'², D. Neureiter ${ }^{* 2}$}

'Universitätsklinik für Chirurgie, Salzburger Landeskliniken, Paracelsus Medizinische Privatuniversität, Salzburg, Austria, ${ }^{2}$ Institute of Pathology, Salzburger Landeskliniken/Paracelsus Medizinische Privatuniversität, Salzburg, Austria

Aims. Primary neuroendocrine tumors of the liver, gallbladder and intra- or extrahepatic bile ducts are utterly rare and therefore often left out of consideration in the process of clinical differential diagnosis.

Methods. We describe clinicopathological and immunohistochemical features of a patient with a primary neuroendocrine carcinoma originating from the extrahepatic bile duct.

Results. A 74-year-old, male jaundiced patient was diagnosed with a perihilar malignant stricture in a primary care hospital and considered inoperable. He was treated palliatively with bile duct stenting and upfront chemotherapy. Radiological re-evaluation because of recurrent cholangitis at our department revealed a Bismuth type I lesion which was resected with right hemihepatectomy, bile duct resection and en bloc lymphadenectomy. Postoperative histological examination showed a circumscribed lesion of the extrahepatic bile duct with typical solid and trabecular tumor formations of relatively uniform tumor cells. Local invasiveness with lymph node metastasis was detectable. Extensive immunohistochemical analysis highlighted a neuroendocrine differentiation with expression of chromogranin A and synaptophysin. Utilizing a broad range of functional markers (glucagon, insulin, VIP, somatostatin, serotonin, gastrin, calcitonin) a co-expression of soma- 
tostatin was verified, an immunostaining score of 3 for somatostatin receptor type $2 \mathrm{~A}$ was found [1], and the $\mathrm{Ki}-67$ associated proliferation rate reached up to $45 \%$. All transcription factors applied (CDX-2, TTF-1, PDX-1) displayed no expression pattern. These results led to the final diagnosis of a primary neuroendocrine carcinoma (NEC G3) of the extrahepatic bile duct.

Conclusions. In a series of 8305 NET cases of different origins [2] only one bile-duct NET was described, indicating the rarity of this disease entity. Therefore, no proposal for TNM/ staging classification exists to date. Despite the scarcity of an extrahepatic bile duct NEC, this differential diagnosis should be included in clinical-therapeutic and pathohistological considerations.

\section{Literatur}

1. Volante $\mathrm{M}$ et al (2007) Somatostatin receptor type $2 \mathrm{~A}$ immunohistochemistry in neuroendocrine tumors: a proposal of scoring system correlated with somatostatin receptor scintigraphy. Modern Pathology 20:1172-1182 2. Modlin IM, Sandor A (1997) An analysis of 8305 cases of carcinoid tumors. Cancer 79:813-829

\section{FR.1-011}

\section{Eukaryotic elongation factor 2 (EEF2) as a prognostic marker with} increased kinase activity in hepatocellular carcinoma

H. Reis*1, S. Hagemann ${ }^{2}$, K. Lorenz ${ }^{3}$, J. Wohlschläger ${ }^{2}$, D. Möllmann², B. Sitek ${ }^{4}$, J. Schlaak ${ }^{5}$, H. Meyer ${ }^{4}$, M. Eisenacher', D. Megger',

\section{A.-C. Hoffmann ${ }^{6}$, F. Weber, H. Baba}

'Institute of Pathology, University Hospital of Essen, University of Duisburg-Essen, Essen, Germany, ${ }^{2}$ Institute for Pathology, University Hospital of Essen, University of Duisburg-Essen, Essen, Germany, ${ }^{3}$ Institute of Pharmacology, University of Wuerzburg, Wuerzburg, Germany, ${ }^{4}$ Medical Proteom-Center, Ruhr-University, Bochum, Germany, ${ }^{5}$ Department of Gastroenterology and Hepatology, University Hospital of Essen, University of Duisburg-Essen, Essen, Germany, ${ }^{6}$ Department of Medicine (Cancer Research), University Hospital of Essen, University of Duisburg-Essen, Essen, Germany, ${ }^{7}$ Department of General, Visceral and Transplantation Surgery, University Hospital of Essen, University of Duisburg-Essen, Essen, Germany

Aims. Hepatocellular carcinoma (HCC) is a major lethal cancer worldwide. Recently, multikinase inhibitor therapy has proven efficacy in advanced HCC, but further treatments are needed. Protein phosphorylations are vital mechanisms in cell homeostasis including proliferation and apoptosis thus making kinases attractive drug targets. Aim of the study was to detect and validate differentially expressed proteins as possible new targets in phosphoprotein-enriched lysates of HCC and corresponding non-tumorous liver (NT) samples.

Methods. Phosphoproteomes were analyzed using $2 \mathrm{D}$ Fluorescence Difference Gel Electrophoresis (2D-DIGE; 7xHCC/NT). Protein spots with a change of abundance of at least \pm 1.5 -fold and $\mathrm{p}<0.05$ were further identified by mass-spectrometry. Phosphorylation of 3 candidate proteins was indicated by dephosphorylation with $\lambda$-proteinphosphatase and off-gel fractionation. Expression and distribution of candidate proteins was determined by immunohistochemistry in 93 HCC and NT samples. Activity of the phosphorylation-responsible kinase was compared by in-vitro kinase assays.

Results. 34 differentially regulated proteins in phosphoprotein enriched lysates were identified. Total concentrations of eEF2 and of eEF2 phosphorylated at threonine 56 [peEF2 $\left.\left(\mathrm{T}_{5} 6\right)\right]$ proved to be prognostic markers for overall survival of HCC. Immunohistochemical expression of (cytoplasmic) pERK1/2 (threonine 202; tyrosine 204), phospho-p7oS6kinase and Cyclin D1 positively correlated with eEF2 and p-eEF2(T56). Activity of eEF2-kinase, responsible for phosphorylation of eEF2, was more than 4 times higher in tumor tissues.

Conclusions. $\mathrm{eEF}_{2}$ and $\mathrm{p}-\mathrm{eEF}_{2}\left(\mathrm{~T}_{5} 6\right)$ were detected to be prognostic markers in HCC for overall survival. eEF2-kinase might prove as a valuable target in HCC therapy.
FR.1-012

Intraductal tubulopapillary neoplasm with clear cell morphology: a new entity?

M.G. Ahls ${ }^{* 1}$, M. Niedergethmann ${ }^{2}$, D. Dinter ${ }^{3}$, C. Sauer ${ }^{4}$, J. Lüttges ${ }^{5}$, S. Post ${ }^{6}$, A. Marx', T. Gaiser

'Institute of Pathology, University Medical Center Göttingen, Göttingen, Germany, ${ }^{2}$ Alfried Krupp Krankenhaus, Department of Surgery, Essen, Germany, ${ }^{3}$ Universitätsmedizin Mannheim, Department of Radiology, Mannheim, Germany, ${ }^{4}$ Institute of Pathology, University Medical Center, Mannheim, Germany, ${ }^{5}$ Marienhospital, Pathology, Hamburg, Germany, ${ }^{6}$ Department of Surgery, University Medical Center, Mannheim, Germany

Aims. We analysed the first case of an intraductal tubulopapillary neoplasm of the pancreas with clear cell morphology. Intraductal tubulopapillary neoplasms of the pancreas are very rare tumors.

Methods. A 43-year-old woman visited the surgical clinic with epigastric pain, increased levels of lipase, alpha-amylase and c-reactive protein. Based on the clinical findings, diagnosis of acute pancreatitis of unknown etiology was rendered. A subsequent MRI scan revealed dilatation of the main pancreatic duct with a minor contrast enhancing intraductal tumor of approximately $3.0 \mathrm{~cm}$. The tumor was partially duct-obstructing and suggestive for the diagnosis of intraductal neoplasms of the pancreas.

Results. Surgical resection revealed a $8.0 \times 3.5 \times 2.5 \mathrm{~cm}$ pancreas head with a $17.0 \times 3.0 \mathrm{~cm}$ duodenum segment. Macroscopical examination showed an intraductal, multilocular cystic tumor with a diameter of $2.6 \mathrm{~cm}$ in thepancreatic head (distance to the ampulla $4,0 \mathrm{~cm}$ ) occluding $90 \%$ of the lumen of the main duct leading to aprestenotic dilatation. Histologically, the neoplasm was composed of tubulopapillary glands lined by pseudostratified cells showing abundant clear cytoplasm without any indication of invasiveness. The tumor cell nuclei were enlarged, hyperchromatic and pleomorphic. Mitotic figures were scarce (approx. 1 per 10 high-power fields). Only a few mucin droplets in the cytoplasm of the clear cells were detectable with histochemistry; Di-PAS and alcian blue. No metastases were detected in eight examined lymph nodes from the hepatic artery region. The pancreatic tissue surrounding the tumor showed signs of chronic, fibrotic obstructive pancreatitis with atrophy of the exocrine parenchyma. Immunohistochemistry revealed positivity for Pan-CK, CK7, CK8/18, MUC1, MUC6, carbonic anhydrase IX, CD10, EMA, $\beta$-catenin and e-cadherin. Sanger sequencing did not detect mutations for $\beta$-catenin, BRAF, KRAS, PIK 3 CA and GNAS.

Conclusions. Altogether, histology, immunohistochemical expression profile (MUC1+, MUC6+, MUC2-, MUC5AC-, thrypsin-, chymotrypsin-, CDX2-) and molecular pathology led to the diagnosis of intraductal tubulopapillary neoplasm, clear cell type. So far all reports of ITPN describe the cytoplasm of the ITPN cells as eosinophilic to amphophilic. To the best of our knowledge, this is the first report of ITPN with clear cell morphology.

\section{FR.1-013}

GOT1 expression status in primary resection specimen is a prognostic biomarker in human pancreatic ductal adenocarcinoma (PDAC)

\section{F.M. Feld ${ }^{* 1}$, P.D. Nagel', S.E. Weissinger', C. Welke', P. Möller', J.K. Lennerz'} ${ }^{1}$ Institute of Pathology, University Ulm, Ulm, Germany, ${ }^{2}$ Comprehensive Cancer Center Ulm, Ulm, Germany

Aims. Recently, a metabolic function of the KRAS mutations in PDAC has been established, whereby the mutated KRAS molecule acts as a modulator of the cancer cell's redox maintenance system via an increase in the Glutamic-Oxalacetic Transaminase 1 (GOT1) expression. The clinical relevance of the metabolic KRAS-GOT link is unknown in primary human patient samples. Here we studied whether the GOT1 expression status (a) correlates with the KRAS mutation status, (b) can 
be identified via specific clinicopathological phenotypes, and (c) has prognostic relevance.

Methods. We analyzed two independent PDAC-cohorts: 1. 'International Cancer Genome Consortium'; (ICGC, 57 patients with whole-exome sequencing, genome wide expression profile, clinicopathological data, follow-up) and 2. 'Ulm' (124 patients with molecular genetic workup, tissue-microarray, clinicopathological data, follow up). GOT1-overexpression was assessed via RNA level (ICGC) and immunohistochemistry (Ulm).

Results. (a) In both cohorts the GOT1 expression status was not predictive of the KRAS mutation status ( $\mathrm{p}=0.2$, ICGC; $\mathrm{p}=0.8$, Ulm), indicating that GOT1 assessment cannot serve as a surrogate marker for KRAS mutation testing and that additional modulating factors are at play. (b) Phenotype comparison showed that GOT1 high/positive subgroups do not differ by age, sex, T-, N-, M-status, AJCC-stage grouping or tumor grading $(\mathrm{p}=0.16-1.00)$. (c) Outcome analysis revealed longer overall survival times for patients with GOT1 high-expressing tumors $(p=0.093$, ICGC; $p=0.049$, Ulm). After the combination of both cohorts and multivariate testing, the overall survival of GOT1 high-expressing tumors is significantly longer compared to low-expressing tumors $(\mathrm{p}=0.009)$ and GOT1 may serve as an independent prognostic marker in up to $68 \%$ of patients.

Conclusions. Our data show no correlation between the GOT1 expression status and the KRAS mutation, or specific clinicopathological phenotypes. These findings argue for a rather complex KRAS-GOT1 biology in primary human samples and indicate that GOT1 expression status is a valuable prognostic biomarker in pancreatic ductal adenocarcinoma.

\section{FR.1-014}

Molecular and immunohistochemical subtyping of 200 pancreatic ductal adenocarcinomas (PDAC): a step towards personalized treatment and individual prognosis

\author{
A.M. Schlitter ${ }^{* 1}$, A. Segler', B. Konukiewitz', B. Kong ${ }^{2}$, K. Specht ${ }^{1}$, K. Specht', \\ C. Jäger ${ }^{2}$, J. Kleeff', C. Michalski², I. Esposito', I. Esposito' \\ 'Institute of Pathology, Technical University of Munich, Munich, Germany, \\ ${ }^{2}$ Department of Surgery, Technical University of Munich, Munich, Germany
}

Aims. PDAC is an aggressive tumor with poor overall prognosis and drug resistance. Recent data have shown that molecular subtypes with differences in clinical outcome and drug response exist (Collisson et al. 2011). In addition, recent data indicate an association between mutational status of key tumor suppressor and oncogenes and survival rates (Rachakonda et al. 2013).

Methods. Surgical resection material and detailed clinical data of 200 PDAC patients were collected. Conventional histomorphological analyses and immunohistochemistry for $\mathrm{p}_{53}, \mathrm{SMAD}_{4}$ and $\mathrm{CDKN} 2 / \mathrm{p}_{16}$ were performed. Mutation analysis of KRAS (exon 2 and 3 ) and $\mathrm{TP}_{53}$ were performed and 16 promoter methylation was analyzed.

Results. Patients had a median age of 68.2 years, median overall survival was 19 months. KRAS mutations were identified in $91 \%$ of patients (146/160; $83 \%$ exon $2,8 \%$ exon 3 ). Wild type KRAS was identified in $9 \%$ of patients (14/160) and was associated with significantly better survival estimations (three-year survival rate $26 \%$ versus $56 \%$ months, $p=0.01$ ). Immunohistochemical loss of SMAD 4 and 16 expression was identified in $38 \%(65 / 167)$ and $78 \%(69 / 88)$, respectively. Strong nuclear accumulation of $\mathrm{p}_{53}$ or/and $\mathrm{TP}_{53}$ mutations were detected in $70 \%(103 / 147)$. Most patients (97\%) carried alterations in three genes (44\%; alterations in four genes: $23 \%$, two: $21 \%$, one: $9 \%$ ).

Conclusions. Molecular alterations in key tumor suppressors and oncogenes (KRAS, CDKN2/p16, $\mathrm{TP}_{53}$ and $\mathrm{SMAD}_{4}$ ) are common in PDAC and define molecular subtypes. Wild type KRAS status identifies a molecular subgroup of patients with a favorable outcome.
FR.1-015

HER2 amplification is rare and often heterogeneous in pancreatic cancer

\section{P.R. Stahl*}

University Medical Center, Hamburg-Eppendorf, Hamburg, Germany

Aims. Tumor heterogeneity is probably one of the main reasons for limited efficacy of standard chemotherapy and targeted drugs in certain cases. Though resected tumors are often large, molecular tumor analysis is usually restricted to one tissue block. In this project we introduce a new tool for a high-throughput heterogeneity analysis of pancreatic cancer.

Methods. A heterogeneity tissue microarray (TMA) was manufactured from tissue of 224 patients with pancreatic cancer. Six tissue spots were taken from as many different cancer blocks per patient as possible (at least 3 different cancer containing blocks). Furthermore, tissue cores from one to three corresponding lymph node metastases of 108 patients were added. A heterogeneity analysis for HER2 was performed using fluorescence in situ hybridization. A tumor was considered amplified if the HER2/centromere 17 ratio was $>\mathbf{2} .0$ or if tight HER2 gene clusters were present. Amplifications were considered "high level" if the HER2/ centromere 17 ratio was $>4.0$ or if tight HER 2 gene clusters were present. Results. At least 3 interpretable primary tumor tissue samples were available from 181 patients. A total of 9 patients showed HER2 amplifications. Five patients showed homogeneous HER2 amplifications (3 with high-level amplifications, 2 with low-level amplifications). Four patients showed heterogeneous HER2 amplifications (2 with high-level amplifications, 2 with low-level amplifications).

Conclusions. The data demonstrate that HER2 amplification is rare in pancreatic cancer and shows a highly heterogeneous distribution pattern. This TMA is a valid tool for the analysis of molecular heterogeneity in pancreatic cancer.

\section{FR.1-016}

Intraductal tubulopapillary neoplasms of the bile ducts: clinicopathologic and immunohistochemical analysis of 19 cases

A.M. Schlitter*1, K.-T. Jang ${ }^{2}$, B. Saka ${ }^{3}$, S.-M. Hong ${ }^{4}$, H. Choi ${ }^{3}$, G.J. Offerhaus ${ }^{5}$, R.H. Hruban ${ }^{6}$, Y. Zen', B. Konukiewitz', M. Allgäuer', S. Balci', M. Reid',

\section{G. Klöppel', V. Adsay ${ }^{3}$, I. Esposito}

'Institute of Pathology, Technical University of Munich, Munich, Germany, ${ }^{2}$ Department of Pathology, Samsung Medical Center, Sungkyunkwan University School of Medicine, Seoul, South Korea, ${ }^{3}$ Department of Pathology, Emory University School of Medicine, Atlanta, United States, ${ }^{4}$ Department of Pathology, Asan Medical Center, University of Ulsan College of Medicine, Seoul, South Korea, ${ }^{5}$ Department of Pathology, University Medical Center, Utrecht, Netherlands, ${ }^{6}$ Department of Pathology, Johns Hopkins University School of Medicine, Baltimore, United States, ${ }^{7}$ Institute of Liver Studies, King's College Hospital, London, United Kingdom

Aims. In the pancreas, intraductal tubulopapillary neoplasm (ITPN) is now a well-established category, also recognized by WHO in 2010. Its biliary counterpart is poorly characterized.

Methods. 19 biliary intraductal neoplasms composed almost exclusively of non-mucinous tubular units with/without sheet-like growth, with no or minimal abortive papilla formation, occurring in extra- and intrahepatic bile ducts, were analyzed. Cases with overt papillary growth or mucinous pyloric gland pattern and/or MUC5 AC expression were excluded.

Results. Clinical data: Mean age $64(42-82) ; \mathrm{F} / \mathrm{M}=1.6$. Mean size $=6.1 \mathrm{~cm}$ (1.5-15). Intrahepatic $63 \%$, extrahepatic $16 \%$, and perihilar $21 \%$. Morphology: In addition to characteristic tubular pattern: solid/poorly formed $68 \%$, abortive papillae $47 \%$, clear-cell parathyroid-like areas $16 \%$, luminal calcifications/psammoma-bodies $16 \%$, acidophilic (colloid-like) secretions creating thyroid follicular pattern $11 \%$, oncocytoid cytology 
$11 \%$, focal spotty necrosis $42 \%$, necrosis with "comedocarcinoma-like pattern" $37 \%$, extensive necrosis $11 \%$, and "mallory-like" hyaline globules $11 \%$ were observed. Invasive carcinoma: Present in 14 cases (79\%): $53 \%$ conventional tubular, $27 \%$ in situ-like with comedonecrosis, $13 \%$, tubular-cystic, and $7 \%$ clear cell. Invasive component constituted 5-90\% of the tumor (mean: 52\%). IHC: MUC1 expression was common (79\%) while MUC6 was less common (32\%). MUC2 was not expressed. p53 was uncommon and focal (17\%), SMAD4 was retained, and no $\beta$ catenin nuclear expression was found. Outcome: Follow-up information was available for 15 patients ( $83 \%)$ with a mean follow-up 48 months (range 1-150). One patient with non-invasive disease died at 31 months Two patients with invasive disease had perioperative mortality and were excluded from the analysis. No significant difference in survival $(\mathrm{p}=0.5)$ was found between non-invasive and invasive cases. Overall combined survival rates showed favorable prognosis: 1 yr 93\%, 3 yr 86\%, 5 yr $86 \%$. Conclusions. Biliary ITPNs show striking similarities to their pancreatic counterpart, both morphologically and immunophenotypically . Despite the relatively high incidence of invasive carcinoma (79\%), biliary ITPNs seem to have an indolent behavior significantly better than that of conventional adenocarcinomas (cholangiocarcinomas).

\section{FR.1-017}

Histological and immunohistochemical phenotypes in pancreatic ductal adenocarcinoma-clinical and prognostic relevance?

\section{F. Bergmann ${ }^{* 1}$, L. Schlicht', W. Hartwig' ${ }^{2}$, J. Werner ${ }^{2}$, P. Schirmacher IInstitute of Pathology, Heidelberg, Germany, ${ }^{2}$ Department of Surgery, University of Heidelberg, Heidelberg, Germany}

Aims. Pancreatic ductal adenocarcinomas comprise a spectrum of diverse histological and immunohistochemical phenotypes. Their reported frequency relies on small numbers of cases and their potential clinical relevance has been poorly investigated.

Methods. In the present study, 950 resected pancreatic ductal adenocarcinomas have been characterized microscopically to define and quantify various phenotypes. Furthermore, immunohistochemical analyses were performed, using a multitissue array. The findings were correlated with clinical and pathological features and with follow-up data.

Results. As a result, histological and corresponding immunohistochemical phenotypes are defined in a large, comprehensive series of pancreatic ductal adenocarcinomas. Among others, clear cell and mucinous phenotypes, as well as the expression of cytokeratins 5/6 and 19 and CDX-2 are shown to significantly correlate with the biological tumor behavior and prognosis.

Conclusions. Our findings suggest that a phenotypical subclassification of pancreatic ductal adenocarcinoma is useful and can be achieved with very simple methods.

\section{FR.1-018}

Nup155 regulates a subset of p53 target genes post-transcriptionally in HCC

\section{K. Holzer*1, A. Ori' ${ }^{2}$, J. Winkler', E. Eiteneuer', M. Beck², P. Schirmacher ${ }^{1}$, S. Singer ${ }^{1,2}$}

'Institute of Pathology, University Hospital Heidelberg, Heidelberg, Germany, ${ }^{2}$ European Molecular Biology Laboratory, Structural and Computational Biology, Heidelberg, Germany

Aims. The nuclear pore complex (NPC) is composed of approximately 30 different nucleoporins (Nups) and is embedded in the nuclear envelope. Almost all signaling cascades of oncogenic and tumor-suppressive pathways have to pass the NPC representing the only gate for nucleocytoplasmic transport. There is emerging evidence that NPC components can modulate cancer-relevant pathways on different levels. We could previously show that knockdown of a variety of Nups can affect the $\mathrm{p}_{53}$ pathway by altering $\mathrm{p} 53$ target gene expression. In this study we aim to analyze the requirement of Nup155 for $\mathrm{p} 53$ target gene induction and the underlying mechanism.

Methods. The impact of siRNA-mediated Nup155 knockdown on p53 target gene expression was investigated in different cell lines on protein and mRNA level by immunoblotting and qRT-PCR. Subsequent mechanistic analyses involved protein half-life experiments, subcellular and polysome fractionation followed by qRT-PCR, and quantitative mass spectrometry.

Results. Depletion of Nup155 in HepG2 cells (p53 wild type) by RNAi was followed by a significantly reduced p21 protein accumulation upon Nutlin- and Camptothecin (CPT)-treatment. A selective impact of Nup155 depletion on p21 induction was also observed in $\mathrm{Hep}_{3} \mathrm{~B}-4 \mathrm{Bv}$ cells and in a cell line expressing p21 from a tet-sensitive cDNA construct ("tet-off"). Interestingly, p21 mRNA induction was not significantly affected in these cells. No obvious changes of p21 protein half-life upon Nup155 depletion could be observed in cycloheximide chase experiments. Furthermore, p21 mRNA export was not significantly altered by Nup155 knockdown indicated by an unchanged nuclear/cytoplasmic p21 mRNA-ratio. Polysome fractionation experiments indicated that Nup155 depletion increases the pool of subpolysomal p21 mRNA. Quantitative mass spectrometry revealed distinct changes of protein expression after Nup155 depletion with the cytoplasmic FMR1 interacting protein 2 (CYFIP2) being another $\mathrm{p} 53$ target dependent on Nup155. Conclusions. Our data suggest that Nup155 is required for full induction of a subset of $\mathrm{p}_{53}$ targets such as $\mathrm{p}_{21}$ and CYFIP2. The underlying posttranscriptional mechanism involves mRNA translation and may represent a novel aspect in p53 target gene regulation by NPC components.

\section{FR.1-019}

\section{Is hepatocellular carcinoma a timeless disease?}

N. Elgohary',2, R. Pelegrino', O. Neumann', R. Geffers' ${ }^{3}$, P. Schirmacher', T. Longerich ${ }^{* 1}$

'Institute of Pathology, University Hospital Heidelberg, Heidelberg, Germany, ${ }^{2}$ Department of Medical Oncology (NCl), Cairo University, Cairo, Egypt, ${ }^{3}$ Helmholtz Center for Infection Research, Braunschweig, Germany

Aims. Many biological processes are controlled by an endogenous clock that synchronizes with the phases of the solar day. The mammalian Timeless (TIM) protein interacts with clock proteins and has been shown to be essential for generation of a circadian rhythm in flies. In addition, TIM has been shown to be involved in maintenance of chromosome integrity, growth control and development. Here we analyzed the potential protumorigenic function of TIM in human hepatocarcinogenesis. Methods. We determined the expression of TIM in human HCCs and normal liver using expression profiling, real-time RT-PCR, and immunohistochemistry. Functional analyses were carried out in HepG2 and $\mathrm{Hep}_{3} \mathrm{~B}$ cells following siRNA-mediated TIM knockdown. Pathways altered after TIM inhibition was analyzed on RNA- and protein level. Results. TIM was overexpressed in a subset of human HCCs both on mRNA- and protein level. siRNA-mediated knockdown of TIM reduced cell viability, which was associated by the induction of apoptosis. In addition, induction of a G2 arrest was observed and siRNA-treated cells showed a significantly reduced migratory capacity. The protumorigenic function of TIM seems to be at least partially mediated by the activation of EEF1A2 signaling and $\mathrm{CHEK}_{2}$ inactivation.

Conclusions. Overexpression of TIM exerts oncogenic functions in human HCCs and cooperatively alters other signaling cascades in a protumorigenic fashion. 


\section{AG Gastroenteropathologie \\ Postersession - Unterer GI-Trakt}

\section{FR-019}

Analysis of KRAS mutation, KRAS copy number and numerical alterations of chromosomes 4,10 and 12 in human colon cancer

\section{K. Beyer*, I. Petersen, C. Wölfel}

Institute of Pathology, University Hospital Jena, Friedrich Schiller University Jena, Jena, Germany

Aims. KRAS mutation status has predictive significance in EGFR-antibody treatment of colorectal adenocarcinoma. The aim of the study was the evaluation of KRAS mutation status in correlation to KRAS copy numbers and ploidy status, in particular the presence of triploidy.

Methods. Colorectal adenocarcinomas $(\mathrm{n}=54)$, mostly with known KRAS mutation status, were assembled into a tissue microarray (TMA) and analysed by fluorescence in situ hybridization (FISH). As chromosomes 4 and 10 rarely show alteration these centromere probes were used to analyse the ploidy status. In addition, a dual color FISH probe set for the centromere of chromosome 12 and the KRAS gene was applied to the TMA to analyse numerical alterations of chromosome 12 and KRAS gene copy numbers.

Results. One case was not evaluable, three additional ones were informative for only one FISH probe set. KRAS mutation status was known in 51 cases, 25 with and 26 without mutation. The majority of cases showed a near diploid chromosome content. Near triploid chromosome numbers were observed in only two cases, about 12 cases were hypodiploid, seven were hyperdiploid and the remaining cases were near diploid. In six cases trisomy 12 could be ascertained. Only four tumor samples showed clearly increased KRAS copy numbers. One of them was combined with trisomy 10 and another with triploidy. Diploid tumors seemed to be associated with KRAS wild type status (9 versus 16 cases), whereas KRAS mutation equally appeared in diploid and in aneuploid tumors (12 versus 12 cases).

Conclusions. FISH analysis can be used to analyse the ploidy status and specific chromosomal imbalances. The data suggest that a wild typeKRAS status might be associated with a lower frequency of aneuploidy in colorectal adenocarcinoma. We could not show a correspondent correlation for positive KRAS-mutation and a higher prevalence of aneuploidy so far.

\section{FR-020}

Overexpression and Y435-phosphorylation of Abelson interactor 1 (Abi1) promotes extracellular matrix degradation and invasion by colorectal carcinoma cells

\section{K. Steinestel ${ }^{* 1,2}$, S. Brüderlein ${ }^{2}$, C. Pröpper ${ }^{3}$, P. Möller ${ }^{2}$}

'Bundeswehr Institute of Radiobiology, Munich, Germany, ${ }^{2}$ Institute of Pathology, University Ulm, Ulm, Germany, ${ }^{3}$ Institute of Anatomy and Cell Biology, University of Ulm, Ulm, Germany

Aims. Abelson interactor 1 (Abi1) is a key regulator of actin reorganization during synaptic maturation in neurons as well as in cellular migration. Colorectal carcinoma (CRC) is one of the leading causes of death from cancer, and infiltrating tumor growth pattern and tumor cell "budding" are associated with metastasis and poor prognosis. Since we have previously shown upregulation of Abi1 upon KRAS mutation and during CRC tumorigenesis, our goal was to investigate the role of Abi1 in extracellular matrix (ECM) degradation and CRC invasion.

Methods. Expression of Abi1 at the invasion front of $56 \mathrm{CRC}$ specimens as well as in cultured CRC cells (CHD1) was evaluated applying immunohistochemistry/ western immunoblotting, respectively. Subcellular distribution of Abi1 was visualized using immunofluorescence (IF) microscopy. To analyze the role of phosphorylated Abiı in ECM lysis, we combined a gelatine-based ECM degradation assay with RNA interference experiments and pharmacological treatment with the Abl kinase inhibitor STI571(Glivec). Cell invasion was measured with a modified transwell migration assay, while levels of matrix-metalloproteinase 9 (MMP-9) were analyzed with ELISA.

Results. We found overexpression of Abi1 at the invasive margin of CRCs with infiltrating phenotype and high-grade tumor cell budding compared to tumors that displayed an expanding growth pattern. Strong expression of Abi1 and Laminin 5 \% 2, but loss of E-cadherin expression was observed in CHD1 colorectal carcinoma cells carrying a KRAS G13D mutation. Abi1 localized to sites of matrix degradation in a fluorescent gelatine-based assay, and matrix dissolution was suppressed by RNAi knockdown of Abi1 as well as upon treatment with STI571 that decreased levels of Y435-phosphorylated Abi1. First results show that invasion of CHD1 cells through reconstituted ECM is suppressed in a dose-dependent manner by STI571, while the role of phosphorylated Abi1 in MMP-9 secretion has still to be clarified.

Conclusions. Our results show that overexpression and phosphorylation of Abi1 contribute to ECM degradation and invasion of CRC cells. In ongoing research, we will further analyze the role of Abi1 phosphorylation in MMP-9 secretion and matrix degradation by CRC to evaluate a possible role for $\mathrm{Abl}$ kinase inhibition to prevent the gain of an invasive phenotype in colorectal carcinoma.

\section{FR-021}

The tissue stem cell marker Olfactomedin 4 (OLFM4) is not involved in cancer cell stemness but correlates with differentiation in colorectal cancer

\section{J. Neumann ${ }^{* 1}$, S. Jaitner', A. Schäffauer', K. Blumrath', S. Schwitalla',} F. Greten', L. Kriegl', D. Horst', T. Kirchner', A. Jung'

IInstitute of Pathology of the University of Munich, Munich, Germany, ${ }^{2}$ Institute of Molecular Immunology, Klinikum rechts der Isar, Technical University Munich, Munich, Germany

Aims. Olfactomedin $4\left(\mathrm{OLFM}_{4}\right)$ has been described as a tissue stem cell marker in the normal intestinal mucosa. However, it is unclear whether $\mathrm{OLFM}_{4}$ is also a marker of cancer stem cells (CSCs). Therefore we tested the functional relevance of $\mathrm{OLFM}_{4}$ for cancer stem cell properties in vitro and in situ.

Methods. We analyzed the functional impact of OLFM4 4 on cancer stemness, metastasis and differentiation at RNA- and protein-levels by applying RNA interference (RNAi) and additional functional read outs. Interaction with components of the $\mathrm{Wnt} / \beta$-catenin-pathway was examined by immunofluorescence and luciferase-reporter-assay. The impact on progression, survival and distant spread was tested in three independent collections of CRC and colonic adenomas $(n=60, n=252$ and $\mathrm{n}=106$ ) by use of immunohistochemistry.

Results. We found that forced expression of $\mathrm{OLFM}_{4}$ does not influence cancer stem cell characteristics including chemoresistance in vitro. Furthermore, OLFM 4 did not interact with Wnt/ $\beta$-catenin signaling. Neither the nuclear localization of $\beta$-catenin nor the functional activity of Wnt-signaling was affected in vitro. OLFM4 expression did not exert the metastasis associated characteristics of migration or of anchorageindependent growth of CRC cell lines. Immunohistochemistry revealed no correlation of OLFM4 expression with overall survival or distant metastases in CRC. Adenomas and well differentiated epithelial tubules of grade 1 and 2 CRCs showed, however, high OLFM 4 expression, and the OLFM4 expression was reduced with the loss of tubular differentiation. Furthermore, OLFM4 was low or absent in poorly differentiated CRC.

Conclusions. The expression of the tissue stem cell marker OLFM4 was not involved in the cancer stem cell properties tested and did not correlate with survival or metastasis in human CRC. OLFM4 expression was, however, significantly correlated with well differentiated tubules and hence with the structural epithelial organization in colorectal ade- 
nomas and carcinomas. Thus, we conclude that OLFM4 might be relevant for stemness in normal tissues, which coordinates proliferation and differentiation in crypts as well as in colorectal adenomas and low grade carcinomas, but it is not involved in cancer cell stemness enabling CRC progression and distant metastasis.

\section{FR-022}

\section{The impact of Neuropilin 2 (NRP2) on CXCL12/CXCR4 signaling and} lymph node metastases in colon cancer

\section{H. Schneider ${ }^{* 1}$, P. Hönscheid', R. Pal Singh', B. Wielockx', C. Jakob', G. Baretton', K. Datta' ${ }^{2}$, M. Muders' \\ ${ }^{1}$ Institute of Pathology, Dresden, Germany, ${ }^{2}$ Department of Biochemistry, University of Nebraska Medical Center, Omaha, United States}

Aims. Dissemination of cancer cells to regional lymph nodes is one of the most important prognostic factors for colorectal cancer. To be able to form lymph node metastases tumor cells need a supportive microenvironment. One important part of this niche are lymph vessels that are located inside the lymph node. These intranodal lymph vessels produce chemoattractant factors like CXCL12 that can bind to CXCR4-positive tumor cells and mediate migration. We investigated whether the receptor Neuropilin-2 (NRP2) mediates lymph node metastasis by modulating the CXCR4/CXCL12 signaling pathway.

Methods. In a cohort of 113 node-negative colon cancer patients the lymph vessel density in regional lymph nodes was quantified by standard immunohistochemistry (IHC) staining against $\mathrm{D}_{2}-40$. In vitro experiments were performed with the cell lines SW480 and SW620. Protein and mRNA levels were assessed by western blot and qRT-PCR Boyden Chamber Migration Assays were performed after stimulation with CXCL12, RNAi-mediated depletion of NRP2 as well as CXCR4, and $\mathrm{NRP}_{2}$ blockade by Semaphorin ${ }_{3} \mathrm{~F}$ (Sema3 F). To confirm our in vitro results, a cohort of 78 patients with advanced node positive colon cancer was analyzed for $\mathrm{NRP}_{2}$ expression in primary tumors and lymph node metastases by standard IHC.

Results. In node-negative colon cancer patients intranodal lymphangiogenesis correlated with disease-free survival $(\mathrm{p}<0.05)$. Depletion of $\mathrm{NRP}_{2}$ decreased $\mathrm{CXCR}_{4}$ expression, while $\mathrm{CXCR}_{4}$ had no influence on NRP2 expression. Blocking NRP2 abrogated CXCL12-induced AKT phosphorylation. The increase of CXCR 4 mRNA after stimulation with CXCL12 was inhibited after NRP2 depletion. Tumor cell migration towards a CXCL12-gradient was reduced when NRP2 function was blocked by siRNA or Sema3F $(\mathrm{p}<0.001)$. Our in vitro results were confirmed by an up-regulation of $\mathrm{NRP}_{2}$ in lymph node metastases in advanced colon cancer $(\mathrm{p}<0.001)$.

Conclusions. $\mathrm{NRP}_{2}$ modulates the $\mathrm{CXCL}_{4} / \mathrm{CXCL}_{12}$ signaling axis in colorectal cancer cells and influences cell migration. Therefore, $\mathrm{NRP}_{2}$ might have an impact on the metastatic potential of these cells. Additionally, the detection of intranodal lymph vessels in node negative colon cancer patients by standard IHC against D2-40 might offer a new tool to decide whether an adjuvant treatment is recommended.

This project was funded by a "Else-Kröner-Promotionsstipendium" and has been presented at the "23rd Annual Conference of the German Society for Cytometry" and won a award at the "1oth International Medical Postgraduate Conference".

\section{FR-023 \\ $\mathrm{PBX} 3$ expression indicates high risk colon cancers and is regulated by WNT signaling}

\section{S. Lamprecht*, A. Jung, T. Kirchner, D. Horst}

Institute of Pathology, Ludwig-Maximilians-University, Munich, Germany

Aims. Increased WNT signaling activity, caused by pathway activating mutations, is characteristic for colorectal cancers and drives tumor pro- gression and metastasis. Here, we identify pre-leukemia transcription factor $3\left(\mathrm{PBX}_{3}\right)$ as a gene regulated in part by WNT signaling in colon cancers and assess its prognostic value.

Methods. Gene expression data of colon cancer cells with differential WNT activity were analyzed for $\mathrm{PBX}_{3}$ and the $\mathrm{PBX}_{3}$ promoter region was screened for $\mathrm{TCF}_{4}$ binding sites. Effects of modified WNT signaling on $\mathrm{PBX}_{3}$ expression were tested in cell culture. Clinical correlations and the association of $\mathrm{PBX}_{3}$ expression and $\beta$-catenin were assessed in colon cancer case collections stratified for patient survival, tumor progression and liver metastasis.

Results. Gene expression data revealed consistently overexpressed $\mathrm{PBX}_{3}$ in colon cancer cells with high WNT activity, ChIP data suggested $\mathrm{TCF}_{4}$ binding within the $\mathrm{PBX}_{3}$ promoter, and inhibition of WNT signaling by $\beta$-Catenin knockdown reduced $\mathrm{PBX}_{3}$ levels in colon cancer cell lines. Surprisingly, WNT stimulation on the contrary had limited effects on $\mathrm{PBX}_{3}$ expression. In a colon cancer case collection, $\mathrm{PBX}_{3}$ expression correlated with nuclear $\beta$-Catenin and high $\mathrm{PBX}_{3}$ levels were associated with decreased patient survival and an increased risk for tumor relapse and metastasis. Additionally, an independent case control study confirmed the association of high $\mathrm{PBX}_{3}$ expression and colon cancer metastasis to the liver.

Conclusions. We provide evidence that $\mathrm{PBX}_{3}$ is partially regulated by WNT signaling in colon cancer and that it may be a promising marker for additional risk stratification of colorectal cancer patients.

\section{FR-024}

WNT signaling and distant metastasis in human colon cancer through transcriptional activity of nuclear $\beta$-catenin depend on active $\mathrm{PI} 3 \mathrm{~K}$ signaling

\section{S. Ormanns*, J. Neumann, D. Horst, T. Kirchner, A. Jung}

Institute of Pathology of the University of Munich, Munich, Germany

Aims. We addressed whether activity of $\mathrm{PI}_{3} \mathrm{~K}$ signaling is necessary to fully activate canonical WNT signaling and examined the association of both WNT- and $\mathrm{PI}_{3} \mathrm{~K}$ signaling pathways with colon cancer progression.

Methods. To uncouple effects on nuclear transport and transcriptional activation of $\beta$-catenin, a $\beta$-Catenin-ER fusion gene was cloned which allows for its forced nuclear accumulation using tamoxifen. Additionally, AKT site phosphomimetic $\left(S_{552} \mathrm{D}\right)$ active $\beta$-catenin mutants were employed. WNT signaling pathway activity was measured by reporter gene assays and WNT target gene expression. $\beta$-catenin subcellular level was assessed using immunofluorescence and fractionated cell lysates in Western Blot analysis. $\mathrm{PI}_{3} \mathrm{~K}$ signaling was modulated using the inhibitor LY294002. To study the prognostic value of $\mathrm{PI}_{3} \mathrm{~K}$ pathway activation (indicated by activating $\mathrm{PIK}_{3} \mathrm{CA}$ gene mutations or loss of PTEN protein expression) and nuclear $\beta$-catenin expression, both variables were determined in 110 right sided colon cancer cases with or without distant metastasis.

Results. WNT signaling and target gene expression required $\mathrm{PI}_{3} \mathrm{~K}$ activity which had no effect on the nuclear localization of total $\beta$-catenin protein. Phosphomimetic $S_{552} \mathrm{D}-\beta$-catenin mutants were unable to reconstitute signaling capacities upon $\mathrm{PI}_{3} \mathrm{~K}$ inhibition. Transcriptional activity of nuclear $\beta$-Catenin depended on active $\mathrm{PI}_{3} \mathrm{~K}$ as nuclear accumulation of $\beta$-catenin failed to induce WNT signaling when inhibiting $\mathrm{PI}_{3} \mathrm{~K}$. In human tumors activating mutations in the $\mathrm{PIK}_{3} \mathrm{CA}$ gene or loss of PTEN expression did not correlate with distant metastasis. In contrast the combination of high nuclear $\beta$-catenin expression and activation of the $\mathrm{PI}_{3} \mathrm{~K}$ pathway significantly identified cases in which distant metastasis had occurred. Like in cell culture, activation of the $\mathrm{PI}_{3} \mathrm{~K}$ pathway and nuclear $\beta$-catenin expression were not associated. Conclusions. We conclude that the transcriptional activity of nuclear $\beta$ catenin depends on $\mathrm{PI}_{3} \mathrm{~K}$ activity. $\mathrm{PI}_{3} \mathrm{~K}$ inhibition does not affect the subcellular localization of total $\beta$-catenin protein. As $S_{552} \mathrm{D}$-phosphomimetic mutants failed to restore WNT signaling, $\beta$-catenin transcrip- 
tional activation depends on $\mathrm{PI}_{3} \mathrm{~K}$ activity beyond phosphorylation at serine 552. Both factors synergize for full WNT signaling activity and are associated with distant metastasis in colon cancer. Thus, the detection of high nuclear $\beta$-catenin expression and simultaneous $\mathrm{PI}_{3} \mathrm{~K}$ pathway activation identifies colon cancer patients with a high risk for distant metastasis.

\section{FR-025}

Expression of ARC (apoptosis repressor with caspase recruitment domain) in colorectal cancer liver metastasis and its association with clinical and molecular characteristics of tumorsa preliminary tissue micro array study

\section{Tóth ${ }^{* 1}$, E. Herpel ${ }^{2,3}$, W. Roth ${ }^{2}$, J. Derix ${ }^{4}$, P. Schirmacher ${ }^{2}$, S. Heikaus ${ }^{4}$ IInstitute of Pathology, University Hospital Cologne, Cologne, Germany, ${ }^{2}$ Institute of Pathology, University Clinic Heidelberg, Heidelberg, Germany, ${ }^{3}$ Institute of Pathology, Ruprecht-Karls-University, Heidelberg, Germany, ${ }^{4}$ Institute of Pathology, Heinrich Heine University, Düsseldorf, Germany}

Aims. ARC (apoptosis repressor with caspase recruitment domain) plays a major role in the inhibition of apoptosis in differentiated tissues but also in cancer tissues. The expression of ARC is almost exclusively cytoplasmatic in normal tissues. However, ARC is found both cytoplasmatic and nuclear in tumors. There is no study about the role of ARC in the colorectal cancer liver metastasis in association with tumor characteristics or molecular pathways.

Methods. Hundred liver resection specimens with colorectal cancer metastasis were randomly selected to make tissue micro array blocks with $1 \mathrm{~mm}$ cores in diameter. Each tumor was twice punched. Age of the patients ranged from 33 to 82 years among which are $61 \%$ male and $39 \%$ female. On TMA slides immunohistochemical stainings was performed for established prognostic markers (i.e. $\mathrm{MLH}_{1}, \mathrm{MSH}_{2}, \mathrm{PMS}_{2}, \mathrm{p}_{53}, \mathrm{CDX}_{2}$ and MGMT). Additionally we stained TMA slides for ARC. Cytoplasmatic and nuclear staining was separately scored. Data were analysed by means of linear regression analysis (Software SPSS).

Results. We had valid results in all 100 cases $(100 \%)$ at least in one TMA spot for ARC staining. Weak or no nuclear staining could be detected only in $2 \%$ of the cases, whereas weak or no cytoplasmatic stain was found in $14 \%$. Score 1 expression were found in $25 \%$ and $38 \%$ in the cytoplasma and nuclei, respectively. However, $60 \%$ of the cases showed a diffuse nuclear overexpression (score 2 ) and $61 \%$ had cytoplasmatic overexpression (score 2 and 3, 21\% and 40\%, respectively). No correlation could be detected between ARC expression and tumor characteristics or clinical data (i.e. sex, age, number or size of metastasis, grade or mucinous differentiation). In contrast, cytoplasmatic ARC expression strongly correlated with $\mathrm{MSH}_{2}$ expression $(\mathrm{p}=0.001)$ till nuclear ARC expression showed a similar strong positive correlation with MSH6 $(\mathrm{p}=0.014)$. Any other markers ( $\mathrm{p}_{53}, \mathrm{CDX}_{2}, \mathrm{MGMT}, \mathrm{MLH} 1$ and PMS2) had no correlation with ARC.

Conclusions. This is the first study which shows a correlation between ARC expression, $\mathrm{MSH}_{2}$ and MSH6. These findings support the role of $\mathrm{MSH}_{2}$ and MSH6 in promotion of apoptosis. Tumor cells with intact $\mathrm{MSH} 2$ or MSH6 showed a significant higher expression of ARC. To evaluate the exact relation between $\mathrm{ARC}, \mathrm{MSH} 2$ and $\mathrm{MSH} 6$ functional studies are needed. Furthermore we can state that ARC expression seems to be an independent marker, but further investigations are needed to declare its role and function in colorectal cancer metastasis formation.

\section{FR-026}

Expression of ARC (apoptosis repressor with caspase recruitment domain) in familiar adenomatous polyposis (FAP) adenomasa preliminary tissue micro array study

\section{Tóth' ${ }^{1}$ E. Herpel2,3, W. Roth², J. Derix ${ }^{* 4}$, P. Schirmacher' ${ }^{2}$ S. Heikaus ${ }^{4}$} 'Institute of Pathology, University Hospital Cologne, Cologne, Germany, ${ }^{2}$ Institute of Pathology, University Clinic Heidelberg, Heidelberg, Germany, ${ }^{3}$ Institute of Pathology, Ruprecht-Karls-University, Heidelberg, Germany, ${ }^{4}$ Institute of Pathology, Heinrich Heine University, Düsseldorf, Germany

Aims. ARC is involved in the inhibition of apoptosis in differentiated tissues inhibiting apoptotic pathways. Expression of ARC is almost exclusively cytoplasmatic in normal tissues, whereas ARC is found both cytoplasmatic and nuclear in tumor tissues. Until now no study has been published about the role of ARC in FAP adenomas in association with tumor characteristics or molecular pathways.

Methods. 332 adenomas from 197 FAP patients were punched for tissue microarray blocks with $0.6 \mathrm{~mm}$ diameter. On TMA slides immunohistochemical staining was performed for established prognostic markers (i.e. $\mathrm{MLH}_{1}, \mathrm{MSH}_{2}, \mathrm{PMS}_{2}, \mathrm{p} 53, \mathrm{MGMT}$ and ARC). Cytoplasmatic and nuclear staining were separately scored. For cytoplasmatic and nuclear staining different scores were used. Data were analysed by means of linear regression (Software SPSS).

Results. We had valid results in 324 adenomas (97.5\%) for ARC. Cytoplasmatic ARC expression showed the following distribution: score $\mathrm{O}-4$ cases (1.2\%), score $1-41$ cases (12.6\%), score $2-163$ cases (50.4\%) and score 3-116 cases $(35.8 \%)$. For nuclear ARC staining the distribution was similar: score $0-21$ cases $(6.4 \%)$, score $1-138$ cases $(42.6 \%)$ and score $2-165$ cases (51\%). Regarding MMR proteins, cytoplasmatic ARC expression correlates with $\mathrm{MSH} 2$ expression $(\mathrm{p}=0.002)$ and nuclear ARC expression shows a statistically significant correlation with $\mathrm{MLH}_{1}$ and $\mathrm{MSH}_{2}$ ( $\mathrm{p}=0.003$ and $\mathrm{p}=0.019$, respectively) but not with MSH6 or PMS2. Furthermore, the nuclear overexpression revealed a positive correlation with $\mathrm{p} 53$ overexpression $(\mathrm{p}=0.007)$. In addition, intact MGMT expression was statistically significant associated with nuclear overexpression of ARC.

Conclusions. This is the first study which investigates the ARC expression in FAP adenomas and detected a correlation with DNA-damage repair systems. We can state that if one of the DNA repair systems is damaged the nuclear expression of ARC will be significantly altered. Nuclear ARC expression will be even higher by intact $\mathrm{MLH}_{1}, \mathrm{MSH}_{2}$ and MGMT expression. p53 overexpression is also associated with significantly stronger nuclear ARC expression. In conclusion, microsatellite stable and/or p 53 mutant adenomas from FAP patients are significantly associated with nuclear ARC overexpression. One explanation could be that $\mathrm{ARC}$ binds to $\mathrm{p}_{53}$ tetrameric domain and wild-type $\mathrm{p}_{53}$ regulates ARC negatively. Thus mutant $\mathrm{p} 53$ cannot repress ARC and ARC will be overexpressed and its antiapoptotic effect on $\mathrm{p} 53$ will be enforced.

\section{FR-027}

Changes in DNA methylation of SEPT9 and SHOX2 during transition from normal to malignant colorectal tissue

\section{A. van Ellen*, S. Meller, G. Kristiansen, D. Dietrich}

Institute of Pathology, University Hospital Bonn, Bonn, Germany

Aims. Methylated SEPT9 is a powerful blood-based biomarker for the detection of colorectal cancer (CRC) but with low sensitivity for the detection of precursor lesions and cancers at early stages. DNA methylation of $\mathrm{SHOX}_{2}$ is a biomarker for various cancers. This study investigated if there is a correlation between the state of colorectal carcinogenesis and the DNA methylation of SEPT9 and SHOX2.

Methods. Tissue samples were obtained from patients who underwent surgery at the University Hospital Bonn. 166 FFPET specimens were analysed including normal adjacent tissue (NAT) from the tumorblock 
$(\mathrm{n}=19)$ and excision margins $(\mathrm{n}=15)$ as well as colorectal tissue diagnosed with diverticulosis $(n=6)$ and inflammation $(n=23)$. As neoplastic tissues the subtypes of adenomas (tubular $n=16$, tubulovillous $n=21$, villous $\mathrm{n}=18$, serrated $\mathrm{n}=20$ ) and cancerous colorectal tissue $(\mathrm{n}=28)$ were included. DNA methylation levels of the biomarkers SEPT9 and SHOX 2 were analysed using quantitative and sensitive methylation-specific qPCR assays. The protein expression of SEPT9 and $\mathrm{SHOX}_{2}$ was evaluated using immunohistochemistry (IHC).

Results. DNA methylation of SEPT9 and $\mathrm{SHOX}_{2}$ in adenomas is significantly higher as compared to normal tissues $(\mathrm{p}<0.001)$. No differences were found between CRC and adenomas. Furthermore, qPCR data indicate that transition from adenomas to CRC is accompanied by gene amplification. Serrated adenomas showed significant lower SEPT9 DNA methylation as compared to villous, tubulovillous adenomas $(\mathrm{p}<0.001)$. No significant correlation was found between protein expression and DNA methylation.

Conclusions. Aberrant DNA methylation is an early epigenetic event in carcinogenesis and can therefore also be detected in precursor lesions. Further, amplification of SEPT9 is a late event in malignant transformation representing the main difference between malignant and benign state. Interestingly, SEPT9 DNA methylation might play a specific role in the serrated pathway.

\section{FR-028 \\ 3D-reconstruction reveals two distinct forms of early tubular adenomas in FAP_-implications for modelling the initiation and growth of early adenomas in humans}

\section{J. Kantonen ${ }^{* 1,2}$, N. Zerbe ${ }^{1,3}$, D. Heim ${ }^{1,3}$, P. Hufnagl ${ }^{1,3}$, H. Bläker ${ }^{* 1}$ \\ ${ }^{1}$ Institute of Pathology, Charité University Hospital, Berlin, Germany, ${ }^{2}$ Haart- man Institute, Department of Pathology, University of Helsinki, Helsinki, Finland, ${ }^{3}$ Institute of Pathology, Dept. Digital Pathology, Charité University Hospital, Berlin, Germany}

Aims. Current research on the initiation and growth of adenomatous lesions of the colon is mainly carried out with animal models and organoid cultures. However, the applicability of these results to human patients is far from clear. Novel methods using patient-derived tissues can be developed to either corroborate or refute findings inferred from animal models. Here we aim to show, that the creative use of tissues from FAP-patients can still provide new clues for colorectal cancer research and lead to new insights on human pathology.

Methods. Serial sections ranging from 40 to 230 sections of colonic FFPE-tissue from FAP-patients and a case of SSA/P were cut and stained routinely with $\mathrm{H} \& \mathrm{E}$. Consent for use of the material was provided by the individual patients and/or the Ethics committee of the Charité. Whole-slide images (WSIs) were scanned using 3 DHISTECHs Pannoramic 250 Flash II, and regions of interest were defined by a pathologist in one slide of each series. The WSIs were aligned automatically using a novel computational algorithm. Stack-videos and ${ }_{3} \mathrm{D}$-models were subsequently created based on annotations by a pathologist using opensource and self-written software. Additionally, detailed images were captured using an Olympus BX53 microscope, $\mathrm{DP}_{25}$ digital camera, and the cellSens Dimension software.

Results. The methods of visualization used demonstrate the existence of two different types of early tubular adenomas (eTAs) in FAP-patients. Common features are cellular crowding, hyperchromasia, paucity of goblet cells, crypt fission and a clear-cut border between normal and adenomatous cells on the luminal surface of the mucosa. Differing features are height of the mucosa and the depth that adenomatous crypts reach down into the mucosa. The majority of eTAs have crypts that never reach the vicinity of the muscularis mucosae, where the stem cellharboring crypt-bases normally are located. The FAP-adenomas are then compared to a representative lesion of SSA/P, with similarities seen between rare flat eTAs and SSA/P.
Conclusions. The existence of two distinct early tubular adenomas in the same FAP-patients raises questions about their molecular make-up and cells of origin. A testable model of adenoma initiation is created using these findings and the literature, with a Lgr5+ and Lgr5- cellular origin providing the most straightforward explanation to the differing patterns observed. Further research using human material is warranted to elucidate our findings.

\section{FR-029}

IQGAP2 is involved migration of colorectal cancer (CRC) cells

\section{B. Gröschl*, T. Widmann, M. Bettstetter, P. Rümmele, F. Hofstädter, W. Dietmaier*}

Institute of Pathologie, University of Regensburg, Regensburg, Germany

Aims. IQGAP2, a member of three IQGAP scaffolding proteins, is involved in diverse cellular processes and particularly in cytoskeletal reorganization and adhesion of cells. It has been reported to act as a putative tumor suppressor and may have influence on tumor development and progression. In this study our aim was to investigate IQGAP2 expression and function in colorectal cancer (CRC).

Methods. We analyzed IQGAP2 mRNA expression by RT-qPCR in 17 MSI-H (high microsatellite instability) and 19 MSS (microsatellite stable) colorectal cancers compared to matched normal tissue as well as in three MSI, three MSS and three Maspin overexpressing CRC cell lines. Using Methy-QESD (quantification of endonuclease-resistant DNA) we quantitatively investigated promotor methylation of the IQGAP2 gene in normal and tumor tissue of 10 CRC patients and six CRC cell lines. Subcellular localization of IQGAP2 was determined using immunofluorescent stainings in colorectal cancer cell lines and functional migration assays were performed with IQGAP2 overexpressing and suppressing cell lines using the real-time cell analyzer (RTCA).

Results. In $86 \%$ (30/36) IQGAP2 expression was downregulated in tumor $(\mathrm{p}<\mathrm{o.001})$ and was lower in MSS tumors compared to MSI-H tumors $(\mathrm{p}<0.001)$. Median expression in tumors was 4.3 -fold lower than in normal tissue samples. Accordingly, IQGAP2 mRNA levels were also downregulated in CRC cell lines compared to a normal tissue pool. Stably overexpressing Maspin transfectants showed IQGAP2 loss on mRNA and protein level compared to negative controls. No IQGAP2 promoter methylation could be detected in cell lines, normal or in tumor samples. By immunofluorescence analysis IQGAP2 was distributed in plasma membranes and particularly stained in regions of cell-cell contacts. Migration assays showed that knockdown of IQGAP 2 increased cellular migration, whereas overexpression of IQGAP2 impaired cellular migration.

Conclusions. Our data indicate that IQGAP2 is regulated by Maspin and is involved in cellular migration in colorectal cancer.

\section{FR-030}

BRAF mutations are absent in the perineurial-like stromal component of serrated colorectal fibroblastic polyps (so-called mucosal perineuriomas)

\section{K. Erlenbach-Wünsch" ${ }^{* 1}$, M. Bihl' ${ }^{2}$, M. Vieth ${ }^{3}$, A. Hartmann ${ }^{1}$, A. Agaimy'} 'Institute of Pathology, Friedrich-Alexander-University, Erlangen-Nuremberg, Germany, ${ }^{2}$ Institute of Pathology, University of Basel, Basel, Switzerland, ${ }^{3}$ Institute of Pathology, Hospital of Bayreuth, Bayreuth, Germany

Aims. Benign serrated colorectal fibroblastic polyps are rare benign mucosal lesions characterized by aggregates of spindle cells surrounding serrated mucosal crypts. To date, <200 cases have been reported. However, the experience of the authors suggests a higher incidence than the paucity of reported cases implies, probably indicating under-recognition of these lesions in routine practice. In recent studies a high percentage of serrated fibroblastic polyps showed a V6ooE BRAF mutation 
(63-100\%). However, there exists no study that analyzed possible BRAF mutations in the perineurial-like stromal component of fibroblastic polyps in consideration of its pathogenesis (neoplastic versus reactive). Methods. With a laser we microdissected the stromal and hyperplastic epithelial components of 15 new cases of benign serrated colorectal fibroblastic polyps for separate BRAF mutation analysis.

Results. The patients were 10 women and 5 men with a mean age of 60 years (range: $45-70$ ) The lesions were predominantly located in the rectosigmoid colon and had a mean size of $3.5 \mathrm{~mm}$. Histomorphologically the polyps showed inter-pericryptic and/or micronodular aggregates of spindle cells surrounding the mucosal crypts. Immunohistochemical investigations indicated the expression of at least one perineurial cell marker in all polyps (EMA, GLUT1 and/or Claudin-1). Molecular analyses of epithelial, stromal or both components were successful in 13, 9 and 7 cases, respectively. BRAFV6ooE mutation rates were $54 \%$ for the epithelial and $\mathrm{o} \%$ for stromal component.

Conclusions. Although limited by the small number of evaluable stromal cases, the results of our study are consistent with the postulated reactive nature of the perineurial-like stromal component of serrated colorectal fibroblastic polyps.

\section{FR-031}

Genetic background of colorectal cancer-detection of presumably low penetrance germ line mutations in MMR genes and APC gene by ng Sequencing comprehensively combined with molecular MSI testing and tumor genetics

T.T. Rau ${ }^{* 1}$, C. Kraus' ${ }^{2}$, P. Lux ${ }^{3}$, K. Erlenbach-Wünsch', R. Stoehr', A. Agaimy', R.S. Croner ${ }^{3}$, M. Stürzl ${ }^{3}$, W. Hohenberger ${ }^{3}$, A. Reis ${ }^{2}$, A. Hartmann

IInstitute of Pathology, Friedrich-Alexander-University, Diagnostic Molecular Pathology, Erlangen-Nuremberg, Germany, ${ }^{2}$ Department of Human Genetics, Friedrich-Alexander-University, Erlangen-Nuremberg, Germany, ${ }^{3}$ Department of Surgery, Friedrich-Alexander-University, Erlangen-Nuremberg, Germany

Aims. The pathologist has an important to detect hereditary colorectal cancer in polyposis syndromes and HNPCC syndrome. Germline mutation testing is typically offered only to a subset of patients fulfilling defined diagnostic criteria. Ng Sequencing is a sensitive tool for discovery of possible germline mutations. The aim of our interdisciplinary study was to determine, whether unbiased screening of colorectal cancer cases increased the overall detection rate of germline mutations and to correlate the mutation status to clinical history and molecular pathology of tumors.

Methods. 152 consecutive unselected patients with CRC were enclosed prospectively. Ng sequencing was performed on the Ion Torrent PGM (LifeTech) with an 18 gene panel (AmpliSeq) associated with colorectal cancer. In parallel, all tumors were investigated for MSI and for the $\mathrm{BRAF}^{*} \mathrm{~V} 60 \mathrm{oE}$ somatic mutation.

Results. 11 cases with conspicuous genetic alterations (in silico predictive for CRC) were detected. 1 out of these occurred in APC gene and 10 in known MMR genes. The latter should therefore correspond to MSIstatus. However, analysis of the tumor showed that only 1 out of these was MSI-high with loss of MLH1 immunohistochemically and lack of BRAF mutation or MLH1 hypermethylation. Additionally 6 sporadic MSI carcinomas were found with MLH1 hypermethylation and no germline mutations. Further on we sequenced the same gene panel in tumor DNA from the 11 patients with predictive causative mutations, from 12 patients with unclassified variants and from 5 sporadic MSI (BRAF* V6ooE positive, MLH1 hypermethylated) tumors. All functionally MSI tumors including these 5 sporadic and one germline affected MLH1 case showed a hypermutated phenotype indicative of defective MMR in ngSeq. All other tumors investigated showed a non-hypermutated phenotype despite the predisposing germ line mutation indicative of a sporadic cancer.
Conclusions. In conclusion somatic mutation pattern fits to tumor IHC and MSI testing. Only one of the 1o MMR mutation cases was confirmed as a HNPCC case, while in the other 9 cases the tumor was presumably sporadic, indicating non-penetrance of the germline mutation. Overall, in 3 cases ( $2 \%$ ) a fully penetrant germ line mutation was identified by unbiased sequencing of which only one would have been diagnosed in daily routine. Thus by the use of unbiased NGS screening in CRC cases we were able to identify low penetrant germline variants in MMR and APC genes in cases who did not fulfil current diagnostic criteria.

\section{FR-032}

Phenotype and genotype of polypoid precursor lesions in comparison to synchronous colorectal carcinomas in a consecutive cohort of patients sequenced for 18 colorectal cancer-related genes

K. Erlenbach-Wünsch*1, A. Agaimy', R. Stoehr', P. Lux², C. Kraus ${ }^{3}$, R.S. Croner' ${ }^{2}$, M. Stürz' ${ }^{2}$, A. Reis' ${ }^{3}$, W. Hohenberger ${ }^{2}$, A. Hartmann', T.T. Rau'

IInstitute of Pathology, Friedrich-Alexander-University, Diagnostic Molecular Pathology, Erlangen-Nuremberg, Germany, ${ }^{2}$ Department of Surgery, Friedrich-Alexander-University, Erlangen-Nuremberg, Germany, ${ }^{3}$ Department of Human Genetics, Friedrich-Alexander-University, Erlangen-Nuremberg, Germany

Aims. Little is known about the impact of germline mutations on the phenotype and genotype of synchronous precursor lesions and colorectal cancer. Our project is based on a consecutive interdisciplinary cohort study investigating the genetic background of unselected CRC patients using an 18 gene panel related to CRC. Overall 152 patients were investigated. These were distributed into three categories: no genetic aberrations $(n=129)$, definite genetic aberrations $(n=11)$ and unclassified genetic aberrations $(n=12)$, respectively. The question was whether genetic similarities between primary CRC and synchronous precursor lesions exist dependent from the germline mutational status.

Methods. In the cohort of 152 patients with CRC 29 patients additionally showed 46 polyps. Frequency of polyps did not allow diagnosis of a polyposis syndrome. All 152 carcinomas and 46 polyps were tested for MSI, BRAF and KRAS mutations. All patients with definite germ line mutation were checked for a possible second hit mutation in all carcinomas and synchronous polyps using an 18 gene NGS-panel (AmpliSeq) on the Ion Torrent PGM (LifeTech).

Results. We found 35 classical adenomas, 9 hyperplastic polyps, 1 SSA and 1 TSA: 29 polyps in patients with no genetic aberrations (21 CAD, $6 \mathrm{HP}, 1$ SSA and 1 TSA), 10 polyps in patients with definite genetic aberrations (8 CAD and $2 \mathrm{HP}$ ) and 7 polyps in patients with unclassified genetic aberrations (6 CAD and $1 \mathrm{HP}$ ), respectively. Similarities between carcinoma and polyp were analyzed regarding BRAF and KRAS mutation. Most of the cases did not show a mutation neither in CRC nor in precursor lesions. 10 cases of carcinomas were KRAS mutated, corresponding polyps not. 5 polyps showed KRAS and 2 polyps BRAF mutations, but no mutation in corresponding carcinoma. For KRAS as well as for BRAF there was only one case with both lesions mutated simultaneously. $\chi^{2}$ test revealed no significantly changed frequencies regarding the subgroups of germline genetic background. Therefore we searched for additional genetically second hit mutations in polyps within the subgroup of definite germline mutation by ng sequencing. Only one CAD showed an additional APC gene mutation, but the corresponding carcinoma not.

Conclusions. Our results showed that in determined germline mutations additional somatic mutations showed an incidental pattern comparing precursor lesions and adjacent CRCs. Therefore precursor lesions could not substitute cancer specimen for genetic diagnostic purposes. 


\section{FR-033}

Immunohistochemical panel diagnostics of Hirschsprung's disease with Map2, Calretinin, Glut1 and S100

\section{Bachmann ${ }^{* 1}$, M. Besendörfer', P. Lux², R. Carbon ${ }^{2}$, A. Agaimy', A. Hartmann', T.T. Rau'}

'Institute of Pathology, Friedrich-Alexander-University, Diagnostic Molecular Pathology, Erlangen-Nuremberg, Germany, ${ }^{2}$ Department of Surgery, Friedrich-Alexander-University, Erlangen-Nuremberg, Germany

Aims. Since the report of Meier-Ruge et al. in 1972 Hirschsprung's disease is diagnosed by detection of aganglionosis and hypertrophic nerve fibres in cryosections of the ntestine using acetylcholinesterase staining. This method is time consuming, laborious and requires submission of unfixed specimens. Cryo artefacts impede morphologic evaluation and the diagnostics is offered only by specialized laboratories. However, the knowledge of the enteric nervous system has grown within the last decades and several immunohistochemical markers were proposed to have diagnostic potential. The aim of this study was to find a way to reliably diagnose Hirschsprung's disease using an immunohistochemical panel targeting ganglia as well as nerve fibres.

Methods. We used Map-2 and Calretinin as immunohistochemical markers for ganglia detection and S-10o and Glut-1 for nerve fibre evaluation, respectively. We applied this panel of four antibodies on 70 intestinal FFPE specimens taken from 36 patients. 24 of the samples had been previously diagnosed as aganglionotic, 27 as normal and 19 are regarded as transition zone. We used the current gold standard proposed by Bruder et al. to identify ganglia in H\&E-staining and nerve fibres in acethylcholin esterase staining on cryo sections.

Results. Map2 and Calretinin stained submucosal as well as myenteric ganglia reliably. The number of positive neurons per ganglion is slightly higher in Map2 than in Calretinin staining. The loss of ganglia in Hirschsprung's disease is significantly visible in immunohistochemistry (unpaired Student's t-test, $\mathrm{p}<0.001$ ). At least 5 HPF of definite submucosal tissue seems to be sufficient for diagnosis. The thickness and number of nerve fibres detected with Glut1 and S1oo staining contrasts this loss. Interestingly the uncoated nerve fibres in Hirschsprung's disease develop a perineurium of size and intensity like in deeper subserosal tissue highlighted with the Glut-1 staining. A strong perineurium was not visible in normal submucosal tissue to that extent ( $\chi^{2}$ test, $\mathrm{p}<0.001$ )

Conclusions. Using this panel of immunohistochemical markers the diagnosis of Hirschsprung's disease seems as accurate as with conventional cryo techniques. Especially the examination of loss of ganglia in combination with detection of a prominent perineurium around nerve fibres could be an alternative method and especially applicable on FFPE material.

\section{FR-034}

Dipeptidase 1 (DPEP1) is a marker for the transition from low-grade to high-grade intraepithelial neoplasia and an adverse prognostic factor in colorectal cancer

\section{B. Sipos*1, P. Eisenach ${ }^{2}$, C. Röder ${ }^{3}$, G. Klöppel ${ }^{4}$, H. Kalthoff ${ }^{3}$}

'Department of Pathology, University Hospital, Eberhard Karls University, Tübingen, Germany, ${ }^{2}$ Max Planck Institute of Biochemistry, Department of Molecular Medicine, Martinsried, Germany, ${ }^{3}$ Division of Molecular Oncology, Institute of Experimental Cancer Research, University Hospital Schleswig Holstein, Kiel, Germany, ${ }^{4}$ Institute of Pathology, Technical University of Munich, Munich, Germany

Aims. Colorectal cancer (CRC) is the second leading cause of cancerrelated deaths worldwide. Improvements in the understanding of its molecular mechanism and the characterisation of CRC-specific biomarkers facilitating early detection are considered to increase overall survival.
Methods. A meta-analysis of microarray and Serial Analysis of Gene Expression (SAGE) has been performed to identify differentially regulated genes in CRC. Dipeptidase 1 (DPEP1/MDP/RDP) and Syntenin-2 (SDCBP $2 /$ SITAC18) were found to be differentially expressed in tumour tissue compared to normal mucosa. DPEP1 expression was assessed in a validation set of 87 normal mucosa samples, 20 hyperplastic polyps, 46 $\mathrm{CR}$ adenomas with low- and high-grade intraepithelial neoplasia (IEN) and 217 well-documented CRCs by immunohistochemistry and partially by immunoblotting and real-time-PCR.

Results. DPEP1 expression was specifically increased in human CRC tissue samples compared to normal mucosa ( $\mathrm{p}<0.0001$, Mann-Whitney- $\mathrm{U}$ test), showing a striking upregulation in high-grade compared to lowgrade IEN. Furthermore, high DPEP1 expression was found to strongly correlate with histological stage ( $\mathrm{p}<0.0001$, chi-square test) as well as localisation ( $\mathrm{p}<0.0001$, chi-square test) and has been recognised as an independent adverse prognostic factor, showing significant prognostic values with a ROC-AUC of 0.9230.

Conclusions. DPEP1 has been identified as an excellent marker of highgrade IEN and CRC, and may thus be applied for screening of early neoplastic lesions and for prognostic stratification.

\section{FR-035}

Apoptotic colopathy caused by antimetabolites and TNF- $\alpha$ antagonists

\section{Soldini', A. Gaspert', M. Montani', T. Reineke' ${ }^{2}$, G. Rogler ${ }^{3}$, R. Odze ${ }^{4}$, A. Weber ${ }^{* 1}$}

${ }^{1}$ Institute for Surgical Pathology, Zurich, Switzerland, ${ }^{2}$ Institute for Surgical Pathology, Bern, Switzerland, ${ }^{3}$ Division of Gastroenterology and Hepatology, Zurich, Switzerland, 'Department of Pathology, Brigham and Women's Hospital, Boston, United States

Aims. Gastrointestinal toxicity leading to diarrhea is an adverse effect of several drugs, and diagnostic work-up frequently includes biopsies of the gastrointestinal tract. The combination of watery diarrhea, normal or near to normal colonoscopy, and histological findings resembling graft-versus-host disease-like patterns, has been previously associated with mycophenolic acid and ipilimumab. Here, we present four patients who developed watery diarrhea due to drug-induced apoptotic enteropathy (three cases from colon and one case from ileal pouch) after intake of antimetabolites (methotrexate and capecitabine) and/or tumor necrosis alpha inhibitors (etanercept and infliximab).

Methods. Our patients, and their colon biopsies, were retrieved from the clinical and surgical pathology files of the Institute for Surgical Pathology, University of Zurich, between July 2009 and March 2010. For all patients, the clinical charts, endoscopy reports, and all colonic biopsies from all endoscopies were reviewed for a wide variety of clinical and pathologic information. All biopsies were evaluated semiquantitatively for apoptosis of basal crypts, dilated damaged crypts and mucosal architecture distortion. Also recorded was the presence of intraepithelial lymphocytes, chronic inflammatory cells in the laminar propria, and mucosal ulcerations. In addition, immunohistochemistry for human cytomegalovirus was performed all cases and for herpes simplex virus in three cases.

Results. Endoscopic examination revealed normal mucosa in two patients, whereas the other two showed focal ulcerations. Histological changes included increased apoptosis of basal crypts, dilated and degenerated crypts, defined as cystically dilated cysts with flattened degenerated epithelium containing apoptotic debris and few neutrophils, and architecture distortion. In all cases, onset of diarrhea was temporally association with between drug intake and/or dose increase, and no convincing evidence of other potentially underlying causes of colitis/ enteritis was found, including infections.

Conclusions. Pathologists should be aware of the expanding spectrum of drugs that can cause apoptotic enteropathy in order to report these findings to clinicians and seek for an adequate treatment approach. 


\section{FR-036}

\section{HMGB1 controls energy metabolism in colon cancer cells}

\section{G. Gdynia* ${ }^{* 1}$, J. Fuller' ${ }^{2}$ M. Enders ${ }^{3}$, S. Sauer', J. Kopitz', W. Roth} ${ }^{1}$ Institute of Pathology and German Cancer Research Center, Heidelberg, Germany, ${ }^{2}$ Heidelberg Institute for Theoretical Studies (HITS), Heidelberg, Germany, ${ }^{3}$ Institute of Inorganic Chemistry, University, Heidelberg, Germany, ${ }^{4}$ Metabolic Center Heidelberg, University Hospital, Heidelberg, Germany, ${ }^{5}$ Institute of Pathology, Division of Applied Tumor Biology, Heidelberg, Germany

Aims. Energy metabolism is important for cancer cell proliferation, resistance to therapy and immunological surveillance. The utilization of predominantly glucose as an energy substrate is common for most fast proliferating cancer entities. Therefore we investigated the effect of HMGB1 on glycolysis in colon cancer.

Methods. Electron microscopy, in silico docking studies, ${ }_{13} \mathrm{C}-\mathrm{NMR}$ measurements, $14 \mathrm{C}-$, $3 \mathrm{H}_{2} \mathrm{O}$-tracer experiments, ATP luciferase assay, western blot, in vitro pull down, generation of rho zero cells, $\mathrm{O}_{2}$-consumption, vibratome generated colon carcinoma tissue slice culture, tissue micro array.

Results. HMGB1 induced cell death in different colon carcinoma cell lines (SW480, HCT116, HT29) was accompanied by the formation of giant mitochondria. Via isotope tracing we could show that HMGB1 forced the cancer cells to rely on glycolysis whereas mitochondrial respiration was potently blocked. The responsible mechanism for this metabolic shift was the inhibition of a major glycolytic enzyme as shown in docking studies, enzyme kinetic experiments and in vitro pull down assays. Up-regulation of glutaminolysis could rescue HMGB1-treated cancer cells from the HMGB1-induced cell death whereas down-regulation of a key enzyme of glutaminolysis, ME1 (malic enzyme 1), via siRNA sensitized the cells to this metabolic cell death. Moreover, ME1 was overexpressed in advanced colon carcinomas as shown in a cohort of 1,260 colon carcinoma patients.

Conclusions. HMGB1 blocks the aerobic branch of glycolysis in cancer cells by specific enzyme inhibition. Cancer cells resistant to HMGB1 bypass the glycolytic block by up-regulation of glutaminolysis. Administration of both anti-metabolites and HMGB1 could be a promising approach in targeting the energy metabolism of colon cancer.

\section{AG Hämatopathologie Postersession}

\section{FR.1-020}

\section{A human lymphohaematopoietic engraftment model for assess- ment of haematopoietic radiation injury}

\section{K. Steinestel*1,2, G. Marka ${ }^{3}$, K. Soller ${ }^{3}$, M.E. Hotz', V. Meineke', H. Geiger ${ }^{3}$ 'Bundeswehr Institute of Radiobiology, Munich, Germany, ${ }^{2}$ Institute of Pathology, University Ulm, Ulm, Germany, ${ }^{3}$ Institute of Molecular Medicine, University of Ulm, Ulm, Germany}

Aims. A life-threatening consequence of exposure to ionizing radiation lies in severe impairment of hematopoiesis. However, research possibilities regarding underlying biologic mechanisms and possible mitigating pharmaceutics are limited due to the fact that samples from exposed patients are rare, have to be regarded in a retrospective way and lack precise data on dose and duration of exposure. Prospective rodent or in vitro models, on the other hand, lack comparability with the human in vivo situation. Since successful engraftment of human hematopoietic stem cells (hHSCs) in immunodeficient mice has been previously demonstrated, our goal was to evaluate the usability of this system to assess the human haematopoietic radiation injury.

Methods. For this proof-of-concept study, 18 immunodeficient NODscid-IL2rynull mice were engrafted with $\mathrm{CD}_{34}+$ hHSCs as previously described. After FACS analysis showing successful engraftment, mice were subjected to single-dose $\gamma$-irradiation (o, 1, 3 or $5 \mathrm{~Gy}$ ), and vital parameters, cell counts and cytokine levels as well as survival was monitored for a follow-up period of 5 weeks. At the end of the study, surviving animals were euthanized and all mice underwent histopathologic examination.

Results. Successful engraftment with $\mathrm{CD}_{34}+$ hHSCs gave rise to multi-lineage human lymphohematopoietic cell populations in all murine hosts. All sham-irradiated and $66 \%$ of the 1 Gy-irradiated, but none of the 3- and 5 Gy-irradiated mice survived until the end of the study. FACS analyses showed a decrease in $\mathrm{CD}_{19} / \mathrm{CD}_{33}$ cell populations upon irradiation, while there was an increase in IL-8 secretion and upregulation of proteins involved in DNA repair and apoptosis (pATM, BAX). Histopathologic evaluation of bone marrow sections showed reduced cellularity and dilated vessel spaces consistent with acute radiation syndrome (ASR), while the spleen showed a strong reduction of lymphatic follicles in 3 and 5 Gy-irradiated mice. Currently, we are performing comprehensive immunohistochemical analyses to achieve a more detailed insight into quantitative changes among cell populations. Conclusions. Our first results indicate that NOD-scid-IL2r $\gamma$ null mice engrafted with $\mathrm{CD}_{34}+$ hHSCs represent a valuable tool for the assessment of human haematopoietic radiation injury. The model might also prove helpful in the evaluation of new radio-mitigating agents for the treatment of acute radiation syndrome (ASR) upon possible radiation accidents as well as military or terrorist attacks.

\section{FR.1-021}

Periproctal abscess as initial manifestation of acute myeloid leukemia

\section{H. Geddert ${ }^{* 1}$, A. Pick-May², A. Stenzinger ${ }^{3}$, A. Dimmler}

'Institute of Pathology, St. Vincent's Hospital, Karlsruhe, Germany, ${ }^{2}$ Department for general, visceral and vascular surgery, Deaconess Hospital, Karlsruhe, Germany, ${ }^{3}$ Institute of Pathology, University Hospital Heidelberg, Heidelberg, Germany

Aims. A 56-year-old male patients presents with a periprocal mass, clinically diagnosed as abscess. The findings during operation point to an old anorectal abscess without fistula formation. After good initial postoperative recovery, a further abscess formation with surrounding phlegmonous inflammation requires a surgical revision.

Methods. Histological examination of periproctal tissues reveals-beside granulating inflammation-an infiltrate of immature tumor cells. Immunohistochemically, the tumor cells are identified as myeloid blast. Intermediately, myeloid blasts are detected within the peripheral blood and bone marrow investigation confirms involvement by AML $\mathrm{M}_{2}$ (round 50\%). Finally, an acute myeloid leukemia with extramedullary mass formation in the anorectal region is diagnosed.

Results. The patients dies from fungal pneumonia during chemotherapy.

Conclusions. This case report underlines the need to examine all human issues, even when removed in supposedly banal operations.

\section{FR.1-022}

Intra- and peritumoral Epstein-Barr virus activation in Epstein-Barr virus-negative diffuse large $B$-cell lymphoma

\section{Stuhlmann-Laeisz ${ }^{* 1}$, M. Szczepanowski', A. Borchert ${ }^{1}$, M. Brüggemann ${ }^{2}$, W. Klapper ${ }^{1}$}

'Department of Pathology, Hematopathology Section and Lymph Node Registry, Christian-Albrecht University, Kiel, Germany, ${ }^{22}$ nd Department of Internal Medicine, Christiane-Albrechts Universität, Kiel, Germany

Aims. The vast majority of diffuse large B-cell lymphomas (DLBCL) in European countries are negative for the Epstein-Barr virus (EBV). Only a subset of DLBCL is EBV associated. This is usually seen in the scena- 
rio of immunosuppression or is associated with higher age. In all cases of EBV-positive DLBCL, EBV is detectable in virtually all lymphoma cells, indicating that EBV was present at an initial phase of lymphomagenesis. We aimed to determine the clonal relation, diagnostic and clinical significance of scattered EBV-positive intra- and peritumoral B-cells in EBV-negative DLBCL.

Methods. Four selected cases of consultation files of the Lymph Node Registry Kiel screened for EBV by EBER in situ hybridization were noticed with scattered EBV-positive intra- and peritumoral B-cells in EBVnegative DLBCL. From these cases approximately 2000 EBV-positive blasts were collected by laser-assisted microdissection. Comparative immunoglobulin rearrangement analysis was performed from EBV-negative DLBCL and the microdissected EBV-positive B-cells according to the BIOMED2 protocol.

Results. In all four lymphomas the majority of B-cell blasts were EBVnegative, but were accompanied by a small number of scattered EBVpositive B-cells with blastic morphology comprising less than $10 \%$ of all B-cells of the lesion. All patients were above 46 years, but no clinically evident immunosuppression was reported. In one patient the EBV-positive blasts occurred in the gastric mucosa and resembled an EBV-positive mucocutaneous ulcer adjacent to an EBV-negative DLBCL in the deeper layers of the gastric wall. In the other three cases EBV-positive B-cells occurred in small loose clusters within or adjacent to the EBVnegative DLBCL infiltrate in lymph nodes. In three specimens the EBVpositive cells displayed virus latency type III and in one case latency type II. Immunoglobulin rearrangement analysis was evaluable in one case and yielded clonal amplicons in the microdissected EBV-positive peritumoral B-cells and in the EBV-negative DLBCL that were clonally unrelated.

Conclusions. We conclude that intra- and peritumoral EBV-positive Bcells can occur in close proximity to EBV-negative DLBCL. The EBVpositive intra- and peritumoral B-cells in one informative case were clonally unrelated to the EBV-negative DLBCL. Localized EBV-reactivation may occur due to local immune escape mechanisms and represents a diagnostic pitfall in the differential diagnosis of EBV-positive DLBCL of the elderly and EBV-positive mucocutaneous ulcer.

\section{FR.1-023}

Epstein-Barr virus-positive diffuse large B-cell lymphoma of the elderly is rare in Central Europe and associated with poor outcome if positive for EBNA2

\section{A. Borchert ${ }^{* 1}$, C. Stuhlmann-Laiesz', L. Quintanilla-Martinez ${ }^{2}$, S. Hoeller ${ }^{3}$, A. Tzankov', I. Oschlies', M. Kreuz', R.U. Trappe ${ }^{5}$, W. Klapper \\ 'Department of Pathology, Hematopathology Section and Lymph Node Registry, Christian-Albrecht University, Kiel, Germany, ${ }^{2}$ Institute of Patholo- gy, Eberhard-Karls-University, Tübingen, Germany, ${ }^{3}$ Institute of Pathology, University Hospital, University of Basel, Basel, Switzerland, ${ }^{4}$ Institute for Medical Informatics, Statistics and Epidemiology, Leipzig, Germany, ${ }^{52}$ nd Department of Internal Medicine, Christian-Albrecht University, Kiel, Germany}

Aims. Epstein-Barr virus(EBV)-positive diffuse large B-cell lymphoma (DLBCL) of the elderly is a newly recognized subgroup of EBV-positive lymphomas. EBV-positive DLBCL of the elderly occurs by definition in patients above 50 years, who are not immunocompromized. An age-related decline of immunocompetence leading to re-activation of EBV has been suggested to be important for its pathogenesis. This DLBCL subgroup has been primarily described in Asian patient cohorts, where the incidence seems to be higher compared to Central Europe. We aimed to study the incidence and clinical relevance of EBV-positive DLBCL of the elderly in Central Europe.

Methods. Two previously published series, from registry files of Basel, Switzerland and Tübingen, Germany, were combined with a patient cohort from Kiel, Germany, reflecting a patient cohort representative for DLBCL in Central Europe. These cohorts encompassed 634 diagnosed
DLBCL of patients above 50 years, the median was 71 years (range $50-98$ years). All specimens were analyzed for EBV by EBER in situ hybridization and the overall number of EBV-positive DLBCL of the elderly was calculated from these data. In addition we evaluated the influence of the EBNA2 expression on the overall survival within the group of EBVpositive DLBCL of the elderly. For this purpose, the 15 cases identified in the cohort of Basel, Tübingen and Kiel were combined with 15 additional cases identified among consultation files. These cases $(n=30)$ were further analyzed for EBNA2 expression by immunohistochemistry. Results. Among the 634 DLBCL, 15 EBV-positive lymphomas fulfilling the criteria of EBV-positive DLBCL of the elderly were identified (2.4\%), the median age was 71 years (range $57-88$ years). 8 of 28 evaluable EBVpositive DLBCL cases expressed EBNA2. EBV-positive DLBCL of the elderly expressing EBNA2 showed a significantly worse overall survival compared to EBNA2 negative EBV-positive DLBCL of the elderly (median survival 274 and 4383 days, respectively, $\mathrm{p}=0.0124$ ).

Conclusions. The incidence of EBV-positive DLBCL of the elderly is much lower in Central Europe compared to Asia, where it is reported to be $8-14 \%$. EBNA2 expression in such lymphomas seems to be associated with worse outcome.

\section{FR.1-024}

\section{Targeting the BRAF V600E mutation in multiple myeloma}

M. Andrulis*1, N. Lehners ${ }^{2}$, D. Capper ${ }^{3,4}$, R. Penzel', C. Heining ${ }^{5}$, T. Zenz ${ }^{5}$, A. von Deimling ${ }^{4}$, P. Schirmacher', A.D. Ho ${ }^{2}$, K. Neben ${ }^{2}$, M.S. Raab ${ }^{2}$

IInstitute of Pathology, Ruprecht-Karls-University, Heidelberg, Germany, ${ }^{2}$ Department of Hemathology, Ruprecht-Karls-University, Heidelberg, Germany, ${ }^{3}$ Clinical Cooperation Unit Neuropathology, Heidelberg, Germany, ${ }^{4}$ Institut für Pathologie, Abteilung für Neuropathologie, Heidelberg, Germany, ${ }^{5}$ Department of Medical Oncology, National Center for Tumor Diseases (NCT), Heidelberg, Germany

Aims. Our clinical experience with specific therapeutic targeting of oncogenic mutations in multiple myeloma (MM) is still very limited. Whole-genome sequencing have recently disclosed that a subset of MM patients harbor an activating BRAF V6ooE mutation. The aim of our study was to explore the clinical significance of this mutation.

Methods. We used a BRAF-V6ooE-mutation-specific antibody to screen primary tumor samples from 379 patients with MM. The study cohort included 421 samples (391 bone marrow biopsies, 30 soft tissue plasmacytomas). 37 patients of the cohort had two or more consecutive biopsies available. Mutation was confirmed by direct sequencing.

Results. BRAF V6ooE was detected in seven myeloma patients. In three patients the mutation was absent at diagnosis and only became detectable during the relapse indicating clonal evolution. BRAF mutation was significantly more frequent in patients with extramedullary disease $(\mathrm{p}=0.02)$. The mutation carriers had a significantly shorter median overall survival of 45 months compared to 105 months $(p=0.04)$ of patients without the mutation. One patient with BRAF V6ooE mutation and extensive extramedullary disease was considered for off-label treatment with the mutation-specific BRAF inhibitor, vemurafenib, given the resistance of the disease to standard lines of therapy. Low dose of vemurafenib induced a rapid and stable remission of the disease.

Conclusions. Taken together, we provide evidence for the acquisition of the BRAF V6ooE mutation during the clonal evolution of multiple myeloma and report on the therapeutic and prognostic significance of this drugable mutation. 


\section{FR.1-025}

\section{Lysine-specific demethylase 1 (LSD1) in hematopoietic neoplasms}

D. Niebel ${ }^{* 1}$, J. Kirfel', V. Janzen ${ }^{2}$, M. Majores ${ }^{3}$, T. Höller', G. Kristiansen', I. Gütgemann ${ }^{1}$

'Institute for Pathology, University of Bonn, Bonn, Germany, ${ }^{2}$ Department of Hematology and Oncology, University of Bonn, Bonn, Germany, ${ }^{3} \mathrm{Heinz}-$ Werner-Seifert-Institute for Dermatopathology, Bonn, Germany, ${ }^{4}$ Institute of Medical Biometry, University Medical Center, Bonn, Germany

Aims. Lysine-specific demethylase 1 (LSD1) is a histone $\mathrm{H}_{3} \mathrm{~K}_{4} / \mathrm{K} 9$ demethylase which acts as an epigenetic regulator with roles in both, gene activation and repression and is expressed in several neoplasms such as breast and prostate carcinomas and neuroblastomas. Recently, inhibition of LSD-1 has gained attention as a potential novel treatment in acute myeloid leukaemia via reactivation of the all-trans-retinoic acid differentiation pathway. Furthermore, loss of LSD-1 leads to anaemia and granulocytopenia indicating a role in normal function of hematopoietic precursor cells. Our aim was to investigate the expression of LSD1 in a large number of different hematologic neoplasms in order to possibly widen the range of hematologic malignancies that may be targeted using LSD1 inhibitors.

Methods. 152 bone marrow trephine biopsies were analyzed for LSD1 expression by immunohistochemistry and compared to standard morphology and cell lineage markers.

Results. In myeloproliferative neoplasias (MPN) excluding CML, LSD1 was expressed in more than half of cases $(23 / 39=59.0 \%)$, showing strong nuclear staining in atypical megakaryopoiesis and erythropoiesis and to a lesser degree, in myelopoiesis. In CML LSD1 was expressed less frequently $(6 / 18=33.3 \%)$. In myelodysplastic neoplasms (MDS) about half of cases demonstrated nuclear expression in dysplastic megakaryopoiesis and erythropoiesis $(6 / 11$ (54\%), in CMMoL atypical myelo- and monopoiesis as well as megakaryopoiesis appeared positive [3/9 (33.3\%)] and in primary and secondary acute myeloid leukaemias LSD1 was expressed in a blast specific nuclear pattern [15/37 (40.5\%)]. Bone marrow trephine biopsies of patients with acute lymphoblastic leukaemias (ALL) also demonstrated strong blast-specific LSD1 expression in about half of the cases [7/12 (58.3\%)]. On the other hand in reactive erythropoiesis and megakaryopoiesis LSD1 was expressed in a substantial number of cases $(11 / 26=42.3 \%)$.

Conclusions. We conclude that LSD1 is not only expressed in acute myeloid leukemia, but in a wide range of neoplastic hematologic diseases especially MDS, MPN and acute leukaemia. Expression in reactive hematopoesis needs to be taken into account during the development of specific therapies using LSD1 inhibitors as severe hematologic side orders might result.

\section{FR.1-026}

\section{Expression of insulin-like growth factor-I in classical and nodular lymphocyte-predominant Hodgkin's lymphoma}

\section{E. Eppler ${ }^{* 1}$, E. Janas' ${ }^{1}$ L. Weidmann', M. Wenger', M. Tinguely-Kovarik',} H. Moch', C. Fellbaum ${ }^{3}$

${ }^{1}$ Research Group Neuro-endocrine-immune Interactions, Institute of Anatomy, University of Zurich, Zürich, Switzerland, ${ }^{2}$ Institute of Surgical Pathology, University Hospital, Zürich, Switzerland, ${ }^{3}$ Institute of Pathology, Hegau-Bodensee Clinic, Singen, Germany

Aims. Hodgkin's lymphoma (HL) is among the most frequent nodal lymphomas in the Western world. HL is classified in two disease entities: nodular lymphocyte-predominant Hodgkin's lymphoma (NLPHL) and classical Hodgkin's lymphoma (cHL, 95\% of all HL). HL lesions are characterized by a minority of clonal neoplastic cells, namely in cHL Hodgkin and Reed-Sternberg cells and their variants, and in NLPHL the lymphocyte-predominant cells, respectively, both within a microenvironment of, e.g., reactive $\mathrm{T}$ and $\mathrm{B}$ cells, macrophages, and granulocytes assumed to support proliferation and maintenance of the neoplastic cells through cytokines, chemokines and growth factors. Insulin-like growth factor I (IGF-I) is an important growth factor involved in proliferation, differentiation, apoptosis and cell survival of numerous-including immune-tissues, and there exist indications for a role in tumour pathogenesis and sustainment. Although in recent years evidence has accumulated that HL is characterized by a profound disturbance of cell differentiation and apoptosis mechanisms, a potential involvement of IGF-I in HL has not been systematically investigated to date.

Methods. IGF-I was localized using specific antibodies for HL and immune cells by double-immunofluorescence investigation of pathological lymph node specimens.

Results. We have localized IGF-I in frequent neoplastic cells of all cHL and NLPHL cases investigated. Additionally, IGF-I immunoreactivity was detected in high endothelial venules and in granulocytes and macrophages of the tumour microenvironment.

Conclusions. We assume that IGF-I plays an anti-apoptotic role in tumour pathogenesis and in shaping the tumour microenvironment.

\section{FR.1-027}

EBV infection under Sjögren associated MALT lymphoma: an underestimated risk?

J.E. Strunk ${ }^{* 1}$, C. Schüttler ${ }^{2}$, J. Ziebuhr ${ }^{2}$, M. Stowasser ${ }^{3}$, M. Noethe ${ }^{4}$, K. Mayer ${ }^{5}$, A. Bräuninger', S. Gattenlöhner

IInstitut für Pathology, Giessen, Germany, ${ }^{2}$ Medical Virology, Universitätsklinikum Giessen und Marburg, Standort Giessen, Giessen, Germany, ${ }^{3}$ Diagnostic and interventional radiology, Universitätsklinikum Giessen und Marburg, Standort Giessen, Giessen, Germany, ${ }^{4}$ Medical Clinic III, Universitiy Clinic Giessen and Marburg, Giessen, Giessen, Germany, ${ }^{5}$ Department of Internal Medicine II, Justus-Liebig University Giessen, Giessen, Germany

Aims. Patients with Sjögren's Syndrome, a systemic autoimmune disease of the salivary and lacrimal glands, are especially afflicted with sicca symptoms like xerostomia and xerophthalmia. It is critical to note, that these patients, caused by the chronic inflammation, have an increased risk to develop malignant lymphomas, predominantly MALT lymphomas. The Association of MALT lymphomas with autoimmune disease and/or bacterial infection has been described several times in the literature, whereas an involvement of viral infections in the pathogenesis of Sjögren-associated lymphomas is unknown. Such an aggravation causing viral infection in the context of malignant lymphomas constitutes the Epstein-Barr virus.

Methods. Histomorphological, immunhistochemical and molecular pathological analysis, clinical Chemistry, radiology.

Results. We report a fatal case of a 42-year-old woman with pre-existing Sjögren's syndrome with extranodal marginal B-cell lymphoma (MALT lymphoma) of the salivary glands with secondary EBV-associated high-grade transformation with diffuse Infiltration of lung, liver, lymphnodes und bone marrow.

Conclusions. In clinical daily routine EBV infections of patients under immunsuppressive therapy suffering of Sjögren's associated MALT lymphomas are often underestimated with the risk of fatal outcome. A careful monitoring of the EBV infection status and of other signs for EBV-associated PTLD-like malignancies has to become a standard procedure in Sjögren's syndrome immunuosuppressive therapy. 


\section{FR.1-028}

\section{MET expression in primary mediastinal B-cell lymphoma}

\section{P.D. Nagel*, O. Ritz, P. Möller, J.K. Lennerz}

Institute of Pathology, University Hospital, Ulm, Germany

Aims. Primary mediastinal B-cell lymphoma (PMBL) is a specific subset of diffuse large B-cell lymphoma (DLBCL) that shares several clinicopathological as well as molecular features with classical Hodgkin lymphoma (cHL). Recent studies have demonstrated the proto-oncogene MET as a good prognostic biomarker in CHL and DLBCL with an overexpression in $52 \%$ and $73 \%$ of patients, respectively. MET as a biomarker in PMBL has not been examined.

Methods. Protein detection employed Western Blot and immunohistochemical screening in three currently available PMBL cell lines (Karpas 1106, MedB-1, U2940) as well as 30 primary human tissue samples from an established PMBL patient cohort. Oncogenic function was tested using si-RNA knockdown followed by flow-cytometric cell cycle analysis. Phenotype contingency testing (age, sex, stage and Ki-67 positivity) and statistical outcome comparison employed t-test, Fisher's, chisquare and log-rank tests using graphpad prism and $\mathrm{R}$.

Results. One of three cell lines (Karpas 1106) expressed MET and showed strong cytoplasmic and membranous MET immunoreactivity. Two independent si-RNA probe sets targeting MET (vs. one control si-RNA probe) revealed no differences in cell-cycle fractions. In contrast to existing data in CHL or DLBCL cell lines with partial oncogenic dependence, our data indicate that MET in Karpas 1106 does not alter proliferation and survival. In primary patient samples, five of 30 tumors (17\%) demonstrated strong MET immunoreactivity. Notably, the percentage of MET-positive cases in PMBL is lower than that reported in $\mathrm{cHL}(\mathrm{p}=0.003)$ and DLBCL $(\mathrm{p}=0.001)$ Comparison of the clinicopathological phenotype and the Ki- 67 index between MET+ and MET- tumor samples showed no differences ( $\mathrm{p}$-range $=0.314-1.0$ ). As to outcome, no events occurred in the MET+ subgroup whereas two of the 25 METpatients succumbed to their disease. Thus, similar to CHL and DLBCL, MET positivity in PMBL conveys a survival advantage although this difference did not reach statistical significance $(\mathrm{p}=0.268)$.

Conclusions. For the time being, the specific role of MET in the oncobiology of PMBL remains elusive. However, our data indicate that MET is expressed in a clinically relevant number of PMBL cases $(17 \%)$, and may play a role unrelated to the pro-proliferative oncogenic properties of MET.

\section{FR.1-029}

Nodal reactive and neoplastic proliferations of IRTA1- and/or T-betpositive cells in the marginal- and interfollicular zones: are these cells more closely related to monocytoid B cell than to marginal zone cells?

\section{R. Bob ${ }^{* 1,2}$, H. Stein}

IInstitute for Pathodiagnostik Berlin, Berlin, Germany, ${ }^{2}$ Berlin, Germany

Aims. Marginal-zone B-cells (MZCs) and monocytoid B-cells (MBCs) appear to be related lymphoid cells taking part in reactive and neoplastic proliferations which are not yet well characterized. The aim of the study is to find better diagnostic criteria and to elucidate the cellular origin of these lesions.

Methods. 60 nodal lesions with marginal and interfollicular zone proliferations were analysed for their morphological, immunophenotypical, molecular genetics and IG gene rearrangement features. Based on the results of the rearrangement assay, the cases were divided into reactive and neoplastic ones.

Results. Among the neoplastic ones, two morphologically distinct subgroups emerged. Group I was characterized by a partial nodal effacement with a polymorphic cell population containing MBCs, centrocytoid and transformed large lymphocytes and plasma cells with a marginal zone/perifollicular or interfollicular distribution; group II contained totally effaced lymph nodes with back to back nodules composed of monomorphic small MBCs. All reactive lesions had morphologic features as in group I. By immunohistochemistry, IRTA1 and/or T-bet expression was found in all reactive and in $95 \%$ of the neoplastic cases. PD1 positive T cells were unexpectedly expanded in extrafollicular spaces side by side with MZCs/MBCs.

Conclusions. Expression of IRTA1 and T-bet is very helpful for the identification of marginal zone lesions and suggest that most of these lesions are more closely related to MBC than to marginal zone cells. Most morphological and immunophenotypical patterns in reactive and neoplastic nodal marginal zone expansions were overlapping. Therefore, the only reliable technique to differentiate between neoplastic and reactive marginal zone proliferations is the PCR clonality assay of both the IGHand IGL loci.

\section{AG Pneumopathologie Postersession}

\section{FR-037}

MET detection in non-small cell lung cancer (NSCLC): a comparison of two diagnostic methods and correlation with clinical data

P. Jurmeister*1, D. Lenze', E. Berg', S. Mende', F. Schäper², U. Kellner', H. Herbst ${ }^{4}$, M. Dietel', M. Hummel', M. von Laffert ${ }^{1}$

'Institute of Pathology, Charité University Hospital, Berlin, Germany, ${ }^{2}$ Institute of Pathology, Bioptisches Institut Gemeinschaftspraxis für Pathologie, Berlin, Germany, ${ }^{3}$ Institute of Pathology, Johannes Wesling Klinikum Minden, Minden, Germany, ${ }^{4}$ Institute of Pathologie, Vivantes Klinikum Berlin, Berlin, Germany

Aims. MET-alterations in NSCLC were reported to contribute to acquired resistance to epidermal growth factor receptor (EGFR) tyrosine kinase inhibitors. Furthermore, first results of clinical trials with METinhibitors point towards MET as an emerging important predictive and prognostic marker. Its implementation in the algorithm of molecular lung cancer diagnostics seems of urgent priority.

Methods. Using tissue microarrays, a cohort of 609 surgically resected lung cancers was screened for MET-alterations by immunohistochemistry (IHC: SP44, Roche) and fluorescence in-situ hybridization (FISH: $\mathrm{MET} / \mathrm{CEP}_{7}$ dual-color probe, Abbott). MET-expression was classified as IHC- (IHC o and 1) or IHC+ (IHC 2 and 3). MET-gene status was classified as FISH- (disomy, low trisomy, high trisomy, low polysomy) or FISH+ (high polysomy, gene amplification) according to the UCCC criteria. Furthermore, the mean MET gene copy number (Cappuzzo score) as well as the ratio of MET-signals and chromosome 7 (PathVysion scoring system) was recorded. IHC and FISH were correlated and compared to clincopathological data.

Results. 533 cases (87.5\%) were evaluable by IHC and FISH. IHC+ was seen in $15.4 \%$. According to the UCCC scoring system FISH+ was observed in $9.4 \% .3 .8 \%$ and $1.1 \%$ of cases were considered positive determined by Cappuzzo and PathVysion criteria, respectively. Using the UCCC scoring system the correlation of IHC and FISH showed homogenous results in 90.6\% (IHC-/FISH-: 82.9\%; IHC+/FISH+: 7.7\%). IHC-/FISH+ was observed in $1.7 \%$ and IHC+/FISH- in $7.7 \%$ of cases. MET-alterations occur in both men and women and tend to be more frequent in patients younger than 65 years. They are common in both main types of NSCLC (MET IHC+/FISH+ in squamous cell carcinoma: 4.1\%; adenocarcinoma: 9.3\%) especially in cases with lymphatic vessel permeation, lymph node metastasis or pleural invasion. However MET-alterations also occur in lower stages, as $45.7 \%$ of all IHC+/FISH+ specimens had a stage I disease.

Conclusions. In the majority of cases MET IHC and MET FISH determined by UCCC criteria show a strong correlation. However future studies need to show the clinical role (e.g. therapy response, EGRF-resistance) 
of discrepant MET evaluations and should point out the most reliable test-algorithm. MET-testing should be performed in all NSCLC subtypes. Although pleural infiltration, lymph node metastasis and lymphatic vessel permeation seem to be common in MET-altered cases, lower stages should not be excluded from MET-testing.

\section{FR-038}

\section{Prospective molecular profiling in human pulmonary allografts}

N. Izykowski*1, J. Rische', B. Rath', L. Maegel', J. Gottlieb', T. Welte', A. Haverich', H. Kreipe', F. Laenger', D. Jonigk ${ }^{\text {? }}$

'Institute of Pathology, Hannover Medical School, Hannover, Germany, ${ }^{2}$ Department of Pneumolgoy, Hannover Medical School, Hannover, Germany, ${ }^{3}$ Department of Cardiothoracic, Transplantation and Vascular Surgery, Hannover Medical School, Hannover, Germany

Aims. Lung transplantation (LTx) provides the only therapeutic option for a variety of terminal lung diseases. One of the main problems after transplantation is the obliterative remodelling of the small airways (bronchiolitis obliterans, $\mathrm{BO}$ ), which is considered to be the morphological correlate of chronic allograft dysfunction. Transbronchial biopsies do not reproducibly detect BO because of its discontinuous and patchy nature. For these reasons, modern biomarkers are required to improve the value of tissue-based testing to predict/diagnose graft failure.

Methods. First we analyzed the mRNA expression of 45 fibrosis-associated genes using reverse transcription and TaqMan-based low-density/ high throughput arrays (LDA) in transbronchial biopsies from clinically clearly defined cohorts: lung-transplanted patients with progressive bronchiolitis obliterans syndrome (BOS; $\mathrm{n}=48$ biopsies, 19 patients) and patients with long-term survival without $\mathrm{BOS}(\mathrm{n}=57$ biopsies, 18 patients). An exploratory random analysis was performed to identify genes relevant for the classification of $\mathrm{BO}$ and the survivor group. The top ranked genes from the random forest analysis were selected for prospective testing in biopsies of lung-transplanted patients.

Results. Low-density/high throughput (qRT-PCR) array-based analyses of transbronchial biopsies yielded reproducible and reliable results. We could identify characteristic expression patterns in a set of markers, among which are the matrix metalloproteinases (MMPs), bone morphogenic proteins (BMPs), Sma and Mad-related proteins (SMADs), members of the thrombospondin family, collagen family and cytokines. Conclusions. The world-wide first prospective study on molecular profiling in transbronchial biopsies from human lung-transplanted patients is presented. A distinct mRNA signature could be established which detects and predicts airway remodelling in lung-transplanted patients, even in morphologically inconspicuous biopsies.

\section{FR-039}

\section{Characterization of the inflammatory infiltrate in bacterial pneu- monia in young and elderly patients}

\section{T. Menter*1, C. Giefing-Kroell' ${ }^{2}$ B. Grubeck-Loebenstein², A. Tzankov' 'Institute of Pathology, University Hospital Basel, Basel, Switzerland, ${ }^{2}$ Institute of Biomedical Ageing Research, Innsbruck, Austria}

Aims. Pneumonia caused by Streptococcus pneumoniae is still a major health problem with an increased susceptibility and mortality in the elderly. We evaluated the composition of the inflammatory infiltrate in both young and elderly Streptococcus pneumoniae pneumonia patients in order to shed new in situ and in vivo insights of the age-dependent inflammatory response in such conditions and to correlate our findings with the available data of serological and in vitro studies from the literature.

Methods. Lung tissue of young ( $n=5$; mean age 8.4 years) and elderly $(n=11$; mean age 82.1 years) patients with pneumonia with microbiologically proven presence of Streptococcus pneumoniae from autopsies and control tissue of partial lung resections $(n=10)$ were retrieved from the archive and immunohistochemically stained for markers of neutrophilic granulocytes and various lymphocyte and macrophage subtypes. The composition of the inflammatory infiltrate was numerically assessed and compared between the two groups.

Results. We could demonstrate a higher percentage of neutrophilic granulocytes in the elderly patients ( $95 \%$ versus $75 \%, \mathrm{p}=0.013$ ). In contrast, the relative amount of alveolar macrophages (marked by CD11c) and M1-macrophages (marked by CD14 and HLA-DR) was higher in the young patients (CD11c: $20 \%$ versus $9 \%, \mathrm{p}=0.019$; $\mathrm{CD} 14: 30 \%$ versus $10 \%$, $\mathrm{p}=0.009$; HLA-DR: $52 \%$ versus $11 \%, \mathrm{p}=0.009)$. No difference was observed concerning the percentages of M2 macrophages and lymphocytes. Conclusions. This is the first study characterizing in situ the inflammatory infiltrate of pneumococcal pneumonia in lung tissue. We could confirm earlier in vitro and serological studies showing a reduced $\mathrm{M}_{1}$ macrophage response in elderly patients with a higher proportion of neutrophilic granulocytes. Our findings improve the understanding of mechanisms of response to pneumococcal infection and might give hints to new therapy options such as monoclonal antibodies lowering the influx of neutrophilic granulocytes.

\section{FR-040}

Plakophilin 1 is downregulated in human lung cancer by DNA methylation

\section{Y. Chen*, L. Yang, T. Cui, I. Petersen}

Institute of Pathology, Friedrich Schiller University, Jena, Germany

Aims. Desmosomes fortify cell adhesion and are involved in tumour progression. Our previous studies showed that desmosomal proteins such as desmocollins, desmogleins, as well as desmoplakin are tumour suppressors. In this study, we analysed the role of one of the desmosomal plaque proteins plakophilin ( $\left.\mathrm{PKP}_{1}\right)$ in human lung cancer.

Methods. RT-PCR and Western blot analysis were performed to analyse the expression of PKP1 in lung cancer cell lines. The protein expression of PKP1 in more than 100 primary lung tumours was evaluated by immunohistochemistry on tissue microarray. To investigate methylation status of $\mathrm{PKP}_{1}$, demethylation test and bisulfate sequencing were carried out.

Results. PKP1 was downregulated in 6 out of 8 lung cancer cell lines at both mRNA and protein levels. Primary lung tumour tissues exhibited a heterogeneous expression of PKP1 protein. After treatment with demethylation agent 5'-Aza-2'-deoxycytidine, restoration of PKP1 mRNA expression was found in 4 lung cancer cell lines. bisulfate sequencing revealed a heterogeneous methylation pattern of PKP1 in promoter region and exon 1, which is associated with mRNA downregulation. Right now an expression vector containing the full-length cDNA of PKP1 has been constructed and the functional role of PKP1 in lung cancer cells will be investigated.

Conclusions. Downregulation of plakophilin 1 in human lung cancer cells may be explained by DNA methylation. 


\section{FR-041}

Metabolic switch in non-small cell lung cancer-different impact in different age groups

\section{A. Csanadi*1, C. Otto', M. Donauer', V. Gumpp², J. Rawluk ${ }^{3}$, B. Passlick', M. Werner', G. Kayser'}

'Department Pathology, Universtity Medical Center Freiburg, Freiburg, Germany, ${ }^{2}$ Clinical Cancer Registry, Comprehensive Cancer Center Freiburg, University Medical Center Freiburg, Freiburg, Germany, ${ }^{3}$ Department of Oncology and Hematology, University Medical Center, Freiburg, Germany, ${ }^{4}$ Department of Thoracic Surgery, University Medical Center, Freiburg, Germany

Aims. Changes in carbohydrate metabolism are a common phenomenon in malignant diseases and do occur in non-small cell lung cancer (NSCLC), too. The main goal of this metabolic switch is generation of basic cellular building blocks. ATP-citrate lyase (ACLY) and malic enzyme (ME) are key players connecting citric acid cycle and fatty acid synthesis. We therefore investigated the expression of ACLY and ME in NSCLC.

Methods. 260 NSCPC patients were included in this study. Of these TMAs with a core diameter of $2 \mathrm{~mm}$ were constructed. Of each tumor 3 cores were included. For immunohistochemical stains polyclonal rabbit anti-human ACLY antibody (Cell signaling, 1:400) and monoclonal mouse anti-human ME1 antibody (Abnova Biozol ${ }_{3} \mathrm{H}_{5}$, 1:200o) were used. Specific reactivity included nuclear (ACLY) as well as cytoplasmic (ME and ACLY) staining. For each core staining intensity and percentage of positive cells were assessed.

Results. Both, ACLY and ME were significantly overexpressed in NSCLC compared to non-neoplastic lung tissue $(\mathrm{p}<0.001)$. Survival analysis showed that overexpression of ACLY was significantly associated with a favorable outcome in young patients $(p=0.029)$. ME overexpression revealed a statistical trend towards a poorer survival in old patient group $(\mathrm{p}=0.093)$. Young patients in who's tumors either ACLY and/or ME was overexpressed did have a significantly better outcome $(\mathrm{p}=0.007)$. This was proven to be an independent prognostic factor in our cohort. On the other hand, overexpression of either ACLY and/or $\mathrm{ME}$ in older patients hardly missed statistical significance towards a poorer prognosis $(\mathrm{p}=0.058)$.

Conclusions. Different age groups of NSCLC patients inhabit biologically different tumors e.g. incidences of driver mutations. Our results further indicate that biology of NSCLC in young patients may differ from those of old patients. Complexity of metabolic changes in tumors is further highlighted and contradictory results can at least partly be explained by our findings. Since elective ACLY antagonists are under investigation our results may open new therapeutic options for NSCLC patients.

\section{FR-042}

Expression of GLUT1, PKM2 and ME in primary lung carcinoma and corresponding lymph node metastases

\section{A. Csanadi ${ }^{* 1}$, C. Otto', N. Hörter', A. Oser', J. Rawluk' , B. Passlick, M. Werner', G. Kayser'}

'Department Pathology, University Medical Center Freiburg, Freiburg, Germany, ${ }^{2}$ Department of Oncology and Hematology, University Medical Center, Freiburg, Germany, ${ }^{3}$ Department of Thoracic Surgery, University Medical Center, Freiburg, Germany

Aims. The metabolic switch of tumor cells, necessary for cell surviva and also for metastasis formation, is subject of intensive research. Although up to $90 \%$ of all cancer deaths are associated with metastases, the metabolic state of tumor tissue at metastatic sites is yet scarcely investigated. We, therefore, evaluated expression patterns of GLUT1, PKM2 and ME in primary non-small cell lung cancer (NSCLC) and their corresponding lymph node metastases.
Methods. TMAs were constructed from primary tumor and nodal metastases of 145 NSCLC patients. Expression of GLUT1, PKM2 and ME were assessed by immunohistochemistry. For statistical analysis the $\mathrm{H}$-score was calculated from the percentage of positive cells multiplied by staining intensity. Non-parametric tests and Kaplan-Meier survival analysis were performed.

Results. GLUT1 as well as ME expression in nodal metastases showed a significant positive correlation with the corresponding primary. GLUT1 expression was significantly upregulated in squamous cell carcinoma (SCC) compared to adenocarcinoma (LAC; $(\mathrm{p}<0.001)$ both in the primary tumor and at the metastatic site. While PKM2 expression in primary NSCLC and their corresponding lymph node metastases did not show a significant correlation, overexpression in nodal metastases of $\mathrm{PKM} 2$ was associated with poor patient survival $(\mathrm{p}=0.029)$.

Conclusions. Metastatic NSCLC predominantly seem to preserve their fitting of metabolic enzymes. Different expression patterns of GLUT1 in LAC and SCC were independent from the location of malignant cells. This indicates a permanent biological difference of these two histological entities. PKM2 expression differed in primary and metastatic tumor cells. Since PKM2 overexpression in lymph node metastases was associated with poor outcome this finding can be indicative for metabolic adaption towards increasing aggressiveness of NSCLC.

\section{FR-043}

Activation of angiogenesis differs strongly between pulmonary carcinoids and neuroendocrine carcinomas and is crucial for carcinoid tumorgenesis

F. Mairinger ${ }^{* 1,2}$, R.F.H. Walter ${ }^{3}$, R. Werner', S. Ting', T. Hager', C. Vollbrecht', D. Theegarten', D. Christoph ${ }^{5}$, K.W. Schmid', J. Wohlschlaeger

'Institute of Pathology, University Hospital Essen, University of DuisburgEssen, Essen, Germany, Institut for Pathology, University Hospital Essen, University of Duisburg-Essen, Essen, Germany, ${ }^{3}$ Ruhrlandklinik, University Hospital Essen, University of Duisburg-Essen, Essen, Germany, ${ }^{4}$ Institute of Pathology, University Hospital, Cologne, Germany, ${ }^{5}$ Department of medical Oncology, University Hospital Essen, University of Duisburg-Essen, Essen, Germany

Aims. Neuroendocrine lung tumors are clearly divided in low-grade and high-grade tumors with significant different clinical outcome. The low grade group encompasses typical (TC) and atypical (AC) pulmonary carcinoids, the high grade group is built by large-cell neuroendocrine carcinoma (LCNEC) and small-cell lung cancer (SCLC). Angiogenesis is a hallmark of cancer and has an impact on metastatic potential and biological aggressiveness in a broad range of human malignancies. The present study tries to discover a potential association of a different activation of angiogenesis and potential of malignancy in this class of tumors.

Methods. The gene expression profile from 20 samples of each TC, AC, LCNEC as well as SCLC was discovered via Nanostring nCounter. Six genes (CRHR2, FIGF, FLT4, HIF1A, KDR, MMP3) concerning the angiogenesis were included in this profile.

Results. In the entire cohort, HIF1A $(p=0.0185)$, FIGF $(p=0.0014)$ and $\mathrm{FLT}_{4}(\mathrm{p}=0.0059)$ are significantly different expressed between low- and high-grade tumors. Within the carcinoid only, FIGF is highly significant to differ $\mathrm{AC}$ and $\mathrm{TC}(\mathrm{p}=0.0031)$, but also $\mathrm{FLT}_{4}, \mathrm{MMP}_{3}$ and $\mathrm{CRHR} 2$ shows differences in their expression profiles between this two entities. For pulmonary neuroendocrine carcinomas, FIGF shows some differences in the expression between LCNEC and SCLC $(p=0.0233)$. $\mathrm{COXPH}$ model for OS identifies CRHR2 as prognostic marker in this group of lung tumors ( $\mathrm{p}=0.0396)$. HIF1A and FIGF seem to be associated with PFS.

Conclusions. Our study shows, that activation of angiogenesis plays a crucial role in tumorgenesis in the investigated entities. Angiogenic activation is differently regulated between this tumortypes. Furthermore, the study underlines that FIGF can be a potent diagnostic marker 
to differentiate TC from AC. CRHR2 gene expression can support the prognostic decision of high grade neuroendocrine tumors of the lung.

\section{FR-044}

Folic acid metabolism and DNA-mismatch repair in pulmonary neuroendocrine tumors: identification of potential chemotherapyresistance mechanisms

F. Mairinger ${ }^{* 1,2}$, R.F.H. Walter ${ }^{3}$, R. Werner', C. Vollbrecht', S. Ting', T. Hager', K.W. Schmid', J. Wohlschlaeger', D. Christoph ${ }^{5}$

'Institute of Pathology, University Hospital Essen, University of DuisburgEssen, Essen, Germany, ${ }^{2}$ Institut for Pathology, University Hospital Essen, University of Duisburg-Essen, Essen, Germany, ${ }^{3}$ Ruhrlandklinik, University Hospital Essen, University of Duisburg-Essen, Essen, Germany, ${ }^{4}$ Institute of Pathology, University Hospital, Cologne, Germany, ${ }^{5}$ Department of medical Oncology, University Hospital Essen, University of Duisburg-Essen, Essen, Germany

Aims. Neuroendocrine tumors of the lung account for more than $25 \%$ of all lung cancer cases and include typical (TC) and atypical carcinoids (AC) as well as large cell neuroendocrine (LCNEC) and small cell neuroendocrine carcinomas (SCLC). In contrast to carcinoids, high-grade neuroendocrine tumors are associated with poor prognosis. For neuroendocrine lung tumors a lack of diagnostic, prognostic and predictive biomarkers impairs the improvement of current therapies. A significant amount of these tumors shows a resistance to platinum containing therapeutic regimes. The aim of the study was to investigate this resistance mechanism. Furthermore, expression levels of members of the folic acid metabolism were tested with respect to the possibility of an antifolatebased chemotherapy.

Methods. Eighty patients with histopathologically confirmed, pulmonary neuroendocrine tumors (each 20 TC, AC, LCNEC and SCLC) were used for NanoString nCounter mRNA expression analysis with a custom CodeSet. This CodeSet contains eight genes out of the folic acid metabolism (ATIC, DHFR, FOLR1, FPGS, GART, GGT1, SLC19A1, TYMS) and five genes associated with DNA-repair (ERCC1, MLH1, $\mathrm{MSH}$ 2, MSH6, XRCC1).

Results. FOLR1, FPGS, MLH1 and TYMS are most significant differentially expressed between the four investigated tumor entities (each $\mathrm{p}<0.0001$ ). Additionally, ERCC1 and MSH6 are highly significant between these groups $(\mathrm{p}<0.001)$. Also GGT1 and XRCC1 show an association to entity. FOLR1 and TYMS show most significant associations to biological aggressiveness; also ERCC1, FPGS, MLH1 and XRCC 1 were found to be related to this parameter. Survival analysis shows a highly significant association of FPGS and FOLR 1 to OS as well as FOLR 1 and XRCC1 to PFS. MSH6 and TYMS seem to correlate with the appearance of distant metastasis, FOLR1, FPGS and MLH1 are associated with lymph node invasion (FOLR1: $\mathrm{p}=\mathrm{o} .0001$ ).

Conclusions. Expression of members of both, DNA-repair and folic acid metabolism, shows strong correlations to tumor type in neuroendocrine tumors of the lung. Also predictive and prognostic biomarkers could be identified from these. Both pathways seem to have a strong impact on tumorgenesis and aggressiveness in this group of tumors.

\section{FR-045}

Bronchuswall-thickness in frozen and paraffin sections of porcine lungs - comparative studies on histological and $\mathrm{x}$-ray imaging data

C. Brochhausen ${ }^{* 1}$, C. Tapprich*2, V.H. Schmitt ${ }^{2}$, D. Hollemann ${ }^{2}$, O. Weinheimer ${ }^{3}$, A. Mamilos' ${ }^{2}$ C.J. Kirkpatrick ${ }^{2}$

'Institute of Pathology, University Medical Centre of the Johannes Gutenberg-University, Mainz, Germany, ${ }^{2}$ Institute of Pathology, University Medical Centre, REPAIR-lab, Mainz, Germany, ${ }^{3}$ Diagnostic Radiology, University Heidelberg, Heidelberg, Germany

Aims. Tissue shrinkage before and after fixation and processing is well known. The degree of shrinkage varies significantly with the tissue type. However, the degree of shrinkage and comparison of different processing procedures in various tissues is not well studied. This knowledge is important for comparative studies with histological specimens from frozen and paraffin sections. Therefore, the thickness of bronchus-walls obtained from paraffin and frozen sections was compared. Methods. Four explanted pig lungs were frozen in ventilated condition in a liquid nitrogen bath. The lungs were dissected and 36 bronchi isolated. Of these, frozen sections of $5 \mu \mathrm{m}$ thickness were performed. The surface of the cutting edge was marked on the remaining tissue which was then fixed in $4 \%$ formalin and embedded in paraffin. One frozen and one paraffin section from the same cutting edge were analysed after haematoxylin and eosin staining. The wall thickness of the bronchi was measured using high power fields of 400 -fold magnification. In each bronchus 40 measurements were implemented at different wall positions. Hence, in each group 1440 wall measurements were performed in total. Statistical analysis was conducted using SPSS Statistics 20. Wilcoxon test and t-test were used with a significance level at 0.05 .

Results. The thickness of the bronchial wall was significantly $(\mathrm{p}<0.001)$ smaller in the frozen sections (median: $0.50 \mathrm{~mm}$; min: $0.37 \mathrm{~mm}$; max: $0.97 \mathrm{~mm}$ ) compared to the paraffin sections (median: $0.58 \mathrm{~mm}$; min: $0.35 \mathrm{~mm}$; max: $1.06 \mathrm{~mm}$ ). For the median values a divergence of $0.05 \mathrm{~mm}$ was found between paraffin and frozen sections (min: $-0.11 \mathrm{~mm}$; max: $0.22 \mathrm{~mm}$ ). The ratio of the median wall thickness of both groups was the following: Frozen/Paraffin section=0.8609. Thus, the divergence between paraffin and frozen sections amounted to $13.91 \%$.

Conclusions. The bronchus wall thickness of paraffin and frozen section was compared and gave a divergence of nearly $14 \%$. These results are important regarding the pathological evaluation of bronchus tissue by different methods and their comparison with X-ray analyses, for example, in clinical trials. To elucidate the method with minimal size difference compared to the original in situ size or to develop mathematical conversion formulae, further studies will be performed to compare histological data with prior collected in situ measurement methods e.g. using MRI.

\section{FR-046}

Development of protocols for specific determination of autophagy markers in formalin fixed paraffin embedded tissue by immunohistochemistry

\section{S. Berezowska*, A.M. Schläfli, R. Langer, M.P. Tschan \\ Institute of Pathology, University of Bern, Bern, Switzerland}

Aims. Autophagy is a mechanism for degradation of cellular components, which are engulfed in autophagosomes following fusion with lysosomes. Autophagy assures cellular homeostasis. In cancer, its deregulation is important in carcinogenesis, propagation of the malignant phenotype, treatment response and development of resistance. To date tissue based analysis of autophagy by immunohistochemistry (IHC) remains poorly standardized. We evaluated the feasibility of assessing autophagy markers by IHC in FFPE-tissue.

Methods. $\mathrm{LC}_{3} \mathrm{~B}$ and $\mathrm{p} 62$ are frequently used as autophagy markers and were evaluated due to their predominant association with autophagoso- 
mal membranes. LC 3 B and p62 were knocked down in HEK-293T and human H1299 lung cancer cells using lentivirally delivered small hairpin RNAs with subsequent induction of autophagy by treatment with everolimus or starvation, and bafilomycin A. FFPE-cell pellets of treated cells and controls were stained with anti-LC $3 \mathrm{~B}$ and p62 antibodies. Western Blot experiments validated antibody specificity, knock-down efficiency and autophagy induction in cells prior to fixation. Then, p62 and $\mathrm{LC}_{3} \mathrm{~B}$ stainings were applied on a tissue microarray with 81 human malignant and non-tumorous lung and stomach tissue samples.

Results. Specificity and sensitivity of the $\mathrm{LC}_{3} \mathrm{~B}$ and $\mathrm{p} 62$ antibodies in FFPE tissue was confirmed by Western Blot and knock-down experiments. Dot-like staining was significantly decreased in the knockdowns compared to controls. Tissue IHC showed dot-like staining in various degrees in 16/41 ( $\mathrm{LC}_{3} \mathrm{~B}$ ) and 18/41 (p62) tumors, but only in one non-neoplastic sample. For $\mathrm{LC}_{3} \mathrm{~B}$ a predominantly weak cytoplasmic staining was seen in both neoplastic and non-neoplastic epithelium. In contrast, p62 showed a stronger cytoplasmic staining in neoplastic tissue and a comparable nuclear staining in tumor and non-tumorous tissue.

Conclusions. We have established a protocol for specific and reliable $\mathrm{LC}_{3} \mathrm{~B}$ - and $\mathrm{p} 62$-IHC staining in FFPE-tissue by preceding functiona cell culture based experiments. Our first results show successful and dependable application of IHC to stain dot-like autophagy structures in FFPE tissue. Dots were differentially and significantly more frequently expressed in tumors, suggesting either increased or defective autophagy pathways in cancer cells. The next step comprises refinement of the scoring system and application to larger case collections, with evaluation of a possible predictive and prognostic value.

\section{FR-047}

Secondary resistance to tyrosine kinase inhibitor therapy in EGFRmutant lung cancer with phenotypic change to small cell carcinoma: a case report with large-scale molecular analysis

\section{F. Haller*1, F. Fuchs'², E.A. Moskalev', R. Stöhr', A. Agaimy', R.J. Rieker?, A. Hartmann}

'Institute of Pathology, Friedrich-Alexander-University, Erlangen-Nuremberg, Germany, ${ }^{2}$ Department of Gastroenterology, Pneumology and Endocrinology, Friedrich-Alexander-University, Erlangen-Nuremberg, Germany

Aims. Approximately $10 \%$ of non-small cell lung cancers harbor activating mutations in the EGFR gene. While these tumors generally respond well to selective therapy with tyrosine kinase inhibitors, the majority of the tumors will acquire secondary therapy resistance. Different mechanisms of acquired resistance have been described, including secondary mutations in the kinase domain of the EGFR gene, as well as amplifications of other genes (e.g. MET) with a switch of receptor tyrosine kinase signaling. Rarely, a phenotypic change to small cell carcinoma has been reported. We here describe a case of acquired resistance to tyrosine kinase inhibitor therapy in an EGFR mutant lung cancer with large-scale molecular analysis of primary tumor and recurrence by massive parallel sequencing.

Methods. A 70-year-old female was diagnosed with non-small cell lung cancer, and initial mutation analysis by Sanger sequencing revealed the classical L858R activating mutation in the EGFR gene. Therapy with a selective tyrosine kinase receptor inhibitor was started according to extensive disease. After six months, multifocal progressive disease was observed, and biopsy of a bone metastasis revealed a phenotypic change to small cell carcinoma. Large-scale molecular analysis of both the primary biopsy and the recurrence were performed using the TrueSight Tumor gene panel of 26 cancer-related genes with subsequent massive parallel sequencing on a MiSeq machine.

Results. The L858R mutation in the EGFR gene was present in both samples with an allele frequency of $39 \%$ and $23 \%$, respectively. Additionally, an activating mutation in the MET oncogene was observed at allele frequencies of $37 \%$ and $47 \%$, respectively. There was no additional mutation among the 26 cancer-related genes found in the small cell carcinoma.
Conclusions. In the current case, acquired resistance to tyrosine kinase inhibitor therapy in an EGFR-mutant tumor was observed, and correlated with potential amplification of the MET oncogene detected by massive parallel sequencing. Phenotypically, a switch to small cell carcinoma was observed. Biopsy of progressive tumor under tyrosine kinase inhibitor therapy is relevant for decision of second line therapy regimen.

\section{FR-048}

Is the mRNA expression profile of apoptosis and cell cycle related genes a marker for the aggressiveness of neuroendocrine tumors of the lung?

R.F.H. Walter ${ }^{* 1,2}$, F.D. Mairinger ${ }^{2}$, R. Werner', S. Ting ${ }^{3}$, C. Vollbrecht ${ }^{4}$, D. Theegarten ${ }^{2}$, G. Stamatis ${ }^{5}$, D. Zwanziger ${ }^{6}$, W. Eberhardt ${ }^{7}$, M. Schuler', D.C. Christoph ${ }^{7}$, L. Freitag ${ }^{8}$, K.W. Schmid ${ }^{2}$, J. Wohlschlaeger ${ }^{2}$

${ }^{1}$ Ruhrlandklinik, West German Cancer Center, University Hospital Essen, University of Duisburg-Essen, Essen, Germany, ${ }^{2}$ Institute of Pathology and Neuropathology, University of Duisburg-Essen, University Hospial, Essen, Germany, ${ }^{3}$ Institute of Pathology and Neuropathology, University of Duisburg-Essen, University Hospial, Division of molecular Pathology, Essen, Germany, ${ }^{4}$ Institute of Pathology, University Hospital, Cologne, Germany, ${ }^{5}$ Ruhrlandklinik, West German Cancer Center, University Hospital Essen, Division of Thoracic Surgery and Endoscopy, University of Duisburg-Essen, Essen, Germany, ${ }^{6}$ University Hospital Essen, Division of Laboratory Research, Department of Endocrinology and Metabolic Diseases, University of Duisburg-Essen, Essen, Germany, ${ }^{7}$ Department of Oncology, University of Duisburg-Essen, University Hospital, Essen, Germany, ${ }^{8}$ Department of interventional Pneumology, University of Duisburg-Essen, University Hospital, Essen, Germany

Aims. Neuroendocrine tumors of the lung comprise four entities (typical and typical carcinoids, large cell neuroendocrine cancer and small cell lung cancer) with varying aggressiveness regarding clinicopathology. Cell cycle and apoptosis are key pathways of multicellular homeostasis and deregulation of these pathways is associated with cancerogenesis.

Methods. Twenty representative FFPE-specimens of each tumor entity (TC, AC, LCNEC, and SCLC) were used for mRNA expression analysis using the NanoString technique. Based on recent literature a total of 91 mRNA targets were investigated as potential tumor markers. Eight genes related to apoptosis and ten genes regulating key points of cell cycle were investigated in this subset.

Results. The NanoString technique was able to specifically and sensitively analyze $91 \mathrm{mRNA}$ targets in 80 FFPE samples using $100 \mathrm{ng}$ of total RNA. ASCL1 $(p=0.00164), B C L 2(p=2.93 E-05)$, CASP8 $(p=0.003016)$, $\mathrm{CCNE}_{1}(\mathrm{p}=0.01354), \mathrm{CDK}_{1}(\mathrm{p}=0.01459), \mathrm{CDK}_{2}(\mathrm{p}=0.01136), \mathrm{CDKN}_{1} \mathrm{~A}$ $(\mathrm{p}=0.01072)$ and $\mathrm{CDKN} 2 \mathrm{~A}(\mathrm{p}=0.0002186)$ showed lower expression in carcinoids compared to carcinomas. In contrast, $\mathrm{CCNE} 1(\mathrm{P}=0.01354)$ and CDK6 ( $\mathrm{p}=3.66 \mathrm{E}-\mathrm{o6})$ showed elevated expression in carcinoids compared to carcinomas. The calculated $\mathrm{BCL}_{2} / \mathrm{BAX}$ ratio $(\mathrm{p}=0.006305)$ showed increasing values from TC to SCLC. Between SCLC and LCNEC $\mathrm{CDK}_{2}(\mathrm{p}=0.01421), \mathrm{CDKN}_{1} \mathrm{~B}(\mathrm{p}=0.003182), \mathrm{CDKN}_{2} \mathrm{~A}(\mathrm{p}=0.02913)$ and $\mathrm{PNN}(\mathrm{p}=0.0008087)$ expression was significantly different with higher expression in SCLC.

Conclusions. Regarding cell cycle control, the results imply that carcinoids have upregulated $\mathrm{CDK}_{4} / 6$ and CCND1 expression controlling RB1 phosphorylation via this signaling cascade. $\mathrm{CDK}_{2}$ and $\mathrm{CCNE}_{1}$ were upregulated in carcinomas showing that they use the opposite way to control $\mathrm{RB} 1$. Regarding apoptosis, $\mathrm{BAX}$ and $\mathrm{BCL} 2$ are antagonists in regulating this cell process. BCL2 expression increased over BAX expression with increasing malignancy of the tumor. 


\section{AG Oralpathologie Postersession}

\section{FR.1-030}

Slice cultures from head and neck squamous cell carcinoma: a novel test system for drug susceptibility and mechanisms of resistance

\section{Kubick*1, M. Gerlach ${ }^{2}$}

'institute of Pathology, Medicine, University of Leipzig, Leipzig, Germany, ${ }^{2}$ Institute of Anatomy, Anatomy, University of Leipzig, Leipzig, Germany

Aims. Human head and neck squamous cell carcinoma (HNSCC) fundamentally vary in their susceptibility to different cytotoxic drugs and treatment modalities. Today there is no clinically accepted test system to predict the most effective therapy for an individual patient.

Methods. Therefore we established tumour-derived slice cultures which can be kept in vitro for at least 6 days. Upon treatment with guideline conform cytotoxic drugs, slices were fixed and paraffin sections were cut for histopathological analysis. Moreover quantitative analysis of cell death by the determination of lactatedehydrogenase (LDH) concentration was done.

Results. Slice cultures stay ex vivo in an excellent morphological condition and show mitotic activity in a period of 6 days. The treatment with Cisplatin or Docetaxel has a significant impact on the viability, shown by appearing apoptotic cells. The LDH-measurement confirms our observations after HE-staining. Apoptotic fragmentation, activation of caspase 3, and cell loss were observed in treated tumour slices. The number of nuclei per field in untreated slices was compared with treated slices derived from the same tumour. This allowed estimation of the anti-neoplastic activity of individual drugs on an individual tumour. Conclusions. These were the first steps to predict the best therapy for an individual patient. HNSCC-derived slice cultures survive well in vitro and may serve not only to improve personalised therapies but also to detect mechanisms of tumour resistance by harvesting surviving tumour cells after treatment [1]. Furthermore the use of HE-stains on slice cultures insures that real carcinoma tissue is treated by cytotoxic drugs and examined afterwards.

\section{Literatur}

1. Gerlach M (2013) Slice cultures from head and neck squamous cell carcinoma: a novel test system for drug susceptibility and mechanisms of resistance. British Journal of Cancer doi:10.1038/bjc.2013.700, 2013-01-02

\section{FR.1-031}

Role of Harvey Ras (HRAS) mutations in head and neck squamous cell carcinoma (HNSCC)

\section{K. Endhardt ${ }^{* 1}$, A. Khattri ${ }^{2}$, M. Keck ${ }^{2}$ D. Rieke ${ }^{2}$, A. Ress ${ }^{2}$, J. Braegelmann ${ }^{2}$, D. Mahmutoglu' ${ }^{2}, K$. Leung ${ }^{2}$, T. Seiwert ${ }^{2}$}

IInstitute of Pathology, Augsburg, Germany, ${ }^{2}$ Section of Hematology/Oncology, Department of Medicine \& University of Chicago Cancer Research Center, University of Chicago, Chicago, United States

Aims. Harvey Ras (HRAS) was recently identified being mutated in a small fraction of head and neck squamous cell carcinomas (HNSCC) and likely plays an important role as an oncogenic driver. We aimed to verify the occurrence and determine the precise role of HRAS mutations for signaling and carcinogenesis of HNSCC.

Methods. We completed mutational screening (Sanger Sequencing) in cell lines and tumor tissues for the known RAS mutation hotspots G12X, G13X and Q61X and determined the impact on cell viability by siRNA-mediated knockdown of the HRAS gene. Furthermore we performed viability testing followed by immunoblotting for various mutated cell lines and visualized the signaling effects in downstream cascades. Cells were analyzed untreated, in presence of $\mathrm{PI}_{3} \mathrm{~K}$ inhibitors, EGFR inhibitors and likewise in combination. Further viability assays were performed after transient transfection of HRAS(G12D)-expression vectors in wild-type cell lines.

Results. In 49 HNSCC cell lines and 52 HNSCC tumor tissues we identified 3 cell lines, $\mathrm{HN}_{4}\left(\mathrm{G}_{12} \mathrm{D}\right), \mathrm{BB}_{49}(\mathrm{Q} 61 \mathrm{~L})$ and $\mathrm{H}_{357}\left(\mathrm{G}_{13} \mathrm{~S}\right)($ rate $=6.1 \%)$ as well as 2 tumor tissue samples (mutations: $\mathrm{Q} 61 \mathrm{~L}$ and $\mathrm{G} 13 \mathrm{~V}$; rate $=3.8 \%$ ) with canonical HRAS mutations. HRAS gene knockdown was highly efficacious at suppressing viability in HRAS mutated cell lines. Viability assays revealed resistance to EGFR inhibition to a different degree. Immunoblotting revealed that mutant HRAS signals exclusively via $\mathrm{PI}_{3} \mathrm{~K}-\mathrm{AKT}$ and not via the MAPK pathway. By contrast $\mathrm{PI}_{3} \mathrm{~K}$ inhibition was highly effective and induced apoptosis. Combinations of $\mathrm{PI}_{3} \mathrm{~K}$ and EGFR inhibition indicated synergy in wild-type cell lines only. Accordingly to previous results, transient transfection of HRAS(G12D) vectors in wild-type cell lines showed a clear tendency for increased resistance to EGFR inhibition.

Conclusions. Previous clinical studies have shown that EGFR targeting agents often remain insufficient as single targeted therapy in HNSCC. Canonical HRAS mutations seem to be strong oncogenic drivers. They associate with near complete EGFR-resistance in HNSCC cell lines and near complete dependence on $\mathrm{PI}_{3} \mathrm{~K}$ signaling. In conclusion, our data indicates, that $\mathrm{PI}_{3} \mathrm{~K}$ inhibitors may be effective in HNSCC.

\section{FR.1-032}

Evaluation of prognostically and therapeutically relevant genomic alterations in salivary gland carcinomas

I. Grünewald ${ }^{* 1}$, C. Vollbrecht ${ }^{2}$, M. Meyer ${ }^{3}$, J. Meinrath ${ }^{2}$, L.C. Heukamp ${ }^{2}$, W. Hartmann'2, E. Wardelmann', U. Drebber', D. Beutner', R. Büttner'2, M. Stenner', M. Odenthal ${ }^{2}$

'Gerhard-Domagk-Institute for Pathology, University Hospital Münster, Münster, Germany, ${ }^{2}$ Institute of Pathology, University Hospital Cologne, Cologne, Germany, ${ }^{3}$ Department of Ear, Nose and Throat, Head and Neck Surgery, University Hospital Cologne, Cologne, Germany, ${ }^{4}$ Department of Ear, Nose and Throat, University Hospital Münster, Münster, Germany

Aims. Salivary gland carcinomas (SGC) pose a challenge for diagnostics and therapy because of their low incidence and a variety of histological subtypes. In many other types of malignant tumors, e.g. lung cancer, colon carcinoma and tumors arising in histologically similar organs as breast or pancreas, analyses employing new molecular biological techniques offer new opportunities for an individualized tumor therapy. Because of the aforementioned reasons only little is known on therapeutically relevant genetic alterations in SGC.

Methods. In this study we analyzed genetic alterations in about 100 salivary gland carcinomas of different tumor types which were collected from 1998 to 2011 at the University Hospital of Cologne and performed next generation sequencing (NGS) using the Ion AmpliSeq Cancer Panel which covers the relevant regions of 46 cancer genes.

Results. In this collection of SGC we detected very few KRAS and NRAS mutations, however almost $30 \%$ of the tumors carried HRAS mutations with much higher frequency of the p.Q61R substitution than substitutions at codon 12/13. Furthermore, we found several genetic variants in some salivary gland carcinomas, including alterations of the $\mathrm{PIK}_{3} \mathrm{CA}$, KIT, BRAF, ATM, FGFR2, PTEN, APC, AKT, NOTCH1 and RET genes and diverse alterations of the $\mathrm{P}_{53}$ gene.

Conclusions. For the future, analyses of larger collections of salivary gland carcinomas and functional characterization of the involved signaling pathways are required to evaluate opportunities for an individualized tumor therapy in SGC. 


\section{FR.1-033}

Receptor tyrosine kinase signaling is involved in salivary gland tumorigenesis and might suit as an approach for an individualized tumor therapy in salivary gland carcinomas

I. Grünewald*1, J. Meinrath'², M. Meyer ${ }^{3}$, D. Beutner ${ }^{2}$, M. Stenner', U. Drebber', S. Huss', E. Wardelmann', R. Büttner', W. Hartmann ${ }^{2}$

${ }^{1}$ Gerhard-Domagk-Institute for Pathology, University Hospital Münster, Münster, Germany, ${ }^{2}$ Institute of Pathology, University Hospital Cologne, Cologne, Germany, ${ }^{3}$ Department of Ear, Nose and Throat, Head and Neck Surgery, University Hospital Cologne, Cologne, Germany, ${ }^{4}$ Department of Ear, Nose and Throat, University Hospital Münster, Münster, Germany

Aims. Salivary gland carcinomas (SGC) represent a heterogeneous group of malignant tumors with a low incidence and a high histological diversity. The definition of appropriate diagnostic and therapeutic algorithms therefore is particularly challenging. Only little is known on the role of oncogenic signaling pathways in SGC tumorigenesis.

Methods. We used a set of about 100 salivary gland carcinomas of different tumor types collected from 1998 to 2011 at the University Hospital of Cologne to analyze the relevance of receptor tyrosine kinase signaling pathways in SGC. Immunohistochemical stainings and fluorescence in situ hybridization of Her-2, EGFR and c-Met as well as downstream kinase pathway effectors were performed, since overexpression of these receptor tyrosine kinases is associated with the opportunity for an individualized tumor therapy in malignant tumors of many other organs.

Results. Subgroups of SGC showed overexpression and/or amplification of Her-2, EGFR or c-Met pointing to a potential activation of the downstream signaling pathways in SGC tumorigenesis. These findings might reveal options for an individualized tumor therapy also in salivary gland carcinomas.

Conclusions. For the future, analyses of larger collections of SGC and further evaluation of receptor tyrosine kinase signaling as a putative target for an individualized tumor therapy in salivary gland carcinomas is necessary.

\section{FR.1-034}

\section{$\mathrm{PI} 3 \mathrm{~K} / \mathrm{AKT}$ signaling is involved in salivary gland tumorigenesis}

I. Grünewald ${ }^{* 1}$, J. Meinrath ${ }^{2}$, M. Meyer ${ }^{3}$, D. Beutner ${ }^{2}$, M. Stenner', U. Drebber' ${ }^{2}$ S. Huss', E. Wardelmann', R. Büttner' ${ }^{2}$, W. Hartmann ${ }^{2}$ 'Gerhard-Domagk-Institute for Pathology, University Hospital Münster, Münster, Germany, ${ }^{2}$ Institute of Pathology, University Hospital Cologne, Cologne, Germany, ${ }^{3}$ Department of Ear, Nose and Throat, Head and Neck Surgery, University Hospital Cologne, Cologne, Germany, ${ }^{4}$ Department of Ear, Nose and Throat, University Hospital Münster, Münster, Germany

Aims. Salivary gland carcinomas (SGC) represent a heterogeneous group of malignant tumors with a low incidence and a high histological diversity. According to this, SGC show variable courses of disease and pose a challenge for diagnostics and therapy. Only little is known on the role of oncogenic signaling pathways in SGC tumorigenesis.

Methods. A set of about 100 salivary gland carcinomas of different tumor types collected from 1998 to 2011 at the University Hospital of Cologne was used to analyze the relevance of the $\mathrm{PI}_{3} \mathrm{~K} / \mathrm{AKT}$ signaling pathway in SGC. Immunohistochemical stainings of central pathway effectors including p-(Ser473)-AKT, p-(Ser9)-GSK-3 $\beta$ and PTEN and fluorescence in situ hybridization of the $\mathrm{PIK}_{3} \mathrm{CA}$ gene were performed. Results. Subgroups of SGC displayed loss of PTEN expression, overexpression of $\mathrm{p}-\mathrm{AKT}$ or $\mathrm{p}-\mathrm{GSK}-3 \beta$ or an amplification of the $\mathrm{PIK}_{3} \mathrm{CA}$ gene pointing to an activation of the $\mathrm{PI}_{3} \mathrm{~K} / \mathrm{AKT}$ cascade in SGC tumorigenesis.

Conclusions. Further molecular analyses are required to understand the functional role of $\mathrm{PI}_{3} \mathrm{~K} / \mathrm{AKT}$ signaling in SGC.

\section{FR.1-035}

Malignant solitary fibrous tumor of the nasal cavity

H. Geddert ${ }^{* 1}$, S. Barthelmess' ${ }^{2}$, F. Anders ${ }^{3}$, U. Werner', A. Dunsche ${ }^{5}$, A. Dimmler ${ }^{1}$

'Institute of Pathology, St. Vincent's Hospital, Karlsruhe, Germany, ${ }^{2}$ Institute of Pathology, Diagnostic Molekular Pathology, University Hospital, Erlangen, Germany, ${ }^{3}$ St. Vincent's Hospital, ENT Department, Karlsruhe, Germany, ${ }^{4}$ Institut of Pathology, Municipal Hospital, Karlsruhe, Germany, ${ }^{5}$ Oral and Maxillofacial Surgery and Dentistry, Municipal Hospital, Karlsruhe, Germany

Aims. A 69-year-old male complains of nasal obstruction. His medical history is significant for hypertension, diabetes, chronic heart failure, obstructive sleep apnea syndrome and spinal canal stenosis. Nasoendoscopy reveals a soft mass filling the right nasal cavity. Computed tomography shows an extension into the maxillary sinus with destruction of the medial wall, floor of orbit and nasal septum. The tumor grows through the cribriform plate into the anterior skull base, cavernous sinus and dura.

Methods. The patient undergoes an endonasal tumor resection combined with an anterior craniotomy. A CSF leakage has to be closured twice. Eight months later, a tumor recurrence is treated by re-operation and radiation. More than three years later, the second relapse in the upper nasal cavity, orbit and roof of orbit after cannot be removed completely by another combined transnasal and neurosurgical operation. After another year the tumor progress requires an enucleation of the eye and debulking of the upper nasal cavity. After further 16 month or six years after first diagnosis, respectively, the patient dies from epistaxis.

Results. The first intranasal manifestation constitutes a non-encapsulated proliferation of spindle cells with low pleomorphism and low proliferation. Immunohistochemically, the spindle cells are strongly positive for $\mathrm{CD}_{34}$ and bcl-2, while other marker (e.g. S10o, CD117, aktin, keratin) are negative. These findings meet the rare diagnosis of a solitary fibrous tumor of the nasal cavity. A benign biological nature is assumed, while no invasive growth or other malignant features are evident. In contrast, the intracranial parts are diagnosed as malignant SFT due to focal atypia, necrosis and increased mitotic ratio. The pathology of the latest relapse is characterized by a dedifferentiation with brisk pleomorphism, markedly increased proliferation and loss of $\mathrm{CD}_{34}$. All manifestations of the tumor show the newly described somatic fusions of the NAB2 gene (NGFI-A binding protein 2) and STAT6 gene (signal transducer and activator of transcription 6). The most frequent variant with exon 4 $\left(\mathrm{NAB}_{2}\right)$ and exon 2 (STAT6) is identified.

Conclusions. Rarely a solitary fibrous tumor is diagnosed in the nasal cavity. The prediction of the biological behavior can be challengingat least not because of the intratumor heterogeneity. The NAB2ex4STAT6ex2 fusion is a frequent and early event in tumorigenesis of SFT. 
AG Herz-, Gefäß-, Nephro- und

Transplantatpathologie Postersession

\section{FR.1-036}

Comprehensive analysis of clinicopathological data reveals heterogeneous relations between atherosclerosis and cancer

J. Budczies ${ }^{* 1}$, M. von Winterfeld', F. Klauschen', A.-C. Kimmritz',

J.-M. Daniel', A. Warth', V. Endris ${ }^{3}$, C. Denkert', H. Pfeiffer', W. Weichert ${ }^{3}$,

M. Dietel', D. Wittschieber', A. Stenzinger ${ }^{3}$

'Institute of Pathology, Charité - University medicine of Berlin, Berlin, Germany, ${ }^{2}$ Department Cardiac and Thoracic Surgery, Hannover Medical School, Hannover, Germany, ${ }^{3}$ Institute of Pathology, Ruprecht-Karls-University, Heidelberg, Germany, ${ }^{4}$ Institute of Legal Medicine, University Hospital Münster, Münster, Germany

Aims. Atherosclerosis and cancer share common risk factors and involve similar molecular pathomechanisms. Most clinical and epidemiological studies show a positive correlation between atherosclerosis and smoking-related cancers and heterogeneous results for non smoking-related cancers. However, up-to date large-scale autopsy studies including a detailed analysis of cancer types are lacking. Therefore, we sought to investigate the relation between major cancer types and the grade of atherosclerosis in a recent well-powered autopsy cohort.

Methods. In 2101 patients both, autopsy data and clinical data including demographics, disease groups, tumor type, cause of death, and grade of atherosclerosis were reviewed and statistically analyzed.

Results. We found cancer in general associated with less atherosclerosis (OR: o.6o, $\mathrm{p}<0.0001)$. In particular, hematologic neoplasm and sarcomas were associated with much less atherosclerosis $(\mathrm{OR}=0.45, \mathrm{p}<0.0001$ and $\mathrm{OR}=0.43, \mathrm{p}=0.087$ ), while carcinomas were associated with moderately less atherosclerosis $(\mathrm{OR}=0.72, \mathrm{p}=0.002)$. Furthermore, non smoking-related cancers were associated with much less atherosclerosis $(\mathrm{OR}=0.41, \mathrm{p}<0.0001)$, while possibly smoking-related cancers and smoking-related cancer showed no significant association. In a comprehensive analysis 21 cancer types, biliary tract cancer, lymphomas/ lymphoid leukemias and kidney cancer were associated with much less atherosclerosis $(\mathrm{OR}=0.19, \mathrm{p}<0.0001, \mathrm{OR}=0.41, \mathrm{p}<0.0001$ and $\mathrm{OR}=0.48$, $\mathrm{p}=0.029$ ). In an exploratory analysis of treatment strategies, we found that tumors with a recommendation of oxazaphosphorines and pyrimidine antagonist treatment were significantly associated with less atherosclerosis $(\mathrm{OR}=0.33, \mathrm{p}=0.0068$ and $\mathrm{OR}=0.58, \mathrm{p}=0.012)$.

Conclusions. In conclusion, the study showed an inverse association between cancer and atherosclerosis post mortem that depends on the cancer type and suggests a possible impact of chemotherapy regimens.

\section{FR.1-037}

Histological and immunohistological findings of chronic thromboembolic pulmonary hypertension (CTEPH)

\section{Brochhausen', S. Krajnak ${ }^{* 1}$, C. Wiedenroth', V.H. Schmitt', A. Mamilos', M. Wurzel', E. Mayer', C.J. Kirkpatrick' \\ 'Institute of Pathology, University Medical Centre of the Johannes Guten- berg-University, Mainz, Germany, ${ }^{2}$ Thoracic Surgery, Kerckhoff Clinic, Thoracic Surgery, Bad Nauheim, Germany}

Aims. Chronic thromboembolic pulmonary hypertension (CTEPH) is characterised by the presence of organising thromboemboli in the proximal pulmonary arteries (PA) and by arteriopathy in the distal PA. Both processes lead to vascular remodeling and fibrous stenosis up to complete obliteration of PA. In consequence, the pulmonary vascular resistance increases resulting in right-heart failure. It is assumed that CTEPH pathophysiology contains dysfunction of endothelial integrity followed by increased endothelial permeability and access of several mediators to PA wall cells, which leads to an altered regulation of both vascular and circulating cells. Aim of this study was to characterise lining endothelium, newly formed vessels and single cells in the thrombotic proximal and distal tissue.

Methods. Pulmonary endarterectomy (PEA) tissue samples from 20 CTEPH patients were treated according to standardized procedures for histological (HE-, EvG) staining and immunohistological evaluation for nestin, $\mathrm{CD}_{31}, \mathrm{CD}_{34}$ and SM-alpha-actin expression by use of monoclonal antibodies.

Results. Histologically, the proximal samples were characterised by homogenous fibrous tissue with low amounts of spindle cells, whereas the distal samples showed a heterogeneous cell-rich tissue with numerous small vessels. Immunohistologically, Nestin-, CD31- positive single cells were detected in both proximal and distal lesions of PA with a higher extend in proximal tissue samples. Furthermore, Nestin, $C_{31}$ and $\mathrm{CD}_{34}$ expression could be found in endothelial cells in the neointima as well as in recanalized vessels of the proximal and distal specimens. The positivity for SM-alpha-actin was more often detectable in distal tissue samples than in proximal ones.

Conclusions. In the current study significant histomorphological differences between proximal and distal PA lesions could be observed given by fibrous tissue in the proximal samples and a vessel-rich tissue in the distal samples. Recanalized vessels showed expression of Nestin, CD31and $\mathrm{CD}_{34}$. Interestingly, some $\mathrm{CD}_{31} / \mathrm{CD}_{34}$-positive vessels were negative for nestin. Aim for our future studies will be the investigation of the molecular mechanisms which are involved in the impaired regulation of cells in CTEPH and how to prevent them in the context of targeted therapy.

\section{FR.1-038}

Detection of de-novo expressed ED-A+ Fibronectin after human cardiac transplantation: promising therapeutic target and potential novel serum biomarker for the early detection of allograft rejection?

M. Franz ${ }^{* 1}$, B. Ziffels', M. Matusiak-Brückner', K. Grün', H. Maschek², P. Richter ${ }^{3}$, U. Schulz', J. Gummert ${ }^{4}$, H.R. Figulla', A. Renner', A. Berndt ${ }^{3}$ 'Department of Internal Medicine I, University Hospital Jena, Friedrich Schiller University Jena, Jena, Germany, ${ }^{2}$ Institute of Pathology, "Pathologie am Tiergarten", Hannover, Germany, Institute of Pathology, University Hospital Jena, Friedrich Schiller University Jena, Jena, Germany, ${ }^{4}$ Clinic for Thoracic and Cardiovascular Surgery, Heart Center North Rhine-Westphalia, Ruhr University of Bochum, Bad Oeynhausen, Germany

Aims. Chronic cardiac rejection, represented by allograft vasculopathy (CAV) and interstitial fibrosis (CIF), is accompanied by tissue remodelling which entails the de-novo expression of fetal ED-A+ Fibronectin (Fn). In a rat model, ED-A+ Fn has been proven to be re-expressed in clear association to CAV and CIF. Since the availability of human antiED-A+ Fn antibodies usable as vehicles for targeted delivery of diagnostic agents or drugs, it is of great interest to investigate the ED-A+ Fn expression also in human cardiac allografts. Thus, the study was aimed 1) to analyse the ED-A+ Fn deposition in human cardiac biopsies and 2) to establish an ELISA method to specifically assess serum levels of the molecule.

Methods. Tissue specimens $(\mathrm{n}=69)$ from 48 heart-transplanted patients were available. Histopathological analysis was performed according to the ISHLT guidelines. Protein expression of ED-A+ and other markers (B+ Tenascin- $\mathrm{C}$ and $\alpha$-smooth muscle actin) was assessed by immunohistochemistry and, exemplarily, also by Western Blot. Furthermore, an ELISA method for the specific detection of ED-A+ Fn serum levels in humans and rats was developed.

Results. Histopathological analysis of the 69 biopsies revealed different ISHLT grades ( $\mathrm{OR}$ in 36 cases, $1 \mathrm{R}$ in 21 cases and $2 \mathrm{R}$ in 8 cases). In the majority of samples, there was a distinct and quantitatively relevant reoccurrence of ED-A+ Fn. There was a significant correlation between 
$\mathrm{ED}-\mathrm{A}+\mathrm{Fn}$ and $\mathrm{B}+$ Tenascin $-\mathrm{C}$ protein deposition $(\mathrm{p}=\mathrm{o} .000)$. A spatial association of ED-A+ Fn to CAV and CIF could be proven by immunofluorescence double labelling. Using Western Blot analysis, ED-A+ Fn could be detected in diseased human cardiac tissue. Using a newly established ELISA protocol, a specific detection of ED-A+ Fn could be evidenced both in human and rat serum.

Conclusions. There is an awesome re-occurrence of ED-A+ Fn following human cardiac transplantation. Referring to the correlation of ED-A+ Fn expression to chronic rejection in animal models, it can be postulated that patients showing an early re-occurrence might have an increased risk to develop CAV and CIF. Human anti-ED-A+ Fn antibodies for targeted delivery of diagnostics or bioactive payloads might pave the way for novel strategies to image, prevent or treat the chronic rejection. Specific quantitative assessment of serum ED-A+ Fn by ELISA enables studies to identify its possible role as a biomarker to improve surveillance of heart transplant recipients.

\section{FR.1-039}

\section{Glomerular miRNA expression in the Fischer to Lewis rat model of acute humoral rejection}

P. Zeuschner*1, S. Zell', H. Höflich', C.L. Bockmeyer ${ }^{* 1}$, K. Säuberlich', J. Wittig', V. Grau', J.U. Becker'

'Institute of Pathology, Hannover Medical School, Hannover, Germany, ${ }^{2}$ Sektion Experimentelle Chirurgie, Klinik für Allgemein-, Viszeral-, Thorax-, Transplantations- und Kinderchirurgie, Universitätsklinikum Gießen, Gießen, Germany

Aims. Acute humoral rejection is currently the most important diagnostic and therapeutic problem in renal transplantation. The Fischer to Lewis rat model is a well established model of acute humoral rejection due to MHC and non-MHC alloantibodies. In order to identify diagnostically useful glomerular miRNA expression patterns and to identify novel pathogenetic concepts and therapeutic targets we quantified the glomerular miRNA expression of this model.

Methods. 6 weeks after life-sustaining transplantation without immunosuppression 8 allografts were harvested for histologic examination and glomerular miRNA quantification by RT-PCR. Histologic changes and miRNA expression patterns were correlated and compared to 8 isografts.

Results. Glomerular capillaries contained significantly more monocytes in allografts than in isografts, consistent with transplant glomerulitis. In allograft glomeruli miR-451 and miR-192 were significantly downregulated compared to isografts. Both were inversely correlated with glomerular $\mathrm{C}_{4} \mathrm{~d}$ deposition and monocyte content.

Conclusions. With miR-192 and miR-451 we identified two micro-RNAs which are downregulated in the glomeruli of a rat model of acute humoral rejection. Whereas little is known about miR-192, miR-451 is known to be involved in inflammation. Both could be valid target molecules in acute humoral rejection. Our next projects will determine the exact cell type expressing both miRNAs, their relevance for human acute humoral rejection, their function and potential as therapeutic targets.

\section{FR.1-040}

Glomerular miRNA expression in the Fischer to Lewis rat model of transplant glomerulopathy

\section{P. Zeuschner"', S. Zell', H. Höflich', J. Wittig', K. Säuberlich', C.L. Bockmeyer', V. Grau', J.U. Becker'}

'Institute of Pathology, Hannover Medical School, Hannover, Germany, ${ }^{2}$ Sektion Experimentelle Chirurgie, Klinik für Allgemein-, Viszeral-, Thorax-, Transplantations- und Kinderchirurgie, Universitätsklinikum Gießen, Gießen, Germany

Aims. Chronic humoral rejection manifesting as transplant glomerulopathy is currently the most important factor limiting long-term allograft survival. The Fischer to Lewis rat model is a well established model of chronic humoral rejection due to MHC and non-MHC alloantibodies. In order to identify diagnostically useful glomerular miRNA expression patterns and to identify novel pathogenetic concepts and therapeutic targets we quantified the glomerular miRNA expression of this model.

Methods. 6 months after life-sustaining transplantation without immunosuppression 8 allografts were harvested for histologic examination and glomerular miRNA quantification by RT-PCR. Histologic changes $(n=8)$ and miRNA expression patterns $(n=4)$ of allografts were correlated and compared to 4 isografts.

Results. Glomerular $\mathrm{C}_{4} \mathrm{~d}$ staining, glomerular basement membrane double contours and focal and segmental glomerulosklerosis were more extensive in allografts than in isografts. Glomerular miR-155 and miR222 were upregulated, glomerular miR-192, miR-451, miR-466b downregulated in allografts compared to isografts. All 5 miRNAs correlated significantly with glomerular $\mathrm{C}_{4} \mathrm{~d}$ deposition, glomerular basement membrane double contours and glomerulosclerosis.

Conclusions. We identified glomerular 5 miRNAs differentially regulated in a rat model of transplant glomerulopathy. In silico pathway analysis reveals involvement of these miRNAs in inflammation and fibrosis. We will next identify their glomerular expression site, their mRNA targets and their potential for therapeutic use.

\section{FR.1-041}

Glomerular miRNA expression profile derived from models of acute humoral rejection is diagnostic of chronic active humoral rejection but currently inefficient in monitoring therapy response

H. Höflich*1, S. Zell', P. Zeuschner', J. Wittig', C.L.Bockmeyer', C. Süsal', H. Billing ${ }^{3}$, A. Fichtner ${ }^{3}$, B. Tönshoff ${ }^{3}$, J.U. Becker ${ }^{* 1}$

IInstitute of Pathology, Hannover Medical School, Hannover, Germany, 2Department of Transplantation Immunology, Institute of Immunology, University of Heidelberg, Heidelberg, Germany, ${ }^{3}$ Department of Pediatrics I, University Children's Hospital, Heidelberg, Germany

Aims. Humoral rejection remains a challenge for diagnosis and therapy in renal transplantation. Through analysis of various human and animal models of humoral rejection we have identified a panel of $18 \mathrm{miR}$ NAs that could be useful for the diagnosis of humoral rejection and for the prediction of therapy response. This study is the first validation of this panel.

Methods. We examined 9 biopsies from patients with chronic active humoral rejection before therapy (pre-Rx) and 8 biopsies from these patients after therapy (post-Rx) with plasmapheresis, intravenous immunoglobulin and rituximab. Glomerular miRNA expression of a panel of 18 miRNAs was quantified by RT-PCR after microdissection from paraffin sections. Results were compared to 8 controls without evidence of humoral rejection.

Results. Glomerular expression of miR-let7c, miR-125a-5p, miR-28-3p and miR-99b was higher in the pre-Rx biopsies than in the controls, glomerular miR-548 was lower. No significant difference could be found 
for the glomerular expression of the 18 examined miRNAs between pre$\mathrm{Rx}$ and post-Rx biopsies or between responders and non-responders. Conclusions. Glomerular miRNA expression profiling could be a helpful anxillary technique for the diagnosis of humoral rejection. In contrast to mRNA profiling, glomerular miRNA profiling can be performed after thorough histologic workup of the biopsy core. Prospective biopsy studies and in vitro studies will show the diagnostic, prognostic and predictive potential of glomerular miRNA profiling and could identify novel pathophysiologic pathways in humoral rejection.

\section{AG Dermatopathologie Postersession}

\section{FR.1-042 \\ Pathologic features of uveal melanomas after radiotherapy: a proposal for a regression grading}

A. Zimpfer ${ }^{* 1}$, C. Dahmen ${ }^{1}$, K. Wolff ${ }^{2}$, A. Zhivov' ${ }^{2}$, R. Guthoff', G. Hildebrandt ${ }^{3}$, N. Andratschke ${ }^{3}$, A. Erbersdobler ${ }^{1}$

'Institute of Pathology, University of Rostock, Rostock, Germany, 2Department of Ophthalmology, University of Rostock, Rostock, Germany, ${ }^{3}$ Department of Radiotherapy, University of Rostock, Rostock, Germany

Aims. Uveal melanoma (UM) is the most common primary intraocular tumour in Caucasians. Several therapeutic options are available, among those resection or enucleation, and bulbus-sparing modalities as brachytherapy with ruthenium-106 radioactive plaques, fractionated high-dose gamma knife stereotactic irradiation or fractionated linearaccelerator-based stereotactic teletherapy. The aims of this study were to estimate and grade histomorphological reactions in UM after radiotherapy and compare the effects with untreated UM. A proposal for a regression grading system (not yet described in the literature) is given. Methods. A total of 85 bulbus enucleates or iridectomies comprising 62 choroidal melanomas and 23 melanomas of the iris and/or ciliary body were analyzed. In 22 and 2 cases brachytherapy and fractionated Cyberknife radiotherapy had been administered, respectively. The following histomorphological features were assessed and graded: necrosis, nuclear swelling, pleomorphic nuclei, pyknosis and karyorrhexis, mitotic figures, inflammation, fibrosis and hyalinization, and vascular changes. Regression was assessed especially by integrating necrosis, vascular changes and scaring. Additionally, microvessel density and macrophage content were determinded by $\mathrm{CD}_{34}$ and CD68 immunohistochemistry, respectively. Statistical calculations were done using SPSS 15.0.

Results. 85 UM cases were retrieved from the institutional files. 43 (53.1\%) patients were male (range $40-88$ years, median 67 years) and $38(44.7 \%)$ patients were female (range $44-87$ years, median 66 years). Nuclear swelling, necrosis, fibrosis and vascular changes as well as macrophage content were significantly correlated with prior treatment ( $\mathrm{p}=0.005, \mathrm{p}=0.040, \mathrm{p}=0.020, \mathrm{p}=0.026, \mathrm{p}=0.033$ ), respectively. The own regression index as well as regression grading indices retrieved from the literature differed significantly between the treated and untreated UM group ( $\mathrm{p}>0.0001)$.

Conclusions. The systematic assessment of conventional histomorphological features into a regression grading system might help to discover radiosensitive UM cases and to stratify prognostic groups of $\mathrm{UM}$.

\section{AG Informatik, innovative Bildgebung und Biobanking Postersession}

\section{FR.1-043}

Evaluation of historical and recent autopsy records from university and community hospitals in Vienna

D. Höflmayer ${ }^{* 1}$, E. Winter ${ }^{2}$, T. Wasserscheid ${ }^{2}$, K. Vig-Kuna ${ }^{3}$, W. Feig/ ${ }^{3}$

'Department of Pathology and Bacteriology, Emperor Franz Josef-Hospital, Vienna, Austria, ${ }^{2}$ Pathologic-anatomical Collection in the Narrenturm, Museum of Natural History, Vienna, Austria, ${ }^{3}$ Deparment of Pathology, Medical University of Vienna, Vienna, Austria

Aims. The post-mortem examination represents the very last step in the diagnostic work-up of a patient. The aim of the study was to describe the development of autopsy patterns and diagnoses from the early 19th century until present with special emphasis on comparing university and community hospitals in Vienna.

Methods. Autopsy records from the Clinical Institute of Pathology of the Medical University Vienna and three community hospitals in Vienna were used in this study. For selected years, 120 autopsy records from university and community based institutes were reviewed. Data collected included whether the patient was dissected or not, age, sex, and the pathologic diagnosis or diagnoses leading to death, or-if specified-the cause(s) of death. To overcome the heterogeneous wording for further analysis, the pathologic conditions were coded using the ICD10-GM Version 2013.

Results. In Vienna until the late 1960 os virtually all patients dying at university or community hospitals were dissected. By the year 2000, autopsy rates dropped to $37.5 \%$ at the community hospital and to $52.5 \%$ at the university hospital. Throughout the centuries, the median age of patients dying at the university hospital was lower than of those dying at community hospitals. In the year 2000 the mean age differed significantly between those being dissected and those not being dissected, the former being younger than the latter. However this was only observed in the community hospital and not at the university hospital, where, in contrast, the mean age of those being dissected was slightly higher than those being not dissected. Infectious diseases like tuberculosis were most prominent as leading cause of death during the 19th century and early 2oth century, while after 1950 cardiovascular diseases and cancer increased. However, in many cases death was rather caused by a sum of pathologic conditions than by a single one. Not surprisingly the wording changed dramatically during the centuries, as was the formal aspect of the autopsy record. It was not until the 1970s that cause of death, underlying disease and other relevant diseases were mentioned separately in the record.

Conclusions. Due to the unique situation of the availability of autopsy records from more than 150 years from both university and community hospitals, comparisons of epidemiological data of patients as well as an insight into changing formal aspects of autopsy records-the basis of any mortality statistic_-are possible.

\section{FR.1-044}

OpenClinica based data acquisition for pseudonymized biomaterial at RWTH CBMB biobank: linking clinical and scientific domains

P. Leusmann ${ }^{* 1}$, C. Spreckelsen'2, J. Veeck', J. Jäkel', S. Förster', R. Knüchel-

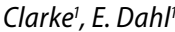

${ }^{1}$ RWTH cBMB/Institute of Pathology, Uniklinik RWTH Aachen, Aachen, Germany, ${ }^{2}$ Institute of Medical Informatics, RWTH Aachen University, Aachen, Germany

Aims. RWTH cBMB is the centralized biomaterial bank of the Uniklinik RWTH Aachen and strives to offer biobanking excellence by its 
high quality standards and state of the art IT solutions. To reach this goal medical data has to be connected to stored biomaterial samples in an efficient way while complying with data protection requirements. Depending on the specific research project, it may be beneficial to connect the data directly to the sample or to keep a more general case- or patient-related association like it is common in clinical practice. Besides the data present in clinical information systems (CIS), it is often desired to acquire additional data in a research project-related database. Consequently, there is a demand for a suitable pseudonymized data acquisition system for the scientific context and need for a way to connect it to existing medical data.

Methods. To create a suited identifier for a data record, the affiliation of the provided sample to a treatment case stored in an external system as well as a foreign key to this case is required. To compute the identifier, the foreign key will be encrypted asymmetrically. Since the resulting cipher is too big for a manual input, it will be associated with a fixed length placeholder value, building the core of the identifier. Including a checksum for detecting input errors, the identifier can be represented as manually usable string.

Results. The presented solution facilitates the enrichment of biomaterial samples with data of the treatment context as well as with the pseudonymized data acquired in the research-context. To provide a data input system, the RWTH cBMB currently evaluates the provision of the OpenClinica electronic data capture system. In this system the user is able to conduct the data acquisition with the help of an electronic case report form.

Conclusions. The identifier described offers the benefit of hiding personally identifying data while maintaining the link to the data source. However, the decryption of data is still possible with the assistance of the external data trustee keeping the private encryption key. The identifier is robust against typing errors and thus can be used manually. These characteristics show the applicability of the described technique to be used as an inter-domain identifier for pseudonymized biomaterial. By enabling a link between available research data and medical information in a data warehouse, this projects helps to leverage the research value of samples retrieved from RWTH cBMB.

\section{FR.1-045}

Biobanking within the business framework-requirements and implications of fee for service based funding

\section{A. Maier ${ }^{* 1,2}$, R. Kirsten ${ }^{1,2}$, P. Schirmacher ${ }^{1,2}$, E. Herpe ${ }^{2,3}$ 'BMBH at the Institute of Pathology, University Clinics Heidelberg, Heidel- berg, Germany, ${ }^{2}$ Institute of Pathology, University Hospital Heidelberg, Heidelberg, Germany, ${ }^{3} \mathrm{NCT}$ Tissue Bank at the Institute of Pathology, University Clinics Heidelberg, Heidelberg, Germany}

Aims. Operating a biobank that is partly funded by revenues from a feefor-service-model is tied to a variety of preconditions and implications that do not apply to the same extent to biobanks that do not charge service fees. In order to successfully implement a fee based compensation system, these factors have to be investigated and addressed.

Methods. To identify the relevant factors, the implications that usually apply to new companies or organisations that undergo changes in their distribution channels were analysed and the results transferred to the biobanking framework.

Results. Internal cost analysis and cost calculation are the basis for any venture that is dependent on a cost-covering operation. Only with an exact knowledge of costs and cost structures, economic viable models can be established. This, too, affects biobanks with their diverse cost drivers. In order to operate a biobank at least partly in a business environment it is necessary to develop a basic business strategy including an elaborate business plan as one of the most important planning and communication tools. Controlling systems that are adapted to the organisational structure are another central prerequisite. With controlling instruments in place, operating numbers can be defined and continu- ously compared to actual figures. In the case of deviations corrective measures can be initiated. Results from controlling systems can also be used for reporting. Modifications in the financing basis should be supported by a targeted change management approach. In order to optimise the change process, the focus should be based on the organisational structures as well as on all internal and external stakeholders. This will help to insure a better understanding why compensation in exchange for provided samples and services is necessary. Other long-range implications concern the competitive relationship between individual biobanks with the same or a similar portfolio, as well as the legal status between biobanks and their clients which might require an adjustment in their contractual relationships.

Conclusions. To insure the smooth introduction of fee-for-service models, knowledge of internal costs, customized business models, derived controlling systems as well as a general service orientation and active change management during the conversion period are necessary. In the course of the operational phase, issues like competition and legal status may constitute further points for consideration.

\section{FR.1-046}

\section{Development and implementation of a quality management sys-} tem for the BioMaterialBank Heidelberg

\section{S. Schmitt ${ }^{* 1,2}$, R. Kirsten ${ }^{1,2}$, P. Schirmacher ${ }^{1,2}$, E. Herpe $^{2,3}$}

'BMBH at the Institute of Pathology, Heidelberg University Hospital, Heidelberg, Germany, ${ }^{2}$ Institute of Pathology, University Hospital Heidelberg, Heidelberg, Germany, ${ }^{3} \mathrm{NCT}$ Tissue Bank at the Institute of Pathology, Heidelberg University Hospital, Heidelberg, Germany

Aims. One of the central goals of the BioMaterialBank Heidelberg, a BMBF-funded infrastructure project, is to implement a harmonized quality management system for all partner biobanks in Heidelberg. Furthermore, accreditation is intended for tissue banks in accordance with DIN EN ISO/IEC 17020 as inspection body type C and certification for liquid biobanking according to DIN EN ISO 9oo1. Accreditation will be performed by Deutsche Akkreditierungsstelle GmbH (DAkkS, national accreditation body for the Federal Republic of Germany). A basis for implementation is already provided by the quality management system of the NCT Tissue Bank at the Institute of Pathology which has been accredited in 2009 and reaccredited in 2013 [1].

Methods. To implement a harmonized quality management system for the BioMaterialBank Heidelberg a comprehensive survey of all existing procedures and equipment was performed in all seven partner biobanks. To this end, complex questionnaires to assess already existing documents and basic parameters were elaborated, accompanied by personal site inspections of local biobanks. Based on this data, individual measures were taken to implement the harmonized Quality Management System (QMS) at participating biobanks. Resulting QM documents for the standardized processes and techniques will be compiled in a common QM documentation for the whole biobanking infrastructure and made individually available via a password protected cloud system.

Results. The basic structure of the quality management system could be established in five of seven biobanks and first standardized processes are already being applied. Shortly after introduction of the QMS, employees of participating biobanks report sustainable improvements in the workflow and structure of biobanking processes. First internal audits and management reviews will be performed in two participating biobanks in the first half of 2014, and the registration process for accreditation or certification, respectively, will be accomplished in 2014 . Conclusions. For biobanking in scientific and biomedical research, a standardized and harmonized QM system, which guarantees an equal and careful treatment of all biospecimens is of particular relevance, rendering the achieved results in clinical research projects more reliable and reproducible. 


\section{Literatur}

1. Herpel E, Rocken C, Manke H, Schirmacher P, Flechtenmacher C (2010) Quality management and accreditation of research tissue banks: experience of the National Center for Tumor Diseases (NCT) Heidelberg, Virchows Archiv, http:// link.springer.com/article/10.1007\%2Fs00428-010-0998-1

\section{FR.1-047}

\section{The DKTK Biobanking Platform}

\section{Herzog ${ }^{* 1}$, R. Kirsten ${ }^{2,3}$, P. Schirmacher ${ }^{2,3}$}

${ }^{1}$ German Cancer Consortium (DKTK), German Cancer Research Center, Heidelberg, Germany, ${ }^{2} \mathrm{BMBH}$ at the Institute of Pathology, Heidelberg University Hospital, Heidelberg, Germany, ${ }^{3}$ Institute of Pathology, University Hospital Heidelberg, Heidelberg, Germany

Aims. As the incidence of cancer in Germany is around half a million cases per year, early detection, diagnosis, treatment and prevention stil remain the major challenges in health research. For this purpose the Federal Ministry of Education and Research (BMBF) established the German Consortium for Translational Cancer Research (DKTK) as one of the German Centers for Health Research (DZG). The key task of the DKTK is to provide optimal research conditions to accelerate the translational process of basic research and its clinical application. The consortium is composed of seven translational research programs and five comprehensive research platforms. The Biobanking Platform is one component of the Clinical Communication Platform (CCP) which re presents the organizational backbone of the DKTK. The mission of the Biobanking Platform is to harmonize processes and regulations concer ning biomaterials within the DKTK. By establishing an internal project management, the Biobanking Platform will provide access to homogenously collected and quality-assured biomaterials which are mandatory for successful cancer research.

Methods. To implement these harmonized structures within the DKTK, detailed information of the participating biobanks concerning quality management (QM), information technology (IT), ethical, legal and social issues (ELSI) and project management is required. To this end, complex questionnaires to assess these data are compiled during personal visits of the coordination office at the partner sites.

Results. After systematic analysis, data will be used for the definition of regulations for multisite projects, for the determination of an uniform minimal data set for biomaterial banks and to identify already existing prioritized collections. Moreover, the Biobanking Platform will provide support when required, e.g. for the introduction of a quality management system.

Conclusions. The Biobanking Platform will act as a trustee for all DKTK researchers by assuring a considerate handling and an efficient use of the asservated biomaterials.

\section{FR.1-048}

Measures to improve cryopreserved biospecimens quality for translational research: the RWTH centralized biomaterial bank (RWTH (BMB)

\section{J. Jäkel', P. Leusmann', C. Spreckelsen², S. Förster', R. Knüchel', E. Dahl',} J. Veeck*1

${ }^{1}$ Institute of Pathology, RWTH University, Aachen, Germany, ${ }^{2}$ RWTH Aachen University, Institute of Medical Informatics, Aachen, Germany

Aims. Process standardization in qualified biobanking is required not only for pre-analytical sample preservation but should cover all steps from obtainment to sample provision and be guided by evidence-based SOPs. Current efforts at RWTH cBMB aim at the development of an
ISO-certified quality management system which facilitates high quality translational research for biomedical investigators.

Methods. Basic SOPs were defined before acquiring any type of human biomaterial. Active research on preserved biospecimens, e.g. stability of molecular analytes in relation to handling time or freezing method, further refines SOPs and improves biospecimens' quality. Pathological sample characterization is performed before samples are provided to researchers.

Results. More than 20,000 biospecimens, of which $18 \%$ are solid tissue samples $(\sim 3,600)$, have been currently preserved at RWTH cBMB according to SOPs adopted from guidelines of two specialized organizations, i.e. ISBER and OBBR. The high number of SOPs and diversity of personnel involved led to current investigations whether SOP management will be improved by the standard of "Business Process Model and Notation" (BPMN). The freezing method (snap versus gentle) showed morphological differences mainly in protein-rich liver tissue, but only slightly in duodenal tissue, whereas nucleic acid yield and purity remained unaffected. RNA has been selected as a quality marker analyte of molecular decomposition in various tissues. While RNA quality, as demonstrated by the RNA integrity number (RIN, Agilent) of liver tissue was stable at room temperature over 240 minutes (RIN8) it decreased rapidly in duodenal samples (RIN3) rendering these unsuitable for transcriptomic research. Consequently, protective measures are taken to improve the quality of those samples, and SOPs are modified accordingly. Ultimately, any solid tissue is reviewed on H\&E sections by board-certified pathologists. Diagnosis must be re-confirmed and cellular content be appropriate before samples are finally provided to researchers. Own assessments revealed that up to $20 \%$ of samples may contain suboptimal quality, including tumorous cells in normal tissue or lack of representative tumor cells in cancer tissue.

Conclusions. Establishment of a quality management system at RWTH $\mathrm{cBMB}$ is an iterative, integrative and interdisciplinary task involving clinicians, biologists, technicians and computer scientists, but is required to provide biospecimens quality suitable for high impact biomedical research.

\section{FR.1-049}

The BMBF Initiative to build up centralized biomaterial banks in Germany: the popgen 2.0-Network

\section{A. Wolf*1, W. Lieb', A. Günsel', G. Jacobs', M. Krawczak²}

'Institute of Epidemiology, Christian-Albrechts-University Kiel, Kiel, Germany, ${ }^{2}$ Institute of Medical Informatics and Statistics, Christian-Albrechts-University Kiel, Kiel, Germany

Aims. Funded by the Federal Ministry of Education and Research as one of the five facilities supported within the National Biobank Initiative, the popgen 2.0-Network $\left(\mathrm{P}_{2} \mathrm{~N}\right)$ has started to merge seven data and probe collections existing at the Medical Faculty of the Christian-Albrechts-University (CAU) Kiel. This network aims to establish a uniform and centralised research infrastructure for biosamples and related data in order to enhance scientific usability of quality-controlled data and probes within an appropriate legal and ethical framework.

Methods. $\mathrm{P}_{2} \mathrm{~N}$ will combine data and biosamples of seven collections, namely of popgen, the Cancer Centre North, the Department of Neurosurgery, the Institutes of Pathology and Pharmacology, the University Lung Centre North and the Centre of Family Medicine. In order to harmonise, standardise and ease access to these manifold probe types together with the both comprehensive and sensible data attached to them, $\mathrm{P}_{2} \mathrm{~N}$ is presently transferring sample data to their central biobank information management system (BIMS) and purifying existing associated documents-like SOPs and ICs-with respect to a unified quality management system (QMS).

Results. A shared network of various biobanks with centralised access is merely as useful as the consistent and uniform quality of the probes and data collected and provided by the network partners. Due to the broad 
heterogeneity of the participating biosample and data collections the usage of one common efficient albeit flexible BIMS is a crucial ingredient for a properly functioning biobank network. Equally important is standardised handling of the probes, the accompanying data and auxiliary documents. Ideally, these routines must adequately be supported by the BIMS and a uniform set of SOPs and ICs should suitably be channelled by an appropriate QMS/DMS.

Conclusions. We are currently in the process of setting up one of Germany's largest biosample and data collections, including approx. $2 \mathrm{G}$ biosamples from more than $800 \mathrm{~K}$ individuals. Standardised IT and quality control infrastructures will facilitate an efficient and data safety conform access.

\section{FR.1-050}

Biobanking activities in the German Center for Infection Research (DZIF)

\section{Schmidt*1, B. Abt ${ }^{2}$, G. Anton ${ }^{3}$, F. Lasitschka', J. Overmann ${ }^{2}$, H.-E. Wich- mann $^{3}$, P. Schirmacher}

'Institute of Pathology, University Hospital Heidelberg, Heidelberg, Germany, ${ }^{2}$ Leibniz Institute, DSMZ German Collection of Microorganisms and Cell Culture, Braunschweig, Germany, ${ }^{3}$ Molecular Epidemiology, HMGU-Helmholtz Center, Munich, Germany

Aims. A central goal of the biobanking infrastructure inside BMBF-funded German Center for Infection Research (DZIF) is to provide fast and standardized access to biomaterials which is nowadays mandatory for multi-site and translational research. So far, there are no existing harmonized biobanking structures for infectious diseases in Germany and also a lack of biobanking structures and expertise at several DZIF partner sites. To build up an early-on working biobanking platform, preexisting biomaterials as well as respective expertise and technologies at different partner sites will be integrated. During implementation, structures like quality and project management, IT infrastructure and ELSI aspects will be harmonized within DZIF biobanking wherever possible. Methods. The DZIF biobank structure will be governed by a board consisting of the coordinators of the three columns tissue (Heidelberg), liquid (Munich) and pathogens and microbial producers (Braunschweig) and the biobanking representatives of all other DZIF partner sites. Central coordination (Heidelberg) of the platform is essential for further development, harmonization, and coordinative embedding in national and international research activities of DZIF biobanking.

Results. After the initial phase all positions at the main sites are filled and there is a regular exchange of information between the biobanking representatives of all DZIF sites. A DZIF Biobanking homepage (www. dzif-biobanken.de) and catalogue has been developed at Munich site and up to now 27 partner biobanks are registered. Future work includes extending the catalogue and networking within the national (DZGs and Ag BMB TMF) and international (Infectious Diseases Biobank London, ESBB) biobanking community.

Conclusions. Moreover, in future harmonized DZIF standard operating procedures (SOPs) are of central importance to give the opportunity to establish biomaterial banking as a common DZIF resource with a consistent high standard.

\section{AG Molekularpathologie Postersession}

\section{SA-070}

The over expression of c-FLIP in thymoma and thymic carcinoma through promoter hypomethylation

\section{A. Grass" ${ }^{* 1}$ D. Belharazem ', B. Schalke², P. Ströbel', A. Marx'}

'Department of Pathology, Mannheim, Germany, ${ }^{2}$ Department of Neurology, Regensburg, Germany, ${ }^{3}$ Department of Pathology, Göttingen, Germany

Aims. Epigenetic modifications, especially methylation or demethylation, of pro-apoptotic and anti-apoptotic genes are described in many different diseases, such as cancer. Cellular FLICE like inhibitory protein (cFLIP) is an anti-apoptotic protein, which is able to modulate activation of procaspase- 8 and in this way c-FLIP prevents induction of apoptosis mediated by death receptors. We focus on the methyl status of the c-FLIP gene promoter and its role as an anti-apoptotic protein in thymomas and thymic carcinomas.

Methods. We analyzed bisulfite-treated DNA isolated from cryopreserved whole tissue samples from 82 patients aged between 21 and 83 via methyl specific PCR (MSP). This patient group is composed of 8 A-thymomas, 22 AB-thymomas, 18 B2-thymomas, 19 B3-thymomas and 15 thymic carcinomas. Twenty whole tissues from healthy thymuses aged between 60 and 85 are used as a reference. The gene expression and protein detection were determined by real time PCR and western blot. Results. Based on MSP analysis, both the benign and the malignant thymomas and thymic carcinomas exhibit a permanent hypomethylation of the cFLIP gene promoter, which is also associated with a significant over expression of cFLIP mRNA $\left({ }^{* * * *} \mathrm{p}<0.0001\right)$ and protein levels. Normal thymuses are characterized by a hemimethylation of the c-FLIP gene promoter.

Conclusions. The hypomethylation of the gene promoter and the corresponding over expression of the anti-apoptotic cFLIP indicates an apoptosis blockade. The epigenetic modification of the c-FLIP gene could be involved in the development and aggressiveness of thymomas and thymic carcinomas. It is possible that novel c-FLIP targeted agents could be used in the treatment of thymoma and thymic carcinoma.

\section{SA-071}

Comparison of pre-analytical FFPE sample preparation methods and the impact on next generation sequencing in routine diagnostics

\section{Heydt ${ }^{* 1}$, H. Künstlinger', M.A. Ihle', L. Li' ${ }^{2}$, J. Fassunke', M. Odenthal',} R. Büttner', S. Merkelbach-Bruse'

'Institute of Pathology, University Hospital Cologne, Cologne, Germany, ${ }^{2}$ Institute of Pathology, University Hospital Göttingen, Göttingen, Germany

Aims. Over the last years next generation sequencing (NGS) has rapidly evolved and is at the edge of transitioning from research use into routine diagnostics. NGS has matured to an attractive platform in molecular oncology due to its sensitivity as well as the possibility of analysing multiple genes at the same time. For accurate and reliable NGS results not only the performance itself and the data analysis are important, but also the pre-analytical methods can have an influence on the outcome. Therefore we need robust, sensitive, efficient and validated pre-analytical methods and instruments for formalin-fixed, paraffin-embedded (FFPE) DNA extraction and quantification. This study aims to compare and evaluate pre-analytical methods to find the most suitable workflow for NGS in routine diagnostics.

Methods. Five different quantification methods were tested and compared. DNA was quantified by UV absorbance, fluorescent dye-based quantitation and quantitative PCR. Additionally a cohort of FFPE samples from small biopsies to large resections was analysed with five different DNA extraction systems and quantified by two different met- 
hods. DNA was extracted with the BioRobot M48, the QIAcube and the QIAsymphony SP all from Qiagen, the Maxwell ${ }^{\circ} 16$ from Promega and the InnuPure ${ }^{\oplus} \mathrm{C} 16$ from Analytik Jena. On all instruments the recommended FFPE extraction kits were used and the elution volume was set to $50 \mu \mathrm{l}$. The extracted and quantified FFPE DNA was subsequently amplified by multiplex PCR with customized AmpliSeq PCR primers from Life Technologies to evaluate if the eluates are free of inhibiting factors like high salt concentrations.

Results. DNA from FFPE samples was extracted with five different DNA extraction systems and compared. Significant differences could be seen in DNA quality and quantity as well as the impact on PCR amplification. There is more than a tenfold difference in DNA quantity between some of the systems. Especially the eluates of one system showed poor DNA quality and quantity resulting in inhibition of PCR amplification. Slight differences in the five analysed quantification methods could be seen but the results tended to be in the same range.

Conclusions. This study showed that it is not only important to focus on the NGS method and the data analysis but also on pre-analytical steps. It is important to choose the most reliable and constant DNA extraction system, especially when using small biopsies and low elution volumes for NGS runs in routine diagnostics.

\section{SA-072 \\ Efficacy of PCR for the differential diagnosis of tuberculosis (TB) in various forms of granulomatous lesions in paraffin-embedded tissues (PET)}

S. Montenegro', C. Delgado, , , S. Pineda ${ }^{2}$, C. Reyes ${ }^{2}$, A. Herrera ${ }^{* 1,2}$, T. de la Barra $^{2}$, C. Cabezas ${ }^{2}$, L. Spencer ${ }^{1,2}$, C. Palma ${ }^{2}$, F. Mucientes ${ }^{1}$

'Sección de Patología, Facultad de Medicina, Universidad de Concepción, Chile, Concepción, Chile, ${ }^{2}$ Hospital Guillermo Grant Benavente, Unidad de Anatomía Patologica, Concepcion, Chile

Aims. The morphology of tuberculous lesions varies and histologically the granulomas may be classified into necrobiotic, tuberculoid, suppurative, sarcoidal and foreign body miscellaneous granulomas. Granulomatous inflammation in PET samples occur in conditions such as TB, other infections, toxic, allergic, autoimmune, tumors and conditions of unknown etiology. The histologic diagnosis is suggestive but never confirmatory of TB in absence of a positive Acid-Fast Bacilli (AFB) stain. Hence, the molecular confirmation of TB is necessary for differential diagnosis due to the low sensitivity of AFB stain. We demonstrated the utility of a combination of molecular tests for the sensitive and specific detection of mycobacterial infection and we correlated those findings with the histologic features of the granuloma and with AFB staining.

Methods. PET samples: we studied 380 varied PETs types, recent to older than 9 years: 123 lymph nodes, 49 skin, 39 lung, 43 pleura, 31 intestine, 21 breast and 74 others tissues (bone marrow, prostate, liver, brain, kidney, larynx, and others) that were submitted for the histopathology diagnosis of TB. The PETs were grouped into 5 histological granuloma types: 1 . with caseous necrosis $(n=201) ; 2$. without caseous necrosis $(n=60)$; 3. sarcoidal $(n=25)$; 4. suppurative $(n=30)$; 5 . chronic nonspecific $(n=64)$. DNA extraction: sections (4) of $5 \mu \mathrm{m}$ of identified lesion were deparaffinized, digested and DNAs extracted following standard procedure. Only samples with amplifiable DNA were included. Molecular methods: two nested PCR-IS6110 amplified a 123bp and a 95bp, respectively for MTB complex detection. Nested pan-Mycobacterium of $65 \mathrm{kda}$ gene sequences amplified a $142 \mathrm{bp}$ of other mycobacterial DNA detection.

Results. Absolute sensitivities of PCR vs AFB staining for MTB for archival PETs granulomas were: Type 1: PCR 70.6\%, AFB staining $28.4 \%$ Type 2: PCR $36.7 \%$, AFB 8.3\%. Type 3: PCR 8\%, AFB staining 4\%. Type 4: PCR $16.7 \%$, AFB staining 6.7\%. Type 5: PCR 6.3\%, AFB staining o\%. Clinical validity for PCR was estimated considering as most probable TB those PET with caseous necrosis granulomas and any positive AFB stain. PCR: sensitivity $73 \%$, specificity $94 \%$, PPV $95 \%$, and NPV $70 \%$. AFB staining: sensitivity $31 \%$, specificity/PPV $100 \%$ and NPV $54 \%$. Conclusions. Use of a sensitive and specific combination of PCR-based testing of mycobacterial infections increased the accuracy of histological diagnosis associated with granulomatous tissue lesions.

SA-073

Comparison of three massive parallel sequencing platforms by interlaboratory testing of 30 lung cancer samples and their applicability for routine diagnostics

J. Fassunke ${ }^{* 1}$, F. Haller', R. Penzeß', S. Hebele', E.A. Moskalev', L. Heukamp', N. Pfarr ${ }^{3}$, S. Merkelbach-Bruse', V. Endris ${ }^{3}$

'Institute of Pathology, University Hospital, Cologne, Germany, ${ }^{2}$ Institute of Pathology, Friedrich-Alexander-University, Erlangen-Nuremberg, Germany, ${ }^{3}$ Institute of Pathology, Ruprecht-Karls-University, Heidelberg, Germany

Aims. In the growing field of personalised medicine, the analysis of numerous potential targets is becoming a challenge in terms of work load, tissue availability as well as costs. The molecular analysis of non-small cell lung cancer (NSCLC) has shifted from the analysis of the EGFR mutation status to the analysis of different gene regions including also resistance mutations or translocations. Massive parallel sequencing allows rapid comprehensive mutation testing in routine molecular pathological diagnostics even on small formalin-fixed, paraffin-embedded (FFPE) biopsies. This study aims to compare and evaluate current parallel sequencing platforms for their usage in routine diagnostics.

Methods. A cohort of 30 FFPE NSCLC samples was assembled by the institutes of Cologne (C), Erlangen (E) and Heidelberg (H). Tumor areas were marked on $\mathrm{H} \& \mathrm{E}$ stained sections by experienced pathologists in each institute. After macrodissection, DNA was extracted with the BioRobot M48 (C), the QIAsymphony (H) and manually with spin columns (E) according to the manufacturer's guidelines. Sequencing libraries were prepared by focused multiplex PCR using center-specific gene panels. Massive parallel sequencing was carried out by using three next generation sequencing systems: MiSeq (Illumina, C), GS Junior (Roche, E) and the PGM Ion Torrent (Life Technologies, H). In two institutes data analysis was performed with the platform specific software $(\mathrm{E}, \mathrm{H})$ and the integrative genome viewer. In Cologne, data analysis was carried out with an in-house software system.

Results. DNA from 30 NSCLC samples was extracted with three different DNA extraction systems and analysed on three different parallel sequencing platforms. 26 out of 30 samples were analysable by all institutes. Concerning the mutation status, we found concordance in 26 out of 26 samples. In two samples minor variants could be detected. Few samples were not analysable due to poor DNA quality in alternating institutes. One small tissue sample was not analysable at all. Characteristic nucleotide transitions could be identified as fixation artefacts by reanalysing samples with Sanger sequencing.

Conclusions. This study showed good concordance in the detection of mutations with different parallel sequencing platforms. Failure of samples can be traced back to different DNA extraction systems and DNA quality. Exact measuring of DNA concentration enhances the feasibility of parallel sequencing. Further analysis is needed regarding the validation of minor variants. 


\section{SA-074}

Simultaneous analysis of microsatellite stability and somatic mutations in colorectal cancer using Illumina's terminator-based sequencing

\section{Kloth*1, K. König', L. Ozretic', C. Vollbrecht', M. Odenthal', J. Fassunke', S. Merkelbach-Bruse', T. Zander', L. Heukamp', R. Büttner' \\ 'Institute of Pathology, University Hospital, Cologne, Germany, ${ }^{2}$ Department I of Internal Medicine, University Hospital Cologne, Cologne, Germany}

Aims. The recent advances in the molecular characterization of colorectal cancer (CRC) and associated therapeutic regimes have led to a substantial increase in diagnostic tests. This tendency is particularly noticeable in the case of extended RAS diagnostics predicting the response to EGFR-targeted therapy. Beyond that, the genomic landscape of CRC is essentially defined by two distinct phenotypes harboring different mutational profiles-cancers characterized by microsatellite instability (MSI) in contrast to microsatellite stability (MSS). While hereditary non-polyposis colorectal cancer (HNPCC) could be suspected by taking into account the revised Bethesda criteria, the initiation of further analyses in sporadic MSI relies on histological appearance. To facilitate the diagnostic process of colorectal cancers, we intended to develop a diagnostic approach for the combined analysis of microsatellite stability and somatic mutations.

Methods. We designed a multiplex PCR AmpliSeq custom panel (Life Technologies) for therapeutically relevant somatic mutations and five quasi-monomorphic mononucleotide repeats (MNR). Multiplex PCR was performed with DNA extracted from FFPE material of MSI-H, MSI-L and corresponding normal tissues as well as stable colorectal cancers. To reduce the high sequencing error rate in areas of homopolymers we used Illumina's terminator-based sequencing, in which just a single nucleotide is incorporated at the same time.

Results. Terminator-based sequencing of quasi-monomorphic MNRs can be used to determine the microsatellite stability in multiplex PCR approaches with a high sensitivity and specificity. The mean read coverage of each investigated MNR marker was higher than 10oo. By defining cut-off values in the allelic size distribution of the investigated markers in normal tissue, we were able to determine the MSI status without the need for corresponding normal tissue. Cut-off values were validated in a cohort of 100 samples with known MSI status. Detected mutations could be validated by Sanger sequencing.

Conclusions. Our study confirms the suitability of quasi-monomorphic MNRs in the combined analysis of microsatellite stability and somatic mutations by multiplex PCR and subsequent terminator-based paralle sequencing. This approach can facilitate the diagnostic process of CRC, but also of other cancers prone to microsatellite instability, e.g. endometrial cancer.

\section{SA-075}

Impact of routinely employed procedures for tissue processing on the proteomic analysis of formalin-fixed paraffin-embedded tissue

\section{P. Bronsert ${ }^{* 1,2}$, J. Weißer ${ }^{3}$, M. Kühs', V. Drendel', S. Timme', S. Küsters', U. Wellner ${ }^{5}$, S. Lassmann', M. Werner', O. Schilling ${ }^{6}$}

'Department of Pathology, Universtity Medical Center Freiburg, Freiburg, Germany, ${ }^{2}$ Department Pathology, Universtity Medical Center Freiburg, Freiburg, Germany, ${ }^{3}$ Research Center for Molecular Medicine of the Austrian Academy of Sciences, Vienna, Austria, ${ }^{4}$ Department of Surgery, Clinic for General and Visceral Surgery, University Medical Center Freiburg, Freiburg, Germany, ${ }^{5} \mathrm{Clinic}$ for Surgery, University Clinic of Schleswig-Holstein Campus Lübeck, Lübeck, Germany, ${ }^{6}$ Institute of Molecular Medicine and Cell Research, Albert-Ludwigs-University Freiburg, Freiburg, Germany

Aims. Proteomic analysis of formalin fixed, paraffin embedded (FFPE) tissues is gaining increasing interest for the in-depth analysis of ab- errant proteome composition in disease processes. For FFPE tissues, different processing procedures are often used in an intermittent and loosely documented manner. We have used mass-spectrometry based proteomic analysis of three differentially processed FFPE and one cryopreserved tumor samples to investigate the impact of tissue processing techniques on the quality of the proteomic analysis. We focused on three processing systems (xylene based, ethanol/vacuum based or microwave based), which are frequently used in European institutes of pathology.

Methods. In our study, specimens of identical tissue resections were processed simultaneously with the four methods outlined above. Tissue specimens of each patient were serially sliced, processed with the aforementioned techniques, and subjected to mass-spectrometry based proteomic analysis. The results were compared to corresponding cryopreserved tissue samples.

Results. All processing techniques achieved very good proteome coverage similar to the cryopreserved counterpart. Gene ontology profiles and peptide modifications such as methionine oxidation or proteolytic truncation were highly similar for all techniques as well as for the cryopreserved samples. No significant differences, also in relation to cryopreserved samples, were detected with regard to peptide identification rates and composition, methionine oxidation, lysine unblocking, and proteolytic processing.

Conclusions. Our results strongly underline robustness of proteomic analysis of FFPE tissues and highlights that different tissue processing techniques yield equivalent proteome coverage upon proteomic analysis. The study is of particular relevance since different processing techniques are often used in parallel and without detailed documentation.

\section{SA-076}

Analysis of intratumoral genomic heterogeneity of breast cancer reveals clonal populations with lower levels of aberrations but a high capacity to progress and metastasize

\section{Ruiz ${ }^{* 1}$, J. Gsponer', V. Perrina', A. Krause', A. Rufle', T. Lorber', D. Juskevicius', S. Eppenberger', E. Obermann', E. Obermann', L. Terracciano', M. Barrett' ${ }^{2}$, L. Bubendorf'}

'Institute of Pathology, University Hospital Basel, Basel, Switzerland, ${ }^{2}$ The Translational Genomics Research Institute (TGen), Phoenix, United States

Aims. Breast cancers, as well as many other tumor entities, are composed of different clonal tumor populations that have evolved during the progression of the tumor. The presence of these populations in a tumor provides the basis for the existence of intratumoral heterogeneity. Aim of this study was the detection and genomic analysis of clonal tumor populations from patients with breast cancer in order to infer their clonal evolution and to determine the population responsible for progression and metastasis.

Methods. Eight breast cancer patients with multiple matched tumor biopsies (either primary/recurrence and/or primary/metastasis) were selected. Clonal tumor populations were sorted based on their DNA content by usage of the BD Influx FACS. DNA from sorted populations was subjected to Array-CGH analysis and next-generation sequencing by usage of the Agilent microarray platform and the Ion Torrent PGM system, respectively.

Results. Six out of the eight analyzed primary breast tumors consisted of multiple clonal tumor populations. A diploid or near-diploid tumor population was detected in all analyzed tissue samples. The different clonal tumor populations were characterized by shared as well as by population-specific genomic aberrations. Interestingly, despite the long time intervals between the biopsies of up to 12 years, the (near-)diploid tumor populations were characterized by a rather stable genome that did not undergo larger genomic changes over time.

Conclusions. Our data provides further evidence that the (near-)diploid tumor populations that are found in the primary tumor and that are characterized by a less aberrant genome have a stronger capability for 
progression and metastasis. In the era of personalized cancer medicine, in which genomic aberrations are more and more predictive for specific treatments, the detection and analysis of these (near-) diploid tumor cells is of utmost importance. These cells are often outnumbered in the primary tumor by a large number of aneuploid cells and thus may be overlooked if non-sorted or non-enriched bulk tumor biopsies are used for genomic profiling.

\section{SA-077 \\ Improved PCR performance using DNA from formalin-fixed and paraffin-embedded tissues by overcoming PCR inhibition}

\section{Dietrich, B. Uh'*, V. Sailer, E.E. Holmes, M. Jung, S. Meller, G. Kristiansen Institute of Pathology, University Hospital Bonn, Bonn, Germany}

Aims. Formalin-fixed and paraffin-embedded (FFPE) tissues is a valuable source for genetic and epigenetic studies. Since the fixation process degrades nucleic acids, PCR robustness, which is mandatory for the clinical utility of genetic and epigenetic biomarkers, is either impaired or limited to short amplicons ( $300 \mathrm{bp})$. Consequently, methods to improve PCR performance using nucleic acids from FFPE tissue are necessary Methods. In this study, the effects of genomic and bisulfite-converted DNA derived from FFPE tissues on PCR performance was investigated employing qPCR and conventional PCR.

Results. We found that poor PCR amplification was partly caused by inhibition of the DNA polymerase by fragmented DNA from FFPE tissue and not only due to the absence of sufficient intact template molecules. The adoptions of the PCR condition (increased concentration of polymerase and dNTP, extended PCR elongation time) successfully minimized the inhibition and results in a robust amplification of up to $600 \mathrm{bp}$ amplicons. This observation was valid for genomic DNA from FFPE tissue and bisulfite-converted DNA required for DNA methylation analyses.

Conclusions. In summary, PCR using DNA from FFPE tissue suffers from inhibition which can be reduced by adaptation of the PCR conditions, therefore allowing for a significant improvement of PCR performance with regard to variability and the generation of larger amplicons. To overcome this hitherto unknown PCR inhibition is of tremendous value for FFPE based research.

\section{SA-078 \\ Scale up of next generation sequencing sample preparation by automation}

\section{Vollbrecht*, U. Koitzsch, K. König, M. Kloth, R. Büttner, M. Odenthal} Institute of Pathology, University Hospital, Cologne, Germany

Aims. Next Generation Sequencing (NGS) gains more importance in the different fields of research and also in the diagnostics. The great potential of the NGS systems opens up the way to a personalized therapy not only because mutations present in a small clonal subset can be detected, but it also can help to divide patients into individual risk profiles or biological subgroups so that they can be treated according to their mutational status. A lot of different NGS sample preparation protocols exist and even if they accelerate the sample processing by the opportunity of sample multiplexing, they mainly consist of many single time consuming, error-prone steps. Protocols include pipetting of small volumes and many samples as well as a lot of incubation steps. Automation of the sample preparation would help to decrease the risk of errors and can save time. Our aim was to automate most of the sample preparation steps for a current NGS technology namely MiSeq (Illumina) sequencing method.

Methods. Three different NGS sample preparation protocols (Qiagen Multiplex PCR + NEBNext, Qiagen GeneRead Panels + NEBNext, AmpliSeq + NEXTflex) were carried out with a Biomek FXP (Beckamn
Coulter) system. All protocols included diverse steps of magnetic bead based purification and size selection of DNA. Each procedure was performed with three up to 96 samples. Sample preparation with manual handling served as a control. Quality check of the steps was done with Bioanalyzer 2100 (Agilent Technologies). Finally samples were sequenced with MiSeq instrument (Illumina).

Results. Automated sample preparation showed no significant difference between sample quality and quantity. Each sample either prepared by manual handling or by automated process showed good sequencing results.

Conclusions. Automated sample processing for NGS helps to avoid errors that can occur due to manual handling. It can help to scale up the sample preparation by processing up to 96 samples, simultaneously, thereby accelerating the sample NGS preparation. In addition it provides the opportunity of sample tracking and an improved process control, resulting in a powerful and efficient tool for eclectic approaches in future molecular pathology.

\section{SA-079}

Loss of centrosome integrity during mammalian cardiomyocyte terminal differentiation

\section{D.C. Zebrowski ${ }^{1,2}$, F. Ricciardi', F.B. Engel*2}

'Max Planck Institut for Heart and Lung Research, Bad Nauheim, Germany, ${ }^{2}$ Institute of Pathology, Department of Nephropathology, University Hospital Erlangen, Erlangen, Germany

Aims. In contrast to cardiomyocytes of adult mammals, cardiomyocytes of adult zebrafish and newt are capable of proliferation and regeneration. The mechanism underlying loss of proliferative-competence in mammalian cardiomyocytes, and what molecular phenotype distinguishes mammalian cardiomyocytes from those of zebrafish and newt, is unknown.

Methods. One important regulator of cell cycle progression, differentiation, and genomic stability is the centrosome. The centrosome, a solitary organelle in resting animal cells, consists of two closely paired centrioles embedded in the pericentriolar matrix. During S-phase, the centrosome duplicates whereby a single daughter centriole forms from each parental centriole. At $\mathrm{G}_{2} / \mathrm{M}$, parental centrioles disassociate from one another, thereby facilitating the formation of a bipolar mitotic spindle. Upon cytokinesis, each daughter cell receives a single centrosome. Traditionally, the centrosome is known as the primary microtubule organizing center in animal cells where, in addition to promoting the formation of a mitotic spindle, defines cytoskeletal architecture. Recently, the centrosome has been shown act as a scaffold for a number of cell-cycle regulatory proteins. Consistent with this role, loss of centrosome integrity results in various cell cycle perturbations. Therefore, we have here investigated the centrosome integrity in cardiomyocytes from rats, zebrafish and newts based on immunofluorescence stainings.

Results. Our results indicate that rat neonatal cardiomyocytes undergo precocious loss of centriole cohesion (i.e. prior to $\mathrm{G} 2 / \mathrm{M}$ ) before they enter their final cell cycle and that centriole cohesion is thereafter not restored. The absence of centriole cohesion was associated with p38-mediated Go/G1 cell cycle arrest. Suppression of p38 activity permitted cell cycle progression, however, this correlated with mitotic abnormalities. Underlying the loss of centriole cohesion was a reassignment of centriole-cohesion proteins $\mathrm{CDK}_{5} \mathrm{RAP}_{2}$ and Pericentrin as well as the microtubule organizing center to the nuclear envelope. In contrast to adult rat cardiomyocytes, centriole cohesion was maintained in adult zebrafish and newt cardiomyocytes.

Conclusions. These findings implicate centrosome integrity as a critical component for cardiomyocyte proliferative and regenerative capacity. 


\section{SA-080}

Different micro-RNA expression profiles distinguish subtypes of neuroendocrine tumours of the lung: results of a profiling study

F. Mairinger*1,2, S. Ting', R. Werner', R.F.H. Walter ${ }^{3}$, T. Hager', C. Vollbrecht', D. Christoph ${ }^{5}$, K. Worm' ${ }^{1}$, T. Mairinger6, S.-Y. Sheu-Grabellus', D. Theegarten', K.W. Schmid', J. Wohlschlaeger ${ }^{1}$

'Institute of Pathology, University Hospital Essen, University of DuisburgEssen, Essen, Germany, ${ }^{2}$ Institut for Pathology, University Hospital Essen, University of Duisburg-Essen, Essen, Germany, ${ }^{3}$ Ruhrlandklinik, University Hospital Essen, University of Duisburg-Essen, Essen, Germany, ${ }^{4}$ Institute of Pathology, University Hospital, Cologne, Germany, ${ }^{5}$ Department of medical Oncology, University Hospital Essen, University of Duisburg-Essen, Essen, Germany, ${ }^{6}$ Department of Pathology, Helios Klinikum Emil von Behring, Berlin, Germany

Aims. MicroRNAs (miRNAs) are a class of small (approx. 22 nucleotides), non-coding, highly conserved single-stranded RNAs with posttranscriptional regulatory features including the regulation of cell proliferation, differentiation, survival and apoptosis. They are deregulated in a broad variety of tumours showing characteristic expression patterns and can thus be used as a diagnostic tool. In contrast to nonsmall cell carcinoma (NSCC) of the lung neuroendocrine lung tumours, encompassing typical and atypical carcinoids (TC/AC), small cell lung cancer (SCLC) and large cell neuroendocrine lung cancer (LCNELC), no data about deregulation of tumour entity specific miRNAs are available to date. miRNA expression differences might give useful information about the biological characteristics of these tumour as well as serve as helpful markers.

Methods. In 12 pulmonary neuroendocrine tumours classified as either TC, AC, LCNELC, or SCLC, screening for 763 miRNAs known to be involved in pulmonary cancerogenesis was conducted by performing 384 well TaqMan low-density array real-time qPCR.

Results. In the entire cohort, 44 miRNAs were identified which showed a significantly different miRNA expression. For 11 miRNAs the difference was highly significant $(\mathrm{p}<0.01)$. Seven miRNAs showed a negative (miR-22, miR-29a, miR29b, miR-29c, miR-367*; miR-504, miR-513C, miR-120o) and four miRNAs a positive (miR-18a; miR-15 $\mathrm{b}^{*}, \mathrm{miR}-335^{*}$, miR-1201) correlation to the grade of tumour biology. The miRNAs let7d; miR-19; miR-576-5p; miR-340*; miR-1286 are significantly associated with survival. Members of the miR-29 family seem to be extremely important in this group of tumours.

Conclusions. We found a number of miRNAs which showed a highly significant deregulation in pulmonary neuroendocrine tumours. Moreover some of these deregulated miRNAs seem to a allow discrimination of the various subtypes of pulmonary neuroendocrine tumours. Thus the analysis of specific sets of miRNAs can be proposed as diagnostic and/or predictive markers in this group of neoplasias.

\section{SA-081 \\ Clinicopathological and molecular features of GIST of the oesopha- geal region}

S. Huss ${ }^{* 1}$, W. Hartmann', H. Künstlinger' ${ }^{2}$, M.A. Ihle' ${ }^{2}$, S. Merkelbach-Bruse', M. Trautmann', I. Grünewald', F. Wötzel', J. Zustin', R. Büttner', H.-U. Schildhaus ${ }^{3}$, E. Wardelmann

${ }^{1}$ Gerhard-Domagk-Institute for Pathology, University Hospital Münster, Münster, Germany, ${ }^{2}$ Institute of Pathology, University Hospital, Cologne, Germany, ${ }^{3}$ Institute of Cellular and Molecular Immunology, Georg August University of Göttingen, Göttingen, Germany

Aims. Gastrointestinal stromal tumors (GISTs) are the most common mesenchymal tumors of the gastrointestinal tract. Most tumors are driven by ontogenetic KIT or PDGFRA mutations. In 2002 a risk classification was introduced by Fletcher et al. based on size and mitotic count. The current risk classification (according to Miettinen et al. 2006) addi- tionally takes localization into consideration. However, for oesophageal GIST, no such risk classification could yet be established so far, due to their very low incidence. We therefore were encouraged to report on a series of GISTs situated in the oesophageal region that were diagnosed in our referral center.

Methods. We screened our GIST and Sarcoma Registry Münster (formerly Cologne/Bonn) for GIST in the oesophageal region. Clinicopathological characteristics and molecular data were collected.

Results. Altogether, 27 cases were identified. There was no sex predominance (14 male vs. 13 female patients). Mean tumor size was $5.7 \mathrm{~cm}$ (range: $0.2-12.0 \mathrm{~cm}$; median: $6.0 \mathrm{~cm}$ ). Mitotic count ranged from $0-26$ per 50 HPFs or $5 \mathrm{~mm} 2$ (median: 11, mean: 8). 17 cases displayed a spindled cell morphology and 4 exhibited an epitheloid phenotype. One case showed mixed cell morphology. Among 17 cases with available data, 7 cases were classified as being at intermediate and 5 cases at high risk of progressive disease according to Fletcher (2002) which was used because Miettinen classification was not applicable. 5 cases belonged to the very low/ low categories. In 17 cases a mutation in KIT exon 11 was found, predominantly involving codons 557 and $558(\mathrm{n}=10)$.

Conclusions. We report on a large and unique cohort of patients with gastrointestinal stromal tumors of the oesophageal region. These tumors predominantly displayed a spindled phenotype, showed an intermediate/high risk of progressive disease [according to Fletcher (2002)] and revealed a KIT exon 11 mutation (mostly involving codons 557 and 558). According to these data the majority of oesophageal GIST has obviously a more aggressive phenotype than gastric GIST.

\section{SA-082}

\section{The prognostic value of BRAF mutations in a cohort of wild-type} GISTs

S. Huss" ${ }^{* 1}$, H. Künstlinger ${ }^{2}$, M.A. Ihle', S. Merkelbach-Bruse ${ }^{2}$, I. Grünewald', W. Hartmann ${ }^{1}$, R. Büttner ${ }^{2}$, E. Wardelmann ${ }^{1}$, H.-U. Schildhaus ${ }^{3}$

'Gerhard-Domagk-Institute for Pathology, University Hospital Münster, Münster, Germany, Institute of Pathology, University Hospital, Cologne, Germany, ${ }^{3}$ Institute of Cellular and Molecular Immunology, Georg August University of Göttingen, Göttingen, Germany

Aims. Gastrointestinal stromal tumors (GISTs) are the most common mesenchymal tumors of the gastrointestinal tract. The majority is driven by oncogenic mutations in KIT or PDGFRA. However, about $10-15 \%$ of GISTs show wild-type sequences in KIT exons 8, 9, 11, 13 and 17 , as well as in PDGFRA exons 12, 14 and 18. They are referred to as "wild-type GISTs" (wt-GISTs). wt-GISTs are a heterogeneous group of GISTS of which some are affected by BRAF muations. In this study we therefore aimed at analyzing a large cohort of wt-GISTs for BRAF mutations to update the knowledge about a possible genotype-phenotype correlation.

Methods. More than 3,00o GIST samples from Caucasian patients were available in the GIST and Sarcoma Registry Münster (formerly Cologne/Bonn). Out of these, we selected 172 wild-type GISTs to evaluate the frequency of BRAF mutations by means of high resolution melting (HRM) analysis and subsequent Sanger sequencing.

Results. BRAF mutations were found molecularly in 7 cases (3.9\%) of our wt-GIST cohort. In two cases with multiple tumors all tumors harbored the same mutation. Concerning clinicopathological parameters, 2 GISTs occurred in the stomach, 4 were localized in the small bowel and one in the rectum. The GIST patients' mean age was 57.4 years (median: 60.0 years, range: $36-69$ years). Gender was known in 5 cases (ratio: 3 male $/ 2$ female). The size of the tumors ranged from $1.0-8.3 \mathrm{~cm}$ in diameter (mean: $3.9 \mathrm{~cm}$, median: $4.0 \mathrm{~cm}$ ). Mitotic count ranged from o to 2 (median: o, mean: o.6). According to Miettinen et al., the risk classification was as follows: no risk: 3 ; very low risk: o; low risk: 3 ; intermediate risk: 1; high risk. o.

Conclusions. In our wt-GIST cohort $(n=172)$, only $3.9 \%$ of the cases investigated were BRAF p.V6ooE mutated. This frequency is in the lower 
range of previously reported series in the literature (2-13\%). In our series, all but one GIST were classified as being at no/very low or low risk for progression. This could lead to the hypothesis of a "benign" phenotype of BRAF mutated cases. However, in the current literature the majority of BRAF mutated GISTs was reported as being at intermediate or high risk of progression. Therefore the question of a prognostic value of BRAF mutations in GISTs seems to remain open.

\section{SA-083}

Flow-sorting and genomic profiling of pure clonal tumor populations from fresh-frozen (FF) and from formalin fixed and paraffinembedded (FFPE) tissue specimens

\section{S. Rau*1, T. Lorber', V. Perina', D. Juskevicius', J. Gsponer', M. Rämö', A. Rufle', M. Barrett' ${ }^{2}$, C. Ruiz', L. Bubendorf' \\ 'Institute of Pathology, University Hospital, Basel, Switzerland, ${ }^{2}$ The Translational Genomics Research Institute (TGEN), Scottsdale, Arizona, United States}

Aims. Recent studies have suggested that human tumors are composed of different clonal populations and that their presence is reflected as intratumoral heterogeneity. Whereas most of the genomic studies are performed with fresh-frozen material, the vast majority of the biopsies are archived as formalin fixed and paraffin embedded (FFPE) tissue blocks. Aim of this study was to establish of an approach that allows for the detection and separation of clonal tumor populations from FFPE biopsies in order to subject pure tumor DNA of the sorted populations to genomic profiling.

Methods. Scrolls from FFPE biopsies from malignant melanoma were de-waxed and subjected to nuclei extraction by enzymatic digestion. Extracted nuclei were stained with DAPI and in addition, with an antibody against a specific tumor marker highly expressed in the tissue of interest, such as Sox1o for melanoma. Stained nuclei were sorted according to their DNA content (DAPI) as well as to the positivity of the selected tumor marker by usage of the BD Influx FACS equipment. DNA from sorted populations was subjected to genomic profiling by means of Array-CGH. Data resulting from the FACS and the CGH microarrays were analyzed with the FlowJo and the DNA Genomic Workbench software, respectively.

Results. We were able to detect and sort distinct clonal populations based on DNA content. As expected, detected aneuploid populations were positive for the expression of the tumor marker used (Sox10), but the diploid population consisted of Soxı positive and negative cells. Application of sorted subpopulations to CGH microarrays revealed that the diploid cells not expressing Sox1o were normal by means of ArrayCGH. Only the diploid Soxio positive as well as the aneuploid cells were characterized by genomic aberrations.

Conclusions. In this study, we show that flow-sorting of clonal tumor populations cannot only be used for the analysis of intratumoral genomic heterogeneity, but by including a second parameter, it might be used for the stratification of diploid cells into normal cells and tumor cells. This approach allows for a robust separation of diploid tumor cells even if the biopsies are heavily admixed with normal cells. Further, the sorted normal cells (diploid and tumor marker negative) may be used as internal ploidy control and even as potential reference material (germline) for next-generation sequencing studies.

\section{SA-084}

Mutations in polymerase $\varepsilon$ per se as key molecular alteration of the novel ultramutator phenotype do not define a clinically distinct entity of colorectal cancer

N. Pfarr*', A. Stenzinger', V. Endris', R. Penzel', L. Jansen', E. Herpel', A. Warth', F. Klauschen ${ }^{3}$, W. Roth', H. Bläker', J. Chang-Claude ${ }^{4}$, H. Brenner ${ }^{2,5}$, M. Hoffmeister ${ }^{2}$, W. Weichert ${ }^{1,5,6}$

'Institute of Pathology, Ruprecht-Karls-University, Heidelberg, Germany, ${ }^{2}$ Division of Clinical Epidemiology and Aging Research, German Cancer Research Center (DKFZ), Heidelberg, Germany, ${ }^{3}$ Institute of Pathology, Charité University Hospital, Berlin, Germany, ${ }^{4}$ Unit of Genetic Epidemiology, German Cancer Research Center (DKFZ), Heidelberg, Germany, ${ }^{5}$ German Consortium for Translational Cancer Research (DKTK), Heidelberg, Germany, ${ }^{6}$ National Center for Tumor Diseases (NCT), Heidelberg, Germany

Aims. Recent molecular profiling studies reported a new class of ultramutated colorectal cancers (CRC), which are likely caused by exonuclease domain (END) mutations in DNA polymerase $\varepsilon$ (POLE). However, data on the clinical implications of these findings as to whether these mutations in analogy to endometrial cancer define a unique CRC entity with distinct clinical outcome is entirely lacking.

Methods. We performed Sanger sequencing of the POLE END in 431 well annotated microsatellite stable (MSS) CRCs of a population-based epidemiological case-control study. Mutation data were correlated with major epidemiological, clinical, genetic and pathological parameters including overall and disease-specific survival.

Results. In 373 out of 431 MSS CRC all exons of POLE END were analyzable. 47 mutations were identified in 41 of these samples (11\%). Besides already reported mutations, we found many new mutations in exons 13 and 14 (corresponding to codons 411-491) as well as in exon 9 (corresponding to codons $268-301$ ). However, we found no associations of POLE END mutations with clinicopathological parameters, including sex, age, tumor location and tumor stage, CIMP, RAS and BRAF mutations. Survival analysis revealed adjusted (for sex, age, tumor location and therapy) HRs of 1.38 and 1.44 for disease-specific and overall survival indicating slightly impaired survival of patients with mutations in POLE END. However, these differences were not statistically significant. This was also true for subgroups of mutations.

Conclusions. In contrast to endometrial cancer, somatic POLE mutations do not appear to define a clinically strongly distinct disease entity in MS-stable CRC.

\section{SA-085}

\section{SRC represents a potential therapeutic target in liposarcoma}

E. Sievers ${ }^{* 1}$, M. Trautmann ${ }^{2}$, D. Kindler ${ }^{2}$, I. Grünewald ${ }^{3}$, S. Huss ${ }^{3}$, F. Pedeutour', P. Aman ${ }^{5}$, J. Nishio ${ }^{6}$, M. Renner', G. Mechtersheimer', E. Wardelmann ${ }^{3}$, R. Büttner', W. Hartmann ${ }^{3}$

'Institute of Pathology, University Hospital Cologne, Cologne, Germany, ${ }^{2}$ Institute of Pathology, University Hospital, Cologne, Germany, ${ }^{3}$ Institute of Pathology, University Medical Center, Münster, Germany, ${ }^{4}$ University Hospital Nice, Nice, France, ${ }^{5}$ Institute for Pathology, Göteborg University, Göteborg, Sweden, ${ }^{6}$ Faculty of Medicine, Fukuoka University, Fukuoka, Japan, ${ }^{7}$ Institute of Pathology, Ruprecht-Karls-University, Heidelberg, Germany

Aims. Liposarcomas (LS) represent the most common malignant soft tissue tumors in adults. Therapeutic outcome of LS is mainly determined by the efficiency of surgery as a high tendency for local relapse is seen. Chemo- and radiotherapy represent further therapeutic options, however, specifically targeted therapies are currently not available. The oncoprotein SRC is a tyrosine kinase, which has been shown to be activated in a variety of human cancers. SRC activation plays an important role in cancer cell proliferation, survival, angiogenesis and motility. In this work we analyzed LS biopsies comprising the main different subgroups, i.e. myxoid (MLS), well/dedifferentiated (WDLS/DDLS) and 
pleomorphic (PLS) LS and corresponding cell lines regarding SRC activation and studied the biological effects of SRC inhibition.

Methods. Based on data derived from phospho-kinase screens in LS cells, immunhistochemical stainings of (Tyr416)-phosphorylated (p)SRC were performed in primary LS biopsies. LS cell lines treated with the SRC inhibitor dasatinib were analyzed for phosphorylation of SRC and its downstream signaling molecules, cell proliferation, migration and apoptosis. Furthermore, synergistic effects of simultaneous treatments with dasatinib and conventional cytotoxic drugs were investigated.

Results. Particularly WDLS/DDLS and MLS tumor cells showed a significant expression of p-SRC. In LS cell lines, inhibition of SRC with dasatinib resulted in a substantially impaired cellular growth accompanied by a decreased phosphorylation of SRC and its downstream targets. Flow cytometric analysis showed that effects of SRC inhibition were due to an increase in apoptosis [cleaved PARP(Asp 214)] and a decrease of cellular proliferation [phospho-(Ser1o) Histone $\mathrm{H}_{3}$ ]. Scratch and Boyden chamber assays indicated a diminished migratory potential of the tumor cells due to SRC inactivation. Simultaneous treatment of liposarcoma cell lines with dasatinib and chemotherapeutic drugs resulted in additive effects.

Conclusions. Our data suggest that SRC kinase activation represents an important biological feature of LS which might be successfully addressed in targeted therapeutic approaches.

\section{SA-086}

\section{WDFY-1/FENS-1-a new player in the regulation of autophagy}

M. Muders ${ }^{* 1}$, S. Dutta ${ }^{2}$, P. Hönscheid', S. Haberlau' ${ }^{3}$ S. Schellenburg ${ }^{4}$, K. Datta ${ }^{5}$, G. Baretton

'Institute of Pathology of University Hospital Carl Gustav Carus, Medical Center TU Dresden, Dresden, Germany, ${ }^{2}$ Institute of Biochemistry, Nebraska Medical Center, Omaha, NE, United States, ${ }^{3}$ Radiation Oncology/Oncoray, University Hospital Carl Gustav Carus, Dresden, Germany, ${ }^{4}$ Institute of Pathology of University Hospital Carl Gustav Carus, Dresden, Germany, ${ }^{5}$ Department of Biochemistry, University of Nebraska Medical Center, Omaha, United States

Aims. We have already shown that Neuropilin-2 (NRP-2) and the vascular endothelial growth factor-C (VEGF-C) regulate autophagy and chemotherapy resistance in different cancer types (Muders et al.; Cancer Res 2009; Stanton et al., Cancer Res 2013). In gene array analysis we characterize the protein WDFY-1/FENS-1 as a downstream target of this signaling axis. In this study, we characterize the role of WDFY-1/ FENS-1 in regulating NRP-2 induced autophagy.

Methods. Affymetrix gene array analysis was used to characterize proteins that are deregulated after depletion of NRP-2 and VEGF-C. WDFY-1/FENS-1 regulation by $\mathrm{NRP}_{2}$ and VEGF-C was confirmed by qRT-PCR and Western Blot in different cancer types like prostate cancer and pancreatic cancer. Functional assays like fluorescence microscopy and competitive binding assays were executed to study the function of WDFY-1/FENS-1 in lysosome trafficking.

Results. WDFY-1/FENS-1 is upregulated after depletion of NRP-2 or VEGF-C in prostate and pancreatic cancer. The upregulation of WDFY1/FENS-1 reduces Docetaxel and Gemcitabine resistance induced by NRP2. One of the mechanisms is the inhibition of autophagy by WDFY1/FENS-1. This inhibition is caused by deregulated maturation of endosomes and lysosomes which results from the reduced efficiency of the proteins important for the transformation of early to late endosomes that are blocked by WDFY-1/FENS-1.

Conclusions. WDFY-1/FENS-1 is a poorly characterized protein that is regulated by NRP-2 and VEGF-C. It is important for the regulation of autophagy. It affects the maturation of lysosomes by competing with proteins important for the transition of early to late endosomes and therefore blocks the formation of mature lysosomes.

\section{SA-087}

Detection of rearrangements and transcriptional up-regulation of ALK in FFPE lung cancer specimens using a novel sensitive quantitative reverse transcription PCR assay

K. Gruber ${ }^{* 1,2}$, H. Horn ${ }^{1,2}$, J. Kalla ${ }^{3}$, P. Fritz ${ }^{1,2}$, A. Rosenwald ${ }^{4}$, M. Kohlhäufl, G. Friedel $l^{5}$, M. Schwab, G. Ott ${ }^{1,2}$, C. Kalla, $a^{1,2}$

${ }^{1}$ Dr. Margarete Fischer-Bosch-Institute of Clinical Pharmacology, Stuttgart, Germany, ${ }^{2}$ Department of Clinical Pathology, Robert-Bosch-Krankenhaus, Stuttgart, Germany, ${ }^{3}$ Institute of Pathology, Schwarzwald-Baar-Klinikum, Villingen-Schwenningen, Germany, ${ }^{4}$ Institute of Pathology, University of Würzburg, Würzburg, Germany, ${ }^{5}$ Center for Pulmonology and Thoracic Surgery, Klinik Schillerhöhe, Stuttgart, Germany

Aims. The approved dual-color FISH test for the detection of ALK gene rearrangements in lung cancer (NSCLC) is complex and represents a low-throughput assay difficult to use in daily diagnostic practice. We devised a sensitive and robust routine diagnostic test for the detection of rearrangements and transcriptional upregulation of ALK.

Methods. We developed a quantitative RT-PCR assay adapted to RNA isolated from routine FFPE material and applied it to 652 NSCLC specimens. The reliability of this technique to detect ALK dysregulation was shown by comparison with FISH and immunohistochemistry.

Results. qRT-PCR analysis detected unbalanced ALK expression indicative of a gene rearrangement in $24(4.6 \%)$ and full-length ALK transcript expression in six (1.1\%) of 523 interpretable tumors. Among 182 tumors simultaneously analyzed by FISH and qRT-PCR, the latter accurately typed $97 \%$ of 19 rearranged and 158 nonrearranged tumors and identified ALK deregulation in two cases with insufficient FISH. Six tumors expressing full-length ALK transcripts did not show rearrangements of the gene. Immunohistochemistry detected ALK protein over-expression in tumors with gene fusions and transcriptional up-regulation, but did not distinguish between the two. One case with full-length ALK expression carried a heterozygous point mutation (S122oY) within the kinase domain potentially interfering with kinase activity and/or inhibitor binding.

Conclusions. Our qRT-PCR assay reliably identifies and distinguishes ALK rearrangements and full-length transcript expression in FFPE material. It is an easy-to-perform, cost-effective and high-throughput tool for the diagnosis of ALK activation. The expression of full-length ALK transcripts may be relevant for ALK inhibitor therapy in NSCLC.

\section{SA-088}

Anti-tumor activity of GABARAP-deficient mice treated with 7,12-Dimethylbenz(a)anthracene (DMBA)

\section{F.S. Salah ${ }^{* 1}$, M. Ebbinghaus ${ }^{2}$, R. Bräuer', I. Petersen'}

'Institute of Pathology, University Hospital, Friedrich-Schiller University, Jena, Germany, ${ }^{2}$ Institute of Physiology ', Friedrich-Schiller University, Jena, Germany

Aims. GABARAP (gamma-aminobutyric acid receptor associated protein) belongs to a protein family which is involved in the autophagy machinery. There is an increasing number of studies indicating a role of autophagy in tumour initiation and progression as well as DNA damage response (DDR). DMBA is a potent carcinogen by inducing DNA damage, and it is well known that DMBA-treatment leads to immunosuppression in mice by suppression of various cytokines which are playing an essential role in anti-tumor activity, for instance IL-2 and IFN. The importance of GABARAP and autophagy in anti-tumor immune response and DDR after treatment with a carcinogen (DMBA) is not clarified yet.

Methods. We treated the transgenic mice with DMBA to induce tumor. Peritoneal macrophages and splenic lymphocytes from GABARAP knockout $(\mathrm{KO})$ and wild-type $(\mathrm{Wt})$ mice treated with DMBA and from untreated control mice were isolated, cultured and stimulated. Cytoki- 
ne levels were determined by ELISA. The expression of surface markers was analyzed using Flow Cytometry in order to determine the population of splenocytes. Mouse embryonic fibroblasts (MEFs) are isolated from $\mathrm{KO}$ and Wt mice and cultured, and then treated with DMBA and CPT (camptothecin, a DNA double-strand break inducer) to investigate the DNA damage, cell cycle and apoptosis.

Results. GABARAP KO mice exhibited lower tumor formation compared with Wt mice. We found elevated levels of IL-1 $\beta$, TNF- $\alpha$, IL-6, IL-2 and IFN- $\gamma$ in GABARAP KO mice after treating with DMBA. These cytokines play an important role in anti-tumor activity and/or death, mediated by tissue injury and lethal shock. In contrast, Wt mice showed immunosuppression through decreased IL-2 production after treatment. Furthermore, DNA damage in KO MEFs accumulated in a timedependent manner, in contrast to Wt MEFs, and this triggered cell cycle arrest and apoptosis.

Conclusions. In our model, GABARAP deficiency inhibits tumor formation and activates the immune response, in addition to trigger cell death in vitro through impaired DDR. These results support a role of GABARAP in tumorigenesis. Moreover, the data improve the understanding of anti-tumor immune responses and its contribution to the therapeutic handling of neoplasia by regulation of cytokine secretion and autophagy. Otherwise DDR insufficiency could induce genomic instability resulting in tumorigenesis. However, extremely increased DNA damage may also induce cell death which is the rationale of using drugs like camptothecin in chemotherapy.

\section{SA-089 \\ A rare case of an ALK-positive lung adenocarcinoma missed by ALK-in situ-hybridization but detected by RT-PCR for EML4-ALK}

\section{A. Streubel ${ }^{*}$, A. Roth, S. Stephan-Falkenau, T. Mairinger, F. Wagner Institut für Gewebediagnostik/MVZ, Berlin, Germany}

Aims. Fluorescence in situ hybridization (FISH) for the anaplastic lymphoma kinase (ALK) gene is currently regarded as the gold standard to detect ALK break apart-positive lung carcinoma. A positive test is required for administration of the FDA-approved ALK-inhibitor Crizotinib. We report here a rare case of an ALK-positive lung adenocarcinoma missed by ALK-ISH (CISH and FISH) but detected by quantitative reverse transcription PCR (RT-PCR) and unambigously confirmed as EML4-ALK fusion variant 1 by Sanger sequencing.

Methods. A 52-year-old female patient presented with a large pleural effusion. A cytological specimen as well as several biopsies from the costal pleura were investigated morphologically and by standard immunohistochemistry and diagnosed as manifestation of a lung adenocarcinoma. Dual-colour CISH and FISH using break-apart probes for the ALK gene on 2 p 23 were conducted with $3 \mu \mathrm{m}$ paraffin sections. RT-PCR for the detection of EML4-ALK variants was carried out on the LightCycler 2.0 instrument following extraction of total RNA from a $20 \mu \mathrm{m}$ section of the FFPE tissue.

Results. CISH as well as FISH for detection of ALK break-apart events were clearly negative ( $3 \%$ of ALK positive cells). In contrast, RT-PCR was positive for EML4-ALK fusion variant 1 (exon 13 EML4; exon 20 ALK), which was unequivocally confirmed by Sanger sequencing. Conclusions. Although FISH is regarded as the gold standard to detect ALK-positive lung carcinoma, rare cases occur which are missed by this method. This is probably a result of both complex rearrangements and losses of genomic DNA camouflaging the break apart of the ALK gene.

\section{SA-090}

The specific $\mathrm{p} 38$ isoform induced gene expression panel in malign thymoma and thymic carcinoma

S. Küffer ${ }^{* 1}$, P. Krone ${ }^{2}$, V. Venkataramani', D. Belharazem², C. Sauer ${ }^{2}$, A. Marx², P. Ströbel'

'Institute of Pathology, University Medical Center Göttingen, Göttingen, Germany, ${ }^{2}$ Institute of Pathology, University Medical Center, Mannheim, Germany, ${ }^{3}$ Department of Hematology and Oncology, University Medical Center, Göttingen, Germany

Aims. Maligne tyhmoma (TH) and thymic carcinoma (TC) are rare tumours and therefore randomized studies and verified therapies are largely missing. Preliminary analysis of TH and TC showed an increased activity of the p38 family. Moreover, the relatively unknown p38 isoforms gamma and delta were specifically activated in a subgroup of $\mathrm{TH}$ and TC. Activation of the $\mathrm{p} 38$ pathway plays an important role in invasion and angiogenesis of tumors and might therefore be a promising new therapeutic target in TH and TC. However, genes activated by the single p38 isoforms are relatively unknown. In this study we investigate the downstream gene expression signature of the $\mathrm{p}_{3} 8$ isoforms in cell culture systems for a better characterization and potential therapeutic implementation in TH and TC.

Methods. p38 isoform activation analyses in TH/TC were performed by specific MAPK Phospho-Protein-Arrays. $\mathrm{P}_{3} 8$ expression and activation in cell lines were analyzed by Western Blot with isoform specific antibodies and qRT-PCR. For $\mathrm{p}_{3} 8$ isoform induction and activation the prostate cell lines $\mathrm{PC}_{3}, 22 \mathrm{RV} 1, \mathrm{DU}_{145}$ and $\mathrm{BPH} 1$ were incubated with 10 micro molar sodium arsenite for 24 hours. For transcriptome analysis (RNA-Seq) of $\mathrm{p}_{3} 8$ specific genes isoforms were knocked down with specific siRNAs during Arsenite stress induction. After 24h RNA was isolated using TRIZOL method and RNA was processed for sequencing on a HISEQ 2000.

Results. The analysis of MAPKs in $44 \mathrm{TH}$ und TC revealed a strong activation of at least one of the $\mathrm{p} 38$ isoforms in more than half of the cases. In more detail, in about one third of the samples when p 38 gamma is activated the other isoforms are mostly inactive. Arsenite stress induction in the prostate cancer cell lines $\mathrm{PC}_{3}, 22 \mathrm{RV}_{1}, \mathrm{DU}_{145}$ and $\mathrm{BPH} 1$ activated rather specifically p 38 alpha, beta, gamma and delta respectively. The comparisons of the $\mathrm{p} 38$ isoform specific siRNA knock down to ctrl. siRNA during arsenite stress induction revealed a distinct isoform induced gene panel.

Conclusions. The intrinsic expression of p38 isoforms, especially p38 alpha and gamma, in TH/TC indicate to be good therapeutic targets. The validation of the isoform specific gene panel in TH and TC will hopefully reveal a molecular sub-classification and estimate the potential impact of therapeutic treatment.

\section{SA-091}

Nuclear apoptosis repressor with caspase recruitment domain (ARC) overexpression is associated with poor survival in patients with metastatic head and neck squamous cell carcinoma (HNSCC)

\section{Tóth ${ }^{* 1}$, N. Reulen ${ }^{2}$, D. Köhler ${ }^{3}$, N. Stoecklein ${ }^{4}$, C. Sproll ${ }^{3}$}

'Institute of Pathology, University Hospital Cologne, Cologne, Germany, ${ }^{2}$ Biomaterialbank, Comprehensive Cancer Center Düsseldorf, University Cancer Center Düsseldorf, Düsseldorf, Germany, ${ }^{3}$ Department of Oral-, Maxillo- and Plastic Facial Surgery, University Clinic Düsseldorf, Düsseldorf, Germany, ${ }^{4}$ Department of General-, Visceral and Pediatric Surgery, University Clinic Düsseldorf, Düsseldorf, Germany

Aims. In many solid cancers, ARC-expression levels increase with grade and are associated with poor prognosis. ARC-overexpression thereby renders both radio- and chemoresistance to tumor cells. There is so far no publication about the expression and the potential prognostic role of ARC in HNSCC. We intended to investigate the expression pattern 
of ARC in association to p53 using TMA technology, and to correlate to prognostic parameters like overall and disease-free-survival (OS and DFS).

Methods. 54 patients ( 29 male/25 female, between ages of $47-95$ years) with node-positive HNSCC were enrolled in this study. Tissue microarray (TMA) blocks with $4.0 \mathrm{~mm}$ cores were designed. Normal mucosa, tumor tissue and lymph node metastases were punched. On routinely processed slides $\mathrm{p}_{53}$ and ARC immunostaining were performed. ARC staining was graded separately for tumor and metastasic tissue. Cytoplasmatic (four-grade scale) and nuclear (three-grade scale) ARC expression was also separately evaluated. Immunohistochemical and clinical follow-up data were statistically analysed (Software SPSS).

Results. Strong nuclear overexpression of p53 could be detected in 14 cases $(27 \%)$. In tumor tissue samples cytoplasmatic ARC overexpression (score 2 and 3 ) could be observed in 31 cases (59\%), whereas strong nuclear overexpression (score 2 -more than $75 \%$ of the nuclei are positive) was detected only in 8 cases (15\%). Nuclear expression of ARC was found in 38 cases (73\%). Cytoplasmatic ARC expression had no association with any histopathological or clinical data neither in tumor nor in metastasic tissue. However, nuclear ARC overexpression was significantly correlated with nuclear $\mathrm{p} 53$ overexpression in tumor samples $(\mathrm{p}=0.028)$, but not in lymph node metastasis. Regarding the nuclear ARC expression only in metastases a significant negative correlation could be observed with disease-free and overall survival (DFS, $\mathrm{p}=0.049$ and OS, $\mathrm{p}=0.047$, respectively).

Conclusions. We have observed an association between the expression of ARC and its negative regulator $\mathrm{p} 53$. Overexpression of $\mathrm{p} 53$ points to a functional loss of the protein upon which this loses its inhibitory action on ARC and therefore ARC will be upregulated. Consequently, upregulated ARC can inhibit p53. In our panel, nuclear ARC overexpression in lymph node metastasis is associated with poorer disease-free and overall survival among patients with metastatic HNSCC. The exact mechanism in which way ARC leads to worse prognosis should be elucidated in functional studies.

\section{SA-092}

Massive parallel sequencing of KIT kinase domain in primary and metastatic gastrointestinal stromal tumors (GISTs) for detection of low-frequent secondary resistance mutations

\section{N. Kumm*, A. Agaimy, E.A. Moskalev, A. Hartmann, F. Haller} Institute of Pathology, Friedrich-Alexander-University, Erlangen-Nuremberg, Germany

Aims. Gastrointestinal stromal tumors (GISTs) are the most common mesenchymal tumors of the gastrointestinal tract. Approximately $70 \%$ harbor activating mutations in the KIT gene, which is the basis for selective therapy with tyrosine kinase inhibitors. Although GISTs with KIT mutations respond well to selective TKI therapy, a majority of the tumors will acquire secondary resistance due to secondary mutations in the kinase domain of the KIT gene. However, the time to secondary therapy resistance varies between few months to several years. The aim of the current study was to evaluate if secondary resistance mutations can be found at low allele frequencies $(<5 \%)$ already in the tumor tissue before treatment. The detection of low frequent resistance mutations in a metastatic GIST would probably predict the time to secondary resistance, and could help to manage therapy regimen and follow-up.

Methods. Thirty GISTs were evaluated for mutations of the KIT kinase domain by massive parallel sequencing using a 454 Junior GS. The sensitivity of the assay was determined to be $1 \%$. Additionally, tissue from 10 metastases with acquired therapy resistance was analysed.

Results. There was $100 \%$ concordance between primary activating mutations between Sanger sequencing and massive parallel sequencing The observed allele frequencies varied between 40 and $80 \%$. Secondary mutations could be found in the metastases with clinical acquired therapy resistance. In contrast, no "low frequency" resistance mutations were found by massive parallel sequencing in the untreated primary tumors.

Conclusions. Although secondary resistance mutations occur under tyrosine kinase inhibitor therapy, they cannot be detected in the primary tumors before therapy. These mutations are either present at frequencies $<1 \%$, or occur de-novo under therapy. Radiologic evaluation remains the gold standard for the monitoring of progressive disease in GISTs undergoing tyrosine kinase inhibitor therapy.

\section{SA-093}

Evaluation of BRAF V600E mutation status by massive parallel sequencing and immunohistochemistry in different subtypes of thyroid carcinoma

\section{Kapsner*, E.A. Moskalev, A. Agaimy, A. Hartmann, F. Haller}

Institute of Pathology, Friedrich-Alexander-University, Erlangen-Nuremberg, Germany

Aims. Mutation rates of BRAF V6ooE in Papillary thyroid carcinoma (PTC) range between $28 \%$ and $77 \%$. This mutation can also be found in $25 \%$ of anaplastic thyroid carcinoma (ATC). We wanted to investigate the incidence of BRAF V6ooE mutations on PTC and ATC and rare subtypes of thyroid carcinoma. Furthermore, we examine the specificity and sensitivity of the BRAF V6ooE immunohistochemical (IHC) antibody staining, and if it could be used in diagnostic routine of thyroid carcinomas.

Methods. The BRAF mutation status was analyzed by massive parallel sequencing using a 454 GS Junior. Additionally, tissue microarrays were created from the tumor samples and stained with the BRAF V6ooEspecific antibody. Sequencing results were correlated with the immunostaining and clinic-pathological parameters.

Results. The cohort contained 196 cases of thyroid carcinomas (40 follicular thyroid carcinoma (FTC), 39 PTC, 29 PTC follicular variant (FVPTC), 14 medullary thyroid carcinoma (MTC), 9 FTC minimally invasive, 7 ATC, 7 oxyphilic adenocarcinoma, 3 PTC columnar cell, 2 undifferentiated carcinomas (UTC), 2 insular carcinomas, 2 mixed medullary-follicular carcinomas and 1 case each of carcinoma not otherwise specified, FTC trabecular, papillary microcarcinoma, PTC oxyphilic cell, MTC with amyloid stroma. Additionally, 37 follicular adenomas (FA) were analysed. Metastases were found in $43 \%$ of the ATC, $18 \%$ of the FTC, $8 \%$ of the PTC and $7 \%$ of the FVPTC. Affected lymph nodes were found in $100 \%$ of the UTC, $43 \%$ of the ATC, $39 \%$ of the PTC, $36 \%$ of the MTC, $8 \%$ of the FTC and $7 \%$ of the FVPTC. From their disease died $71 \%$ of the ATC, $10 \%$ of the PTC, $10 \%$ of the FTC and $3 \%$ of the FVPTC. The BRAF V6ooE IHC staining correlated significantly with the mutation status determined by massive parallel sequencing.

Conclusions. Both massive parallel sequencing and immunostaining are suitable for analysis of BRAF V60oE mutation status in thyroid cancer. Different subtypes of thyroid cancer are characterized by distinct frequency of BRAF mutations. 


\section{SA-094}

\section{FFPE tissue as a feasible source for gene expression analysis-} a comparison of three reference genes and one tumor marker

R.F.H. Walter ${ }^{* 1,2}$, F.D. Mairinger ${ }^{2}$, J. Wohlschlaeger ${ }^{2}$, K. Worm ${ }^{2}$, S. Ting ${ }^{3}$, C. Vollbrecht ${ }^{4}$, K.W. Schmid ${ }^{2}$, T. Hager ${ }^{2}$

${ }^{1}$ Ruhrlandklinik, West German Cancer Center, University Hospital Essen, University of Duisburg-Essen, Essen, Germany, ${ }^{2}$ Institute of Pathology and Neuropathology, University of Duisburg-Essen, University Hospital, Essen, Germany, ${ }^{3}$ Institute of Pathology and Neuropathology, University of Duisburg-Essen, University Hospital, Division of molecular Pathology, Essen, Germany, ${ }^{4}$ Institute of Pathology, University Hospital, Cologne, Germany

Aims. Formalin-fixation, paraffin-embedding is the standard processing technique for tumor tissue in modern pathology. New techniques such as cryoconservation allow rapid fixation and long-time storage but come along with increased costs and enlarged storage complexity. However, formalin-fixed, paraffin-embedded (FFPE) tissue is available in a large quantity making it the ideal material for retrospective studies. The following study was designed to investigate the influence of formalin-fixation on the quality of mRNA and applicability of FFPE derived mRNA for gene expression analysis. Three potential reference genes for pulmonary tumors with neuroendocrine differentiation were included and tested for their robust expression.

Methods. Eighty specimens collected from 2005 to 2012 at the Institute for Pathology and Neuropathology at the University Hospital Essen were analyzed for their gene expression by using TaqMan ${ }^{\circledR}$ gene expression assays on demand (AoD). Three distinct potential reference genes (ACTB, GAPDH, HPRT1) were evaluated for their expression and a proteasome subunit (PSMA1) was included in the analysis as tumor marker and functioned as an internal technical control.

Results. For GAPDH and ACTB a highly significant correlation and consistent expression between the investigated entities was found making them reliable reference genes for further research. Additionally, the feasibility for a FFPE tissue-based gene expression analysis was verified by showing that the mRNA quality is sufficient.

Conclusion. When standardized FFPE preparation is performed carefully, sufficient mRNA can be isolated for reliable and successful gene expression analysis. That provides the basis the door for large, retrospective studies that correlate molecular and clinical follow-up data.

\section{SA-095 \\ Inhibition of MDM2 via Nutlin-3-the next therapy for pleural mesothelioma with poor prognosis?}

R.F.H. Walter ${ }^{* 1,2}$, F.D. Mairinger' ${ }^{2}$, S. Ting ${ }^{3}$, C. Vollbrecht', T. Hager ${ }^{2}$, K. Worm ${ }^{2}$, W. Eberhardt ${ }^{5}$, J. Kollmeier ${ }^{6}$, T. Mairinger ${ }^{6}$, D.C. Christoph ${ }^{5}$, K.W. Schmid ${ }^{2}$, L. Freitag', J. Wohlschlaeger ${ }^{2}$

${ }^{1}$ Ruhrlandklinik, West German Cancer Center, University Hospital Essen, University of Duisburg-Essen, Essen, Germany, ${ }^{2}$ Institute of Pathology and Neuropathology, University of Duisburg-Essen, University Hospital, Essen, Germany, ${ }^{3}$ Institute of Pathology and Neuropathology, University of Duisburg-Essen, University Hospital, Division of molecular Pathology, Essen, Germany, ${ }^{4}$ Institute of Pathology, University Hospital, Cologne, Germany, ${ }^{5}$ Department of Oncology, University of Duisburg-Essen, University Hospital, Essen, Germany, ${ }^{6}$ Department of Pathology, Heliosklinikum Emil von Behring, Berlin, Germany, ${ }^{7}$ Department of interventional Pneumology, University of Duisburg-Essen, University Hospital, Essen, Germany

Aims. Malignant pleural mesothelioma (MPM) is a highly aggressive tumor that is treated with a standard regimen of cisplatin in combination with pemetrexed. But this treatment cannot be considered a tailored therapy because predictive and prognostic biomarkers are lacking. MPM show an inactivation of $\mathrm{TP}_{53}$ but mutations of this gene are rare so other mechanisms like deletion of the locus, methylation and inhibi- tion by regulators might be responsible for the inactivation. MDM2 and $\mathrm{P}_{14} / \mathrm{ARF}$ are upstream regulators of $\mathrm{TP}_{53}$ activity.

Methods. In this study, the mRNA or immunoexpression of $\mathrm{TP}_{53}$, MDM2 and P14/ARF were investigated in three MPM-cell lines. These three cell lines represent the major subtypes of MPM patients that were investigated in a previous study.

Results. Nutlin- 3 is an inhibitor of MDM2 and is a non-genotoxic drug with little toxicity in animal models showing lower drug resistance risk than conventional drugs. In in-vitro experiments Nutlin-3 in combination with cisplatin showed an up to 9.75 times higher induction of senescence and up to 5 times higher apoptosis rate compared to the cisplatin and pemetrexed regimen. Moreover, Nutlin-3 seems to restore $\mathrm{TP}_{53}$ activity in $\mathrm{TP}_{53}$ wild type mesotheliomas leading to senescence. Conclusions. A combination of cisplatin and Nutlin-3 triggers apoptosis and senescence more efficiently than pemetrexed and cisplatin making it a potent innovative tailored therapy for patients with MPM.

\section{SA-096}

Screening 80 neuroendocrine lung cancer patients by using the NanoString technology implies several targets for new tailored therapies

R.F.H. Walter ${ }^{* 1,2}$, F.D. Mairinger ${ }^{2}$, R. Werner', S. Ting ${ }^{3}$, C. Vollbrecht, D. Theegarten ${ }^{2}$, G. Stamatis ${ }^{5}$, D. Zwanziger ${ }^{6}$, W. Eberhardt ${ }^{7}$, M. Schuler? D.C. Christoph 7 , L. Freitag ${ }^{8}$, K.W. Schmid ${ }^{2}$, J. Wohlschlaeger ${ }^{2}$

${ }^{1}$ Ruhrlandklinik, West German Cancer Center, University Hospital Essen, University of Duisburg-Essen, Essen, Germany, ${ }^{2}$ Institute of Pathology and Neuropathology, University of Duisburg-Essen, University Hospital, Essen, Germany, ${ }^{3}$ Institute of Pathology and Neuropathology, University of Duisburg-Essen, University Hospital, Division of molecular Pathology, Essen, Germany, ${ }^{4}$ Institute of Pathology, University Hospital, Cologne, Germany, ${ }^{5}$ Ruhrlandklinik, West German Cancer Center, University Hospital Essen, Division of Thoracic Surgery and Endoscopy, University of Duisburg-Essen, Essen, Germany, ${ }^{6}$ University Hospital Essen, Division of Laboratory Research, Department of Endocrinology and Metabolic Diseases, University of Duisburg-Essen, Essen, Germany, ${ }^{7}$ Department of Oncology, University of Duisburg-Essen, University Hospital, Essen, Germany, ${ }^{8}$ Department of interventional Pneumology, University of Duisburg-Essen, University Hospital, Essen, Germany

Aims. Neuroendocrine lung cancer (NELC) accounts for more than $25 \%$ of all lung cancer cases and includes typical and atypical carcinoids, large cell and small cell neuroendocrine carcinomas. For most NELC no approved drug regimen is available and if there is any therapy chemoresistance is a frequent event.

Methods. Twenty representative FFPE-specimens of each tumor entity (TC, AC, LCNEC, and SCLC) were used for mRNA expression analysis using the NanoString technique. Based on recent literature a total of 91 mRNA targets were investigated as potential tumor markers. Twenty-three of these targets are known as targets of tailored therapies or are tested in recent phase trials.

Results. The NanoString technique was able to specifically and sensitively analyze 91 mRNA targets in 8o FFPE samples using $100 \mathrm{ng}$ of total RNA. EGFR ( $\mathrm{p}=0.0003175)$, FGFR1 ( $\mathrm{p}=0.003596)$, and FPGS $(\mathrm{p}=0.000000129)$ showed elevated expression in carcinoids compared to carcinomas. Instead, BCL2 ( $\mathrm{p}=0.00002929)$ and TYMS $(\mathrm{p}=0.0003175)$ showed higher expression in carcinomas compared to carcinoids. Conclusions. For several tumor entities approved therapy regimens exist, but for most NELC tailored therapies are lacking. Patients with carcinoid-tumors may benefit from targeted therapies against overexpression of EGFR (e.g. Cetuximab, Gefitinib), FGFR1 (e.g. Regorafenib, Brivanib) and FPGS (e.g. methotrexate-phosphinate, Suramin). Patients with carcinomas showed elevated expression of TYMS that can be inhibited by Raltitrexed and other folate analogues. BCL2 also showed elevated expression in carcinomas and in recent in vivo studies 
ABT-199 was found as promising therapeutic agent. Our results provide the molecular biological grounds for the development of novel tailored treatment strategies for patients suffering from NELC and to overcome chemoresistance of current therapies.

\section{SA-097}

Fibroblast growth factor receptor 1 (FGFR1) potentials as therapeutic target in head and neck squamous cell cancer (HNSCC)

T. Clauditz ${ }^{* 1}$, A. Münscher ${ }^{2}$, M. Blessmann ${ }^{3}$, W. Wilczak', G. Sauter ${ }^{1}$, T. Grob 'University Medical Center, Hamburg-Eppendorf, Hamburg, Germany, ${ }^{2}$ Department of Otorhinolaryngology and Head and Neck Surgery, Hamburg, Germany, ${ }^{3}$ Department of Oral and Maxillofacial Surgery; University Medical Center Hamburg-Eppendorf, Hamburg, Germany

Aims. FGFR1 has recently been described as a potential target for antibody-based treatment of squamous cell carcinoma of the lung successful in advanced disease as well as in adjuvant setting. To determine the potential applicability of such therapies squamous cell carcinomas of the head and neck region were analyzed for amplification of the FGFR1 gene locus. Additionally a possible association of HPV infection and FGFR1 gene amplification were determined.

Methods. A tissue microarray was constructed from 453 squamous cell carcinomas of the head and neck region (including tumors of the oral cavity, oropharynx and larynx). FGFR1 gene copy number was assessed by fluorescence in situ hybridization (FISH). Possible HPV infection of all amplified cases was analysed via polymerase chain reaction (PCR) and p16 immunohistochemistry.

Results. Amplification of the FGFR 1 gene was found in all analyzed subgroups (oral cavity: $10 \%$, oropharynx: $1 \%$ and larynx: $5 \%$ ) with a significant predominance in the subset of carcinomas of the oral cavity. Additional analyzes on HPV infection showed one case being infected with HPV type 16 . No correlations between FGFR1 gene copy number and clinicopathologic data as well as HPV infection were found.

Conclusions. Regarding future therapy options FGFR1 might become a serious therapy target in HNSCC. Possible effects of HPV infection on FGFR1 gene locus have to be evaluated in further studies.

\section{SA-098 \\ ATF knockdown reinforces oxidative stress-induced apoptosis in TE-7 cancer cells}

D. Walluscheck ${ }^{* 1}$, A. Poehlmann', R. Hartig², U. Lendeckel ${ }^{3}$, P. Schönfeld ${ }^{4}$, A. Hotz-Wagenblatt ${ }^{5}$, K. Reissig', K. Bajbouj ${ }^{6}$, A. Roessner', R. SchneiderStock

'Institute of Pathology, University Hospital, Otto-von-Guericke-University, Magdeburg, Germany, ${ }^{2}$ Institute of Molecular and Clinical Immunology, University Hospital, Otto-von-Guericke-University, Magdeburg, Germany, ${ }^{3}$ Institute of Medical Biochemistry and Molecular Biology, University Medicine Greifswald, Greifswald, Germany, ${ }^{4}$ Department of Biochemistry and Cell Biology, University Hospital, Otto-von-Guericke University, Magdeburg, Germany, ${ }^{5}$ Genomics Proteomics Core Facility, German Cancer Research Center (DKFZ), Heidelberg, Germany, ${ }^{6}$ University of Hawaii Cancer Center, University of Hawaii, Honolulu, United States, ${ }^{7}$ Experimental Tumor Pathology, Institute of Pathology, Friedrich-Alexander-University ErlangenNürnberg, Erlangen, Germany

Aims. Various oxidative stress-based anti-cancer therapies aim at improving cell death sensitivity. In this context, the switch from cell cycle arrest to increased apoptosis plays an important role. Therefore, we focused on identifying a universal molecule which functions as a mediator of growth arrest. The target molecule should be inhibited to enhance apoptosis.
Methods. The oxidative stress-inducing therapy was mimicked by the addition of $\mathrm{H}_{2} \mathrm{O}_{2}$ and CDNB to TE-7 cells. Western blot analyses served to detect DNA damage, cell cycle and apoptosis regulating proteins. Cell death and cell cycle progression were measured by FACS. To identify a target molecule, we carried out cDNA array analyses. Fluorescence immunolabeling analyses and co-immunoprecipitation were performed to confirm its activation. The inhibition of the target molecule was realized by gene knockdown. Promoter binding studies were done by ChIP analyses.

Results. After induction of DNA damage by $\mathrm{H}_{2} \mathrm{O}_{2}$, TE-7 cells showed $\mathrm{S}$ and $\mathrm{G} 2$ arrests accompanied by p21WAF1 induction and low apoptosis. In this cell line, we identified ATF-2 as a target molecule involved in DNA damage, cell cycle and apoptosis regulation. The transcription factor ATF-2 induced $\mathrm{G}_{2}$ arrest and $\mathrm{p}_{2} \mathrm{WAF}_{1}$ in the $\mathrm{H}_{2} \mathrm{O}_{2}$ response. The p21WAF1 promoter was bound by activated ATF-2 to a hitherto unknown ATF-2 binding sequence, suggesting transactivation via this sequence. We could show that the ATF-2 knockdown caused impaired G2 arrest and p21WAF1 expression and notably an increase in apoptosis. Finally, CDNB treatment also provoked an ATF-2-dependent G2/M arrest.

Conclusions. We improved the apoptosis sensitivity of TE-7 cells after $\mathrm{H}_{2} \mathrm{O}_{2}$ stress through knockdown of the transcription factor ATF-2. The knockdown caused a switch from G2 growth arrest to apoptosis, and this switch was induced by $2{ }_{2} 1 W A F 1$ downregulation. The mechanism of ATF-2-induced arrest and inhibition of apoptosis seems to be a general feature of oxidative stress as shown by CDNB treatment. Therefore, we propose ATF-2 as a target to improve cell death sensitivity of oxidative stress-based anti-cancer therapy.

\section{SA-099}

Evaluation of colon cancer morphology: a comparison between conventional formalin and novel PAXgene tissue fixation by an international ring trial

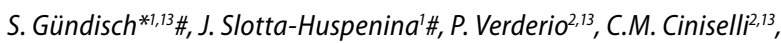
S. Pizzamiglio ${ }^{2,13}$, C. Schott ${ }^{1,13}$, E. Drecoll', C. Viertler ${ }^{3,13}$, K. Zatlouka $^{3,13}$, M. Kap ${ }^{4,13}$, P. Riegman ${ }^{4,13}$, I. Esposito', K. Specht', G. Babaryka', M. Asslaber ${ }^{3}$, K. Bodó, M. den Bakker', J. den Hollander ${ }^{5}$, F. Fend ${ }^{6}$, J. Neumann', S. Reu', A. Perren ${ }^{8}$, R. Langer ${ }^{8}$, A. Lugli ${ }^{8}$, I. Becker ${ }^{9}$, T. Richter ${ }^{9}$, G. Kayser ${ }^{10}$, A.M. May ${ }^{10}$, F. Carneiro ${ }^{11}$, J.M. Lopes ${ }^{11}$, L. Sobin' ${ }^{12}$, H. Höfler', K.-F. Becker,13 IInstitute of Pathology, Technical University of Munich, Munich, Germany, 2Unit of Medical Statistics, Biometry and Bioinformatics, Fondazione IRCCS, Istituto Nazionale dei Tumori, Milan, Italy, Institute of Pathology, Medical University, Graz, Austria, ${ }^{4}$ Department of Pathology, Erasmus Medical Center, Rotterdam, Netherlands, ${ }^{5}$ Institute of Pathology, Erasmus Medical Center, Rotterdam, Netherlands, ${ }^{\circ}$ Institute of Pathology, Eberhard-KarlsUniversity, Tübingen, Germany, ${ }^{7}$ Institute of Pathology, Ludwig-Maximilians-University, Munich, Germany, ${ }^{8}$ Institute of Pathology, University of Bern, Bern, Switzerland, ${ }^{9}$ Pathology Rosenheim, Rosenheim, Germany, ${ }^{10} \mathrm{In}$ stitute of Pathology, Freiburg, Germany, "IPATIMUP, Institute of Molecular Pathology and Immunology of the University of Porto, University of Porto, Porto, Portugal, ${ }^{12}$ Frederick National Laboratory for Cancer Research, The Cancer Human Biobank, National Cancer Institute, Rockville, United States, ${ }^{13}$ The SPIDIA Consortium, www. spidia.eu. \# Both authors contributed equally to this work.

Aims. Molecular pathology is of increasing importance and requires high quality tissue samples which allow simultaneous molecular and histopathological analysis. The suitability of the PAXgene tissue preservation technology for molecular analyses has previously been shown. However, application in histopathological routine diagnostics has not been comprehensively tested yet. Thus, the aim of our study was to evaluate the quality of histo- and cytomorphological features of PAXgene fixed specimens and the suitability of the novel fixative for histomorphological classification in comparison to formalin fixation. 
Methods. Fifteen colon cancer tissues were collected, divided into two mirrored samples and either formalin fixed (FFPE) or PAXgene fixed (PFPE) before paraffin-embedding. HE- and PAS-stained sections were scanned and evaluated in a blinded, randomized ring trial by $20 \mathrm{pa}-$ thologists from Europe and the US using virtual microscopy. The pathologists evaluated histological grading according to the WHO classification criteria, histological subtype, presence of adenoma, presence of lymphovascular invasion, quality of histomorphology, and nuclear details.

Results. Comprehensive statistical analysis revealed that the reproducibility concerning grading between both fixation methods was satisfactory ( $\mathrm{kw}=0.73,95 \% \mathrm{CI}$ 0.41-0.94), with a higher agreement between the study reference and the PFPE samples ( $\mathrm{kw}=0.86,95 \% \mathrm{CI} 0.67-1.00)$. The inter-observer reproducibility was rather low $(\mathrm{kw}=0.60)$, with assessment of tumor differentiation (i.e. $G_{2}$ vs. $G_{3}$ ) as the most critical category. Histomorphological quality parameters were scored equal or better for PFPE than for FFPE samples. In detail, overall quality and mucus preservation were judged significantly better for PFPE cases. In contrast, significant retraction artefacts were observed more frequently in PFPE samples. Nuclear details, especially the detection of mitosis, were also scored equal or better for PFPE samples due to i.e. increased contrast in HE- and PAS-stainings. Other considered parameters were not significantly related to the fixation method.

Conclusions. In conclusion, our findings suggest that the PAXgene tissue system leads to excellent preservation of histomorphology and nuclear details and allows sophisticated morphological diagnosis. The study warrants morphological evaluation of the PAXgene tissue system in other diseases.

This work has received funding from the European project SPIDIA (www. spidia.eu).

\section{SA-100}

\section{About discrimination of adenocarcinomas with MALDI imaging}

M. Sperling ${ }^{* 1}$, O. Klein', G. Nebrich², H. Thiele', I. Kollecker', A. Dellmann', N. aßler ${ }^{1}$, K. Donhuijsen ${ }^{1}$

'Academic Hospital Braunschweig, Department of Pathology, Braunschweig, Germany, ${ }^{2}$ Berlin/Brandenburg Center for Regenerative Therapies, Campus Virchow-Klinikum, Charité Berlin, Berlin, Germany, ${ }^{3}$ Fraunhofer MEVIS Inst. for Medical Imaging Computing, Lübeck, Steinbeis Innovation Center SCiLS Research, Bremen, Lübeck, Germany

Aims. Despite their different sites of origin sinonasal adenocarcinoma of intestinal type (SNAIT) bear a strong histological resemblance to colorectal carcinomas (CRAIT). Even in immunohistology no major differences were detected so far. Previously we could show differences in peptide profiles using MALDI imaging. Therefore we further investigated both types of carcinomas.

Methods. Paraffin sections of SNAIT and CRAIT were mounted on ITO-coated conductive glass slides for MALDI analysis. After Paraffin removal antigen retrieval was done via water bath for $20 \mathrm{~min}$, in $20 \mathrm{mmol}$ citrate buffer ( $\mathrm{pH}$ 6). Additional, we performed a tryptic digestion of tissue section. Trypsin solution was applied directly onto the section by an automated spraying device. Tissue incubation with the trypsin solution was performed for two hours at $37^{\circ} \mathrm{C}$. The tissue section was coated with alpha-cyano-4-hydroxycinnamic acid. The MALDI Imaging data sets were acquired in the mass ranges $\mathrm{m} / \mathrm{z} 400$ Da to 4.000 $\mathrm{Da}$ and evaluated by Wilcoxon test $(\mathrm{p}<0.05)$.

Results. We obtained good peptide profiles, confirming previous results as well as showing differences in additional masses between SNAIT and CRAIT. Identification by "topt dow" mass spectrometry of masses was tried and revealed one mass $\mathrm{m} / \mathrm{z} 944 \mathrm{Da}$ as being a Histone $\mathrm{H}_{2} \mathrm{~A}$ protein. Conclusions. We were able to confirm, that situ MALDI imaging is suitable for detecting characteristic differences even in tumors with high morphological similarities. Further research to identify the different molecules is needed to classify the peptide profile differences in detail.

\section{SA-101}

\section{Genome-wide chromosomal aberration analysis reveals clonally} unrelated relapses in diffuse large B-cell lymphoma

\section{Juskevicius*, A. Rufle, C. Ruiz, S. Dirnhofer, A. Tzankov}

Institute of Pathology, University Hospital Basel, Basel, Switzerland

Aims. Generally, lymphoma recurrences are considered to represent a relapse of the original neoplasm, but this concept has been recently challenged by demonstration of clonally unrelated relapses by immunoglobulin (heavy chain) gene (IGH) rearrangement analysis. We applied a genome-wide clonality testing on 17 paired primary and relapsed DLBCL aiming to provide an unambiguous answer about the existence of clonally unrelated recurrences in this entity. Additionally, we profiled 10 cases of non-relapsing DLBCL and compared their profiles to the relapsing ones searching for genetic markers at diagnosis, which could predict lymphoma recurrence.

Methods. Genomic DNA extracted from formalin-fixed paraffin-embedded tissue with at least $70 \%$ tumor cell content was used for the analysis. Array-comparative genomic hybridization was utilized to detect chromosomal copy number aberrations and to determine clonal relationship status of each primary-relapse pair. This result was then verified by IGH gene fragment length analysis. Directed deep-sequencing was performed on a custom hot-spot panel consisting of most frequently mutated genes in DLBCL.

Results. Among 17 cases of recurring DLBCL, 3 relapses (17\%) were identified, which were clonally unrelated to the primary disease by copy number aberration and IGH fragment length analysis. Different modes of tumor progression could be detected within clonally related set of relapses, providing evidence of a common early progenitor cell population in some patients. Moreover, some DLBCL exhibited substantial intra- and inter-tumoral heterogeneity, which is probably a source of variation in patient outcomes and clonal progression. Comparison of copy number aberration and point mutation profiles between relapsing and non-relapsing tumors yielded significant differences, which could be useful for understanding the genetic basis of DLBCL recurrence. Conclusions. We provide a clear evidence for the existence of clonally unrelated relapses in DLBCL. The existence of clonally unrelated relapses might be of clinical importance since relapses are usually treated more aggressively than primary neoplasms with a significant treatment-related morbidity, and this could be by-passed in the former instances. Moreover, the high definition clonal analyses provide new insights into the molecular profiles of tumors in vivo, and therefore might contribute to the development of more personalized approaches for cancer treatment.

\section{SA-102}

\section{FGFR1 dual-color chromogenic and silver in-situ hybridization-} a new tool for the detection of the FGFR1 copy number status

M. Braun ${ }^{* 1,2}$, D. Böhm ', W. Vogel', A. Franzen', A. Schröck ${ }^{1,3}$, F. Bootz', L. Heaseley ${ }^{4}$, S. Perner ${ }^{1,2}$

'Department of Prostate Cancer Research, Pathology, University of Bonn, University of Bonn, Bonn, Germany, ${ }^{2}$ Institute of Pathology, University Hospital Bonn, Bonn, Germany, ${ }^{3}$ Department of Otorhinolaryngology/Head and Neck Surgery, University Hospital Bonn, Bonn, Germany, ${ }^{4}$ Department of Craniofacial Biology, University of Colorado at Denver, Aurora, United States

Aims. Recently, the fibroblast growth factor receptor 1 (FGFR1) was described as the first actionable target in squamous cell lung cancers. Clinical trials testing specific FGFR inhibitors are in process and patients are selected based on their FGFR1 copy number status. Fluorescence 
in-situ Hybridization (FISH) is the most commonly used method for detecting FGFR1 amplifications, but it has its limitations. Here, we established a new long lasting and easily assessable FGFR1 chromogenic and silver in situ hybridization (CS-ISH) assay.

Methods. We assessed 394 patients diagnosed with head and neck squamous cell carcinoma with the FGFR1 CS-ISH assay and compared these results with those obtained by FGFR1 FISH.

Results. The FISH assay was assessable in $86.8 \%$ (342/394) of cases, whereas the CS-ISH was successful in $79.4 \%(313 / 394)$ of cases. FGFR1 amplification was detected by FISH in $12.6 \%(43 / 342)$. In detail, $7.6 \%$ (26/342) showed a low level amplification (LLA) and 5.0\% (17/342) a high level amplification (HLA). $87.7 \%$ (317/351) of our investigated specimens displayed no FGFR1 amplification. By CS-ISH, 10.2\% (32/313) showed an amplification. In detail 5.7\% (18/313) showed a LLA, $4.5 \%(14 / 313)$ a HLA and $89.8 \%(281 / 313)$ of our investigated specimens displayed no FGFR 1 amplification. A high concordance (Pearson's correlation coefficient= $0,971, \mathrm{p}<0.01$ ) was observed comparing both techniques.

Conclusions. The ease of bright field microscopy, fully automated workflow, oil free evaluation and stable, light-insensitive signals are features that open up CS-ISH based FGFR1 amplification assays for the widespread use in clinical routine and research laboratories. Hence, we emphasise CS-ISH as a new tool for the identification of gene amplifications.

\section{SA-103 \\ Phosphorylation of plakophilin 3 at Y195 is performed by reactive oxygen species (ROS)-activated c-Src kinase but not by growth factors}

\section{S. Neuber*, P. Rexin, M. Meyer, V. Wischmann, R. Moll, A. Schmidt Institute of Pathology, Philipps University, Marburg, Germany}

Aims. An essential component of desmosomal adhesion comprises the three related plakophilins ( $\mathrm{PKP}_{1}$ to $\mathrm{PKP}_{3}$ ). PKPs are desmosomal plaque proteins that are also thought to be involved in particular signaling events and possibly also in malignant transformation. Modifications especially of tyrosine residues are of importance for the regulation of the junctional und cytoskeletal remodeling and may be deregulated in malignant processes. Here we analyze a modification of $\mathrm{PKP}_{3}$ by a specific tyrosine phosphorylation related to some of these aspects.

Methods. Deduced from phosphotomic databases, we generated and purified antibodies that specifically detect Tyr-phosphorylated $\mathrm{PKP}_{3}$ at Y195 that may be of special interest for $\mathrm{PKP}_{3}$ regulation. These antibodies were employed for the immunobiochemical and immunohistochemical analysis of the respective Tyr-phosphorylation of $\mathrm{PKP}_{3}$ and the identification of the responsible kinase.

Results. Tyr-phosphorylation of $\mathrm{PKP}_{3}$ appears only transiently under normal conditions in epithelial cells. Treatment of cells with phosphatase inhibitor pervanadate revealed that $\mathrm{PKP}_{3}$ is actually phosphorylated at Y195. To identify conditions that lead to Y195 modification we incubated cells with different growth factors (EGF, IFG1, HGF) which did, however, not lead to a specific phosphorylation. Using specific inhibitors of Src-family kinases on cultured cells we were able to identify Src-kinase acting at $\mathrm{Y}_{195}$ of $\mathrm{PKP}_{3}$. As a proof, isolated Src kinase was able to phosphorylate recombinant $\mathrm{PKP}_{3}$ polypeptide at $\mathrm{Y}_{195}$ in an invitro assay. Additionally, siRNA-mediated knockdown of Src in HaCaT cells obviously reduced Y195 phosphorylation after stimulation. When looking for conditions that trigger Y195-phosphorylation by Src-kinase, we identified reactive oxygen species (ROS) as one factor. Incubation of cells with hydrogen peroxide led to a rapid phosphorylation at Y195 that could be eliminated by Src-kinase inhibitors. Finally, we detected Y195-phosphorylation immunohistochemically in tumor areas of poorly differentiated prostate adenocarcinomas.

Conclusions. In conclusion, we demonstrate for the first time that $\mathrm{PKP}_{3}$ is an additional junctional substrate of Src-kinase. Nevertheless, further studies are needed to completely understand the functional relevance of this mechanism on non-neoplastic and neoplastic conditions.

\section{SA-104}

Imaging mass spectrometry (IMS) to discriminate metastasis of breast from pancreatic cancer in FFPE tissues

\section{R. Casadonte ${ }^{* 1}$, M. Kriegsmann ${ }^{2}$, K. Friedrich ${ }^{3}$, G. Baretton ${ }^{3}$, M. Otto ${ }^{1,4,5}$,} S.-O. Deininger ${ }^{6}$, D. Suckau ${ }^{6}$, J. Kriegsmann ${ }^{1,4,5}$

${ }^{1}$ Proteopath GbR, Trier, Germany, ${ }^{2}$ University of Heidelberg, Trier, Germany, ${ }^{3}$ University of Dresden, Department of Pathology, Dresden, Germany, ${ }^{4}$ Center for Histology, Cytology and Molecular Diagnostics, Trier, Germany, ${ }^{5}$ Institute of Molecular Pathology, Trier, Germany, ${ }^{6}$ Bruker Daltonik GmbH, Bremen, Germany

Aims. Personalized cancer therapy of metastatic disease relies on accurate classification of the tumor. The primary site of cancer usually dictates the treatment, expected outcome, and overall prognosis. At present, histopathological diagnosis is based on clinical information, morphology, immunohistochemistry, and may include molecular methods. This process is complex, expensive, requires an experienced pathologist and may be time consuming. The purpose of this study is to establish IMS as a new tool to classify metastases in regard to the tumor of origin in FFPE tissues.

Methods. 96 breast and 92 pancreatic biopsies in a tissue microarray from different patients, were included in our study. Additionally, six liver metastases, including three breast and three pancreatic carcinomas were used for proteomic classification. All samples were subjected to trypsin and matrix deposition using the ImagePrep device (Bruker, Bremen), and were subsequently analyzed by MALDI IMS using a Bruker Autoflex Speed mass spectrometer. Statistical analysis was performed by ClinProTools 3.0.

Results. 29 breast and 32 pancreas tumor samples were used as training set to build a classification model using a support vector machine algorithm (SVM). $17 \mathrm{~m} / \mathrm{z}$ peptide species allowed to establish SVM classifiers, determined through statistical comparison by means of a Wilcoxon/Kruskal-Wallis test (PWKW<0.05), which could discriminate breast from pancreas carcinomas, with high recognition capability $(100 \%)$ and an overall cross validation of $99.21 \%$. These classifiers were applied to two test sets including 67 breast and 60 pancreas primary carcinoma patients. SVM model classified breast from pancreas carcinoma with an overall accuracy of $83.38 \%$, a sensitivity of $85.95 \%$ and a specificity of $76.96 \%$. We applied our model to classify three breast and three pancreatic individual liver metastasis samples. Classification results were concordant to the histopathological diagnosis made by pathologists.

Conclusions. We have developed an IMS classification model using FFPE breast and pancreatic tumor specimens and found proteomic signatures that accurately could discern breast from pancreatic carcinomas. Furthermore, this classification model was applied to metastasis of breast and pancreatic carcinoma with correct identification of the adequate tumor type.

\section{SA-105}

\section{Chromogenic in situ hybridization (CISH) is a reliable method to} detect ALK-positive non-small cell lung carcinomas

\section{F. Wagner*, A. Streubel, A. Roth, S. Stephan-Falkenau, T. Mairinger Institut für Gewebediagnostik/MVZ, Berlin, Germany}

Aims. We assessed the potential of a chromogenic in situ hybridization (CISH) assay in comparison with quantitative RT-PCR (qPCR) to detect anaplastic lymphoma kinase (ALK) break apart-positive lung carcinomas.

Methods. Dual-colour CISH using a break-apart probe for the ALK gene on 2p23 was performed with 181 FFPE tissue and agar block sections from 175 cases of non-small cell lung carcinomas (NSCLC). Stained slides were analyzed with a standard bright-field microscope at 10oox magnification by counting signals from 60 non-overlapping nuclei 
from three different tumour areas. Samples with $\geq 15 \%$ of positive nuclei were judged as ALK break apart-positive. All samples were simultaneously analyzed by qPCR for EML4-ALK to validate CISH results, and positive samples were subject to Sanger sequencing.

Results. CISH was successful with 173 of 181 hybridized samples (96\%), and 7 ALK break apart positive cases were detected. CISH signals were specific and distinct for both colours. All positive cases were confirmed by qPCR and Sanger sequencing, and concordance between CISH and qPCR was $100 \%$. Nearly all samples (9/10) which failed by qPCR were accessible to CISH analysis.

Conclusions. CISH is a very reliable, convenient and inexpensive method to detect ALK positive NSCLC. CISH success rate is comparably high as with qPCR, and it detects all ALK break-apart events in a single assay. It is of special value when RNA quality is poor, or when small biopsies with a very limited amount of tumour cells have to be analyzed.

\section{SA-106 \\ Genomic heterogeneity and clonal evolution in malignant melano- mas \\ T. Lorber ${ }^{* 1}$, S. Rau', V. Perrina' , T. Dietsche', M. Barrett'², K. Glatz', C. Ruiz', L. Bubendorf' \\ IInstitute for Pathology, Basel, Switzerland, ${ }^{2}$ Translational Genomics Re- search Institute, Scottsdale, United States}

Aims. Recent improvements in genomic analysis tools allow research groups to intensively deep-sequence different tumor entities, including malignant melanomas. However, most of the studies performed have to deal with single biopsies per patient, which limits the insight into genomic tumor evolution. In this study, we subjected sorted clonal tumor populations from several specimens per patient to genomic profiling in order to infer genomic heterogeneity in malignant melanomas and genomic evolution during tumor progression.

Methods. A multi-parameter sorting approach was used to flow-sort multiple biopsies from individual melanoma patients. Therefore, nuclei were extracted from both frozen and FFPE biopsies and sorted according to DNA-ploidy. To achieve sorting of pure tumor, we additionally applied an anti-SOX10 antibody that is specific for melanoma nuclei. Resulting clonal tumor populations were genomically characterized by usage of high resolution CGH microarrays and targeted deep-sequencing with NGS.

Results. CGH microarray and NGS analysis of flow-sorted clonal tumor populations revealed the clonal relationship between multiple tumor biopsies from individual patients. The melanoma marker SOX10 allowed us to uncover and sort pure tumor populations, eliminating a contamination of non-neoplastic cells in downstream analysis of aCGH and NGS. Combining the knowledge from ploidy analysis, aCGH and NGS data, we were able to detect chromosomal imbalances and allelic frequencies from these populations. Furthermore our approach allows us to track the evolution of clonal populations across time and organs and in the context of therapeutic interventions.

Conclusions. Human malignant melanomas are composed of different clonal populations with population-specific genomic aberrations and mutations. By applying aCGH and NGS we were able to characterize these sorted clonal tumor populations. Further bioinformatical investigations of these sorted clonal tumor populations are fundamental for the understanding of clonal relationship and genomic heterogeneity and their potential impact on metastasis and therapy response in malignant melanomas.

\section{SA-107}

\section{Carbacylin stimulates cardiomyocyte cell cycle progression via the} PPAR delta

\section{A. Magadum ', T. Kim², Q. Yang ${ }^{2}$, F.B. Engel*3}

${ }^{1}$ Max Planck Institut for Heart and Lung Research, Bad Nauheim, Germany, ${ }^{2}$ Department of Nutrition Sciences, University of Alabama at Birmingham, Birmingham, United States, ${ }^{3}$ Institute of Pathology, Department of Nephropathology, University Hospital Erlangen, Erlangen, Germany

Aims. Newt, zebrafish and newborn mice can regenerate their heart through cardiomyocyte proliferation. In contrast, the postnatal mammalian heart has only a limited capacity to repair cardiac injury. Thus, it is important to identify new inducer of mammalian cardiomyocyte proliferation.

Methods. Here, our goal was to establish a fluorescence-based live imaging-screening assay to identify a novel inducer of adult mammalian cardiomyocyte cell cycle re-entry. For this purpose we employed the so-called Fucci system, which utilizes the fact that the two cell cycle oscillators, Cdt1 and Geminin, are subjected to cell cycle-dependent proteolysis. We screened two libraries: a library containing antagonists and agonists of nuclear receptors and a library with compounds affecting enzymes modifying histones.

Results. Geminin is actively degraded in non-cycling cells. Accordingly, adenoviral overexpression of Geminin fused to Azami Green resulted in less than $1.5 \%$ Azami Green-positive postnatal cardiomyocytes. In contrast, stimulation with fibroblast growth factor (FGF) 1 and p38 mitogen-activated protein kinase inhibitor (p38i), known to induce cardiomyocyte proliferation, increased the number of Azami-Green-positive postnatal cardiomyocytes by 18 -fold. Utilizing this system we identified carbacyclin, a known agonist of peroxisome proliferator-activated receptor delta (PPAR delta). Compared to untreated cells, carbacyclin increased DNA synthesis by more than 10-fold as measured by BrdU incorporation. Moreover, immunofluorescence confocal microscopy showed a more than 10-fold increase in the number of cardiomyocytes undergoing mitosis and cytokinesis. Our data indicate that carbacyclin acts via the PPAR delta/PDK1/p308Akt/pGSK $3 / \beta$-catenin-pathway and induces preferentially cell cycle re-entry of mono-nucleated postnatal cardiomyocytes. Importantly, carbacyclin induced also cell cycle progression of adult cardiomyocytes in vitro and inducible cardiomyocyte-specific overexpression of constitutively active PPAR delta was sufficient to induce adult cardiomyocyte mitosis in-vivo.

Conclusions. Taken together, we established a cardiomyocyte proliferation screening platform and identified carbacyclin as a new inducer of postnatal cardiomyocyte proliferation via the PPAR delta/PDK1/ p308Akt/pGSK $3 / \beta$-catenin-pathway with the potential to regenerate the mammalian heart.

\section{SA-108}

Prognostic and diagnostic value of SEPT9 and SHOX2 DNA methylation and cytology in malignant, paramalignant and benign pleural effusions

M. Jung ${ }^{* 1}$, D. Dietrich', S. Puetzer', A. Leisse', E.E. Holmes', S. Meller', B. Uhl', P. Schatz ${ }^{2}$, C. Ivascu' ${ }^{3}$, G. Kristiansen'

'Institute of Pathology, University Hospital Bonn (UKB), Bonn, Germany, ${ }^{2}$ Metanomics Health GmbH, Berlin, Germany, ${ }^{3}$ Roche Pharma AG, Hematology/Oncology, Grenzach-Wyhlen, Germany

Aims. The accurate identification of tumor cells in pleural effusions is crucial for the discrimination between benign, malignant and paramalignant pleural effusions and thus for determining an appropriate patient treatment. The diagnosis of pleural effusions is based on cytological analyses as a gold-standard-method. However, its sensitivity can suffer from low abundance of tumor cells and the fact, that actively dividing mesothelial cells can mimic adenocarcinomas. Aberrant DNA 
methylation is common in cancer cells and its diagnostic potential in comparison to cytology is only insufficiently characterized. The aim of the study was the development and validation of a diagnostic test based on the DNA methylation biomarkers $\mathrm{SHOX}_{2}$ and SEPT9. According to our previous studies, this assay should allow for a highly specific detection of malignant cells.

Methods. A case control study was conducted comprising pleural effusions from 114 patients $(58$ cancer patients, 56 control patients with benign diseases). The presence of malignant disease was confirmed by histology based on biopsy or surgical specimen. Pleural effusions were analyzed by conventional cytology and a quantitative real-time PCR assay for DNA methylation assessment.

Results. $21 \%$ of the cases were cytologically positive and $26 \%$ were $\mathrm{SHOX}_{2}$ or SEPT9 methylation positive. The combination of cytology and methylation data allowed detecting $36 \%$ of the cancer patients correctly. Both, cytological and methylation analyses, showed $100 \%$ specificity. Interestingly, DNA methylation analyses but not cytological analyses showed a prognostic value in Kaplan-Meier-Analyses regarding the overall survival of cancer patients [ $\log$ rank test, $\mathrm{p}=0.02\left(\mathrm{SHOX}_{2}\right)$, $\mathrm{p}=0.02(\mathrm{SEPT} 9)]$.

Conclusions. The DNA methylation biomarkers $\mathrm{SHOX}_{2}$ and SEPT9 show a diagnostic and even a prognostic value in this pilot study and might be a helpful adjunct to cytology and other existing clinical investigations in patients suffering from pleural effusions.

\section{SA-109}

Evaluation of single- and double-strand paired-end sequencing of formalin-fixed paraffin-embedded tumor biopsies for the detection of single nucleotide variants and complex mutations for routine diagnostics

\section{S. Bartels*, B. Hasemeier, E. Schipper, H. Kreipe, U. Lehmann Institute of Pathology, Hannover Medical School, Hannover, Germany}

Aims. This project addresses the question whether high-throughput single- and double-strand paired-end sequencing can be implemented into the routine molecular diagnostic workup of tumour biopsies and evaluated this new methodology in comparison to alternative sequencing methods.

Methods. Genomic DNA isolated during the routine work-up of 75 biopsies from 75 patients (bone marrow, lung, colorectal, breast, skin). 150 to $3000 \mathrm{ng}$ was used for target enrichment and library preparation. For target enrichment a custom-designed gene panel covering 34 genes related to myeloid neoplasia and a pre-designed cancer panel (TruSight Tumor from Illumina.Inc) were used. $151 \mathrm{bp}$ and $121 \mathrm{bp}$, respectively, pairedend sequencing was performed using v2 chemistry. Technical replicates for 12 samples were performed. For identification of sequence variants BAM-files were loaded into the IGV browser or vcf-files were loaded into the VariantStudio-software from Illumina (version 2.1). Identified sequence variants were compared with prior results obtained by bi-directional Sanger sequencing or pyrosequencing. For K-ras, N-ras, and B-raf an alternative target enrichment protocol was applied using the same paired-end sequencing approach. A subset of samples was analyzed in parallel using a semiconductor-based sequencing device.

Results. All sequence variants identified before by Sanger sequencing or pyrosequencing were unequivocally confirmed by high-throughput amplicon sequencing. Sequence depths correlated linearly with DNA input. Technical replicates were $100 \%$ identical with respect to sequence variants only if mean sequence depth was above 10oo. The number of sequence variants correlated inversely with sequence depth. The amount of amplifiable DNA before library preparation using quantitative realtime PCR correlated only weakly with sequence output. Alternative target enrichment protocols compare favorable in terms of hands-on time and costs per sample. Seminconductor-based high throughput sequencing displays different strengths representing a complementation rather than an alternative.
Conclusions. With a sequence depth of at least 1000 reads per amplicon target-resequencing of gene panels enables the reliable identification of mutations in routinely processed biopsies. Different target enrichment protocols and sequencing devices display different strengths and weaknesses. The time-consuming sequence variants evaluation requires a high level of experience and knowledge and reduces the throughput of the system.

\section{SA-110}

Loss of miR expression results in increased tumor initiation but slower growth of adenomas in the small intestine in an APC-mouse model

\section{S. Jaitner*, T. Kirchner, A. Jung}

Institute of Pathology, Ludwig-Maximilians-University, Munich, Germany

Aims. Intestinal carcinogenesis depends on the activation of the canonical Wnt-signalling pathway most often by mutations in the tumorsuppressor gene APC (adenomatous polyposis coli). Additionaly, a large group of micro-RNAs (miR) are known to play an important role in the process of carcinogenesis (onco-miR). Currently it is unknown how a deregulation in miR influences APC driven intestinal carcinogenesis. Here, we present a mouse model which combines an activation of the Wnt signaling pathway (loss of APC function) with the loss of miR (loss of Dicer function-Dicer is an essential endonuclease in the process of miR maturation).

Methods. Lgr5-EGFP-IRES-Cre ERT2 X LSL-Rosa26-lacZ X APCfl X Dicerfl and LSL-Rosa26-lacZ X APCfl X Dicerfl control mice were bred in a $\mathrm{C}_{57} \mathrm{BL} / 6$ background. In these mice Cre-recombinase expression is under the control of the promoter of the intestinal stem cell marker Lgr5. Moreover, Cre has been fused to the estrogen receptor gene (ERT2) which allows the control of the nuclear localization and thus activity of Cre by injecting tamoxifen into the mice. Recombination can then be traced by expression of beta-galactosidase (Rosa26-lacZ). After induction or recombination the number and size of adenomas was determined. Thereby adenomas of three different genotypes with respect to Dicer zygosity were analyzed: Lgr5-EGFP-IRES-Cre ERT2 X APCfl/fl X (1) Dicerwt/wt, (2) Dicerfl/wt, and (3) Dicerfl/fl.

Results. All mice developed adenomas in the small intestine as it was described for the parental APCfl/fl strain indicating that the breeding of our transgenic mice had no influence on the gross biology of the carcinogenesis. Significant more adenomas were found in the absence of Dicer in a dose dependent manner (1) Dicerwt/wt (346 \pm 118 adenomas per animal [apa]), (2) Dicerfl/wt (643 \pm 228 apa; $p=0.02)$, and (3) Dicerfl/ fl ( $918 \pm 365$ apa; $p=0.01)$. Moreover, the absence of Dicer resulted in significantly smaller sized adenomas $(\mathrm{p}=0.001)$.

Conclusions. Absence of Dicer and thus miRs in the context of active canonical Wnt-Signalling affects intestinal carcinogenesis. It seems that the tumor initiation rate might be increased (more adenomas) but proliferation reduced (smaller size). More detailed analyses are currently done on these mice.

\section{SA-111}

Implementing MALDI TOF for RAS mutation testing in routine FFPE tissue samples-a new powerful, sensitive and cost-effective tool for molecular diagnostics

N. Arens', M. Kriegsmann², M. Otto 1,3,4, J. Kriegsmann ${ }^{* 1,3,4}$

${ }^{1}$ Institute of Molecular Pathology, Trier, Germany, ${ }^{2}$ University of Heidelberg, Trier, Germany, ${ }^{3}$ Center for Histology, Cytology and Molecular Diagnostics, Trier, Germany, ${ }^{4}$ Proteopath GbR, Trier, Germany

Aims. RAS gene mutation testing has emerged as an important tool for diagnosis, prognosis, treatment, and predicting patient outcome in response to targeted therapy for colorectal carcinoma. Current therapy 
guidelines recommend testing of KRAS and NRAS exons 2, 3 and 4. A wide spectrum of methods is applied in diagnostic molecular pathology, each providing advantages and disadvantages. We established a Sequenom MALDI TOF DNA analysis platform that efficiently and precisely measures the amount of genetic target material and/or variations. Operational reliability of the mass spectrometric RAS mutation detection assay was proven by PCR/chip hybridization and Sanger sequencing. Methods. DNA samples isolated from 85 colorectal carcinomas were investigated by mass spectrometry of multiplex PCR products and sequencing techniques. KRAS exon 2 was analyzed by PCR and subsequent chip hybridization, exons 3 and 4 and NRAS exons 2, 3 and 4 were examined by Sanger sequencing.

Results. All mutations detected by PCR/chip hybridization or Sanger sequencing respectively could be verified by MALDI TOF analyses. Mass spectrometry analysis combines high reliability with reduced laboratory turnaround time and comparatively low costs per test.

Conclusions. MALDI TOF technology is a reliable, fast, sensitive and cost-effective tool for mutation detection in FFPE tumor samples.

\section{SA-112 \\ Performance evaluation of kits for bisulfite conversion of DNA from various sources (extracted DNA, tissues and cell lines, FFPE tissues, aspirates, plasma, serum, urine)}

\section{E.E. Holmes*, M. Jung, B. Uhl, S. Meller, G. Kristiansen, D. Dietrich Institut of Pathology, Bonn, Germany}

Aims. The analysis of DNA methylation generally requires a preceding bisulfite conversion. During this conversion cytosines are deamined to uraciles whereas methyl-cytosines remain unaffected. The choice of an appropriate conversion kit for a specific application can be difficult and should be based on the specific performance requirements and the applied sample material. In this study, nine bisulfite conversion kits were evaluated: EpiTect ${ }^{\circledR}$ Fast FFPE Bisulfite Kit, EpiTect ${ }^{\circledR}$ Bisulfite Kit, EpiTect $^{\circledR}$ Fast DNA Bisulfite Kit (Qiagen), EZ DNA Methylation-Gold Kit, EZ DNA Methylation-Direct Kit, EZ DNA Methylation-Lightning Kit (Zymo Research), innuCONVERT Bisulfite All-In-One Kit, innuCONVERT Bisulfite Basic Kit, innuCONVERT Bisulfite Plasma Kit (Analytik Jena).

Methods. The performance of the kits was compared with regard to DNA yield, degradation, purity, conversion efficiency, stability and handling by means of qPCR, UV, clone sequencing, HPLC, and agarose gel electrophoresis.

Results. The study showed that all nine kits yielded highly pure DNA. The converted DNA was suitable for PCR analyses without inhibiting the PCR reaction. The EZ DNA Methylation-Gold Kit and the innuCONVERT Bisulfite kits showed significantly higher yields of bisulfite converted DNA as compared to the other kits. All kits showed bisulfite conversion efficiency above $97 \%$. The inappropriate conversion of methylated cytosines to thymines ranged between 0.9\% (innuCONVERT Bisulfite kits) and 2.7\% (EZ DNA Methylation-Direct Kit). Time-to-result ranged from $131 \mathrm{~min}$ (innuCONVERT kits) to $402 \mathrm{~min}^{\text {(EpiTect }}{ }^{\circ}$ Bisulfite Kit). Hands-on-time varied between 66 min (EZ DNA Methylation-Lightning Kit) and 104 min (EpiTect ${ }^{\oplus}$ Fast FFPE and Fast DNA Bisulfite kits). The highest DNA yield from formalin-fixed and paraf fin-embedded (FFPE) tissue sections without prior extraction were obtained using the innuCONVERT Bisulfite All-In-One Kit. The EZ DNA Methylation-Direct Kit only yielded DNA with only low PCR-amplifiability. The innuCONVERT Bisulfite Plasma Kit allowed for the analysis of up to $3 \mathrm{ml}$ plasma, serum, ascites, pleural effusions and urine.

Conclusions. In summary, the innuCONVERT Bisulfite All-In-One Kit exhibited the highest versatility regarding the input of different sample materials (extracted DNA, tissue, FFPE tissue, cell lines, urine sediment, and cellular fractions of bronchial aspirates, pleural effusions, ascites).

\section{SA-113}

Das Alzheimer-Amyloid-Precursor-Protein schützt Tumorstammzellen vor ER-Stress

V. Venkataramani*1, A. Frosch' ${ }^{2}$, S. Küffer ${ }^{2}$, C. Kiecke ${ }^{1}$, R. Koch ${ }^{1}$, T. Visakorpi ${ }^{3}$, J.S. Rhim ${ }^{4}$, L. Trümper', P. Ströbel', G.G. Wulf'

'Department of Hematology and Oncology, University Medical Center, Göttingen, Germany, ${ }^{2}$ Institute of Cellular and Molecular Immunology, Georg August University of Göttingen, Göttingen, Germany, ${ }^{3}$ Institute of Medical Technology, Molecular Biology of Prostate Cancer Group, University of Tampere, Tampere, Finland, ${ }^{4}$ Department of Surgery, Center for Prostate Disease Research, Uniformed Services University of the Health Sciences, Bethesda, United States

Aims. We have previously established that the amyloid precursor protein (APP) is overexpressed in several solid cancer types, including prostate cancer. In the current study, we highlight the essential involvement of APP in the "side population" (SP), representing the putative cancer stem cell (CSC) fraction of several prostate cancer cell lines. We could show that APP-Levels are significantly enriched in the SP-Fraction, correlating with the amount of established stem cell markers, such as Oct$3 / 4$ and NANOG. Using gain-of-function and loss-of-function studies we could demonstrate that APP essentially impairs the colony formation ability without affecting the quantitative amount of CSC. In order to elucidate the molecular mechanism(s), we show that loss-of-APP is not inducing apoptosis, autophagy or DNA-Damage. Interestingly, ERstress markers, such as the molecular chaperone GRP 78 and calreticulin are significantly up-regulated in several APP-knock-down cell lines, including AR-overexpressing variants.

Methods. Following PCa cell lines were used: LNCaP cells expressing $\mathrm{AR}$ in different expression levels as well as $22 \mathrm{Rv} 1$ and primary prostate cell lines. Following functional assays such as cell proliferation, colony forming assays and Western blot analyses were performed. A FACS-based technique known as SP-analysis was established in all cancer cell lines, distinguishing the putative CSC-population. Using CAM-Assay, tumor weight and IHC were performed.

Results. Down-regulation of APP expression using shRNA approach significantly reduced cell growth and clonality. Using SP-analysis, we could demonstrate increased tumor clonality of SP in contrast to the non-SP population. Interestingly, APPshRNA cells had the same amount of SP. However, the SP-population of APPshRNA cells presented a loss of colony formation ability. Analysing the molecular basis of this phenotype, we could exclude induction of apoptosis, autophagy or DNA damage in APPshRNA cell lines, whereas ER stress markers, like $\mathrm{GRP} 78$ and calreticulin were significantly up-regulated. Selective APP/ sAPPa-Inhibitors not only mimicked loss of SP-potency but also induced ER-stress without inducing apoptosis. Similar results could also be obtained with the ER-stress-inducer Thapsigargin. Supporting our results, down-regulation of ER-stress using TUDA rescued the APPshRNA phenotype and increased tumor clonality.

Conclusions. In conclusion, our results highlight the functions of APP in the CSC-population, protecting these cells against ER-stress.

\section{SA-114}

Laser capture microdissection enables a reliable molecular analysis of biomarkers in NSCLC patients with tiny amounts of tissue available

M. Demes ${ }^{* 1}$, A. Fisseler-Eckhoff ${ }^{1,2}$

'Group Practice of Pathology Wiesbaden, Molecular Pathology, Wiesbaden, Germany, ${ }^{2}$ Dr. Horst-Schmidt-Kliniken (HSK), Institute of Pathology and Cytology, Wiesbaden, Germany

Aims. Laser Capture Microdissection (LCM) permits the enrichment of tumor cells from formalin fixed paraffin embedded (FFPE) sections 
through a focused laser beam which cuts accurately defined tumor areas for further DNA analysis.

Methods. Archival FFPE sections ( $3 \mu \mathrm{m}$ in size) of adenocarcinomas are transferred and heat-fixed on routinely used slides. After that, the slides are deparaffinized and stained with hematoxylin and eosin (HE). For visualization the slides are covered by liquid cover glass. Cut energy of 70 and cut focus of 71 was applied to transfer the tumor cells into an Eppendorf tube containing $15 \mu \mathrm{L}$ ATL buffer. After protein digestion the DNA is isolated through the use of QIAamp DNA Micro Kit (Qiagen, Deutschland) according to the manufacture's protocol "Isolation of Genomic DNA from Laser-Microdissected Tissues". KRAS mutations as well as EGFR mutations in exon 18, 19 and 21 were assayed.

Results. The DNA of FFPE material of distinct adenocarcinomas was isolated by manual and laser capture microdissection to gain further insides into the valid assessment of the EGFR and KRAS mutation status. This paper presents to you a valid method which enables an accurate DNA isolation of tiny amounts from formalin fixed embedded tissue available. As compared to manual microdissection, the mutation status could be also confirmed through laser capture microdissection despite of using tiny amounts of tumorous tissue. 400,00o $\mu \mathrm{m} 2$ (o.4 mm2) tumor material is sufficient to get reliable result. In contrast to most other publications, we used normal routinely used slides (Marienfeld) and normal ATL buffer for sample capturing visualized by HE stain and liquid cover glass.

Conclusions. Laser capture microdissection can be routinely used for critical cases or biopsies which comprises of few cells only. Therefore by using this specialized method tumor cells can be isolated and further be enriched through polymerase chain reaction. Necrosis and inflammatory cells trigged also in case of therapeutic agents, can be excluded which may have an influence on the final result. To analytically differentiate the heterogeneous growth pattern of the primary tumor (proportion of solide, acinary, lepidic, micropapillary or papillary adenocarcinoma), metastases or lymph node involvements, this method is a useful tool. With respect to personalized medicine laser capture microdissection will get more attention in the future.

\section{SA-115}

Quantitative detection of amplifiable genomes in the DNA obtained from FFPE tissue: proposal of an improved diagnostic algorithm for EGFR mutation analyses in NSCLC

\section{A. Roth ${ }^{* 1}$, A. Streubel', O. Landt' ${ }^{2}$ S. Stephan-Falkenau', T. Mairinger' 'Institut für Gewebediagnostik, MVZ des HELIOS Klinikum Emil von Beh- ring, Berlin, Germany, ${ }^{2}$ TIB Molbiol Berlin, Berlin, Germany}

Aims. To analyze data obtained using pyrosequencing and a real-time PCR for EGFR and KRAS mutational testing in 3075 NSCLC patients, respectively. We studied factors that may have had an influence on sensitivity with the aim to develop an ideal diagnostic algorithm for accurate and evidence-based EGFR mutational detection reporting. Methods. DNAs obtained from macro-dissected formalin paraffin embedded tissue from 3049 patients were analyzable by PCR and tested for EGFR and KRAS mutations. In addition to pyrosequencing, we tested specimens in parallel by dideoxy sequencing, clamped real-time PCR, E746_A75odel-specific PCR and $\beta$-globin-based absolute quantification of amplifiable genomes.

Results. The detection rate for EGFR mutations $(n=201)$ was $11 \%$ in KRAS wild type adenocarcinomas $(n=1825)$. Our findings show a high sensitivity of sequencing methods. We found that additional testing using more sensitive targeted PCRs did not increase the detection rate for EGFR mutations. Additional testing of the quantity of amplifiable genomes in the DNA provided the important information on which specimens may fail in the diagnostic process independently from determination of tumor cellularity. Following these findings, a clearly laid out diagnostic algorithm devised to quantitatively identify specimens prone to detection failure was developed.
Conclusions. Determining the quantity of amplifiable genomes in conjunction with estimates of tumor cellularity enables to accurately identify patients with specimens inadmissible to sensitive and reliable mutational testing. Given its impact on treatment outcome we envision the proposed improved diagnostic algorithm will pave the way towards higher quality EGFR mutation testing in routine practice and more accurate clinical data in future trials involving molecular cancer mutation testing and personalized medicine in general.

\section{AG Knochen-, Gelenk- und Weichgewebspathologie Postersession}

\section{SA.1-001}

The macrophage inflammatory proteins MIP1 a (CCL3) and MIP2 a (CXCL2) in implant-associated osteomyelitis

\section{U. Dapunt', S. Maurer², G. Hänsch², M.M. Gaida*3,4}

'Department of Orthopaedics, Ruprecht-Karls-University, Heidelberg, Germany, ${ }^{2}$ Institute of Immunology, Ruprecht-Karls-University, Heidelberg, Germany, ${ }^{3}$ Institute of Pathology, Ruprecht-Karls-University, Heidelberg, Germany, ${ }^{4}$ National Cancer Institute, National Institutes of Health, Laboratory of Immune Cell Biology, Bethesda, United States

Aims. Bacterial bone infections remain a serious complication of endoprosthetic surgery or osteosynthesis following fractures. These infections are difficult to treat, because different species bacteria form biofilms on the implants, which are relatively resistant to antibiotics, but elicit a profound local inflammatory response and eventually loss of bone. In the present study we addressed the question how the local inflammatory response is linked to osteolysis.

Methods. Biopsies were taken during surgery from osteolytic sites, and for comparison from more distant tissue and muscle. By quantitative RT-PCR gene expression of macrophage inflammatory proteins MIP1 a $\left(\mathrm{CCL}_{3}\right)$ and MIP2 a (CXCL2) was tested, as were cell typical markers. Bone degradation, cellular infiltrates and cytokine expression was confirmed by conventional and immune histology. Furthermore, in vitro we tested production of $\mathrm{CCL}_{3}$ and $\mathrm{CXCL}_{2}$ by macrophages and by cultivated osteoblasts and also the induction of osteoclast generation by these cytokines.

Results. CCL3 and CXCL2 were found in osteolytic tissue, and to a lesser degree at more distant sites or in muscle. Gene expression correlated closely with expression of CD14, which was used as marker for monocytes/macrophages. Co-localisation of MIP with monocytic cells could be confirmed by histology. Our in vitro experiments confirmed data in the literature showing that monocytes produce MIPs following stimulation. We now found that also cultivated primary osteoblasts synthesised MIPs when co-cultivated with lipoteichoic acid (LTA) as an example for a bacteria-derived entity, and also when infected with Staphylococcus epidermidis. Co-cultivation of monocytes, isolated from the peripheral blood of healthy donors, induced a differentiation of these cells to osteoclasts, identified as giant cells with multiple nuclei expressing cathepsin $\mathrm{K}$ and tartrate-resistant acid phosphates.

Conclusions. In response to bacterial infection, bone pro-inflammatory cytokines are produced locally, among others the multifunctional chemokines $\mathrm{CCL}_{3}$ and $\mathrm{CXCL}_{3}$. These cytokines can induce generation of bone resorbing osteoclasts, thus providing a link between bacterial infection and osteolysis. 


\section{SA.1-002}

Influence of decalcification on the expression of CD 15 and CD 20 in osteocartilage tissue

\section{J. Schilling*, S. Müller, M. Martin, V. Krenn \\ MVZ für Histologie, Zytologie und molekulare Diagnostik Trier, Trier, Germany}

Aims. In orthopedic pathology the immunohistochemical characterisation of hematopoietic cells is especially in cases of osteoarthritis (OA) of great importance. In this context it is often necessary to this discriminate between a reactive lymphocytosis from a B-cell lymphoma and to evaluate the structure of the granulocytopoesis in decalcified tissues. Therefore a stabile detection of CD 15 and CD 20 is relevant for a definitive diagnosis. In order to evaluate the stability of CD 15 and $C D$ 20 expression in osteocartilage tissue, tissue samples were decalcified under different conditions and the expression-intensity of CD 15 and $\mathrm{CD} 20$ was analysed immunohistochemically.

Methods. Tissues samples from 15 patients were collected within the frame work of histopathological routine diagnosis on the basis of anonymized tissue samples. Grading of cartilage degeneration was done according to the Otte-Score in EDTA decalcified tissue samples, synovialitis classification was done a cording to the Synovialitis-score. Two experimental EDTA decalcification settings were performing. EDTAsetting 1: Room temperature $\left(22^{\circ} \mathrm{C}\right)$. Decalcification periods ranged from 1 day, to 3 days, to 6 days, and to 9 days. EDTA-setting 2: Temperature $37^{\circ} \mathrm{C}$. Decalcification periods 1 day, to 3 days, to 6 days, and to 9 days. Immunohistochemical analysis was carried out with Ventana Benchmark XT with DAB-Substrat, counterstaining with Hämalaun. The intensity of CD 15 and CD 20 expression was evaluated according to the immunohistochemical Her-2 expression Score (o to $3^{+}$).

Results. In both experimental settings the expression of CD 15 was steady and intense (3+). There was no reduction of the intensity in neutrophil granulocytes. By contrast CD 20 showed an intense expression only on day $1(3+)$ and a strong reduction of the intensity after day 3 (from $2+$, to $1+$ and to 0 ).

Conclusions. CD 15 may be regarded in respect to EDTA-decalcification procedures as a robust $\mathrm{CD}$ antigen whereas $\mathrm{CD} 20$ is sensitive to EDTA decalcification-time and decalcification-temperature. This finding has implications concerning the detection of $\mathrm{CD} 20$ positive intraosseous lymphatic infiltrates especially in respect of B-cell lymphoma detection and lymphoma diagnosis.

\section{SA.1-003 \\ Particle algorithm for joint implant material characterisation in the SLIM with focus on lymphocytic adverse reactions}

F. Hopf ${ }^{* 1}$, M. Huber', M. Thomsen ${ }^{3}$, S. Müller', B. Kölbel', J. Kretzer', P. Thomas ${ }^{5}$, T. Gehrke ${ }^{6}$, V. Krenn ${ }^{7}$

${ }^{1} M V Z$ für Histologie, Zytologie und molekulare Diagnostik Trier, Trier, Germany, ${ }^{2} \mathrm{SMZ}$ Otto Wagner Spital, Pathologisch-Bakteriologisches Institut, Wien, Austria, ${ }^{3}$ Klinik für Orthopädie, DRK Klinik, Baden-Baden, Baden-Baden, Germany, ${ }^{4}$ Labor für Biomechanik und Implantatforschung, Klinik für Orthopädie und Unfallchirurgie, Universitätsklinikum Heidelberg, Heidelberg, Germany, ${ }^{5}$ Klinik und Poliklinik für Dermatologie und Allergologie der LMU, München, München, Germany, ${ }^{6} \mathrm{HELIOS}$ ENDO-Klinik, Hamburg, Hamburg, Germany

Aims. In histopathological SLIM diagnostics (synovial-like interface membrane) the particle identification is due to the heterogeneity of implant materials, due to its differential diagnosis to the non prosthesis-material particles a great challenge. Even more the pathogenetic relevance of lymphocytic infiltrates in the SLIM as a possible substrate for an allergic type IV reaction to implant materials is unclear and no defined criteria exist for adverse reactions.
Methods. This particle algorithm includes: 1) conventional light microscopy characteristics with a guide to sizing, shaping and coloring, 2) polarization optical criteria, 3) enzyme-histochemical characteristics (oil red staining and Prussian blue reaction) and 4) a semiquantitative evaluation of $\mathrm{CD}_{3}$ positive lymphocytes and CD68 positive macrophages in the SLIM.

Results. 45 SLIM-cases had been classified according to the SLIM-consensus classification. A complete spectrum of morphological detectable particles (metals, ceramics and polymers) could be defined. The immunohistochemical evaluation showed a high content of CD68 cells and a rather small content of $\mathrm{CD}_{3}$ cells. $\mathrm{CD}_{3}$ positive lymphoyctes where focally distributed 1-in the in the vicinity of small blood vessels, 2-close to necrotic areas and 3-close to macroparticles especially to PMMA vacuoles.

Conclusions. In all SLIM Typ I cases, obviously independent from the implant material qualities, $\mathrm{CD}_{3}$ positive infiltrates could be detected in three different infiltrate patterns. The focal association of the $\mathrm{CD}_{3}$ infiltrates with implant particles suggest a functional relationship of the $\mathrm{CD} 3$ response and the material. Since a direct abrasion particle-induced inflammatory effect in the SLIM cannot be differentiated from a hypersensitivity type IV reaction a correlation with allergological data is needed.

\section{SA.1-004 \\ Consensual SLIM classification concerning endoprosthetical dys- functions after total ankle prosthesis}

S. Müller ${ }^{* 1}$, R. Frhr. von Welser', M. Walther ${ }^{3}$, B. Kölbel', F. Hopf', V. Krenn ${ }^{1}$ 'MVZ für Histologie, Zytologie und molekulare Diagnostik Trier, Trier, Germany, ${ }^{2}$ Chirurgische Klinik, Endocentrum, Schongau, Germany, ${ }^{3}$ Schön Klinik, Fußchirurgie. Sprunggelenkchirurgie, München, Germany

Aims. Histopathological standardisation concerning endoprosthetical dysfunctions of the talocrural joint does not exist. The histopathological particle algorithm of the synovia like interface membrane (SLIM) was originally developed for periprosthetical membranes of knee and hip. This enables us to clarify any causes of endoprosthetical dysfunction including clinical prognosis by standardised diagnostic procedure techniques in detail. For aetiological investigation, the aim is to develop a subclassification especially for periprosthetical membranes of the talocrural joint by method of transfer.

Methods. We examined 100 cases of periprosthetical membranes of the talocrural joint with the standardised histopathological Consensus Classification. Therefor a particle algorithm had been created which is based on 1) conventional transmitted light microscopy with a guide to sizing, shaping and coloring 2) polarization optical criteria and 3) enzyme-histochemical properties (Prussian blue reaction).

Results. According to the Consensual SLIM-Classification, we mostly identified abrasion-induced types of the synovia like interface membrane and secondly the indifference-types. One remarkable characteristic of the specific localisation was the detection of small cuboidal particles in a brown matrix, which we identified as Calcium-Pyrophosphatedepositions (CPPD). Pseudo-cystic structures and necrosis were over average.

Conclusions. The Consensual Classification of Synovia like Interface membrane fundamentally applies to periprosthetic membranes of the ankle joint. In all cases $(\mathrm{n}=100)$ we found a similar distribution of SLIMsubtypes (Types I-IV) in comparison to other anatomical regions. One important difference is the detection of Calcium-Pyrophosphate-Depositions (CPPD), necrosis and pseudocystic structures. Calcium-Pyrophosphate-Particles are discussed as possible pathogenetic factor of third-body-abrasion: In this specific localisation detected Calcium-Pyrophosphate-Depositions (CPPD) may be seen as independent substrate of endoprosthetical surface destruction, which could be examined by further biomechanical analysis. 


\section{SA.1-005}

\section{A case of a malignant bone tumor in Iron Age Switzerland}

\section{N. Moghaddam*1, R. Langer', S. Ross ${ }^{3}$, F. Müller', S. Lösch}

${ }^{1}$ Institute of Legal Medicine, Department of Physical Anthropology, University of Bern, Bern, Switzerland, 'Institute of Pathology, University of Bern,

Bern, Switzerland, ${ }^{3}$ Institute of Legal Medicine, Center of Forensic Imaging, University of Bern, Bern, Switzerland, ${ }^{4}$ Bernisches Historisches Museum, Bern, Switzerland

Aims. The La Tène burial site of Münsingen Rain, in the Canton of Bern in Switzerland, is regarded as one of the most important Iron Age burial sites in Europe. One of the 77 individuals showed an unusual alteration of the left proximal humerus, so this bone and additionally the scapula, the skull, and the right femur were retained after discovery in 1904. We tried to classify this lesion by using modern radiographic and histopathologic methods.

Methods. Anthropological examination of sex and age was performed. Radiological examinations included plain x-ray imaging and a multislice CT-scan. For histological analysis, representative samples of the lesion were taken and hard-cut sections were prepared. Histopathologic processing included formalin fixation, decalcification, and paraffin embedding with subsequent cutting and staining with hematoxylin/ eosin and elastica van gieson.

Results. The individual was male. The age at death is classified as over 60 years. There is a bone forming tumor at the left proximal humerus with extraosseous growth and involvement of the adjacent scapula. Radiologic examination showed a large, mainly sclerotic bone tumor with "sunburst" appearance of the periphery. Histology showed an irregular bone formation, consistent with osteoid matrix.

Conclusions. In summary, there are two major differential diagnoses: given that the tumor in the humerus represents a single lesion, it might be considered as a primary malignant bone tumor. Due to the irregular matrix formation resembling osteoid the most likely diagnosis is an osteoblastic osteosarcoma despite the age of the patient and the rather uncommon site, followed by chondrosarcoma with osteoblastic features (e.g. dedifferentiated chondrosarcoma). An osteosclerotic plasmocytoma (which would be very unlikely) should also be included in the differential diagnosis, as well as an osteoblastic metastasis of a tumor of different primary location.

\section{SA.1-006 \\ Occurrence of secondary aneurysmal bone cyst within myositis ossificans}

\section{S. Söder*1, S. Sesselmann' ${ }^{2}$ A. Hartmann', A. Agaimy ${ }^{1}$}

${ }^{1}$ Institute of Pathology, Friedrich-Alexander-University, Erlangen-Nuremberg, Germany, ${ }^{2}$ Institute of Orthopedic surgery, Erlangen-Nuernberg, Erlangen, Germany

Aims. Secondary aneurysmal bone cysts can be found in a broad range of both benign and malignant bone tumors. For primary aneurysmal bone cysts an association with USP6 locus rearrangement has been found, but so far no specific genetic alteration has been postulated for secondary aneurysmal bone cysts. Despite being quite common in the skeleton, primary and secondary bone cysts outside the skeleton are extremely rare.

Methods. We received a tumor specimen measuring $5.2 \mathrm{~cm}$ from a 43-year-old women with a painful lump in the right thigh. The specimen was formalin fixed, decalcified, paraffin embedded, sectioned and hematoxylin-eosin stained. Immunohistochemical stains for $\mathrm{CD}_{34}$, vimentin and Ki67/Mib-1 were also performed.

Results. The resection specimen displayed the typical histology of myositis ossificans with zonal maturation and entrapped skeletal muscle fibers. The center of the myositis ossificans contained blood filled cystic spaces with hemorrhages, mononuclear fibrous stroma and multiple multinucleated osteoclastic giant cells in the adjacent stroma, features consistent with a typical aneurysmal bone cyst. Medical history of the patient revealed a trauma in the area of the tumor several months before the onset of the pain.

Conclusions. Aneurysmal bone cysts can occur outside of the skeleton, but their occurrence within myositis ossificans is exceedingly rare. They may cause diagnostic confusion or challenge as they can display untypical clinical and especially radiologic features.

\section{SA.1-007}

\section{Clinicopathological parameters of tumor regression}

\section{A. Mohammed* ${ }^{*}$ l. Petersen}

Institute for Pathology, University Hospital Jena, Jena, Germany

Aims. To evaluate clinicopathological parameters of tumor regression based on published reports.

Methods. A retrospective analysis on published cases with (spontaneous) tumor regression was performed. The study was based on a PubMed literature search using the key word "spontaneous tumor regression". From the list of published reports the first 300 were analyzed in details regarding the following parameters: (complete or partial) spontaneous tumor regression, sex, histological tumor type and in particular factors that were associated with regression. The parameters were analyzed with respect to different age groups.

Results. In total, 4987 cases were found in PubMed being associated with the search key words. Of these, 300 cases were assessed in details. Patients' age ranged from newborns to adults with a maximum age of 91. The collective harboured more males $(58.67 \%)$ than females $(40 \%)$. In (1.33\%) of cases, the sex remained unknown. Regression was reported particularly in malignant tumors (80.33\% malignant versus $19.67 \%$ benign). The majority was non-metastatic $(64.67 \%$ versus $35.33 \%)$. A subgroup of 201 had no documented treatment. SR was categorized into complete or partial regression, age group and tumor type. We found complete SR most commonly at age group $21-30(84 \%)$, while partial SR commonly in older patients, e.g. aged $81-90$ (50\%). The most common tumor type with spontaneous regression at young ages (o-10) was osteochondroma (22.58\%). Reports on regression being associated with treatment or drug intake were found in 99 cases. Radiotherapy was reported in 22 cases (7.33\%), regression occurred most frequently in seminoma. In 19 cases (6.33\%), conventional chemotherapy was associated with regression, notably in lymphoma. Surgery leading to tumor regression was reported in 20 cases $(6.67 \%)$, the most frequent tumor type was renal cell carcinoma. Drug treatment other than conventional chemotherapy was applied in 50 cases $(16.67 \%)$, again the most frequent tumor type was lymphoma.

Conclusions. Complete SR was more frequent than partial SR. The tumor types showing regression different according to the age groups. The study revealed some interesting finding on the still enigmatic phenomenon of tumor regression.

\section{SA.1-008}

The neovascularization in degenerated intervertebral discsits pathophysiological and diagnostic role

\section{Brochhausen*1, D. Blaschke ${ }^{2}$, C.J. Kirkpatrick ${ }^{2}$}

${ }^{1}$ Institute of Pathology, University Medical Centre of the Johannes Gutenberg-University, Mainz, Germany, ${ }^{2}$ Institute of Pathology, University Medical Centre, REPAIR-Iab, Mainz, Germany

Aims. Herniated discs are a common condition and are almost exclusively caused by degenerative changes in intervertebral discs. Despite several histological score-systems for the analyses of disc degeneration, the "histologic degeneration score" (HDS) by Weiler et al. is so far the single classification system for pathological assessment of material from 


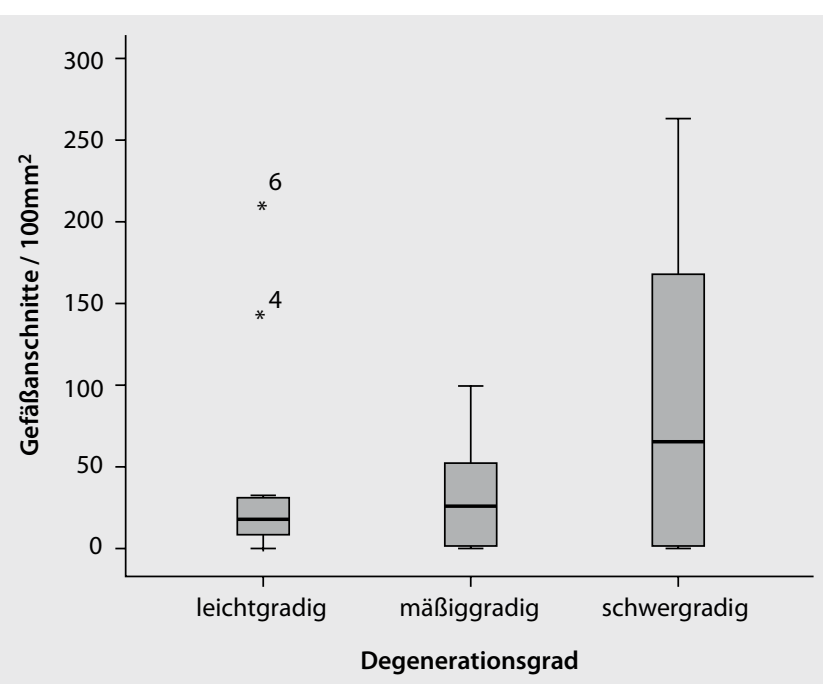

Fig. 1 | SA.1-008 $\Delta$ Distribution of vessel cross section $/ 100 \mathrm{~mm}^{2}$ differentiated by degree of degeneration

nuclectomies. Therefore it is currently used as guidance in routine diagnostics since the evaluation system is very complex. The HDS does not include evaluation of vascular ingrowth into this avascular tissue under physiological conditions. The presence, extent and significance of neovascularization in degenerated intervertebral discs are controversial discussed for a long time in literature. Aim of the present study was to compare the quantitative extent of vascular invasion and the degree of degeneration.

Methods. 35 samples from nuclectomies were analysed histologically. The determination of the degree of degeneration (mild $=9$, moderate $=11$, severe $=15$ ) was based on HE and Alzian staining based on the HDS of Weiler et al. Blood vessels were visualized by immunohistological staining against $\mathrm{CD}_{31}$ with monoclonal antibodies. The cross sections of vessels were quantified and compared with the histological degree of degeneration

Results. Totally, in $74.3 \%$ of all samples vessels could be detected regardless of the degree of degeneration. Furthermore, there was a visible tendency occurring with increasing degree of degeneration to increasing ingrowth of vessels. This finding was not statistically significant ( $\mathrm{rs}=0.17, \mathrm{p}=0.165$ ). In addition, an average of $16 \%$ (range $5-40 \%$ ) of all vessels exhibited activated endothelial cells.

Conclusions. To our knowledge, this study is the first quantitative analyses of vessel in degenerative disc degeneration, which revealed a tendency to a higher amount of vessels with increasing level of degeneration. Previous studies raised the level semi-quantitatively. The finding of activated endothelial cells indicates the potential role of inflammation in the pathogenesis of degenerative disc disease. Further prospective studies should illuminate the potential role of the inflammatory reaction for the neovascularization (• Fig. 1).

\section{SA.1-009}

Assessment of the relationship between the histopathological degree of temporo-mandibular joint disc degeneration and clinical and imaging findings

\section{S. Söder ${ }^{* 1}$, M. Kramer' ${ }^{2}$ E. Nkenke ${ }^{3}$, A. Hartmann'}

'Institute of Pathology, Friedrich-Alexander-University, Erlangen-Nuremberg, Germany, ${ }^{2}$ Institute of diagnostic Radiology, University of ErlangenNuernberg, Erlangen, Germany, ${ }^{3}$ Institute of mandibulofacial surgery, University of Erlangen-Nuernberg, Erlangen, Germany

Aims. Lack of lubrification, trauma, functional overloading, joint laxity, hyperactivity of the lateral pterygoid muscle as well as occlusal factors can damage the temporomandibular joint (TMJ) and lead to subsequent disc degeneration. The exact mechanisms leading to the joint damage are still not completely understood. The aim of the present study was to apply a histological scoring system to TMJ joint disc specimens and to check if there is a correlation to clinical and imaging findings.

Methods. Twenty patients (age 32-84) undergoing TMJ discectomy were included in the study. All patients suffered from intolerable unilateral joint pain and joint dysfunction which failed to respond to at least 12 months of nonsurgical treatment. MRI imaging of the TMJ region was performed with a 1.5-Tesla Magnetom Aera device. Discectomy was performed in all cases following a standardized surgical procedure. The joint disc specimen was fixed in formalin, embedded in paraffin and stained with hematoxylin-eosin. For evaluation of iron deposits classic Prussian blue reaction was used. Pathological evaluation was performed according to the three-tiered histological degeneration grading score system for menisci proposed by Krenn et al. 2010 which ranges from low grade (grade 1) to high grade (grade 3). The statistical data evaluation included clinical, radiological (MRI) and histopathological findings.

Results. Correlation between histopathological findings and MRI showed a high level of concordance. Scarring of the disc could be detected in every sample, and scarring was significantly more pronounced in male patients compared to female patients. All samples showed significant signs of degeneration, with most samples demonstrating medium to high grade degeneration (average grade 2.4 \pm 0.5 . There was no significant difference between younger $(<50)$ and older patients regarding the degree of degeneration.

Conclusions. Discectomy leads to a good outcome in about $60 \%$ of the cases. To minimize the number of unfavorable outcomes it is essential to increase our understanding of the mechanisms leading to the damage of the TMJ. So far the usage of non standardized or too complex histologic scoring systems has made it difficult to compare results between different studies and different treatments. The standardized use of a straightforward scoring system for disc degeneration would make it easier to evaluate new treatments and would also be very helpful for future research into the development of degeneration of the TMJ.

\section{SA.1-010}

Correlation of histopathology and microbiology: defined criteria of non-specific bacterial infection in synovitis and osteomyelitis improves the diagnostic safety

B. Kölbel*1, V. Krenn', S. Müller', A. Tiemann'², U. Illgner ${ }^{3}$

'MVZ für Histologie, Zytologie und molekulare Diagnostik Trier, Trier, Germany, ${ }^{2}$ Berufsgenossenschaftliche Kliniken Bergmannstrost, Halle, Germany, ${ }^{3}$ St. Josef-Stift Sendenhorst, Sendenhorst, Germany

Aims. Especially in joint tissue samples microbiological detection of bacteria is not always successful especially when an antibiotic treatment has already been started or in cases of low virulent bacteria. Therefore, the histopathological diagnosis of an inflammatory reaction as substrate of a bacterial infection, especially of non-specific bacterial infections is very important. 
Methods. In this retrospective analysis tissue samples (synovial tissue/ periarticular tissue, $\mathrm{n}=2 \mathrm{O}$ and bone tissue samples; $\mathrm{n}=25$ ) were collected within the framework of histopathological routine diagnosis. Histopathological diagnosis was carried out by conventional enzyme techniques (PAS) and immunohistochemical techniques (CD15). Infectious bacterial synovitis was defined by: 1) diffuse infiltration of neutrophil granulocytes, 2) grouped position of at least five neutrophil granulocytes (micro-abscesses), 3) necrosis, 4) ulceration of synovial lining and 5-Fibrin-insudations. Infectious bacterial osteomyelitis was defined by points 1 and 2 of bacterial synovitis criteria and 3 ) necrotic bone tissue/ bone trabecula, 4) intramedullar fibrin extravasations, 5) macrophage-, lymphocyte- and plasmacell infiltrations and 6) loss of haematopoesis Results. Staphylococcus aureus was the most commonly detected bacteria by microbiology, in 4 cases bacteria could not be demonstrated. The correlation between the histopathological diagnosis of infection and positive microbiology was $93 \%$.

Conclusions. Especially in patients with Rheumatoid arthritis the combination of histopathology and microbiology significantly increased the safety of detecting an infection or tissue contamination. We therefore recommend that bacterial infection diagnosis (non-specific bacterial infection) of joints should be carried out according following defined criteria: 1) diffuse infiltration of neutrophil granulocytes, 2) grouped position of at least five neutrophil granulocytes (micro-abscesses), 3) necrosis and in cases of osteomyelitis, 4) loss of haematopoesis.

\section{AG Urologische Pathologie Postersession I}

\section{SA-116 \\ A combined dual-color chromogenic and silver in situ hybridization (PTEN/Ch10) assay improves the detection of PTEN loss in patients with prostate cancer} Torres $^{2}$, T. Ruffalo' ${ }^{2}$, K. Sallam ${ }^{2}$, H. Moch', W. Jochum ${ }^{3}$, P.J. Wild'

'Department of Pathology, University Hospital, Zurich, Switzerland, ${ }^{2}$ Roche Ventana Medical Systems Inc., Tucson, Arizona, United States, ${ }^{3}$ Institute of Pathology, Cantonal Hospital St. Gallen, Switzerland

Aims. Inactivation of the tumor suppressor gene PTEN (phosphatase and tensin homologue) has been found as a recurrent and prevalent event in prostate cancer with prognostic utility, qualifying for routine clinical use. To detect PTEN deletions, fluorescence in situ hybridization (FISH) is the method of choice. FISH, however, shares some disadvantages for widespread and routine application in daily clinical practice. Chromogenic in-situ hybridization (CISH) using organic chromogens and enzymatic metallography silver in-situ hybridization have emerged as promising bright-field alternatives.

Methods. We established a dual-color assay using combined silver in situ hybridization for detection of PTEN loss (black signals) and chromogenic in situ hybridization for detection of chromosome 10 centromeric region (red signals). We compared these results with those obtained by PTEN FISH. Furthermore, each specimen was reviewed and scored for viable DNA by ALU II staining. The Alu II probe detects Alu repeat sequences of human DNA present in formalin-fixed, paraffin-embedded human tissues by silver in situ hybridization. Alu, an important group of widely distributed sequence repeats in the human genome, provides a positive control for viable DNA in in-situ hybridization detection systems. A tissue microarray with 87 prostate tissue cores was assessed for their PTEN loss by applying ALU II, dual-color FISH and dual-color CISH assays, respectively.

Results. Of the 87 tissue cores, 15 (17\%) were excluded from further analyses due to unviable DNA as shown by lack of ALU II staining or lack of tissue. In only 7 (9.7\%) out of final 72 analyzable tissue cores the CISH assay was not adequate, mainly due to weak read signals of the centro- meric probe. A high concordance between FISH and CISH based results could be found.

Conclusions. Our findings demonstrate that the PTEN status can be reliably detected by CISH. The CISH technique combines the accuracy and precision of FISH with the ease of bright-field microscopy, and may allow the fast and routine detection of PTEN loss also in prostate needle biopsies with minute cancer infiltrates.

\section{SA-117}

\section{CD57 expression in prostate cancer}

H. Wangerin*1, G. Kristiansen ${ }^{2}$, T. Schlomm 3 , C. Stephan ${ }^{4}$, S. Gunia ${ }^{5}$, A. Zimpfer', W. Weichert ${ }^{6}$, G. Sauter ${ }^{7}$, A. Erbersdobler ${ }^{1}$

'Institute of Pathology, University Medicine Rostock, Rostock, Germany, ${ }^{2}$ Institute for Pathology, University of Bonn, Bonn, Germany, ${ }^{3}$ Martini-Clinic, Prostate Cancer Center, University Medical Center Hamburg Eppendorf, Hamburg, Germany, ${ }^{4}$ Department of Urology, Charité University Hospital, Berlin, Germany, ${ }^{5}$ Institute of Pathology, Stendal, Germany, ${ }^{6}$ Institute of Pathology, Ruprecht-Karls-University, Heidelberg, Germany, ${ }^{7}$ Institute of Pathology, University Medical Center Hamburg Eppendorf, Hamburg, Germany

Aims. CD57 is an antigen normally expressed on natural killer lymphocytes. However, an expression of CD57 has also been reported in prostate cancer by some studies from the nineteen-eighties, but this fact has nearly fallen in oblivion by pathologists today. The aim of this study was to analyze $\mathrm{CD}_{57}$ expression in a large set of prostate cancers with modern techniques.

Methods. We performed a comprehensive immunohistochemical investigation on CD57 expression in 3262 prostate carcinomas, using five sets of tissue microarrays from 3 different institutions comprising incidental-, and clinically manifest tumors, as well as lymph node metastases and benign prostatic tissue. The results were compared with Gleason grade, pT-classification, tissue-based PSA expression, and clinical follow-up.

Results. Overall, $87 \%$ of prostate cancers showed a moderate or strong expression of $\mathrm{CD}_{57}$. There was no significant difference to corresponding benign prostatic tissue. Loss of $\mathrm{CD}_{57}$ expression slightly increased from incidental over clinically manifest cancers to metastases, and correlated significantly with Gleason grade, pT-classification, and PSA recurrence after prostatectomy. CD57 was not in every case coexpressed with PSA. It showed a significant heterogeneity, especially in large, high-grade tumors.

Conclusions. There is a peculiar expression of $\mathrm{CD}_{57}$ in carcinomas and benign tissues from the prostate gland. Loss of $\mathrm{CD}_{57}$ expression seems to be associated with dedifferentiation and tumor aggressiveness. The marker is suitable for the identification of prostatic tissue, and for prognostic considerations in prostate cancer. However, its usefulness is hampered by the heterogenous expression in advanced tumors.

\section{SA-118}

\section{Integrins are differentially expressed in prostate cancer}

\section{K. Heß ${ }^{* 1}$, C. Böger², H.-M. Behrens' ${ }^{2}$, C. Röcken ${ }^{2}$}

'Institute of Pathology, Kiel, Germany, ${ }^{2}$ Institute of Pathology, Kiel, Germany

Aims. Integrins are transmembrane receptors which mediate cell signaling pathways and play important roles in the pathology of tumor progression and metastasis. In this retrospective study we evaluated and characterized integrin expression in prostate cancer (PC) and correlated the expression with various clinicopathological patient characteristics.

Methods. Information on 1284 patients (median age 65 years) with PC who had undergone radical prostatectomy spanning the period from 
1997 to 2011 was retrieved from the archive of the Department of Pathology of the University of Kiel. Tissue micro arrays (TMA) were constructed from formalin-fixed and paraffin-embedded (FFPE) tissue samples. Integrin expression was assessed using monoclonal antibodies against $\alpha v \beta_{3}$ (EM22703), $\alpha v \beta_{5}$ (EMo9902), $\alpha v \beta 6$ (EMo5201), $\alpha v \beta 8$ (EM13309), $\alpha$ v-chain (EMo1309) and $\beta_{3}$-chain (EMoo212) separately for 52 whole block sections and for TMAs. Immunostaining was evaluated using the Histo Score (Hscore). Date and cause of patient death was obtained from the Epidemiological Cancer Registry of the state of Schleswig-Holstein, Germany. Fisher's exact test, the Kendall's tau and the log rank test were used to correlate integrin expression with clinical data.

Results. The expression of the integrins was variable with regard to the histo-/cytoanatomical localization (basal, membranous, cytoplasmic), cell type (prostate-cancer cells, non-neoplastic epithelium, stroma cells, perineurium), intensity of immunolabeling, and Gleason pattern. Integrin $\alpha v \beta_{3}$ and $\beta_{3}$ were found in prostate vessels. Anti- $\alpha v \beta 6 \mathrm{immu}-$ nostained the basal layer of benign glands. Anti- $\alpha v \beta 8$ showed a strong staining of peripheral nerve sheaths. Integrin $\alpha v \beta_{5}$ and $\alpha v-p a n$ were almost ubiquitously expressed in prostate cancer cells. Membranous immunostaining with the anti- $\alpha v-$ pan antibody correlated significantly inversely with the Gleason pattern $(\mathrm{p}<0.001)$. Cyoplasmatic immunostaining of tumor cells with anti- $\alpha v \beta_{5}$ correlated significantly with the Gleason pattern $(\mathrm{p}=\mathrm{o} .001) .154$ patients died during the study period, out of which 24 due to prostate cancer. Overall patient survival correlated with membranous $\alpha v \beta 5$-expression $(\mathrm{p}=0.012)$.

Conclusions. Our retrospective study provides strong evidence that integrins are differentially expressed in prostate cancer. Interestingly, differentiation of prostate cancer cells and subcellular distribution of integrins seem to be interrelated. Further studies into this topic are warranted.

\section{SA-119 \\ MUC1 in lymph node metastases independently predicts survival in advanced prostate cancer}

\section{Genitsch*1, I. Zlobec', G.N. Thalmann', A. Fleischmann \\ 'Institute of Pathology, University of Bern, Bern, Switzerland, ${ }^{2}$ Department of Urology, University of Bern, Bern, Switzerland}

Aims. MUC1 is a membrane-bound glycoprotein that belongs to the mucin family. It is involved in cell adhesion and also in intracellular signaling. Aberrant expression of MUC1 has been observed in different carcinomas including prostate cancer, where it might serve as a therapeutic target. Our objective was to investigate MUC1 expression in a cohort of prostate cancer patients with lymph node metastases and correlate it with their clinicopathological features and clinical outcome, since its significance in metastasizing prostate cancer is still unknown.

Methods. MUC1 expression was evaluated on tissue microarrays constructed from 119 nodal positive prostate cancer patients treated by radical prostatectomy and extended lymphadenectomy. MUC1 status was correlated with various tumor features and biochemical recurrence-free (bRFS), disease-specific (DSS) and overall (OS) survival.

Results. In primary tumors, high MUC1 expression was significantly correlated with higher tumor volume $(\mathrm{p}=0.005)$ and T-stage $(\mathrm{p}=0.009)$. Furthermore high MUC1 expression in lymph node metastases corresponded with greater total size of metastases $(\mathrm{p}<0.001)$ and total number of metastases $(\mathrm{p}=0.014)$. High MUC1 expression in lymph node metastases predicted an unfavorable outcome compared to patients with low MUC1 expression (5-year bRFS $\mathrm{p}<0.023$, DSS and OS $\mathrm{p}<0.001$ ). In multivariate analysis high expression level of MUC1 in primary prostate cancer was an independent risk factor for bRFS ( $\mathrm{p}<0.046)$, while in lymph node metastases MUC1 independently predicted DSS ( $\mathrm{p}<0.0038)$.

Conclusions. MUC1 in either primary tumor or lymph node metastases correlates significantly with more unfavorable clinicopathological features. Its relevance on survival is primarily inherent in lymph node metastases from prostate cancer. This indicates an important role of ad-

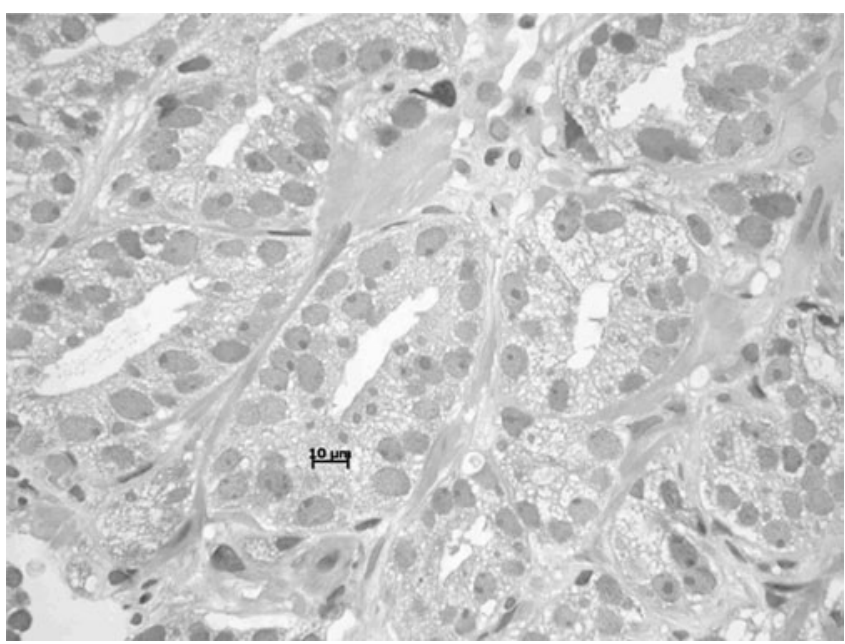

Fig. 1 | SA-120 $\Delta$ Brightly eosinophilic globules in the cytoplasm of carcinoma cells with atypical nuclei and prominent nucleoli

ditional tumor sampling from metastases to utilize the prognostic value of MUC1 in prostate carcinoma. In addition, as MUC1 is a druggable target in prostate cancer it could act as a therapeutic target in metastasized disease.

\section{SA-120}

Adencarcinoma of the prostate with eosinophilic intracytoplasmic globules. First description

\section{R. Bauer*}

Praxis für Pathologie, Bad Oldesloe, Germany

Aims. Eosinophilic intracytoplasmic globules (hyline globules) occur in many benign as well as malignant lesions of various organs. However, in the prostate gland such globules have only been found in benign lesions [1], typically in basal cell hyperplasia [2]. Although the detection of these globules is believed to rule out malignancy, we encountered the following case. A 78-year-old man with a PSA level of $10.3 \mathrm{ng} / \mathrm{ml}$ suffered from chronic urinary obstruction.

Methods. 10 core biopsies were analyzed by histology and immunohistochemistry.

Results. On histology, carcinmatous infiltrates were found in 7 of 10 biopsies. Approximately $20 \%$ of the tumor infiltrates revealed bright eosinophilic globular structures in the apical cytoplasm of the tumour cells, measuring on average $5 \mu \mathrm{m}$ in diameter. The diagnosis of prostatic adenocarcinoma was straightforward in terms of morphology (small tightly packed acini, atypical cells with abundant cytoplasm and prominent nucleoli, basophilic intraluminal secretions) as well as immunohistochemistry (PSA+, PSAP+, AMACR+, HMW-CK-, p63-). Basal cell hyperplasia as well as a non-prostatic adenocarcinoma could be excluded with certainty.

Conclusions. In conclusion, the detection of eosinophilic intracytoplasmic globules in a prostatic lesion is not a unique feature of basal cell hyperplasia and does not rule out malignancy (- Fig. 1).

\section{Literatur}

1. Dikov, D, Roland, J, Chatelet, FP et al (2003) Hyaline globules (Thanatosomes) in prostate disease. Am J Surg Pathol 27(5):700-702

2. Rioux-Leclercq N, Epstein Jl (2002) Unusual morphologic patterns of basal cell hyperplasia of the prostate. Am J Surg Pathol 26(2):237-243 


\section{SA-121}

\section{Ceramic foam plates-a new tool for processing fresh radical prostatectomy specimens}

\section{T. Vlajnic ${ }^{* 1}$, M. Oeggerli', A. Rufle', C. Rentsch ${ }^{2}$, C. Ruiz', L. Bubendorf' 'Institute of Pathology, University Hospital Basel, Basel, Switzerland, 2Department of Urology, University Hospital Basel, Basel, Switzerland}

Aims. There is an increasingly high demand for fresh prostate cancer tissue to study the molecular mechanism of tumor development and progression at high resolution and quality. Fresh cancer tissue is also a precondition to generate preclinical xenograft models as an important step towards new therapeutic strategies in advanced prostate cancer. However, handling of fresh radical prostatectomy specimens and collecting fresh tumor tissue has been notoriously challenging given the distinctive physical properties of prostate tissue and the fact that prostate cancer is often multifocal, heterogeneous and difficult to identify on gross examination. Here, we have developed a novel approach using ceramic foam plates for processing freshly cut whole mount sections from radical prostatectomy specimens without compromising further diagnostic assessment.

Methods. Twenty radical prostatectomy specimens were processed during intraoperative frozen section examination and sectioned from the apex to the base in whole mount slices. Prior to sampling, putative carcinoma foci were morphologically verified by frozen section analysis. The fresh whole mount slices were then pinched between two ceramic foam plates and fixed overnight in 10\% buffered formalin. To test tissue preservation after this procedure, formalin-fixed and paraffin embedded whole mount sections were stained with $\mathrm{H} \& \mathrm{E}$ and analyzed by immunohistochemistry, fluorescence and silver in situ hybridization (FISH and SISH, respectively), and DNA extraction was performed.

Results. There were no morphological artefacts on H\&E stained whole mount sections from slices that had been fixed between two plates of ceramic foam, and the histoarchitechture was fully preserved and unaffected. The quality of immunohistochemistry, FISH or SISH and the extracted DNA was equivalent to or superior as compared to traditionally processed radical prostatectomy specimens.

Conclusions. Fixation with ceramic foam plates in combination with frozen section examination is an excellent method for processing fresh radical prostatectomy specimens, allowing for a precise identification and collection of tumor tissue without compromising further diagnostic analysis.

\section{SA-122}

\section{Incidental prostate cancer prevalence at radical cystoprostatecto-} my-importance of the histopathological work-up

T. Vlajnic ${ }^{* 1}$, C. Wetterauer ${ }^{2}$, M. Weibel' ${ }^{2}$ J. Gsponer', T. Zellweger ${ }^{3}$, S. Bütikofer', G. Müller', H. Püschel', A. Bachmann'2, T. Gasser', L. Bubendorf', C. Rentsch ${ }^{2}$

IInstitute of Pathology, University Hospital Basel, Basel, Switzerland, 2Department of Urology, University Hospital Basel, Basel, Switzerland, ${ }^{3}$ Clara Hospital Basel, Basel, Switzerland

Aims. The reported incidental prostate cancer prevalence rates at radical cystoprostatectomy (CP) cover a broad range, from $4 \%$ to $60 \%$. The histopathological work-up has been suggested to have an influence on prostate cancer detection rates.

Methods. We identified 114 patients who had undergone CP for bladder cancer between 2000 and 2012. Complete histopathological assessment was defined as follows: (i) complete embedding of the prostate gland; (ii) sectioning of 15 or more prostate sections; and (iii) processing as whole mount slides. Prostate cancer prevalence rates derived from complete and incomplete histopathological assessments were compared.

Results. Overall prostate cancer detection rate was $59.6 \%$. A mean of 14.4 macroscopic tissue sections (thickness 3-5 $\mathrm{mm}$ ) were sectioned.
Sectioning 15 or more sections ( $52.6 \%$ of the samples) resulted in a prostate cancer detection rate of $75 \%$, compared to $42.6 \%$ when sectioning fewer than 15 sections $(\mathrm{p}<0.001)$. Complete embedding yielded a prostate cancer detection rate of $72.3 \%$ and of $23.1 \%$ for partly embedded samples $(\mathrm{p}<0.0001)$. Prostate cancer was detected in $68.8 \%$ of the whole mounted samples and in $38.2 \%$ of the samples sectioned as standard slides $(\mathrm{p}<0.01) .44 .1 \%$ of the incidental prostate cancers were clinically relevant.

Conclusions. The quality of the histopathological work-up significantly influences prostate cancer detection rates and might at least partially explain the highly variable reported incidental prostate cancer prevalence rates at $\mathrm{CP}$. The high proportion of significant prostate cancer found in our series calls for a careful surgical approach to the prostate during CP.

\section{SA-123}

Myoglobin expression in prostate cancer is correlated to androgen receptor expression and markers of tumor hypoxia

S. Meller ${ }^{* 1}$, A. Bicker ${ }^{2}$, M. Montani ${ }^{3}$, K. Ikenberg ${ }^{4}$, B. Rostamzahdeh', P. Wild ${ }^{4}$, D. Dietrich', T. Sulser', H. Moch ${ }^{4}$, T.A. Gorr ${ }^{5,6}$, C. Stephan 7 , K. Jung ${ }^{7,8}$, T. Hankeln ${ }^{2}$, G. Kristiansen'

${ }^{1}$ Institute of Pathology, University Hospital Bonn, Bonn, Germany, ${ }^{2}$ Institute of Molecular Genetics, Johannes Gutenberg University, Mainz, Germany, ${ }^{3}$ Institute of Pathology, University of Bern, Bern, Switzerland, ${ }^{4}$ Institute of Surgical Pathology, University Hospital Zurich, Zurich, Switzerland, ${ }^{5}$ Institute of Veterinary Physiology, Vetsuisse Faculty, University Zurich, Zurich, Switzerland, ${ }^{6}$ Clinic IV, Div. of Pediatric Hematology and Oncology, University Medical Center, Freiburg, Germany, ${ }^{7}$ Department of Urology, Charité—Universitätsmedizin Berlin, Berlin, Germany, ${ }^{8}$ Berlin Institute of Urologic Research, Berlin, Germany

Aims. Recent studies identified unexpected expression and transcriptional complexity of the hemoprotein myoglobin (MB) in human breast cancer but its role in prostate cancer is still unclear.

Methods. Expression of MB was immunohistochemically analysed in two independent cohorts of radical prostatectomy specimens $(n=220$ and $n=625$ ). MB expression data was correlated to clinicopathological parameters and molecular parameters of androgen and hypoxia signalling. Expression levels of novel tumor-associated MB transcript variants and the VEGF gene were analyzed using qRT-PCR.

Results. $40 \%$ and $50 \%$ of the prostate cancer cases, respectively, were $\mathrm{MB}$ positive and significantly correlated with androgen receptor (AR) expression $(\mathrm{p}=0.018$ and $\mathrm{p}=0.001)$ and in one cohort even with a better prognosis (log rank test, $\mathrm{p}=0.018$ ). The positive correlation with CAIX $(\mathrm{p}<0.001)$ and FASN $(\mathrm{p}=0.047$ and $\mathrm{p}=0.056)$ as well as the paralleled increased expression of the tumor-associated $\mathrm{MB}$ transcript variants and VEGF suggest that hypoxia participates in MB expression regulation. Conclusions. Analogous to breast cancer, MB expression in prostate cancer is associated with steroid hormone signalling, markers of hypoxia and a possibly less aggressive clinical behaviour. Further studies to elucidate the new functional roles of $\mathrm{MB}$ in human carcinomas beyond the intra-muscular function of oxygen storage are clearly warranted. 


\section{SA-124}

\section{Prognostic grade grouping of prostatic carcinoma after combined} histological and cytological grading

\section{Ringli*1, J. Tonhauser', I. Poser ${ }^{3}$, H.-H. Seifert', J. Breul', B. Helpap ${ }^{1,5,6}$ 'Department of Pathology, HBH-Hospital, Singen, Germany, ${ }^{2}$ Department of Urology, HBH-Hospital, Singen, Germany, ${ }^{3}$ Dept. Urology, Lorretto Hospital, Frfeiburg, Germany, ${ }^{4}$ Dept. Urology, Lorretto Hospital, Freiburg, Germany, ${ }^{5}$ Department of Pathology, HBH-Hospital, Singen, Germany, ${ }^{6}$ Department of Pathology, HBH-Hospital, Singen, Germany}

Aims. Former outcome studies mainly base on the old grading system of Gleason. Modification of grading of Gleason by the International Society of Uropathology (ISUP; 2005/2010) lead to a distinct separation of pattern 3 and 4 . Three prognostic groups of low, intermediate and high grade malignancy result from that. This three-part separation is base of the prognostic grade grouping by Pierorazio, Carter, and Epstein 2012. Methods. The five-part prognostic grade grouping after radical prostatectomy was examined by means of combination of histological-cytological grading of Gleason and Helpap. Data of progress of PSA and survival or cancer specific death were registered between 2005 and 2013 Results. In group I/V there exists a clear situation of grading and prognosis for low risk carcinomas Gleason score 6/Helpap 2a without progress of PSA and without cases of death specific for carcinomas with active surveillance as option of therapy. The most often existing group II/V $\left(\mathrm{GS}_{3}+4=7 \mathrm{a} / 2 \mathrm{~b}\right)$ shows a prognostic demarcation of the intermediary group of prognosis with low progress of PSA $(5 \%)$ without death of disease (DoD) of low risk and high risk carcinomas with active options of therapy i.e. radical prostatectomy. Regarding high grade (risk) carcinomas a prognostic demarcation of GS $7 \mathrm{~b}, 8$, and 9-10 carcinomas concerning TNM and rates of death specific for carcinomas is necessary. The prognostic situation of GS $7 \mathrm{~b}$ carcinomas is clearly more favorable without DoD. The rate of biochemical recurrence does not differ significantly within the group of high risk carcinomas.

Conclusions. This scale of prognosis of carcinoma of prostate is a good pattern for urologists as well as for pathologists.

\section{SA-125 \\ Prognostic significance of IMP3 tissue and serum levels in prostate cancer}

H. Reis*1, S. Tschirdewahn'2, C. Niedworok', G. Kramer', S. Sevcenco ${ }^{3}$, S.F. Shariat ${ }^{3}$, H. Rübben ${ }^{2}$, F. vom Dorp², T. Szarvas ${ }^{3}$

'Institute of Pathology, University Hospital of Essen, University of DuisburgEssen, Essen, Germany, ${ }^{2}$ Clinic for Urology, University Hospital of Essen, University of Duisburg-Essen, Essen, Germany, ${ }^{3}$ Department of Urology, Medical Faculty, Vienna, Austria

Aims. The prognostic influence of protein tissue expression levels of the oncofetal protein insuline-like growth factor-II mRNA-binding protein-3 $\left(\mathrm{IMP}_{3}\right)$ has been demonstrated in various human malignancies while its serum levels yet have not been analyzed. Aim of this study was to assess the prognostic significance of both serum and tissue levels of $\mathrm{IMP}_{3}$ in prostate cancer (PC).

Methods. $\mathrm{IMP}_{3}$ protein expression was analyzed in $124 \mathrm{PC}$ and 13 benign prostate hyperplasia (BPH) patients using immunohistochemistry. Gene expression levels of IMP 3 and its molecular target IGF2 were analyzed by real-time PCR in 29 frozen tissue samples of PC. ELISA was used to assess serum $\mathrm{IMP}_{3}$ levels in $94 \mathrm{PC}, 20 \mathrm{BPH}$ and 20 controls. Clinicopathological and follow-up data were collected and correlated with the results.

Results. IMP 3 immunostaining was present in $0 \%$ of BPHs, $15 \%$ of clinically localized PCs and $65 \%$ of palliatively treated metastatic PCs ( $\mathrm{p}<\mathrm{0.001)}$. Accordingly, lowest serum $\mathrm{IMP}_{3}$ concentrations were detected in controls, intermediate in BPH patients, higher in PC $(\mathrm{p}<0.001$ each) and highest in metastatic PC patients $(\mathrm{p}=0.036)$. Poor cancer- specific survival was independently associated with high $\mathrm{IMP}_{3}$ serum levels in patients who underwent radical prostatectomy.

Conclusions. $\mathrm{IMP}_{3}$ levels were identified to be higher in tissue and serum samples of $\mathrm{PC}$ patients compared to non-PC thus allowing $\mathrm{IMP}_{3}$ serum levels to identify a group of high-risk $\mathrm{PC}$. $\mathrm{IMP}_{3}$ serum levels were additionally of prognostic value being associated with metastasis and PC cancer-specific survival.

\section{SA-126}

The use of different prostate-specific markers in the diagnosis of metastatic prostate cancer

\author{
A. Queisser*1,2, S. Hagedorn ${ }^{1,2}$, W. Vogel ${ }^{1,2}$, N. Wernert ${ }^{1}$, G. Kristiansen ${ }^{1}$, \\ S. Perner ${ }^{1,2}$ \\ 'Institute for Pathology Bonn, Bonn, Germany, ${ }^{2}$ Institute of Pathology, \\ Department of Prostate Cancer Research, Bonn, Germany
}

Aims. Prostate cancer (PCa) is the most commonly diagnosed cancer and second most common cause of cancer death in the western world. While treatment is often successful in early tumor stages, some cancers are initially diagnosed in an advanced metastatic stage. In the scenario of a cancer of unknown primary origin (CUP) it is important to determine the tumor origin in order to allow a specific treatment. Requirements for diagnostic cancer markers are a high sensitivity and specificity. PSA is used as the standard tumor marker for PCa and is highly expressed in primary PCa but the expression level declines with advanced tumor stage. The aim of this study was to identify the most potent prostate-specific marker or marker constellation which is able to detect metastatic PCa at high sensitivity and specificity. Therefore known prostate-specific markers like prostate-specific membrane antigen (PSMA), prostate-specific antigen (PSA), androgen receptor (AR), prostate secretory protein (PSP) and prostein were included in this study.

Methods. Expression of PSMA, PSA, AR, PSP and prostein was determined on paraffin-embedded tissue of a patient cohort consisting of lymph node metastases $(n=54)$ and distant metastases $(n=32)$. The analysis included triplicate biopsy cores of each patient. Staining against PSMA, PSA, AR, PSP and prostein were performed with immunohistochemistry and analyzed by two independent pathologists. Staining was assessed as positive for PSMA and PSA if the staining was apical, for AR if the staining was nuclear and for PSP and prostein if the staining was cytoplasmic.

Results. We could show that for the identification of metastatic PCa a combined staining of three prostate-specific markers should be used. In detail, $92.2 \%$ of lymph node metastases were positive for PSMA, $88.9 \%$ for PSA, $72.2 \%$ for AR, $85.2 \%$ for PSP and $81.5 \%$ for prostein. In contrast, distant metastases showed an expression rate of $93.7 \%$ for PSMA, 93.7\% for PSA, $90.6 \%$ for AR, $71.9 \%$ for PSP and $84.4 \%$ for prostein. The best marker combination in our cohort with a sensitivity of $97.5 \%$ were PSMA, PSA and AR in metastatic PCa.

Conclusions. Our data show that a combined staining of at least three prostate-specific markers should be utilized to identify PCa metastases. Moreover a positive staining of this combination could also assign CUP as cancers of prostate origin. 


\section{AG Urologische Pathologie Postersession II}

\section{SA-127 \\ Clear cell papillary renal cell carcinoma-a new distinct entity of renal cell carcinomas}

K.-F. DemI*1, H.-U. Schildhaus' ${ }^{2}$, E. Compérat ${ }^{3}$, A. von Teichmann', M. Storz', P. Schraml', M. Anlauf', F. Fend', B. Fleige ${ }^{6}$, A. Förster', N. Gaßler ${ }^{8}$, C. Girardet $^{9}$, R. Grobholz ${ }^{10}$, S. Hailemariam ${ }^{11}$, R. Hinze ${ }^{12}$, R. Knüchel-Clarke ${ }^{8}$, B. Lhermitte ${ }^{13}$, G. Nesi ${ }^{14}$, T. Rüdiger ${ }^{15}$, G. Rühl ${ }^{16}$, M.A. Tiab ${ }^{9}$, H. Moch ${ }^{1}$ 'Institute of Surgical Pathology, University Hospital Zurich, Zurich, Switzerland, 'Department of Pathology, University Medical Center, Göttingen, Germany, ${ }^{3}$ Service d'Anatomie et Cytologie Pathologiques ', Groupe Hospitalier Pitié-Salpêtrière, Paris, France, ${ }^{4}$ Institute of Pathology, Heinrich Heine University, Düsseldorf, Germany, ${ }^{5}$ Institute of Pathology and Neuropathology, University Hospital, Tübingen, Germany, ${ }^{6}$ Institute of Pathology, HELIOS Klinikum Berlin-Buch, Berlin, Germany, ${ }^{7}$ Institute of Pathology Bogenhausen, Städtisches Klinikum München GmbH, Munich, Germany, ${ }^{8}$ Institute of Pathology, RWTH University, Aachen, Germany, ${ }^{9}$ Institut Central des Hospitaux Valaisans, Service d'Histocytopathologie, Sion, Switzerland, ${ }^{10}$ Cantonal Hospital, Institute of Pathology, Aarau, Switzerland, ${ }^{11}$ Institute of Pathology Aarau, Aarau, Switzerland, ${ }^{12}$ Institute of Pathology, HELIOS Kliniken Schwerin, Schwerin, Germany, ${ }^{13}$ Institut universitaire de pathologie de Lausanne, Lausanne, Switzerland, ${ }^{14}$ Dipartimento di Chirurgia e Medicina Traslazionale, Università degli Studi di Firenze, Florenz, Italy, ${ }^{15}$ Institute of Pathology, Städtisches Klinikum Karlsruhe gGmbH, Karlsruhe, Germany, ${ }^{16}$ Institute of Pathology, Klinikum Niederberg, Velbert, Germany

Aims. Clear cell papillary renal cell carcinoma (CCPRCC) has been proposed as a potential new tumor entity at the Vancouver consensus conference by the International Society of Urological Pathology (ISUP) Initially, CCPRCC was described in end-stage renal disease. Recently, sporadic CCPRCC was also reported. The aim of our study was to characterize CCPRCC by immunohistochemistry and to analyze the VHL gene mutation status. The findings were compared with clear cell RCC and papillary RCC.

Methods. We investigated 28 CCPRCC specimens using immunohistochemistry for $\mathrm{CK}_{7}, \mathrm{CA}-9$, GLUT-1, CD-10, E-cadherin, vimentin, $\beta$ catenin, estrogen receptor, progesterone receptor, parafibromin, PAX2, PAX-8, CD133, OCT3/4, MET and PTEN. VHL gene mutations and allele loss were investigated by direct sequencing and fluorescence in situ hybridization (FISH). Clinical follow-up data were obtained from a subset of CCPRCC patients.

Results. Immunohistochemical and genetic analyses showed a profile different from clear cell RCC and papillary RCC. No recurrence of tumor or tumor-related death was observed.

Conclusions. CCPRCC represents a new distinct entity of renal cell carcinomas and should be included into a future WHO classification of renal cancer.

\section{SA-128}

Markers of urothelial carcinoma in normal urothelium of smokers and non-smokers: aberrant expression of $\mathrm{mcm} 2$ and CHEK 2 is associated with tobacco use

\section{W. Otto ${ }^{* 1}$, S. Denzinger' ${ }^{2}$ S. Bertz' ${ }^{1}$ M. Burger ${ }^{2}$, A. Hartmann'}

Institute of Pathology, University Hospital Erlangen, Erlangen, Germany, ${ }^{2}$ Department of Urology, University of Regensburg, Regensburg, Germany

Ziele. Der Genuss von Tabakerzeugnissen gilt als wichtigster klinischer Risikofaktor für die Entwicklung eines Urothelkarzinoms der Harnblase, wobei die entscheidenden Mechanismen, die zur Entstehung des Karzinoms führen, nach wie vor nicht im Detail geklärt sind. Hilfestellung bei der Suche nach den zellulären Wirkungen des Rauchens, die zur Krebsentstehung führen, könnte eine Untersuchung von Effekten des Tabakkonsums auf das histomorphologisch noch gesunde Urothel bringen. Wir analysierten daher erstmals die etablierten immunhistochemischen Marker des Harnblasenkarzinoms 553 und CK2O, die Proliferationsmarker ki67 und $\mathrm{Mcm} 2$ sowie $\mathrm{CHEK} 2$ als Protein aus dem DNA-Reparatur-Mechanismus im Normalurothel gesunder Probanden ohne Harnblasenkarzinom.

Methoden. Nach entsprechender Aufklärung, basierend auf einem positiven Ethikvotum der Universität Regensburg, entnahmen wir bei männlichen Patienten, die wegen Benigner Prostatahyperplasie einer transurethralen Resektion der Prostata zugeführt wurden, eine Probe von makroskopisch unauffälligem Urothel der Harnblase. Wir fertigten nach histopathologischer Begutachtung durch einen erfahrenen Uropathologen vom Paraffin eingebetteten Material einen Tumor Microarray an und führten immunhistochemische Färbungen mit p53, $\mathrm{CK}_{20}$, ki-67, Mcm2 und CHEK2 durch. Die mikroskopische Auswertung erfolgte nach den für die entsprechenden Marker gängigen Regelungen und Cut-off-Werten.

Ergebnisse. Von den 169 männlichen Patienten (Altersmedian: 69 Jahre, IQR 64-75) waren $43 \%$ Nichtraucher $(n=72), 57 \%(n=97)$ gaben in der Anamnese einen regelmäßigen Tabakkonsum an (12\% aktiv, $45 \%$ sistiert). Während sich die Expression von p $53 \geq 10 \%$, ki- $67 \geq 5 \%$ und ein abnormales Expressionsmuster (negativ, $\geq 5 \%$ ) von $\mathrm{CK}_{2} \mathrm{O}$ nicht statistisch signifikant zwischen Patienten mit und ohne Raucheranamnese unterschied, zeigten aktive und ehemalige Raucher häufiger eine Expression von $\mathrm{Mcm} 2 \geq 20 \%$ ( $31 \%$ vs. $15 \%$; $\mathrm{p}=0,038$ ). Moderate und starke Expression von $\mathrm{CHEK}_{2}$ war häufiger bei Nichtrauchern als bei Rauchern festzustellen ( $49 \%$ vs. $21 \%$; $\mathrm{p}=0,001$ ).

Schlussfolgerung. Wir konnten statistisch signifikante Unterschiede in der Expression von $\mathrm{Mcm}_{2}$ und $\mathrm{CHEK}_{2}$ im Normalurothel zwischen Rauchern und Nichtrauchern feststellen. Dies könnte auf eine bereits beginnende erhöhte Proliferationstendenz des histomorphologisch normalen Urothels hinweisen, in dem zelluläre Reparaturmechanismen herunterreguliert sind. Weitere Vergleichsstudien zur Markerexpression im Urothel gesunder Raucher, Nichtraucher und Ex-Raucher sollten folgen.

\section{SA-129}

$\mathrm{PI} 3 \mathrm{~K} / \mathrm{AKT} / \mathrm{mTOR}$ pathway plays a major pathogenetic role in glycogen accumulation and tumor development in renal distal tubuli of diabetic rats and men

\section{S. Ribback ${ }^{* 1,2}$, D.F. Calvisi', A. Cigliano' ${ }^{2}$, M.G. Pilo' ${ }^{2}$ L. Terracciano ${ }^{3}$, N. Kroeger', F. Dombrowski}

IInstitut für Pathologie, Greifswald, Germany, ${ }^{2}$ Institut für Pathologie, Universitätsmedizin Greifswald, Greifswald, Germany, ${ }^{3}$ Institute of Pathology, Molecular Pathology Division, University Hospital, Basel, Switzerland, ${ }^{4}$ Klinik für Urologie, Universitätsmedizin Greifswald, Greifswald, Germany

Aims. We previously showed that metabolic changes in diabetic rats affect the renal distal tubular epithelium and lead to the development of glycogen storing clear cell tubuli, which represent principal precursor lesions of renal cell carcinoma. Histologically, these lesions resemble human glycogenotic distal tubules and clear cell renal cell carcinoma (ccRCC). In human ccRCC, the upregulation of the $\mathrm{PI}_{3} \mathrm{~K} / \mathrm{AKT} / \mathrm{mTOR}$ signaling pathway and the lipogenic phenotype are associated to tumor progression and are suggested as potential therapeutical targets.

Methods. Human glycogenotic tubules and tissue microarrays of ccRCC $(n=102)$ were examined in terms of morphologic, metabolic and molecular alterations, and compared to glycogenotic clear cell tubuli and RCC developed in the rat following N-Nitrosomorpholine administration or streptozotocin-induced diabetes. In addition, diabetic and non-diabetic Lewis rats $(\mathrm{n}=111)$ were subjected to daily administration of the dual $\mathrm{PI}_{3} \mathrm{~K}$ and mTOR inhibitor, NVP-BEZ235, for 1 (40 mg/kg) or 4 weeks (10 mg/kg), respectively. Effects of NVP-BEZ235 were also investigated in CAKI1 and RCC4 human ccRCC cell lines. 
Results. Rat RCC and human ccRCC as well as rat and human glycogenotic tubuli revealed a strong induction of the $\mathrm{PI}_{3} \mathrm{~K} / \mathrm{AKT} / \mathrm{mTOR} \mathrm{pa}-$ thway and related metabolic alterations (glycolysis, de novo fatty acid and cholesterol synthesis) compared to the unaltered renal tissue. Of note, dual inhibition of $\mathrm{PI}_{3} \mathrm{~K}$ and mTOR resulted in a marked reduction of proliferation in human ccRCC cell lines and in rat glycogenotic tubules, which was paralleled by downregulation of the proteins involved in glycolysis and de novo lipogenesis in vitro and in vivo. Furthermore, NVP-BEZ235 administration led to increased volume fraction of autophagic vacuoles in rat glycogenotic tubules.

Conclusions. Altogether, the present data indicate that human glycogenotic distal tubules reveal a pattern of morphologic and metabolic alterations resembling that of the rat model of diabetes-induced nephrocarcinogenesis and human ccRCC. Importantly, these morphologic and metabolic changes are effectively inhibited by NVP-BEZ235 administration, thus indicating that suppression of the $\mathrm{PI}_{3} \mathrm{~K} / \mathrm{AKT} /$ mTOR pathway might be highly beneficial to prevent diabetes-induced nephrocarcinogenesis.

\section{SA-130 \\ miRNA profiling identifies candidate miRNAs for bladder cancer diagnosis and clinical outcome}

E. Kilic ${ }^{* 1}$, N. Ratert' ${ }^{2}$, H.-A. Meyer ${ }^{2}$, M. Jung ${ }^{2}$, P. Lioudmer ${ }^{2}$, H.-J. Mollenkopf ${ }^{3}$, I. Wagner ${ }^{3}$, K. Miller', A. Erbersdobler 5 , S. Weikert ${ }^{4}$, K. Jung ${ }^{4}$

'Institute of Pathology, Charité - University medicine of Berlin, Berlin, Germany, ${ }^{2}$ Department of Urology, University Hospital Charité, Berlin, Germany, ${ }^{3}$ Max Planck Institute for Infection Biology, Berlin, Germany, ${ }^{4}$ Department of Urology, Charité University Hospital, Berlin, Germany, ${ }^{5}$ Institute of Pathology, University Hospital, Rostock, Germany

Aims. The current prognosticators such as tumor grade, stage, size, and multifocality do not accurately reflect the clinical outcome. It is of clinical interest to identify biomarkers that could improve diagnostic and/ or prognostic predictions. The objectives of this study were to identify deregulated miRNAs in bladder cancer samples and evaluate their potential as diagnostic and prognostic biomarkers.

Methods. We screened 723 miRNAs by microarray and selected a subset of 15 distinctively deregulated miRNAs for further validation by realtime quantitative RT-(q)PCR.

Results. Seven miRNAs (miR-20a, miR-106b, miR-13ob, miR-141, miR200a, miR-200a*, and miR-205) were found to be up-regulated and eight miRNAs (miR-10o, miR-125b, miR-130a, miR-139-5p, miR-145*, miR-199a-3p, miR-214, and miR-222) were found to be down-regulated in malignant bladder tissue samples compared to healthy tissue. Four miRNAs that have already been described in the literature (miR-141, miR-199a-3p, miR-205, and miR-214) were significantly differentially expressed between nonmuscle-invasive and muscle-invasive bladder cancer. Furthermore, real-time RT-qPCR of all miRNAs provided high overall correct classification $(>75 \%)$ of bladder cancer diagnosis. Two miRNAs (miR-141 and miR-205) were associated with overall survival time.

Conclusions. The verification of tumor-specific miRNA expression profile, together with the observed association of miR-141 and miR-205 expression with overall survival, underline the potential of miRNAs to function as diagnostic and/or prognostic markers of bladder cancer.

\section{SA-131}

\section{CK19 is specifically expressed in yolk sack tumours of the testis}

\section{F. Bremmer*, P. Ströbel, J. Strecker, H.J. Radzun, C.L. Behnes Institut für Pathologie, University Medical Center, Göttingen, Germany}

Aims. Malignant germ cell tumours are the most common malignant tumours in young men. They are histologically subdivided into semino- mas and non-seminomas. Non-seminomas are furthermore subdivided in embryonic carcinomas, yolk sack tumours, chorioncarinomas and teratomas. The differentiation of the histological subtypes is essential for the therapeutic management. For histological diagnosis various immunohistochemical markers are suitable to distinguish the different histological tumour entities. For the diagnosis of yolk sack tumours the immunohistochemical analysis of alpha-feto-protein, cytokeratin, and glypican-3 is helpful.

Methods. We investigated 156 malignant germ cell tumours of the testis composed of 67 seminomas, 56 embryonic carcinomas, 29 yolk sack tumours and 18 teratomas for their expression of CK19. We furthermore compared these findings with the immunohistochemical analysis of Glypican-3, alpha feto protein, $\mathrm{CD}_{3} \mathrm{O}$, and cytokeratin.

Results. All investigated seminomas and embryonic carcinomas did not express CK19. In contrast all 29 investigated yolk sack tumours strongly expressed CK19 protein. These findings were also observed in mixed germ cell tumour consisting of embryonic carcinomas and yolk sack tumours.

Conclusions. CK19 is a highly specific marker to distinguish yolk sack tumours from other malignant germ cell tumours of the testis. Furthermore, $\mathrm{CK}_{19}$ could be established as a new tumour serum marker in cases of yolk sack tumours. In addition to alpha feto-protein and Glypican-3, the positive CK19 expression could be an indicator for a hepatic differentiation.

\section{SA-132}

FAM96A is down-regulated in bladder tumors and expression loss is associated with worse outcome in advanced bladder carcinomas

\section{A. Rogler ${ }^{* 1}$, I. Edling', R.J. Rieker', A. Hartmann', M. Zörnig ${ }^{2}$}

IInstitute of Pathology, University Hospital Erlangen, Erlangen, Germany, ${ }^{2}$ Institute for Tumor Biology and Experimental Therapy, Georg-SpeyerHaus, University of Frankfurt, Frankfurt, Germany

Aims. In 2010, 13,000 cases of non-invasive papillary bladder cancers and 15,000 cases of invasive bladder carcinomas occurred in Germany. This was associated with 5516 deaths. Despite a relatively low mortality rate, bladder cancer is a chronic disease which needs life-long surveillance and costs therefore are immense. Therefore, it is essential to find adequate biomarkers for prediction of outcome and progression of disease to provide the best appropriate treatment for every patient. Thus, we analyzed the promising marker FAM96A in a cohort of bladder cancer patients. FAM96A (family with sequence similarity 96, member A) is located at chromosome 15 and encodes a mammalian DUF59 protein which is part of the cytosolic iron-sulphur protein assembly machinery. Methods. We immunohistochemically analyzed two series of tissue micro arrays with tumor specimen from 91 patients with papillary tumors (mainly pTa and $\mathrm{pT} 1$ ) and from 331 patients with advanced bladder carcinomas (characterized trough $\mathrm{pT}_{3} / 4$ and/or $\mathrm{pN} 1$ ) and compared it with the expression in normal urothelium of 21 patients (control group) with chi2 test. Protein expression was also correlated with patient outcome using Kaplan-Meier analysis.

Results. FAM96A showed particularly strong and moderate expression in normal urothelium, whereas expression was rather weak or lost in bladder tumors. There was a significant difference in protein expression between normal tissue and the papillary tumors as well as between normal tissue and advanced tumors ( $\mathrm{p}$-values $<0.0001$ ). We found a significantly better overall $(p=0.017)$ and disease-specific survival $(p=0.003)$ for patients with moderate or strong FAM96A expression compared to those with weak or lost expression in the advanced bladder carcinoma cohort.

Conclusions. In this study we identified FAM96A as a novel predictive marker for advanced bladder carcinoma. FAM96A seems to be a tumor suppressor gene as it is weaker expressed or lost in bladder tumors compared to normal urothelium. It is important to clarify the biological function of FAM96A in the bladder mucosa and its role in carcinogene- 
sis. It seems likely that aberrant expression of this iron-sulphur cofactor has an impact on cell metabolism, DNA maintenance or translation, which might increase the malignant potential of the cell.

\section{SA-133}

Fibroblast growth factor 3 (FGFR3) rearrangements and amplifications in bladder cancer-a tissue microarray-based FISH study.

U. Zinnal ${ }^{* 1}$, R. Stöhr', S.V. Williams ${ }^{2}$, K. Schmitz ${ }^{3}$, S. Bertz', W. Anna', M. Knowles ${ }^{4}$, B. Wullich ${ }^{5}$, A. Hartmann', H.-U. Schildhaus ${ }^{6}$

${ }^{1}$ Institute of Pathology, Friedrich-Alexander-University, Diagnostic Molecular Pathology, Erlangen-Nuremberg, Germany, ${ }^{2}$ Section of Experimental Oncology, Leeds Institute of Molecular Medicine, Leeds, United Kingdom, ${ }^{3}$ Department of Pathology, University Medical Center, Göttingen, Germany, ${ }^{4}$ Leeds Institute of Cancer and Pathology, Leeds, United Kingdom, ${ }^{5}$ Department of Urology, Friedrich-Alexander-University, Erlangen-Nuremberg, Germany, ${ }^{6}$ Department of Pathology, Georg-August-University, Göttingen, Germany

Aims. Although most bladder cancers are non-invasive tumors, muscle-invasive and metastasizing disease shows high mortality and limited response to conventional therapy. Targeted therapy studies have shown limited response rates underscoring the need for new tumor-specific therapies. FGFR3- mutations are the most frequent genetic alterations in bladder cancer, occurring predominantly in noninvasive low-grade papillary tumors. Recently specific chromosomal re-arrangements involving FGFR 3 were detected in $5-10 \%$ of bladder cancer cell lines and primary tumor samples. Cell lines with $\mathrm{FGFR}_{3}$ fusion proteins with $\mathrm{TACC}_{3}$ or BAIAP $\mathrm{L}_{1}$ are extremely sensitive to $\mathrm{FGFR}_{3}$-selective agents providing a possible rationale for target-specific therapy in a subset of bladder cancer patients. The aim of the current study was to evaluate the frequency of the FGFR3 re-arrangement by FGFR3-FISH analysis using a bladder cancer tissue microarray.

Methods. An unselected consecutive cohort of 529 bladder cancers was retrieved from the files of the Institute of Pathology, University Erlangen and histopathological and clinical features were re-evaluated. The cohort includes 239 pTa tumors, 123 pT1 tumors, 101 pT2 tumors and 62 $\mathrm{pT}_{3}$-pT4 tumors. 202 patients showed low grade and 326 patients high grade disease and 398 patients showed an at least partially papillary and 131 patients a only solid growth pattern. FISH analyses were done using a specific $\mathrm{FGFR}_{3}$ break apart probe set with green and orange probes spanning the 3 ' and 5 ' flanking regions of FGFR 3 . Overall 390 tumors of these 529 bladder cancers were analyzed.

Results. In total we found 11 bladder cancers with abnormalities. 6/11 tumors showed re-arrangements. 5 of these 11 tumors had FGFR 3 amplifications. The majority of patients (9/11) with FGFR 3 translocations or amplifications were non-muscle invasive tumors. All but one case showed a papillary growth pattern and absence of carcinoma in situ. However, 9 of 11 tumors with abnormalities were high grade tumors. The exact breakpoints of the translocations and the clinical characteristics of the tumors are evaluated in the moment.

Conclusions. In summary, FGFR 3 translocations and amplifications can be detected by FISH in approximately $3 \%$ of an unselected bladder cancer cohort. Our data argue for a possible involvement in development or progression of papillary bladder cancer with high-grade. Therapy with selective agents against FGFR 3 could be a possible option for a subset of bladder cancer patients.

\section{SA-134}

Prognostic impact of the amount of micropapillary component in urothelial carcinomas of the bladder on cancer specific survival in patients treated with TURB and adjuvant radiochemotherapy

S. Bertz ${ }^{* 1}$, B. Keck ${ }^{2}$, H. Taubert ${ }^{3}$, S. Wach ${ }^{3}$, S. Krause ${ }^{4}$, S. Schick ${ }^{5}$, O.J. Ott ${ }^{6}$, E. Weigert', O. Dworak', C. Rödel' ${ }^{8}$, B. Wullich' ${ }^{2}$, R. Fietkau ${ }^{6}$, A. Hartmann ${ }^{7}$

'Institute of Pathology, Friedrich-Alexander-University, Diagnostic Molecular Pathology, Erlangen-Nuremberg, Germany, ${ }^{2}$ Department of Urology, Friedrich-Alexander-University, Erlangen-Nuremberg, Germany, ${ }^{3}$ Division of Molecular Urology, Clinic of Urology, University of Erlangen-Nuremberg, Erlangen, Germany, ${ }^{4}$ Clinic of Urology, AKH Linz, Linz, Austria, ${ }^{5}$ Tumor Registry of the University of Erlangen-Nuremberg, Erlangen, Germany, ${ }^{6} \mathrm{De}-$ partment of Radiation Oncology, University Hospital Erlangen, Erlangen, Germany, ${ }^{7}$ Institute for Pathology, City Hospital Fürth, Fürth, Germany, ${ }^{8}$ Department of Radiation Oncology, University Hospital Frankfurt, Frankfurt, Germany

Aims. To evaluate the prognostic impact of rare variants of urothelial bladder cancer in patients treated with radiochemotherapy (RCT).

Methods. Data of 145 patients with muscle-invasive urothelial carcinoma (UC) of the bladder treated with TURB and RCT with curative intent between 1982 and 2007 were evaluated. Clinical characteristics including long-term follow-up (median 69 months, range 4 to 279 months) were documented. Histopathological reevaluation emphasized on rare histological variants. The percentage of variant histology was assessed for each tumour. Kaplan-Meier-analyses and Cox's regression hazard analyses were performed to identify factors predictive of survival.

Results. Variable amounts of variant histology were found in 40 of 145 cases. The mere presence of a variant histology did not affect prognosis. However, at considering the amount of variant histology, patients with $\geq 30 \%$ of micropapillary component $(n=10)$ in their tumours showed a poor prognosis. In Kaplan-Meier analysis cancer specific survival (CSS) was significantly worse in tumours with at least 30\% micropapillary morphology compared to conventional UC without variant histology $(\mathrm{p}=0.012)$. Mean survival rates were 97 months for patients with micropapillary tumours compared to 220 months for patients with conventional tumour histology. In cases with additionally smaller amounts of micropapillary morphology there was no significant difference in CSS compared to conventional UC patients. In univariate Cox's proportional hazards regression analysis $\geq 30 \%$ micropapillary morphology demonstrated a hazard ratio (HR) of 3.70 (95\% CI 1.231-11.094) for CSS $(\mathrm{p}=0.020)$. Multivariate Cox's regression analysis revealed age and distant metastasis but not micropapillary morphology $(\geq 30 \%)$ as independent prognostic factors.

Conclusions. Increased amounts $(\geq 30 \%)$ of micropapillary morphology represent univariately a prognostic factor for reduced CSS in patients with UC of the bladder treated by TURB and RCT. Further studies are needed to evaluate a potential benefit from different treatment strategies for bladder cancers with micropapillary but also other rare variant morphologies.

\section{SA-135}

\section{A rare case of urinary schistosomiasis}

M. Gajda*1, M. Mireskandari', F. Hartmann', C. Seeliger ${ }^{3}$, B. Schleenvoigt', M. Baier ${ }^{5}$, H. Oppel', I. Petersen

IInstitute of Pathology, Jena, Germany, ${ }^{2}$ Department of Urology, FriedrichSchiller-University, Jena, Germany, ${ }^{3}$ Department of Gynecology and Obstetrics, Friedrich-Schiller-University, Jena, Germany, ${ }^{4}$ Center of Infectious Diseases and Infection Control, FSU, Jena, Germany, ${ }^{5}$ Institute of Medical Microbiology, FSU, Jena, Germany

Aims. Schistosomiasis is an infection with trematode parasites and is a prevalent tropical disease, ranking second to malaria and posing a great public health and social economic threat in Africa, Latin America and 
Asia. In some regions, Schistosoma haematobium is one of the principal causes of hematuria. More than 300 million people in 76 countries have these parasites. We report a patient who had a journey to Malawi and contact with fresh water while swimming in Lake Malawi one year prior to the occurrence of hematuria.

Methods. Detailed clinical, cytological and histopathological review of a clinical case and review of the literature using PUBMED.

Results. The 28-year-old woman developed hematuria in the 21st week of pregnancy. A TUR was performed to rule out bladder cancer. Urinary cytology revealed no malignant cells. Eosionophilia and single parasite egg was identified. The histological diagnosis of Schistosomiasis was established by finding egg of Schistosoma haematobium and manifestations of eosinophilc cystitis in biopsy samples. After histological confirmation of diagnosis the patient underwent the standard therapy with a single injection of praziquantel. The following pregnancy course was unremarkable and ended to the vacuum assisted birth of a mature girl in the week $40+5$ of pregnancy. Histologic examination of placenta showed isolated and probably avital Schistosoma eggs. The infant had adequate growth and development. Both are currently regarded as free of schistosomiasis clinically and parasitologically.

Conclusions. Infection with Schistosoma haematobium at any part of the urinary tract, particularly infection of urinary bladder (cystitis), can cause hematuria. In a patient with hematuria and history of recent travel to tropical destinations schistosomiasis has to be considered in the differential diagnoses. If not properly treated, infection with Schistosoma causes longstanding urinary complications and may result in serious sequelae such as renal failure or bladder carcinoma.

\section{SA-136}

\section{Frequency of FGFR2 exon 7 mutations in bladder cancer}

R. Stöhr ${ }^{* 1}$, C. Spiegelberg', J. Giedl', A. Rogler', M.-O. Riener', T. Filbeck², M. Burger ${ }^{3}$, P. Rümmele ${ }^{4}$, A. Hartmann

'Institute of Pathology, University Hospital Erlangen, Erlangen, Germany, ${ }^{2}$ Via Medis, Department of Nephrology and Urology, Eichstätt, Germany, ${ }^{3}$ St. Josef Medical Centre, Department of Urology, University of Regensburg, Regensburg, Germany, ${ }^{4}$ Institute of Pathology, University of Regensburg, Regensburg, Germany

Aims. The FGF/FGFR-system plays an important role in embryogenesis, tissue homeostasis and carcinogenesis. Mutational activation of FGFR2 resulting in aberrant FGFR2 signaling activation is known from both hereditary germ line alterations and somatic mutations in various malignancies (e.g. breast, gastric or ovarian cancer). FGFR2 mutations are mainly located within the hinge between Ig-like domains (exon 7), around the $3 \mathrm{rd}$ Ig-like domains and within the kinase domain. For bladder cancer only sparse data on FGFR2 mutations are available. Recently, a case of early-onset papillary carcinoma of the bladder showing a FGFR2 p.Pro253Arg mutation in exon 7 in a patient with Apert Syndrome was reported. The aim of this study was the further evaluation of FGFR2 exon 7 alterations in bladder cancer.

Methods. Sections from formalin-fixed, paraffin-embedded bladder tumors were used for DNA isolation. After precise microdissection, exon 7 of the FGFR2 gene was analysed by direct Sanger sequencing. Overall, a cohort of 215 bladder tumors [cohort 1: unselected cases: $n=106$; cohort 2: early-onset bladder cancer cases (age at time of diagnosis $\leq 45$ years): $\mathrm{n}=109]$ was analysed so far.

Results. All cases could be analysed successfully. Mutations in exon 7 of FGFR2 could not be detected in any of the cases. All tumors showed wild type sequence.

Conclusions. So far, the recently reported association between early-onset papillary carcinoma of the bladder with germ line FGFR2 p.Pro253Arg mutation could not be found in our cohorts of sporadic bladder tumors. These data indicate that FGFR2 gene mutations might only play a minor role in bladder carcinogenesis.

\section{SA-137}

MITF (microphthalmia-associated transcription factor) p.E318K mutation does not play a major role in sporadic renal cell carcinoma

C. Stöhr* ${ }^{* 1}$, B. Walter ${ }^{2}$, F. Hofstädter ${ }^{3}$, M. Burger', P. Ghiorzo ${ }^{5}$, R. Sturm ${ }^{6}$, R. Hinze', H. Moch ${ }^{8}$, K. Junker ${ }^{9,10}$, R. Stöhr', A. Hartmann ${ }^{1}$

IInstitute of Pathology, University Hospital Erlangen, Erlangen, Germany, ${ }^{2}$ Department of Urology, Kreiskliniken Altötting-Burghausen, AltoettingBurghausen, Germany, ${ }^{3}$ Department of Pathology, University of Regensburg, Regensburg, Germany, ${ }^{4}$ Department of Urology, St. Josef Medical Centre, University Regensburg, Regensburg, Germany, ${ }^{5}$ Department of Internal and Medical Specialties, Universtiy of Genoa, Genoa, Italy, ${ }^{6}$ Institute of Molecular Biosciences, University of Queensland, Brisbane, Australia, ${ }^{7}$ Institute of Pathology, Helios Hospitals Schwerin, Schwerin, Germany, ${ }^{8}$ Institute of Surgical Pathology, Universtiy Hospital Zurich, Zurich, Switzerland, ${ }^{9}$ Department of Urology and Pediatric Urology, Saarland University Medical Center, Homburg/Saar, Germany, ${ }^{10}$ Department of Urology, University Hospital Jena, Jena, Germany

Aims. MITF gene encodes for a basic helix-loop-helix zipper transcription factor of the Myc superfamily. MITF is known to induce expression of HIF1-alpha (hypoxia inducible factor 1-alpha) which is involved in renal carcinogenesis. MITF p.E $318 \mathrm{~K}$ mutation leads to insufficient small ubiquitin-like modifier (SUMO)-ylation of MITF and results in enhanced and uncontrolled activation of its target genes. A recent study on control patients and melanoma patients who coincidentally were affected by renal cell carcinoma (RCC) has revealed a more than five-fold elevated risk for mutation carriers to be affected by one or both of these malignancies. This suggests a possible role for MITF p.E318K in renal carcinogenesis. To date there is no data on this mutation in patients affected by sporadic RCC. We therefore tested a collection of sporadic renal tumours for MITF $\mathrm{pE} 318$.K mutation status.

Methods. DNA was extracted from 426 formalin fixed paraffin embedded sporadic renal tumours that had been classified according to the 2004 WHO classification of renal tumours and staged according to the 2002 TNM classification. DNA was tested for MITF p.E318K by pyrosequencing.

Results. Of 403 analyzable tumours, 402 renal tumours were wild-type, only one case carried the MITF p.E $318 \mathrm{~K}$ mutation. The allele frequency of the mutation therefore was 0.0012. The tumour harbouring the p.E $318 \mathrm{~K}$ mutation was a clear cell RCC, $\mathrm{pT}_{3} \mathrm{~b}$ No Mo G3 (according to TNM classification 2002). The affected patient was male, 61 years old and had no known coexisting malignancies.

Conclusions. The allele frequency in our study population is in line with reported allele frequencies of the general population in the UK or France. MITF p.E318K mutation therefore does not play a major role in sporadic RCC carcinogenesis, but is obviously restricted to a rare subpopulation of inherited RCC.

\section{SA-138}

Microvascular architecture and PDGFRb expression in renal cell carcinoma

\section{F. Pfister ${ }^{* 1}$, K. Amann 1 , C. Stöhr ${ }^{2}$, A. Hartmann ${ }^{3}$}

Institute of Pathology, Friedrich-Alexander-University, Nephropathology, Erlangen-Nuremberg, Germany, ${ }^{2}$ Institute of Pathology, Friedrich-Alexander-University, Erlangen-Nuremberg, Germany, ${ }^{3}$ Institute of Pathology, Friedrich-Alexander-University, Erlangen-Nürnberg, Germany

Aims. Angiogenesis has been studied in detail in clear cell renal cell carcinoma and substantial clinical activity of anti-angiogenic drugs including inhibitors of vascular endothelial growth factor receptor (VEGFR) and/or platelet-derived growth factor receptor (PDGFR) has been shown. However, comparative quantitative data on the expression of the pericyte marker PDGFRb in renal cell carcinoma (RCC) are rare. 
In this study, vascular density and diameter as well as PDGFRb expression were investigated in different RCC subtypes.

Methods. Tissue microarrays used in this study included 431 biopsies of RCC ( 336 clear cell, 20 papillary type 1, 42 papillary type 2,33 chromophobe). Vascular density and diameters were measured in $\mathrm{CD}_{31}$-stained paraffin sections and staining signal intensity of PDGFRb immunohistochemistry was quantified using an image analysis software $\left(\right.$ cellF $^{\oplus}$, Olympus).

Results. Clear cell RCC were characterized by highest vascular density ( $+47 \%$ to $+230 \%$ compared to others subtypes) and highest PDGFRb expression in pericytes $(+179 \%$ to $+304 \%)$ of all analyzed tumor groups. In contrast, papillary type 1 carcinoma showed lowest vascular density and diameter. Vascular diameters were comparable between clear cell, papillary type 2 and chromophobe RCC and PDGFRb expression did not differ between other tumor groups than clear cell RCC. Interestingly, vascular density was significantly lower in poorly differentiated $\left(\mathrm{G}_{3}\right)$ clear cell $(>-30 \%)$ and chromophobe $(>-49 \%)$ RCC and vascular diameter was significantly increased in poorly differentiated $\left(\mathrm{G}_{3}\right)$ clear cell $(>+29 \%)$ and papillary $(>+22)$ RCC when compared to better differentiated (G1 or G2) tumors. Furthermore, PDGFRb expression was significantly reduced in $\mathrm{G}_{2}$ and $\mathrm{G}_{3}$ clear cell RCC $(-52 \%$ and $-54 \%)$ and increased in $\mathrm{G}_{2}$ and $\mathrm{G} 3$ papillary RCC $(158 \%$ and $+81 \%)$ when compared to $\mathrm{G} 1$ tumors.

Conclusions. Subtypes of RCC show significant, potentially diagnostic helpful differences in their vascular architecture. Clear cell RCC are characterized by highest vascular density and highest expression of PDGFRb in pericytes, indicating the importance of angiogenesis in this tumor entity. Furthermore, our data indicate that well differentiated clear cell RCC might be especially susceptible to anti-angiogenic agents targeting the PDGF/receptor-pathway.

\section{SA-139 \\ Positive FGFR3 immunoreactivity defines low-grade urothelial bladder cancer}

K.-F. Dem/*1, C. Poyet ${ }^{2}$, T. Hermanns' ${ }^{2}$, Q. Zhong', M.-O. Riener', C. Fankhauser', M. Burger ', F. Hofstaedter', A. Hartmann ${ }^{5}$, R. Stöhr' ${ }^{5}$, E.C. Zwarthoff', T. Sulser' ${ }^{2}$, H. Moch', P.J. Wild ${ }^{1}$

'Institute of Surgical Pathology, University Hospital Zurich, Zurich, Switzerland, ${ }^{2}$ Department of Urology, University Hospital, Zurich, Switzerland, ${ }^{3}$ St. Josef Medical Centre, Department of Urology, University of Regensburg, Regensburg, Germany, ${ }^{4}$ Institute of Pathology, University of Regensburg, Regensburg, Germany, ${ }^{5}$ Institute of Pathology, FriedrichAlexander-University, Erlangen-Nuremberg, Germany, ${ }^{6}$ Department of Pathology, Erasmus Medical Center, Rotterdam, Netherlands

Aims. Molecular markers superior to conventional clinicopathologic parameters are needed to predict disease courses in patients with urothelial bladder cancer. We investigated the immunoreactivity of FGFR 3 in primary urothelial bladder tumours with regard to clinicopathological and known molecular features.

Methods. Tissue microarrays were used to analyse FGFR 3 expression immunohistochemically in 255 unselected patients with primary urothelial carcinoma (rabbit polyclonal (E1023), Spring Bioscience, Fremont, CA, dilution 1:50). FGFR 3 immunoreactivity was scored semiquantitatively as negative (score o) or positive (score 1+). FGFR 3 mutations were detected by $\mathrm{SNaPshot}$ analysis.

Results. Positive FGFR3 immunoreactivity was found in 113 of 207 analyzable cases $(54.6 \%)$, and was significantly associated with FGFR mutation $(\mathrm{p}<0.001)$, lower tumour stage $(\mathrm{p}<0.001)$, low histologic grade $(\mathrm{p}<0.001)$, papillary growth pattern $(\mathrm{p}<0.001)$, and $\mathrm{Ki}-67$ proliferation rate $<25 \%(\mathrm{p}<0.001)$. Positive $\mathrm{FGFR}_{3}$ immunostaining $(\mathrm{p}=0.002)$ and FGFR $_{3}$ mutation $(\mathrm{p}=\mathrm{0.002})$ were significantly associated with longer disease-specific survival in univariate analysis. However, they were not independent from classical pathologic parameters in multivariate analysis. None of the molecular markers was associated with tumour recurrence.

Conclusions. Low grade non-invasive papillary urothelial tumours are defined by the presence of an FGFR 3 immunoreactivity. Therefore, immunohistochemical $\mathrm{FGFR}_{3}$ expression may represent an early event in the carcinogenesis of papillary urothelial cancer, which is lost during tumor progression.

\section{AG Gynäko- und Mammapathologie Postersession}

\section{SA-140}

Breast carcinoma with lobular and micropapillary component: Tumor heterogeneity demonstrated by Endopredict ${ }^{\circledast}$

\section{B. Döring ${ }^{* 1}$, M. Otto ${ }^{1,2,3}$, J. Kriegsmann ${ }^{1,2,3}$}

${ }^{1}$ Center for Histology, Cytology and Molecular Diagnostics, Trier, Germany, ${ }^{2}$ Proteopath GbR, Trier, Germany, ${ }^{3}$ Institute of Molecular Pathology, Trier, Germany

Aims. Tumor heterogeneity of breast carcinomas is well known. Estrogen and progesterone receptor as well as proliferation index and Her2 expression are very heterogeneous in a number of tumors and can cause problems gaining a concordant result. Multigene tests have emerged for the prediction of prognosis in breast carcinomas. Although large areas of the tumor tissue are investigated in multigene tests, tumor heterogeneity is not remarked. In this case study two clear-cut different areas of tumor can be demonstrated by H\&E. The aim of this case study was to evaluate if the Endopredict ${ }^{\oplus}$ test achieves different results if only one tumor component was studied or if the whole tumor was investigated. Methods. Endopredict ${ }^{\oplus}$ test was applied according to the manufacturer's protocol. RNA of the different areas of the tumor was isolated (either the lobular component or the micropapillary component). Furthermore, RNA of the whole tumor was isolated and investigated in another Endopredict ${ }^{\oplus}$ test.

Results. Investigation of the micropapillary component showed an EP score of 4.5 and an EPclin score of 3.0. The lobular component showed an EP score of 6.0 and an EPclin score of 3.4. By examining the whole tumor the respective values were 5.1 for the EP score and 3.1 for the EPclin score. Interestingly, the micropapillary area produces a low risk EPclin score while the lobular area produces a high risk case with a risk for metastasis of $10 \%$ in 10 years. The examination of the whole tumor results in a low-risk category.

Conclusions. According to the rules of general pathology- a tumor is as malignant as its worst part- the tissue that has to be investigated should be well considered when applying multigene assays in breast carcinomas.

\section{SA-141}

Immunohistochemical and FISH-based Investigation of androgen receptor in invasive breast cancer

\section{N.J. Rupp*, D. Kradolfer, W. Jochum, C. Öhlschlegel}

Institute of Pathology, Cantonal Hospital St. Gallen, St. Gallen, Switzerland

Aims. Androgen receptor (AR) expression is observed in female invasive breast cancer. Specific targeted therapies (e.g. tamoxifen) are used in the adjuvant therapy of estrogen (ER) and progesterone receptor (PR) positive breast carcinoma. In ER/PR negative or triple negative carcinomas, AR may be an alternative target. Aim of our study was to determine the frequency of AR expression in invasive breast cancer and to analyse AR gene amplification as a potential mechanism for AR expression.

Methods. We investigated $n=100$ invasive breast cancer cases based on a tissue microarray (TMA) for immunohistochemical expression of androgen receptor (AR), estrogen receptor (ER), progesterone receptor 
(PR) and HER2/neu (ErbB2) using commercially available antibodies and evaluation criteria applied in routine diagnostics. Fluorescence in situ hybridization (FISH) was performed to analyse the AR and HER2/ neu amplification status.

Results. AR was expressed in $>50 \%$ of the tumor cells in $67 \%$ of the cases. AR expression was associated with ER and PR expression ( $\mathrm{p}<0.001)$. However, 5 ER/PR negative cases showed AR expression. AR amplification was detected in 1 case which also showed moderate to strong AR and ER expression, weak PR expression, HER2/neu negativity and morphological features of invasive-apocrine carcinoma (IAC). Positive HER2/neu gene amplification was found in $20.9 \%$ of the cases. AR expression was not associated with the HER2/neu amplification.

Conclusions. AR expression is frequent in invasive breast cancer. In contrast, AR gene amplification is rare suggesting that other mechanisms than AR gene amplification induce AR expression in invasive breast cancer. AR gene amplification status may be helpful in the diagnosis of invasive apocrine carcinoma of the breast.

\section{SA-142}

\section{Low BRCA1 expression and impaired BRCA1 foci formation iden- tifies homologous recombination deficiency in sporadic breast cancer}

H. Gevensleben*1, F. Daley ${ }^{2}$, M.K. Graeser ${ }^{2}$, M. Parton ${ }^{3}$, J. Reis-Filho ${ }^{4}$, A. Ashworth ${ }^{2}$, I.E. Smith ${ }^{3}$, M. Dowsett ${ }^{3}$, N.C. Turner ${ }^{2}$ Institute of Pathology, University Hospital Bonn, Bonn, Germany, ${ }^{2}$ The Breakthrough Breast Cancer Research Centre, Institute of Cancer Research, London, United Kingdom, ${ }^{3}$ Breast Unit, Royal Marsden Hospital, London, United Kingdom, ${ }^{4}$ Department of Pathology, Memorial Sloan-Kettering Cancer Center, New York, United States

Aims. Defective homologous recombination (HR) is a determinant of sensitivity to DNA-damaging chemotherapy, in particular platinum chemotherapy, and Poly (ADP-ribose) polymerase (PARP) inhibition. We investigated whether loss of BRCA1 or PTEN expression is a surrogate marker for defective HR in sporadic breast cancer.

Methods. Biopsies from 57 sporadic breast cancers taken prior to and 24 hours after the first cycle of neoadjuvant chemotherapy were assessed for BRCA1 and PTEN expression by immunohistochemistry (IHC). Expression was correlated with proliferation markers and RAD 51 foci formation in 24 hours biopsies as assessment of HR competence.

Results. BRCA1 expression, assessed by staining intensity and foci formation, increased post-chemotherapy $(\mathrm{p}=0.0019$ and $\mathrm{p}=0.0003$, respectively), suggesting the upregulation of BRCA1 in response to DNA damage. Further, BRCA1 expression was shown to correlate with geminin expression $(\mathrm{r}=0.49, \mathrm{p}=0.0002)$, confirming its $\mathrm{S}-\mathrm{G} 2$ phase cell cycle regulated expression. To investigate the association with RAD51 foci formation, BRCA1 expression was assessed in post-chemotherapy cores, and adjusted for proliferative rate. Cancer subgroups with high proliferation but low BRCA1 expression or low BRCA1 foci formation had reduced $\mathrm{RAD}_{51}$ foci formation $(\mathrm{p}=0.0252$ and $\mathrm{p}=0.0005$, respectively), and both subgroups were more frequently triple-negative breast cancers ( $\mathrm{p}=0.0142$ and $\mathrm{p}=0.0009$, respectively). BRCA1 expression, without considering proliferation, did not associate with impaired RAD 51 foci formation, and PTEN expression was neither associated with proliferation markers nor RAD 51 foci formation.

Conclusions. Cancers with high proliferation and low BRCA1 expression post-chemotherapy are associated with a functional defect in HR and identify a group of patients who may benefit from platinum chemotherapy or PARP inhibitors.

\section{SA-143}

Glutamate enrichment as new diagnostic opportunity in breast cancer

J. Budczies*1, B.M. Pfitzner', B. Györffy' ${ }^{2}$, K.-J. Winzer ${ }^{3}$, C. Radke ${ }^{4}$, M. Dietel', O. Fiehn ${ }^{5}$, C. Denkert ${ }^{1}$

'Institute of Pathology, Charité - University medicine of Berlin, Berlin, Germany, ${ }^{2}$ Research Laboratory of Pediatrics and Nephrology, Hungarian Academy of Sciences, Budapest, Hungary, ${ }^{3}$ Interdisciplinary Breast Center, Charité University Hospital, Berlin, Germany, ${ }^{4}$ Pathology, DRK Hospital Berlin, Berlin, Germany, ${ }^{5}$ Genome Center, University of California, Davis, United States

Aims. Exogenous glutamine is an important source of energy and molecular building blocks for many tumors. There is a renewed interest in therapeutically targeting glutamine metabolism due to the recent discovery of two novel glutaminase inhibitors.

Methods. To quantify the dysregulation of the glutamine-glutamate equilibrium in breast cancer, we analyzed a set of 367 clinical breast cancer and normal breast samples using gas chromatography combined with time-of-flight mass spectrometry.

Results. A positive correlation between glutamine and glutamate in normal breast tissues switched to a negative correlation between glutamine and glutamate in breast cancer tissues. Compared to the level of glutamate/glutamine in normal tissues, we found $56 \%$ of the ER+ tumor tissues and $88 \%$ of the ER- tumors tissues glutamate-enriched. The glutamate/glutamine ratio (GGR) significantly correlated with ER status $(\mathrm{p}=8.0 \mathrm{E}-09)$ and with tumor grade $(\mathrm{p}=3.3 \mathrm{E}-05)$. Higher levels of GGR were associated with shorter overall survival $(\mathrm{HR}=1.30, \mathrm{p}=0.027)$. In a supervised analysis of metabolomics data and of genome-wide expression data, replacement of GGR by metabolite surrogate markers performed very well, while replacement of GGR by RNA markers had a limited accuracy. Functional analysis of the gene expression data showed a negative correlation between glutamate enrichment and activation of peroxisome proliferator-activated receptor (PPAR) pathway.

Conclusions. Our findings may have important implications for patient stratification related to utilization of glutaminase inhibitors. 


\section{SA-144}

Evaluation of breast cancer morphology in PAXgene tissue fixed compared to formalin fixed samples by an international ring trial

C. Viertler ${ }^{* 1}$, P. Verderio' ${ }^{2}$, C.M. Ciniselli2 ${ }^{2}$ S. Gündisch ${ }^{3}$, M. Kap ${ }^{4}$, I. Amendoeira ${ }^{5}$, J. Andreu ${ }^{6}$, M. den Bakker', S. Bianchi', P. Branton ${ }^{8}$, G. Bussolati ${ }^{9}$, G. Callagy ${ }^{10}$, I. Castellano ${ }^{9}$, E. Chmielik' ${ }^{11}$, A. Cordoba' ${ }^{12}$, G. Cserni ${ }^{13}$, C. van Deurzen ${ }^{4}$, M.P. Foschini ${ }^{14}$, S.F. Grazio ${ }^{15}$, J. Kulka ${ }^{16}$, I. Liepniece-Karele ${ }^{17}$, F. Moinfar', A. Reiner ${ }^{18}$, A. Ryska ${ }^{19}$, A. Sapino ${ }^{9}$, F. Tavassoli20 , Z. Varga ${ }^{21}$, V. Zolota ${ }^{22}$, P. Riegman ${ }^{4}$, K.-F. Becker ${ }^{3}$, K. Zatloukal', P. Regitnig'

${ }^{1}$ Institute of Pathology, Medical University, Graz, Austria, ${ }^{2}$ Unit of Medical Statistics, Biometry and Bioinformatics, Fondazione IRCCS, Istituto Nazionale dei Tumori, Milan, Italy, ${ }^{3}$ Institute of Pathology, Technical University of Munich, Munich, Germany, ${ }^{4}$ Department of Pathology, Erasmus Medical Center, Rotterdam, Netherlands, ${ }^{5}$ Institute of Molecular Pathology and Immunology, University of Porto and CHS, Joao, Porto, Portugal, ${ }^{6}$ Department of Pathology, Hospital Parc Taulí, Barcelona, Spain, ${ }^{7}$ Pathological Anatomy Unit, Department of Surgery and Translational Medicine, University of Florence, Florence, Italy, ${ }^{8}$ Biorepositories and Biospecimen Research Branch, National Cancer Institute, Bethesda, MD, United States, ${ }^{9}$ Department of Medical Sciences, University of Turin, Turin, Italy, ${ }^{10} \mathrm{Galway}$ Clinical Science Institute, National University of Ireland, Galway, Ireland, "Department of Pathology, Oncology Center and Cancer Institute, Gliwice, Poland, ${ }^{12 B r e a s t}$ Pathology Section, Complejo Hospital of Navarra, Pamplona, Spain, ${ }^{13}$ Department of Pathology, Bacs-Kiskun County Teaching Hospital, Kecskemet, Hungary, ${ }^{14}$ Department of Biomedical and Neuromotor Sciences, Section of Anatomic Pathology, University of Bologna, Bologna, Italy, ${ }^{15}$ Department of Gynecological Pathology and Cytology, Division of Gynecology University Medical Center Ljubljana, Ljubljana, Slovenia, ${ }^{162}$ nd Department of Pathology, Semmelweis University, Budapest, Hungary, ${ }^{17}$ Pathology Center, Riga East Clinical University Hospital, Riga, Latvia, ${ }^{18}$ Institute of Pathology, Danube Hospital, Sociomedical Center East, Vienna, Austria, ${ }^{19}$ The Fingerland Department of Pathology, Charles University Medical Faculty Hospital, Hradec Kralove, Czech Republic, ${ }^{20}$ Department of Pathology, Yale School of Medicine, New Haven, United States, ${ }^{21}$ Institute of Surgical Pathology, University Hospital Zurich, Zurich, Switzerland, ${ }^{22}$ Department of Pathology, Medical School, University of Patras, Patras, Greece

Aims. Characterization of human disease in modern pathology requires analysis of multiple parameters ranging from histopathology to a broad spectrum of molecular biomarkers. The histomorphological characterization is based on the analysis of formaldehyde-fixed and paraffin-embedded (FFPE) tissues but this is known to hinder molecular analyses compared to frozen tissue samples. Within the European $\mathrm{FP}_{7}$ project "Standardisation and improvement of generic pre-analytical tools and procedures for in-vitro diagnostics" (SPIDIA) we demonstrated the simultaneous high-quality preservation of biomolecules and morphology by a new fixation technology (PAXgene Tissue). The aim of this study is to evaluate the quality of histomorphology and suitability of PAXgene-fixed, paraffin-embedded (PFPE) breast cancer samples for routine diagnostics in comparison to FFPE samples.

Methods. Tissue samples of 18 breast cancer cases were divided into two mirrored samples for PFPE and FFPE. All hematoxylin and eosin stained glass slides were scanned and evaluated for histopathological tumor type, tumor grade, and the quality of morphology and cellular details in a blinded, randomized ring trial study by 23 participants from the European Working Group for Breast Screening Pathology, the European Society of Pathology and the United States National Cancer Institute using virtual microscopy.

Results. Comprehensive statistical analysis showed a high level of reproducibility in tumor grading $(\mathrm{Kwj}=0.92,95 \% \mathrm{CI}$ 0.76-1.00) between PFPE and FFPE samples. The inter-observer reproducibility for grading (and its scoring components) was not completely satisfactory, with grade 2 tumors as the most critical category, but showed a similar performance level in the two randomized groups of participants (median $\mathrm{Kw}$ of 0.67 and 0.64 ). Although substantial retraction artefacts were observed more frequently in PFPE samples, other morphology preservation parameters e.g. the quality of epithelial tumor cells, chromatin structure, cytoplasmic details and the overall quality of morphology were scored better in PFPE than in FFPE samples. Other considered parameters were not significantly altered by the fixation method.

Conclusions. These findings suggest that the PAXgene tissue system facilitates state-of-the-art histopathological analysis, comparable to formalin fixed tissues.

This work received funding from the European project SPIDIA (grant agreement $n^{\circ}$ 222916).

\section{SA-145}

Breast cancer in Ethiopia and Sudan —a different disease compared to breast carcinoma in Germany?

\section{S. Hauptmann*1, A. Omer ${ }^{2}$, T. Wakuma ${ }^{3}$, E. Erichsen ${ }^{3}$, E. Kantelhardt ${ }^{4}$} 'MVZ für Histologie, Zytologie und molekulare Diagnostik Trier, Trier, Germany, ${ }^{2}$ Institute of Pathology, University of Gezira, Wad Medani, Sudan, ${ }^{3}$ Department of Surgery, EECMY Aira Hospital, Aira, Ethiopia, ${ }^{4}$ Dptm for Gynecology, University Hospital Halle, Halle (Saale), Germany

Aims. Breast Cancer is an emerging burden of disease worldwide. Recent surveys show fundamental differences concerning the presentation of the disease in African countries. The aim of this study is to compare a small series of breast carcinomas treated at a remote rural hospital (Aira hospital) in Ethiopia and at the University Hospital in Sudan (Wad Medani) with a similar series of breast carcinomas diagnosed at the University Hospital Halle (Saale).

Methods. We have analyzed 37 cases from Ethiopia, 35 cases from Sudan and 137 cases from Germany. Histological type and grade was evaluated and expression of estrogen receptor, progesterone receptor, HER2 and $\mathrm{Ki}-67$ were analyzed by routine immunohistochemical staining.

Results. The tumors from both Ethiopia and Sudan frequently had a more undifferentiated, blast-like, solid histology than the tumor from Germany. This is reflected by the histological grading in these three cohorts with grade 1 in $5 \%, 18 \%, 25 \%$, grade 2 in $28 \%, 55 \%, 58 \%$, and grade 3 in $67 \%, 27 \%$, and $18 \%$, respectively. The rates of triple negative cases were $15 \%, 41 \%$, and $10 \%$, respectively. HER $23+$ positivity was found in $21 \%$, $25 \%$, and $9 \%$, respectively. The rate of ER- and PR-negativity was also different with $69 \%, 67 \%$, and $15 \%$ of the former, and $55 \%, 82 \%$, and $24 \%$ of the latter. Moreover, African patients present late with T1 tumors in $8 \%$ and $15 \%$ compared to $52 \%$ in Germany. Another intriguing feature is the high proliferation rate observed in the African samples.

Conclusions. Breast cancer in Ethiopia and Sudan presents late, with a high percentage of poorly differentiated, highly proliferative and frequently triple negative tumors, suggesting that their biology is different to tumors occur in Germany.

\section{SA-146}

\section{Aberrant expression of VE-cadherin in breast cancer cells}

M. Großer ${ }^{* 1}$, A. Schmidt', M. Rezaei', A. Klawitter', K. Friedrich', G. Baretton', G. Breier*1

'Institute of Pathology, University Hospital Carl Gustav Carus, Dresden, Germany, ${ }^{2}$ Anatomie, Münster, Germany

Aims. Loss of E-cadherin and gain of N-cadherin are thought to promote tumor progression and tumor cell invasion in carcinomas. We have recently observed aberrant expression of vascular endothelial (VE-) cadherin in a mouse model of breast cancer. In this model, VE-cadherin induction paralleled epithelial-mesenchymal transition and enhanced transforming growth factor-beta signaling in breast cancer cells. Here, we examined the expression of classical cadherins in human breast cancer specimens and studied the interplay of cadherins in cell lines. 
Methods. Tissue microarray. Expression analyses by reverse transcription-polymerase chain reaction (RT-PCR), western blot and immunofluorescence microscopy. RNA interference. Cell migration assays. Tumor experiments.

Results. Vascular endothelial (VE-)cadherin was expressed in 61 of the $79(77 \%)$ of the invasive carcinomas examined. $82 \%$ of the tumors expressed $\mathrm{N}$-cadherin protein, and $62 \%$ of the specimens were positive for both VE- and N-cadherin. Membrane localization of N-cadherin in tumors significantly correlated with smaller tumor size whereas nuclear expression of $\mathrm{N}$-cadherin was observed primarily in poorly differentiated tumors. VE-cadherin expression correlated with HER-2 status and with estrogen receptor expression. Expression of VE-cadherin was detected in various human breast carcinoma cell lines. In several cell lines, co-expression of E-, N- and VE-cadherin was detected. Silencing of VE-cadherin or N-cadherin in murine breast cancer cells led to reduced tumor growth. $\mathrm{N}$-cadherin was required for maintaining VE-cadherin expression and the mesenchymal cell phenotype, whereas VE-cadherin prevented localization of $\mathrm{N}$-cadherin at cell-cell contacts and promoted cell migration and invasion in vitro.

Conclusions. Our findings provide evidence that VE-cadherin and $\mathrm{N}$ cadherin act in concert to promote breast cancer progression.

\section{SA-147}

\section{Characterization of PARP-1 inhibition response in breast cancer cells}

\section{S.B. Bartmann', C. Geisler', C. Henkel', R. Knüchel', J. Veeck*1}

${ }^{1}$ Institute of Pathology, RWTH University, Aachen, Germany, ${ }^{2}$ Ruhr University Bochum, Medical Proteome Center, Bochum, Germany

Aims. PARP inhibitors (PARibs) lead to synthetic lethality in BRCA-associated breast cancer. Despite being effective in an infrequent subset of tumors, knowledge is lacking whether sensitive subsets exist in sporadic breast cancer as well. Also, the rapid acquisition of resistance in BRCAassociated cancer cells needs further investigation of their potential in sporadic breast cancer cells.

Methods. Response to two PARibs (AG14361, olaparib) was characterized in two models, i.e. five breast cancer cell lines of increasing PA$\mathrm{Rib}$ resistance and isogenic breast cancer cells sensitive (UACC 3199 ) or rendered resistant (UACC $3199-R$ ) to AG14361. Upon PARibs, cells were assayed for loss of PARylation and DNA damage response by $\gamma-\mathrm{H}_{2} \mathrm{AX}$ and $\operatorname{Rad}_{51}$ staining. Two-dimensional difference gel electropheresis (2D-DIGE) was applied to isogenic UACC $3199 / \mathrm{R}$ cells to identify proteins associated with acquired PARib resistance.

Results. All tested breast cancer cell lines induced $\gamma-\mathrm{H}_{2} \mathrm{AX}$ foci, demonstrating increased DNA double-strand breaks (DSBs), upon irradiation or treatment with AG14361 and olaparib in concentrations which inhibited total protein PARylation. In PARib sensitive cells only, Rad51 focalization, indicating error-free DSB repair, failed to be induced upon PARib treatment or irradiation while $\operatorname{Rad}_{51}$ foci were induced by those treatments in PARib resistant cells. Resistant UACC $3199-\mathrm{R}$ cells

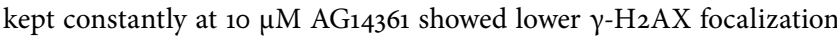
when compared to sensitive UACC 3199 cells at $10 \mu \mathrm{M}$ AG14361. However, Rad51 foci were not inducible in both cells upon treatment. DIGE analysis of the cell line pair preliminarily revealed several differentially expressed proteins involved in DNA damage response (DNA-PKc, Annexin $\mathrm{A} 1$ ), aggressiveness (Vimentin) or stress response (TNFR-associated protein 1). Their protein expression is currently being validated in breast cancer cell lines and explored in primary breast cancer tissues. Conclusions. PARP-1 inhibition leads to DSBs in breast cancer cells, yet cytotoxicity correlates with their failure to repair DSBs by homologous recombination, as indicated by lacking Rad51 foci induction upon PA$\mathrm{Rib}$ treatment. Cells rendered resistant were not increasingly capable of Rad51-mediated DSB repair, indicating that molecular mechanisms of acquired and intrinsic resistance to PARibs may differ. Characterization of differentially expressed proteins will further clarify the molecular mechanism of acquired resistance in originally sensitive, sporadic breast cancer cells.

\section{SA-148}

Lipidomic profiling of oestrogen positive and negative breast cancer cell lines shows distinct differences in the lipid profile

\section{S.F. Brockmoeller*1,2, Z. Áment ${ }^{2}$, P. Kreuzaler ${ }^{2}$, M. Dietel ${ }^{3}$, C. Denkert ${ }^{3}$, J. Griffin ${ }^{2}$}

'Leeds University, Pathology, Leeds, United Kingdom, ²Department of Biochemistry and Cambridge Systems Biology Centre, Department of Biochemistry and Cambridge Systems Biology Centre, University of Cambridge, Cambridge, United Kingdom, ${ }^{3}$ Institute of Pathology, Charité University Hospital, Berlin, Germany

Aims. Alterations in lipid metabolism-specifically in choline metabolism-are an important but poorly characterised, early and universal characteristic of cancer cells. De novo synthesis of fatty acids is heightened in cancer progenitor-(Ackerstaff et al, 2003) cancer cells which makes the pathway attractive as a therapeutic target. The aim of this study was to characterise the changes in the lipidome in oestrogen (ER) positive and negative breast cancer cell lines (Hilvo et al. 2011).

Methods. MCF-7, BT-549 were grown, harvested and extracted as described (ATCC, Ament et al. 2013; Le Belle et al. 2002). The samples were analysed on a Xevo G2 Quadrupole Time-of-flight mass spectrometer and using MassLynx software (Waters Ltd, Elstree, Hertfordshire, UK). Lipids were identified using the MS/MS fragmentation pattern of the specific $\mathrm{m} / \mathrm{z}$ ratio of the single ion result, which was compared with databases (e.g. LIPIDmaps). Multivariate statistical analysis was performed (SIMCA-P 12+ package; Umetrics, Umea, Sweden) and the data mean centred. Pareto scaling and multivariate statistical models were used.

Results. Over 30 different lipids were detected and identified using exact masses from the LC-MS positive mode data. A clear separation was observed between the two cell lines in the PLS-DA model. The following metabolites were significant higher in the MCF-7 cell line: $\mathrm{PE}$ (34:2), PE (36:1), PE (36:2), PE (38:2), PA (36:4), PE (38:2) and during the growth curve: LPC (22:1), PC (27:1) and PC (33:1). The following metabolites showed increases during cell growth for the cell line BT-549: PC (32:0), PC (33:1), PC (34:1), PC (34:2) and PC (36:3).

Conclusions. These results suggest that the $\mathrm{MCF}-7$ cell lines contain more polyunsaturated fatty acids in contrast to BT- 549 were an increase of de novo synthesis of phospholipids was observed. PE (34:2), PE (36:1), $\mathrm{PE}$ (36:2) was increased in the ER + cell lines (Hilvo et al. 2011). The ERcell line (BT-549) showed significant changes in the de novo synthesis of phospholipids during the growth curve including PC (32:0; Hilvo et al. 2011), PC (34:1). This study found that PC (36:2) was increased both during proliferation and in the hormone receptor negative group. These results suggest that de novo lipogenesis could be interesting for target therapies (Rysman et al. 2010).

\section{SA-149}

Expression of different cell cycle regulating proteins in cervical and vulvar squamous cell neoplasms

\section{Leibold ${ }^{* 1}$, A. Cassataro', R. Knüchel', T. Braunschweig ${ }^{2}$}

'Institute of Pathology, RWTH University, Aachen, Germany, ${ }^{2}$ Institute of Pathology, Aachen, Germany

Aims. Cervical epithelial squamous cell neoplasms are generally based on human papilloma virus high-risk (HPV-HR) infections. In vulva it is discussed controversially, but in most cases HPV-HR infections seem to be responsible. Expression levels of the splice variants of CDKN2A p14 and p16 are not yet sufficiently described in cervical and vulvar epithelial neoplasms, expression levels of other cell cycle regulation proteins: p21, p27 and p53 are either not yet characterized properly.

Methods. Immunohistochemical stainings against p14, p16, p21, p27 and p53 were performed on tissue micro arrays containing 100 CIN III samples (mean age: 36 years), 50 invasive cervical squamous cell carcinoma 


\section{Abstracts}

\begin{tabular}{|lllllll}
\hline Tab. 1 & SA-149 & IHC expression levels of cell cycle regulation proteins in cervix and vulvar epithelial neoplasms \\
\hline & Normal Vulva & Normal Cervix & VIN III & CIN III & SCC Vulva & SCC Cervix \\
\hline p16 & 0.37 & 0.03 & 1.07 & 2.85 & 1.24 & 2.76 \\
\hline p14 & 0.04 & 0.00 & 0.13 & 2.71 & 1.02 & 2.65 \\
\hline p21 & 1.43 & 1.75 & 2.83 & 2.73 & 2.75 & 2.75 \\
\hline p27 & 2.08 & 1.92 & 2.55 & 2.26 & 1.75 & 2.27 \\
\hline p53 & 1.17 & 1.88 & 1.93 & 1.20 & 1.96 & 2.21 \\
\hline
\end{tabular}

(SCC; mean age: 53 years), 75 VIN III (mean age 54 years) and 50 invasive vulva SCC (mean age: 66 years).

Results. The provided table (- Tab. 1) shows similar mean values of epithelial expression of p14 and p16 in CIN III and invasive cervical SCC; in VIN III and vulva SCC values are similar, but less intense. In normal epithelium of both sides, values are low. p21 is slightly higher expressed in both invasive carcinoma groups. p27 is in general higher expressed in non invasive lesions, but less present in cervical SCC. p53 shows a higher expression in cervical SCC but in CIN III; vulva lesions are both stronger stained than normal vulva epithelium.

Conclusions. Differences in expression of $\mathrm{p}_{16}$ and 14 in cervical and vulva neoplasms are remarkable. Results with low levels of p16 in vulvar lesions lead to a lower percentage of HPV-HR related neoplasms. Low levels of p14 in VIN III are likely based on the origin of CIN III as it is discussed to originate of metaplastic cylindric epithelium in contrast to original squamous epithelium, as in vulva. Increase of p21 in all lesions seems to be due to an increased p53. p53 or p21 may accumulate as a malor nonfunctional protein or is an effect of insufficient antiregulation. The higher expression of p27 in both pre-invasive neoplasms and invasive cervical carcinoma in respect to vulva carcinoma could be based on AKT regulation. Further evaluation on regulatory mechanisms of p27 is in progress.

\section{SA-150}

Dual-color in situ hybridization for detection of CCNE1/URI amplification in ovarian cancer

\section{A. Noske ${ }^{* 1}$, L.A. Henricksen ${ }^{2}$, B. LaFleur ${ }^{2}$, A.-K. Zimmermann', S. Singh ${ }^{2}$, A. Tubbs' ${ }^{2}$ H. Moch \\ 'Institute of Surgical Pathology, University Hospital, Zurich, Switzerland ${ }^{2}$ Ventana Medical Systems, Tucson, United States}

Aims. CCNE1 and URI are part of the frequent genomic amplification site on $19 q 12$ in epithelial ovarian cancer and both are potential candidates for targeted therapy. Purpose of the study was the establishment of a dual-color in situ hybridization technique for automated detection of CCNE1/URI. CCNE1/ URI status was further compared with clinicopathological factors and patient outcome.

Methods. Development and validation of a novel ISH assay for detection of CCNE1/URI copy numbers and Chromosome 19 as a surrogate on ovarian cancer samples using Ventana BenchMark XT Platform and conventional bright field microscopy. The association of the novel ISH probe was compared with URI amplification status previously assessed by fluorescence ISH (FISH).

Results. The ISH assay was established on a control set $(n=40)$ and full cohort of 136 epithelial ovarian carcinomas. CCNE1/URI amplification was significantly associated with URI amplification status previously assessed by FISH. Shorter survival times were seen in patients with amplified carcinomas.

Conclusions. We show that the novel dual-color ISH technique is feasible to detect CCNE1/URI amplification. This assay may serve to monitor CCNE1/URI amplification status in ovarian cancer patients which may benefit from CCNE1/Cyclin E1 and/or CDK2 targeted therapies.

\section{SA-151}

Incidence and characterization of tubal dysplasia in cases with serous ovarian carcinoma

\section{E. Schmoeckel*1, T. Kirchner ${ }^{1}$, A.Y. Nikitin², D. Mayr ${ }^{1}$}

'Institute of Pathology, Ludwig-Maximilians-University, Munich, Germany, ${ }^{2}$ Department of Biomedical Sciences and Cornell Stem Cell Program, Cornell University, Ithaca, United States

Aims. Fallopian tube fimbria is considered to be among the main places of origin of high-grade serous ovarian carcinoma and primary peritoneal carcinoma. According to this hypothesis, exfoliated cells of tubal intraepithelial carcinoma or occult tubal carcinoma migrate to the ovarian surface where main tumor-mass develops. Recently expression of stem cell markers in ovarian carcinomas has been reported. However, the role of stem cells in pathogenesis of these malignancies remains unclear. In our study we examined cancer-free fallopian tubes with the aim to identify and characterize dysplastic lesions by a panel of stem cell and differentiation-related markers.

Methods. Serial sections of formalin-fixed paraffin-embedded material of 50 patients with ovarian carcinoma and cancer-free uterine (fallopian) tubes were immunohistochemically stained for tumor suppressor proteins $\mathrm{p} 53$ and p16, differentiation markers Pax8 and Wilms-tumor (WT-1), and stem cell markers CD133, CD44 and CD49f. Cell density and location were semiquantitatively evaluated and correlation with survival was performed.

Results. Severe dysplasia characterized by p53-overexpression was detected in 4 out of 50 cases in the tubal fimbria. In contrast, a weak and focal expression of p53 was seen in 35/50 cases. WT-1- and p16-expression were low and lacked any correlation to dysplasia. CD44 was detected in small groups of cells mainly located in the fimbrial part of uterine tube, while CD133 was present mainly in the epithelia of the proximal part. The majority of tubal epithelium cells expressed CD49f. Majority of carcinomas had no or less than $1 \%$ of $\mathrm{CD}_{4} 4$ positive cells. However, 8 cases had areas containing between 15 and $70 \%$ of positive cells. Dysplastic lesions also contained some $\mathrm{CD}_{44}$ positive cells, whereas $\mathrm{CD}_{49} \mathrm{f}$ was present in the majority of neoplastic cells. A substantial proportion of the examined serous ovarian cancer cases showed p53-negativity (17/50), which was associated with a worse survival.

Conclusions. Our study suggests that only a minor fraction of cancers currently regarded as of primary ovarian origin may arise in the fimbriated end of the fallopian tube. $\mathrm{CD}_{44}$ may be a promising marker for detection of cells with stem cell properties present in high-grade serous ovarian carcinoma and in dysplastic tubal epithelium. 


\section{SA-152}

\section{Epithelial-mesenchymal transition (EMT) in vulvar cancer with and} without inguinal lymph node metastases

\section{Eckey ${ }^{* 1}$, M. Höckel' , R. Scherling ${ }^{2}$, B. Hentschel, M.A. ${ }^{3}$, L.-C. Horn ${ }^{* 4}$}

${ }^{1}$ Institute of Pathology, Division of Breast, Gynecologic \& Perinatal Pathology, University of Leipzig, Leipzig, Germany, ${ }^{2}$ Department of Obstetrics and Gynecology, Division of Gynecologic Oncology, University of Leipzig, Leipzig, Germany, ${ }^{3}$ Institute for Medical Informatics, Statistics and Epidemiology, University of Leipzig, Germany, Leipzig, Germany, ${ }^{4}$ Institute of Pathology, Leipzig, Germany

Aims. During process of invasion cancer cells may usurp a normal embryological process, epithelial-mesenchymal transition (EMT), as a means of acquiring migratory capacity (Stewart and Mccluggage 2013). The knowledge of EMT in vulvar cancer is very limited (Rodrigues et al. 2013), especially in comparing cases with and without inguinal metastatic disease.

Methods. 15 cases with and 15 cases without inguinal metastatic disease were obatiend from our vulval cancer registry. Representative tumor block was immunohistochemically satined against vimentin, p53 and cyclin D1. An immunoreactive score (IRS) was calculated by summarizing the percent of positive stained cells and their staining intensity. Staining intensity (SI) was scores as weak, moderate and strong. The percent of positive stained tumor cells at the front of invasion was scored as $0=0 \%$ positive stained tumor cells, $1=1 \%$ positive cells, $2=2-$ $<10 \%$ positive cells, $3=10-<33 \%$ positive cells, $4=33-<66 \%$ positive cells, $5=66-100 \%$ positive cells. The median values of IRS in the group with and without metastatic disease were compared for statistical analyses. The zonal staining comparing the front of invasion and centre of the tumor was evaluated as well. Additionally the pattern of invasion and the grade of peritumoral desmoplastic change (DSR) and peritumoral inflammatory response (PER) was compared within the cases with and without pelvic lymph node involvement.

Results. There was a pronounced staining at the front of invasion within the tumor cells for vimentin and cyclin D1. But there were no differences in IRS when cases with and without inguinal lymph node metastases were compared. The median IRS-values were for vimentin 2.5 for pNo versus 4 for $\mathrm{pN}+(\mathrm{p}=0.87)$, for $\mathrm{p} 536$ for $\mathrm{pNo}$ versus 6.5 for $\mathrm{pN}+$ $\mathrm{p}=0.93$ ) and for cyclin $\mathrm{D}_{1} 5$ for pNo versus 7 for $\mathrm{pN}+(\mathrm{p}>0.05)$. There were no differences in the pattern of invasion (spray-like versus fingerlike) between the cases with and without inguinal lymph node involvement $(\mathrm{p}=0.1)$, as was for DES $(\mathrm{p}=0.1)$ and PER $(\mathrm{p}=0.28)$.

Conclusions. EMT occurs in vulva cancer and may be highlighted by different staining for vimentin, $\mathrm{p} 53$ and cyclin $\mathrm{D}_{1}$ at the front of invasion. There were no differences in EMT and peritumoral stromal remodelling as well as for tumor cell dissociation analysing cases with and without inguinal lymph node metastases.

\section{SA-153}

\section{Ovarian hemangiomas with associated hilus cell hyperplasia}

\section{L.-C. Horn', R. Handzel', C. Göpel', J. Einenkel*2}

${ }^{1}$ Institute of Pathology, Leipzig, Germany, ${ }^{2}$ Department of Obstetrics and Gynecology, Division of Gynecologic Oncology, University of Leipzig, Leipzig, Germany, ${ }^{3}$ Department of Obstetrics and Gynecology, Martin-Luther-University, Halle/Saale, Halle/Saale, Germany

Aims. Vascular tumors are rare in the female genital tract, particularly in the ovary. Lower than 50 cases of true ovarian hemangiomas have been reported (Uppal et al. 2004).

Methods. Here we report three cases.

Results. The first consisted of a 66-year-old woman with the clinical diagnoses of an ovarian cysts, the endometrium was unremarkable. The resection specimen showed a heavily regressive changed luteinised cyst and at hilus of the ovary a capillary hemangioma with associated hilus cell proliferation of $1.4 \mathrm{~cm}$ in largest dimension. The second represented in an 81-year-old women with an hysterectomy 5 years before because of simple endometrial hyperplasia. Now she presented with ovarian cysts and a bilateral BSO was performed. One ovary presented a peritoneal cyst and a $3.5 \mathrm{~cm}$ hemangioma with hilus cell proliferation. The $3 \mathrm{rd}$ case consisted in an 8o-year-old lady with postmenopausal bleeding and suspicion of granulosa cell tumor of the ovary. The D\&C represented an endometrial polyp with simple and atypical endometrial hyperplasia and one ovary a $1.5 \mathrm{~cm}$ hemangioma with hilus cell hyperplasia.

Conclusions. True hemangiomas of the ovary are rare lesions, especially those with associated hilus cell proliferation (Savargoankar et al. 1994). The differential diagnoses include reactive vascular proliferation of the ovarian hilus, steroid cell tumor with prominent vascularisation and angiosarcoma. About $5 \%$ of the cases may be associated with hormonal function and therefore associated with endometrial hyperplasia.

\section{SA-154}

Vulvo-vaginal intestinal/enteric heterotopia-report of two cases

L.-C. Horn ${ }^{* 1}$, U.K. Bauerfeind ${ }^{2,3}$, I. Karbe ${ }^{3}$, R. Handzel ${ }^{4}$, J. Einenkel ${ }^{4}$

${ }^{1}$ Institute of Pathology, Leipzig, Germany, ${ }^{22}$ nd Department of Pathology, Semmelweis University, Budapest, Hungary, ${ }^{3}$ Department of Obstetrics and Gynecology, Martin-Luther-University, Halle/Saale, Halle/Saale, Germany, ${ }^{4}$ Department of Obstetrics and Gynecology, Division of Gynecologic Oncology, University of Leipzig, Leipzig, Germany

Aims. The occurrence of ectopic intestinal/enteric type epithelium at the vulva is a rare entity sometimes mimicking intraepithelial neoplasia or malignant disease.

Methods. Here we report two cases.

Results. The first consisted of an 82-year-old woman with a long standing (10 years) white papillary lesion with some reddish areas at her left labium, extending into the vaginal introitus. The second case occurred in a 30 -year-old women at the vaginal introitus. Biopsies represented colonic-type glandular epithelium with positive immunostaining against CDX-2, $\mathrm{p} 53, \mathrm{CK} 7$ and CEA, whereas staining against estrogen and progesterone receptor, mammoglobin, GCDFP-15 and CK 20 was negative. Conclusions. The occurrence of coelomic-type glandular epithelium at the vulva may represent the result of dysontogenetic replacement of embryologic stem cells which undergo mucinous differentiation. So, the proper diagnostic term may glandular heterotopia. However, some lesions, especially in the proximity to orthotopic vulval glands, may be of metaplastic origin. Immunohistochemical staining patterns (CEA, CK 7 positive, CK 20 negative) indicate an intestinal/enteric phenotype (i.e. intestinal/enteric heterotopia). Because of the reported increased risk of malignant transformation of glandular vulval lesions, close clinical follow is recommended.

\section{SA-155}

Tumor associated macrophages in breast cancer and their potential role in tumor prognosis

H. Hessel ${ }^{* 1}$, J. Neumann', G. Dietmann², H.-J. Müller', T. Jarutat ${ }^{2}$, F. Feuerhake ${ }^{2}$, T. Kirchner ${ }^{1}$

IInstitute of Pathology, Ludwig-Maximilians-University, Munich, Germany, ${ }^{2}$ Roche Pharma, Research and Early Development (pRED), Pathology, Penzberg, Germany

Aims. Tumor associated macrophages (TAM) are relevant components of the tumor microenvironment. Based on the macrophages polarization model TAM can be differentiated in vitro into M1 macrophages with tumoricidal and M2 macrophages with tumorigenic capacity. In this study we investigated in vivo the prognostic relevance of M2-like, CD68+CD163+ macrophages within breast cancer specimens including 
several histopathological subtypes by comparative analyses of TAM, tumor vascularization and histopathological diagnosis parameters. Methods. Analyses were performed on formalin-fixed paraffin-embedded tissue of 74 breast cancer specimens composed of 57 invasive ductal and 10 invasive lobular carcinomas. 20 of all carcinomas showed a triple-negative phenotype. M1-like and M2-like macrophages were characterized by automated immunohistochemical (IHC) duplex staining for CD68 and CD163. The macrophages expression pattern was determined by the degree of overall macrophages and M2-like macrophages. Additionally, all tumor samples were characterized with regard to the degree of vascularization by $\mathrm{CD}_{34}$ IHC staining. Nonparametric statistical analyses were done to analyze the correlation between the number of overall macrophages/M2-like macrophages, tumor vascularization and various histopathological parameters (tumor grading, tumor size, lymph node status, tumor staging). Fisher's exact test of independence was applied using SPSS software version 21.

Results. Statistical analyses revealed a significant association between the increased number of overall macrophages and high tumor grading $(\mathrm{p}=\mathrm{0.045})$. Furthermore, we could demonstrate a strong correlation between the number of M2-like macrophages and the degree of tumor vascularization $(\mathrm{p}<0.0005)$. Comparative analyses of different histopathological subtypes revealed an increased quantity of overall macrophages at invasive ductal breast cancer patients in contrast to invasive lobular breast cancer patients $(\mathrm{p}=0.002)$. Additionally, triple negative breast cancer specimen showed a significant higher level of overall macrophages as well as M2-like macrophages compared to non triple negative breast cancer specimens $(\mathrm{p}=0.002$ respective $\mathrm{p}=0.014)$.

Conclusions. In breast cancer the overall quantity of macrophages as well as the M2-like macrophages subpopulation correlate with variable prognostic parameters suggesting macrophages typing/subtyping could serve as additional prognostic parameter in this indication.

\section{AG Paidopathologie Postersession}

\section{SA-156}

23-month-old boy with monomorphic PTLD after liver transplantation-a case report

\section{Sändig*1, M. Siekmeyer', L.-C. Horn', C. Wickenhauser ${ }^{1}$ IInstitute of Pathology, University Hospital, Leipzig, Germany, ${ }^{2}$ Hospital for Children and Adolescents, University of Leipzig, Leipzig, Germany}

Aims. Introduction: Post-transplant lymphoproliferative disorders (PTLD) develop as a consequence of immunosuppression in a recipient of a solid organ or a bone marrow/stem cell allograft. They comprise a spectrum ranging from clearly Epstein-Barr virus (EBV)-driven infectious mononucleosis-like polyclonal proliferations to EBV- positive or EBV-negative proliferations resembling a subset of B-cell lymphoma in immunocompetent individuals. In childhood, PTLD has most frequently been reported after kidney-, liver- or intestinal transplantation as well as hematopoietic stem cell transplantation.

Methods. Case report.

Results. Here, we report a case of a 23-month-old boy who received a liver transplantation due to extrahepatic biliary atresia. Seven months af ter transplantation the patient shortly developed a rapid increasing dysfunction of his liver graft and finally he died by multiorgan failure. By autopsy we found an EBV-associated monomorphic PTLD, presented as a diffuse large B-cell lymphoma, with multiple organ involvement. Conclusions. PTLD may be a severe complication of organ transplantation in childhood. Due to the inevitable necessary severe immunosuppressive therapy EBV reactivation and/or EBV infection leads to B-cell proliferation and finally to autonomous B-cell neoplasia. As symptoms of PTLD are not characteristic diagnosis may be hampered. On the other hand reduction of immunosuppressive therapy combined by rituxi- mab therapy may be lead to cure at least in EBV-driven polymorphous PTLD. In the present case PTLD was not diagnosed until autopsy was performed.

\section{SA-157 \\ Congenital immature teratoma of the nasopharynx with intra- cranial extension-a case report and review of the literature}

\section{S. Opitz*1, C. Horn' ${ }^{2}$, H. Stephan ${ }^{3}$, R. Faber}

'pathology, medicine, university of Leipzig, Leipzig, Germany, ${ }^{2}$ Institute of Pathology, Dept. of Breast, Gynecologic and Perinatal Pathology, University Hospital, Dept. of Breast, Gynecologic and Perinatal Pathology, Leipzig, Germany, ${ }^{3}$ Division of Perinatal Pathology, and Department of Obstetric \& Gynecology, Division of Perinatal Diagnostics, University Hospital, Leipzig, Germany, ${ }^{4}$ Centre of Prenatal Medicine, Leipzig, Germany

Aims. Teratoma is the predominant histologic subtype of fetal and neonatal germ cell tumors. They are divided in gonadal or extragonadal lesions. Overall, the sacrococcygeal region is the most common site of origin in the newborn period, accounting for more than $50 \%$. In contrast naso/oropharyngeal teratomas are exceptionally rare (2-3\%). Histologically, the majority represents mature teratomatous lesions. Here we report a stillborn fetus with a congenital immature teratoma grad 3 of the nasopharynx with an intracranial extension.

Methods. Case report: A 38-year-old woman, gravida 4, para 3 presented at the 26.5th weeks of gestation after ultrasound screening because of cranio-facial dysmorphia and severe polyhydramnion. Results of specialized ultrasound examination revealed an extra- and intracorporal teratoma originating most likely from naso-ethmoidal region. Due to complications of severe polyhydramnion the patient went into labour prematurely and delivered a stillborn female fetus.

Results. Fetal autopsy showed a nasopharyngeal immature teratoma (grade 3) with a maximum diameter of $21 \mathrm{~cm}$ and intracranial extension together with facial dysmorphia without additional malformations.

Conclusions. Immature teratoma of the naso/oropharynx with intracranial extension is a very rare lesion. To the best of our knowledge, there are less than ten reported cases in the literature. In the present case, the teratoma was associated with a severe polyhydramnion and consecutive cardiac decompensation of the fetus. There were no metastatic deposits.

\section{SA-158}

Twin pregnancy with a male "early mole" and co-existent female fetus. A case report and review of the literature

S. Scheil-Bertram ${ }^{* 1}$, D. Mosch', K. Hauptmann ${ }^{3}$, T. Fink', A. Fisseler-Eckhoff' IInstitute of Pathology and Cytology, Wiesbaden, Germany, ${ }^{2}$ Dept. Gynecology \& Obstetrics, Bad Soden, Taunus, Germany, ${ }^{3}$ Institute of Pathology, Charité - University medicine of Berlin, Berlin, Germany

Aims. Early mole and co-existent fetus is rarely reported in the literature. In general, a hydatidiform mole co-existing with a fetus is estimated with an incidence of one in 22,000-100,00o pregnancies (Vimercati et al. 2013). Twin pregnancies with a complete hydatidiform mole and coexistent fetus are generally diagnosed between the 26 th and 40 th week of gestation (for review see Piura et al. 2008).

Methods. A 39-year-old patient was referred to us at 9 weeks of gestation due to abnormal ultrasound and a question of gestational trophoblastic disease. Prior pregnancies were normal. Liver biochemical parameters were within normal range. The patient had no chemotherapies before hysterectomy. Chemotherapy followed surgery because of persisting elevated beta-hCG. After chemotherapy, $\beta$-hCG was within normal range.

Results. Hysterectomy was performed. The pathological and molecular examination revealed a twin pregnancy with a male complete hydatidiform mole ("early" mole) with tumorous mass of $12 \mathrm{~cm}$ diameter and 


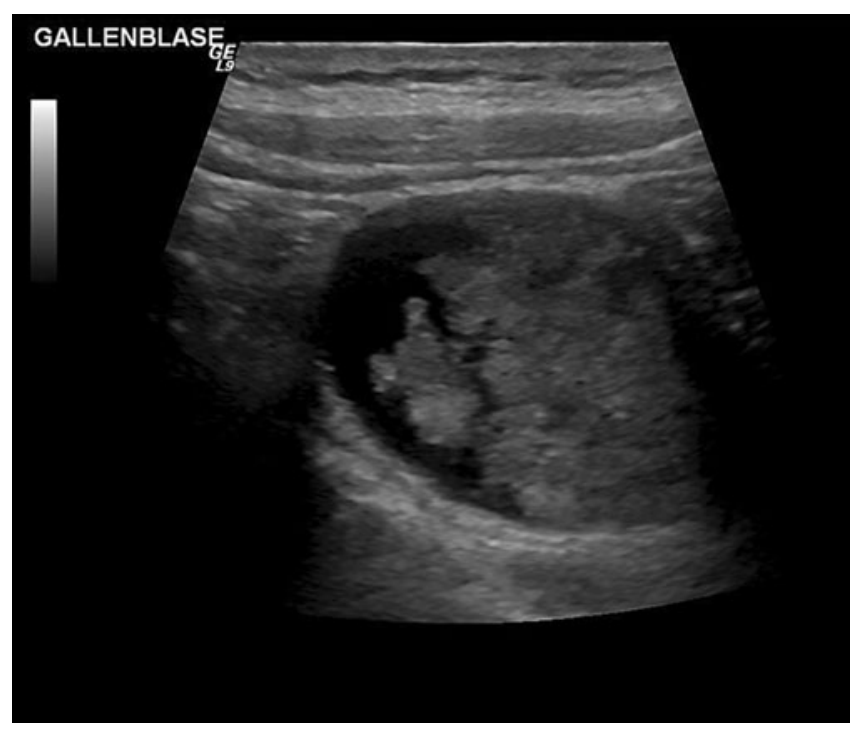

Fig. 1 | SA-159 $\Delta$ Ultrasonic image of a papillary gallbladder tumor

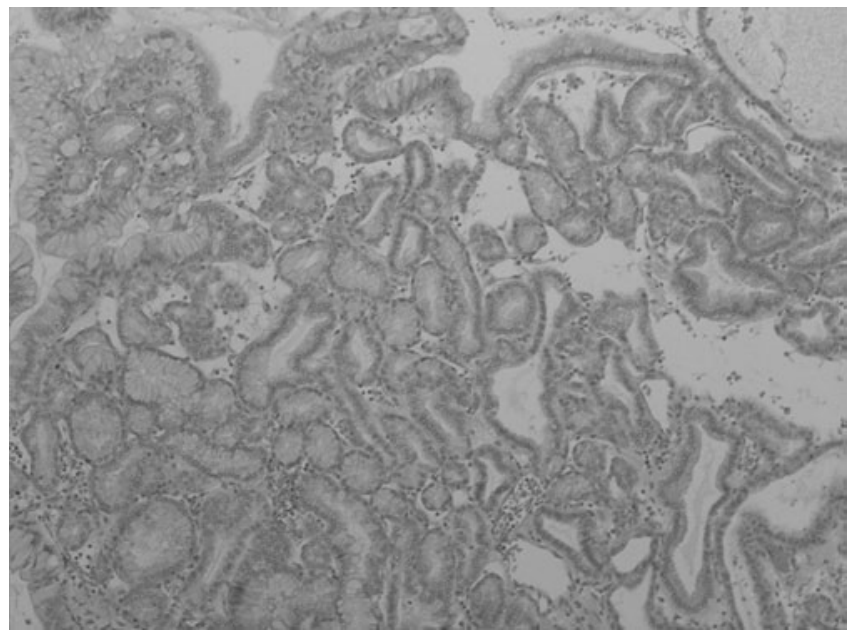

Fig. 2 | SA-159 $\Delta$ Pyloric gland adenoma

a co-existent female fetus (amniotic sac with fetus: $5 \mathrm{~cm}$ diameter). We diagnosed an invasive complete hydatidiform mole.

Conclusions. Our case represented the case of a twin gestation with a complete hydatidiform mole and co-existent fetus diagnosed in the 9 th week of gestation. Because of the early diagnosis the prognosis is favourable/low-risk situation.

\section{SA-159}

\section{Case report: pyoloric gland adenoma of the gallbladder in a 14-year-old boy}

B. Märkl*1, M. Frühwald², A. Gnekow', K. Vollert ${ }^{3}$, T. Schuster', G. Klöppel', H. Arnholdt ${ }^{1}$

IInstitute of Pathology, Augsburg, Germany, ${ }^{2}$ Children's Hospital of Augsburg, Augsburg, Germany, ${ }^{3}$ Department of Radiology, Medical Center, Augsburg, Germany, ${ }^{4}$ Klinikum rechts der Isar München, München, Germany

Aims. Gallbladder polyps of any cause are exceedingly rare lesions in children. Basically, they can be classified into two groups: tumorous and non-tumorous. Inflammatory and cholesterol polyps are the main non-tumorous entities. Tumorous polyps consist of pseudotumors, adenoma and carcinoma

Methods. Case report: We report the case of 14-year-old boy who was under clinical surveillance due to aplasia of the left kidney and a congenital heart anomaly. Abdominal ultrasound revealed an asymptomatic gallbladder polyp (Fig. 1). The haematological investigations and liver function tests were normal. Due to the large size of the polyp cholecystectomy was performed.

Results. Macroscopically, the specimen showed a sessile polyp with a diameter of $4.5 \mathrm{~cm}$. The mucosa in the surrounding of the lesion was normal. The histologic evaluation revealed a papillary and small-cystic lesion with densely packed glandular structures and intestinal differentiation. The mucin producing cells demonstrated sometimes the typical morphology of goblet cells but no atypia. Immunohistochemically the cells expressed MUC5 and MUC6 and did not express MUC1 and MUC2 which confirmed the diagnosis of a pyloric gland adenoma (Fig. 2). After a follow up of 42 month clinical and radiological investigations revealed no pathologic findings in the abdomen.

Conclusions. Gallbladder polyps are rare findings in childhood. In this case, the lesion showed worrisome radiological features leading to cholecystectomy. The final diagnosis of a pyloric gland adenoma has been confirmed by immunophenotyping. Until now the patient is recurrence and symptom free (- Fig. 1, 2). 


\section{Autorenindex}

A

Affolter, A.

Agaimy, A.

Ahls, M.G.

Ahrens, T.D.

Anagnostopoulos, I.

Andreou, D.

Andrulis, M.

Arens, N.

Aumann, K.

Awan, S.

B

Bachmann, L.

Bartels, $S$.

Barthelmeß, S.

Bartmann, S.B.

Bauer, R.

Bauer, U.

Baumhoer, D.

Behnes, C.L.

Behrens, H.-M.

Berezowska, S.

Berger, J.

Bergmann, F.

Berndt, A.

Bertz, S.

Beyer, K.

Bierhoff, E.

Birkenmeier, K.

Bob, R.

Böge, Y.

Böger, $C$.

Böhm, F.

Böhm, M.

Bohnenberger, $\mathrm{H}$.

Borchert, A.

Bovée, J.

Braun, M.

Bremmer, F.

Brobeil, A.

Brochhausen, $C$.

Brockmeyer, P.

Brockmoeller, S.F.

Bronsert, $\mathrm{P}$.

Bubendorf, L.

Budczies, J.

Bure, I.

\section{C}

Calvisi, D.

Casadonte, R.

Cernat, L.

Chen, J.
DO-056

DO-061, SO-005,

SO-048, SO-053,

SO-055, FR-010,

FR-K061

FR.1-012

DO-002, FR-005

DO-019

SA-016

FR.1-024

SA-111

SO-036

SO-032

FR-033

DO-022, SA-109

DO-078

SA-147

SA-120

SO-017

SA-017

DO-092

FR-004

FR-046

DO-039

FR.1-017

DO-057

SA-134

FR-019

SO-004

DO-034

FR.1-029

FR.2-055

DO-003

FR.2-056

DO-076

DO-071

FR.1-023

SA-K015

SA-102

DO-094, SA-131

DO-023

FR-045, FR.1-037,

SA-008, SA-012

DO-054

SO-035, SA-148

DO-009, SA-075

FR-K071

FR.1-036, SA-143

FR-008

DO-010, FR.1-003

DO-067, SA-104

DO-015

SO-068
Chen, Y.

Christgen, M.

Clauditz, T.

Csanadi, A.

SO-041, FR-040

FR-055

SA-097

FR-041, FR.1-042

D

Dagher, G. $\quad$ FR-074

Dahl, E. FR-073

Dapunt, U. SA-001

Darb-Esfahani, S. FR-056

Decker, J. SO-071

Demes, M. SA-114

Deml, K.-F.

Diamantis-

Karamitopoulou, E. DO-001

Dietrich, D. SA-077

Döllinger, $C . \quad$ DO-052

Döring, B. SA-140

\section{E}

Eckey, C.

Ehling, J.

El-Baba, C.

Elgohary, N.

Ellert, E.

Endhardt, K.

Eppler, E.

Erlenbach-Wünsch, K.

SA-152

DO-012

DO-072

FR.1-019

DO-027

FR.1-031

FR.1-026

FR-030, FR-032

F

Fassunke, J.

Feld, F.M.

Fichter, C.D.

Flechtenmacher, $\mathrm{C}$.

Focke, C.

Franz, M.

Frauenschläger, $\mathrm{K}$.

Friedrich, $\mathrm{K}$.

Friemel, J.

Fröhlich, J.A.

Fukayama, M.

SA-073

FR.1-013

FR-006

DO-065

SO-024, SO-030

FR.1-038

FR-069

SO-034

FR-018

DO-059

FR-K052

\section{G}

Gaida, M.M.

Gaisa, N.T.

FR.2-053

Gajda, M.

DO-096

SA-135

Gassler, N.

Gdynia, G.

Gebauer, N.

Geddert, $\mathrm{H}$.

Genitsch, V.

Gevensleben, $\mathrm{H}$.

Gilg, M.M.

Goeppert, B.

Grass, A.

Grob, T.

$\begin{array}{ll}\text { Großer, M. } & \text { SA-146 } \\ \text { Gruber, K. } & \text { SA-087 } \\ \text { Gröschl, B. } & \text { FR-029 } \\ \text { Grünewald, I. } & \text { FR.1-032, FR.1-033, } \\ & \text { FR.1-034 } \\ \text { Grünmüller, L. } & \text { SO-007 } \\ \text { Gsponer, J. } & \text { DO-090 } \\ \text { Gündisch, S. } & \text { SA-099 }\end{array}$

H

Haberlau, S. DO-088

Häberle, L. FR-070

Hagedorn, S. DO-073

Hager, T. SO-014

Haller, F. FR-011, FR-047

Hartmann, S. DO-035, SA-011

Hashani, M. DO-068

Hauptmann, S. SO-023, SA-145

Hermanns-Sachweh, B. SO-050

Hermeking, $\mathrm{H}$. SO-065

Herzog, M. FR.1-047

Hessel, $\mathrm{H}$. SA-155

Heublein, S. SO-022

Heukamp, L. FR-072

Heydt, C. SA-071

$\mathrm{Heß,K}$. SA-118

Hilbert, E. SO-011

Höflich, H. FR.1-041

Höflmayer, D. FR.1-043

Hoffmann, W. SA-021

Holmes, E.E. SA-112

Hopf, F. SA.003

Horn, $\mathrm{H}$. DO-032

Horn, L.-C. SA-153, SA-154

Huss, $\mathrm{S}$.

Hwang, S.J.

SA-081, SA-082

DO-K053

I

Ihrler, S. DO-058

Ivanovska, J. DO-079

Izykowski, N. FR-038

\section{J}

Jaitner, S. SA-110

Jeon, M.K. DO-075

Jiang, $X$. FR.2-064

Jonigk, D. SA-013

Jung, $M$. SA-108

Jurmeister, $\mathrm{P}$. FR-037

Juskevicius, D. SA-101

Jäkel, J. FR.1-048

K

Kampmann, E. FR-065

Kantonen, J. FR-028

Kapsner, L. SA-093 


\begin{tabular}{|c|c|c|c|c|c|}
\hline Kayser, G. & DO-038 & Metzig, M. & FR.2-058 & Renner, M. & FR-067 \\
\hline Keck, B. & DO-095 & Moghaddam, N. & SA.2-005 & Ribback, S. & DO-037, FR.1-002, \\
\hline Kilic, E. & SA-130 & Mogler, C. & FR.1-005 & & SA-129 \\
\hline Kiss, A. & SO-060 & Mohammed, A. & SA-007 & Richter, G. & SO-001 \\
\hline Kovalszky, I. & SO-062 & Montenegro, S. & SA-072 & Ringli, D. & SA-124 \\
\hline Klauschen, F. & SO-031, FR-058 & Montgomery, E. & SO-057 & Röcken, C. & FR-K056 \\
\hline Klebe, $S$. & SO-046, SO-047 & Morawietz, L. & SO-054 & Rogler, A. & SA-132 \\
\hline Klempert, I. & DO-047 & Moskalev, E. & DO- 005, SO-039 & Rose, M. & DO-098 \\
\hline Klieser, E. & FR-017 & Muckenhuber, A. & DO-008 & Roth, A. & SA-115 \\
\hline Kloth, M. & SA-074 & Muders, M. & SA-086 & Ruiz, C. & SA-076 \\
\hline Knas, T. & DO-020 & Müller, A.M. & SO-010, SO-012 & Rüegger, C. & SO-018 \\
\hline Knisely, A. & so-020 & Müller, S. & SA-004 & Rüschoff, J. & DO-070, SA-116 \\
\hline Knösel, T. & FR-K062 & Münst, S. & SO-028 & Rupp, N.J. & SA-141 \\
\hline Kölbel, B. & SA-010 & & & & \\
\hline König, K. & DO-081 & & & & \\
\hline Korsching, E. & DO-043 & $\mathbf{N}$ & & & \\
\hline Kosmidis, P. & DO-030 & Nagel, P.D. & FR.1-028 & $\mathbf{S}$ & \\
\hline Krenn, V. & SA-K024 & Nass, N. & so- 033 & Sändig, l. & SA-156 \\
\hline Kriegsmann, J. & FR.2-060, SA-K025 & Neuber, S. & SA-103 & Salah, F.S. & SA-088 \\
\hline Kriegsmann, M. & SO-052, FR.1-006, & Neumann, J. & DO-014, FR-021 & Schaaf, C. & DO-006 \\
\hline & FR.1-009, FR.2-063 & Niebel, D. & FR.1-025 & Scheil-Bertram, S. & SA-158, SA-018 \\
\hline Kristiansen, G. & DO-091 & Noske, A. & SA-150 & Schierle, $\mathrm{K}$. & FR-012 \\
\hline Kubick, C. & FR.1-030 & Nowak, M. & DO-086, DO-087 & Schilling, J. & SA.002 \\
\hline Küffer, S. & SA-090 & Nusshold, E. & SO-009 & Schirmacher, P. & FR-K051 \\
\hline Künstlinger, $\mathrm{H}$. & DO-084 & & & Schlageter, M. & FR.1-004 \\
\hline Kumm, N. & SA-092 & & & Schlitter, A.M. & FR.1-014, FR.1-016 \\
\hline Kunze, K. & DO-013 & 0 & & Schmidt, L. & FR.1-050 \\
\hline & & Offermann, A. & DO-062, DO-089 & Schmitt, S. & FR.1-046 \\
\hline & & Opitz, S. & SA-157 & Schmitt, V.H. & FR-015 \\
\hline $\mathbf{L}$ & & Ormanns, S. & FR-024 & Schmitt, W.D. & DO-049 \\
\hline Lamprecht, S. & FR-023 & Otto W. & DO-097 SA-128 & Schmoeckel, E. & SA-151 \\
\hline Lasitschka, F. & DO-048, SO-042, & otto, w. & DU-09/, SA-128 & Schneider, $\mathrm{H}$. & FR-022 \\
\hline & FR.2-049, SO-073 & & & Schneider, N. & FR-001 \\
\hline Leedham, S. & SA-K023 & & & Schneider, R.K. & DO-024 \\
\hline Leibold, C. & SA-149 & $\mathbf{P}$ & & Schneider-Stock, R. & SA-022 \\
\hline Leich, $\mathrm{E}$. & DO-029 & & & Schoner, K. & SO-019 \\
\hline Lennerz, J.K. & FR-057 & Pellegrino, R. & FR.1-007 & Schrader, T. & DO-046 \\
\hline Lenz, P. & DO-016 & Petersen, I. & FR-K060 & Schröder, J.A. & DO-040 \\
\hline Leusmann, P. & FR.1-044 & Pfarr, N. & SA-084 & Schumann, G. & SO-002 \\
\hline Lichtmannegger, I. & FR-003 & Pfister, F. & SA-138 & Schwindenhammer, B. & DO-074 \\
\hline Lin, J. & FR.2-065 & Pfitzner, B.M. & SO-027 & Sievers, E. & SA-085 \\
\hline Lippert, C. & DO-004 & Pflüger, D. & DO-077 & Sinn $P$. & SO-026 \\
\hline Lohmann, C. & SA-020 & Picci, P. & FR-K063 & Sipos, B. & FR-034, SO-059 \\
\hline Lorber, $\mathrm{T}$. & SA-106 & Pincus, M. & SO-015 & Sobottka-Brillout, B. & FR.2-057 \\
\hline Lutz, L. & DO-017 & Pöhlmann, A. & SA-024 & Söder, S. & SA-006, SA-009 \\
\hline & & Poremba, C. & SO-029 & Soldini, D. & FR-035 \\
\hline & & Porubsky, S. & DO-066, SA-014 & Soltermann, A. & so-043 \\
\hline & & Poulsom, R. & SO-058 & Sommer, U. & FR.2-062 \\
\hline M & & Primavesi, F. & FR.1-010 & Sperling, M. & SA-100 \\
\hline Macher-Göppinger, S. & DO-093 & & & Stahl, P.R. & FR.1-015 \\
\hline Magadum, A. & SA-107 & 0 & & Staiger, A.M. & DO-031 \\
\hline Maier, A. & DO-050, DO-051, & & & Steiger, K. & DO-007 \\
\hline & FR.1-045, FR-075 & Quagliata, L. & FR.1-008 & Steinestel, K. & FR-020, FR.1-020 \\
\hline Mairinger, $\mathrm{F}$. & FR-043, FR-044, & Queisser, A. & SA-126 & Steinmann, S. & DO-085 \\
\hline & SA-080 & & & Stenzinger, A. & DO-055 \\
\hline Makovitzky, J. & SO-064 & & & Stiegelbauer, V. & SO-016 \\
\hline Matheis, P. & FR-007 & $\mathbf{R}$ & & Stöhr, C. & SA-137 \\
\hline Mathiak, M. & FR-002 & Rämö, M. & DO-099 & Stöhr, R. & SA-136 \\
\hline May, A.M. & DO-028 & Rau, S. & SA-083 & Straub, B.K. & SO-061 \\
\hline Mechtersheimer, G. & SO-051 & Rau, T.T. & FR-031 & Straub, D. & DO-060 \\
\hline Meder, L. & DO-083 & Rechsteiner, M. & DO-021, DO-080 & Straub, M. & FR-068 \\
\hline Meller, S. & SA-123 & Regauer, $\mathrm{S}$. & FR.2-054, FR.2-061 & Streubel, A. & SA-089 \\
\hline Menter, $\mathrm{T}$. & SO-038, FR-039 & Reis, $\mathrm{H}$. & FR.1-011, SA-125 & Strunk, J.E. & FR.1-027 \\
\hline Merten, L. & FR-009 & Reissig, K. & SO-066 & Stuhlmann-Laeisz, C. & FR.1-022 \\
\hline Merz, C. & FR.2-050 & Ren, $Z$. & FR.2-069 & Süsal, C. & DO-063 \\
\hline Mesteri, I. & DO-018 & Rengstl, B. & DO-036 & Szendröi, M. & SA-019 \\
\hline
\end{tabular}




\section{Autorenindex}

T

Taube, E.T. SO-021

ten Haaf, A. SO-044

Travis, W.D. SO-056

Tschaharganeh, D.F. FR.1-001

Tur, M.K. FR.2-059

Turowski, G. SO-013

Tóth, C. FR-025, FR-026,

SA-091, SO-K063

V

van Ellen, A. $\quad$ FR-027

Varga, Z. SO-025

Venkataramani, V. SA-113

Viertler, C. SA-144

Vlajnic, T. SO-003, SA-121,

SA-122

Vogler, N. FR-014

Vollbrecht, C. $\quad$ DO-082, SA-078

von Laffert, M. SO-040

von Witzleben, A. FR-066

W

Wagner, $\mathrm{F}$. SA-105

Waldburger, N. SO-069

Walluscheck, D. SA-098

Walter, R.F.H. FR-048, SA-094,

SA-095, SA-096

Wang, $\mathrm{M}$. SO-070

Wangerin, $\mathrm{H}$. SA-117

Wardelmann, E. FR-064

Warth, A. SO-037

Wehling, J. SO-045

Weis, C.-A. DO-042

Weissinger, S.E. SO-006

Wen, $\mathrm{H}$. FR.2-067

Wielockx, B. FR.2-052

Winkler, J. FR.2-051

Wittekind, C. SO-049

Wolf, A. FR.1-049

Wolf, $\mathrm{M}$. DO-011

Wolf, $T$ DO-044

Wright, N. FR-059

X

$\begin{array}{ll}\text { Xiu-Wu, B. } & \text { FR.2-068 }\end{array}$

7

Zebrowski, D.C. $\quad$ SA-079

Zerbe, N. DO-041

Zeuschner, P. DO-064, FR.1-039,

Zhang, W. SO-072

Zhong, Q. DO-045

Zimpfer, A. SO-008, FR-042

Zinnal, U. SA-133 